\title{
Cu-Catalyzed C-H Alkenylation of Benzoic Acid and Acrylic Acid Derivatives with Vinyl Boronates
}

\author{
Jian-Jun $\mathrm{Li}^{\dagger}$, Cheng-Gang Wang ${ }^{\dagger}$, Jin-Feng $\mathrm{Yu}^{\dagger}$, Peng Wang ${ }^{*}, \dagger$, , and Jin-Quan $\mathrm{Yu}^{*}, \S$ \\ 'State Key Laboratory of Organometallic Chemistry, Center for Excellence in Molecular Synthesis, Shanghai \\ Institute of Organic Chemistry, CAS 345 Lingling Road, Shanghai 200032, P.R. China \\ ${ }^{*}$ CAS Key Laboratory of Energy Regulation Materials, Shanghai Institute of Organic Chemistry, CAS 345 \\ Lingling Road, Shanghai 200032, P.R. China \\ ${ }^{\S}$ The Scripps Research Institute (TSRI) 10550 North Torrey Pines Road, La Jolla, CA 92037, USA
}

\section{Table of Contents}

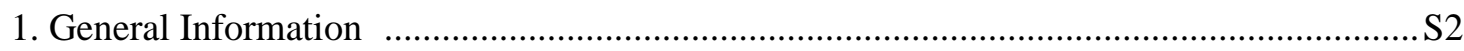

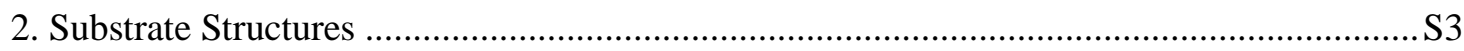

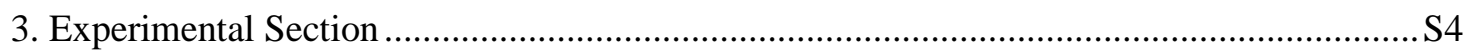

3.1 General Procedure for Preparation of Copper Salts .................................................. S4

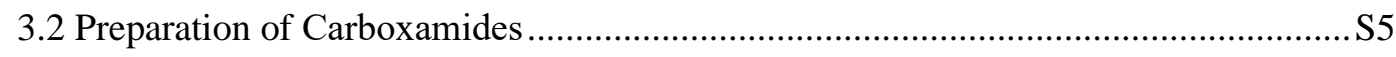

3.3 Preparation of Alkenylboronates........................................................................ 13

3.4 Optimizations of the Reaction Conditions ……..................................................... S16

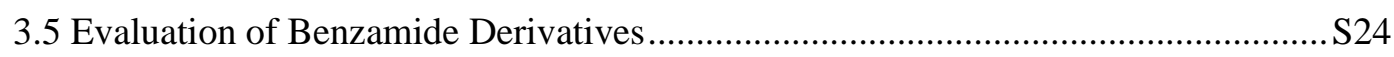

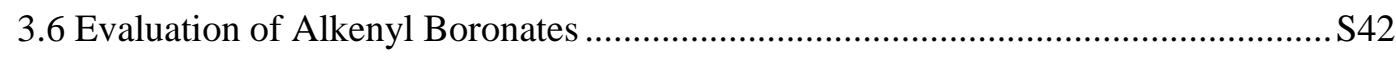

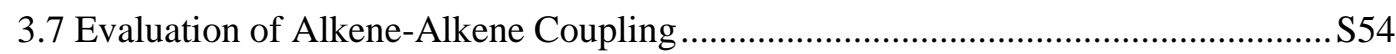

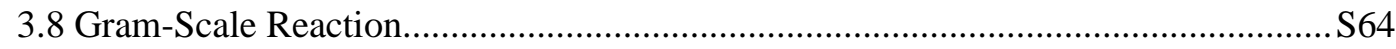

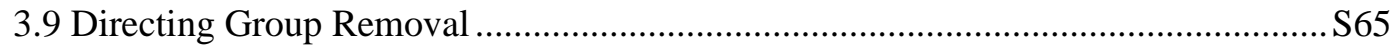

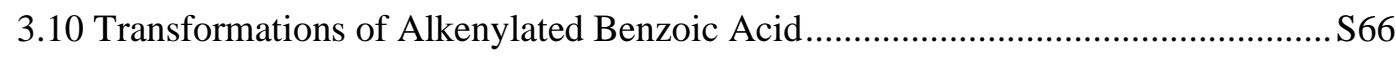

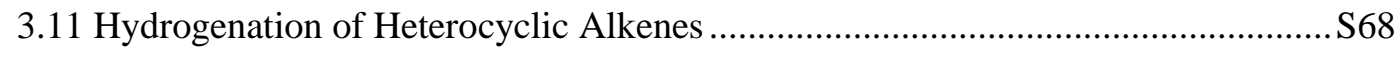

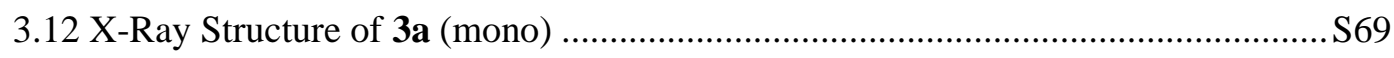

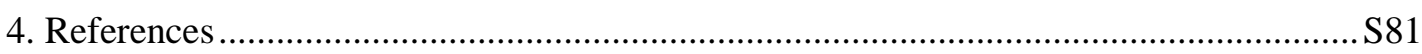

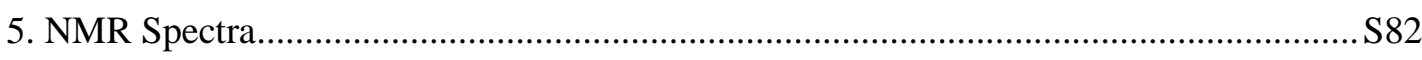




\section{General Information}

All the commercially available chemicals were obtained accordingly. Copper acetate was purchased from Strem. Dimethyl sulfoxide (DMSO) was purchased from J\&K Scientific. Other reagents were purchased from Alfa Aesar, Sigma-Aldrich, Aladdin, Adamas-beta, Bidepharm, Macklin, 9Dingchem, and Energy Chemical of the highest purity grade and used without further purification, unless otherwise indicated. Tetrahydrofuran (THF), acetonitrile $\left(\mathrm{CH}_{3} \mathrm{CN}\right)$, dichloromethane $\left(\mathrm{CH}_{2} \mathrm{Cl}_{2}\right)$ and $N, N$-dimethylformamide (DMF) were dried using the solvent purification system. Other anhydrous solvents were purchased from J\&K Scientific. The extent of reaction was monitored by thin-layer chromatography (TLC), was performed on $0.25 \mathrm{~mm}$ silica gel HSGF254 plates. Visualization was carried out with ultraviolet light $(254 \mathrm{~nm})$ or stained with potassium permanganate followed by gentle heating if necessary. ${ }^{1} \mathrm{H},{ }^{13} \mathrm{C}$, and ${ }^{19} \mathrm{~F}$ NMR spectra were recorded at room temperature on a Varian 400, Bruker 400, Agilent $400\left(400 \mathrm{~Hz}\right.$ for ${ }^{1} \mathrm{H} ; 375 \mathrm{~Hz}$ for ${ }^{19} \mathrm{~F} ; 100 \mathrm{~Hz}$ for $\left.{ }^{13} \mathrm{C}\right)$, Bruker Avance Neo 500 instrument (500 MHz), Bruker Avance Neo 600 instrument (600 MHz), or JEOL JNM-ECZ600R/S1 (600 $\mathrm{MHz}$ ) spectrometer. The chemical shifts $(\delta)$ were quoted in parts per million (ppm) referenced to tetramethylsilane (TMS) (0.0 ppm for $\left.{ }^{1} \mathrm{H} \mathrm{NMR}\right)$ and $\mathrm{CDCl}_{3}\left(77.0 \mathrm{ppm}\right.$ for $\left.{ }^{13} \mathrm{C} \mathrm{NMR}\right)$. The following abbreviations were used to explain multiplicities: $\mathrm{s}=$ singlet, $\mathrm{d}=$ doublet, $\mathrm{t}=$ triplet, $\mathrm{q}=$ quartet, $\mathrm{m}=$ multiplet, br = broad, and coupling constants $(\mathrm{J} \mathrm{Hz}) .{ }^{13} \mathrm{C}$ NMR spectra were fully decoupled by broad band proton decoupling. High-resolution mass spectra (HRMS) were recorded on an Agilent Mass spectrometer using ESITOF or CI/EI. 


\section{Substrate Structures}

\section{(Hetero)Arene Carboxamides}

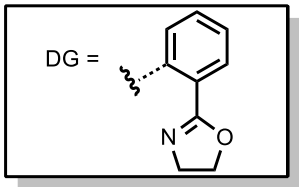<smiles>O=C(NC(=O)c1ccccc1)c1ccccc1</smiles>

$1 \mathrm{a}$<smiles>Cc1ccc(C(=O)N[O+][Na])cc1</smiles>

$1 b$

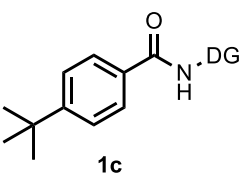

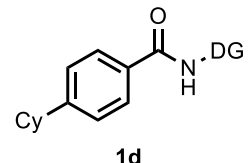<smiles>C=Cc1ccc(C(=O)N[N+](=O)[O-])cc1</smiles>

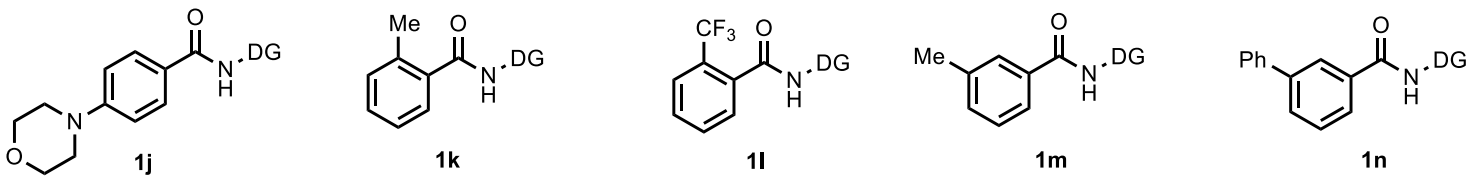<smiles>COc1cccc(C(=O)NNC(=O)c2cccc(OC(F)(F)F)c2)c1</smiles><smiles>O=C(N[O+])c1ccc2c(c1)OCCO2</smiles><smiles>O=C(NO)c1ccncc1</smiles><smiles>[13CH3]NC(=O)c1cccs1</smiles>

$1 \mathrm{v}$<smiles>O=C(N[O+])c1ccc(-c2ccccn2)cc1</smiles>

Vinyl Carboxamides

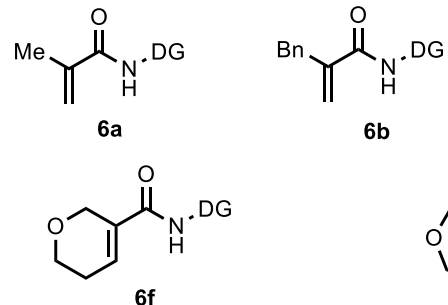<smiles>C/C(=C\c1ccccc1)C(=O)N[O+]</smiles><smiles>O=C(N[O+])C1=CCCCC1</smiles><smiles></smiles><smiles>O=C(NO)C1=CCCOC1</smiles>

$6 c$

$6 d$<smiles></smiles>

$6 \mathrm{~g}$<smiles>O=C(N[O+])C1=CCN(Cc2ccccc2)CC1</smiles>

$6 \mathrm{~h}$

$6 i$

Alkenyl Boronates
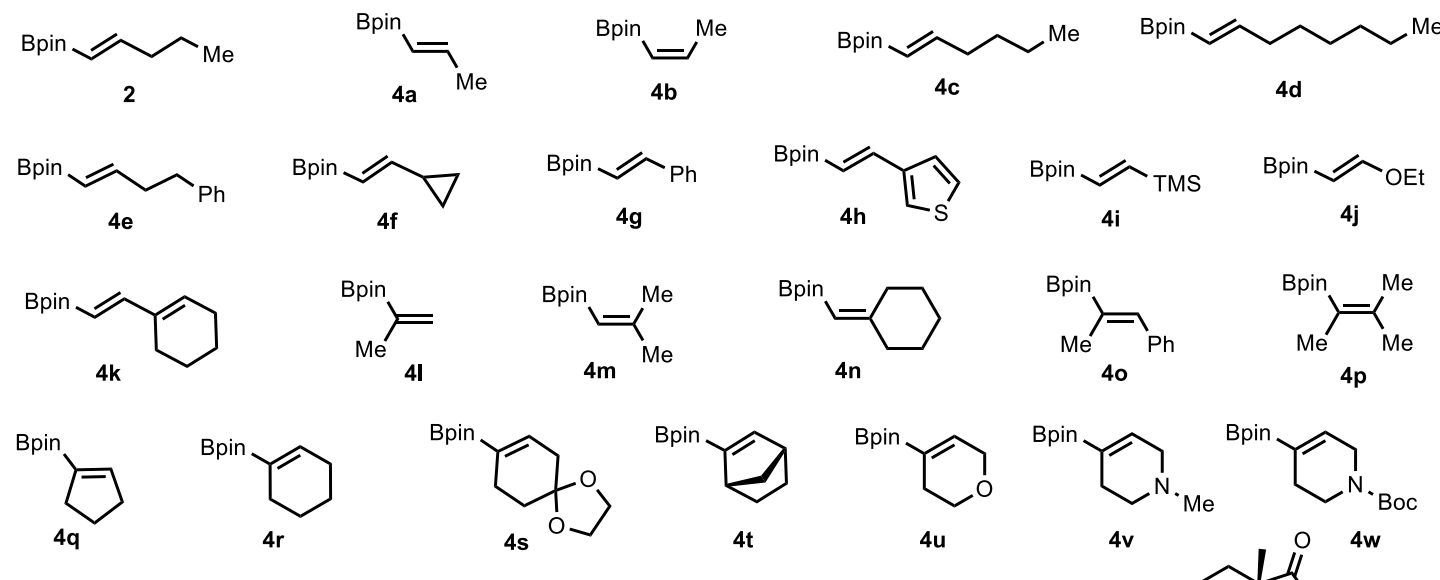

Bpin

4x Boc<smiles>Brc1ccccc1</smiles>

$4 y$<smiles>[C+]C1=CC2CC(C1)[NH+]2</smiles>

$4 z$
(Npin) 


\section{Experimental Section}

\subsection{General Procedure for Preparation of Copper Salts}

Copper(II) benzoate was synthesized following literature procedure. ${ }^{1}$

$$
\mathrm{Cu}(\mathrm{OH})_{2}+\mathrm{PhCO}_{2} \mathrm{H} \underset{\text { reflux }}{\stackrel{\mathrm{EtOH}}{\longrightarrow}} \mathrm{Cu}\left(\mathrm{PhCO}_{2}\right)_{2}
$$

An oven dried $100 \mathrm{~mL}$ sealed tube charged with $\mathrm{Cu}(\mathrm{OH})_{2}(2.93 \mathrm{~g}, 30 \mathrm{mmol}), \mathrm{PhCO}_{2} \mathrm{H}(7.69 \mathrm{~g}, 63 \mathrm{mmol}), \mathrm{EtOH}$ $(30 \mathrm{~mL})$, and the reaction mixture was placed into a pre-heated oil bath and reflux for $24 \mathrm{~h}$. The reaction mixture was filtered immediately, and the filter cake was washed with hot EtOH. The filtrate was concentrated under reduced pressure, and the resulting residue was recrystallized in the small amount of EtOH to afford $\mathrm{Cu}\left(\mathrm{PhCO}_{2}\right)_{2}$ as a light-blue crystalline solid $(5.58 \mathrm{~g}, 61 \%)$. 


\subsection{Preparation of Carboxamides}
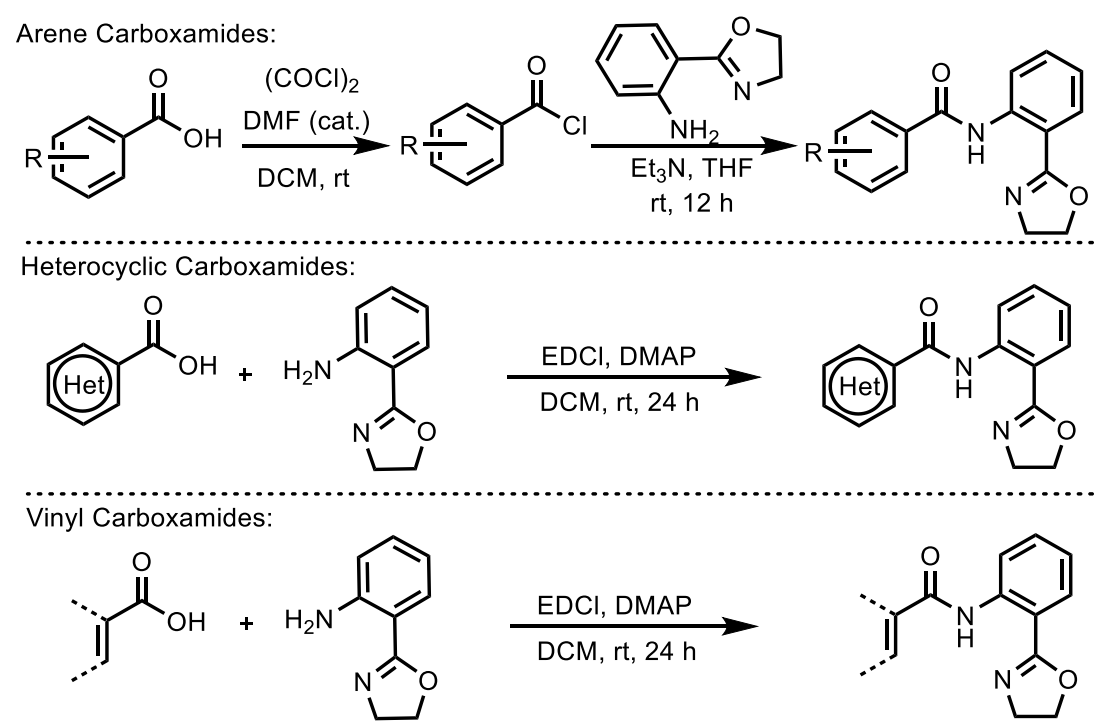

The benzoic acid substrates were synthesized following known procedure. ${ }^{2} \mathbf{1 a - 1 c}, \mathbf{1 f - 1 g}, \mathbf{1 k - 1 m}, \mathbf{1 o}, \mathbf{1 q}$, and 1u-w are known in literature.

\section{General Procedure for Synthesis of Arene Carboxamides:}

To a solution 2-(4,5-dihydrooxazol-2-yl)aniline $(5.0 \mathrm{mmol}), \mathrm{Et}_{3} \mathrm{~N}(7.5 \mathrm{mmol})$ in THF $(20 \mathrm{~mL})$ was slowly added the tetrahydrofuran solution of acid chloride $(5.0 \mathrm{mmol})$, prepared from the corresponding carboxylic acid and oxalyl chloride, under nitrogen at room temperature. The reaction mixture was stirred at room temperature for $12 \mathrm{~h}$. Upon completion, the reaction was quenched with saturated $\mathrm{NaHCO}_{3}(100 \mathrm{~mL})$ and extracted with EtOAc $(3 \times 150 \mathrm{~mL})$. The combined organic layers were washed with saturated brine, dried over $\mathrm{Na}_{2} \mathrm{SO}_{4}$, concentrated under vacuum. The crude product was purified by column chromatography on silica gel with a gradient eluent of petroleum ether/ethyl acetate or recrystallized from petroleum ether/ethyl acetate to provide the desired product (1d-e, 1h-j, 1n, 1p, 1r-t).

\section{General Procedure for Synthesis of Heterocyclic Carboxamides:}

To a solution of heterocyclic carboxylic acid $(4.0 \mathrm{mmol}), 2-(4,5$-dihydrooxazol-2-yl)aniline $(5.0 \mathrm{mmol})$ and DMAP $(0.5 \mathrm{mmol})$ in DCM $(20 \mathrm{~mL})$ was added EDCI $(6.0 \mathrm{mmol})$ under nitrogen at $0{ }^{\circ} \mathrm{C}$. The mixture was stirred for $24 \mathrm{~h}$ at room temperature. Saturated $\mathrm{NaHCO}_{3}$ was added to quench reaction, and then extracted with EtOAc $(3 \times 100 \mathrm{~mL})$. The combined organic layers were washed with saturated brine, dried over $\mathrm{Na}_{2} \mathrm{SO}_{4}$, concentrated under reduced pressure. The crude product was recrystallized from petroleum ether/ethyl acetate or isolated by column chromatography on silica gel with a gradient eluent of petroleum ether/ethyl acetate or recrystallized from petroleum ether/ethyl acetate to provide the desired product $(\mathbf{1} \mathbf{u}-\mathbf{w})$.<smiles>O=C(Nc1ccccc1C1=NCCO1)c1ccc(Cl)cc1</smiles>

4-Cyclohexyl- $N$-(2-(4,5-dihydrooxazol-2-yl)phenyl)benzamide (1d) 
1d was obtained by recrystallization from petroleum ether/ethyl acetate (50/1) as white solid (450 $\mathrm{mg}, 65 \%)$. mp 164-166 ${ }^{\circ} \mathrm{C} ;{ }^{1} \mathrm{H}$ NMR $\left(400 \mathrm{MHz}, \mathrm{CDCl}_{3}\right) \delta 12.96(\mathrm{~s}, 1 \mathrm{H}), 8.97(\mathrm{~d}, J=8.4 \mathrm{~Hz}, 1 \mathrm{H}), 8.01(\mathrm{~d}, J=8.3 \mathrm{~Hz}, 2 \mathrm{H})$, $7.88(\mathrm{~d}, J=7.9 \mathrm{~Hz}, 1 \mathrm{H}), 7.50(\mathrm{t}, J=7.9 \mathrm{~Hz}, 1 \mathrm{H}), 7.32(\mathrm{~d}, J=8.3 \mathrm{~Hz}, 2 \mathrm{H}), 7.08(\mathrm{t}, J=7.6 \mathrm{~Hz}, 1 \mathrm{H}), 4.38(\mathrm{t}, J=$ $9.1 \mathrm{~Hz}, 2 \mathrm{H}), 4.17(\mathrm{t}, J=9.2 \mathrm{~Hz}, 2 \mathrm{H}), 2.61-2.52(\mathrm{~m}, 1 \mathrm{H}), 1.90-1.82(\mathrm{~m}, 4 \mathrm{H}), 1.79-1.73(\mathrm{~m}, 1 \mathrm{H}), 1.51-1.34(\mathrm{~m}$, $4 \mathrm{H}), 1.32-1.23(\mathrm{~m}, 1 \mathrm{H}) ;{ }^{13} \mathrm{C}$ NMR $\left(100 \mathrm{MHz}, \mathrm{CDCl}_{3}\right) \delta 166.0,164.8,152.0,140.2,132.7,132.5,129.2,127.8$, 127.0, 122.1, 119.7, 113.4, 66.2, 54.6, 44.5, 34.2, 26.7, 26.0; HRMS (ESI-TOF) $m / z$ Calcd for $\mathrm{C}_{22} \mathrm{H}_{24} \mathrm{~N}_{2} \mathrm{O}_{2} \mathrm{Na}$ $[\mathrm{M}+\mathrm{Na}]^{+}: 371.1730$, found: 371.1727 .<smiles>O=C(Nc1ccccc1C1=NCCO1)c1ccc(-c2ccccc2)cc1</smiles>

\section{$N$-(2-(4,5-Dihydrooxazol-2-yl)phenyl)-[1,1'-biphenyl]-4-carboxamide (1e)}

1e was obtained by recrystallization from petroleum ether/ethyl acetate (50/1) as yellow solid (410 $\mathrm{mg}, 65 \%)$. mp 137-139 ${ }^{\circ} \mathrm{C} ;{ }^{1} \mathrm{H}$ NMR $\left(400 \mathrm{MHz}, \mathrm{CDCl}_{3}\right) \delta 13.08(\mathrm{~s}, 1 \mathrm{H}), 8.99(\mathrm{~d}, J=8.5 \mathrm{~Hz}, 1 \mathrm{H}), 8.16(\mathrm{~d}, J=8.3 \mathrm{~Hz}, 2 \mathrm{H})$, $7.90(\mathrm{~d}, J=7.9 \mathrm{~Hz}, 1 \mathrm{H}), 7.72(\mathrm{~d}, J=8.5 \mathrm{~Hz}, 2 \mathrm{H}), 7.64(\mathrm{~d}, J=7.4 \mathrm{~Hz}, 2 \mathrm{H}), 7.52(\mathrm{t}, J=7.9 \mathrm{~Hz}, 1 \mathrm{H}), 7.46(\mathrm{t}, J=$ $7.5 \mathrm{~Hz}, 2 \mathrm{H}), 7.38(\mathrm{t}, J=7.3 \mathrm{~Hz}, 1 \mathrm{H}), 7.11(\mathrm{t}, J=8.0 \mathrm{~Hz}, 1 \mathrm{H}), 4.40(\mathrm{t}, J=9.0 \mathrm{~Hz}, 2 \mathrm{H}), 4.20$ (t, $J=9.0 \mathrm{~Hz}, 2 \mathrm{H})$; ${ }^{13} \mathrm{C}$ NMR $\left(100 \mathrm{MHz}, \mathrm{CDCl}_{3}\right) \delta 165.8,164.9,144.3,140.2,140.0,133.9,132.6,129.3,128.8,128.2,127.9$, 127.2, 127.2, 122.3, 119.8, 113.5, 66.2, 54.7; HRMS (ESI-TOF) $m / z$ Calcd for $\mathrm{C}_{22} \mathrm{H}_{18} \mathrm{~N}_{2} \mathrm{O}_{2} \mathrm{Na}[\mathrm{M}+\mathrm{Na}]^{+}$: 365.1260, found: 365.1256 .<smiles>COc1ccc(C(=O)Nc2ccccc2C2=NCCO2)cc1</smiles>

\section{$N$-(2-(4,5-Dihydrooxazol-2-yl)phenyl)-4-(methylthio)benzamide (1h)}

1h was obtained by recrystallization from petroleum ether/ethyl acetate (50/1) as white solid (410 $\mathrm{mg}, 67 \%)$. mp 106-108 ${ }^{\circ} \mathrm{C}$; ${ }^{1} \mathrm{H}$ NMR $\left(400 \mathrm{MHz}, \mathrm{CDCl}_{3}\right) \delta 12.98(\mathrm{~s}, 1 \mathrm{H}), 8.94(\mathrm{~d}, J=8.5 \mathrm{~Hz}, 1 \mathrm{H}), 8.00(\mathrm{~d}, J=8.5 \mathrm{~Hz}, 2 \mathrm{H})$, $7.88(\mathrm{~d}, J=7.9 \mathrm{~Hz}, 1 \mathrm{H}), 7.51(\mathrm{t}, J=8.0 \mathrm{~Hz}, 1 \mathrm{H}), 7.31(\mathrm{~d}, J=8.6 \mathrm{~Hz}, 2 \mathrm{H}), 7.09(\mathrm{t}, J=8.0 \mathrm{~Hz}, 1 \mathrm{H}), 4.40(\mathrm{t}, J=$ $9.2 \mathrm{~Hz}, 2 \mathrm{H}), 4.18(\mathrm{t}, J=9.2 \mathrm{~Hz}, 2 \mathrm{H}), 2.53(\mathrm{~s}, 3 \mathrm{H}) ;{ }^{13} \mathrm{C} \mathrm{NMR}\left(100 \mathrm{MHz}, \mathrm{CDCl}_{3}\right) \delta 165.5,164.9,143.6,140.2$, 132.6, 131.4, 129.2, 128.1, 125.3, 122.3, 119.7, 113.4, 66.2, 54.6, 14.9; HRMS (ESI-TOF) $\mathrm{m} / \mathrm{z}$ Calcd for $\mathrm{C}_{17} \mathrm{H}_{16} \mathrm{~N}_{2} \mathrm{O}_{2} \mathrm{SNa}[\mathrm{M}+\mathrm{Na}]^{+}: 335.0825$, found: 335.0821 .<smiles>CNc1ccc(C(=O)Nc2ccccc2C2=NCCO2)cc1</smiles>

\section{4-Acetamido- $N$-(2-(4,5-dihydrooxazol-2-yl)phenyl)benzamide (1i)}

$1 \mathbf{i}$ was obtained by recrystallization from petroleum ether/ethyl acetate (50/1) as white solid (120 mg, 19\%). mp 
240-242 ${ }^{\circ} \mathrm{C} ;{ }^{1} \mathrm{H}$ NMR (400 MHz, $\left.\mathrm{CDCl}_{3}\right) \delta 13.01(\mathrm{~s}, 1 \mathrm{H}), 8.95(\mathrm{~d}, J=8.4 \mathrm{~Hz}, 1 \mathrm{H}), 8.08(\mathrm{~d}, J=8.7 \mathrm{~Hz}, 2 \mathrm{H})$, $7.91(\mathrm{~d}, J=8.0 \mathrm{~Hz}, 1 \mathrm{H}), 7.66(\mathrm{~d}, J=8.1 \mathrm{~Hz}, 2 \mathrm{H}), 7.52(\mathrm{t}, J=8.4 \mathrm{~Hz}, 1 \mathrm{H}), 7.33(\mathrm{~s}, 1 \mathrm{H}), 7.11(\mathrm{t}, J=7.5 \mathrm{~Hz}, 1 \mathrm{H})$, $4.43(\mathrm{t}, J=9.5 \mathrm{~Hz}, 2 \mathrm{H}), 4.22(\mathrm{t}, J=9.6 \mathrm{~Hz}, 2 \mathrm{H}), 2.23(\mathrm{~s}, 3 \mathrm{H}) ;{ }^{13} \mathrm{C} \mathrm{NMR}\left(125 \mathrm{MHz}, \mathrm{CDCl}_{3}\right) \delta 168.4,165.4$, 165.0, 140.9, 140.2, 132.7, 130.8, 129.3, 128.9, 122.3, 119.8, 119.0, 113.5, 66.3, 54.7, 24.8; HRMS (ESI-TOF) $m / z$ Calcd for $\mathrm{C}_{18} \mathrm{H}_{17} \mathrm{~N}_{3} \mathrm{O}_{3} \mathrm{Na}[\mathrm{M}+\mathrm{Na}]^{+}: 346.1162$, found: 346.1160 .<smiles>O=C(Nc1ccccc1C1=NCCO1)c1ccc(N2CCOCC2)cc1</smiles>

\section{$N$-(2-(4,5-Dihydrooxazol-2-yl)phenyl)-4-morpholinobenzamide (1j)}

$\mathbf{1 j}$ was obtained by column chromatography on silica gel with a gradient eluent of petroleum ether/ethyl acetate (2/1) as yellow solid (260 mg, 37\%). mp 154-156 ${ }^{\circ} \mathrm{C} ;{ }^{1} \mathrm{H}$ NMR $\left(400 \mathrm{MHz}, \mathrm{CDCl}_{3}\right) \delta 12.86(\mathrm{~s}, 1 \mathrm{H}), 8.96(\mathrm{~d}, J=$ $8.4 \mathrm{~Hz}, 1 \mathrm{H}), 8.03(\mathrm{~d}, J=9.0 \mathrm{~Hz}, 2 \mathrm{H}), 7.89(\mathrm{dd}, J=7.9,1.3 \mathrm{~Hz}, 1 \mathrm{H}), 7.50(\mathrm{t}, J=7.9 \mathrm{~Hz}, 1 \mathrm{H}), 7.08(\mathrm{t}, J=8.0$ $\mathrm{Hz}, 1 \mathrm{H}), 6.95(\mathrm{~d}, J=8.9 \mathrm{~Hz}, 2 \mathrm{H}), 4.41(\mathrm{t}, J=9.7 \mathrm{~Hz}, 2 \mathrm{H}), 4.20(\mathrm{t}, J=9.2 \mathrm{~Hz}, 2 \mathrm{H}), 3.90-3.84(\mathrm{~m}, 4 \mathrm{H}), 3.32$ $3.25(\mathrm{~m}, 4 \mathrm{H}) ;{ }^{13} \mathrm{C}$ NMR $\left(100 \mathrm{MHz}, \mathrm{CDCl}_{3}\right) \delta 165.8,164.9,153.4,140.5,132.6,129.2,129.2,125.5,121.9$, 119.7, 114.0, 113.2, 66.6, 66.2, 54.7, 47.9; HRMS (ESI-TOF) $m / z$ Calcd for $\mathrm{C}_{20} \mathrm{H}_{21} \mathrm{~N}_{3} \mathrm{O}_{3} \mathrm{Na}[\mathrm{M}+\mathrm{Na}]^{+}: 374.1475$, found: 374.1478 .<smiles>O=C(Nc1ccccc1C1=NCCO1)c1cccc(-c2ccccc2)c1</smiles>

\section{$N$-(2-(4,5-Dihydrooxazol-2-yl)phenyl)-[1,1'-biphenyl]-3-carboxamide (1n)}

1n was obtained by recrystallization from petroleum ether/ethyl acetate (50/1), as white solid (430 $\mathrm{mg}, 78 \%$ ). mp 160-162 ${ }^{\circ} \mathrm{C} ;{ }^{1} \mathrm{H}$ NMR (400 MHz, $\left.\mathrm{CDCl}_{3}\right) \delta 13.14$ (s, 1H), 8.99 (d, $\left.J=8.4 \mathrm{~Hz}, 1 \mathrm{H}\right), 8.37$ (s, 1H), 8.07 (d, $J$ $=7.7 \mathrm{~Hz}, 1 \mathrm{H}), 7.86(\mathrm{~d}, J=7.8 \mathrm{~Hz}, 1 \mathrm{H}), 7.74(\mathrm{~d}, J=7.6 \mathrm{~Hz}, 1 \mathrm{H}), 7.66(\mathrm{~d}, J=7.5 \mathrm{~Hz}, 2 \mathrm{H}), 7.58-7.41(\mathrm{~m}, 4 \mathrm{H})$, $7.37(\mathrm{~d}, J=7.2 \mathrm{~Hz}, 1 \mathrm{H}), 7.07(\mathrm{t}, J=7.6 \mathrm{~Hz}, 1 \mathrm{H}), 4.36(\mathrm{t}, J=9.5 \mathrm{~Hz}, 2 \mathrm{H}), 4.15(\mathrm{t}, J=9.4 \mathrm{~Hz}, 2 \mathrm{H}) ;{ }^{13} \mathrm{C} \mathrm{NMR}$ $\left(100 \mathrm{MHz}, \mathrm{CDCl}_{3}\right) \delta 165.7,164.9,141.3,140.3,140.1,135.6,132.6,130.1,129.3,129.1,128.8,127.6,127.1$, 126.7, 126.3, 122.3, 119.7, 113.4, 66.2, 54.6; HRMS (ESI-TOF) $m / z$ Calcd for $\mathrm{C}_{22} \mathrm{H}_{18} \mathrm{~N}_{2} \mathrm{O}_{2} \mathrm{Na}[\mathrm{M}+\mathrm{Na}]^{+}$: 365.1260 , found: 365.1255 .<smiles>O=C(Nc1ccccc1C1=NCCO1)c1cccc(OC(F)(F)F)c1</smiles>

\section{$N$-(2-(4,5-Dihydrooxazol-2-yl)phenyl)-3-(1,1,2,2-tetrafluoroethoxy)benzamide (1p)}

1p was obtained by recrystallization from petroleum ether/ethyl acetate (50/1), as yellow solid (501 $\mathrm{mg}, 77 \%)$. mp 84-86 ${ }^{\circ} \mathrm{C} ;{ }^{1} \mathrm{H}$ NMR $\left(400 \mathrm{MHz}, \mathrm{CDCl}_{3}\right) \delta 13.18(\mathrm{~d}, J=5.4 \mathrm{~Hz}, 1 \mathrm{H}), 8.93(\mathrm{dd}, J=8.4,2.2 \mathrm{~Hz}, 1 \mathrm{H}), 8.05-$ 
$7.97(\mathrm{~m}, 2 \mathrm{H}), 7.93-7.83(\mathrm{~m}, 1 \mathrm{H}), 7.56-7.46(\mathrm{~m}, 2 \mathrm{H}), 7.40-7.33(\mathrm{~m}, 1 \mathrm{H}), 7.14-7.06(\mathrm{~m}, 1 \mathrm{H}), 5.96(\mathrm{tt}, J=53.1$, $2.7 \mathrm{~Hz}, 1 \mathrm{H}), 4.47-4.33(\mathrm{~m}, 2 \mathrm{H}), 4.24-4.09(\mathrm{~m}, 2 \mathrm{H}) ;{ }^{13} \mathrm{C}$ NMR $\left(150 \mathrm{MHz}\right.$, decoupled for $\left.{ }^{19} \mathrm{~F}, \mathrm{CDCl}_{3}\right) \delta 165.0$, 164.4, 148.9, 139.9, 137.2, 132.7, 129.9, 129.3, 126.1, 124.8, 122.6, 120.9, 119.7, 116.5, 113.6, 107.6, 66.3, 54.4; ${ }^{19} \mathrm{~F}$ NMR (375 MHz, $\left.\mathrm{CDCl}_{3}\right) \delta-88.10-88.17(\mathrm{~m}, 2 \mathrm{~F}),-136.81\left(\mathrm{dt}, J_{F-F}=53.1,5.6 \mathrm{~Hz}, 2 \mathrm{~F}\right)$; HRMS (ESITOF) $m / z$ Calcd for $\mathrm{C}_{18} \mathrm{H}_{14} \mathrm{~F}_{4} \mathrm{~N}_{2} \mathrm{O}_{3} \mathrm{Na}[\mathrm{M}+\mathrm{Na}]^{+}: 405.0833$, found: 405.0823 .<smiles>COc1cccc(C(=O)Nc2ccccc2C2=NCCO2)c1[N+](=O)[O-]</smiles>

$N$-(2-(4,5-Dihydrooxazol-2-yl)phenyl)-3-methoxy-2-methylbenzamide (1r)

1r was obtained by recrystallization from petroleum ether/ethyl acetate (50/1) as white solid (390 $\mathrm{mg}, 53 \%)$. mp 166-168 ${ }^{\circ} \mathrm{C} ;{ }^{1} \mathrm{H}$ NMR $\left(400 \mathrm{MHz}, \mathrm{CDCl}_{3}\right) \delta 12.53(\mathrm{~s}, 1 \mathrm{H}), 8.95(\mathrm{~d}, J=8.4 \mathrm{~Hz}, 1 \mathrm{H}), 7.88(\mathrm{~d}, J=8.0 \mathrm{~Hz}, 1 \mathrm{H})$, $7.51(\mathrm{t}, J=8.0 \mathrm{~Hz}, 1 \mathrm{H}), 7.25-7.16(\mathrm{~m}, 2 \mathrm{H}), 7.10(\mathrm{t}, J=7.6 \mathrm{~Hz}, 1 \mathrm{H}), 6.93(\mathrm{~d}, J=8.4 \mathrm{~Hz}, 1 \mathrm{H}), 4.36-4.27(\mathrm{~m}$, $2 \mathrm{H}), 4.04-3.96(\mathrm{~m}, 2 \mathrm{H}), 3.85(\mathrm{~s}, 3 \mathrm{H}), 2.39$ (s, 3H); ${ }^{13} \mathrm{C} \mathrm{NMR}\left(100 \mathrm{MHz}, \mathrm{CDCl}_{3}\right) \delta 168.7,164.4,158.0,139.9$, 138.5, 132.4, 129.1, 126.4, 125.3, 122.4, 119.7, 119.3, 113.4, 111.5, 66.1, 55.6, 54.6, 12.7; HRMS (ESI-TOF) $m / z$ Calcd for $\mathrm{C}_{18} \mathrm{H}_{18} \mathrm{~N}_{2} \mathrm{O}_{3} \mathrm{Na}[\mathrm{M}+\mathrm{Na}]^{+}: 333.1210$, found: 333.1203 .<smiles>COc1ccc(C(=O)Nc2ccccc2C2=NCCO2)cc1OC</smiles>

$N$-(2-(4,5-Dihydrooxazol-2-yl)phenyl)-3,4-dimethoxybenzamide (1s)

$1 \mathrm{~s}$ was obtained by recrystallization from petroleum ether/ethyl acetate (50/1) as white solid (550 $\mathrm{mg}, 71 \%)$. mp 179-181 ${ }^{\circ} \mathrm{C} ;{ }^{1} \mathrm{H}$ NMR $\left(400 \mathrm{MHz}, \mathrm{CDCl}_{3}\right) \delta 12.92(\mathrm{~s}, 1 \mathrm{H}), 8.96(\mathrm{~d}, J=8.5 \mathrm{~Hz}, 1 \mathrm{H}), 7.89-7.83(\mathrm{~m}, 1 \mathrm{H})$, 7.74-7.69 (m, 1H), $7.67(\mathrm{~s}, 1 \mathrm{H}), 7.50(\mathrm{t}, J=7.9 \mathrm{~Hz}, 1 \mathrm{H}), 7.12-7.04(\mathrm{~m}, 1 \mathrm{H}), 6.96-6.89(\mathrm{~m}, 1 \mathrm{H}), 4.37(\mathrm{dt}, J=$ 9.3, $4.7 \mathrm{~Hz}, 2 \mathrm{H}), 4.17(\mathrm{dd}, J=9.2,3.7 \mathrm{~Hz}, 2 \mathrm{H}), 3.97(\mathrm{~d}, J=2.1 \mathrm{~Hz}, 1 \mathrm{H}), 3.94(\mathrm{~d}, J=2.0 \mathrm{~Hz}, 1 \mathrm{H}) ;{ }^{13} \mathrm{C}$ NMR $\left(100 \mathrm{MHz}, \mathrm{CDCl}_{3}\right) \delta 165.4,165.0,151.8,148.6,140.3,132.6,129.2,127.6,122.0,121.0,119.5,113.2,110.5$, 110.4, 66.1, 55.9, 55.8, 54.6; HRMS (ESI-TOF) $\mathrm{m} / z$ Calcd for $\mathrm{C}_{18} \mathrm{H}_{18} \mathrm{~N}_{2} \mathrm{O}_{4} \mathrm{Na}[\mathrm{M}+\mathrm{Na}]^{+}: 349.1159$, found: 349.1155 .<smiles>O=C(Nc1ccccc1C1=NCCO1)c1ccc2c(c1)OCCO2</smiles>

\section{$N$-(2-(4,5-Dihydrooxazol-2-yl)phenyl)-2,3-dihydrobenzo[b][1,4]dioxine-6-carboxamide (1t)}

1t was obtained by recrystallization from petroleum ether/ethyl acetate (50/1) as white solid (510 $\mathrm{mg}, 66 \%) . \mathrm{mp}$ 167-169 ${ }^{\circ} \mathrm{C}$; ${ }^{1} \mathrm{H}$ NMR $\left(400 \mathrm{MHz}, \mathrm{CDCl}_{3}\right) \delta 12.89(\mathrm{~s}, 1 \mathrm{H}), 8.93(\mathrm{~d}, J=8.4 \mathrm{~Hz}, 1 \mathrm{H}), 7.84(\mathrm{dd}, J=7.9,1.5 \mathrm{~Hz}$, $1 \mathrm{H}), 7.63(\mathrm{~d}, J=2.1 \mathrm{~Hz}, 1 \mathrm{H}), 7.59(\mathrm{dd}, J=8.5,2.1 \mathrm{~Hz}, 1 \mathrm{H}), 7.48(\mathrm{t}, J=8.0 \mathrm{~Hz}, 1 \mathrm{H}), 7.06(\mathrm{t}, J=7.6 \mathrm{~Hz}, 1 \mathrm{H})$, 
$6.92(\mathrm{~d}, J=8.4 \mathrm{~Hz}, 1 \mathrm{H}), 4.35(\mathrm{t}, J=9.7 \mathrm{~Hz}, 2 \mathrm{H}), 4.27(\mathrm{q}, J=5.0 \mathrm{~Hz}, 4 \mathrm{H}), 4.16(\mathrm{t}, J=9.2 \mathrm{~Hz}, 2 \mathrm{H}) ;{ }^{13} \mathrm{C} \mathrm{NMR}$ $\left(100 \mathrm{MHz}, \mathrm{CDCl}_{3}\right) \delta 165.2,164.7,146.6,143.2,140.2,132.4,129.1,128.4,122.0,121.1,119.5,117.2,117.1$, 113.3, 66.1, 64.5, 64.0, 54.5; HRMS (ESI-TOF) $\mathrm{m} / z$ Calcd for $\mathrm{C}_{18} \mathrm{H}_{16} \mathrm{~N}_{2} \mathrm{O}_{4} \mathrm{Na}[\mathrm{M}+\mathrm{Na}]^{+}: 347.1002$, found: 347.0996.

The vinyl carboxylic acids (5,6-dihydro-2H-pyran-3-carboxylic acid (6f-s), 1-methyl-1,2,5,6tetrahydropyridine-3-carboxylic acid (6g-s), and 2, 3, 6, 7-tetrahydrooxepine-4-carboxylic acid (6i-s)) were synthesized following the known procedure. ${ }^{3}$ Others are commercially available.

\section{General Procedure for Synthesis of Vinyl Carboxamides:}

To a solution of vinyl carboxylic acid (4.0 mmol), 2-(4,5-dihydrooxazol-2-yl)aniline (5.0 mmol) and DMAP $(0.5 \mathrm{mmol})$ in DCM $(20 \mathrm{~mL})$ was added EDCI $(6.0 \mathrm{mmol})$ under nitrogen at $0{ }^{\circ} \mathrm{C}$. The mixture was stirred for $24 \mathrm{~h}$ at room temperature. Saturated $\mathrm{NaHCO}_{3}$ was added to quench reaction, and then extracted with EtOAc (3 $\times 100 \mathrm{~mL}$ ). The combined organic layers were washed with saturated brine, dried over $\mathrm{Na}_{2} \mathrm{SO}_{4}$, concentrated under reduced pressure. The crude product was recrystallized from petroleum ether/ethyl acetate or isolated by column chromatography on silica gel with a gradient eluent of petroleum ether/ethyl acetate to provide the desired product (6a-i).<smiles>C=C(C)C(=O)Nc1ccccc1C1=NCCO1</smiles>

\section{$N$-(2-(4,5-Dihydrooxazol-2-yl)phenyl)methacrylamide (6a)}

6a was obtained by recrystallization from petroleum ether/ethyl acetate (50/1) as white solid (195 $\mathrm{mg}, 43 \%)$. mp 78-80 ${ }^{\circ} \mathrm{C} ;{ }^{1} \mathrm{H} \mathrm{NMR}\left(400 \mathrm{MHz}, \mathrm{CDCl}_{3}\right) \delta 12.54(\mathrm{~s}, 1 \mathrm{H}), 8.84(\mathrm{~d}, J=8.5 \mathrm{~Hz}, 1 \mathrm{H}), 7.85(\mathrm{~d}, J=8.0 \mathrm{~Hz}, 1 \mathrm{H})$, $7.46(\mathrm{t}, J=8.0 \mathrm{~Hz}, 1 \mathrm{H}), 7.07(\mathrm{t}, J=8.0 \mathrm{~Hz}, 1 \mathrm{H}), 6.01(\mathrm{~s}, 1 \mathrm{H}), 5.50(\mathrm{~s}, 1 \mathrm{H}), 4.36(\mathrm{t}, J=9.3 \mathrm{~Hz}, 2 \mathrm{H}), 4.12(\mathrm{t}, J=$ $9.6 \mathrm{~Hz}, 2 \mathrm{H}), 2.10(\mathrm{~s}, 3 \mathrm{H}) ;{ }^{13} \mathrm{C} \mathrm{NMR}\left(100 \mathrm{MHz}, \mathrm{CDCl}_{3}\right) \delta 167.1,164.6,140.9,139.9,132.4,129.1,122.2,120.9$, 119.6, 113.3, 66.1, 54.6, 18.7; HRMS (ESI-TOF) $\mathrm{m} / \mathrm{z}$ Calcd for $\mathrm{C}_{13} \mathrm{H}_{14} \mathrm{~N}_{2} \mathrm{O}_{2} \mathrm{Na}[\mathrm{M}+\mathrm{Na}]^{+}: 253.0947$, found: 253.0943.<smiles>C=C(Cc1ccccc1)C(=O)Nc1ccccc1C1=NCCO1</smiles>

\section{2-Benzyl- $N$-(2-(4,5-dihydrooxazol-2-yl)phenyl)acrylamide (6b)}

6b was obtained by column chromatography on silica gel with a gradient eluent of petroleum ether/ethyl acetate (20/1) as white solid (570 mg, 62\%). mp 76-78 ${ }^{\circ} \mathrm{C}$; ${ }^{1} \mathrm{H}$ NMR $\left(400 \mathrm{MHz}, \mathrm{CDCl}_{3}\right) \delta 12.62(\mathrm{~s}, 1 \mathrm{H}), 8.82(\mathrm{~d}, J=$ $8.5 \mathrm{~Hz}, 1 \mathrm{H}), 7.86(\mathrm{~d}, J=8.0 \mathrm{~Hz}, 1 \mathrm{H}), 7.46(\mathrm{t}, J=8.0 \mathrm{~Hz}, 1 \mathrm{H}), 7.34-7.24(\mathrm{~m}, 4 \mathrm{H}), 7.21(\mathrm{t}, J=6.9 \mathrm{~Hz}, 1 \mathrm{H}), 7.07$ $(\mathrm{t}, J=7.1 \mathrm{~Hz}, 1 \mathrm{H}), 6.09(\mathrm{~s}, 1 \mathrm{H}), 5.37(\mathrm{~s}, 1 \mathrm{H}), 4.38(\mathrm{t}, J=9.4 \mathrm{~Hz}, 2 \mathrm{H}), 4.12(\mathrm{t}, J=9.5 \mathrm{~Hz}, 2 \mathrm{H}), 3.80(\mathrm{~s}, 2 \mathrm{H}) ;{ }^{13} \mathrm{C}$ NMR $\left(100 \mathrm{MHz}, \mathrm{CDCl}_{3}\right) \delta 166.9,164.7,145.4,140.0,138.9,132.5,129.2,129.2,128.4,126.3,122.3,121.1$, 
119.8, 113.4, 66.2, 54.7, 38.1; HRMS (ESI-TOF) $m / z$ Calcd for $\mathrm{C}_{19} \mathrm{H}_{18} \mathrm{~N}_{2} \mathrm{O}_{2} \mathrm{Na}[\mathrm{M}+\mathrm{Na}]^{+}: 329.1260$, found: 329.1254 .<smiles>CC(=Cc1ccccc1)C(=O)Nc1ccccc1C1=NCCO1</smiles>

(E)-N-(2-(4,5-Dihydrooxazol-2-yl)phenyl)-2-methyl-3-phenylacrylamide (6c)

6c was obtained by recrystallization from petroleum ether/ethyl acetate (50/1) as yellow solid (169 $\mathrm{mg}, 28 \%$ ). mp120-122 ${ }^{\circ} \mathrm{C} ;{ }^{1} \mathrm{H}$ NMR $\left(400 \mathrm{MHz}, \mathrm{CDCl}_{3}\right) \delta 12.65(\mathrm{~s}, 1 \mathrm{H}), 8.91(\mathrm{~d}, J=7.9 \mathrm{~Hz}, 1 \mathrm{H}), 7.87(\mathrm{~d}, J=7.9 \mathrm{~Hz}, 1 \mathrm{H})$, $7.62(\mathrm{~s}, 1 \mathrm{H}), 7.48(\mathrm{t}, J=7.9 \mathrm{~Hz}, 1 \mathrm{H}), 7.43-7.37(\mathrm{~m}, 4 \mathrm{H}), 7.33-7.28(\mathrm{~m}, 1 \mathrm{H}), 7.07(\mathrm{t}, J=7.6 \mathrm{~Hz}, 1 \mathrm{H}), 4.36(\mathrm{t}, J$ $=9.6 \mathrm{~Hz}, 2 \mathrm{H}), 4.11(\mathrm{t}, J=9.4 \mathrm{~Hz}, 2 \mathrm{H}), 2.25(\mathrm{~s}, 3 \mathrm{H}) ;{ }^{13} \mathrm{C} \mathrm{NMR}\left(100 \mathrm{MHz}, \mathrm{CDCl}_{3}\right) \delta 168.4,164.7,140.1,136.4$, 135.1, 133.1, 132.5, 129.4, 129.1, 128.2, 127.8, 122.2, 119.8, 113.4, 66.1, 54.6, 14.3; HRMS (ESI-TOF) $\mathrm{m} / z$ Calcd for $\mathrm{C}_{19} \mathrm{H}_{18} \mathrm{~N}_{2} \mathrm{O}_{2} \mathrm{Na}[\mathrm{M}+\mathrm{Na}]^{+}: 329.1260$, found: 329.1258 .<smiles>O=C(Nc1ccccc1C1=NCCO1)C1=CCCCC1</smiles>

$N$-(2-(4,5-Dihydrooxazol-2-yl)phenyl)cyclohex-1-enecarboxamide (6d)

6d was obtained by recrystallization from petroleum ether/ethyl acetate (50/1) as yellow solid (160 mg, 30\%). mp 127-129 ${ }^{\circ} \mathrm{C} ;{ }^{1} \mathrm{H}$ NMR $\left(400 \mathrm{MHz}, \mathrm{CDCl}_{3}\right) \delta 12.30(\mathrm{~s}, 1 \mathrm{H}), 8.85(\mathrm{~d}, J=8.5 \mathrm{~Hz}, 1 \mathrm{H}), 7.85(\mathrm{dd}, J=7.9,1.5$ $\mathrm{Hz}, 1 \mathrm{H}), 7.45(\mathrm{t}, J=8.0 \mathrm{~Hz}, 1 \mathrm{H}), 7.05(\mathrm{t}, J=8.0 \mathrm{~Hz}, 1 \mathrm{H}), 6.93-6.85(\mathrm{~m}, 1 \mathrm{H}), 4.36(\mathrm{t}, J=9.4 \mathrm{~Hz}, 1 \mathrm{H}), 4.13(\mathrm{t}$, $J=9.6 \mathrm{~Hz}, 2 \mathrm{H}), 2.46-2.39(\mathrm{~m}, 2 \mathrm{H}), 2.28-2.21(\mathrm{~m}, 2 \mathrm{H}), 1.78-1.70(\mathrm{~m}, 2 \mathrm{H}), 1.68-1.60(\mathrm{~m}, 2 \mathrm{H}) ;{ }^{13} \mathrm{C}$ NMR $(100$ $\left.\mathrm{MHz}, \mathrm{CDCl}_{3}\right) \delta 167.5,164.6,140.2,135.1,134.4,132.4,129.1,121.8,119.8,113.3,66.1,54.6,25.7,24.2,22.3$, 21.5; HRMS (ESI-TOF) $m / z$ Calcd for $\mathrm{C}_{16} \mathrm{H}_{18} \mathrm{~N}_{2} \mathrm{O}_{2} \mathrm{Na}[\mathrm{M}+\mathrm{Na}]^{+}: 293.1260$, found: 293.1261.<smiles>O=C(Nc1ccccc1C1=NCCO1)C1=COCCC1</smiles>

$N$-(2-(4,5-Dihydrooxazol-2-yl)phenyl)-3,4-dihydro-2H-pyran-5-carboxamide (6e)

6e was obtained by recrystallization from petroleum ether/ethyl acetate (50/1) as white solid (200 mg, 37\%). mp 109-111 ${ }^{\circ} \mathrm{C} ;{ }^{1} \mathrm{H}$ NMR $\left(400 \mathrm{MHz}, \mathrm{CDCl}_{3}\right) \delta 12.14(\mathrm{~s}, 1 \mathrm{H}), 8.82(\mathrm{~d}, J=8.5 \mathrm{~Hz}, 1 \mathrm{H}), 7.83(\mathrm{~d}, J=7.8 \mathrm{~Hz}, 1 \mathrm{H})$, 7.64 (s, 1H), 7.45 (d, $J=7.9 \mathrm{~Hz}, 1 \mathrm{H}), 7.02$ (t, $J=7.6 \mathrm{~Hz}, 1 \mathrm{H}), 4.40-4.30$ (m, 2H), 4.16-4.03 (m, 4H), 2.47$2.40(\mathrm{~m}, 2 \mathrm{H}), 2.01-1.90(\mathrm{~m}, 2 \mathrm{H}) ;{ }^{13} \mathrm{C} \mathrm{NMR}\left(100 \mathrm{MHz}, \mathrm{CDCl}_{3}\right) \delta 166.8,164.6,152.6,140.3,132.3,129.0,121.5$, 119.6, 112.9, 109.8, 66.2, 66.0, 54.5, 21.3, 19.3; HRMS (ESI-TOF) $m / z$ Calcd for $\mathrm{C}_{15} \mathrm{H}_{16} \mathrm{~N}_{2} \mathrm{O}_{3} \mathrm{Na}[\mathrm{M}+\mathrm{Na}]^{+}$: 295.1053, found: 295.1050 . 
<smiles>O=C(Nc1ccccc1C1=NCCO1)C1=CCCOC1</smiles>

\section{$N$-(2-(4,5-Dihydrooxazol-2-yl)phenyl)-5,6-dihydro-2H-pyran-3-carboxamide (6f)}

6f was obtained by column chromatography on silica gel with a gradient eluent of petroleum ether/ethyl acetate (10/1) as yellow solid (595 mg, 22\%). mp 113-115 ${ }^{\circ} \mathrm{C} ;{ }^{1} \mathrm{H}$ NMR (400 MHz, $\left.\mathrm{CDCl}_{3}\right) \delta 12.44(\mathrm{~s}, 1 \mathrm{H}), 8.78(\mathrm{~d}, J$ $=8.4 \mathrm{~Hz}, 1 \mathrm{H}), 7.87(\mathrm{~d}, J=7.7 \mathrm{~Hz}, 1 \mathrm{H}), 7.46(\mathrm{t}, J=7.7 \mathrm{~Hz}, 1 \mathrm{H}), 7.08(\mathrm{t}, J=7.5 \mathrm{~Hz}, 1 \mathrm{H}), 6.96(\mathrm{~s}, 1 \mathrm{H}), 4.52(\mathrm{~s}$, 2H), $4.40(\mathrm{t}, J=9.4 \mathrm{~Hz}, 2 \mathrm{H}), 4.16(\mathrm{t}, J=9.3 \mathrm{~Hz}, 2 \mathrm{H}), 3.81(\mathrm{t}, J=5.5 \mathrm{~Hz}, 2 \mathrm{H}), 2.38(\mathrm{~s}, 2 \mathrm{H}) ;{ }^{13} \mathrm{C}$ NMR $(100$ $\left.\mathrm{MHz}, \mathrm{CDCl}_{3}\right) \delta 164.9,164.7,139.9,134.3,132.6,131.4,129.2,122.3,119.8,113.2,66.2,64.9,63.4,54.6,25.5$; HRMS (ESI-TOF) $m / z$ Calcd for $\mathrm{C}_{15} \mathrm{H}_{16} \mathrm{~N}_{2} \mathrm{O}_{3} \mathrm{Na}[\mathrm{M}+\mathrm{Na}]^{+}: 295.1053$, found: 295.1051.<smiles>O=C(Nc1ccccc1C1=NCCO1)C1=CCCOCC1</smiles>

$N$-(2-(4,5-Dihydrooxazol-2-yl)phenyl)-2,3,6,7-tetrahydrooxepine-4-carboxamide (6g)

6g was obtained by column chromatography on silica gel with a gradient eluent of petroleum ether/ethyl acetate (5/1) as white solid (191 mg, 58\%). mp 100-102 ${ }^{\circ} \mathrm{C} ;{ }^{1} \mathrm{H}$ NMR $\left(400 \mathrm{MHz}, \mathrm{CDCl}_{3}\right) \delta 12.46(\mathrm{~s}, 1 \mathrm{H}), 8.79(\mathrm{~d}, J=$ $8.5 \mathrm{~Hz}, 1 \mathrm{H}), 7.83(\mathrm{~d}, J=7.6 \mathrm{~Hz}, 1 \mathrm{H}), 7.44(\mathrm{t}, J=7.7 \mathrm{~Hz}, 1 \mathrm{H}), 7.04(\mathrm{t}, J=7.6 \mathrm{~Hz}, 1 \mathrm{H}), 6.93(\mathrm{t}, J=6.1 \mathrm{~Hz}, 1 \mathrm{H})$, $4.33(\mathrm{t}, J=9.5 \mathrm{~Hz}, 2 \mathrm{H}), 4.07(\mathrm{t}, J=9.5 \mathrm{~Hz}, 2 \mathrm{H}), 3.79-3.69(\mathrm{~m}, 4 \mathrm{H}), 2.88-2.81(\mathrm{~m}, 2 \mathrm{H}), 2.56-2.49(\mathrm{~m}, 2 \mathrm{H}) ;{ }^{13} \mathrm{C}$ $\operatorname{NMR}\left(100 \mathrm{MHz}, \mathrm{CDCl}_{3}\right) \delta 167.9,164.6,140.9,140.0,136.6,132.4,129.1,121.9,119.4,113.2,69.6,68.9,66.0$, 54.5, 32.0, 31.9; HRMS (ESI-TOF) $m / z$ Calcd for $\mathrm{C}_{16} \mathrm{H}_{18} \mathrm{~N}_{2} \mathrm{O}_{3} \mathrm{Na}[\mathrm{M}+\mathrm{Na}]^{+}: 309.1210$, found: 309.1205 .<smiles>O=C(Nc1ccccc1C1=NCCO1)C1=CCN(Cc2ccccc2)CC1</smiles>

\section{1-Benzyl- $N$-(2-(4,5-dihydrooxazol-2-yl)phenyl)-1,2,3,6-tetrahydropyridine-4-carboxamide (6h)}

6h was obtained by column chromatography on neutral alumina with a gradient eluent of petroleum ether/ethyl acetate (6/1) as yellow solid (500 mg, 51\%). mp 159-161 ${ }^{\circ} \mathrm{C} ;{ }^{1} \mathrm{H}$ NMR (400 MHz, $\left.\mathrm{CDCl}_{3}\right) \delta 12.39(\mathrm{~s}, 1 \mathrm{H}), 8.84$ $(\mathrm{d}, J=8.4 \mathrm{~Hz}, 1 \mathrm{H}), 7.83(\mathrm{~d}, J=7.9 \mathrm{~Hz}, 1 \mathrm{H}), 7.43(\mathrm{t}, J=7.9 \mathrm{~Hz}, 1 \mathrm{H}), 7.38-7.30(\mathrm{~m}, 4 \mathrm{H}), 7.30-7.23(\mathrm{~m}, 1 \mathrm{H})$, $7.03(\mathrm{t}, J=7.6 \mathrm{~Hz}, 1 \mathrm{H}), 6.81(\mathrm{~s}, 1 \mathrm{H}), 4.31(\mathrm{t}, J=9.5 \mathrm{~Hz}, 2 \mathrm{H}), 4.05(\mathrm{t}, J=9.5 \mathrm{~Hz}, 2 \mathrm{H}), 3.62(\mathrm{~s}, 2 \mathrm{H}), 3.18(\mathrm{~d}, J$ $=2.9 \mathrm{~Hz}, 2 \mathrm{H}), 2.67(\mathrm{t}, J=5.5 \mathrm{~Hz}, 2 \mathrm{H}), 2.57(\mathrm{~s}, 2 \mathrm{H}) ;{ }^{13} \mathrm{C} \mathrm{NMR}\left(100 \mathrm{MHz}, \mathrm{CDCl}_{3}\right) \delta 166.1,164.5,140.0,137.9$, $132.7,132.3,132.3,129.1,129.0,128.2,127.1,121.9,119.6,113.2,66.0,62.4,54.5,52.8,49.3,25.2 ; \mathrm{HRMS}$ (ESI-TOF) $m / z$ Calcd for $\mathrm{C}_{22} \mathrm{H}_{24} \mathrm{~N}_{3} \mathrm{O}_{2}[\mathrm{M}+\mathrm{H}]^{+}: 362.1863$, found: 362.1863 . 
<smiles>CN1CCC=C(C(=O)Nc2ccccc2C2=NCCO2)C1</smiles>

$N$-(2-(4,5-Dihydrooxazol-2-yl)phenyl)-1-methyl-1,2,5,6-tetrahydropyridine-3-carboxamide (6i)

6i was obtained by column chromatography on neutral alumina with a gradient eluent of petroleum ether/ethyl acetate $(1 / 1)$ as yellow solid $(530 \mathrm{mg}, 62 \%)$. mp 83-85 ${ }^{\circ} \mathrm{C} ;{ }^{1} \mathrm{H}$ NMR $\left(400 \mathrm{MHz}, \mathrm{CDCl}_{3}\right) \delta 12.46(\mathrm{~s}, 1 \mathrm{H}), 8.80$ $(\mathrm{d}, J=8.3 \mathrm{~Hz}, 1 \mathrm{H}), 7.85(\mathrm{~d}, J=7.8 \mathrm{~Hz}, 1 \mathrm{H}), 7.45$ (t, $J=7.8 \mathrm{~Hz}, 1 \mathrm{H}), 7.06$ (t, $J=7.6 \mathrm{~Hz}, 1 \mathrm{H}), 6.87$ (s, $1 \mathrm{H}), 4.37$ $(\mathrm{t}, J=9.4 \mathrm{~Hz}, 2 \mathrm{H}), 4.13(\mathrm{t}, J=9.0 \mathrm{~Hz}, 2 \mathrm{H}), 3.31(\mathrm{~s}, 2 \mathrm{H}), 2.58-2.52(\mathrm{~m}, 2 \mathrm{H}), 2.48-2.38(\mathrm{~m}, 5 \mathrm{H}) ;{ }^{13} \mathrm{C} \mathrm{NMR}(100$ $\left.\mathrm{MHz}_{\mathrm{CDCl}}\right) \delta 165.5,164.7,140.0,133.5,132.4,131.9,129.1,122.0,119.6,113.2,66.1,54.5,53.5,50.8,45.7$, 26.5; HRMS (ESI-TOF) $m / z$ Calcd for $\mathrm{C}_{16} \mathrm{H}_{19} \mathrm{~N}_{3} \mathrm{O}_{2} \mathrm{Na}[\mathrm{M}+\mathrm{Na}]^{+}: 308.1369$, found: 308.1360 . 


\subsection{Preparation of Alkenylboronates}

The alkenylboronates $(\mathbf{2}, \mathbf{4 c}, \mathbf{4 g}, \mathbf{4 l}-\mathbf{m}, \mathbf{4 q - \mathbf { s }}$, and $\mathbf{4} \mathbf{u}-\mathbf{z})$ are commercially available. The alkenylboronates (4d-f, $\mathbf{4 h}-\mathbf{i}, \mathbf{4 k}, \mathbf{4 0})$ were synthesized following the general procedure below. ${ }^{4}$ Others $\left(\mathbf{4} \mathbf{a}-\mathbf{b},{ }^{8} \mathbf{4 j},{ }^{9} \mathbf{4 n},{ }^{10} \mathbf{4} \mathbf{p},{ }^{11} \mathbf{4 t},{ }^{12}\right)$ were synthesized following the known procedures.

\section{General Procedure for Synthesis of Alkenylboronates (4d-f, $4 \mathrm{~h}-\mathrm{i}, 4 \mathrm{k}, 40)$ :}

A stirring bar, $\mathrm{B}_{2} \mathrm{Pin}_{2}(7.5 \mathrm{mmol}, 1.9 \mathrm{~g})$, micro copper powder $(0.5 \mathrm{mmol}, 32.0 \mathrm{mg})$, and $\mathrm{NaOMe}(1.0 \mathrm{mmol}, 54$ $\mathrm{mg}$ ) were added to an oven dried $100 \mathrm{~mL}$ Schlenk tube. Under the nitrogen, alkyne $(5.0 \mathrm{mmol})$ and EtOH $(20$ $\mathrm{mL}$ ) were added to the mixture, and the tube was capped tightly. Then the reaction mixture was stirred for $24 \mathrm{~h}$ at room temperature. Upon completion, the mixture was poured into water $(100 \mathrm{~mL})$, extracted with DCM $(3 \times$ $100 \mathrm{~mL}$ ). The combined organic phases were dried over $\mathrm{Na}_{2} \mathrm{SO}_{4}$, concentrated under reduced pressure, and purified by flash column chromatography on silica gel to provide the desired product (4d-f, $\mathbf{4 h}-\mathbf{i}, \mathbf{4 k}, \mathbf{4 0})$.

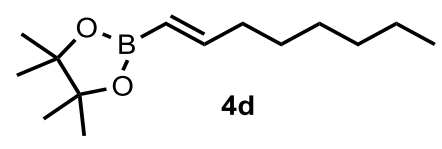

\section{(E)-4,4,5,5-Tetramethyl-2-(oct-1-en-1-yl)-1,3,2-dioxaborolane (4d) ${ }^{4}$}

4d was obtained by flash column chromatography on silica gel (petroleum ether/ethyl acetate $=100 / 1$ ), as colorless oil (535 mg, 45\%). ${ }^{1} \mathrm{H}$ NMR $\left(400 \mathrm{MHz}, \mathrm{CDCl}_{3}\right) \delta 6.68-6.59(\mathrm{~m}, 1 \mathrm{H}), 5.42(\mathrm{~d}, J=18.0 \mathrm{~Hz}, 1 \mathrm{H}), 2.15$ (q, $J=6.9 \mathrm{~Hz}, 2 \mathrm{H}), 1.45-1.35(\mathrm{~m}, 2 \mathrm{H}), 1.34-1.23(\mathrm{~m}, 18 \mathrm{H}), 0.88(\mathrm{t}, J=6.6 \mathrm{~Hz}, 3 \mathrm{H})$.

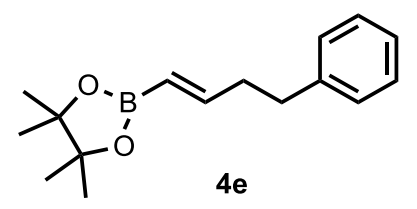

\section{(E)-4,4,5,5-Tetramethyl-2-(4-phenylbut-1-en-1-yl)-1,3,2-dioxaborolane (4e) $)^{4,5}$}

4e was obtained by flash column chromatography on silica gel (petroleum ether/ethyl acetate $=100 / 1$ ), as colorless oil (645 mg, 50\%). ${ }^{1} \mathrm{H}$ NMR (400 MHz, $\left.\mathrm{CDCl}_{3}\right) \delta$ 7.30-7.23 (m, 2H), 7.21-7.15 (m, 3H), 6.75-6.65 $(\mathrm{m}, 1 \mathrm{H}), 5.50(\mathrm{~d}, J=18.0 \mathrm{~Hz}, 1 \mathrm{H}), 2.74(\mathrm{t}, J=8.0 \mathrm{~Hz}, 2 \mathrm{H}), 2.51-2.43(\mathrm{~m}, 2 \mathrm{H}), 1.27(\mathrm{~s}, 12 \mathrm{H})$.<smiles>CC1(C)OB(/C=C/C2CC2)OC1(C)C</smiles>

(E)-2-(2-Cyclopropylvinyl)-4,4,5,5-tetramethyl-1,3,2-dioxaborolane (4f) ${ }^{4}$

4f was obtained by flash column chromatography on silica gel (petroleum ether/ethyl acetate $=200 / 1$ ), as colorless oil (960 mg, 99\%). ${ }^{1} \mathrm{H}$ NMR $\left(400 \mathrm{MHz}, \mathrm{CDCl}_{3}\right) \delta 6.07(\mathrm{dd}, J=17.8,9.3 \mathrm{~Hz}, 1 \mathrm{H}), 5.48(\mathrm{~d}, J=17.8$ $\mathrm{Hz}, 1 \mathrm{H}), 1.57-1.46(\mathrm{~m}, 1 \mathrm{H}), 1.25(\mathrm{~s}, 12 \mathrm{H}), 0.83-0.77(\mathrm{~m}, 2 \mathrm{H}), 0.56-0.50(\mathrm{~m}, 2 \mathrm{H})$. 
<smiles>CC1(C)OB(/C=C/c2ccsc2)OC1(C)C</smiles>

\section{(E)-4,4,5,5-Tetramethyl-2-(2-(thiophen-3-yl)vinyl)-1,3,2-dioxaborolane (4h) ${ }^{4}$}

4h was obtained by flash column chromatography on silica gel (petroleum ether/ethyl acetate $=100 / 1$ ), as yellow solid (908 mg, 77\%). ${ }^{1} \mathrm{H}$ NMR $\left(400 \mathrm{MHz}, \mathrm{CDCl}_{3}\right) \delta 7.38(\mathrm{~d}, J=18.6 \mathrm{~Hz}, 1 \mathrm{H}), 7.32-7.24$ (m, 3H), 5.94 (d, $J=18.1 \mathrm{~Hz}, 1 \mathrm{H}), 1.33-1.27$ (m, 12H).<smiles>CC1(C)OB(/C=C/[Si](C)(C)C)OC1(C)C</smiles>

\section{(E)-Trimethyl(2-(4,4,5,5-tetramethyl-1,3,2-dioxaborolan-2-yl)vinyl)silane $(4 \mathbf{i})^{4,6}$}

4i was obtained by flash column chromatography on silica gel (petroleum ether/ethyl acetate $=100 / 1$ ), as colorless oil (226 mg, 20\%). ${ }^{1} \mathrm{H}$ NMR $\left(400 \mathrm{MHz}, \mathrm{CDCl}_{3}\right) \delta 7.09(\mathrm{~d}, J=21.8 \mathrm{~Hz}, 1 \mathrm{H}), 6.21(\mathrm{~d}, J=21.8 \mathrm{~Hz}, 1 \mathrm{H})$, $1.25(\mathrm{~s}, 12 \mathrm{H}), 0.05(\mathrm{~s}, 9 \mathrm{H})$.<smiles>CC1(C)OB(/C=C/C2=CCCCC2)OC1(C)C</smiles>

\section{(E)-2-(2-(Cyclohex-1-en-1-yl)vinyl)-4,4,5,5-tetramethyl-1,3,2-dioxaborolane $(4 \mathrm{k})^{4,7}$}

4k was obtained by flash column chromatography on silica gel (petroleum ether/ethyl acetate $=100 / 1$ ), as colorless oil (374 mg, 32\%). ${ }^{1} \mathrm{H}$ NMR $\left(400 \mathrm{MHz}, \mathrm{CDCl}_{3}\right) \delta 7.03(\mathrm{~d}, J=18.2 \mathrm{~Hz}, 1 \mathrm{H}), 5.97(\mathrm{~s}, 1 \mathrm{H}), 5.43(\mathrm{~d}, J=$ $18.3 \mathrm{~Hz}, 1 \mathrm{H}), 2.19-2.11(\mathrm{~m}, 4 \mathrm{H}), 1.71-1.54(\mathrm{~m}, 4 \mathrm{H}), 1.28(\mathrm{~s}, 12 \mathrm{H})$.<smiles>C/C(=C\c1ccccc1)OB1OC(C)(C)C(C)(C)O1</smiles>

\section{(Z)-4,4,5,5-Tetramethyl-2-(1-phenylprop-1-en-2-yl)-1,3,2-dioxaborolane (40) ${ }^{4}$}

40 was obtained by flash column chromatography on silica gel (petroleum ether/ethyl acetate $=80 / 1$ ), as colorless oil (744 mg, 61\%). ${ }^{1} \mathrm{H}$ NMR (400 MHz, $\left.\mathrm{CDCl}_{3}\right) \delta$ 7.40-7.36 (m, 2H), 7.35-7.30 (m, 2H), 7.26-7.19 $(\mathrm{m}, 2 \mathrm{H}), 1.99(\mathrm{~s}, 3 \mathrm{H}), 1.30(\mathrm{~s}, 12 \mathrm{H})$.

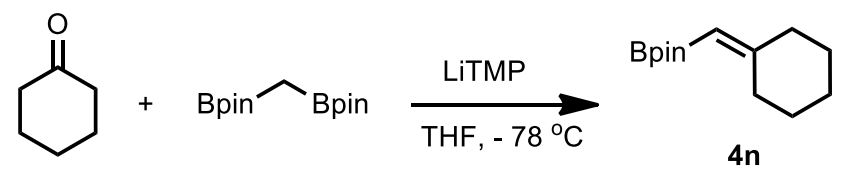

To a solution of 2, 2, 6, 6-tetramethylpiperidine (339 mg, $2.4 \mathrm{mmol})$ in THF (4 mL) was slowly added $n$-BuLi $(1.0 \mathrm{~mL}, 2.5 \mathrm{M}$ in hexane, $2.4 \mathrm{mmol})$ at $0{ }^{\circ} \mathrm{C}$ under nitrogen atmosphere, and the mixture was stirred at the same temperature for $10 \mathrm{~min}$. After cooled to $-78^{\circ} \mathrm{C}$, a solution of bis[(pinacolato)boryl]methane (643 $\left.\mathrm{mg}, 2.4 \mathrm{mmol}\right)$ in THF $(5 \mathrm{~mL})$ was added dropwise, and the resulting mixture was stirred at $-78^{\circ} \mathrm{C}$ for another $30 \mathrm{~min}$. Then, a 
solution of cyclohexanone $(197 \mathrm{mg}, 2.0 \mathrm{mmol})$ in THF $(3 \mathrm{~mL})$ was added dropwise at $-78^{\circ} \mathrm{C}$, and stirred at the same temperature for $4 \mathrm{~h}$. The reaction was allowed to slowly warm up to room temperature in 4 hours. At $0{ }^{\circ} \mathrm{C}$, saturated aqueous $\mathrm{NH}_{4} \mathrm{Cl}(50 \mathrm{~mL})$ was added dropwise to quench this reation. The mixture was stirred for $1 \mathrm{~h}$, filtered and concentrated. Water $(50 \mathrm{~mL})$ was added to the obtained residue, and the aqueous layer was extracted with EtOAc $(3 \times 50 \mathrm{~mL})$. The combined organic phases were washed with brine $(100 \mathrm{~mL})$, dried over $\mathrm{Na}_{2} \mathrm{SO}_{4}$, filtered and evaporated. The crude product was purified by flash column chromatography (petroleum ether/ethyl acetate $=80 / 1)$ to afford $\mathbf{4 n}$ as a colorless oil $(370 \mathrm{mg}, 83 \%) .{ }^{10}{ }^{1} \mathrm{H} \mathrm{NMR}\left(400 \mathrm{MHz}, \mathrm{CDCl}_{3}\right) \delta 5.01(\mathrm{~s}, 1 \mathrm{H}), 2.50$ $(\mathrm{t}, J=6.1 \mathrm{~Hz}, 2 \mathrm{H}), 2.18(\mathrm{t}, J=6.1 \mathrm{~Hz}, 2 \mathrm{H}), 1.61-1.50(\mathrm{~m}, 6 \mathrm{H}), 1.25(\mathrm{~s}, 12 \mathrm{H})$.

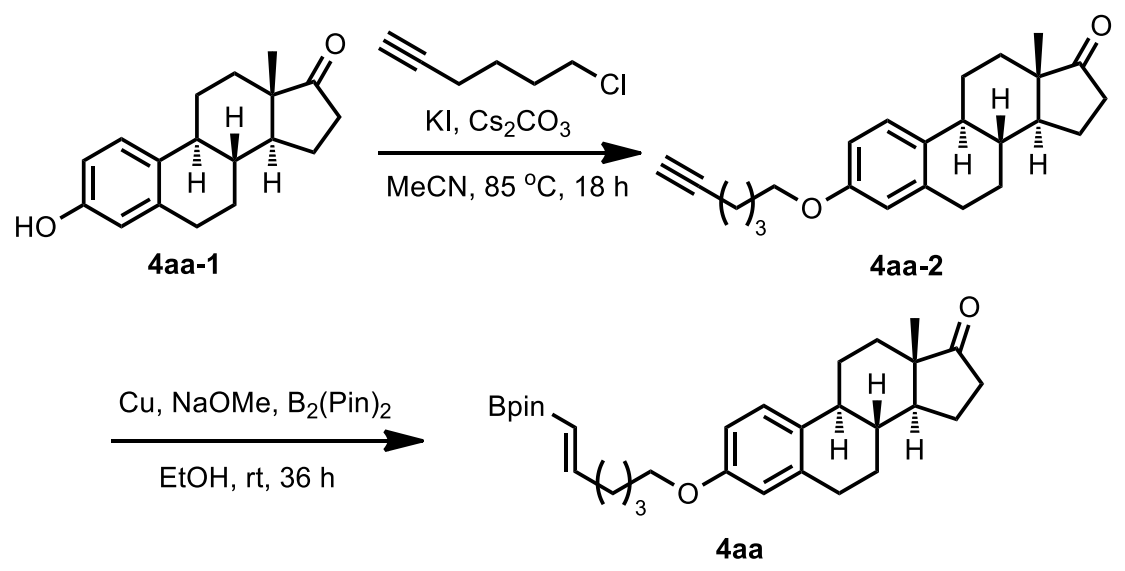

Under nitrogen atmosphere, a reaction mixture of 1,3,5(10)-Estratrien-3-ol-17-one (1.4 g, $5.0 \mathrm{mmol})$, 5-chloropent-1-yne (641 mg, $5.5 \mathrm{mmol}), \mathrm{KI}(83 \mathrm{mg}, 0.5 \mathrm{mmol})$ and $\mathrm{Cs}_{2} \mathrm{CO}_{3}(2.5 \mathrm{~g}, 7.5 \mathrm{mmol})$ in $\mathrm{MeCN}(20 \mathrm{~mL})$ was stirred for $18 \mathrm{~h}$ at $85{ }^{\circ} \mathrm{C}$. The reaction mixture was poured into water $(100 \mathrm{~mL})$ and extracted with EtOAc $(3 \times$ $100 \mathrm{~mL})$. The combined organic phases were washed with brine $(100 \mathrm{~mL})$, dried over $\mathrm{Na}_{2} \mathrm{SO}_{4}$, filtered and evaporated. The crude product was purified by flash column chromatography on silica gel (petroleum ether/ethyl acetate $=5 / 1)$ to afford $\mathbf{4 a a - 2}$ as white solid $(1.56 \mathrm{~g}, 89 \%)$. mp $94-96{ }^{\circ} \mathrm{C} ;{ }^{1} \mathrm{H} \mathrm{NMR}\left(400 \mathrm{MHz}, \mathrm{CDCl}_{3}\right) \delta 7.18$ $(\mathrm{d}, J=8.5 \mathrm{~Hz}, 1 \mathrm{H}), 6.69(\mathrm{~d}, J=8.4 \mathrm{~Hz}, 1 \mathrm{H}), 6.63(\mathrm{~s}, 1 \mathrm{H}), 3.95(\mathrm{t}, J=6.2 \mathrm{~Hz}, 2 \mathrm{H}), 2.92-2.85(\mathrm{~m}, 2 \mathrm{H}), 2.52-$ $2.45(\mathrm{~m}, 1 \mathrm{H}), 2.40-2.35(\mathrm{~m}, 1 \mathrm{H}), 2.28-1.85(\mathrm{~m}, 9 \mathrm{H}), 1.76-1.40(\mathrm{~m}, 8 \mathrm{H}), 0.90(\mathrm{~s}, 3 \mathrm{H}) ;{ }^{13} \mathrm{C} \mathrm{NMR}(100 \mathrm{MHz}$, $\left.\mathrm{CDCl}_{3}\right) \delta 220.8,156.9,137.6,131.9,126.2,114.4,112.0,84.0,68.6,67.1,50.3,47.9,43.9,38.3,35.8,31.5$, 29.6, 28.2, 26.5, 25.8, 25.0, 21.5, 18.1, 13.8; HRMS (EI) $\mathrm{m} / z$ Calcd for $\mathrm{C}_{24} \mathrm{H}_{30} \mathrm{O}_{2}$ [M]: 350.2246, found: 350.2245 .

A stirring bar, $\mathrm{B}_{2} \mathrm{Pin}_{2}(1.36 \mathrm{~g}, 5.35 \mathrm{mmol})$, micro copper powder (29 mg, $\left.0.45 \mathrm{mmol}\right)$, and $\mathrm{NaOMe}(49 \mathrm{mg}, 0.9$ mmol) were added to an oven dried $100 \mathrm{~mL}$ Schlenk tube. The tube was capped and back-filled with nitrogen (3 times). Under the nitrogen flow, 4aa-2 $(1.56 \mathrm{~g}, 4.46 \mathrm{mmol})$ and EtOH $(20 \mathrm{~mL})$ were added to the mixture, and the tube was capped tightly again. Then the reaction mixture was stirred for $36 \mathrm{~h}$ at room temperature. After that, the mixture was poured into water $(100 \mathrm{~mL})$, extracted with DCM $(3 \times 100 \mathrm{~mL})$. The combined organic phases were dried over $\mathrm{Na}_{2} \mathrm{SO}_{4}$, concentrated under vacuum, and purified by column chromatography on silica gel (petroleum ether/ethyl acetate $=30 / 1)$ to afford 4 aa as white solid $(592 \mathrm{mg}, 28 \%) . \mathrm{mp} 115-117{ }^{\circ} \mathrm{C} ;{ }^{1} \mathrm{H} \mathrm{NMR}$ $\left(400 \mathrm{MHz}, \mathrm{CDCl}_{3}\right) \delta 7.17(\mathrm{~d}, J=8.6 \mathrm{~Hz}, 1 \mathrm{H}), 6.73-6.59(\mathrm{~m}, 3 \mathrm{H}), 5.46(\mathrm{~d}, J=18.0 \mathrm{~Hz}, 1 \mathrm{H}), 3.92(\mathrm{t}, J=6.4 \mathrm{~Hz}$, 2H), 2.92-2.84 (m, 2H), 2.53-2.45 (m, 1H), 2.41-2.35 (m, 1H), 2.28-1.91 (m, 7H), 1.82-1.73 (m, 2H), $1.65-$ $1.41(\mathrm{~m}, 8 \mathrm{H}), 1.26(\mathrm{~s}, 12 \mathrm{H}), 0.90(\mathrm{~s}, 3 \mathrm{H}) ;{ }^{13} \mathrm{C} \mathrm{NMR}\left(100 \mathrm{MHz}, \mathrm{CDCl}_{3}\right) \delta 220.7,157.0,153.8,137.5,131.7$, 126.1, 114.4, 112.0, 82.9, 67.5, 50.3, 47.9, 43.9, 38.3, 35.8, 35.3, 31.5, 29.5, 28.7, 26.5, 25.8, 24.7, 24.6, 21.5, 13.8; HRMS (EI) $m / z$ Calcd for $\mathrm{C}_{30} \mathrm{H}_{43}(10) \mathrm{BO}_{4}[\mathrm{M}]: 477.3291$, found: 477.3286 . 


\subsection{Optimizations of the Reaction Conditions}

Table S1. Screening of Bases..$^{a, b}$

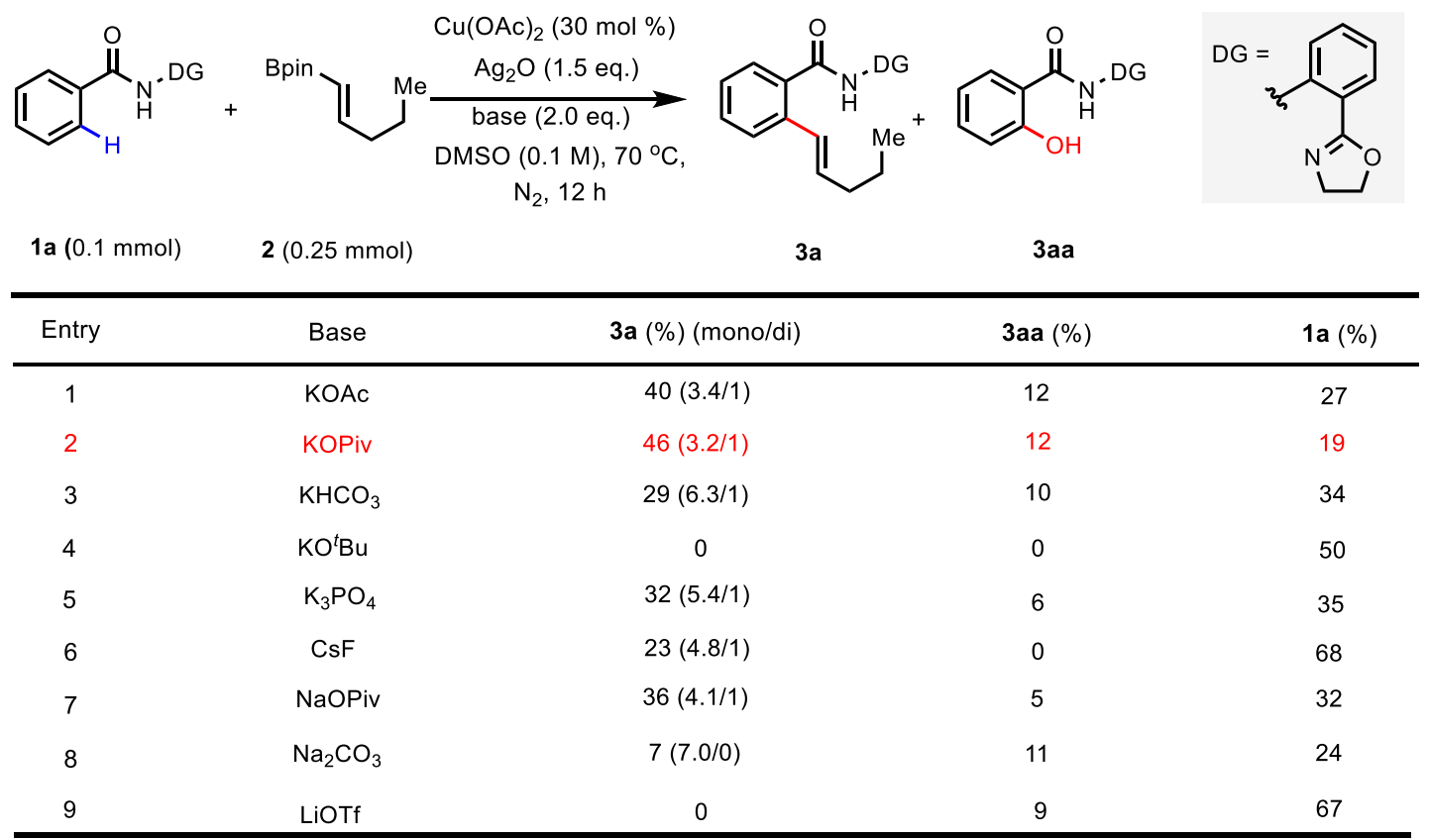

${ }^{a}$ Conditions: 1a $(0.1 \mathrm{mmol}), 2$ (0.25 mmol), $\mathrm{Cu}(\mathrm{OAc})_{2}(0.03 \mathrm{mmol}), \mathrm{Ag}_{2} \mathrm{O}(0.2 \mathrm{mmol})$, base $(0.2 \mathrm{mmol})$, DMSO $(1.0 \mathrm{~mL}), \mathrm{N}_{2}, 70{ }^{\circ} \mathrm{C}, 12 \mathrm{~h} ;{ }^{b}$ The yield was determined by ${ }^{1} \mathrm{H}$ NMR using $\mathrm{CH}_{2} \mathrm{Br}_{2}$ as an internal standard.

Table S2. Screening of Double Bases. ${ }^{a, b}$

\begin{tabular}{|c|c|c|c|c|c|c|c|c|c|}
\hline \multicolumn{2}{|c|}{$1 \mathrm{a}(0.1 \mathrm{mmol})$} & $\underbrace{\mathrm{Bpin}}_{\mathrm{DM}}$ & $\begin{array}{l}\mathrm{Ac})_{2}(30 \\
\mathrm{g}_{2} \mathrm{O}(1.5 \\
\text { Piv }(2.0 \\
\text { ase }(2.0 \mathrm{e} \\
\mathrm{O}(0.1 \mathrm{M}) \\
\mathrm{N}_{2}, 12\end{array}$ & $\begin{array}{l}\text { mol \%) } \\
\text { eq.) } \\
\underset{\text { eq.) }}{\longrightarrow} \\
\text { q.) } \\
\text { h } 70^{\circ} \mathrm{C} \text {, }\end{array}$ & & & 只 & $\mathrm{DG}=$ & \\
\hline Entry & Base & 3a (\%) (mono/di) & 3aa $(\%)$ & $1 \mathrm{a}(\%)$ & Entry & Base & $3 \mathbf{a}(\%)(\mathrm{mono} / \mathrm{di})$ & 3aa $(\%)$ & $1 \mathrm{a}(\%)$ \\
\hline 1 & LiOTf & $26(3.3 / 1)$ & 16 & 50 & 8 & $\mathrm{NaO}^{t} \mathrm{Bu}$ & $10(10 / 0)$ & 0 & 80 \\
\hline 2 & $\mathrm{Na}_{2} \mathrm{CO}_{3}$ & $62(2.4 / 1)$ & 13 & 18 & 9 & $\mathrm{~K}_{2} \mathrm{CO}_{3}$ & $58(2.4 / 1)$ & 14 & 20 \\
\hline 3 & $\mathrm{NaHCO}_{3}$ & $56(2.5 / 1)$ & 13 & 18 & 10 & $\mathrm{KHCO}_{3}$ & $66(2.1 / 1)$ & 12 & 18 \\
\hline 4 & $\mathrm{NaBF}_{4}$ & $49(4.4 / 1)$ & 8 & 36 & 11 & $\mathrm{~K}_{3} \mathrm{PO}_{4}$ & $45(3.5 / 1)$ & 17 & 24 \\
\hline 5 & $\mathrm{HCO}_{2} \mathrm{Na}$ & $5(5 / 0)$ & 0 & 90 & 12 & $\mathrm{CsHCO}_{3}$ & $58(2.2 / 1)$ & 13 & 17 \\
\hline 6 & NaTFA & $27(3.5 / 1)$ & 17 & 30 & 13 & $\mathrm{CsF}$ & $59(2.7 / 1)$ & 13 & 21 \\
\hline 7 & $\mathrm{NaOH}$ & $38(3.8 / 1)$ & 16 & 29 & 14 & DBU & $41(3.6 / 1)$ & 5 & 40 \\
\hline
\end{tabular}

${ }^{a}$ Conditions: $1 \mathrm{a}(0.1 \mathrm{mmol}), 2(0.25 \mathrm{mmol}), \mathrm{Cu}(\mathrm{OAc})_{2}(0.03 \mathrm{mmol}), \mathrm{Ag}_{2} \mathrm{O}(0.2 \mathrm{mmol}), \mathrm{KOPiv}(0.2 \mathrm{mmol})$, base (0.2 mmol), DMSO $(1.0 \mathrm{~mL}), \mathrm{N}_{2}, 70{ }^{\circ} \mathrm{C}, 12 \mathrm{~h} ;{ }^{b}$ The yield was determined by ${ }^{1} \mathrm{H}$ NMR using $\mathrm{CH}_{2} \mathrm{Br}_{2}$ as an internal standard. 
Table S3. Screening of the Ratio of the Double Bases. ${ }^{a, b}$

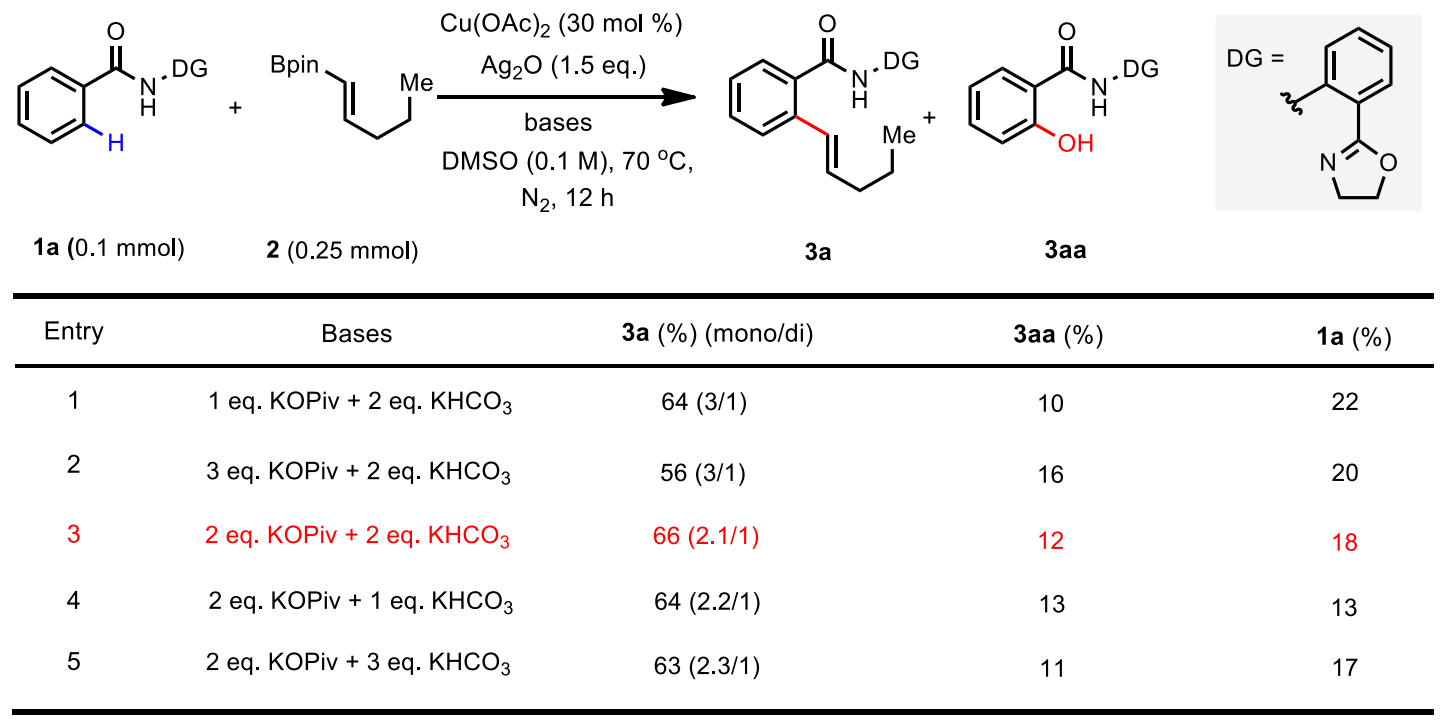

${ }^{a}$ Conditions: 1a $(0.1 \mathrm{mmol}), 2(0.25 \mathrm{mmol}), \mathrm{Cu}(\mathrm{OAc})_{2}(0.03 \mathrm{mmol}), \mathrm{Ag}_{2} \mathrm{O}(0.2 \mathrm{mmol})$, bases, DMSO $(1.0 \mathrm{~mL})$, $\mathrm{N}_{2}, 70{ }^{\circ} \mathrm{C}, 12 \mathrm{~h}$; ${ }^{b}$ The yield was determined by ${ }^{1} \mathrm{H}$ NMR using $\mathrm{CH}_{2} \mathrm{Br}_{2}$ as an internal standard.

Table S4. Screening of Cu Salts. ${ }^{a, b}$

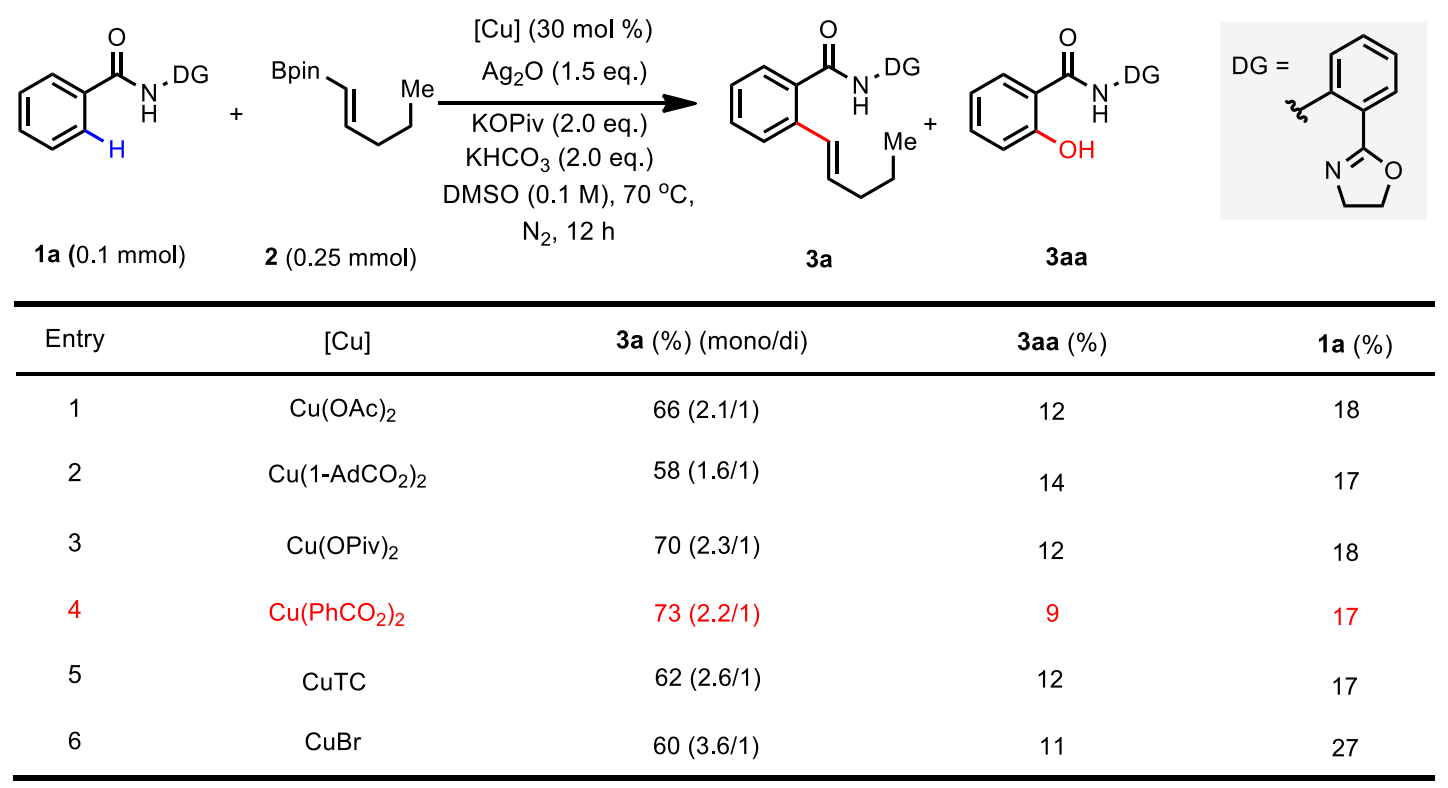

${ }^{a}$ Conditions: $1 \mathrm{a}(0.1 \mathrm{mmol}), 2(0.25 \mathrm{mmol}),[\mathrm{Cu}](0.03 \mathrm{mmol}), \mathrm{Ag}_{2} \mathrm{O}(0.2 \mathrm{mmol}), \mathrm{KOPiv}(0.2 \mathrm{mmol}), \mathrm{KHCO}_{3}$ $(0.2 \mathrm{mmol})$, DMSO $(1.0 \mathrm{~mL}), \mathrm{N}_{2}, 70{ }^{\circ} \mathrm{C}, 12 \mathrm{~h} ;{ }^{b}$ The yield was determined by ${ }^{1} \mathrm{H}$ NMR using $\mathrm{CH}_{2} \mathrm{Br}_{2}$ as an internal standard. 
Table S5. Screening of Temperature..$^{a, b}$

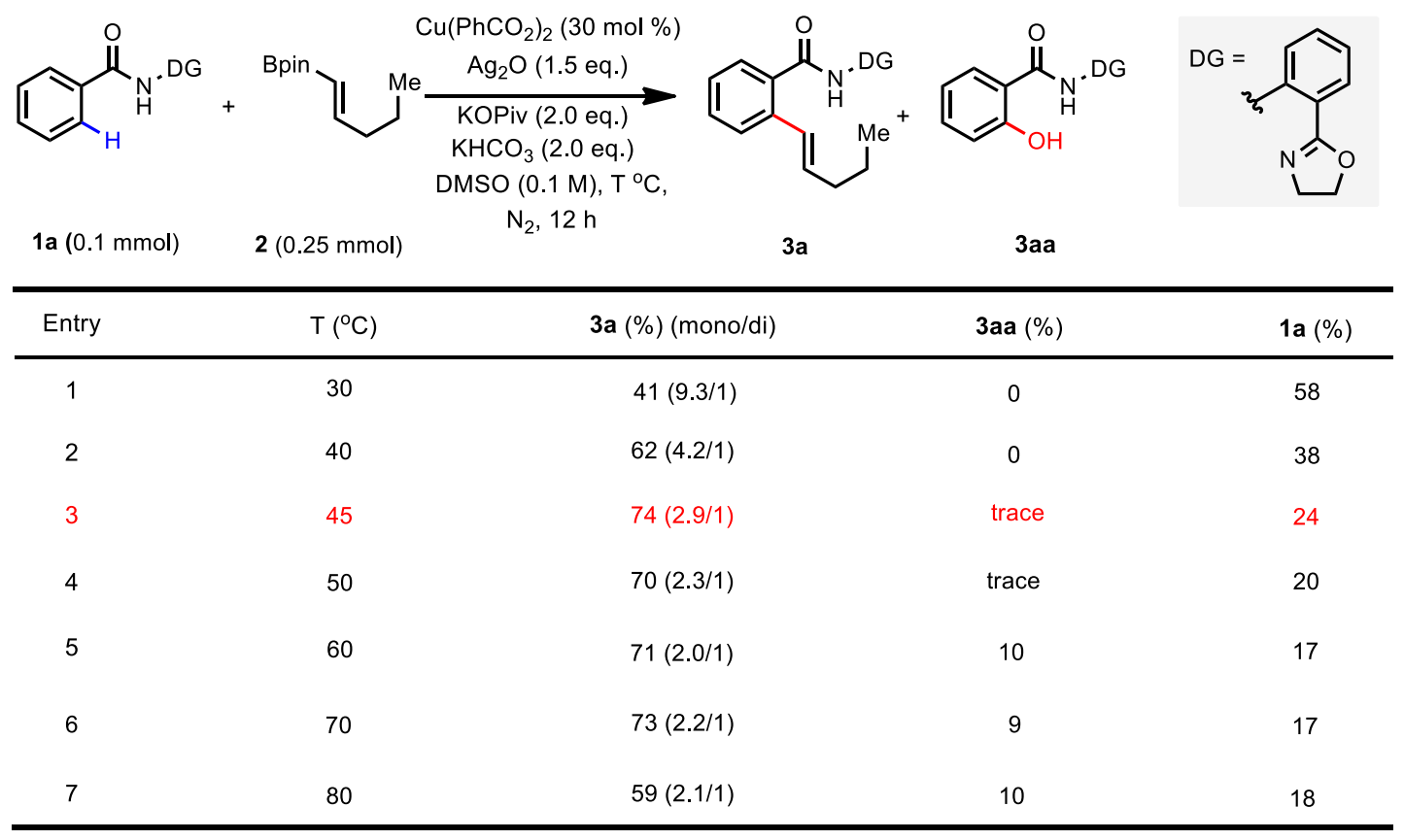

${ }^{a}$ Conditions: $1 \mathrm{a}(0.1 \mathrm{mmol}), 2(0.25 \mathrm{mmol}), \mathrm{Cu}\left(\mathrm{PhCO}_{2}\right)_{2}(0.03 \mathrm{mmol}), \mathrm{Ag}_{2} \mathrm{O}(0.2 \mathrm{mmol}), \mathrm{KOPiv}(0.2 \mathrm{mmol})$, $\mathrm{KHCO}_{3}(0.2 \mathrm{mmol})$, DMSO $(1.0 \mathrm{~mL}), \mathrm{N}_{2}, \mathrm{~T}{ }^{\circ} \mathrm{C}, 12 \mathrm{~h}$; ${ }^{b}$ The yield was determined by ${ }^{1} \mathrm{H}$ NMR using $\mathrm{CH}_{2} \mathrm{Br}_{2}$ as an internal standard.

Table S6. Screening of Solvents. ${ }^{a, b}$

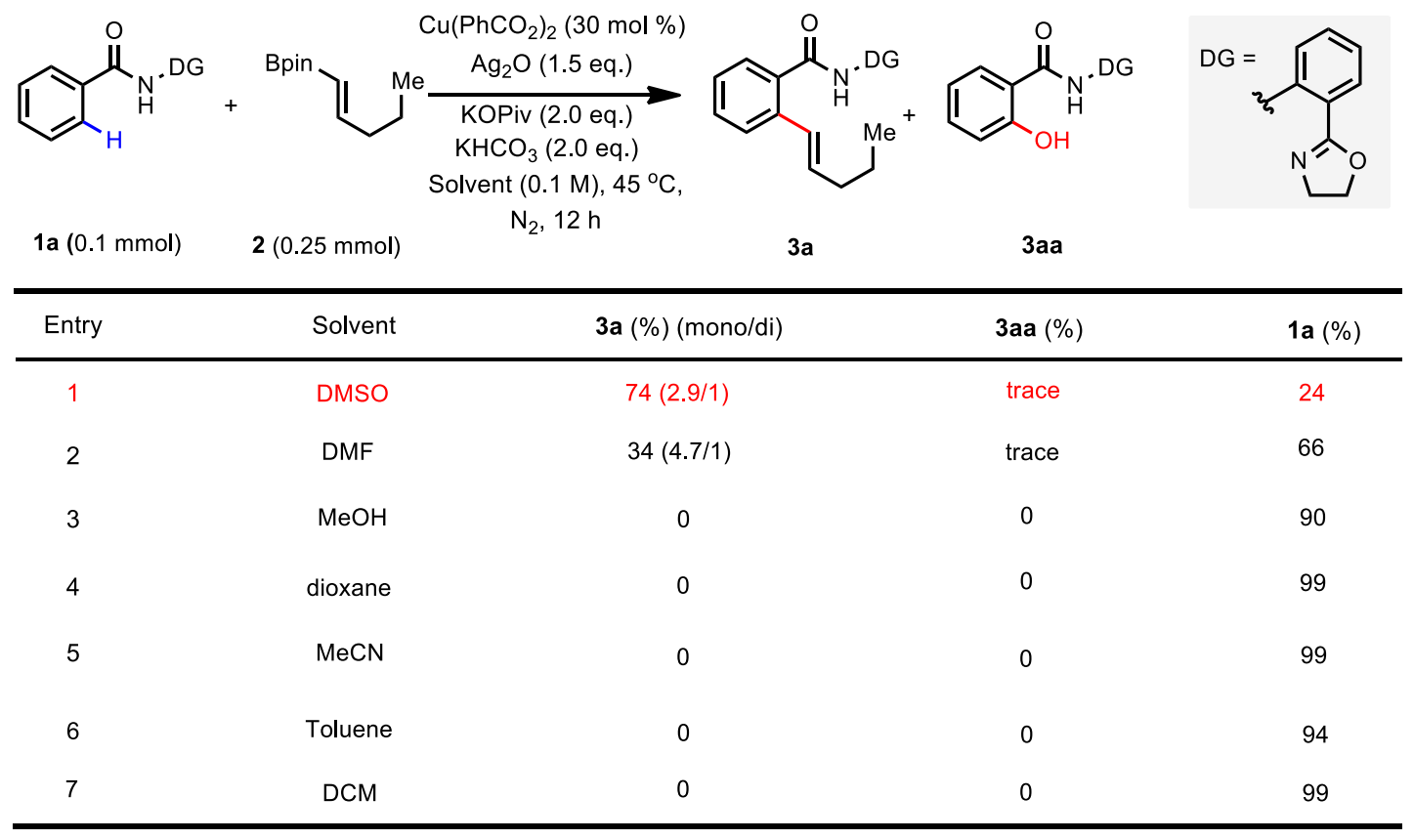

${ }^{a}$ Conditions: 1a $(0.1 \mathrm{mmol}), 2(0.25 \mathrm{mmol}), \mathrm{Cu}\left(\mathrm{PhCO}_{2}\right)_{2}(0.03 \mathrm{mmol}), \mathrm{Ag}_{2} \mathrm{O}(0.2 \mathrm{mmol}), \mathrm{KOPiv}(0.2 \mathrm{mmol})$, $\mathrm{KHCO}_{3}(0.2 \mathrm{mmol})$, Solvent $(1.0 \mathrm{~mL}), \mathrm{N}_{2}, 45{ }^{\circ} \mathrm{C}, 12 \mathrm{~h} ;{ }^{b}$ The yield was determined by ${ }^{1} \mathrm{H}$ NMR using $\mathrm{CH}_{2} \mathrm{Br}_{2}$ as an internal standard. 
Table S7. Screening of Concentration. ${ }^{a, b}$

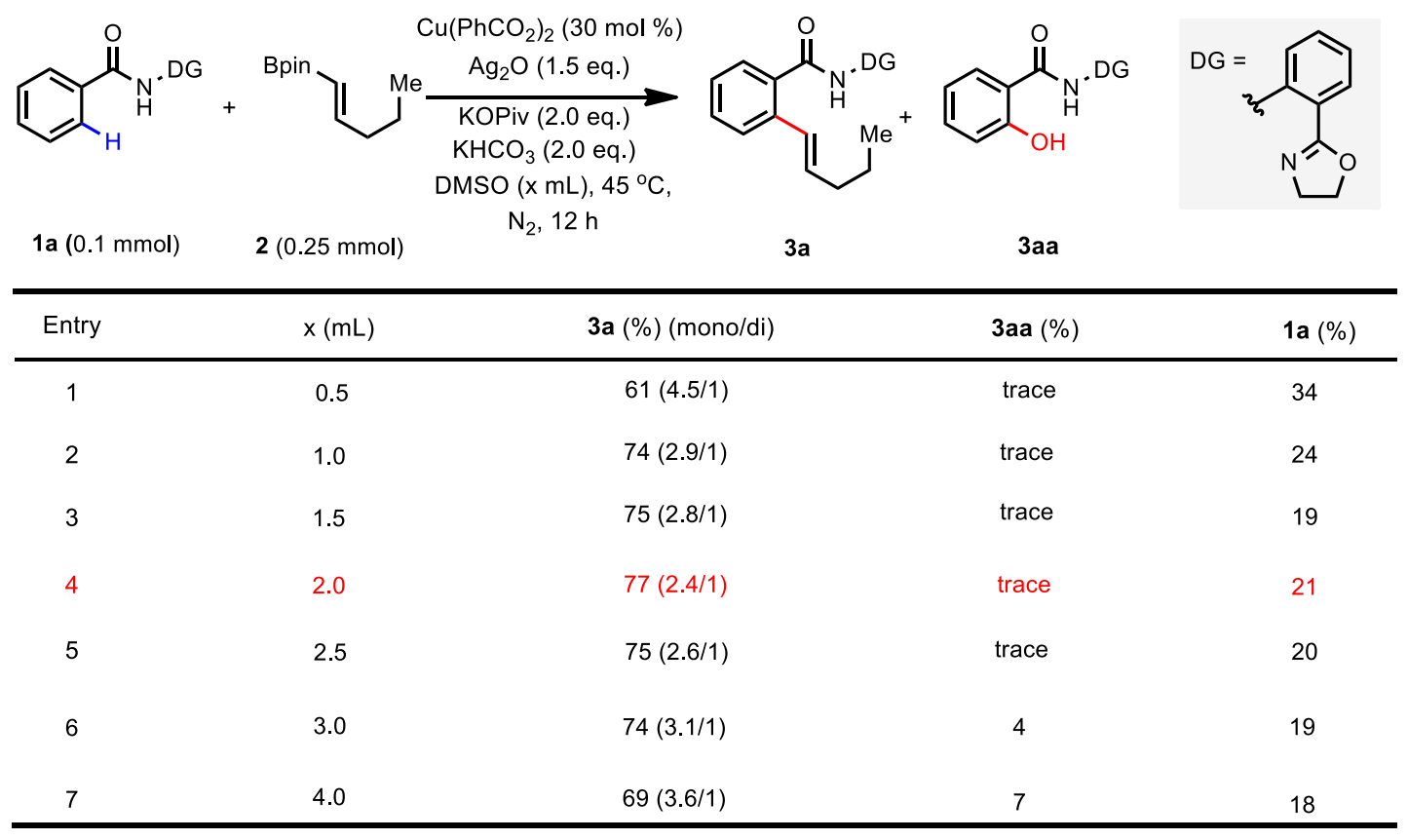

${ }^{a}$ Conditions: 1a $(0.1 \mathrm{mmol}), 2(0.25 \mathrm{mmol}), \mathrm{Cu}\left(\mathrm{PhCO}_{2}\right)_{2}(0.03 \mathrm{mmol}), \mathrm{Ag}_{2} \mathrm{O}(0.2 \mathrm{mmol}), \mathrm{KOPiv}(0.2 \mathrm{mmol})$, $\mathrm{KHCO}_{3}(0.2 \mathrm{mmol})$, DMSO (x mL), $\mathrm{N}_{2}, 45^{\circ} \mathrm{C}, 12 \mathrm{~h}$; ${ }^{b}$ The yield was determined by ${ }^{1} \mathrm{H} \mathrm{NMR}$ using $\mathrm{CH}_{2} \mathrm{Br}_{2}$ as an internal standard.

Table S8. Screening of the Loading of $\mathrm{Cu}\left(\mathrm{PhCO}_{2}\right)_{2}{ }^{a, b}$

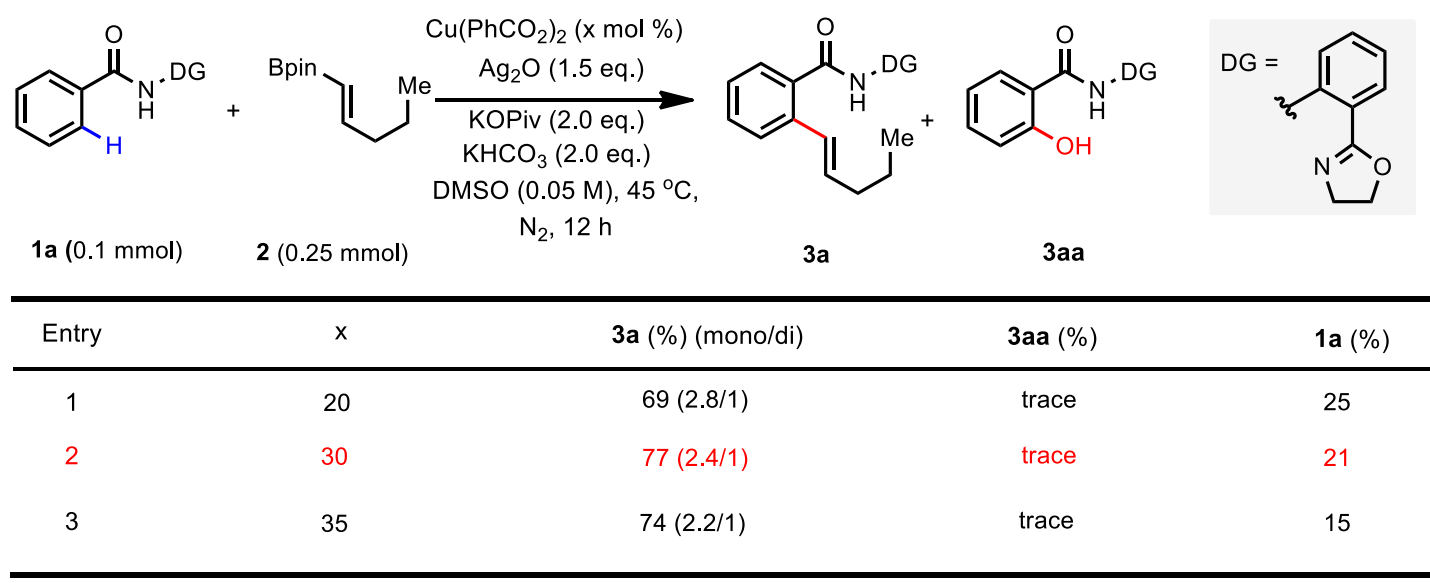

${ }^{a}$ Conditions: $1 \mathrm{a}(0.1 \mathrm{mmol}), 2(0.25 \mathrm{mmol}), \mathrm{Cu}\left(\mathrm{PhCO}_{2}\right)_{2}, \mathrm{Ag}_{2} \mathrm{O}(0.2 \mathrm{mmol}), \mathrm{KOPiv}(0.2 \mathrm{mmol}), \mathrm{KHCO}_{3}(0.2$ mmol), DMSO $(2.0 \mathrm{~mL}), \mathrm{N}_{2}, 45{ }^{\circ} \mathrm{C}, 12 \mathrm{~h}$; ${ }^{b}$ The yield was determined by ${ }^{1} \mathrm{H} \mathrm{NMR} \mathrm{using} \mathrm{CH}_{2} \mathrm{Br}_{2}$ as an internal standard. 
Table S9. Screening of Oxidants. ${ }^{a, b}$

\begin{tabular}{|c|c|c|c|c|}
\hline $\mathrm{N}_{H}$ & $+\underbrace{\mathrm{Bpin}} \underbrace{\mathrm{Me}}_{\mathrm{DMS}} \frac{\mathrm{KC}(\mathrm{Ph}}{\mathrm{KC}}$ & $\begin{array}{l}\text { mol \%) } \\
\underset{\text { eq.) }}{\longrightarrow} \\
\text { eq.) } \\
\text {, } 45^{\circ} \mathrm{C} \text {, }\end{array}$ & لI & \\
\hline Entry & [O] & 3a (\%) (mono/di) & 3aa (\%) & $1 \mathrm{a}(\%)$ \\
\hline 1 & $\mathrm{Ag}_{2} \mathrm{O}$ & $77(2.4 / 1)$ & trace & 21 \\
\hline 2 & $\mathrm{AgOAC}$ & $25(4 / 1)$ & 11 & 5 \\
\hline 3 & $\mathrm{Ag}_{2} \mathrm{CO}_{3}$ & $43(4.4 / 1)$ & 8 & 38 \\
\hline 4 & Selectfluor & 0 & 0 & 99 \\
\hline 5 & NMO & 0 & 0 & 99 \\
\hline 6 & $\mathrm{O}_{2}$ & 0 & trace & 99 \\
\hline 7 & Air & 0 & 23 & 65 \\
\hline 8 & $\mathrm{Ag}_{2} \mathrm{O}+$ Air atmosphere & $65(3.1 / 1)$ & 8 & 20 \\
\hline
\end{tabular}

${ }^{a}$ Conditions: $1 \mathrm{a}(0.1 \mathrm{mmol}), 2(0.25 \mathrm{mmol}), \mathrm{Cu}\left(\mathrm{PhCO}_{2}\right)_{2}(0.03 \mathrm{mmol}),[\mathrm{O}](0.2 \mathrm{mmol}), \mathrm{KOPiv}(0.2 \mathrm{mmol})$, $\mathrm{KHCO}_{3}(0.2 \mathrm{mmol})$, DMSO $(2.0 \mathrm{~mL}), \mathrm{N}_{2}, 45{ }^{\circ} \mathrm{C}, 12 \mathrm{~h}$; ${ }^{b}$ The yield was determined by ${ }^{1} \mathrm{H} \mathrm{NMR}$ using $\mathrm{CH}_{2} \mathrm{Br}_{2}$ as an internal standard.

Table S10. Screening of the Loading of Oxidants. ${ }^{a, b}$

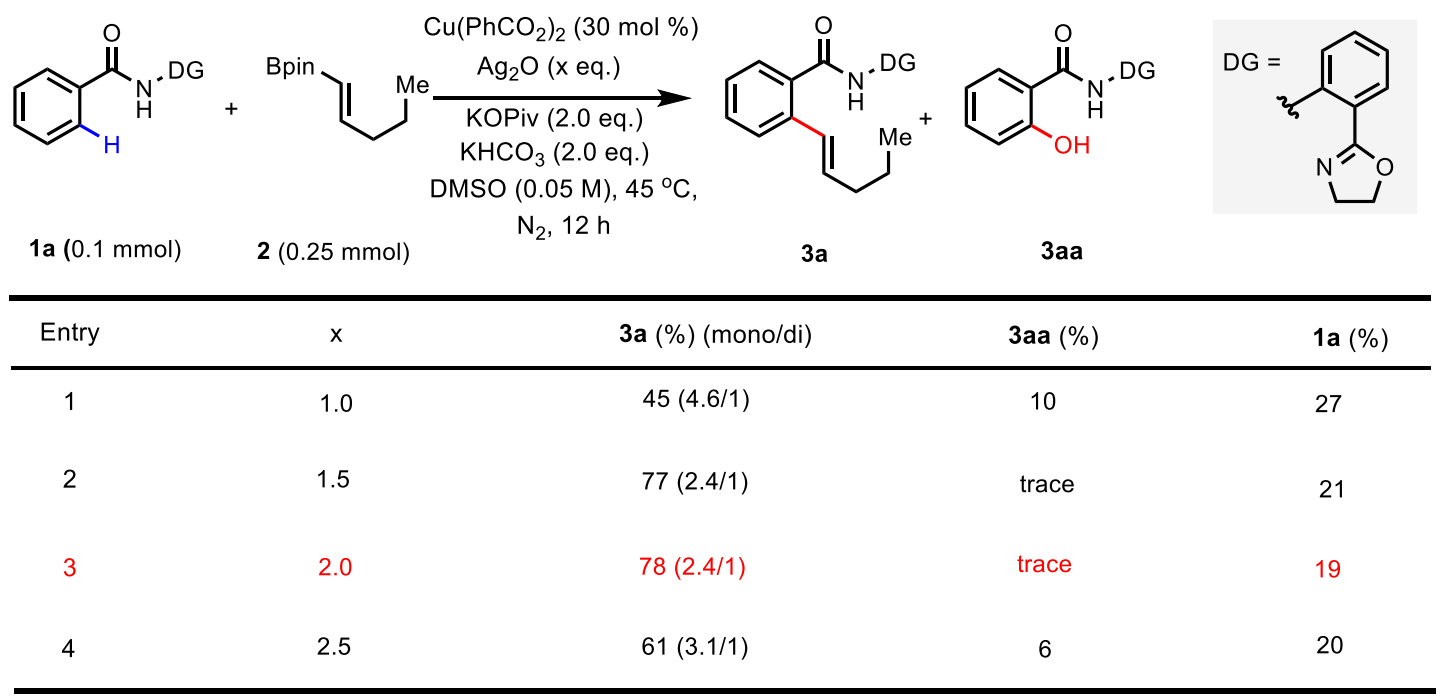

${ }^{a}$ Conditions: $1 \mathrm{a}(0.1 \mathrm{mmol}), 2(0.25 \mathrm{mmol}), \mathrm{Cu}\left(\mathrm{PhCO}_{2}\right)_{2}(0.03 \mathrm{mmol}), \mathrm{Ag}_{2} \mathrm{O}, \mathrm{KOPiv}(0.2 \mathrm{mmol}), \mathrm{KHCO}_{3}(0.2$ mmol), DMSO $(2.0 \mathrm{~mL}), \mathrm{N}_{2}, 45^{\circ} \mathrm{C}, 12 \mathrm{~h}$; ${ }^{b}$ The yield was determined by ${ }^{1} \mathrm{H}$ NMR using $\mathrm{CH}_{2} \mathrm{Br}_{2}$ as an internal standard. 
Table S11. Screening of the Loading of $2^{a, b}$

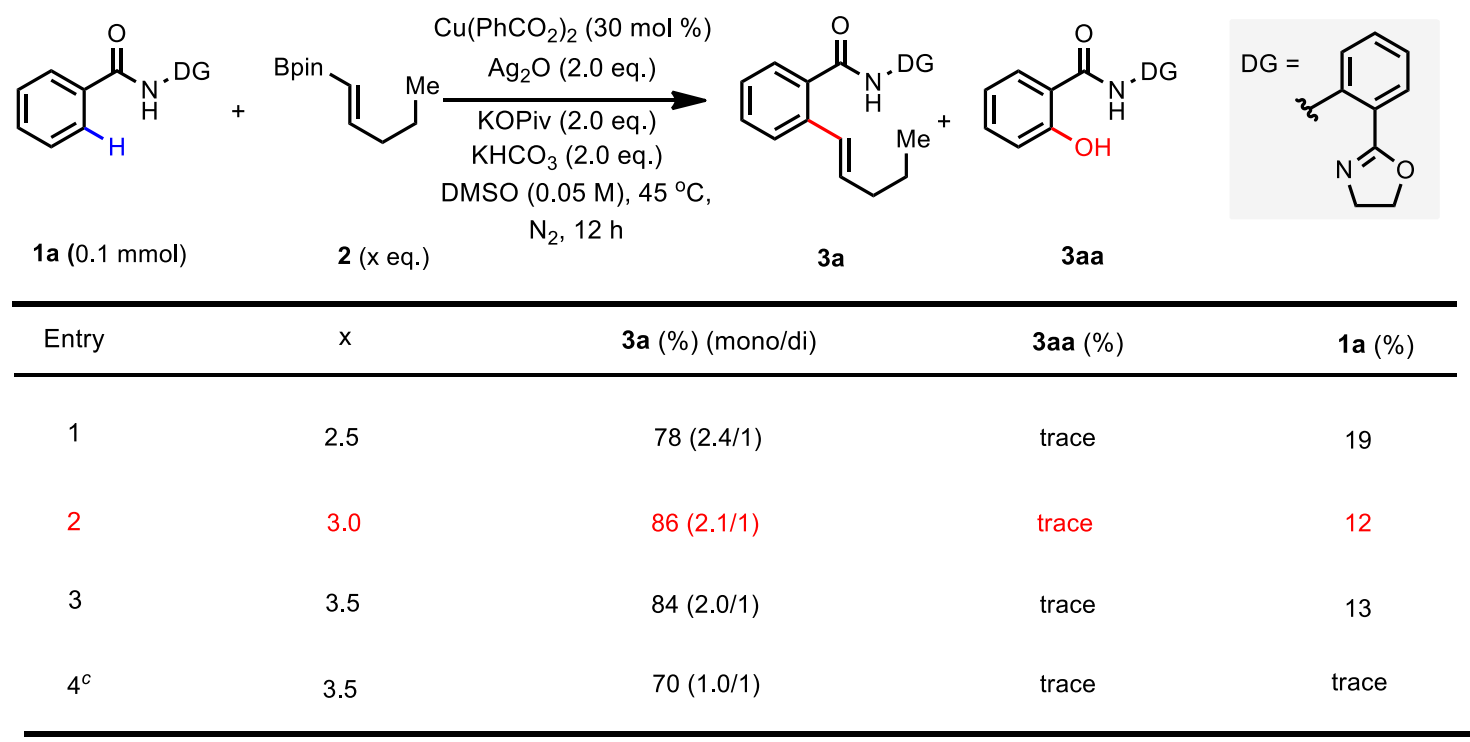

${ }^{a}$ Conditions: $1 \mathrm{a}(0.1 \mathrm{mmol}), 2$ (x eq.), $\mathrm{Cu}\left(\mathrm{PhCO}_{2}\right)_{2}(0.03 \mathrm{mmol}), \mathrm{Ag}_{2} \mathrm{O}(0.2 \mathrm{mmol}), \mathrm{KOPiv}(0.2 \mathrm{mmol}), \mathrm{KHCO}_{3}$ $(0.2 \mathrm{mmol})$, DMSO $(2.0 \mathrm{~mL}), \mathrm{N}_{2}, 45{ }^{\circ} \mathrm{C}, 12 \mathrm{~h}$; ${ }^{b}$ The yield was determined by ${ }^{1} \mathrm{H}$ NMR using $\mathrm{CH}_{2} \mathrm{Br}_{2}$ as an internal standard; ${ }^{c} 36 \mathrm{~h}$.

Table S12. Screening of the Reaction Time. ${ }^{a, b}$

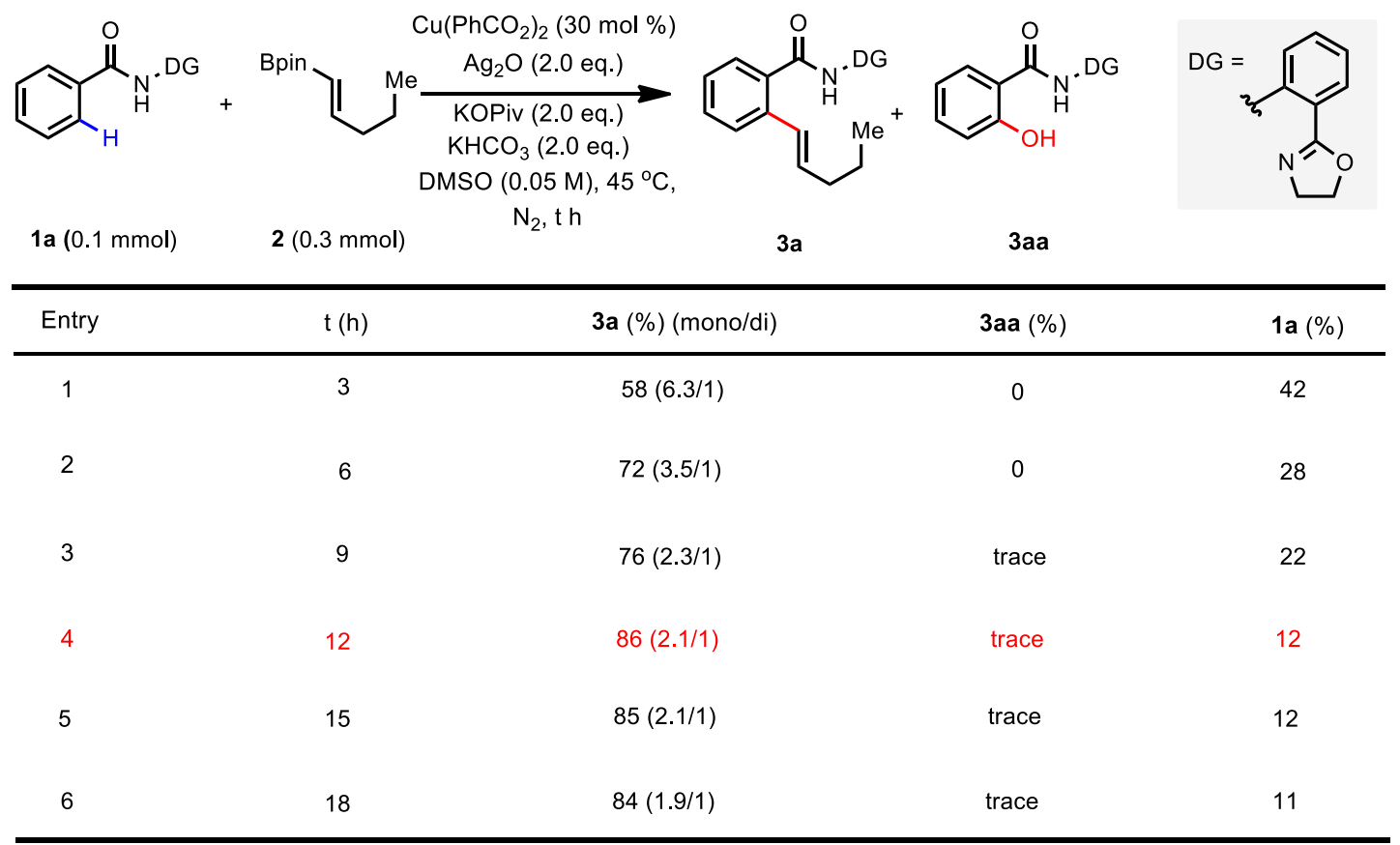

${ }^{a}$ Conditions: 1a $(0.1 \mathrm{mmol}), 2(0.3 \mathrm{mmol}), \mathrm{Cu}\left(\mathrm{PhCO}_{2}\right)_{2}(0.03 \mathrm{mmol}), \mathrm{Ag}_{2} \mathrm{O}(0.2 \mathrm{mmol}), \mathrm{KOPiv}(0.2 \mathrm{mmol})$, $\mathrm{KHCO}_{3}(0.2 \mathrm{mmol})$, DMSO $(2.0 \mathrm{~mL}), \mathrm{N}_{2}, 45{ }^{\circ} \mathrm{C}, \mathrm{t} \mathrm{h} ;{ }^{b}$ The yield was determined by ${ }^{1} \mathrm{H}$ NMR using $\mathrm{CH}_{2} \mathrm{Br}_{2}$ as an internal standard. 
Table S13. Re-comparation of Copper Salts. ${ }^{a, b}$

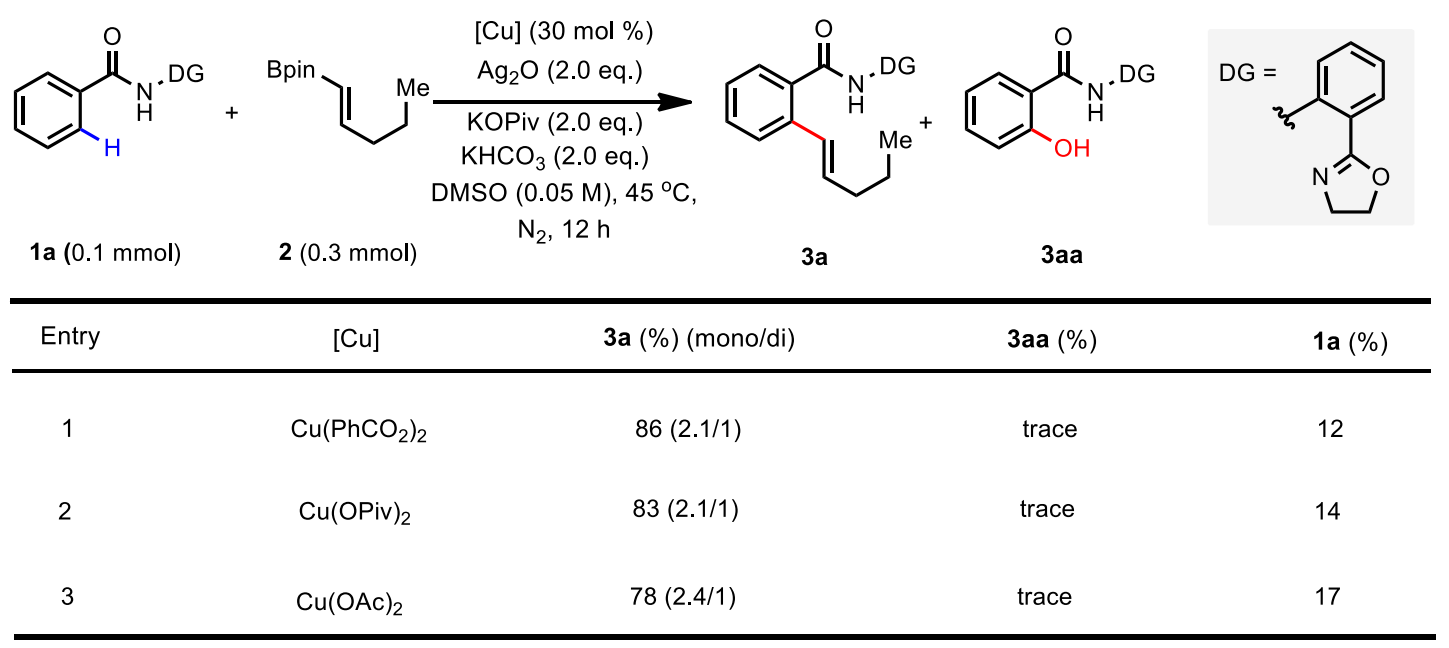

${ }^{a}$ Conditions: 1a $(0.1 \mathrm{mmol}), 2(0.3 \mathrm{mmol}),[\mathrm{Cu}](0.03 \mathrm{mmol}), \mathrm{Ag}_{2} \mathrm{O}(0.2 \mathrm{mmol}), \mathrm{KOPiv}(0.2 \mathrm{mmol}), \mathrm{KHCO}_{3}$ $(0.2 \mathrm{mmol})$, DMSO $(2.0 \mathrm{~mL}), \mathrm{N}_{2}, 45^{\circ} \mathrm{C}, 12 \mathrm{~h}$; ${ }^{b}$ The yield was determined by ${ }^{1} \mathrm{H}$ NMR using $\mathrm{CH}_{2} \mathrm{Br}_{2}$ as an internal standard.

Table S14. Control Experiments. ${ }^{a, b}$

\begin{tabular}{|c|c|c|c|c|c|c|}
\hline$(0.1 \mathrm{~m}$ & $\mathrm{nmol}$ ) & 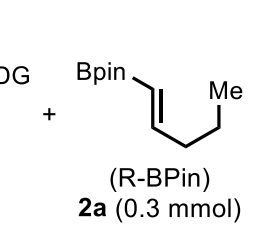 & $\begin{array}{r}\left(\mathrm{PhCO}_{2}\right)_{2} \mathrm{Cu}(30 \mathrm{r} \\
\mathrm{Ag}_{2} \mathrm{O}(2.0 \mathrm{equ} \\
\mathrm{KOPiv}(2.0 \mathrm{eq} \\
\mathrm{KHCO}_{3}(2.0 \mathrm{eq} \\
\mathrm{DMSO}, \mathrm{N}_{2}, 45^{\circ} \mathrm{C}\end{array}$ & 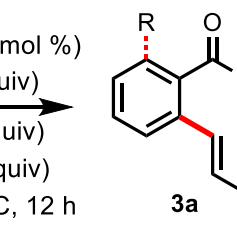 & $\underbrace{1}_{4 a}$ & (1) \\
\hline Entry & & eviation from optimal co & nditions & $(\%)$ of $\mathbf{3 a}(\mathrm{mono} / \mathrm{di})$ & Yield $(\%)$ of $\mathbf{4 a}$ & Yield $(\%)$ of $1 \mathrm{a}$ \\
\hline 1 & & none & & $86(2.1 / 1.0)$ & trace & 12 \\
\hline 2 & & $\mathrm{No}\left(\mathrm{PhCO}_{2}\right)_{2} \mathrm{Cu}$ & & N.D. & 0 & 99 \\
\hline 3 & & No KOPiv & & $6(6.0 / 0)$ & 0 & 93 \\
\hline 4 & & $\mathrm{No} \mathrm{KHCO}_{3}$ & & $66(2.3 / 1.0)$ & 8 & 10 \\
\hline 5 & & No bases & & 0 & 0 & 99 \\
\hline 6 & & No $\mathrm{Ag}_{2} \mathrm{O}$ & & trace & 0 & 97 \\
\hline 7 & & air instead of $\mathrm{N}_{2}$ & & $70(2.6 / 1.0)$ & 6 & 15 \\
\hline 8 & & air instead of $\mathrm{Ag}_{2} \mathrm{O}$ & & 0 & 23 & 65 \\
\hline 9 & & selectfluor instead of & $\mathrm{Ag}_{2} \mathrm{O}$ & 0 & 0 & 99 \\
\hline 10 & & 1 eq. $\mathrm{Ag}_{2} \mathrm{O}+$ air instea & of $\mathrm{Ag}_{2} \mathrm{O}$ & $15(15 / 0)$ & 15 & 60 \\
\hline 11 & & 1 eq. $\mathrm{Ag}_{2} \mathrm{O}+\mathrm{O}_{2}$ instea & $\mathrm{d}$ of $\mathrm{Ag}_{2} \mathrm{O}$ & $10(10 / 0)$ & 20 & 45 \\
\hline 12 & $0.1 \mathrm{eq}$ & q. $\mathrm{Ag}_{2} \mathrm{O}+2$ eq. selectfl & uor instead of $\mathrm{Ag}_{2} \mathrm{O}$ & 0 & 0 & 95 \\
\hline 13 & $0.1 \mathrm{e}$ & eq. $\mathrm{Ag}_{2} \mathrm{O}+2$ eq. $\mathrm{NMO}$ & instead of $\mathrm{Ag}_{2} \mathrm{O}$ & $4(4 / 0)$ & trace & 83 \\
\hline
\end{tabular}

${ }^{a}$ The yield was determined by ${ }^{1} \mathrm{H}$ NMR using dibromomethane as an internal standard. ${ }^{b}$ Isolated yield.

To validate the role of silver salt in our reaction, control experiments were conducted. The reaction cannot proceed in the absence of copper(II) benzoate and bases, and trace amount of the desired alkenylation product 
were observed in the absence of $\mathrm{Ag}_{2} \mathrm{O}$. In addition, 23\% of hydroxylation byproduct was formed using oxygen as oxidant instead of $\mathrm{Ag}_{2} \mathrm{O}$. The formation of hydroxylation byproduct, probably derived from Cu-catalyzed C$\mathrm{H}$ acetoxylation followed by a rapid hydrolysis (J. Org. Chem. 2015, 80, 8843), indicated a Cu-involved C-H activation process rather than that of $\mathrm{Ag}$.

Given that only trace amount of desired product was observed using a catalytic amount of Ag with other oxidants, we hypothesized that the silver could serve as not only an oxidant but also an activator to accelerate the transmetalation of vinyl borons. 


\subsection{Evaluation of Benzamide Derivatives}

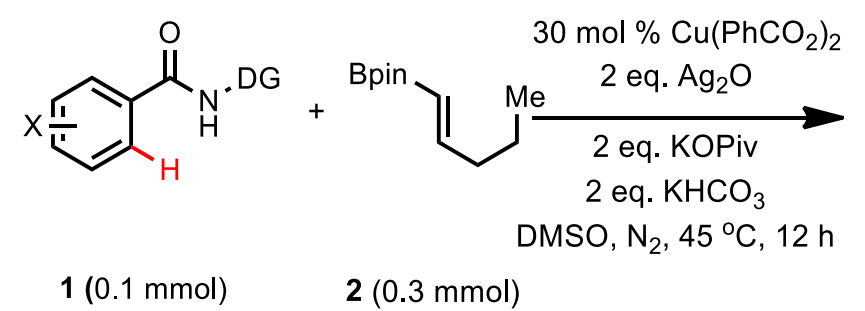

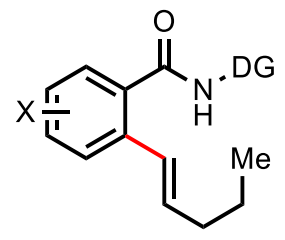

3

\section{General Procedure A for Alkenylation of Benzamide Derivatives:}

An oven dried $25 \mathrm{~mL}$ Schlenk tube was charged with substrate $1(0.1 \mathrm{mmol}), \mathrm{Cu}\left(\mathrm{PhCO}_{2}\right)_{2}(0.03 \mathrm{mmol}), \mathrm{Ag}_{2} \mathrm{O}$ $(0.2 \mathrm{mmol}), \mathrm{KOPiv}(0.2 \mathrm{mmol})$, and $\mathrm{KHCO}_{3}(0.2 \mathrm{mmol})$, and the tube was capped and back-filled with nitrogen (3 times). Under the nitrogen flow, $2(0.3 \mathrm{mmol})$ and DMSO $(2 \mathrm{~mL})$ were added to the mixture, and the tube was capped tightly again. Then the reaction mixture was placed into a pre-heated oil bath and stirred at $45{ }^{\circ} \mathrm{C}$ for $12 \mathrm{~h}$. After cooled to room temperature, EtOAc $(20 \mathrm{~mL})$ was added to quench the reaction, and organic layer was washed with $\mathrm{NH}_{3} \cdot \mathrm{H}_{2} \mathrm{O}(20 \mathrm{~mL})$, and the water phase was extracted by EtOAc $(20 \mathrm{~mL})$. The combined organic layers were washed with saturated brine, dried over $\mathrm{Na}_{2} \mathrm{SO}_{4}$, concentrated under vacuum. The crude product was purified by preparative TLC to afford the desired product 3 .<smiles>CCC/C=C/c1ccccc1C(=O)Nc1ccccc1C1=NCCO1</smiles>

$3 a$ (mono)

(E)-N-(2-(4,5-Dihydrooxazol-2-yl)phenyl)-2-(pent-1-en-1-yl)benzamide (3a (mono))

Substrate 1a was alkenylated with alkenyl boronates (2) following the general procedure A. After purification by preparative thin-layer chromatography (petroleum ether/ethyl acetate $=20 / 1), \mathbf{3 a}($ mono) was obtained as a white solid (17.1 mg, 52\%). mp 98-100 $\left.{ }^{\circ} \mathrm{C} ;{ }^{1} \mathrm{H} \mathrm{NMR} \mathrm{(400} \mathrm{MHz,} \mathrm{CDCl}_{3}\right) \delta 12.57(\mathrm{~s}, 1 \mathrm{H}), 8.94(\mathrm{~d}, J=8.6 \mathrm{~Hz}$, $1 \mathrm{H}), 7.89(\mathrm{~d}, J=8.0 \mathrm{~Hz}, 1 \mathrm{H}), 7.60(\mathrm{~d}, J=7.9 \mathrm{~Hz}, 2 \mathrm{H}), 7.52(\mathrm{t}, J=7.8 \mathrm{~Hz}, 1 \mathrm{H}), 7.40(\mathrm{t}, J=7.6 \mathrm{~Hz}, 1 \mathrm{H}), 7.31-$ $7.27(\mathrm{~m}, 1 \mathrm{H}), 7.12(\mathrm{t}, J=7.7 \mathrm{~Hz}, 1 \mathrm{H}), 6.91(\mathrm{~d}, J=15.5 \mathrm{~Hz}, 1 \mathrm{H}), 6.27-6.17(\mathrm{~m}, 1 \mathrm{H}), 4.34(\mathrm{t}, J=9.5 \mathrm{~Hz}, 2 \mathrm{H})$, $4.02(\mathrm{t}, J=9.5 \mathrm{~Hz}, 2 \mathrm{H}), 2.18(\mathrm{q}, J=7.4 \mathrm{~Hz}, 2 \mathrm{H}), 1.52-1.42(\mathrm{~m}, 2 \mathrm{H}), 0.92(\mathrm{t}, J=7.4 \mathrm{~Hz}, 3 \mathrm{H}) ;{ }^{13} \mathrm{C} \mathrm{NMR}(100$ $\left.\mathrm{MHz} \mathrm{CDCl}_{3}\right) \delta 168.5,164.5,140.1,137.0,135.4,133.4,132.5,130.1,129.2,127.7,127.6,126.6,126.5,122.5$, 119.8, 113.5, 66.2, 54.7, 35.5, 22.5, 13.7; HRMS (ESI-TOF) $\mathrm{m} / z$ Calcd for $\mathrm{C}_{21} \mathrm{H}_{23} \mathrm{~N}_{2} \mathrm{O}_{2}[\mathrm{M}+\mathrm{H}]^{+}: 335.1754$, found: 335.1759 . 


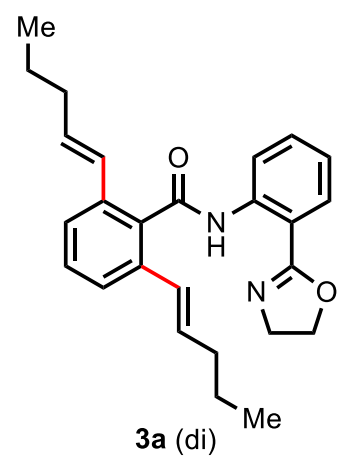

$N$-(2-(4,5-Dihydrooxazol-2-yl)phenyl)-2,6-di((E)-pent-1-en-1-yl)benzamide (3a (di))

Substrate 1a was alkenylated with alkenyl boronates (2) following the general procedure A. After purification by preparative thin-layer chromatography (petroleum ether/ethyl acetate $=20 / 1), \mathbf{3 a}$ (di) was obtained as a white solid $(9.9 \mathrm{mg}, 25 \%) . \mathrm{mp} 55-57{ }^{\circ} \mathrm{C} ;{ }^{1} \mathrm{H}$ NMR $\left(400 \mathrm{MHz}, \mathrm{CDCl}_{3}\right) \delta 12.29(\mathrm{~s}, 1 \mathrm{H}), 8.95(\mathrm{~d}, J=8.3 \mathrm{~Hz}, 1 \mathrm{H}), 7.87$ $(\mathrm{d}, J=8.0 \mathrm{~Hz}, 1 \mathrm{H}), 7.54(\mathrm{t}, J=7.6 \mathrm{~Hz}, 1 \mathrm{H}), 7.41(\mathrm{~d}, J=7.7 \mathrm{~Hz}, 2 \mathrm{H}), 7.31-7.27(\mathrm{~m}, 1 \mathrm{H}), 7.14(\mathrm{t}, J=7.7 \mathrm{~Hz}$, $1 \mathrm{H}), 6.51(\mathrm{~d}, J=15.8 \mathrm{~Hz}, 2 \mathrm{H}), 6.25-6.13(\mathrm{~m}, 2 \mathrm{H}), 4.29(\mathrm{t}, J=9.5 \mathrm{~Hz}, 2 \mathrm{H}), 3.90(\mathrm{t}, J=9.4 \mathrm{~Hz}, 2 \mathrm{H}), 2.10(\mathrm{q}, J$ $=7.2 \mathrm{~Hz}, 4 \mathrm{H}), 1.45-1.35(\mathrm{~m}, 4 \mathrm{H}), 0.86(\mathrm{t}, J=7.4 \mathrm{~Hz}, 6 \mathrm{H}) ;{ }^{13} \mathrm{C} \mathrm{NMR}\left(100 \mathrm{MHz}, \mathrm{CDCl}_{3}\right) \delta 168.8,164.0,139.6$, 135.2, 135.0, 133.4, 132.4, 129.1, 128.8, 127.0, 123.9, 122.7, 120.2, 113.7, 66.1, 54.7, 35.2, 22.4, 13.6; HRMS (ESI-TOF) $m / z$ Calcd for $\mathrm{C}_{26} \mathrm{H}_{31} \mathrm{~N}_{2} \mathrm{O}_{2}[\mathrm{M}+\mathrm{H}]^{+}$: 403.2380, found: 403.2391 .<smiles>CCC/C=C/c1cc(C)ccc1C(=O)Nc1ccccc1C1=NCCO1</smiles>

(E)- $N$-(2-(4,5-Dihydrooxazol-2-yl)phenyl)-4-methyl-2-(pent-1-en-1-yl)benzamide (3b (mono))

Substrate 1b was alkenylated with alkenyl boronates (2) following the general procedure A. After purification by preparative thin-layer chromatography (petroleum ether/ethyl acetate $=20 / 1), \mathbf{3 b}($ mono) was obtained as a white solid (15.7 mg, 46\%). mp 133-135 ${ }^{\circ} \mathrm{C} ;{ }^{1} \mathrm{H} \mathrm{NMR}\left(400 \mathrm{MHz}, \mathrm{CDCl}_{3}\right) \delta 12.52(\mathrm{~s}, 1 \mathrm{H}), 8.93(\mathrm{~d}, J=8.4 \mathrm{~Hz}$, $1 \mathrm{H}), 7.88(\mathrm{~d}, J=7.5 \mathrm{~Hz}, 1 \mathrm{H}), 7.96-7.48(\mathrm{~m}, 2 \mathrm{H}), 7.40(\mathrm{~s}, 1 \mathrm{H}), 7.15-7.05(\mathrm{~m}, 2 \mathrm{H}) 6.92(\mathrm{~d}, J=15.9 \mathrm{~Hz}, 1 \mathrm{H})$, 6.25-6.16 (m, 1H), $4.34(\mathrm{t}, J=9.5 \mathrm{~Hz}, 2 \mathrm{H}), 4.02(\mathrm{t}, J=9.5 \mathrm{~Hz}, 2 \mathrm{H}), 2.39(\mathrm{~s}, 3 \mathrm{H}), 2.18(\mathrm{q}, J=7.3 \mathrm{~Hz}, 2 \mathrm{H})$, $1.52-1.42(\mathrm{~m}, 2 \mathrm{H}), 0.92(\mathrm{t}, J=7.4 \mathrm{~Hz}, 3 \mathrm{H}) ;{ }^{13} \mathrm{C} \mathrm{NMR}\left(100 \mathrm{MHz}, \mathrm{CDCl}_{3}\right) \delta 168.5,164.5,140.2,140.2,137.1$, 133.0, 132.6, 132.5, 129.1, 127.9, 127.8, 127.4, 127.1, 122.3, 119.8, 113.4, 66.1, 54.7, 35.3, 22.5, 21.5, 13.8; HRMS (ESI-TOF) $m / z$ Calcd for $\mathrm{C}_{22} \mathrm{H}_{25} \mathrm{~N}_{2} \mathrm{O}_{2}[\mathrm{M}+\mathrm{H}]^{+}: 349.1911$, found: 349.1920 . 


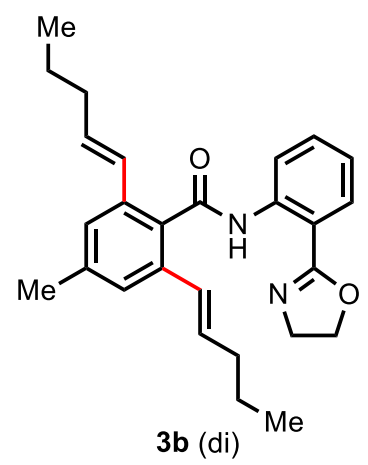

$N$-(2-(4,5-Dihydrooxazol-2-yl)phenyl)-4-methyl-2,6-di((E)-pent-1-en-1-yl)benzamide (3b (di))

Substrate $\mathbf{1 b}$ was alkenylated with alkenyl boronates (2) following the general procedure A. After purification by preparative thin-layer chromatography (petroleum ether/ethyl acetate $=20 / 1), \mathbf{3 b}$ (di) was obtained as a white solid (10.5 mg, 26\%). mp 57-59 ${ }^{\circ} \mathrm{C} ;{ }^{1} \mathrm{H}$ NMR $\left(400 \mathrm{MHz}, \mathrm{CDCl}_{3}\right) \delta 12.24(\mathrm{~s}, 1 \mathrm{H}), 8.95(\mathrm{~d}, J=8.4 \mathrm{~Hz}, 1 \mathrm{H}), 7.87$ $(\mathrm{d}, J=8.1 \mathrm{~Hz}, 1 \mathrm{H}), 7.53(\mathrm{t}, J=7.7 \mathrm{~Hz}, 1 \mathrm{H}), 7.22(\mathrm{~s}, 2 \mathrm{H}), 7.13(\mathrm{t}, J=7.6 \mathrm{~Hz}, 1 \mathrm{H}), 6.50(\mathrm{~d}, J=15.7 \mathrm{~Hz}, 2 \mathrm{H})$, 6.23-6.13 (m, 2H), $4.29(\mathrm{t}, J=9.5 \mathrm{~Hz}, 2 \mathrm{H}), 3.91(\mathrm{t}, J=9.5 \mathrm{~Hz}, 2 \mathrm{H}), 2.36(\mathrm{~s}, 3 \mathrm{H}), 2.09(\mathrm{q}, J=7.0 \mathrm{~Hz}, 4 \mathrm{H})$, $1.44-1.34(\mathrm{~m}, 4 \mathrm{H}), 0.86(\mathrm{t}, J=7.4 \mathrm{~Hz}, 6 \mathrm{H}) ;{ }^{13} \mathrm{C} \mathrm{NMR}\left(100 \mathrm{MHz}, \mathrm{CDCl}_{3}\right) \delta 169.0,164.0,139.7,138.3,135.2$, 133.0, 132.6, 132.4, 129.1, 127.2, 124.6, 122.6, 120.2, 113.7, 66.1, 54.7, 35.2, 22.5, 21.5, 13.6; HRMS (ESITOF) $m / z$ Calcd for $\mathrm{C}_{27} \mathrm{H}_{33} \mathrm{~N}_{2} \mathrm{O}_{2}[\mathrm{M}+\mathrm{H}]^{+}:$417.2537, found: 417.2549 .<smiles>CCC/C=C/c1cc(C(C)(C)C)ccc1C(=O)Nc1ccccc1C1=NCCO1</smiles>

(E)-4-(Tert-butyl)- $N$-(2-(4,5-dihydrooxazol-2-yl)phenyl)-2-(pent-1-en-1-yl)benzamide (3c (mono))

Substrate 1c was alkenylated with alkenyl boronates (2) following the general procedure A. After purification by preparative thin-layer chromatography (petroleum ether/ethyl acetate $=20 / 1), \mathbf{3 c}($ mono) was obtained as a white solid (16.5 mg, 43\%). mp 132-134 ${ }^{\circ} \mathrm{C} ;{ }^{1} \mathrm{H}$ NMR (400 MHz, $\left.\mathrm{CDCl}_{3}\right) \delta 12.53$ (s, 1H), $8.94(\mathrm{~d}, J=8.5 \mathrm{~Hz}$, $1 \mathrm{H}), 7.88(\mathrm{~d}, J=7.8 \mathrm{~Hz}, 1 \mathrm{H}), 7.61-7.55(\mathrm{~m}, 2 \mathrm{H}), 7.51(\mathrm{t}, J=7.9 \mathrm{~Hz}, 1 \mathrm{H}), 7.31(\mathrm{~d}, J=8.1 \mathrm{~Hz}, 1 \mathrm{H}), 7.11(\mathrm{t}, J=$ $7.5 \mathrm{~Hz}, 1 \mathrm{H}), 6.96(\mathrm{~d}, J=15.8 \mathrm{~Hz}, 1 \mathrm{H}), 6.26-6.15(\mathrm{~m}, 1 \mathrm{H}), 4.35(\mathrm{t}, J=9.5 \mathrm{~Hz}, 2 \mathrm{H}), 4.05(\mathrm{t}, J=9.5 \mathrm{~Hz}, 2 \mathrm{H})$, 2.19 (q, $J=7.3 \mathrm{~Hz}, 2 \mathrm{H}), 1.55-1.43(\mathrm{~m}, 2 \mathrm{H}), 1.37$ (s, 9H), 0.93 (t, $J=7.3 \mathrm{~Hz}, 3 \mathrm{H}) ;{ }^{13} \mathrm{C} \mathrm{NMR}\left(100 \mathrm{MHz}, \mathrm{CDCl}_{3}\right)$ $\delta 168.5,164.5,153.3,140.2,136.9,132.9,132.6,132.5,129.2,128.3,127.7,123.8,123.5,122.3,119.9,113.5$, 66.1, 54.7, 35.3, 34.8, 31.2, 22.5, 13.8; HRMS (ESI-TOF) $m / z$ Calcd for $\mathrm{C}_{25} \mathrm{H}_{31} \mathrm{~N}_{2} \mathrm{O}_{2}[\mathrm{M}+\mathrm{H}]^{+}: 391.2380$, found: 391.2390 . 
<smiles>CCC/C=C/c1cc(C(C)(C)C)cc(/C=C/CCC)c1C(=O)Nc1ccccc1C1=NCCO1</smiles>

4-(Tert-butyl)- $N$-(2-(4,5-dihydrooxazol-2-yl)phenyl)-2,6-di((E)-pent-1-en-1-yl)benzamide (3c (di))

Substrate 1c was alkenylated with alkenyl boronates (2) following the general procedure A. After purification by preparative thin-layer chromatography (petroleum ether/ethyl acetate $=20 / 1), \mathbf{3 c}(\mathrm{di})$ was obtained as a white solid (12.3 mg, 27\%). mp 107-109 ${ }^{\circ} \mathrm{C}$; ${ }^{1} \mathrm{H}$ NMR $\left(400 \mathrm{MHz}, \mathrm{CDCl}_{3}\right) \delta 12.23(\mathrm{~s}, 1 \mathrm{H}), 8.95(\mathrm{~d}, J=8.2 \mathrm{~Hz}, 1 \mathrm{H})$, $7.87(\mathrm{~d}, J=7.8 \mathrm{~Hz}, 1 \mathrm{H}), 7.54(\mathrm{t}, J=8.0 \mathrm{~Hz}, 1 \mathrm{H}), 7.41(\mathrm{~s}, 2 \mathrm{H}), 7.13(\mathrm{t}, J=7.6 \mathrm{~Hz}, 1 \mathrm{H}), 6.53(\mathrm{~d}, J=15.8 \mathrm{~Hz}$, 2H), 6.24-6.13 (m, 2H), 4.29 (t, $J=9.5 \mathrm{~Hz}, 2 \mathrm{H}), 3.93(\mathrm{t}, J=9.4 \mathrm{~Hz}, 2 \mathrm{H}), 2.10$ (q, $J=7.1 \mathrm{~Hz}, 4 \mathrm{H}), 1.47-1.34$ $(\mathrm{m}, 13 \mathrm{H}), 0.86(\mathrm{t}, J=7.3 \mathrm{~Hz}, 6 \mathrm{H}) ;{ }^{13} \mathrm{C} \mathrm{NMR}\left(100 \mathrm{MHz}, \mathrm{CDCl}_{3}\right) \delta 169.1,164.0,151.4,139.7,134.8,132.9$, 132.6, 132.4, 129.1, 127.6, 122.6, 121.2, 120.3, 113.7, 66.1, 54.7, 35.2, 34.8, 31.3, 22.5, 13.6; HRMS (ESITOF) $m / z$ Calcd for $\mathrm{C}_{30} \mathrm{H}_{39} \mathrm{~N}_{2} \mathrm{O}_{2}[\mathrm{M}+\mathrm{H}]^{+}: 459.3006$, found: 459.3022 .<smiles>CCC/C=C/c1cc(C2CCCCC2)ccc1C(=O)Nc1ccccc1C1=NCCO1</smiles>

(E)-4-Cyclohexyl- $N$-(2-(4,5-dihydrooxazol-2-yl)phenyl)-2-(pent-1-en-1-yl)benzamide (3d (mono))

Substrate 1d was alkenylated with alkenyl boronates (2) following the general procedure A. After purification by preparative thin-layer chromatography (petroleum ether/ethyl acetate $=20 / 1), \mathbf{3 d}$ (mono) was obtained as a white solid (16.3 mg, 40\%). mp 111-113 ${ }^{\circ} \mathrm{C}$; ${ }^{1} \mathrm{H} \mathrm{NMR}\left(400 \mathrm{MHz}, \mathrm{CDCl}_{3}\right) \delta 12.53$ (s, 1H), $8.94(\mathrm{~d}, J=8.5 \mathrm{~Hz}$, $1 \mathrm{H}), 7.88(\mathrm{~d}, J=7.9 \mathrm{~Hz}, 1 \mathrm{H}), 7.56(\mathrm{~d}, J=7.9 \mathrm{~Hz}, 1 \mathrm{H}), 7.51(\mathrm{t}, J=8.0 \mathrm{~Hz}, 1 \mathrm{H}), 7.42(\mathrm{~s}, 1 \mathrm{H}), 7.14-7.08(\mathrm{~m}, 2 \mathrm{H})$, $6.94(\mathrm{~d}, J=15.8 \mathrm{~Hz}, 1 \mathrm{H}), 6.27-6.16(\mathrm{~m}, 1 \mathrm{H}), 4.35(\mathrm{t}, J=9.5 \mathrm{~Hz}, 2 \mathrm{H}), 4.04(\mathrm{t}, J=9.5 \mathrm{~Hz}, 2 \mathrm{H}), 2.59-2.50(\mathrm{~m}$, $1 \mathrm{H}), 2.18(\mathrm{q}, J=7.3 \mathrm{~Hz}, 2 \mathrm{H}), 1.92-1.85(\mathrm{~m}, 4 \mathrm{H}), 1.77(\mathrm{~d}, J=12.4 \mathrm{~Hz}, 1 \mathrm{H}), 1.55-1.37(\mathrm{~m}, 6 \mathrm{H}), 1.34-1.25(\mathrm{~m}$, $1 \mathrm{H}), 0.93(\mathrm{t}, J=7.4 \mathrm{~Hz}, 3 \mathrm{H}) ;{ }^{13} \mathrm{C} \mathrm{NMR}\left(100 \mathrm{MHz}, \mathrm{CDCl}_{3}\right) \delta 168.5,164.4,150.3,140.2,137.2,132.9,132.8$, $132.5,129.1,128.0,127.9,125.2,125.1,122.3,119.8,113.4,66.1,54.7,44.6,35.3,34.2,26.8,26.1,22.6,13.8$; HRMS (ESI-TOF) $m / z$ Calcd for $\mathrm{C}_{27} \mathrm{H}_{33} \mathrm{~N}_{2} \mathrm{O}_{2}[\mathrm{M}+\mathrm{H}]^{+}: 417.2537$, found: 417.2549. 
<smiles>CCCC=Cc1cc(C2CCCCC2)cc(/C=C/CCC)c1C(=O)Nc1ccccc1C1=NCCO1</smiles>

\section{4-Cyclohexyl- $N$-(2-(4,5-dihydrooxazol-2-yl)phenyl)-2,6-di((E)-pent-1-en-1-yl)benzamide (3d (di))}

Substrate 1d was alkenylated with alkenyl boronates (2) following the general procedure A. After purification by preparative thin-layer chromatography (petroleum ether/ethyl acetate $=20 / 1$ ), 3d (di) was obtained as a white solid (12.2 mg, 26\%). mp 100-102 ${ }^{\circ} \mathrm{C} ;{ }^{1} \mathrm{H}$ NMR $\left(400 \mathrm{MHz}, \mathrm{CDCl}_{3}\right) \delta 12.23(\mathrm{~s}, 1 \mathrm{H}), 8.94(\mathrm{~d}, J=8.0 \mathrm{~Hz}, 1 \mathrm{H})$, $7.87(\mathrm{~d}, J=7.7 \mathrm{~Hz}, 1 \mathrm{H}), 7.53$ (t, $J=7.7 \mathrm{~Hz}, 1 \mathrm{H}), 7.24$ (s, 2H), 7.13 (t, $J=7.4 \mathrm{~Hz}, 1 \mathrm{H}), 6.51(\mathrm{~d}, J=15.5 \mathrm{~Hz}$, 2H), 6.23-6.10 (m, 2H), $4.29(\mathrm{t}, J=9.6 \mathrm{~Hz}, 2 \mathrm{H}), 3.92(\mathrm{t}, J=9.6 \mathrm{~Hz}, 2 \mathrm{H}), 2.57-2.47(\mathrm{~m}, 1 \mathrm{H}), 2.09$ (q, $J=7.1$ $\mathrm{Hz}, 4 \mathrm{H}), 1.95-1.85(\mathrm{~m}, 4 \mathrm{H}), 1.77(\mathrm{~d}, J=12.4 \mathrm{~Hz}, 1 \mathrm{H}), 1.50-1.35(\mathrm{~m}, 9 \mathrm{H}), 0.86(\mathrm{t}, J=7.4 \mathrm{~Hz}, 6 \mathrm{H}) ;{ }^{13} \mathrm{C} \mathrm{NMR}$ $\left(100 \mathrm{MHz}, \mathrm{CDCl}_{3}\right) \delta 169.1,163.9,148.5,139.6,135.1,132.9,132.9,132.4,129.1,127.4,122.6,122.6,120.3$, 113.7, 66.1, 54.7, 44.7, 35.2, 34.3, 26.9, 26.1, 22.5, 13.6; HRMS (ESI-TOF) $m / z$ Calcd for $\mathrm{C}_{32} \mathrm{H}_{41} \mathrm{~N}_{2} \mathrm{O}_{2}[\mathrm{M}+\mathrm{H}]^{+}$: 485.3163, found: 485.3180 .<smiles>CCC/C=C/c1cc(-c2ccccc2)ccc1C(=O)Nc1ccccc1C1=NCCO1</smiles>

(E)-N-(2-(4,5-Dihydrooxazol-2-yl)phenyl)-3-(pent-1-en-1-yl)-[1,1'-biphenyl]-4-carboxamide (3e (mono))

Substrate 1e was alkenylated with alkenyl boronates (2) following the general procedure A. After purification by preparative thin-layer chromatography (petroleum ether/ethyl acetate $=20 / 1), 3 \mathbf{e}($ mono) was obtained as a white solid (17.3 mg, 43\%). mp 94-96 ${ }^{\circ} \mathrm{C}$; ${ }^{1} \mathrm{H}$ NMR (400 MHz, $\left.\mathrm{CDCl}_{3}\right) \delta 12.64$ (s, 1H), 8.96 (d, $J=8.4 \mathrm{~Hz}$, $1 \mathrm{H}), 7.90(\mathrm{~d}, J=8.0 \mathrm{~Hz}, 1 \mathrm{H}), 7.81(\mathrm{~s}, 1 \mathrm{H}), 7.70(\mathrm{~d}, J=8.0 \mathrm{~Hz}, 1 \mathrm{H}), 7.65(\mathrm{~d}, J=7.2 \mathrm{~Hz}, 2 \mathrm{H}), 7.55-7.45(\mathrm{~m}$, 4H), 7.39 (t, $J=7.3 \mathrm{~Hz}, 1 \mathrm{H}), 7.13(\mathrm{t}, J=7.6 \mathrm{~Hz}, 1 \mathrm{H}), 6.99$ (d, $J=15.6 \mathrm{~Hz}, 1 \mathrm{H}), 6.35-6.23(\mathrm{~m}, 1 \mathrm{H}), 4.36$ (t, $J$ $=9.5 \mathrm{~Hz}, 2 \mathrm{H}), 4.05(\mathrm{t}, J=9.5 \mathrm{~Hz}, 2 \mathrm{H}), 2.21(\mathrm{q}, J=7.1 \mathrm{~Hz}, 2 \mathrm{H}), 1.55-1.45(\mathrm{~m}, 2 \mathrm{H}), 0.93(\mathrm{t}, J=7.4 \mathrm{~Hz}, 3 \mathrm{H})$; ${ }^{13} \mathrm{C}$ NMR $\left(100 \mathrm{MHz}, \mathrm{CDCl}_{3}\right) \delta 168.2,164.5,143.0,140.5,140.1,137.7,134.1,133.6,132.6,129.2,128.8$, $128.4,127.8,127.7,127.2,125.4,125.4,122.5,119.9,113.5,66.2,54.7,35.3,22.5,13.8$; HRMS (ESI-TOF) $m / z$ Calcd for $\mathrm{C}_{27} \mathrm{H}_{27} \mathrm{~N}_{2} \mathrm{O}_{2}[\mathrm{M}+\mathrm{H}]^{+}: 411.2067$, found: 411.2082 . 
<smiles>CCC/C=C/c1cc(-c2ccccc2)cc(/C=C/CCC)c1C(=O)Nc1ccccc1C1=NCCO1</smiles>

$N$-(2-(4,5-Dihydrooxazol-2-yl)phenyl)-3,5-di((E)-pent-1-en-1-yl)-[1,1'-biphenyl]-4-carboxamide (3e (di))

Substrate 1e was alkenylated with alkenyl boronates (2) following the general procedure A. After purification by preparative thin-layer chromatography (petroleum ether/ethyl acetate $=20 / 1), \mathbf{3 e}(\mathrm{di})$ was obtained as a white solid (12.5 mg, 26\%). mp 122-124 ${ }^{\circ} \mathrm{C}$; ${ }^{1} \mathrm{H}$ NMR $\left(400 \mathrm{MHz}, \mathrm{CDCl}_{3}\right) \delta 12.36(\mathrm{~s}, 1 \mathrm{H}), 8.97(\mathrm{~d}, J=8.5 \mathrm{~Hz}, 1 \mathrm{H})$, $7.88(\mathrm{~d}, J=7.9 \mathrm{~Hz}, 1 \mathrm{H}), 7.65(\mathrm{~d}, J=7.4 \mathrm{~Hz}, 2 \mathrm{H}), 7.62(\mathrm{~s}, 2 \mathrm{H}), 7.55(\mathrm{t}, J=7.9 \mathrm{~Hz}, 1 \mathrm{H}), 7.47(\mathrm{t}, J=7.5 \mathrm{~Hz}, 2 \mathrm{H})$, $7.38(\mathrm{t}, J=7.3 \mathrm{~Hz}, 1 \mathrm{H}), 7.15(\mathrm{t}, J=7.7 \mathrm{~Hz}, 1 \mathrm{H}), 6.58(\mathrm{~d}, J=15.6 \mathrm{~Hz}, 2 \mathrm{H}), 6.33-6.21(\mathrm{~m}, 2 \mathrm{H}), 4.30(\mathrm{t}, J=9.5$ $\mathrm{Hz}, 2 \mathrm{H}), 3.92(\mathrm{t}, J=9.5 \mathrm{~Hz}, 2 \mathrm{H}), 2.12(\mathrm{q}, J=7.2 \mathrm{~Hz}, 4 \mathrm{H}), 1.49-1.34(\mathrm{~m}, 4 \mathrm{H}), 0.87(\mathrm{t}, J=7.4 \mathrm{~Hz}, 6 \mathrm{H}) ;{ }^{13} \mathrm{C} \mathrm{NMR}$ $\left(100 \mathrm{MHz}, \mathrm{CDCl}_{3}\right) \delta 168.7,164.0,141.7,141.0,139.6,135.7,133.9,133.7,132.5,129.1,128.7,127.5,127.2$, 127.1, 122.9, 122.7, 120.2, 113.7, 66.1, 54.7, 35.2, 22.4, 13.6; HRMS (ESI-TOF) $m / z$ Calcd for $\mathrm{C}_{32} \mathrm{H}_{35} \mathrm{~N}_{2} \mathrm{O}_{2}$ $[\mathrm{M}+\mathrm{H}]^{+}: 479.2693$, found: 479.2709 .<smiles>C=Cc1ccc(C(=O)Nc2ccccc2C2=NCCO2)c(/C=C/CCC)c1</smiles>

(E)-N-(2-(4,5-Dihydrooxazol-2-yl)phenyl)-2-(pent-1-en-1-yl)-4-vinylbenzamide (3f (mono))

Substrate 1f was alkenylated with alkenyl boronates (2) following the general procedure A. After purification by preparative thin-layer chromatography (petroleum ether/ethyl acetate $=20 / 1), \mathbf{3 f}$ (mono) was obtained as a white solid (11.7 mg, 33\%). mp 110-112 ${ }^{\circ} \mathrm{C}$; ${ }^{1} \mathrm{H} \mathrm{NMR}\left(400 \mathrm{MHz}, \mathrm{CDCl}_{3}\right) \delta 12.59$ (s, 1H), $8.93(\mathrm{~d}, J=8.5 \mathrm{~Hz}$, $1 \mathrm{H}), 7.89(\mathrm{~d}, J=7.9 \mathrm{~Hz}, 1 \mathrm{H}), 7.63-7.57(\mathrm{~m}, 2 \mathrm{H}), 7.52(\mathrm{t}, J=7.3 \mathrm{~Hz}, 1 \mathrm{H}), 7.35(\mathrm{~d}, J=8.1 \mathrm{~Hz}, 1 \mathrm{H}), 7.12(\mathrm{t}, J=$ $7.1 \mathrm{~Hz}, 1 \mathrm{H}), 6.93(\mathrm{~d}, J=15.7 \mathrm{~Hz}, 1 \mathrm{H}), 6.76(\mathrm{dd}, J=17.6,10.8 \mathrm{~Hz}, 1 \mathrm{H}), 6.29-6.20(\mathrm{~m}, 1 \mathrm{H}), 5.85(\mathrm{~d}, J=17.6$ $\mathrm{Hz}, 1 \mathrm{H}), 5.35(\mathrm{~d}, J=10.9 \mathrm{~Hz}, 1 \mathrm{H}), 4.35(\mathrm{t}, J=9.6 \mathrm{~Hz}, 2 \mathrm{H}), 4.03(\mathrm{t}, J=9.5 \mathrm{~Hz}, 2 \mathrm{H}), 2.19$ (q, $J=6.9 \mathrm{~Hz}, 2 \mathrm{H})$, $1.53-1.41(\mathrm{~m}, 2 \mathrm{H}), 0.93$ (t, $J=7.4 \mathrm{~Hz}, 3 \mathrm{H}) ;{ }^{13} \mathrm{C} \mathrm{NMR}\left(100 \mathrm{MHz}, \mathrm{CDCl}_{3}\right) \delta 168.1,164.5,140.1,139.2,137.5$, 136.3, 134.6, 133.5, 132.5, 129.2, 128.2, 127.6, 124.7, 124.2, 122.4, 119.8, 115.3, 113.5, 66.2, 54.7, 35.3, 22.5, 13.8; HRMS (ESI-TOF) $m / z$ Calcd for $\mathrm{C}_{23} \mathrm{H}_{25} \mathrm{~N}_{2} \mathrm{O}_{2}[\mathrm{M}+\mathrm{H}]^{+}: 361.1911$, found: 361.1920 . 
<smiles>C=Cc1cc(/C=C/CCC)c(C(=O)Nc2ccccc2C2=NCCO2)c(/C=C/CCC)c1</smiles>

\section{$N$-(2-(4,5-Dihydrooxazol-2-yl)phenyl)-2,6-di((E)-pent-1-en-1-yl)-4-vinylbenzamide (3f (di))}

Substrate 1f was alkenylated with alkenyl boronates (2) following the general procedure A. After purification by preparative thin-layer chromatography (petroleum ether/ethyl acetate $=20 / 1), \mathbf{3 f}(\mathrm{di})$ was obtained as a white solid (10.3 mg, 24\%). mp 78-80 ${ }^{\circ} \mathrm{C} ;{ }^{1} \mathrm{H}$ NMR $\left(400 \mathrm{MHz}, \mathrm{CDCl}_{3}\right) \delta 12.30(\mathrm{~s}, 1 \mathrm{H}), 8.95(\mathrm{~d}, J=8.5 \mathrm{~Hz}, 1 \mathrm{H}), 7.87$ $(\mathrm{d}, J=7.8 \mathrm{~Hz}, 1 \mathrm{H}), 7.54(\mathrm{t}, J=7.3 \mathrm{~Hz}, 1 \mathrm{H}), 7.44(\mathrm{~s}, 2 \mathrm{H}), 7.14(\mathrm{t}, J=7.7 \mathrm{~Hz}, 1 \mathrm{H}), 6.74(\mathrm{dd}, J=17.6,11.0 \mathrm{~Hz}$, $1 \mathrm{H}), 6.51(\mathrm{~d}, J=15.7 \mathrm{~Hz}, 2 \mathrm{H}), 6.29-6.17(\mathrm{~m}, 2 \mathrm{H}), 5.84(\mathrm{~d}, J=17.6 \mathrm{~Hz}, 1 \mathrm{H}), 5.32(\mathrm{~d}, J=10.9 \mathrm{~Hz}, 1 \mathrm{H}), 4.29(\mathrm{t}$, $J=9.5 \mathrm{~Hz}, 2 \mathrm{H}), 3.91(\mathrm{t}, J=9.5 \mathrm{~Hz}, 2 \mathrm{H}), 2.11(\mathrm{q}, J=6.9 \mathrm{~Hz}, 4 \mathrm{H}), 1.48-1.31(\mathrm{~m}, 4 \mathrm{H}), 0.86(\mathrm{t}, J=7.4 \mathrm{~Hz}, 6 \mathrm{H})$; ${ }^{13} \mathrm{C} \mathrm{NMR}\left(100 \mathrm{MHz}, \mathrm{CDCl}_{3}\right) \delta 168.6,164.0,139.5,137.7,136.6,135.5,134.5,133.6,132.4,129.1,127.0,122.7$, $121.8,120.2,114.7,113.6,66.1,54.7,35.2,22.4,13.6$; HRMS (ESI-TOF) $m / z$ Calcd for $\mathrm{C}_{28} \mathrm{H}_{33} \mathrm{~N}_{2} \mathrm{O}_{2}[\mathrm{M}+\mathrm{H}]^{+}$: 429.2537, found: 429.2550 .<smiles>CCC/C=C/c1cc(OC)ccc1C(=O)Nc1ccccc1C1=NCCO1</smiles>

(E)-N-(2-(4,5-Dihydrooxazol-2-yl)phenyl)-4-methoxy-2-(pent-1-en-1-yl)benzamide (3g (mono))

Substrate $1 \mathrm{~g}$ was alkenylated with alkenyl boronates (2) following the general procedure A. After purification by preparative thin-layer chromatography (petroleum ether/ethyl acetate $=20 / 1), \mathbf{3 g}$ (mono) was obtained as a white solid (14.2 mg, 39\%). mp 107-109 ${ }^{\circ} \mathrm{C} ;{ }^{1} \mathrm{H}$ NMR (400 MHz, $\left.\mathrm{CDCl}_{3}\right) \delta 12.52(\mathrm{~s}, 1 \mathrm{H}), 8.91(\mathrm{~d}, J=8.4 \mathrm{~Hz}$, $1 \mathrm{H}), 7.88(\mathrm{~d}, J=7.8 \mathrm{~Hz}, 1 \mathrm{H}), 7.62(\mathrm{~d}, J=8.6 \mathrm{~Hz}, 1 \mathrm{H}), 7.51(\mathrm{t}, J=7.9 \mathrm{~Hz}, 1 \mathrm{H}), 7.14-7.07(\mathrm{~m}, 2 \mathrm{H}), 6.98(\mathrm{~d}, J=$ $15.6 \mathrm{~Hz}, 1 \mathrm{H}), 6.82(\mathrm{dd}, J=8.5,2.5 \mathrm{~Hz}, 1 \mathrm{H}), 6.26-6.14(\mathrm{~m}, 1 \mathrm{H}), 4.35(\mathrm{t}, J=9.5 \mathrm{~Hz}, 2 \mathrm{H}), 4.04(\mathrm{t}, J=9.5 \mathrm{~Hz}$, 2H), 3.87 (s, 3H), 2.19 (q, $J=7.6 \mathrm{~Hz}, 2 \mathrm{H}), 1.54-1.42(\mathrm{~m}, 2 \mathrm{H}), 0.93(\mathrm{t}, J=7.4 \mathrm{~Hz}, 3 \mathrm{H}) ;{ }^{13} \mathrm{C}$ NMR $(100 \mathrm{MHz}$, $\left.\mathrm{CDCl}_{3}\right) \delta 168.1,164.5,160.9,140.2,139.5,133.4,132.5,129.7,129.2,128.1,127.9,122.2,119.7,113.4,112.1$, 111.6, 66.1, 55.3, 54.7, 35.2, 22.5, 13.8; HRMS (ESI-TOF) $m / z$ Calcd for $\mathrm{C}_{22} \mathrm{H}_{25} \mathrm{~N}_{2} \mathrm{O}_{3}[\mathrm{M}+\mathrm{H}]^{+}: 365.1860$, found: 365.1867 . 
<smiles>CCC/C=C/c1cc(OC)cc(/C=C/CCC)c1C(=O)Nc1ccccc1C1=NCCO1</smiles>

\section{$N$-(2-(4,5-Dihydrooxazol-2-yl)phenyl)-4-methoxy-2,6-di((E)-pent-1-en-1-yl)benzamide (3g (di))}

Substrate $1 \mathrm{~g}$ was alkenylated with alkenyl boronates (2) following the general procedure A. After purification by preparative thin-layer chromatography (petroleum ether/ethyl acetate $=20 / 1$ ), $\mathbf{3 g}$ (di) was obtained as a white solid (11.4 mg, 27\%). mp 68-70 ${ }^{\circ} \mathrm{C}$; ${ }^{1} \mathrm{H}$ NMR (400 MHz, $\left.\mathrm{CDCl}_{3}\right) \delta 12.25(\mathrm{~s}, 1 \mathrm{H}), 8.95(\mathrm{~d}, J=8.4 \mathrm{~Hz}, 1 \mathrm{H}), 7.87$ $(\mathrm{d}, J=6.8 \mathrm{~Hz}, 1 \mathrm{H}), 7.53(\mathrm{t}, J=7.9 \mathrm{~Hz}, 1 \mathrm{H}), 7.13(\mathrm{t}, J=7.5 \mathrm{~Hz}, 1 \mathrm{H}), 6.93(\mathrm{~s}, 2 \mathrm{H}), 6.51(\mathrm{~d}, J=15.6 \mathrm{~Hz}, 2 \mathrm{H})$, 6.23-6.13 (m, 2H), 4.29 (t, $J=9.5 \mathrm{~Hz}, 2 \mathrm{H}), 3.91$ (t, $J=9.5 \mathrm{~Hz}, 2 \mathrm{H}), 3.87$ (s, 3H), 2.10 (q, $J=6.9 \mathrm{~Hz}, 4 \mathrm{H})$, $1.46-1.33(\mathrm{~m}, 4 \mathrm{H}), 0.86(\mathrm{t}, J=7.4 \mathrm{~Hz}, 6 \mathrm{H}) ;{ }^{13} \mathrm{C} \mathrm{NMR}\left(100 \mathrm{MHz}, \mathrm{CDCl}_{3}\right) \delta 168.8,164.0,159.6,139.7,137.0$, 133.5, 132.4, 129.1, 128.7, 127.1, 122.5, 120.1, 113.6, 109.3 66.1, 55.3, 54.7, 35.1, 22.4, 13.6; HRMS (ESITOF) $m / z$ Calcd for $\mathrm{C}_{27} \mathrm{H}_{33} \mathrm{~N}_{2} \mathrm{O}_{3}[\mathrm{M}+\mathrm{H}]^{+}: 433.2486$, found: 433.2503 .<smiles>CCC/C=C/c1cc(OC)ccc1C(=O)Nc1ccccc1C1=NCCO1</smiles>

\section{(E)- $N$-(2-(4,5-Dihydrooxazol-2-yl)phenyl)-4-(methylthio)-2-(pent-1-en-1-yl)benzamide (3h (mono))}

Substrate $\mathbf{1 h}$ was alkenylated with alkenyl boronates (2) following the general procedure A. After purification by preparative thin-layer chromatography (petroleum ether/ethyl acetate $=20 / 1), \mathbf{3 h}$ (mono) was obtained as a white solid (13.8 mg, 37\%). mp 83-85 $\left.{ }^{\circ} \mathrm{C} ;{ }^{1} \mathrm{H} \mathrm{NMR} \mathrm{(400} \mathrm{MHz,} \mathrm{CDCl}_{3}\right) \delta 12.56(\mathrm{~s}, 1 \mathrm{H}), 8.91(\mathrm{~d}, J=8.2 \mathrm{~Hz}$, $1 \mathrm{H}), 7.88(\mathrm{~d}, J=8.0 \mathrm{~Hz}, 1 \mathrm{H}), 7.60-7.48(\mathrm{~m}, 2 \mathrm{H}), 7.42(\mathrm{~s}, 1 \mathrm{H}), 7.16-7.08(\mathrm{~m}, 2 \mathrm{H}), 6.92(\mathrm{~d}, J=15.7 \mathrm{~Hz}, 1 \mathrm{H})$, 6.26-6.15 (m, 1H), $4.35(\mathrm{t}, J=9.5 \mathrm{~Hz}, 2 \mathrm{H}), 4.03(t, \mathrm{~J}=9.5 \mathrm{~Hz}, 2 \mathrm{H}), 2.54(\mathrm{~s}, 3 \mathrm{H}), 2.18(\mathrm{q}, J=7.3 \mathrm{~Hz}, 2 \mathrm{H}), 1.52-$ $1.41(\mathrm{~m}, 2 \mathrm{H}), 0.92(\mathrm{t}, J=7.4 \mathrm{~Hz}, 3 \mathrm{H}) ;{ }^{13} \mathrm{C} \mathrm{NMR}\left(100 \mathrm{MHz}, \mathrm{CDCl}_{3}\right) \delta 167.9,164.5,141.4,140.1,137.8,133.8$, 132.5, 131.9, 129.2, 128.4, 127.5, 123.9, 123.8, 122.4, 119.8, 113.5, 66.2, 54.7, 35.2, 22.5, 15.3, 13.8; HRMS (ESI-TOF) $m / z$ Calcd for $\mathrm{C}_{22} \mathrm{H}_{25} \mathrm{~N}_{2} \mathrm{O}_{2} \mathrm{~S}[\mathrm{M}+\mathrm{H}]^{+}: 381.1631$, found: 381.1639 . 
<smiles>CCC/C=C/c1cc(S(C)(=O)=O)cc(/C=C/CCC)c1C(=O)Nc1ccccc1C1=NCCO1</smiles>

$N$-(2-(4,5-Dihydrooxazol-2-yl)phenyl)-4-(methylthio)-2,6-di((E)-pent-1-en-1-yl)benzamide (3h (di))

Substrate $\mathbf{1 h}$ was alkenylated with alkenyl boronates (2) following the general procedure A. After purification by preparative thin-layer chromatography (petroleum ether/ethyl acetate $=20 / 1$ ), $\mathbf{3 h}$ (di) was obtained as a white solid (10.0 mg, 23\%). mp 95-97 ${ }^{\circ} \mathrm{C}$; ${ }^{1} \mathrm{H}$ NMR $\left(400 \mathrm{MHz}, \mathrm{CDCl}_{3}\right) \delta 12.29(\mathrm{~s}, 1 \mathrm{H}), 8.94(\mathrm{~d}, J=8.4 \mathrm{~Hz}, 1 \mathrm{H}), 7.87$ $(\mathrm{d}, J=7.9 \mathrm{~Hz}, 1 \mathrm{H}), 7.53(\mathrm{t}, J=7.8 \mathrm{~Hz}, 1 \mathrm{H}), 7.27-7.26(\mathrm{~m}, 2 \mathrm{H}), 7.14(\mathrm{t}, J=7.7 \mathrm{~Hz}, 1 \mathrm{H}), 6.49$ (d, $J=15.7 \mathrm{~Hz}$, 2H), 6.26-6.13 (m, 2H), 4.29 (t, $J=9.5 \mathrm{~Hz}, 2 \mathrm{H}), 3.92(\mathrm{t}, J=9.6 \mathrm{~Hz}, 2 \mathrm{H}), 2.54(\mathrm{~s}, 3 \mathrm{H}), 2.10$ (q, $J=7.2 \mathrm{~Hz}, 4 \mathrm{H})$, $1.44-1.33(\mathrm{~m}, 4 \mathrm{H}), 0.86(\mathrm{t}, J=7.4 \mathrm{~Hz}, 6 \mathrm{H}) ;{ }^{13} \mathrm{C} \mathrm{NMR}\left(100 \mathrm{MHz}, \mathrm{CDCl}_{3}\right) \delta 168.4,164.0,139.5,139.1,135.9$, $133.9,132.4,132.3,129.1,126.7,122.7,121.7,120.1,113.6,66.1,54.7,35.2,22.4,15.7,13.6$; HRMS (ESITOF) $m / z$ Calcd for $\mathrm{C}_{27} \mathrm{H}_{33} \mathrm{~N}_{2} \mathrm{O}_{2} \mathrm{~S}[\mathrm{M}+\mathrm{H}]^{+}: 449.2257$, found: 449.2273 .<smiles>CNc1ccc(C(=O)Nc2ccccc2C2=NCCO2)c(/C=C/CCNC(C)(C)C)c1</smiles>

(E)-4-Acetamido- $N$-(2-(4,5-dihydrooxazol-2-yl)phenyl)-2-(pent-1-en-1-yl)benzamide (3i (mono))

Substrate 1i was alkenylated with alkenyl boronates (2) following the general procedure A. After purification by preparative thin-layer chromatography (petroleum ether/ethyl acetate $=2 / 1), 3 \mathbf{i}($ mono) was obtained as a white solid (12.6 mg, 33\%). mp 151-153 ${ }^{\circ} \mathrm{C}$; ${ }^{1} \mathrm{H}$ NMR (400 MHz, $\left.\mathrm{CDCl}_{3}\right) \delta 12.62(\mathrm{~s}, 1 \mathrm{H}), 8.90(\mathrm{~d}, J=8.6 \mathrm{~Hz}$, $1 \mathrm{H}), 7.91-7.85(\mathrm{~m}, 2 \mathrm{H}), 7.61(\mathrm{~s}, 1 \mathrm{H}), 7.58-7.48(\mathrm{~m}, 3 \mathrm{H}), 7.12(\mathrm{t}, J=7.6 \mathrm{~Hz}, 1 \mathrm{H}), 6.88(\mathrm{~d}, J=15.6 \mathrm{~Hz}, 1 \mathrm{H})$, 6.20-6.09 (m, 1H), 4.34 (t, $J=9.4 \mathrm{~Hz}, 2 \mathrm{H}), 4.02(\mathrm{t}, J=9.5 \mathrm{~Hz}, 2 \mathrm{H}), 2.18(\mathrm{~s}, 3 \mathrm{H}), 2.13(\mathrm{~d}, J=7.0 \mathrm{~Hz}, 2 \mathrm{H})$, $1.48-1.35$ (m, 2H), 0.89 (t, $J=7.4 \mathrm{~Hz}, 3 \mathrm{H}) ;{ }^{13} \mathrm{C} \mathrm{NMR}\left(100 \mathrm{MHz}, \mathrm{CDCl}_{3}\right) \delta 168.8,168.2,164.4,134.0,139.8$, $138.2,133.8,132.5,131.0,129.2,128.8,127.2,122.5,119.7,117.9,117.3,113.5,66.2,54.7,35.2,24.6,22.4$, 13.7; HRMS (ESI-TOF) $m / z$ Calcd for $\mathrm{C}_{23} \mathrm{H}_{26} \mathrm{~N}_{3} \mathrm{O}_{3}[\mathrm{M}+\mathrm{H}]^{+}: 392.1969$, found: 392.1979 . 
<smiles>CCC/C=C/c1cc(NC=O)cc(/C=C/CCC)c1C(=O)Nc1ccccc1C1=NCCO1</smiles>

4-Acetamido- $N$-(2-(4,5-dihydrooxazol-2-yl)phenyl)-2,6-di((E)-pent-1-en-1-yl)benzamide (3i (di))

Substrate 1i was alkenylated with alkenyl boronates (2) following the general procedure A. After purification by preparative thin-layer chromatography (petroleum ether/ethyl acetate $=2 / 1$ ), $\mathbf{3 i}$ (di) was obtained as a white solid (7.3 mg, 16\%). mp 71-73 ${ }^{\circ} \mathrm{C} ;{ }^{1} \mathrm{H}$ NMR $\left(400 \mathrm{MHz}, \mathrm{CDCl}_{3}\right) \delta 12.37$ (s, 1H), $8.94(\mathrm{~d}, J=8.5 \mathrm{~Hz}, 1 \mathrm{H}), 7.96-$ $7.78(\mathrm{~m}, 2 \mathrm{H}), 7.55(\mathrm{t}, J=7.5 \mathrm{~Hz}, 1 \mathrm{H}), 7.42-7.37(\mathrm{~m}, 2 \mathrm{H}), 7.16(\mathrm{t}, J=7.7 \mathrm{~Hz}, 1 \mathrm{H}), 6.43(\mathrm{~d}, J=15.4 \mathrm{~Hz}, 2 \mathrm{H})$, 6.23-6.06 (m, 2H), 4.29 (t, $J=9.6 \mathrm{~Hz}, 2 \mathrm{H}), 3.91(\mathrm{t}, J=9.5 \mathrm{~Hz}, 2 \mathrm{H}), 2.18(\mathrm{~s}, 3 \mathrm{H}), 2.08(\mathrm{q}, J=7.2 \mathrm{~Hz}, 4 \mathrm{H})$, $1.45-1.30(\mathrm{~m}, 4 \mathrm{H}), 0.84(\mathrm{t}, J=7.4 \mathrm{~Hz}, 6 \mathrm{H}) ;{ }^{13} \mathrm{C} \mathrm{NMR}\left(100 \mathrm{MHz}, \mathrm{CDCl}_{3}\right) \delta 169.1,168.8,163.9,139.4,138.2$, 136.0, 133.9, 132.4, 131.1, 129.2 126.5, 122.8, 120.1, 116.0, 113.8, 66.1, 54.7, 35.2, 24.4, 22.3, 13.6; HRMS (ESI-TOF) $m / z$ Calcd for $\mathrm{C}_{28} \mathrm{H}_{34} \mathrm{~N}_{3} \mathrm{O}_{3}[\mathrm{M}+\mathrm{H}]^{+}: 460.2595$, found: 460.2609 .<smiles>CCC/C=C/c1cc(N2CCOCC2)ccc1C(=O)Nc1ccccc1C1=NCCO1</smiles>

(E)- $N$-(2-(4,5-Dihydrooxazol-2-yl)phenyl)-4-morpholino-2-(pent-1-en-1-yl)benzamide (3j (mono))

Substrate $\mathbf{1 j}$ was alkenylated with alkenyl boronates (2) following the general procedure $\mathbf{A}\left(\mathrm{Cu}(\mathrm{OPiv})_{2}(30\right.$ mol\%)). After purification by preparative thin-layer chromatography (petroleum ether/ethyl acetate $=4 / 1), 3 \mathbf{j}$ (mono) was obtained as a white solid $(14.1 \mathrm{mg}, 34 \%)$ mp 83-85 ${ }^{\circ} \mathrm{C} ;{ }^{1} \mathrm{H}$ NMR $\left(400 \mathrm{MHz}, \mathrm{CDCl}_{3}\right) \delta 12.50(\mathrm{~s}$, $1 \mathrm{H}), 8.91(\mathrm{~d}, J=8.4 \mathrm{~Hz}, 1 \mathrm{H}), 7.87(\mathrm{~d}, J=7.9 \mathrm{~Hz}, 1 \mathrm{H}), 7.62(\mathrm{~d}, J=8.6 \mathrm{~Hz}, 1 \mathrm{H}), 7.50(\mathrm{t}, J=8.0 \mathrm{~Hz}, 1 \mathrm{H}), 7.09$ (t, $J=7.6 \mathrm{~Hz}, 1 \mathrm{H}), 7.05-6.97(\mathrm{~m}, 2 \mathrm{H}), 6.81(\mathrm{dd}, J=8.6,2.4 \mathrm{~Hz}, 1 \mathrm{H}), 6.20-6.10(\mathrm{~m}, 1 \mathrm{H}), 4.35$ (t, $J=9.5 \mathrm{~Hz}$, 2H), 4.05 (t, $J=9.5 \mathrm{~Hz}, 2 \mathrm{H}), 3.91-3.84(\mathrm{~m}, 4 \mathrm{H}), 3.31-3.25(\mathrm{~m}, 4 \mathrm{H}), 2.19$ (q, $J=7.4 \mathrm{~Hz}, 2 \mathrm{H}), 1.52-1.43(\mathrm{~m}$, $2 \mathrm{H}), 0.93(\mathrm{t}, J=7.4 \mathrm{~Hz}, 3 \mathrm{H}) ;{ }^{13} \mathrm{C} \mathrm{NMR}\left(100 \mathrm{MHz}, \mathrm{CDCl}_{3}\right) \delta 168.1,164.5,152.4,140.4,139.2,132.8,132.5$, 129.5, 129.1, 128.7, 126.4, 122.1, 119.7, 113.3, 113.1, 112.7, 66.8, 66.1, 54.7, 48.4, 35.2, 22.5, 13.8; HRMS (ESI-TOF) $m / z$ Calcd for $\mathrm{C}_{25} \mathrm{H}_{29} \mathrm{~N}_{3} \mathrm{O}_{3} \mathrm{Na}[\mathrm{M}+\mathrm{Na}]^{+}: 442.2101$, found: 442.2111 . 
<smiles>CCC/C=C/c1cc(N2CCOCC2)cc(/C=C/CCC)c1C(=O)Nc1ccccc1C1=NCCO1</smiles>

$N$-(2-(4,5-Dihydrooxazol-2-yl)phenyl)-4-morpholino-2,6-di((E)-pent-1-en-1-yl)benzamide (3j (di))

Substrate $\mathbf{1 j}$ was alkenylated with alkenyl boronates (2) following the general procedure $\mathbf{A}\left(\mathrm{Cu}(\mathrm{OPiv})_{2}(30\right.$ mol\%)). After purification by preparative thin-layer chromatography (petroleum ether/ethyl acetate $=4 / 1), \mathbf{3} \mathbf{j}$ (mono) was obtained as a white solid $(9.5 \mathrm{mg}, 20 \%) . \mathrm{mp} 103-105{ }^{\circ} \mathrm{C} ;{ }^{1} \mathrm{H} \mathrm{NMR}\left(400 \mathrm{MHz}, \mathrm{CDCl}_{3}\right) \delta 12.22$ (s, $1 \mathrm{H}), 8.95(\mathrm{~d}, J=8.3 \mathrm{~Hz}, 1 \mathrm{H}), 7.86(\mathrm{~d}, J=7.8 \mathrm{~Hz}, 1 \mathrm{H}), 7.53(\mathrm{t}, J=7.6 \mathrm{~Hz}, 1 \mathrm{H}), 7.12(\mathrm{t}, J=7.7 \mathrm{~Hz}, 1 \mathrm{H}), 6.93$ (s, 2H), $6.52(\mathrm{~d}, J=15.6 \mathrm{~Hz}, 2 \mathrm{H}), 6.20-6.10(\mathrm{~m}, 2 \mathrm{H}), 4.29(\mathrm{t}, J=9.5 \mathrm{~Hz}, 2 \mathrm{H}), 3.97-3.83(\mathrm{~m}, 6 \mathrm{H}), 3.29-3.21$ $(\mathrm{m}, 4 \mathrm{H}), 2.09$ (q, $J=7.1 \mathrm{~Hz}, 4 \mathrm{H}), 1.44-1.33(\mathrm{~m}, 4 \mathrm{H}), 0.85(\mathrm{t}, J=7.4 \mathrm{~Hz}, 6 \mathrm{H}) ;{ }^{13} \mathrm{C} \mathrm{NMR}\left(100 \mathrm{MHz}, \mathrm{CDCl}_{3}\right) \delta$ 168.9, 164.0, 151.3, 139.7, 136.5, 133.1, 132.4, 129.1 128.0, 127.6, 122.5, 120.1, 113.5, 111.3, 66.9, 66.0, 54.7, 49.2, 35.2, 22.4, 13.6; HRMS (ESI-TOF) $m / z$ Calcd for $\mathrm{C}_{30} \mathrm{H}_{37} \mathrm{~N}_{3} \mathrm{O}_{3} \mathrm{Na}[\mathrm{M}+\mathrm{Na}]^{+}: 510.2727$, found: 510.2747.<smiles>CCC/C=C/c1cccc(C)c1C(=O)Nc1ccccc1C1=NCCO1</smiles>

(E)-N-(2-(4,5-Dihydrooxazol-2-yl)phenyl)-2-methyl-6-(pent-1-en-1-yl)benzamide (3k)

Substrate 1k was alkenylated with alkenyl boronates (2) following the general procedure $\mathbf{A}\left(\mathrm{Cu}(\mathrm{OPiv})_{2}(30\right.$ $\mathrm{mol} \%$ ), $60{ }^{\circ} \mathrm{C}$ ). After purification by preparative thin-layer chromatography (petroleum ether/ethyl acetate $=$ 20/1), 3k was obtained as a white solid (16.3 mg, 47\%). mp 83-85 ${ }^{\circ} \mathrm{C} ;{ }^{1} \mathrm{H}$ NMR $\left(400 \mathrm{MHz}, \mathrm{CDCl}_{3}\right) \delta 12.29$ (s, $1 \mathrm{H}), 8.95(\mathrm{~d}, J=8.4 \mathrm{~Hz}, 1 \mathrm{H}), 7.91-7.85(\mathrm{~m}, 1 \mathrm{H}), 7.54(\mathrm{t}, J=7.9 \mathrm{~Hz}, 1 \mathrm{H}), 7.39(\mathrm{~d}, J=7.8 \mathrm{~Hz}, 1 \mathrm{H}), 7.23(\mathrm{~d}, J=$ $7.8 \mathrm{~Hz}, 1 \mathrm{H}), 7.14$ (t, $J=7.6 \mathrm{~Hz}, 1 \mathrm{H}), 7.09$ (d, $J=7.6 \mathrm{~Hz}, 1 \mathrm{H}), 6.51(\mathrm{~d}, J=15.7 \mathrm{~Hz}, 1 \mathrm{H}), 6.25-6.14(\mathrm{~m}, 1 \mathrm{H})$, $4.30(\mathrm{t}, J=9.5 \mathrm{~Hz}, 2 \mathrm{H}), 3.93(\mathrm{t}, J=9.5 \mathrm{~Hz}, 2 \mathrm{H}), 2.38(\mathrm{~s}, 3 \mathrm{H}), 2.10(\mathrm{q}, J=7.2 \mathrm{~Hz}, 2 \mathrm{H}), 1.44-1.34(\mathrm{~m}, 2 \mathrm{H}), 0.86$ $(\mathrm{t}, J=7.4 \mathrm{~Hz}, 3 \mathrm{H}) ;{ }^{13} \mathrm{C} \mathrm{NMR}\left(100 \mathrm{MHz}, \mathrm{CDCl}_{3}\right) \delta 169.0,164.1,139.5,136.6,135.0,134.4,133.2,132.5,129.2$, 128.7, 128.5, 127.1, 122.9, 122.7, 120.1, 113.6, 66.1, 54.7, 35.2, 22.4, 19.5, 13.6; HRMS (ESI-TOF) $\mathrm{m} / z$ Calcd for $\mathrm{C}_{22} \mathrm{H}_{25} \mathrm{~N}_{2} \mathrm{O}_{2}[\mathrm{M}+\mathrm{H}]^{+}$: 349.1911, found: 349.1913 .<smiles>CCC/C=C/c1cccc(C(F)(F)F)c1C(=O)Nc1ccccc1C1=NCCO1</smiles> 
Substrate 11 was alkenylated with alkenyl boronates (2) following the general procedure $\mathbf{A}\left(\mathrm{Cu}(\mathrm{OPiv})_{2}(30\right.$ $\mathrm{mol} \%$ ), $60{ }^{\circ} \mathrm{C}$ ). After purification by preparative thin-layer chromatography (petroleum ether/ethyl acetate $=$ 20/1), 31 was obtained as a white solid (13.0 mg, 33\%). mp 52-54 ${ }^{\circ} \mathrm{C} ;{ }^{1} \mathrm{H}$ NMR $\left(400 \mathrm{MHz}, \mathrm{CDCl}_{3}\right) \delta 12.48(\mathrm{~s}$, $1 \mathrm{H}), 8.88(\mathrm{~d}, J=8.4 \mathrm{~Hz}, 1 \mathrm{H}), 7.88(\mathrm{dd}, J=7.9,1.4 \mathrm{~Hz}, 1 \mathrm{H}), 7.76(\mathrm{~d}, J=8.0 \mathrm{~Hz}, 1 \mathrm{H}), 7.59-7.51(\mathrm{~m}, 2 \mathrm{H}), 7.47$ $(\mathrm{t}, J=7.9 \mathrm{~Hz}, 1 \mathrm{H}), 7.16(\mathrm{t}, J=7.3 \mathrm{~Hz}, 1 \mathrm{H}), 6.55(\mathrm{~d}, J=15.8 \mathrm{~Hz}, 1 \mathrm{H}), 6.34-6.19(\mathrm{~m}, 1 \mathrm{H}), 4.31(\mathrm{t}, J=9.5 \mathrm{~Hz}$, 2H), $3.92(\mathrm{t}, J=8.8 \mathrm{~Hz}, 2 \mathrm{H}), 2.13(\mathrm{q}, J=7.3 \mathrm{~Hz}, 2 \mathrm{H}), 1.46-1.35(\mathrm{~m}, 2 \mathrm{H}), 0.86(\mathrm{t}, J=7.4 \mathrm{~Hz}, 3 \mathrm{H}) ;{ }^{13} \mathrm{C} \mathrm{NMR}$ $\left(100 \mathrm{MHz}, \mathrm{CDCl}_{3}\right) \delta 166.0,164.1,139.3,136.7,135.5,134.0$ (q, $\left.J_{C-F}=2.0 \mathrm{~Hz}\right), 132.5,129.1,129.1,129.0$, $127.3\left(\mathrm{q}, J_{C-F}=31.0 \mathrm{~Hz}\right), 125.8,124.4\left(\mathrm{q}, J_{C-F}=5.0 \mathrm{~Hz}\right), 123.8\left(\mathrm{~d}, J_{C-F}=273.0 \mathrm{~Hz}\right), 123.0,120.2,113.7,66.1$, 54.6, 35.2, 22.3, 13.5; ${ }^{19} \mathrm{~F}$ NMR $\left(375 \mathrm{MHz}, \mathrm{CDCl}_{3}\right) \delta-59.01$ (s, 3F). HRMS (ESI-TOF) $\mathrm{m} / \mathrm{z}$ Calcd for $\mathrm{C}_{22} \mathrm{H}_{22} \mathrm{~F}_{3} \mathrm{~N}_{2} \mathrm{O}_{2}[\mathrm{M}+\mathrm{H}]^{+}: 403.1628$, found: 403.1640 .<smiles>CCCC=Cc1ccc(C)cc1C(=O)Nc1ccccc1C1=NCCO1</smiles>

(E)- $N$-(2-(4,5-Dihydrooxazol-2-yl)phenyl)-5-methyl-2-(pent-1-en-1-yl)benzamide (3m)

Substrate $\mathbf{1 m}$ was alkenylated with alkenyl boronates (2) following the general procedure $\mathbf{A}\left(\mathrm{Cu}(\mathrm{OPiv})_{2}(35\right.$ $\mathrm{mol} \%$ ), $50{ }^{\circ} \mathrm{C}$ ). After purification by preparative thin-layer chromatography (petroleum ether/ethyl acetate $=$ 20/1), 3m was obtained as a white solid $(26.8 \mathrm{mg}, 77 \%)$. mp $130-132{ }^{\circ} \mathrm{C} ;{ }^{1} \mathrm{H}$ NMR $\left(400 \mathrm{MHz}, \mathrm{CDCl}_{3}\right) \delta 12.54$ (s, 1H), $8.93(\mathrm{~d}, J=8.2 \mathrm{~Hz}, 1 \mathrm{H}), 7.89(\mathrm{dd}, J=8.0,4.0 \mathrm{~Hz}, 1 \mathrm{H}), 7.55-7.47(\mathrm{~m}, 2 \mathrm{H}), 7.44(\mathrm{~s}, 1 \mathrm{H}), 7.21(\mathrm{~d}, J=$ $8.2 \mathrm{~Hz}, 1 \mathrm{H}), 7.12(\mathrm{t}, J=7.1 \mathrm{~Hz}, 1 \mathrm{H}), 6.86(\mathrm{~d}, J=15.8 \mathrm{~Hz}, 1 \mathrm{H}), 6.23-6.12(\mathrm{~m}, 1 \mathrm{H}), 4.35(\mathrm{t}, J=9.5 \mathrm{~Hz}, 2 \mathrm{H})$, $4.03(\mathrm{t}, J=9.5 \mathrm{~Hz}, 2 \mathrm{H}), 2.37(\mathrm{~s}, 3 \mathrm{H}), 2.16(\mathrm{q}, J=6.8 \mathrm{~Hz}, 2 \mathrm{H}), 1.50-1.40(\mathrm{~m}, 2 \mathrm{H}), 0.91(\mathrm{t}, J=7.4 \mathrm{~Hz}, 3 \mathrm{H}) ;{ }^{13} \mathrm{C}$ NMR $\left(100 \mathrm{MHz}, \mathrm{CDCl}_{3}\right) \delta 168.6,164.4,140.1,136.3,135.3,134.1,132.5,132.4,130.9,129.1,128.4,127.4$, 126.4, 122.4, 119.8, 113.4, 66.1, 54.6, 35.3, 22.6, 21.1, 13.7; HRMS (ESI-TOF) $m / z$ Calcd for $\mathrm{C}_{22} \mathrm{H}_{25} \mathrm{~N}_{2} \mathrm{O}_{2}$ $[\mathrm{M}+\mathrm{H}]^{+}:$349.1911, found: 349.1914.<smiles>CCC/C=C/c1ccc(-c2ccccc2)cc1C(=O)Nc1ccccc1C1=NCCO1</smiles>

(E)- $N$-(2-(4,5-Dihydrooxazol-2-yl)phenyl)-4-(pent-1-en-1-yl)-[1,1'-biphenyl]-3-carboxamide (3n)

Substrate 1n was alkenylated with alkenyl boronates (2) following the general procedure $\mathbf{A}\left(\mathrm{Cu}(\mathrm{OPiv})_{2}(30\right.$ mol\%)). After purification by preparative thin-layer chromatography (petroleum ether/ethyl acetate $=20 / 1), 3 \mathbf{n}$ was obtained as a white solid (23.4 mg, 57\%). mp 108-110 ${ }^{\circ} \mathrm{C} ;{ }^{1} \mathrm{H} \mathrm{NMR}\left(400 \mathrm{MHz}, \mathrm{CDCl}_{3}\right) \delta 12.70(\mathrm{~s}, 1 \mathrm{H})$, $8.97(\mathrm{~d}, J=8.3 \mathrm{~Hz}, 1 \mathrm{H}), 7.94-7.87(\mathrm{~m}, 2 \mathrm{H}), 7.72-7.62(\mathrm{~m}, 4 \mathrm{H}), 7.53(\mathrm{t}, J=7.9 \mathrm{~Hz}, 1 \mathrm{H}), 7.44(\mathrm{t}, J=7.6 \mathrm{~Hz}$, 2H), $7.34(\mathrm{t}, J=7.3 \mathrm{~Hz}, 1 \mathrm{H}), 7.12(\mathrm{t}, J=7.4 \mathrm{~Hz}, 1 \mathrm{H}), 6.99$ (d, $J=15.7 \mathrm{~Hz}, 1 \mathrm{H}), 6.33-6.22(\mathrm{~m}, 1 \mathrm{H}), 4.34(\mathrm{t}, J$ $=9.5 \mathrm{~Hz}, 2 \mathrm{H}), 4.02(\mathrm{t}, J=9.5 \mathrm{~Hz}, 2 \mathrm{H}), 2.21(\mathrm{q}, J=7.3 \mathrm{~Hz}, 2 \mathrm{H}), 1.54-1.43(\mathrm{~m}, 2 \mathrm{H}), 0.94(\mathrm{t}, J=7.4 \mathrm{~Hz}, 3 \mathrm{H})$; ${ }^{13} \mathrm{C}$ NMR $\left(100 \mathrm{MHz}, \mathrm{CDCl}_{3}\right) \delta 168.2,164.6,140.1,140.1,139.3,136.1,135.5,133.4,132.6,129.2,128.8$, 
128.7, 127.5, 127.2, 127.0, 126.8, 126.4, 122.5, 119.8, 113.5, 66.1, 54.7, 35.3, 22.5, 13.8; HRMS (ESI-TOF) $m / z$ Calcd for $\mathrm{C}_{27} \mathrm{H}_{27} \mathrm{~N}_{2} \mathrm{O}_{2}[\mathrm{M}+\mathrm{H}]^{+}:$411.2067, found: 411.2077.<smiles>CCC/C=C/c1ccc(OC)cc1C(=O)Nc1ccccc1C1=NCCO1</smiles>

(E)-N-(2-(4,5-Dihydrooxazol-2-yl)phenyl)-5-methoxy-2-(pent-1-en-1-yl)benzamide (3o)

Substrate 10 was alkenylated with alkenyl boronates (2) following the general procedure $\mathbf{A}\left(\mathrm{Cu}(\mathrm{OPiv})_{2}(30\right.$ mol\%)). After purification by preparative thin-layer chromatography (petroleum ether/ethyl acetate $=20 / 1$ ), 30 was obtained as a white solid (27.1 mg, 75\%). mp 111-113 ${ }^{\circ} \mathrm{C} ;{ }^{1} \mathrm{H} \mathrm{NMR}\left(400 \mathrm{MHz}, \mathrm{CDCl}_{3}\right) \delta 12.58(\mathrm{~s}, 1 \mathrm{H})$, $8.94(\mathrm{~d}, J=8.4 \mathrm{~Hz}, 1 \mathrm{H}), 7.89(\mathrm{dd}, J=7.9,1.3 \mathrm{~Hz}, 1 \mathrm{H}), 7.57-7.49(\mathrm{~m}, 2 \mathrm{H}), 7.19-7.07(\mathrm{~m}, 2 \mathrm{H}), 6.96(\mathrm{dd}, J=$ $8.7,2.6 \mathrm{~Hz}, 1 \mathrm{H}), 6.83(\mathrm{~d}, J=15.7 \mathrm{~Hz}, 1 \mathrm{H}), 6.10(\mathrm{dt}, J=15.6,7.0 \mathrm{~Hz}, 1 \mathrm{H}), 4.34(\mathrm{t}, J=9.4 \mathrm{~Hz}, 2 \mathrm{H}), 4.02(\mathrm{t}, J=$ $9.5 \mathrm{~Hz}, 2 \mathrm{H}), 3.84(\mathrm{~s}, 3 \mathrm{H}), 2.20-2.10(\mathrm{~m}, 2 \mathrm{H}), 1.50-1.38(\mathrm{~m}, 2 \mathrm{H}), 0.91(\mathrm{t}, J=7.4 \mathrm{~Hz}, 3 \mathrm{H}) ;{ }^{13} \mathrm{C}$ NMR $(100 \mathrm{MHz}$, $\left.\mathrm{CDCl}_{3}\right) \delta 168.1,164.4,158.2,139.9,136.2,132.5,131.4,129.6,129.2,127.8,126.9,122.5,119.8,116.9,113.4$, 112.0, 66.1, 55.4, 54.7, 35.2, 22.6, 13.7; HRMS (ESI-TOF) $m / z$ Calcd for $\mathrm{C}_{22} \mathrm{H}_{25} \mathrm{~N}_{2} \mathrm{O}_{3}[\mathrm{M}+\mathrm{H}]^{+}: 365.1860$, found: 365.1870 .<smiles>CCC/C=C/c1ccc(OC(F)(F)F)cc1C(=O)Nc1ccccc1C1=NCCO1</smiles>

(E)-N-(2-(4,5-Dihydrooxazol-2-yl)phenyl)-2-(pent-1-en-1-yl)-5-(1,1,2,2-tetrafluoroethoxy)benzamide (3p)

Substrate 1p was alkenylated with alkenyl boronates (2) following the general procedure $\mathbf{A}\left(\mathrm{Cu}(\mathrm{OPiv})_{2}(30\right.$ mol\%)). After purification by preparative thin-layer chromatography (petroleum ether/ethyl acetate $=20 / 1$ ), $\mathbf{3 p}$ was obtained as a white solid (23.6 mg, 53\%). mp 84-86 ${ }^{\circ} \mathrm{C}$; ${ }^{1} \mathrm{H}$ NMR $\left(400 \mathrm{MHz}, \mathrm{CDCl}_{3}\right) \delta 12.79(\mathrm{~s}, 1 \mathrm{H}), 8.92$ $(\mathrm{d}, J=8.4 \mathrm{~Hz}, 1 \mathrm{H}), 7.90(\mathrm{dd}, J=7.9,1.2 \mathrm{~Hz}, 1 \mathrm{H}), 7.62(\mathrm{~d}, J=8.7 \mathrm{~Hz}, 1 \mathrm{H}), 7.57-7.50(\mathrm{~m}, 2 \mathrm{H}), 7.26-7.24(\mathrm{~m}$, $1 \mathrm{H}), 7.14(\mathrm{t}, J=7.6 \mathrm{~Hz}, 1 \mathrm{H}), 6.93(\mathrm{~d}, J=15.8 \mathrm{~Hz}, 1 \mathrm{H}), 6.27-6.16(\mathrm{~m}, 1 \mathrm{H}), 5.93(\mathrm{tt}, J=53.1,12.8 \mathrm{~Hz}, 1 \mathrm{H})$, $4.37(\mathrm{t}, J=9.6 \mathrm{~Hz}, 2 \mathrm{H}), 4.04(\mathrm{t}, J=9.5 \mathrm{~Hz}, 2 \mathrm{H}), 2.20(\mathrm{q}, J=7.0 \mathrm{~Hz}, 2 \mathrm{H}), 1.53-1.42(\mathrm{~m}, 2 \mathrm{H}), 0.93(\mathrm{t}, J=7.4$ $\mathrm{Hz}, 3 \mathrm{H}) .{ }^{13} \mathrm{C}$ NMR $\left(150 \mathrm{MHz}\right.$, decoupled for $\left.{ }^{19} \mathrm{~F}, \mathrm{CDCl}_{3}\right) \delta 166.8,164.6,147.0,139.9,136.2,136.0,134.2$, 132.6, 129.2, 128.0, 126.6, 123.6, 122.8, 121.1, 119.8, 116.5, 113.6, 107.7, 66.3, 54.4, 35.2, 22.4, 13.7; ${ }^{19} \mathrm{~F} \mathrm{NMR}$ $\left(375 \mathrm{MHz}, \mathrm{CDCl}_{3}\right) \delta-88.16--88.22(\mathrm{~m}, 2 \mathrm{~F}),-136.78\left(\mathrm{dt}, J_{F-F}=53.1,5.7 \mathrm{~Hz}, 2 \mathrm{~F}\right) ; \mathrm{HRMS}$ (ESI-TOF) $m / z$ Calcd for $\mathrm{C}_{23} \mathrm{H}_{23} \mathrm{~F}_{4} \mathrm{~N}_{2} \mathrm{O}_{3}[\mathrm{M}+\mathrm{H}]^{+}: 451.1639$, found: 451.1652 . 
<smiles>CCC/C=C/c1ccc(Cl)cc1C(=O)Nc1ccccc1C1=NCCO1</smiles>

(E)-5-Chloro- $N$-(2-(4,5-dihydrooxazol-2-yl)phenyl)-2-(pent-1-en-1-yl)benzamide (3q)

Substrate 1q was alkenylated with alkenyl boronates (2) following the general procedure $\mathbf{A}\left(\mathrm{Cu}(\mathrm{OPiv})_{2}(30\right.$ mol\%)). After purification by preparative thin-layer chromatography (petroleum ether/ethyl acetate $=20 / 1$ ), $\mathbf{3 q}$ was obtained as a white solid (13.3 mg, 37\%). mp 134-136 ${ }^{\circ} \mathrm{C}$; ${ }^{1} \mathrm{H}$ NMR $\left(400 \mathrm{MHz}, \mathrm{CDCl}_{3}\right) \delta 12.67(\mathrm{~s}, 1 \mathrm{H})$, $8.88(\mathrm{~d}, J=8.4 \mathrm{~Hz}, 1 \mathrm{H}), 7.89(\mathrm{~d}, J=7.8 \mathrm{~Hz}, 1 \mathrm{H}), 7.62(\mathrm{~d}, J=2.1 \mathrm{~Hz}, 1 \mathrm{H}), 7.56-7.50(\mathrm{~m}, 2 \mathrm{H}), 7.36(\mathrm{dd}, J=8.5$, $1.7 \mathrm{~Hz}, 1 \mathrm{H}), 7.14(\mathrm{t}, J=7.6 \mathrm{~Hz}, 1 \mathrm{H}), 6.85(\mathrm{~d}, J=15.7 \mathrm{~Hz}, 1 \mathrm{H}), 6.27-6.16(\mathrm{~m}, 1 \mathrm{H}), 4.37(\mathrm{t}, J=9.5 \mathrm{~Hz}, 2 \mathrm{H})$, $4.06(\mathrm{t}, J=9.6 \mathrm{~Hz}, 2 \mathrm{H}), 2.20-2.14(\mathrm{~m}, 2 \mathrm{H}), 1.50-1.42(\mathrm{~m}, 2 \mathrm{H}), 0.92(\mathrm{t}, J=7.4 \mathrm{~Hz}, 3 \mathrm{H}) ;{ }^{13} \mathrm{C} \mathrm{NMR}(100 \mathrm{MHz}$, $\left.\mathrm{CDCl}_{3}\right) \delta 166.9,164.5,139.7,136.5,135.5,134.0,132.6,132.2,130.2,129.2,127.8,126.5,122.7,119.8,113.6$, 66.3, 54.6, 35.2, 22.4, 13.7; HRMS (ESI-TOF) $\mathrm{m} / \mathrm{z}$ Calcd for $\mathrm{C}_{21} \mathrm{H}_{22} \mathrm{ClN}_{2} \mathrm{O}_{2}[\mathrm{M}+\mathrm{H}]^{+}:$369.1364, found: 369.1371 .<smiles>CCC/C=C/c1ccc(OC)c([N+](=O)[O-])c1C(=O)Nc1ccccc1C1=NCCO1</smiles>

(E)-N-(2-(4,5-Dihydrooxazol-2-yl)phenyl)-3-methoxy-2-methyl-6-(pent-1-en-1-yl)benzamide (3r)

Substrate $\mathbf{1 r}$ was alkenylated with alkenyl boronates (2) following the general procedure $\mathbf{A}\left(\mathrm{Cu}(\mathrm{OPiv})_{2}(30\right.$ $\mathrm{mol} \%$ ), $60{ }^{\circ} \mathrm{C}$ ). After purification by preparative thin-layer chromatography (petroleum ether/ethyl acetate = 20/1), 3r was obtained as a white solid (15.1 mg, 40\%). mp 104-106 ${ }^{\circ} \mathrm{C} ;{ }^{1} \mathrm{H}$ NMR (400 MHz, $\left.\mathrm{CDCl}_{3}\right) \delta 12.27$ $(\mathrm{s}, 1 \mathrm{H}), 8.94(\mathrm{~d}, J=8.4 \mathrm{~Hz}, 1 \mathrm{H}), 7.88(\mathrm{~d}, J=7.8 \mathrm{~Hz}, 1 \mathrm{H}), 7.53(\mathrm{t}, J=7.8 \mathrm{~Hz}, 1 \mathrm{H}), 7.37(\mathrm{~d}, J=8.6 \mathrm{~Hz}, 1 \mathrm{H})$, $7.14(\mathrm{t}, J=7.6 \mathrm{~Hz}, 1 \mathrm{H}), 6.85(\mathrm{~d}, J=8.6 \mathrm{~Hz}, 1 \mathrm{H}), 6.44(\mathrm{~d}, J=15.6 \mathrm{~Hz}, 1 \mathrm{H}), 6.13-6.01(\mathrm{~m}, 1 \mathrm{H}), 4.30(\mathrm{t}, J=9.5$ $\mathrm{Hz}, 2 \mathrm{H}), 3.95(\mathrm{t}, J=9.5 \mathrm{~Hz}, 2 \mathrm{H}), 3.85(\mathrm{~s}, 3 \mathrm{H}), 2.23(\mathrm{~s}, 3 \mathrm{H}), 2.08$ (q, $J=7.1 \mathrm{~Hz}, 2 \mathrm{H}), 1.44-1.32(\mathrm{~m}, 2 \mathrm{H}), 0.85$ $(\mathrm{t}, J=7.3 \mathrm{~Hz}, 3 \mathrm{H}) ;{ }^{13} \mathrm{C} \mathrm{NMR}\left(100 \mathrm{MHz}, \mathrm{CDCl}_{3}\right) \delta 168.8,164.1,156.5,139.5,137.9,132.4,131.1,129.1,127.5$, 126.8, 123.8, 122.9, 122.7, 120.2, 113.6, 110.9, 66.1, 55.6, 54.7, 35.2, 22.6, 13.6, 12.8; HRMS (ESI-TOF) $\mathrm{m} / z$ Calcd for $\mathrm{C}_{23} \mathrm{H}_{27} \mathrm{~N}_{2} \mathrm{O}_{3}[\mathrm{M}+\mathrm{H}]^{+}: 379.2016$, found: 379.2025 .<smiles>CCC/C=C/c1cc(OC)c(OC)cc1C(=O)Nc1ccccc1C1=NCCO1</smiles>

(E)-N-(2-(4,5-Dihydrooxazol-2-yl)phenyl)-4,5-dimethoxy-2-(pent-1-en-1-yl)benzamide (3s)

Substrate 1s was alkenylated with alkenyl boronates (2) following the general procedure $\mathbf{A}\left(\mathrm{Cu}(\mathrm{OPiv})_{2}(30\right.$ $\mathrm{mol} \%), 9 \mathrm{~h}$ ). After purification by preparative thin-layer chromatography (petroleum ether/ethyl acetate $=20 / 1$ ), 
3s was obtained as a white solid (24.0 mg, 61\%). mp 126-128 ${ }^{\circ} \mathrm{C} ;{ }^{1} \mathrm{H} \mathrm{NMR}\left(400 \mathrm{MHz}, \mathrm{CDCl}_{3}\right) \delta 12.55(\mathrm{~s}, 1 \mathrm{H})$, $8.94(\mathrm{~d}, J=8.5 \mathrm{~Hz}, 1 \mathrm{H}), 7.89(\mathrm{~d}, J=7.8 \mathrm{~Hz}, 1 \mathrm{H}), 7.52(\mathrm{t}, J=7.9 \mathrm{~Hz}, 1 \mathrm{H}), 7.22(\mathrm{~s}, 1 \mathrm{H}), 7.11(\mathrm{t}, J=7.6 \mathrm{~Hz}, 1 \mathrm{H})$, $7.06(\mathrm{~s}, 1 \mathrm{H}), 6.97(\mathrm{~d}, J=15.7 \mathrm{~Hz}, 1 \mathrm{H}), 6.18-6.07(\mathrm{~m}, 1 \mathrm{H}), 4.36(\mathrm{t}, J=9.5 \mathrm{~Hz}, 2 \mathrm{H}), 4.03(\mathrm{t}, J=9.5 \mathrm{~Hz}, 2 \mathrm{H})$, $3.97(\mathrm{~s}, 3 \mathrm{H}), 3.93(\mathrm{~s}, 3 \mathrm{H}), 2.18(\mathrm{q}, J=7.1 \mathrm{~Hz}, 2 \mathrm{H}), 1.58-1.41(\mathrm{~m}, 2 \mathrm{H}), 0.93(\mathrm{t}, J=7.4 \mathrm{~Hz}, 3 \mathrm{H}) ;{ }^{13} \mathrm{C}$ NMR $(100$ $\left.\mathrm{MHz}, \mathrm{CDCl}_{3}\right) \delta 167.8,164.6,150.4,147.6,140.2,132.6,131.6,131.1,129.2,127.6,127.4,122.3,119.6,113.3$, $110.8,108.9,66.1,55.9,54.7,35.2,22.6,13.8$; HRMS (ESI-TOF) $m / z$ Calcd for $\mathrm{C}_{23} \mathrm{H}_{27} \mathrm{~N}_{2} \mathrm{O}_{4}[\mathrm{M}+\mathrm{H}]^{+}: 395.1965$, found: 395.1973 .<smiles>CCC/C=C/c1cc2c(cc1C(=O)Nc1ccccc1C1=NCCO1)OCCO2</smiles>

(E)-N-(2-(4,5-Dihydrooxazol-2-yl)phenyl)-7-(pent-1-en-1-yl)-2,3-dihydrobenzo[b][1,4]dioxine-6carboxamide $\left(3 t\left(\mathrm{C}_{7}\right)\right)$

Substrate 1t was alkenylated with alkenyl boronates (2) following the general procedure $\mathbf{A}\left(\mathrm{Cu}(\mathrm{OPiv})_{2}(30\right.$ $\mathrm{mol} \%)$ ). After purification by preparative thin-layer chromatography (petroleum ether/ethyl acetate $=20 / 1), 3 \mathrm{t}$ $\left(\mathrm{C}_{7}\right)$ was obtained as a white solid $(9.7 \mathrm{mg}, 25 \%)$. mp 138-140 ${ }^{\circ} \mathrm{C} ;{ }^{1} \mathrm{H} \mathrm{NMR}\left(400 \mathrm{MHz}, \mathrm{CDCl}_{3}\right) \delta 12.49(\mathrm{~s}, 1 \mathrm{H})$, $8.89(\mathrm{~d}, J=8.5 \mathrm{~Hz}, 1 \mathrm{H}), 7.87$ (d, $J=7.8 \mathrm{~Hz}, 1 \mathrm{H}), 7.50$ (t, $J=7.9 \mathrm{~Hz}, 1 \mathrm{H}), 7.19(\mathrm{~s}, 1 \mathrm{H}), 7.14-7.06(\mathrm{~m}, 2 \mathrm{H}), 6.85$ $(\mathrm{d}, J=15.6 \mathrm{~Hz}, 1 \mathrm{H}), 6.12-6.00(\mathrm{~m}, 1 \mathrm{H}), 4.41-4.25(\mathrm{~m}, 6 \mathrm{H}), 4.06(\mathrm{t}, J=9.6 \mathrm{~Hz}, 2 \mathrm{H}), 2.15(\mathrm{q}, J=7.2 \mathrm{~Hz}, 2 \mathrm{H})$, $1.50-1.39(\mathrm{~m}, 2 \mathrm{H}), 0.91(\mathrm{t}, J=7.4 \mathrm{~Hz}, 3 \mathrm{H}) ;{ }^{13} \mathrm{C} \mathrm{NMR}\left(100 \mathrm{MHz}, \mathrm{CDCl}_{3}\right) \delta 167.6,164.4,145.2,142.1,140.1$, 132.5, 131.8, 131.6, 129.1, 128.7, 127.1, 122.3, 119.8, 117.1, 115.0, 113.5, 66.2, 64.5, 64.4, 54.7, 35.1, 22.6, 13.7; HRMS (ESI-TOF) $m / z$ Calcd for $\mathrm{C}_{23} \mathrm{H}_{25} \mathrm{~N}_{2} \mathrm{O}_{4}[\mathrm{M}+\mathrm{H}]^{+}: 393.1809$, found: 393.1817 .<smiles>CCCC=Cc1c(C(=O)Nc2ccccc2C2=NCCO2)ccc2c1OCCO2</smiles>

(E)-N-(2-(4,5-Dihydrooxazol-2-yl)phenyl)-5-(pent-1-en-1-yl)-2,3-dihydrobenzo[b][1,4]dioxine-6carboxamide $\left(3 t\left(\mathrm{C}_{5}\right)\right)$

Substrate 1t was alkenylated with alkenyl boronates (2) following the general procedure $\mathbf{A}\left(\mathrm{Cu}(\mathrm{OPiv})_{2}(30\right.$ mol\%)). After purification by preparative thin-layer chromatography (petroleum ether/ethyl acetate $=20 / 1$ ), $3 \mathbf{t}$ $\left(\mathrm{C}_{5}\right)$ was obtained as a white solid $(4.3 \mathrm{mg}, 11 \%) . \mathrm{mp} 136-138{ }^{\circ} \mathrm{C}$; ${ }^{1} \mathrm{H} \mathrm{NMR}\left(400 \mathrm{MHz}, \mathrm{CDCl}_{3}\right) \delta 12.36(\mathrm{~s}, 1 \mathrm{H})$, $8.88(\mathrm{~d}, J=8.7 \mathrm{~Hz}, 1 \mathrm{H}), 7.86(\mathrm{~d}, J=8.3 \mathrm{~Hz}, 1 \mathrm{H}), 7.49(\mathrm{t}, J=8.2 \mathrm{~Hz}, 1 \mathrm{H}), 7.13-7.07(\mathrm{~m}, 2 \mathrm{H}), 6.79(\mathrm{~d}, J=8.5$ $\mathrm{Hz}, 1 \mathrm{H}), 6.57(\mathrm{~d}, J=15.9 \mathrm{~Hz}, 1 \mathrm{H}), 6.25-6.14(\mathrm{~m}, 1 \mathrm{H}), 4.38-4.28(\mathrm{~m}, 6 \mathrm{H}), 4.04(\mathrm{t}, J=9.5 \mathrm{~Hz}, 2 \mathrm{H}), 2.10(\mathrm{q}, J=$ $8.0 \mathrm{~Hz}, 2 \mathrm{H}), 1.36-1.31(\mathrm{~m}, 2 \mathrm{H}), 0.83(\mathrm{t}, J=7.3 \mathrm{~Hz}, 3 \mathrm{H}) ;{ }^{13} \mathrm{C} \mathrm{NMR}\left(100 \mathrm{MHz}, \mathrm{CDCl}_{3}\right) \delta 168.7,164.3,144.7$, 141.4, 140.1, 137.6, 132.5, 130.5, 129.1, 126.3, 122.4, 122.2, 121.1, 119.7, 115.3, 113.3, 66.1, 64.3, 64.1, 54.8, 36.0, 22.3, 13.7; HRMS (ESI-TOF) $m / z$ Calcd for $\mathrm{C}_{23} \mathrm{H}_{25} \mathrm{~N}_{2} \mathrm{O}_{4}[\mathrm{M}+\mathrm{H}]^{+}: 393.1809$, found: 393.1818 . 
<smiles>CCC/C=C/c1cc2c(c(/C=C/CCC)c1C(=O)Nc1ccccc1C1=NCCO1)OCCO2</smiles>

\section{$N$-(2-(4,5-Dihydrooxazol-2-yl)phenyl)-5,7-di((E)-pent-1-en-1-yl)-2,3-dihydrobenzo[b][1,4]dioxine-6-} carboxamide (3t (di))

Substrate 1t was alkenylated with alkenyl boronates (2) following the general procedure $\mathbf{A}$ using $\left(\mathrm{Cu}(\mathrm{OPiv})_{2}\right.$ (30 mol\%)). After purification by preparative thin-layer chromatography (petroleum ether/ethyl acetate $=20 / 1)$, $3 t$ (di) was obtained as a white solid $(6.4 \mathrm{mg}, 14 \%)$. mp 58-60 ${ }^{\circ} \mathrm{C}$; ${ }^{1} \mathrm{H} \mathrm{NMR}\left(400 \mathrm{MHz}, \mathrm{CDCl}_{3}\right) \delta 12.17(\mathrm{~s}, 1 \mathrm{H})$, $8.87(\mathrm{~d}, J=8.5 \mathrm{~Hz}, 1 \mathrm{H}), 7.85(\mathrm{~d}, J=7.3 \mathrm{~Hz}, 1 \mathrm{H}), 7.51(\mathrm{t}, J=7.7 \mathrm{~Hz}, 1 \mathrm{H}), 7.11(\mathrm{t}, J=7.5 \mathrm{~Hz}, 1 \mathrm{H}), 6.96(\mathrm{~s}, 1 \mathrm{H})$, $6.44(\mathrm{~d}, J=15.5 \mathrm{~Hz}, 1 \mathrm{H}), 6.37(\mathrm{~d}, J=16.1 \mathrm{~Hz}, 1 \mathrm{H}), 6.30-6.18(\mathrm{~m}, 1 \mathrm{H}), 6.11-5.99(\mathrm{~m}, 1 \mathrm{H}), 4.35-4.25(\mathrm{~m}, 6 \mathrm{H})$, $3.96(\mathrm{t}, J=9.5 \mathrm{~Hz}, 2 \mathrm{H}), 2.12-2.00(\mathrm{~m}, 4 \mathrm{H}), 1.45-1.33(\mathrm{~m}, 2 \mathrm{H}), 1.29-1.22(\mathrm{~m}, 2 \mathrm{H}), 0.85(\mathrm{t}, J=7.4 \mathrm{~Hz}, 3 \mathrm{H})$, $0.76(\mathrm{t}, J=7.3 \mathrm{~Hz}, 3 \mathrm{H}) ;{ }^{13} \mathrm{C} \mathrm{NMR}\left(100 \mathrm{MHz}, \mathrm{CDCl}_{3}\right) \delta 168.5,164.0,143.6,140.2,139.7,137.0,132.4,131.7$, 129.8, 129.0, 128.7, 126.5, 124.7, 122.5, 122.4, 120.1, 113.6, 112.2, 66.1, 64.5, 64.1, 54.8, 35.9, 35.1, 22.5, 22.3, 13.6, 13.5; HRMS (ESI-TOF) $m / z$ Calcd for $\mathrm{C}_{28} \mathrm{H}_{33} \mathrm{~N}_{2} \mathrm{O}_{4}[\mathrm{M}+\mathrm{H}]^{+}: 461.2435$, found: 461.2447 .<smiles>CCCC=Cc1cnccc1C(=O)Nc1ccccc1C1=NCCO1</smiles>

(E)-N-(2-(4,5-Dihydrooxazol-2-yl)phenyl)-3-(pent-1-en-1-yl)isonicotinamide (3u (mono))

Substrate 1u was alkenylated with alkenyl boronates (2) following the general procedure A. After purification by preparative thin-layer chromatography (petroleum ether/ethyl acetate $=6 / 1), 3 \mathbf{u}($ mono) was obtained as a white solid (7.8 mg, 24\%). mp 122-124 $\left.{ }^{\circ} \mathrm{C} ;{ }^{1} \mathrm{H} \mathrm{NMR} \mathrm{(400} \mathrm{MHz,} \mathrm{CDCl}_{3}\right) \delta 12.79$ (s, 1H), 8.89 (d, J=8.4 Hz, $1 \mathrm{H}), 8.85(\mathrm{~s}, 1 \mathrm{H}), 8.56(\mathrm{~d}, J=5.0 \mathrm{~Hz}, 1 \mathrm{H}), 7.90(\mathrm{~d}, J=7.8 \mathrm{~Hz}, 1 \mathrm{H}), 7.54(\mathrm{t}, J=7.4 \mathrm{~Hz}, 1 \mathrm{H}), 7.46(\mathrm{~d}, J=5.0$ $\mathrm{Hz}, 1 \mathrm{H}), 7.16(\mathrm{t}, J=7.6 \mathrm{~Hz}, 1 \mathrm{H}), 6.82(\mathrm{~d}, J=15.9 \mathrm{~Hz}, 1 \mathrm{H}), 6.37-6.26(\mathrm{~m}, 1 \mathrm{H}), 4.37(\mathrm{t}, J=9.5 \mathrm{~Hz}, 2 \mathrm{H}), 4.03$ $(\mathrm{t}, J=9.5 \mathrm{~Hz}, 2 \mathrm{H}), 2.21(\mathrm{q}, J=6.9 \mathrm{~Hz}, 2 \mathrm{H}), 1.55-1.43(\mathrm{~m}, 2 \mathrm{H}), 0.93(\mathrm{t}, J=7.4 \mathrm{~Hz}, 3 \mathrm{H}) ;{ }^{13} \mathrm{C} \mathrm{NMR}(100 \mathrm{MHz}$, $\left.\mathrm{CDCl}_{3}\right) \delta 166.1,164.6,148.6,147.9,141.2,139.5,135.7,132.6,131.5,129.2,124.5,123.1,120.9,119.9,113.6$, 66.3, 54.5, 35.4, 22.3, 13.7; HRMS (ESI-TOF) $m / z$ Calcd for $\mathrm{C}_{20} \mathrm{H}_{22} \mathrm{~N}_{3} \mathrm{O}_{2}[\mathrm{M}+\mathrm{H}]^{+}: 336.1707$, found: 336.1714 . 


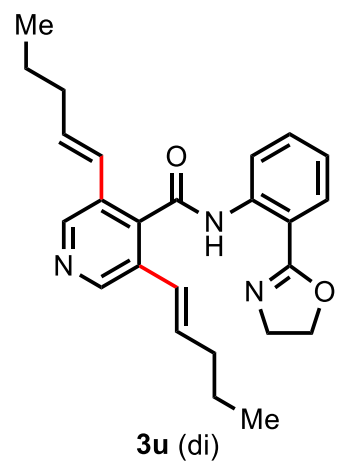

$N$-(2-(4,5-Dihydrooxazol-2-yl)phenyl)-3,5-di((E)-pent-1-en-1-yl)isonicotinamide (3u (di))

Substrate 1u was alkenylated with alkenyl boronates (2) following the general procedure A. After purification by preparative thin-layer chromatography (petroleum ether/ethyl acetate $=6 / 1$ ), $3 \mathbf{u}$ (di) was obtained as a white solid (5.2 mg, 13\%). mp 48-50 ${ }^{\circ} \mathrm{C}$; ${ }^{1} \mathrm{H}$ NMR $\left(400 \mathrm{MHz}, \mathrm{CDCl}_{3}\right) \delta 12.51(\mathrm{~s}, 1 \mathrm{H}), 8.90(\mathrm{~d}, J=8.4 \mathrm{~Hz}, 1 \mathrm{H}), 8.63$ $(\mathrm{s}, 2 \mathrm{H}), 7.89$ (d, $J=7.8 \mathrm{~Hz}, 1 \mathrm{H}), 7.56(\mathrm{t}, J=7.2 \mathrm{~Hz}, 1 \mathrm{H}), 7.18(\mathrm{t}, J=7.3 \mathrm{~Hz}, 1 \mathrm{H}), 6.44(\mathrm{~d}, J=15.8 \mathrm{~Hz}, 2 \mathrm{H})$, 6.34-6.22 (m, 2H), $4.32(\mathrm{t}, J=9.5 \mathrm{~Hz}, 2 \mathrm{H}), 3.92(\mathrm{t}, J=9.5 \mathrm{~Hz}, 2 \mathrm{H}), 2.13(\mathrm{q}, J=6.9 \mathrm{~Hz}, 4 \mathrm{H}), 1.47-1.35(\mathrm{~m}$, $4 \mathrm{H}), 0.87(\mathrm{t}, J=7.4 \mathrm{~Hz}, 6 \mathrm{H}) ;{ }^{13} \mathrm{C} \mathrm{NMR}\left(100 \mathrm{MHz}, \mathrm{CDCl}_{3}\right) \delta 166.1,164.2,145.6,140.1,139.2,135.8,132.6$, 129.6, 129.2, 123.9, 123.1, 120.2, 113.7, 66.2, 54.6, 35.3, 22.2, 13.5; HRMS (ESI-TOF) $\mathrm{m} / \mathrm{z}$ Calcd for $\mathrm{C}_{25} \mathrm{H}_{30} \mathrm{~N}_{3} \mathrm{O}_{2}[\mathrm{M}+\mathrm{H}]^{+}:$404.2333, found: 404.2341 .<smiles>CCC/C=C/c1ccsc1C(=O)Nc1ccccc1C1=NCCO1</smiles>

(E)-N-(2-(4,5-Dihydrooxazol-2-yl)phenyl)-3-(pent-1-en-1-yl)thiophene-2-carboxamide (3v)

Substrate $\mathbf{1 v}$ was alkenylated with alkenyl boronates (2) following the general procedure A. After purification by preparative thin-layer chromatography (petroleum ether/ethyl acetate $=20 / 1$ ), $\mathbf{3 v}$ was obtained as a white solid (12.5 mg, 37\%). mp 53-55 ${ }^{\circ} \mathrm{C} ;{ }^{1} \mathrm{H}$ NMR (400 MHz, $\left.\mathrm{CDCl}_{3}\right) \delta 12.77(\mathrm{~s}, 1 \mathrm{H}), 8.81(\mathrm{~d}, J=8.5 \mathrm{~Hz}, 1 \mathrm{H}), 7.87$ (dd, $J=7.9,1.5 \mathrm{~Hz}, 1 \mathrm{H}), 7.52-7.45(\mathrm{~m}, 1 \mathrm{H}), 7.35-7.29(\mathrm{~m}, 3 \mathrm{H}), 7.13-7.06(\mathrm{~m}, 1 \mathrm{H}), 6.26(\mathrm{dt}, J=15.9,7.0 \mathrm{~Hz}$, $1 \mathrm{H}), 4.39(\mathrm{t}, J=9.4 \mathrm{~Hz}, 2 \mathrm{H}), 4.14(\mathrm{t}, J=9.6 \mathrm{~Hz}, 2 \mathrm{H}), 2.26-2.18(\mathrm{~m}, 2 \mathrm{H}), 1.55-1.44(\mathrm{~m}, 2 \mathrm{H}), 0.95(\mathrm{t}, J=7.4$ $\mathrm{Hz}, 3 \mathrm{H}) ;{ }^{13} \mathrm{C} \mathrm{NMR}\left(100 \mathrm{MHz}, \mathrm{CDCl}_{3}\right) \delta 164.6,161.9,143.8,140.0,134.8,132.4,130.5,129.1,127.3,126.9$, 123.9, 122.4, 120.1, 113.5, 66.3, 54.6, 35.3, 22.5, 13.8; HRMS (ESI-TOF) $m / z$ Calcd for $\mathrm{C}_{19} \mathrm{H}_{21} \mathrm{~N}_{2} \mathrm{O}_{2} \mathrm{~S}[\mathrm{M}+\mathrm{H}]^{+}$: 341.1318, found: 341.1323<smiles>CCCCC=Cc1cc(-c2ccccn2)ccc1C(=O)Nc1ccccc1C1=NCCO1</smiles> 
Substrate 1v was alkenylated with alkenyl boronates (2) following the general procedure A. After purification by preparative thin-layer chromatography (petroleum ether/ethyl acetate $=5 / 1), 3 \mathbf{w}($ mono) was obtained as a white solid (19.4 mg, 48\%). mp 130-132 ${ }^{\circ} \mathrm{C} ;{ }^{1} \mathrm{H} \mathrm{NMR}\left(400 \mathrm{MHz}, \mathrm{CDCl}_{3}\right) \delta 12.67$ (s, 1H), $8.96(\mathrm{~d}, J=8.4 \mathrm{~Hz}$, $1 \mathrm{H}), 8.73(\mathrm{~d}, J=4.6 \mathrm{~Hz}, 1 \mathrm{H}), 8.22(\mathrm{~d}, J=1.6 \mathrm{~Hz}, 1 \mathrm{H}), 7.89(\mathrm{dt}, J=7.9,1.7 \mathrm{~Hz}, 2 \mathrm{H}), 7.80-7.77(\mathrm{~m}, 2 \mathrm{H}), 7.73$ $(\mathrm{d}, J=8.0 \mathrm{~Hz}, 1 \mathrm{H}), 7.56-7.50(\mathrm{~m}, 1 \mathrm{H}), 7.29-7.25(\mathrm{~m}, 1 \mathrm{H}), 7.16-7.10(\mathrm{~m}, 1 \mathrm{H}), 6.98(\mathrm{~d}, J=15.8 \mathrm{~Hz}, 1 \mathrm{H}), 6.37$ $(\mathrm{dt}, J=15.7,7.0 \mathrm{~Hz}, 1 \mathrm{H}), 4.34(\mathrm{t}, J=9.5 \mathrm{~Hz}, 2 \mathrm{H}), 4.01(\mathrm{t}, J=9.5 \mathrm{~Hz}, 2 \mathrm{H}), 2.25-2.17(\mathrm{~m}, 2 \mathrm{H}), 1.54-1.44(\mathrm{~m}$, 2H), $0.93(\mathrm{t}, J=7.4 \mathrm{~Hz}, 3 \mathrm{H}) ;{ }^{13} \mathrm{C}$ NMR $\left(100 \mathrm{MHz}, \mathrm{CDCl}_{3}\right) \delta 168.2,164.4,156.7,149.7,140.9,140.0,137.5$, 136.8, 135.6, 133.9, 132.5, 129.2, 128.3, 127.4, 125.1, 125.0, 122.5, 122.5, 120.9, 119.8, 113.5, 66.2, 54.7, 35.3, 22.5, 13.8; HRMS (ESI-TOF) $\mathrm{m} / z$ Calcd for $\mathrm{C}_{26} \mathrm{H}_{26} \mathrm{~N}_{3} \mathrm{O}_{2}[\mathrm{M}+\mathrm{H}]^{+}: 412.2020$, found: 412.2029 .<smiles>CCC/C=C/c1cc(-c2ccccn2)cc(/C=C/CCCC)c1C(=O)Nc1ccccc1C1=NCCO1</smiles>

$N$-(2-(4,5-Dihydrooxazol-2-yl)phenyl)-2,6-di((E)-pent-1-en-1-yl)-4-(pyridin-2-yl)benzamide (3w (di))

Substrate $\mathbf{1 v}$ was alkenylated with alkenyl boronates (2) following the general procedure A. After purification by preparative thin-layer chromatography (petroleum ether/ethyl acetate $=5 / 1$ ), $\mathbf{3} \mathbf{w}$ (di) was obtained as a white solid (11.7 mg, 25\%). mp 165-167 ${ }^{\circ} \mathrm{C} ;{ }^{1} \mathrm{H}$ NMR (400 MHz, $\left.\mathrm{CDCl}_{3}\right) \delta 12.38(\mathrm{~s}, 1 \mathrm{H}), 8.98(\mathrm{~d}, J=7.9 \mathrm{~Hz}, 1 \mathrm{H})$, $8.73(\mathrm{~d}, J=4.8 \mathrm{~Hz}, 1 \mathrm{H}), 8.01(\mathrm{~s}, 2 \mathrm{H}), 7.88(\mathrm{dd}, J=7.9,1.5 \mathrm{~Hz}, 1 \mathrm{H}), 7.81-7.77(\mathrm{~m}, 2 \mathrm{H}), 7.59-7.52(\mathrm{~m}, 1 \mathrm{H})$, $7.30-7.25(\mathrm{~m}, 1 \mathrm{H}), 7.15(\mathrm{t}, J=7.6 \mathrm{~Hz}, 1 \mathrm{H}), 6.58(\mathrm{~d}, J=15.7 \mathrm{~Hz}, 2 \mathrm{H}), 6.40-6.29(\mathrm{~m}, 2 \mathrm{H}), 4.29(\mathrm{t}, J=9.5 \mathrm{~Hz}$, $2 \mathrm{H}), 3.90(\mathrm{t}, J=9.5 \mathrm{~Hz}, 2 \mathrm{H}), 2.13(\mathrm{q}, J=6.9 \mathrm{~Hz}, 4 \mathrm{H}), 1.47-1.35(\mathrm{~m}, 4 \mathrm{H}), 0.87(\mathrm{t}, J=7.4 \mathrm{~Hz}, 6 \mathrm{H}) ;{ }^{13} \mathrm{C} \mathrm{NMR}$ $\left(100 \mathrm{MHz}, \mathrm{CDCl}_{3}\right) \delta 168.6,164.0,157.3,149.7,139.8,139.6,136.8,135.8,135.3,133.9,132.4,129.1,126.9$, 122.7, 122.6, 122.4, 121.0, 120.2, 113.7, 66.1, 54.7, 35.3, 22.4, 13.6; HRMS (ESI-TOF) $\mathrm{m} / z$ Calcd for $\mathrm{C}_{31} \mathrm{H}_{34} \mathrm{~N}_{3} \mathrm{O}_{2}[\mathrm{M}+\mathrm{H}]^{+}:$480.2646, found: 480.2663 . 


\subsection{Evaluation of Alkenyl Boronates}

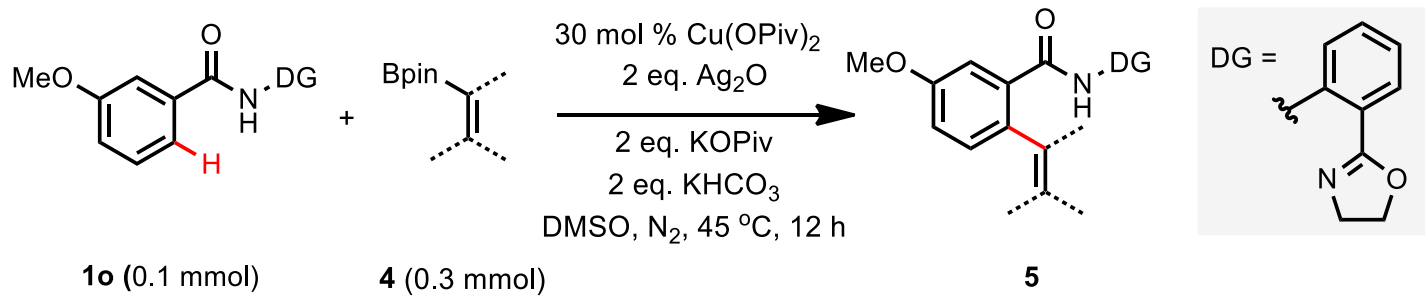

\section{General Procedure B for C-H Alkenylation with Alkenyl Boronates:}

A stirring bar, substrate $10(0.1 \mathrm{mmol}), \mathrm{Cu}(\mathrm{OPiv})_{2}(0.03 \mathrm{mmol}), \mathrm{Ag}_{2} \mathrm{O}(0.2 \mathrm{mmol}), \mathrm{KOPiv}(0.2 \mathrm{mmol})$, and $\mathrm{KHCO}_{3}(0.2 \mathrm{mmol})$ were added to an oven dried $25 \mathrm{~mL}$ Schlenk tube. The tube was capped and back-filled with nitrogen (3 times). Under the nitrogen flow, $4(0.3 \mathrm{mmol})$ and DMSO $(2 \mathrm{~mL})$ were added to the mixture, and the tube was capped tightly again. Then the reaction mixture was placed into a pre-heated oil bath and stirred at $45^{\circ} \mathrm{C}$ for $12 \mathrm{~h}$. After cooled to room temperature, EtOAc $(20 \mathrm{~mL})$ was added to quench the reaction, and organic layer was washed with $\mathrm{NH}_{3} \cdot \mathrm{H}_{2} \mathrm{O}(20 \mathrm{~mL})$, and the water phase was extracted by EtOAc $(20 \mathrm{~mL})$. The combined organic layers were washed with saturated brine, dried over $\mathrm{Na}_{2} \mathrm{SO}_{4}$, concentrated under vacuum. The crude product was purified by preparative TLC or column chromatography (neutral alumina) to afford the desired product 5 .<smiles>C/C=C/c1ccc(OC)cc1C(=O)Nc1ccccc1C1=NCCO1</smiles>

$5 a$

(E)-N-(2-(4,5-Dihydrooxazol-2-yl)phenyl)-5-methoxy-2-(prop-1-en-1-yl)benzamide (5a)

Substrate 10 was alkenylated with alkenyl boronate (4a) following the general procedure B. After purification by preparative thin-layer chromatography (petroleum ether/toluene $=1 / 3$ ), $\mathbf{5 a}$ was obtained as a white solid (27.5 mg, 82\%). mp 93-95 ${ }^{\circ} \mathrm{C} ;{ }^{1} \mathrm{H}$ NMR (400 MHz, $\left.\mathrm{CDCl}_{3}\right) \delta 12.59(\mathrm{~s}, 1 \mathrm{H}), 8.95(\mathrm{~d}, J=8.5 \mathrm{~Hz}, 1 \mathrm{H}), 7.89(\mathrm{~d}$, $J=7.9 \mathrm{~Hz}, 1 \mathrm{H}), 7.56-7.47(\mathrm{~m}, 2 \mathrm{H}), 7.19-7.10(\mathrm{~m}, 2 \mathrm{H}), 6.96(\mathrm{dd}, J=8.7,2.6 \mathrm{~Hz}, 1 \mathrm{H}), 6.85(\mathrm{~d}, J=14.9 \mathrm{~Hz}$, $1 \mathrm{H}), 6.16-6.05(\mathrm{~m}, 1 \mathrm{H}), 4.35$ (t, $J=9.6 \mathrm{~Hz}, 2 \mathrm{H}), 4.03(\mathrm{t}, J=9.5 \mathrm{~Hz}, 2 \mathrm{H}), 3.84(\mathrm{~s}, 3 \mathrm{H}), 1.85$ (dd, $J=6.6,1.5$ $\mathrm{Hz}, 3 \mathrm{H}) ;{ }^{13} \mathrm{C}$ NMR $\left(100 \mathrm{MHz}, \mathrm{CDCl}_{3}\right) \delta 168.1,164.5,158.2,134.0,136.0,132.6,129.8,129.2,128.2,127.8$, 126.1, 122.5, 119.8, 117.0, 113.5, 112.1, 66.2, 55.4, 54.7, 18.7; HRMS (ESI-TOF) $m / z$ Calcd for $\mathrm{C}_{20} \mathrm{H}_{21} \mathrm{~N}_{2} \mathrm{O}_{3}$ $[\mathrm{M}+\mathrm{H}]^{+}:$337.1547, found: 337.1551 .<smiles>CC=Cc1ccc(OC)cc1C(=O)Nc1ccccc1C1=NCCO1</smiles>

5b

(Z)-N-(2-(4,5-dihydrooxazol-2-yl)phenyl)-5-methoxy-2-(prop-1-en-1-yl)benzamide (5b) 
Substrate 10 was alkenylated with alkenyl boronate (4b) following the general procedure $\mathbf{B}$. After purification by preparative thin-layer chromatography (petroleum ether/toluene $=1 / 3$ ), $\mathbf{5} \mathbf{b}$ was obtained as a white solid (22.8 mg, 68\%). mp 73-75 ${ }^{\circ} \mathrm{C} ;{ }^{1} \mathrm{H}$ NMR $\left(400 \mathrm{MHz}, \mathrm{CDCl}_{3}\right) \delta 12.54(\mathrm{~s}, 1 \mathrm{H}), 8.92(\mathrm{~d}, J=8.4 \mathrm{~Hz}, 1 \mathrm{H}), 7.88(\mathrm{~d}$, $J=7.9 \mathrm{~Hz}, 1 \mathrm{H}), 7.50(\mathrm{t}, J=7.9 \mathrm{~Hz}, 1 \mathrm{H}), 7.30-7.26(\mathrm{~m}, 2 \mathrm{H}), 7.11(\mathrm{t}, J=7.6 \mathrm{~Hz}, 1 \mathrm{H}), 6.99(\mathrm{dd}, J=8.5,2.7 \mathrm{~Hz}$, $1 \mathrm{H}), 6.69(\mathrm{~d}, J=11.6 \mathrm{~Hz}, 1 \mathrm{H}), 5.82-5.72(\mathrm{~m}, 1 \mathrm{H}), 4.35(\mathrm{t}, J=9.5 \mathrm{~Hz}, 2 \mathrm{H}), 4.04(\mathrm{t}, J=9.5 \mathrm{~Hz}, 2 \mathrm{H}), 3.86(\mathrm{~s}$, $3 \mathrm{H}), 1.79(\mathrm{dd}, J=7.1,1.4 \mathrm{~Hz}, 3 \mathrm{H}) ;{ }^{13} \mathrm{C}$ NMR $\left(100 \mathrm{MHz}, \mathrm{CDCl}_{3}\right) \delta 167.9,164.5,158.1,139.9,137.5,132.5$, 131.6, 129.2, 128.6, 128.0, 126.6, 122.5, 119.9, 116.2, 113.5, 112.6, 66.1, 55.4, 54.7, 14.5; HRMS (ESI-TOF) $m / z$ Calcd for $\mathrm{C}_{20} \mathrm{H}_{21} \mathrm{~N}_{2} \mathrm{O}_{3}[\mathrm{M}+\mathrm{H}]^{+}: 337.1547$, found: 337.1552 .<smiles>CCC/C=C/c1ccc(OC)cc1C(=O)Nc1ccccc1C1=NCCO1</smiles>

(E)- $N$-(2-(4,5-Dihydrooxazol-2-yl)phenyl)-2-(hex-1-en-1-yl)-5-methoxybenzamide (5c)

Substrate 10 was alkenylated with alkenyl boronate $(\mathbf{4 c})$ following the general procedure $\mathbf{B}\left(50{ }^{\circ} \mathrm{C}\right)$. After purification by preparative thin-layer chromatography (petroleum ether/ethyl acetate $=20 / 1$ ), $\mathbf{5 c}$ was obtained as a white solid $(25.2 \mathrm{mg}, 67 \%) . \mathrm{mp} 102-104{ }^{\circ} \mathrm{C} ;{ }^{1} \mathrm{H}$ NMR $\left(400 \mathrm{MHz}, \mathrm{CDCl}_{3}\right) \delta 12.57(\mathrm{~s}, 1 \mathrm{H}), 8.94(\mathrm{~d}, J=8.4$ $\mathrm{Hz}, 1 \mathrm{H}), 7.89(\mathrm{~d}, J=6.9 \mathrm{~Hz}, 1 \mathrm{H}), 7.52(\mathrm{t}, J=8.3 \mathrm{~Hz}, 2 \mathrm{H}), 7.17-7.09(\mathrm{~m}, 2 \mathrm{H}), 6.96(\mathrm{dd}, J=8.7,2.4 \mathrm{~Hz}, 1 \mathrm{H})$, $6.82(\mathrm{~d}, J=15.8 \mathrm{~Hz}, 1 \mathrm{H}), 6.14-6.04(\mathrm{~m}, 1 \mathrm{H}), 4.34(\mathrm{t}, J=9.5 \mathrm{~Hz}, 2 \mathrm{H}), 4.01(\mathrm{t}, J=9.5 \mathrm{~Hz}, 2 \mathrm{H}), 3.83(\mathrm{~s}, 3 \mathrm{H})$, $2.17(\mathrm{q}, J=6.9 \mathrm{~Hz}, 2 \mathrm{H}), 1.45-1.28(\mathrm{~m}, 4 \mathrm{H}), 0.87(\mathrm{t}, J=7.1 \mathrm{~Hz}, 3 \mathrm{H}) ;{ }^{13} \mathrm{C}$ NMR $\left(100 \mathrm{MHz}, \mathrm{CDCl}_{3}\right) \delta 168.1$, 164.4, 158.2, 140.0, 136.2, 132.5, 131.7, 129.6, 129.2, 127.8, 126.8, 122.5, 119.8, 116.9, 113.5, 112.166 .1 , 55.4, 54.6, 32.8, 31.6, 22.2, 13.9; HRMS (ESI-TOF) $\mathrm{m} / \mathrm{z}$. Calcd for $\mathrm{C}_{23} \mathrm{H}_{27} \mathrm{~N}_{2} \mathrm{O}_{3}[\mathrm{M}+\mathrm{H}]^{+}: 379.2016$ found: 379.2024 .

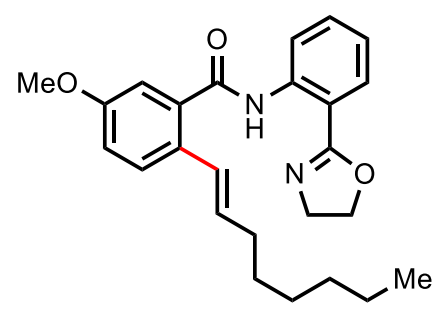

$5 d$

(E)- $N$-(2-(4,5-Dihydrooxazol-2-yl)phenyl)-5-methoxy-2-(oct-1-en-1-yl)benzamide (5d)

Substrate 10 was alkenylated with alkenyl boronate (4d) following the general procedure $\mathrm{B}\left(50{ }^{\circ} \mathrm{C}\right)$. After purification by preparative thin-layer chromatography (petroleum ether/ethyl acetate $=20 / 1), \mathbf{5 d}$ was obtained as a white solid $(27.0 \mathrm{mg}, 67 \%) . \mathrm{mp} 103-105{ }^{\circ} \mathrm{C} ;{ }^{1} \mathrm{H}$ NMR $\left(400 \mathrm{MHz}, \mathrm{CDCl}_{3}\right) \delta 12.58(\mathrm{~s}, 1 \mathrm{H}), 8.94(\mathrm{~d}, J=8.5$ $\mathrm{Hz}, 1 \mathrm{H}), 7.89(\mathrm{~d}, J=6.8 \mathrm{~Hz}, 1 \mathrm{H}), 7.56-7.49(\mathrm{~m}, 2 \mathrm{H}), 7.18-7.08(\mathrm{~m}, 2 \mathrm{H}), 6.96(\mathrm{dd}, J=8.7,2.7 \mathrm{~Hz}, 1 \mathrm{H}), 6.82$ $(\mathrm{d}, J=15.6 \mathrm{~Hz}, 1 \mathrm{H}), 6.15-6.05(\mathrm{~m}, 1 \mathrm{H}), 4.34(\mathrm{t}, J=9.4 \mathrm{~Hz}, 2 \mathrm{H}), 4.02(\mathrm{t}, J=9.5 \mathrm{~Hz}, 2 \mathrm{H}), 3.83(\mathrm{~s}, 3 \mathrm{H}), 2.16(\mathrm{q}$, $J=7.3 \mathrm{~Hz}, 2 \mathrm{H}), 1.47-1.36(\mathrm{~m}, 2 \mathrm{H}), 1.35-1.20(\mathrm{~m}, 6 \mathrm{H}), 0.86(\mathrm{t}, J=6.7 \mathrm{~Hz}, 3 \mathrm{H}),{ }^{13} \mathrm{C} \mathrm{NMR}\left(100 \mathrm{MHz}, \mathrm{CDCl}_{3}\right)$ $\delta$ 168.1, 164.4, 158.2, 140.0, 136.2, 132.5, 131.7, 129.7, 129.2, 127.8, 126.8, 122.5, 119.8, 116.9, 113.4, 112.0, 66.1, 55.4, 54.6, 33.2, 31.7, 29.4, 28.9, 22.6, 14.1; HRMS (ESI-TOF) $m / z$ Calcd for $\mathrm{C}_{25} \mathrm{H}_{31} \mathrm{~N}_{2} \mathrm{O}_{3}[\mathrm{M}+\mathrm{H}]^{+}$: 407.2329, found: 407.2341 . 
<smiles>COc1ccc(/C=C/CCc2ccccc2)c(C(=O)Nc2ccccc2C2=NCCO2)c1</smiles>

(E)-N-(2-(4,5-Dihydrooxazol-2-yl)phenyl)-5-methoxy-2-(4-phenylbut-1-en-1-yl)benzamide (5e)

Substrate 10 was alkenylated with alkenyl boronate (4e) following the general procedure $\mathbf{B}\left(50{ }^{\circ} \mathrm{C}\right)$. After purification by preparative thin-layer chromatography (petroleum ether/ethyl acetate $=20 / 1$ ), $\mathbf{5 e}$ was obtained as a white solid $(21.7 \mathrm{mg}, 51 \%) . \mathrm{mp} 88-90{ }^{\circ} \mathrm{C} ;{ }^{1} \mathrm{H}$ NMR $\left(400 \mathrm{MHz}, \mathrm{CDCl}_{3}\right) \delta 12.59(\mathrm{~s}, 1 \mathrm{H}), 8.94(\mathrm{~d}, J=8.6$ $\mathrm{Hz}, 1 \mathrm{H}), 7.89$ (d, $J=7.9 \mathrm{~Hz}, 1 \mathrm{H}), 7.56-7.47(\mathrm{~m}, 2 \mathrm{H}), 7.29-7.23(\mathrm{~m}, 2 \mathrm{H}), 7.20-7.11(\mathrm{~m}, 5 \mathrm{H}), 6.96(\mathrm{dd}, J=8.6$, $2.4 \mathrm{~Hz}, 1 \mathrm{H}), 6.91(\mathrm{~d}, J=15.6 \mathrm{~Hz}, 1 \mathrm{H}), 6.18-6.07(\mathrm{~m}, 1 \mathrm{H}), 4.35(\mathrm{t}, J=9.6 \mathrm{~Hz}, 2 \mathrm{H}), 4.02(\mathrm{t}, J=9.5 \mathrm{~Hz}, 2 \mathrm{H})$, $3.84(\mathrm{~s}, 3 \mathrm{H}), 2.77-2.71(\mathrm{~m}, 2 \mathrm{H}), 2.54-2.45(\mathrm{~m}, 2 \mathrm{H}) ;{ }^{13} \mathrm{C} \mathrm{NMR}\left(100 \mathrm{MHz}, \mathrm{CDCl}_{3}\right) \delta 168.1,164.5,158.3,141.8$, 140.0, 136.3, 132.6, 130.4, 129.5, 129.2, 128.4, 128.3, 127.9, 127.5, 125.8, 122.6, 119.8, 116.9, 113.5, 112.1, 66.2, 55.4, 54.7, 35.9, 34.9; HRMS (ESI-TOF) $m / z$ Calcd for $\mathrm{C}_{27} \mathrm{H}_{27} \mathrm{~N}_{2} \mathrm{O}_{3}[\mathrm{M}+\mathrm{H}]^{+}$: 427.2016, found: 427.2028.<smiles>COc1ccc(/C=C/C2CC2)c(C(=O)Nc2ccccc2C2=NC3CC3CO2)c1</smiles>

$5 f$

(E)-2-(2-Cyclopropylvinyl)- $N$-(2-(4,5-dihydrooxazol-2-yl)phenyl)-5-methoxybenzamide (5f)

Substrate 10 was alkenylated with alkenyl boronate (4f) following the general procedure B. After purification by preparative thin-layer chromatography (petroleum ether/toluene $=1 / 3), \mathbf{5 f}$ was obtained as a white solid (19.2 mg, 53\%). mp 146-148 ${ }^{\circ} \mathrm{C}$; ${ }^{1} \mathrm{H} \mathrm{NMR}\left(400 \mathrm{MHz}, \mathrm{CDCl}_{3}\right) \delta 12.59(\mathrm{~s}, 1 \mathrm{H}), 8.95(\mathrm{~d}, J=8.4 \mathrm{~Hz}, 1 \mathrm{H}), 7.89(\mathrm{~d}, J=$ $8.0 \mathrm{~Hz}, 1 \mathrm{H}), 7.53(\mathrm{t}, J=7.9 \mathrm{~Hz}, 1 \mathrm{H}), 7.45(\mathrm{~d}, J=8.7 \mathrm{~Hz}, 1 \mathrm{H}), 7.17-7.09(\mathrm{~m}, 2 \mathrm{H}), 6.96-6.90(\mathrm{~m}, 2 \mathrm{H}), 5.58(\mathrm{dd}$, $J=15.7,9.0 \mathrm{~Hz}, 1 \mathrm{H}), 4.35(\mathrm{t}, J=9.5 \mathrm{~Hz}, 2 \mathrm{H}), 4.03(\mathrm{t}, J=9.5 \mathrm{~Hz}, 2 \mathrm{H}), 3.83(\mathrm{~s}, 3 \mathrm{H}), 1.59-1.49$ (m, 1H), 0.79$0.73(\mathrm{~m}, 2 \mathrm{H}), 0.47-0.41(\mathrm{~m}, 2 \mathrm{H}) ;{ }^{13} \mathrm{C} \mathrm{NMR}\left(100 \mathrm{MHz}, \mathrm{CDCl}_{3}\right) \delta 168.2,164.5,158.1,140.0,135.8,135.3,132.5$, $129.5,129.2,127.4,124.5,122.5,119.8,117.0,113.5,112.2,66.2,55.4,54.7,14.7,7.3$; HRMS (ESI-TOF) $\mathrm{m} / 2$ Calcd for $\mathrm{C}_{22} \mathrm{H}_{23} \mathrm{~N}_{2} \mathrm{O}_{3}[\mathrm{M}+\mathrm{H}]^{+}: 363.1703$, found: 363.1710 .<smiles>COc1ccc(/C=C/c2ccccc2)c(C(=O)Nc2ccccc2C2=NCCO2)c1</smiles> 


\section{(E)-N-(2-(4,5-Dihydrooxazol-2-yl)phenyl)-5-methoxy-2-styrylbenzamide (5g)}

Substrate 10 was alkenylated with alkenyl boronate $(\mathbf{4 g})$ following the general procedure $\mathbf{B}\left(50{ }^{\circ} \mathrm{C}\right)$. After purification by preparative thin-layer chromatography (petroleum ether/ethyl acetate $=20 / 1$ ), $\mathbf{5 g}$ was obtained as a white solid $(18.7 \mathrm{mg}, 47 \%) . \mathrm{mp} 135-137{ }^{\circ} \mathrm{C} ;{ }^{1} \mathrm{H}$ NMR $\left(400 \mathrm{MHz}, \mathrm{CDCl}_{3}\right) \delta 12.69(\mathrm{~s}, 1 \mathrm{H}), 8.98(\mathrm{~d}, J=8.4$ $\mathrm{Hz}, 1 \mathrm{H}), 7.88(\mathrm{~d}, J=7.4 \mathrm{~Hz}, 1 \mathrm{H}), 7.71(\mathrm{~d}, J=8.7 \mathrm{~Hz}, 1 \mathrm{H}), 7.61(\mathrm{~d}, J=16.2 \mathrm{~Hz}, 1 \mathrm{H}), 7.54(\mathrm{t}, J=7.8 \mathrm{~Hz}, 1 \mathrm{H})$, $7.44(\mathrm{~d}, J=7.5 \mathrm{~Hz}, 2 \mathrm{H}), 7.29(\mathrm{t}, J=7.5 \mathrm{~Hz}, 2 \mathrm{H}), 7.26-7.23(\mathrm{~m}, 1 \mathrm{H}), 7.20(\mathrm{t}, J=7.3 \mathrm{~Hz}, 1 \mathrm{H}), 7.14(\mathrm{t}, J=7.6$ $\mathrm{Hz}, 1 \mathrm{H}), 7.04(\mathrm{dd}, J=8.7,2.4 \mathrm{~Hz}, 1 \mathrm{H}), 6.98(\mathrm{~d}, J=16.2 \mathrm{~Hz}, 1 \mathrm{H}), 4.19$ (t, $J=9.5 \mathrm{~Hz}, 2 \mathrm{H}), 3.93-3.85(\mathrm{~m}, 5 \mathrm{H})$; ${ }^{13} \mathrm{C}$ NMR $\left(100 \mathrm{MHz}, \mathrm{CDCl}_{3}\right) \delta 167.8,164.4,158.9,139.8,137.6,137.1,132.6,129.2,128.8,128.5,127.6$, 127.4, 126.5, 126.1, 122.6, 119.9, 117.3, 113.6, 112.6, 66.1, 55.5, 54.6; HRMS (ESI-TOF) $\mathrm{m} / \mathrm{z}$ Calcd for $\mathrm{C}_{25} \mathrm{H}_{23} \mathrm{~N}_{2} \mathrm{O}_{3}[\mathrm{M}+\mathrm{H}]^{+}:$399.1703, found: 399.1707 .<smiles>COc1ccc(/C=C/c2ccsc2)c(C(=O)Nc2ccccc2C2=NCCO2)c1</smiles>

(E)-N-(2-(4,5-Dihydrooxazol-2-yl)phenyl)-5-methoxy-2-(2-(thiophen-3-yl)vinyl)benzamide (5h)

Substrate 10 was alkenylated with alkenyl boronate (4h) following the general procedure $\mathbf{B}$. After purification by preparative thin-layer chromatography (petroleum ether/ethyl acetate $=20 / 1$ ), $\mathbf{5} \mathbf{h}$ was obtained as a white solid (21.7 mg, 54\%). mp 68-70 ${ }^{\circ} \mathrm{C}$; ${ }^{1} \mathrm{H}$ NMR (400 MHz, $\left.\mathrm{CDCl}_{3}\right) \delta 12.67(\mathrm{~s}, 1 \mathrm{H}), 8.97(\mathrm{~d}, J=8.5 \mathrm{~Hz}, 1 \mathrm{H}), 7.88$ $(\mathrm{d}, J=7.2 \mathrm{~Hz}, 1 \mathrm{H}), 7.67(\mathrm{~d}, J=8.8 \mathrm{~Hz}, 1 \mathrm{H}), 7.54(\mathrm{t}, J=7.9 \mathrm{~Hz}, 1 \mathrm{H}), 7.45(\mathrm{~d}, J=16.2 \mathrm{~Hz}, 1 \mathrm{H}), 7.27-7.19(\mathrm{~m}$, $4 \mathrm{H}), 7.14(\mathrm{t}, J=7.6 \mathrm{~Hz}, 1 \mathrm{H}), 7.05-6.97(\mathrm{~m}, 2 \mathrm{H}), 4.22(\mathrm{t}, J=9.5 \mathrm{~Hz}, 2 \mathrm{H}), 3.91(\mathrm{t}, J=9.5 \mathrm{~Hz}, 2 \mathrm{H}), 3.87(\mathrm{~s}, 3 \mathrm{H})$; ${ }^{13} \mathrm{C}$ NMR $\left(100 \mathrm{MHz}, \mathrm{CDCl}_{3}\right) \delta 167.9,164.4,158.8,140.5,139.8,136.9,132.6,129.2,128.8,127.4,126.2$, 125.9, 125.2, 123.1, 122.7, 121.9, 119.9, 117.3, 113.6, 112.6, 66.2, 55.5, 54.6; HRMS (ESI-TOF) $\mathrm{m} / \mathrm{z}$ Calcd for $\mathrm{C}_{23} \mathrm{H}_{21} \mathrm{~N}_{2} \mathrm{O}_{3} \mathrm{~S}[\mathrm{M}+\mathrm{H}]^{+}:$405.1267, found: 405.1276.<smiles>COc1ccc(/C=C/[Si](C)(C)C)c(C(=O)Nc2ccccc2C2=NCCO2)c1</smiles>

$5 \mathbf{i}$

\section{(E)-N-(2-(4,5-Dihydrooxazol-2-yl)phenyl)-5-methoxy-2-(2-(trimethylsilyl)vinyl)benzamide (5i)}

Substrate 10 was alkenylated with alkenyl boronate (4i) following the general procedure $\mathbf{B}$. After purification by preparative thin-layer chromatography (petroleum ether/ethyl acetate $=20 / 1$ ), $\mathbf{5 i}$ was obtained as a white solid (12.0 mg, 31\%). mp 141-143 ${ }^{\circ} \mathrm{C}$; ${ }^{1} \mathrm{H} \mathrm{NMR}\left(400 \mathrm{MHz}, \mathrm{CDCl}_{3}\right) \delta 12.59$ (s, 1H), $8.94(\mathrm{~d}, J=8.4 \mathrm{~Hz}, 1 \mathrm{H})$, $7.89(\mathrm{~d}, J=7.8 \mathrm{~Hz}, 1 \mathrm{H}), 7.62(\mathrm{~d}, J=8.7 \mathrm{~Hz}, 1 \mathrm{H}), 7.53(\mathrm{t}, J=7.9 \mathrm{~Hz}, 1 \mathrm{H}), 7.26$ (d, $J=1.8 \mathrm{~Hz}, 1 \mathrm{H}), 7.16(\mathrm{~d}, J$ $=2.6 \mathrm{~Hz}, 1 \mathrm{H}), 7.13(\mathrm{t}, J=7.6 \mathrm{~Hz}, 1 \mathrm{H}), 6.99(\mathrm{dd}, J=8.8,2.6 \mathrm{~Hz}, 1 \mathrm{H}), 6.33(\mathrm{~d}, J=19.0 \mathrm{~Hz}, 1 \mathrm{H}), 4.34(\mathrm{t}, J=9.6$ 
$\mathrm{Hz}, 2 \mathrm{H}), 4.00(\mathrm{t}, J=9.5 \mathrm{~Hz}, 2 \mathrm{H}), 3.86(\mathrm{~d}, J=7.3 \mathrm{~Hz}, 3 \mathrm{H}), 0.07(\mathrm{~s}, 9 \mathrm{H}) ;{ }^{13} \mathrm{C}$ NMR $\left(100 \mathrm{MHz}, \mathrm{CDCl}_{3}\right) \delta 167.9$, 164.5, 159.0, 140.7, 139.9, 137.1, 132.6, 130.2, 129.9, 129.2, 127.8, 122.5, 119.8, 116.9, 113.4, 112.1, 66.1, 55.5, 54.6, -1.3; HRMS (ESI-TOF) $\mathrm{m} / z$ Calcd for $\mathrm{C}_{22} \mathrm{H}_{27} \mathrm{~N}_{2} \mathrm{O}_{3} \mathrm{Si}[\mathrm{M}+\mathrm{H}]^{+}: 395.1785$, found: 395.1794 .<smiles>CCO/C=C/c1ccc(OC)cc1C(=O)Nc1ccccc1C1=NCCO1</smiles>

(E)- $N$-(2-(4,5-Dihydrooxazol-2-yl)phenyl)-2-(2-ethoxyvinyl)-5-methoxybenzamide (5j)

Substrate 10 was alkenylated with alkenyl boronate $(\mathbf{4 j})$ following the general procedure $\mathbf{B}$. After purification by preparative thin-layer chromatography (petroleum ether/ethyl acetate $=20 / 1$ ), $\mathbf{5} \mathbf{j}$ was obtained as a white solid (10.7 mg, 30\%). mp 118-120 ${ }^{\circ} \mathrm{C} ;{ }^{1} \mathrm{H}$ NMR (400 MHz, $\left.\mathrm{CDCl}_{3}\right) \delta 12.57$ (s, 1H), $8.94(\mathrm{~d}, J=8.4 \mathrm{~Hz}, 1 \mathrm{H})$, $7.89(\mathrm{~d}, J=7.9 \mathrm{~Hz}, 1 \mathrm{H}), 7.52(\mathrm{t}, J=7.4 \mathrm{~Hz}, 1 \mathrm{H}), 7.33(\mathrm{~d}, J=8.7 \mathrm{~Hz}, 1 \mathrm{H}), 7.19(\mathrm{~d}, J=2.6 \mathrm{~Hz}, 1 \mathrm{H}), 7.12(\mathrm{t}, J=$ $7.2 \mathrm{~Hz}, 1 \mathrm{H}), 6.94(\mathrm{dd}, J=8.5,2.5 \mathrm{~Hz}, 1 \mathrm{H}), 6.86(\mathrm{~d}, J=12.9 \mathrm{~Hz}, 1 \mathrm{H}), 6.33(\mathrm{~d}, J=12.8 \mathrm{~Hz}, 1 \mathrm{H}), 4.36$ (t, $J=9.5$ $\mathrm{Hz}, 2 \mathrm{H}), 4.04(\mathrm{t}, J=9.5 \mathrm{~Hz}, 2 \mathrm{H}), 3.87-3.80(\mathrm{~m}, 5 \mathrm{H}), 1.28(\mathrm{t}, J=7.0 \mathrm{~Hz}, 3 \mathrm{H}) ;{ }^{13} \mathrm{C} \mathrm{NMR}\left(100 \mathrm{MHz}, \mathrm{CDCl}_{3}\right) \delta$ 168.2, 164.4, 157.6, 148.0, 140.0, 135.6, 132.5, 129.2, 128.0, 127.1, 122.5, 119.8, 117.2, 113.5, 112.6, 103.3, 66.2, 65.0, 55.4, 54.7, 14.7; HRMS (ESI-TOF) $m / z$ Calcd for $\mathrm{C}_{21} \mathrm{H}_{23} \mathrm{~N}_{2} \mathrm{O}_{4}[\mathrm{M}+\mathrm{H}]^{+}:$367.1652, found: 367.1660 .<smiles>COc1ccc(/C=C/C2=CCCCC2)c(C(=O)Nc2ccccc2C2=NCCO2)c1</smiles>

(E)-2-(2-(Cyclohex-1-en-1-yl)vinyl)-N-(2-(4,5-dihydrooxazol-2-yl)phenyl)-5-methoxybenzamide (5k)

Substrate 10 was alkenylated with alkenyl boronate (4k) following the general procedure B. After purification by preparative thin-layer chromatography (petroleum ether/ethyl acetate $=10 / 1$ ), $\mathbf{5 k}$ was obtained as a white solid (26.2 mg, 65\%). mp 103-105 ${ }^{\circ} \mathrm{C}$; ${ }^{1} \mathrm{H}$ NMR (400 MHz, $\left.\mathrm{CDCl}_{3}\right) \delta 12.59(\mathrm{~s}, 1 \mathrm{H}), 8.96(\mathrm{~d}, J=8.4 \mathrm{~Hz}, 1 \mathrm{H})$, $7.88(\mathrm{~d}, J=7.9 \mathrm{~Hz}, 1 \mathrm{H}), 7.58(\mathrm{~d}, J=8.7 \mathrm{~Hz}, 1 \mathrm{H}), 7.53(\mathrm{t}, J=7.9 \mathrm{~Hz}, 1 \mathrm{H}), 7.19(\mathrm{~d}, J=2.7 \mathrm{~Hz}, 1 \mathrm{H}), 7.13(\mathrm{t}, J=$ $7.4 \mathrm{~Hz}, 1 \mathrm{H}), 6.98(\mathrm{dd}, J=8.7,2.6 \mathrm{~Hz}, 1 \mathrm{H}), 6.91(\mathrm{~d}, J=16.1 \mathrm{~Hz}, 1 \mathrm{H}), 6.64(\mathrm{~d}, J=16.1 \mathrm{~Hz}, 1 \mathrm{H}), 5.85(\mathrm{~s}, 1 \mathrm{H})$, $4.33(\mathrm{t}, J=9.4 \mathrm{~Hz}, 2 \mathrm{H}), 3.99(\mathrm{t}, J=9.5 \mathrm{~Hz}, 2 \mathrm{H}), 3.85(\mathrm{~s}, 3 \mathrm{H}), 2.20-2.13(\mathrm{~m}, 4 \mathrm{H}), 1.70-1.60(\mathrm{~m}, 4 \mathrm{H}) ;{ }^{13} \mathrm{C} \mathrm{NMR}$ $\left(100 \mathrm{MHz}, \mathrm{CDCl}_{3}\right) \delta 168.1,164.3,158.3,139.9,136.6,136.4,133.0,132.5,130.1,129.6,129.2,127.4,122.5$, 122.2, 119.9, 117.2, 113.5, 112.4, 66.2, 55.4, 54.6, 26.1, 24.6, 22.6, 22.5; HRMS (ESI-TOF) $\mathrm{m} / \mathrm{z}$ Calcd for $\mathrm{C}_{25} \mathrm{H}_{27} \mathrm{~N}_{2} \mathrm{O}_{3}[\mathrm{M}+\mathrm{H}]^{+}:$403.2016, found: 403.2027. 
<smiles>CNC(=O)c1cc(OC)ccc1C(=O)Nc1ccccc1C1=NCCO1</smiles>

5!

\section{$N$-(2-(4,5-Dihydrooxazol-2-yl)phenyl)-5-methoxy-2-(prop-1-en-2-yl)benzamide (5l)}

Substrate 10 was alkenylated with alkenyl boronate (4I) following the general procedure B. After purification by preparative thin-layer chromatography (petroleum ether/ethyl acetate $=20 / 1$ ), $\mathbf{5} \mathbf{l}$ was obtained as a white solid (18.2 mg, 55\%). mp 101-103 ${ }^{\circ} \mathrm{C} ;{ }^{1} \mathrm{H}$ NMR (400 MHz, $\left.\mathrm{CDCl}_{3}\right) \delta 12.47(\mathrm{~s}, 1 \mathrm{H}), 8.90(\mathrm{~d}, J=8.5 \mathrm{~Hz}, 1 \mathrm{H})$, $7.88(\mathrm{~d}, J=7.9 \mathrm{~Hz}, 1 \mathrm{H}), 7.50(\mathrm{t}, J=7.9 \mathrm{~Hz}, 1 \mathrm{H}), 7.24(\mathrm{~d}, J=8.6 \mathrm{~Hz}, 1 \mathrm{H}), 7.17(\mathrm{~d}, J=2.6 \mathrm{~Hz}, 1 \mathrm{H}), 7.11(\mathrm{t}, J=$ $7.6 \mathrm{~Hz}, 1 \mathrm{H}), 6.96(\mathrm{dd}, J=8.5,2.7 \mathrm{~Hz}, 1 \mathrm{H}), 5.07(\mathrm{~s}, 1 \mathrm{H}), 5.01(\mathrm{~s}, 1 \mathrm{H}), 4.34(\mathrm{t}, J=9.6 \mathrm{~Hz}, 2 \mathrm{H}), 4.02(\mathrm{t}, J=9.4$ $\mathrm{Hz}, 2 \mathrm{H}), 3.84$ (s, 3H), 2.08 (s, 3H); ${ }^{13} \mathrm{C}$ NMR $\left(100 \mathrm{MHz}, \mathrm{CDCl}_{3}\right) \delta 168.6,164.3,158.5,144.3,140.0,137.0$, $134.8,132.5,130.1,129.2,122.4,119.7,116.2,115.0,113.4,112.7,66.1,55.4,54.7,23.9$; HRMS (ESI-TOF) $m / z$ Calcd for $\mathrm{C}_{20} \mathrm{H}_{21} \mathrm{~N}_{2} \mathrm{O}_{3}[\mathrm{M}+\mathrm{H}]^{+}: 337.1547$, found: 337.1553 .<smiles>COc1ccc(C=C(C)C)c(C(=O)Nc2ccccc2C2=NCCO2)c1</smiles>

$N$-(2-(4,5-Dihydrooxazol-2-yl)phenyl)-5-methoxy-2-(2-methylprop-1-en-1-yl)benzamide (5m)

Substrate 10 was alkenylated with alkenyl boronate (4m) following the general procedure $\mathbf{B}$. After purification by preparative thin-layer chromatography (petroleum ether/ethyl acetate $=20 / 1$ ), $\mathbf{5 m}$ was obtained as a white solid (29.0 mg, 83\%). mp 72-74 ${ }^{\circ} \mathrm{C} ;{ }^{1} \mathrm{H}$ NMR (400 MHz, $\left.\mathrm{CDCl}_{3}\right) \delta 12.44(\mathrm{~s}, 1 \mathrm{H}), 8.91(\mathrm{~d}, J=8.4 \mathrm{~Hz}, 1 \mathrm{H}), 7.87$ $(\mathrm{d}, J=7.9 \mathrm{~Hz}, 1 \mathrm{H}), 7.50(\mathrm{t}, J=7.9 \mathrm{~Hz}, 1 \mathrm{H}), 7.25(\mathrm{~d}, J=2.7 \mathrm{~Hz}, 1 \mathrm{H}), 7.20(\mathrm{~d}, J=8.5 \mathrm{~Hz}, 1 \mathrm{H}), 7.10(\mathrm{t}, J=7.6$ $\mathrm{Hz}, 1 \mathrm{H}), 6.97(\mathrm{dd}, J=8.5,2.7 \mathrm{~Hz}, 1 \mathrm{H}), 6.46(\mathrm{~s}, 1 \mathrm{H}), 4.33(\mathrm{t}, J=9.5 \mathrm{~Hz}, 2 \mathrm{H}), 4.02(\mathrm{t}, J=9.5 \mathrm{~Hz}, 2 \mathrm{H}), 3.84$ (s, $3 \mathrm{H}), 1.82(\mathrm{~s}, 3 \mathrm{H}), 1.75(\mathrm{~s}, 3 \mathrm{H}) ;{ }^{13} \mathrm{C} \mathrm{NMR}\left(100 \mathrm{MHz}, \mathrm{CDCl}_{3}\right) \delta 168.1,164.3,157.7,139.9,137.3,135.3,132.5$, $131.9,129.6,129.2,123.0,122.9,122.4,119.9,116.3,113.5,112.5,66.1,55.3,54.8,26.3,19.4$; HRMS (ESITOF) $m / z$ Calcd for $\mathrm{C}_{21} \mathrm{H}_{23} \mathrm{~N}_{2} \mathrm{O}_{3}[\mathrm{M}+\mathrm{H}]^{+}: 351.1703$, found: 351.1711 .<smiles>COc1ccc(C=C2CCCCC2)c(C(=O)Nc2ccccc2C2=NCCO2)c1</smiles> 


\section{2-(Cyclohexylidenemethyl)- $N$-(2-(4,5-dihydrooxazol-2-yl)phenyl)-5-methoxybenzamide (5n)}

Substrate 10 was alkenylated with alkenyl boronate (4n) following the general procedure $\mathbf{B}$. After purification by preparative thin-layer chromatography (petroleum ether/ethyl acetate $=20 / 1$ ), $\mathbf{5 n}$ was obtained as a white solid (28.2 mg, 73\%). mp 128-130 ${ }^{\circ} \mathrm{C} ;{ }^{1} \mathrm{H}$ NMR (400 MHz, $\left.\mathrm{CDCl}_{3}\right) \delta 12.41(\mathrm{~s}, 1 \mathrm{H}), 8.90(\mathrm{~d}, J=8.4 \mathrm{~Hz}, 1 \mathrm{H})$, $7.87(\mathrm{dd}, J=7.9,1.3 \mathrm{~Hz}, 1 \mathrm{H}), 7.50(\mathrm{t}, J=8.0 \mathrm{~Hz}, 1 \mathrm{H}), 7.26-7.23(\mathrm{~m}, 1 \mathrm{H}), 7.16(\mathrm{~d}, J=8.5 \mathrm{~Hz}, 1 \mathrm{H}), 7.10(\mathrm{t}, J=$ $8.0 \mathrm{~Hz}, 1 \mathrm{H}), 6.96(\mathrm{dd}, J=8.5,2.7 \mathrm{~Hz}, 1 \mathrm{H}), 6.42(\mathrm{~s}, 1 \mathrm{H}), 4.34(\mathrm{t}, J=9.5 \mathrm{~Hz}, 2 \mathrm{H}), 4.04(\mathrm{t}, J=9.5 \mathrm{~Hz}, 2 \mathrm{H}), 3.84$ (s, 3H), 2.23-2.13 (m, 4H), 1.55-1.45 (m, 6H); $\left.{ }^{13} \mathrm{C} \mathrm{NMR} \mathrm{(100} \mathrm{MHz,} \mathrm{CDCl}_{3}\right) \delta$ 168.2, 164.3, 157.8, 143.3, 139.9, 137.5, 132.5, 131.9, 129.3, 129.2 122.3, 119.9, 119.7, 116.3, 113.5, 112.5, 66.1, 55.4, 54.8, 37.3, 29.6, 28.4, 27.6, 26.6; HRMS (ESI-TOF) $\mathrm{m} / z$ Calcd for $\mathrm{C}_{24} \mathrm{H}_{27} \mathrm{~N}_{2} \mathrm{O}_{3}[\mathrm{M}+\mathrm{H}]^{+}: 391.2016$, found: 391.2025.<smiles>COc1ccc(/C=C(\[O-])c2ccccc2)c(C(=O)Nc2ccccc2C2=NCCO2)c1</smiles>

(E)-N-(2-(4,5-Dihydrooxazol-2-yl)phenyl)-5-methoxy-2-(1-phenylprop-1-en-2-yl)benzamide (5o)

Substrate 10 was alkenylated with alkenyl boronate (4o) following the general procedure B. After purification by preparative thin-layer chromatography (petroleum ether/ethyl acetate $=20 / 1$ ), $\mathbf{5 0}$ was obtained as a white solid (25.4 mg, 62\%). mp 89-90 ${ }^{\circ} \mathrm{C}$; ${ }^{1} \mathrm{H}$ NMR $\left(400 \mathrm{MHz}, \mathrm{CDCl}_{3}\right) \delta 12.51(\mathrm{~s}, 1 \mathrm{H}), 8.93(\mathrm{~d}, J=8.4 \mathrm{~Hz}, 1 \mathrm{H}), 7.85$ $(\mathrm{d}, J=7.9 \mathrm{~Hz}, 1 \mathrm{H}), 7.50(\mathrm{t}, J=7.9 \mathrm{~Hz}, 1 \mathrm{H}), 7.34(\mathrm{~d}, J=8.5 \mathrm{~Hz}, 1 \mathrm{H}), 7.30-7.22(\mathrm{~m}, 3 \mathrm{H}), 7.23-7.15(\mathrm{~m}, 3 \mathrm{H})$, $7.10(\mathrm{t}, J=7.3 \mathrm{~Hz}, 1 \mathrm{H}), 7.01(\mathrm{dd}, J=8.5,2.6 \mathrm{~Hz}, 1 \mathrm{H}), 6.58(\mathrm{~s}, 1 \mathrm{H}), 4.15(\mathrm{t}, J=9.5 \mathrm{~Hz}, 2 \mathrm{H}), 3.92-3.83(\mathrm{~m}, 5 \mathrm{H})$, $2.17(\mathrm{~s}, 3 \mathrm{H}) ;{ }^{13} \mathrm{C} \mathrm{NMR}\left(100 \mathrm{MHz}, \mathrm{CDCl}_{3}\right) \delta 168.7,164.2,158.5,139.9,138.1,137.6,137.1,136.7,132.6,130.2$, 129.7, 129.2, 128.8, 128.0, 126.3, 122.5, 119.7, 116.6, 113.4, 113.0, 66.0, 55.5, 54.6, 19.7; HRMS (ESI-TOF) $m / z$ Calcd for $\mathrm{C}_{26} \mathrm{H}_{25} \mathrm{~N}_{2} \mathrm{O}_{3}[\mathrm{M}+\mathrm{H}]^{+}: 413.1860$, found: 413.1869 .<smiles>COc1ccc(C(=O)Nc2ccccc2C2=NCCO2)c(C(=O)N(C)C)c1</smiles>

\section{$N$-(2-(4,5-Dihydrooxazol-2-yl)phenyl)-5-methoxy-2-(3-methylbut-2-en-2-yl)benzamide (5p)}

Substrate 10 was alkenylated with alkenyl boronate (4p) following the general procedure B. After purification by preparative thin-layer chromatography (petroleum ether/ethyl acetate $=20 / 1$ ), $\mathbf{5 p}$ was obtained as a white solid (18.3 mg, 51\%). mp 106-108 ${ }^{\circ} \mathrm{C} ;{ }^{1} \mathrm{H}$ NMR $\left(400 \mathrm{MHz}, \mathrm{CDCl}_{3}\right) \delta 12.18(\mathrm{~s}, 1 \mathrm{H}), 8.79(\mathrm{~d}, J=8.4 \mathrm{~Hz}, 1 \mathrm{H})$, $7.87(\mathrm{~d}, J=8.0 \mathrm{~Hz}, 1 \mathrm{H}), 7.49$ (t, $J=7.8 \mathrm{~Hz}, 1 \mathrm{H}), 7.20$ (d, $J=2.7 \mathrm{~Hz}, 1 \mathrm{H}), 7.14-7.05$ (m, 2H), 6.97 (dd, $J=8.4$, $2.7 \mathrm{~Hz}, 1 \mathrm{H}), 4.33(\mathrm{t}, J=9.4 \mathrm{~Hz}, 2 \mathrm{H}), 4.02(\mathrm{t}, J=9.6 \mathrm{~Hz}, 2 \mathrm{H}), 3.84(\mathrm{~s}, 3 \mathrm{H}), 1.95(\mathrm{~s}, 3 \mathrm{H}), 1.70(\mathrm{~s}, 3 \mathrm{H}), 1.53$ (s, 
$3 \mathrm{H}) ;{ }^{13} \mathrm{C} \mathrm{NMR}\left(100 \mathrm{MHz}, \mathrm{CDCl}_{3}\right) \delta 168.5,164.2,157.7,140.0,137.4,136.3,132.4,131.1,129.1,128.3,128.0$, 122.3, 120.1, 116.5, 113.6, 112.3, 66.0, 55.4, 54.8, 22.2, 21.1, 20.3; HRMS (ESI-TOF) $m / z$ Calcd for $\mathrm{C}_{22} \mathrm{H}_{25} \mathrm{~N}_{2} \mathrm{O}_{3}$ $[\mathrm{M}+\mathrm{H}]^{+}: 365.1860$, found: 365.1869 .<smiles>COc1ccc(C2=CCCC2)c(C(=O)Nc2ccccc2C2=NCCO2)c1</smiles>

\section{2-(Cyclopent-1-en-1-yl)- $N$-(2-(4,5-dihydrooxazol-2-yl)phenyl)-5-methoxybenzamide (5q)}

Substrate 10 was alkenylated with alkenyl boronate (4q) following the general procedure $\mathbf{B}$. After purification by preparative thin-layer chromatography (petroleum ether/ethyl acetate $=20 / 1$ ), $\mathbf{5 q}$ was obtained as a white solid (22.4 mg, 62\%). mp 116-118 ${ }^{\circ} \mathrm{C} ;{ }^{1} \mathrm{H}$ NMR (400 MHz, $\left.\mathrm{CDCl}_{3}\right) \delta 12.39(\mathrm{~s}, 1 \mathrm{H}), 8.91(\mathrm{~d}, J=8.4 \mathrm{~Hz}, 1 \mathrm{H})$, $7.87(\mathrm{~d}, J=7.9 \mathrm{~Hz}, 1 \mathrm{H}), 7.51(\mathrm{t}, J=7.8 \mathrm{~Hz}, 1 \mathrm{H}), 7.28(\mathrm{~d}, J=8.7 \mathrm{~Hz}, 1 \mathrm{H}), 7.14-7.08(\mathrm{mz}, 2 \mathrm{H}), 6.94(\mathrm{dd}, J=$ 8.6, $2.7 \mathrm{~Hz}, 1 \mathrm{H}), 5.82(\mathrm{~s}, 1 \mathrm{H}), 4.33(\mathrm{t}, J=9.5 \mathrm{~Hz}, 2 \mathrm{H}), 3.99(\mathrm{t}, J=9.5 \mathrm{~Hz}, 2 \mathrm{H}), 3.83(\mathrm{~s}, 3 \mathrm{H}), 2.62(\mathrm{t}, J=6.5 \mathrm{~Hz}$, $2 \mathrm{H}), 2.35(\mathrm{t}, J=6.1 \mathrm{~Hz}, 2 \mathrm{H}), 1.91-1.80(\mathrm{~m}, 2 \mathrm{H}) ;{ }^{13} \mathrm{C} \mathrm{NMR}\left(100 \mathrm{MHz}, \mathrm{CDCl}_{3}\right) \delta 169.1,164.2,158.3,141.8$, 139.9, 137.2, 132.5, 129.8, 129.2, 129.1, 129.0, 122.4, 119.7, 116.1, 113.4, 112.7, 66.1, 55.4, 54.7, 35.6, 33.5, 24.1; HRMS (ESI-TOF) $m / z$ Calcd for $\mathrm{C}_{22} \mathrm{H}_{23} \mathrm{~N}_{2} \mathrm{O}_{3}[\mathrm{M}+\mathrm{H}]^{+}: 363.1703$, found: 363.1711 .<smiles>COc1ccc(C2=CCCCC2)c(C(=O)Nc2ccccc2C2=NCCO2)c1</smiles>

$N$-(2-(4,5-Dihydrooxazol-2-yl)phenyl)-4-methoxy-2',3',4',5'-tetrahydro-[1,1'-biphenyl]-2-carboxamide (5r)

Substrate 10 was alkenylated with alkenyl boronate (4r) following the general procedure B. After purification by preparative thin-layer chromatography (petroleum ether/ethyl acetate $=20 / 1$ ), $\mathbf{5} \mathbf{r}$ was obtained as a white solid (16.7 mg, 45\%). mp 111-113 ${ }^{\circ} \mathrm{C} ;{ }^{1} \mathrm{H}$ NMR (400 MHz, $\left.\mathrm{CDCl}_{3}\right) \delta 12.28$ (s, 1H), $8.90(\mathrm{~d}, J=8.4 \mathrm{~Hz}, 1 \mathrm{H})$, $7.87(\mathrm{~d}, J=7.9 \mathrm{~Hz}, 1 \mathrm{H}), 7.50(\mathrm{t}, J=7.8 \mathrm{~Hz}, 1 \mathrm{H}), 7.19-7.15(\mathrm{~m}, 2 \mathrm{H}), 7.10(\mathrm{t}, J=7.6 \mathrm{~Hz}, 1 \mathrm{H}), 6.95(\mathrm{dd}, J=8.5$, $2.7 \mathrm{~Hz}, 1 \mathrm{H}), 5.74(\mathrm{~s}, 1 \mathrm{H}), 4.33(\mathrm{t}, J=9.5 \mathrm{~Hz}, 2 \mathrm{H}), 3.99(\mathrm{t}, J=9.5 \mathrm{~Hz}, 2 \mathrm{H}), 3.83(\mathrm{~s}, 3 \mathrm{H}), 2.27(\mathrm{~s}, 2 \mathrm{H}), 2.04-1.97$ $(\mathrm{m}, 2 \mathrm{H}), 1.59-1.45(\mathrm{~m}, 4 \mathrm{H}) ;{ }^{13} \mathrm{C} \mathrm{NMR}\left(100 \mathrm{MHz}, \mathrm{CDCl}_{3}\right) \delta 169.0,164.2,158.1,140.0,137.0,135.5,132.5$, $129.9,129.2,127.0,122.3,119.6,116.5,113.3,112.8,66.1,55.4,54.7,29.9,25.7,23.0,21.9$; HRMS (ESITOF) $m / z$ Calcd for $\mathrm{C}_{23} \mathrm{H}_{25} \mathrm{~N}_{2} \mathrm{O}_{3}[\mathrm{M}+\mathrm{H}]^{+}: 377.1860$, found: 377.1869 . 
<smiles>COc1ccc(C2=CCC3(CC2)OCCO3)c(C(=O)Nc2ccccc2C2=NCCO2)c1</smiles>

$N$-(2-(4,5-Dihydrooxazol-2-yl)phenyl)-5-methoxy-2-(1,4-dioxaspiro[4.5]dec-7-en-8-yl)benzamide (5s)

Substrate 10 was alkenylated with alkenyl boronate (4s) following the general procedure B. After purification by preparative thin-layer chromatography (petroleum ether/ethyl acetate $=10 / 1$ ), $\mathbf{5} \mathbf{s}$ was obtained as a white solid (25.9 mg, 60\%). mp 113-115 ${ }^{\circ} \mathrm{C}$; ${ }^{1} \mathrm{H}$ NMR (400 MHz, $\left.\mathrm{CDCl}_{3}\right) \delta 12.31(\mathrm{~s}, 1 \mathrm{H}), 8.89(\mathrm{~d}, J=8.4 \mathrm{~Hz}, 1 \mathrm{H})$, $7.86(\mathrm{~d}, J=6.9 \mathrm{~Hz}, 1 \mathrm{H}), 7.49(\mathrm{t}, J=7.8 \mathrm{~Hz}, 1 \mathrm{H}), 7.22(\mathrm{~d}, J=8.5 \mathrm{~Hz}, 1 \mathrm{H}), 7.18(\mathrm{~d}, J=2.6 \mathrm{~Hz}, 1 \mathrm{H}), 7.09(\mathrm{t}, J=$ $7.2 \mathrm{~Hz}, 1 \mathrm{H}), 6.95(\mathrm{dd}, J=8.5,2.7 \mathrm{~Hz}, 1 \mathrm{H}), 5.63(\mathrm{~s}, 1 \mathrm{H}), 4.32(\mathrm{t}, J=9.5 \mathrm{~Hz}, 2 \mathrm{H}), 3.98(\mathrm{t}, J=9.5 \mathrm{~Hz}, 2 \mathrm{H}), 3.87-$ $3.82(\mathrm{~m}, 7 \mathrm{H}), 2.55-2.45(\mathrm{~m}, 2 \mathrm{H}), 2.25(\mathrm{~s}, 2 \mathrm{H}), 1.71(\mathrm{t}, J=6.2 \mathrm{~Hz}, 2 \mathrm{H}) ;{ }^{13} \mathrm{C} \mathrm{NMR}\left(100 \mathrm{MHz}, \mathrm{CDCl}_{3}\right) \delta 168.8$, 164.3, 158.4, 140.0, 137.0, 136.6, 134.1, 132.4, 130.0, 129.1, 124.0, 122.3, 119.5, 116.6, 113.5, 112.9, 107.4, 66.0, 64.2, 55.4, 54.7, 36.0, 31.4, 29.2; HRMS (ESI-TOF) $m / z$ Calcd for $\mathrm{C}_{25} \mathrm{H}_{27} \mathrm{~N}_{2} \mathrm{O}_{5}[\mathrm{M}+\mathrm{H}]^{+}: 435.1914$, found: 435.1927.<smiles>COc1ccc(C2=CC3CCC(C2)C3)c(C(=O)Nc2ccccc2C2=NCCO2)c1</smiles>

$5 \mathbf{t}$

2-((1R,4S)-Bicyclo[2.2.1] hept-2-en-2-yl)-N-(2-(4,5-dihydrooxazol-2-yl)phenyl)-5-methoxybenzamide (5t)

Substrate 10 was alkenylated with alkenyl boronate (4t) following the general procedure $\mathbf{B}$. After purification by preparative thin-layer chromatography (petroleum ether/dichloromethane $=1 / 1$ ), $\mathbf{5 t}$ was obtained as a white solid (28 mg, 72\%). mp 88-90 ${ }^{\circ} \mathrm{C} ;{ }^{1} \mathrm{H}$ NMR (400 MHz, $\left.\mathrm{CDCl}_{3}\right) \delta 12.40(\mathrm{~s}, 1 \mathrm{H}), 8.92$ (d, J = 8.4 Hz, 1H), 7.86 $(\mathrm{dd}, J=7.9,1.4 \mathrm{~Hz}, 1 \mathrm{H}), 7.51(\mathrm{t}, J=7.9 \mathrm{~Hz}, 1 \mathrm{H}), 7.22(\mathrm{~d}, J=8.6 \mathrm{~Hz}, 1 \mathrm{H}), 7.14-7.07(\mathrm{~m}, 2 \mathrm{H}), 6.93(\mathrm{dd}, J=$ 8.6, $2.7 \mathrm{~Hz}, 1 \mathrm{H}), 6.03(\mathrm{~d}, J=2.8 \mathrm{~Hz}, 1 \mathrm{H}), 4.36-4.29(\mathrm{~m}, 2 \mathrm{H}), 3.98(\mathrm{t}, J=9.7 \mathrm{~Hz}, 2 \mathrm{H}), 3.83(\mathrm{~s}, 3 \mathrm{H}), 3.10(\mathrm{~s}$, $1 \mathrm{H}), 2.84(\mathrm{~s}, 1 \mathrm{H}), 1.71-1.59(\mathrm{~m}, 2 \mathrm{H}), 1.42(\mathrm{~d}, J=8.0 \mathrm{~Hz}, 1 \mathrm{H}), 1.15-1.00(\mathrm{~m}, 3 \mathrm{H}) ;{ }^{13} \mathrm{C} \mathrm{NMR}\left(100 \mathrm{MHz}, \mathrm{CDCl}_{3}\right)$ $\delta$ 169.1, 164.1, 158.4, 146.1, 140.0, 137.5, 132.5, 131.2, 129.7, 129.2, 127.4, 122.4, 119.7, 116.1, 113.4, 112.9 66.1, 55.4, 54.6, 47.9, 46.3, 43.2, 26.2, 24.7; HRMS (ESI-TOF) $m / z$ Calcd for $\mathrm{C}_{24} \mathrm{H}_{25} \mathrm{~N}_{2} \mathrm{O}_{3}[\mathrm{M}+\mathrm{H}]^{+}: 389.1860$, found: 389.1868 .<smiles>COc1ccc(C2=CCOCC2)c(NC2=NCCO2)c1</smiles>

$5 u$ 
Substrate 10 was alkenylated with alkenyl boronate (4u) following the general procedure $\mathbf{B}$. After purification by preparative thin-layer chromatography (petroleum ether/ethyl acetate $=10 / 1$ ), $\mathbf{5} \mathbf{u}$ was obtained as a white solid (26.3 mg, 70\%). mp 115-117 ${ }^{\circ} \mathrm{C}$; ${ }^{1} \mathrm{H}$ NMR (400 MHz, $\left.\mathrm{CDCl}_{3}\right) \delta 12.45(\mathrm{~s}, 1 \mathrm{H}), 8.88(\mathrm{~d}, J=8.3 \mathrm{~Hz}, 1 \mathrm{H})$, $7.89(\mathrm{~d}, J=7.9 \mathrm{~Hz}, 1 \mathrm{H}), 7.50(\mathrm{t}, J=7.7 \mathrm{~Hz}, 1 \mathrm{H}), 7.23-7.18(\mathrm{~m}, 2 \mathrm{H}), 7.11(\mathrm{t}, J=7.4 \mathrm{~Hz}, 1 \mathrm{H}), 6.98(\mathrm{dd}, J=8.5$, $2.7 \mathrm{~Hz}, 1 \mathrm{H}), 5.75(\mathrm{~s}, 1 \mathrm{H}), 4.35(\mathrm{t}, J=9.5 \mathrm{~Hz}, 2 \mathrm{H}), 4.17(\mathrm{~d}, J=2.4 \mathrm{~Hz}, 2 \mathrm{H}), 4.00(\mathrm{t}, J=9.5 \mathrm{~Hz}, 2 \mathrm{H}), 3.84(\mathrm{~s}$, $3 \mathrm{H}), 3.76(\mathrm{t}, J=5.3 \mathrm{~Hz}, 2 \mathrm{H}), 2.40(\mathrm{~s}, 2 \mathrm{H}) ;{ }^{13} \mathrm{C} \mathrm{NMR}\left(100 \mathrm{MHz}, \mathrm{CDCl}_{3}\right) \delta 168.5,164.4,158.6,139.8,136.9$, 135.0, 133.5, 132.6, 130.0, 129.3, 124.6, 122.5, 119.6, 116.5, 113.3, 113.0, 66.1, 65.7, 64.4, 55.4, 54.7, 29.7; HRMS (ESI-TOF) $\mathrm{m} / \mathrm{z}$ Calcd for $\mathrm{C}_{22} \mathrm{H}_{23} \mathrm{~N}_{2} \mathrm{O}_{4}[\mathrm{M}+\mathrm{H}]^{+}:$379.1652, found: 379.1662 .<smiles>COc1ccc(C2=CCN(C)CC2)c(C(=O)Nc2ccccc2C2=NCCO2)c1</smiles>

$5 \mathrm{v}$

$N$-(2-(4,5-Dihydrooxazol-2-yl)phenyl)-5-methoxy-2-(1-methyl-1,2,3,6-tetrahydropyridin-4-yl)benzamide $(5 \mathrm{v})$

Substrate 10 was alkenylated with alkenyl boronate (4v) following the general procedure B. After purification by column chromatography (neutral alumina, petroleum ether/ethyl acetate $=1 / 1$ ), $\mathbf{5} \mathbf{v}$ was obtained as a white solid (25.6 mg, 66\%). mp 117-119 ${ }^{\circ} \mathrm{C} ;{ }^{1} \mathrm{H}$ NMR (400 MHz, $\left.\mathrm{CDCl}_{3}\right) \delta 12.41(\mathrm{~s}, 1 \mathrm{H}), 8.89(\mathrm{~d}, J=8.5 \mathrm{~Hz}, 1 \mathrm{H})$, $7.89(\mathrm{dd}, J=7.9,1.4 \mathrm{~Hz}, 1 \mathrm{H}), 7.51(\mathrm{t}, J=7.2 \mathrm{~Hz}, 1 \mathrm{H}), 7.21(\mathrm{~d}, J=8.5 \mathrm{~Hz}, 1 \mathrm{H}), 7.18(\mathrm{~d}, J=2.7 \mathrm{~Hz}, 1 \mathrm{H}), 7.12$ $(\mathrm{t}, J=7.6 \mathrm{~Hz}, 1 \mathrm{H}), 6.96(\mathrm{dd}, J=8.5,2.7 \mathrm{~Hz}, 1 \mathrm{H}), 5.69(\mathrm{~s}, 1 \mathrm{H}), 4.35(\mathrm{t}, J=9.5 \mathrm{~Hz}, 2 \mathrm{H}), 4.01(\mathrm{t}, J=9.5 \mathrm{~Hz}, 2 \mathrm{H})$, 3.84 (s, 3H), 2.98-2.95 (m, 2H), 2.52-2.44 (m, 4H), 2.30 (s, 3H); $\left.{ }^{13} \mathrm{C} \mathrm{NMR} \mathrm{(100} \mathrm{MHz,} \mathrm{CDCl}_{3}\right) \delta 168.5,164.4$, 158.4, 140.0, 136.9, 135.5, 134.1, 132.6, 130.3, 129.2, 124.0, 122.5, 119.7, 116.5, 113.4 112.9, 66.1, 55.4, 54.8, 54.7, 52.2, 45.6, 30.6; HRMS (ESI-TOF) $\mathrm{m} / z$ Calcd for $\mathrm{C}_{23} \mathrm{H}_{26} \mathrm{~N}_{3} \mathrm{O}_{3}[\mathrm{M}+\mathrm{H}]^{+}: 392.1969$, found: 392.1978 .<smiles>COc1ccc(C2=CCN(C(=O)OC(C)(C)C)CC2)c(C(=O)Nc2ccccc2C2=NCCO2)c1</smiles>

tert-Butyl 4-(2-((2-(4,5-dihydrooxazol-2-yl)phenyl)carbamoyl)-4-methoxyphenyl)-5,6-di-hydropyridine$1(2 H)$-carboxylate (5w)

Substrate 10 was alkenylated with alkenyl boronate (4w) following the general procedure $\mathbf{B}$. After purification by column chromatography (neutral alumina, petroleum ether/ethyl acetate $=5 / 1$ ), $5 \mathrm{w}$ was obtained as a white solid (38.4 mg, 81\%), rotameric mixture. mp 173-175 ${ }^{\circ} \mathrm{C} ;{ }^{1} \mathrm{H}$ NMR (400 MHz, $\left.\mathrm{CDCl}_{3}\right) \delta 12.45(\mathrm{~s}, 0.5 \mathrm{H}), 12.40$ (s, $0.5 \mathrm{H}), 8.88(\mathrm{~d}, J=8.4 \mathrm{~Hz}, 1 \mathrm{H}), 7.88(\mathrm{dd}, J=7.9,1.4 \mathrm{~Hz}, 1 \mathrm{H}), 7.50(\mathrm{t}, J=7.4 \mathrm{~Hz}, 1 \mathrm{H}), 7.22-7.16(\mathrm{~m}, 2 \mathrm{H})$, 
$7.11(\mathrm{t}, J=7.3 \mathrm{~Hz}, 1 \mathrm{H}), 6.97(\mathrm{dd}, J=8.5,2.7 \mathrm{~Hz}, 1 \mathrm{H}), 5.68(\mathrm{brs}, 1 \mathrm{H}), 4.35(\mathrm{t}, J=9.5 \mathrm{~Hz}, 2 \mathrm{H}), 4.00(\mathrm{t}, J=9.5$ $\mathrm{Hz}, 2 \mathrm{H}), 3.96-3.86(\mathrm{~m}, 2 \mathrm{H}), 3.84(\mathrm{~s}, 3 \mathrm{H}), 3.52-3.38(\mathrm{~m}, 2 \mathrm{H}), 2.50-2.30(\mathrm{~m}, 2 \mathrm{H}), 1.41(\mathrm{~s}, 9 \mathrm{H}) ;{ }^{13} \mathrm{C}$ NMR $(125$ $\left.\mathrm{MHz}, \mathrm{CDCl}_{3}\right) \delta 168.5,164.4,158.6,154.8,139.8,137.0,136.7,136.1,135.8,133.7,133.5,132.6,130.4,129.8$, 129.3, 123.0, 122.8, 122.5, 119.6, 116.6, 113.3, 113.0, 79.5, 66.1, 55.4, 54.6, 43.9, 43.2, 41.1, 39.8, 30.0, 29.7, 28.4; HRMS (ESI-TOF) $\mathrm{m} / z$ Calcd for $\mathrm{C}_{27} \mathrm{H}_{32} \mathrm{~N}_{3} \mathrm{O}_{5}[\mathrm{M}+\mathrm{H}]^{+}: 478.2336$, found: 478.2349 .<smiles>COc1ccc(C2=CCCN(C(=O)OC(C)(C)C)C2)c(C(=O)Nc2ccccc2C2=NCCO2)c1</smiles>

tert-Butyl 3-(2-((2-(4,5-dihydrooxazol-2-yl)phenyl)carbamoyl)-4-methoxyphenyl)-5,6-di-hydropyridine$1(2 H)$-carboxylate $(5 x)$

Substrate 10 was alkenylated with alkenyl boronate $(\mathbf{4 x})$ following the general procedure $\mathbf{B}$. After purification by column chromatography (neutral alumina, petroleum ether/ethyl acetate $=5 / 1$ ), $\mathbf{5} \mathbf{x}$ was obtained as a white solid (38.2 mg, 80\%), rotameric mixture. mp 117-119 ${ }^{\circ} \mathrm{C}$; ${ }^{1} \mathrm{H}$ NMR $\left(400 \mathrm{MHz}, \mathrm{CDCl}_{3}\right) \delta 12.39(\mathrm{~s}, 1 \mathrm{H}), 8.89(\mathrm{~d}$, $J=8.2 \mathrm{~Hz}, 1 \mathrm{H}), 7.87(\mathrm{~d}, J=7.9 \mathrm{~Hz}, 1 \mathrm{H}), 7.50(\mathrm{t}, J=7.8 \mathrm{~Hz}, 1 \mathrm{H}), 7.26-7.15(\mathrm{~m}, 2 \mathrm{H}), 7.10(\mathrm{t}, J=7.6 \mathrm{~Hz}, 1 \mathrm{H})$, $6.98(\mathrm{~d}, J=8.3 \mathrm{~Hz}, 1 \mathrm{H}), 5.84(\mathrm{~s}, 1 \mathrm{H}), 4.34(\mathrm{t}, J=9.5 \mathrm{~Hz}, 2 \mathrm{H}), 4.25-4.05(\mathrm{~m}, 2 \mathrm{H}), 4.02(\mathrm{t}, J=9.3 \mathrm{~Hz}, 2 \mathrm{H}), 3.84$ (s, 3H), 3.37 (s, 2H), 2.13 (brs, 2H), 1.41 (s, 9H); $\left.{ }^{13} \mathrm{C} \mathrm{NMR} \mathrm{(150} \mathrm{MHz,} \mathrm{CDCl}_{3}\right) \delta 168.3,164.5,158.9,154.9$, 140.0, 138.0, 135.1, 132.5, 131.6, 130.3, 129.2, 124.9, 122.4, 119.8, 116.4, 113.4, 113.1, 79.5, 66.1, 55.4, 54.7, 46.7, 39.6, 28.4, 25.4; HRMS (ESI-TOF) $m / z$ Calcd for $\mathrm{C}_{27} \mathrm{H}_{31} \mathrm{~N}_{3} \mathrm{O}_{5} \mathrm{Na}[\mathrm{M}+\mathrm{Na}]^{+}:$500.2156, found: 500.2155.<smiles>COc1ccc(C2=CCCCO2)c(C(=O)Nc2ccccc2C2=NCCO2)c1</smiles>

$5 y$

\section{2-(3,4-Dihydro-2H-pyran-6-yl)-N-(2-(4,5-dihydrooxazol-2-yl)phenyl)-5-methoxybenzamide (5y)}

Substrate 10 was alkenylated with alkenyl boronate (4y) following the general procedure B. After purification by column chromatography (neutral alumina, petroleum ether/ethyl acetate $=10 / 1$ ), $\mathbf{5 y}$ was obtained as a white solid (24.2 mg, 65\%). mp 127-129 ${ }^{\circ} \mathrm{C} ;{ }^{1} \mathrm{H}$ NMR (400 MHz, $\left.\mathrm{CDCl}_{3}\right) \delta 12.38(\mathrm{~s}, 1 \mathrm{H}), 8.93(\mathrm{~d}, J=8.4 \mathrm{~Hz}, 1 \mathrm{H})$, $7.86(\mathrm{dd}, J=7.9,1.4 \mathrm{~Hz}, 1 \mathrm{H}), 7.50$ (t, $J=7.2 \mathrm{~Hz}, 1 \mathrm{H}), 7.37$ (d, $J=8.5 \mathrm{~Hz}, 1 \mathrm{H}), 7.13(\mathrm{~d}, J=2.6 \mathrm{~Hz}, 1 \mathrm{H}), 7.09$ $(\mathrm{t}, J=8.0 \mathrm{~Hz}, 1 \mathrm{H}), 6.92(\mathrm{dd}, J=8.5,2.7 \mathrm{~Hz}, 1 \mathrm{H}), 5.04(\mathrm{t}, J=3.8 \mathrm{~Hz}, 1 \mathrm{H}), 4.33(\mathrm{t}, J=9.5 \mathrm{~Hz}, 2 \mathrm{H}), 4.01(\mathrm{t}, J=$ $9.5 \mathrm{~Hz}, 2 \mathrm{H}), 3.92-3.86(\mathrm{~m}, 2 \mathrm{H}), 3.83(\mathrm{~s}, 3 \mathrm{H}), 2.12-2.05(\mathrm{~m}, 2 \mathrm{H}), 1.80-1.71(\mathrm{~m}, 2 \mathrm{H}) ;{ }^{13} \mathrm{C}$ NMR (100 MHz, $\left.\mathrm{CDCl}_{3}\right) \delta 168.7,164.1,159.3,152.1,140.2,137.5,132.5,129.9,129.0,128.3,122.1,119.6,115.8,113.2,112.7$, 99.9, 66.5, 66.1 55.4, 54.7, 22.1, 20.9; HRMS (ESI-TOF) $m / z$ Calcd for $\mathrm{C}_{22} \mathrm{H}_{23} \mathrm{~N}_{2} \mathrm{O}_{4}[\mathrm{M}+\mathrm{H}]^{+}: 379.1652$, found: 379.1652. 
<smiles>COc1ccc(C2=CC3CCC(C2)N3C(=O)OC(C)(C)C)c(C(=O)Nc2ccccc2C2=NCCO2)c1</smiles>

(1R,5S)-tert-Butyl 3-(2-((2-(4,5-dihydrooxazol-2-yl)phenyl)carbamoyl)-4-methoxyphen-yl)-8-azabicyclo [3.2.1] oct-2-ene-8-carboxylate (5z)

Substrate 10 was alkenylated with alkenyl boronate $(\mathbf{4 z})$ following the general procedure $\mathbf{B}$. After purification by column chromatography (neutral alumina, petroleum ether/ethyl acetate $=10 / 1$ ), $\mathbf{5 z}$ was obtained as a white solid (38.8 mg, 77\%), rotameric mixture. mp 123-125 ${ }^{\circ} \mathrm{C} ;{ }^{1} \mathrm{H}$ NMR (400 MHz, $\left.\mathrm{CDCl}_{3}\right) \delta 12.43(\mathrm{~s}, 0.5 \mathrm{H}), 12.38$ $(\mathrm{s}, 0.5 \mathrm{H}), 8.89(\mathrm{~d}, J=8.4 \mathrm{~Hz}, 1 \mathrm{H}), 7.88(\mathrm{dd}, J=7.9,1.3 \mathrm{~Hz}, 1 \mathrm{H}), 7.51(\mathrm{t}, J=7.9 \mathrm{~Hz}, 1 \mathrm{H}), 7.16-7.07(\mathrm{~m}, 3 \mathrm{H})$, $6.93(\mathrm{dd}, J=8.5,2.6 \mathrm{~Hz}, 1 \mathrm{H}), 6.01(\mathrm{~s}, 1 \mathrm{H}), 4.45-4.18(\mathrm{~m}, 4 \mathrm{H}), 4.01(\mathrm{t}, J=9.6 \mathrm{~Hz}, 2 \mathrm{H}), 3.83(\mathrm{~s}, 3 \mathrm{H}), 3.18-2.83$ $(\mathrm{m}, 1 \mathrm{H}), 2.10(\mathrm{~d}, J=17.2 \mathrm{~Hz}, 1 \mathrm{H}), 2.00-1.80(\mathrm{~m}, 2 \mathrm{H}), 1.73-1.55(\mathrm{~m}, 2 \mathrm{H}), 1.42(\mathrm{~s}, 9 \mathrm{H}) ;{ }^{13} \mathrm{C} \mathrm{NMR}(125 \mathrm{MHz}$, $\left.\mathrm{CDCl}_{3}\right) \delta 168.6,168.4,164.4,158.5,154.0,139.8,137.5,137.4,134.7,134.6,132.9,132.6,131.1,130.8,130.2$, $129.5,129.3,122.5,119.5,116.2,113.2,112.6,79.1,66.1,55.4,54.6,53.5,52.8,52.6,51.8,38.5,37.8,34.6$, 34.0, 29.5, 28.8, 28.3; HRMS (ESI-TOF) $\mathrm{m} / z$ Calcd for $\mathrm{C}_{29} \mathrm{H}_{34} \mathrm{~N}_{3} \mathrm{O}_{5}[\mathrm{M}+\mathrm{H}]^{+}:$504.2493, found: 504.2509. 


\subsection{Evaluation of Alkene-Alkene Coupling}

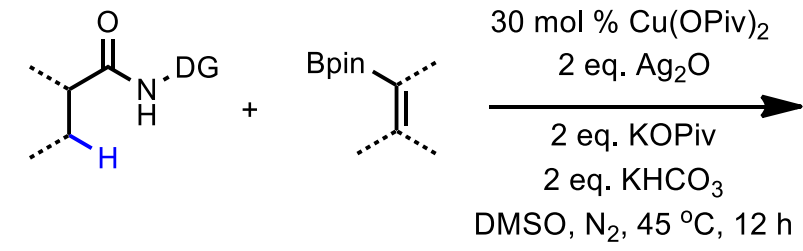

$6(0.1 \mathrm{mmol})$

2 or $4(0.3 \mathrm{mmol})$<smiles>CC(C)=C[C+](C)[C@@H](C)C(=O)N[O-]</smiles><smiles>O=Cc1ccccc1C1=NCCO1</smiles>

7

\section{General Procedure C for Alkene-Alkene Coupling:}

A stirring bar, substrate $6(0.1 \mathrm{mmol}), \mathrm{Cu}(\mathrm{OPiv})_{2}(0.03 \mathrm{mmol}), \mathrm{Ag}_{2} \mathrm{O}(0.2 \mathrm{mmol}), \mathrm{KOPiv}(0.2 \mathrm{mmol})$, and $\mathrm{KHCO}_{3}(0.2 \mathrm{mmol})$ were added to an oven dried $25 \mathrm{~mL}$ Schlenk tube. The tube was capped and back-filled with nitrogen (3 times). Under the nitrogen flow, 2 or $4(0.3 \mathrm{mmol})$ and DMSO $(2 \mathrm{~mL})$ were added to the mixture, and the tube was capped tightly again. Then the reaction mixture was placed into a pre-heated oil bath and stirred at $45{ }^{\circ} \mathrm{C}$. Upon completion (monitored by TLC), the reaction mixture was cooled to room temperature. EtOAc $(20 \mathrm{~mL})$ was added to quench the reaction, and organic layer was washed with $\mathrm{NH}_{3} \cdot \mathrm{H}_{2} \mathrm{O}(20 \mathrm{~mL})$, and the water phase was extracted by EtOAc $(20 \mathrm{~mL})$. The combined organic layers were washed with saturated brine, dried over $\mathrm{Na}_{2} \mathrm{SO}_{4}$, concentrated under vacuum. The crude product was purified by preparative TLC or column chromatography (neutral alumina) to afford the desired product 7 .<smiles>CCC/C=C/C=C(/O)C(=O)Nc1ccccc1C1=NCCO1</smiles>

$7 \mathrm{a}$

(2Z,4E)- $N$-(2-(4,5-Dihydrooxazol-2-yl)phenyl)-2-methylocta-2,4-dienamide (7a)

Substrate 6a was alkenylated with alkenyl boronate (2) following the general procedure $\mathbf{C}(6 \mathrm{~h})$. After purification by preparative thin-layer chromatography (petroleum ether/ethyl acetate $=20 / 1$ ), 7a was obtained as a white solid (15.0 mg, 51\%). mp 87-89 $\left.{ }^{\circ} \mathrm{C} ;{ }^{1} \mathrm{H} \mathrm{NMR} \mathrm{(400} \mathrm{MHz,} \mathrm{CDCl}_{3}\right) \delta 12.29(\mathrm{~s}, 1 \mathrm{H}), 8.87(\mathrm{~d}, J=8.5$ $\mathrm{Hz}, 1 \mathrm{H}), 7.86(\mathrm{~d}, J=7.9 \mathrm{~Hz}, 1 \mathrm{H}), 7.48(\mathrm{t}, J=8.0 \mathrm{~Hz}, 1 \mathrm{H}), 7.08(\mathrm{t}, J=7.6 \mathrm{~Hz}, 1 \mathrm{H}), 6.99-6.90(\mathrm{~m}, 1 \mathrm{H}), 6.27$ (d, $J=11.2 \mathrm{~Hz}, 1 \mathrm{H}), 5.89-5.76(\mathrm{~m}, 1 \mathrm{H}), 4.37(\mathrm{t}, J=9.5 \mathrm{~Hz}, 2 \mathrm{H}), 4.09(\mathrm{t}, J=9.5 \mathrm{~Hz}, 2 \mathrm{H}), 2.14-2.04(\mathrm{~m}, 5 \mathrm{H}), 1.48$ $1.36(\mathrm{~m}, 2 \mathrm{H}), 0.89$ (t, $J=7.4 \mathrm{~Hz}, 3 \mathrm{H}) ;{ }^{13} \mathrm{C} \mathrm{NMR}\left(100 \mathrm{MHz}, \mathrm{CDCl}_{3}\right) \delta 168.1,164.5,140.0,139.3,136.4,132.4$, 129.2, 129.1, 127.5, 122.2, 120.0, 113.4, 66.1, 54.6, 34.9, 22.4, 21.2, 13.7; HRMS (ESI-TOF) $\mathrm{m} / \mathrm{z}$ Calcd for $\mathrm{C}_{18} \mathrm{H}_{23} \mathrm{~N}_{2} \mathrm{O}_{2}[\mathrm{M}+\mathrm{H}]^{+}:$299.1754, found: 299.1758 . 
<smiles>CCC/C=C/C=C(/Cc1ccccc1)C(=O)Nc1ccccc1C1=NCCO1</smiles>

$7 \mathrm{~b}$

(2Z,4E)-2-Benzyl- $N$-(2-(4,5-dihydrooxazol-2-yl)phenyl)octa-2,4-dienamide (7b)

Substrate $\mathbf{6 b}$ was alkenylated with alkenyl boronate (2) following the general procedure $\mathbf{C}(3 \mathrm{~h})$. After purification by preparative thin-layer chromatography (petroleum ether/ethyl acetate $=20 / 1), \mathbf{7 b}$ was obtained as a white solid (14.6 mg, 40\%). mp 67-69 ${ }^{\circ} \mathrm{C}$; ${ }^{1} \mathrm{H}$ NMR (400 MHz, $\left.\mathrm{CDCl}_{3}\right) \delta 12.36(\mathrm{~s}, 1 \mathrm{H}), 8.81(\mathrm{~d}, J=8.5$ $\mathrm{Hz}, 1 \mathrm{H}), 7.84(\mathrm{~d}, J=8.0 \mathrm{~Hz}, 1 \mathrm{H}), 7.45(\mathrm{t}, J=7.9 \mathrm{~Hz}, 1 \mathrm{H}), 7.29-7.24(\mathrm{~m}, 4 \mathrm{H}), 7.21-7.14(\mathrm{~m}, 1 \mathrm{H}), 7.06(\mathrm{t}, J=$ $7.6 \mathrm{~Hz}, 1 \mathrm{H}), 6.82-6.72(\mathrm{~m}, 1 \mathrm{H}), 6.15(\mathrm{~d}, J=11.2 \mathrm{~Hz}, 1 \mathrm{H}), 5.87-5.76(\mathrm{~m}, 1 \mathrm{H}), 4.34(\mathrm{t}, J=9.5 \mathrm{~Hz}, 2 \mathrm{H}), 4.04(\mathrm{t}$, $J=9.5 \mathrm{~Hz}, 2 \mathrm{H}), 3.80(\mathrm{~s}, 2 \mathrm{H}), 2.07(\mathrm{q}, J=7.3 \mathrm{~Hz}, 2 \mathrm{H}), 1.46-1.33(\mathrm{~m}, 2 \mathrm{H}), 0.88(\mathrm{t}, J=7.4 \mathrm{~Hz}, 3 \mathrm{H}) ;{ }^{13} \mathrm{C} \mathrm{NMR}$ $\left(100 \mathrm{MHz}, \mathrm{CDCl}_{3}\right) \delta 167.7,164.3,139.8,139.5,138.9,135.3,134.1,132.4,129.1,128.9,128.4,127.2,126.2$, 122.3, 120.0, 113.3, 66.1, 54.6, 40.5, 34.9, 22.4, 13.7; HRMS (ESI-TOF) $m / z$ Calcd for $\mathrm{C}_{24} \mathrm{H}_{26} \mathrm{~N}_{2} \mathrm{O}_{2} \mathrm{Na}[\mathrm{M}+\mathrm{Na}]^{+}$: 397.1886, found: 397.1881 .<smiles>CCC/C=C/C(=C(/C)C(=O)Nc1ccccc1C1=NCCO1)c1ccccc1</smiles>

(2E,4E)- $N$-(2-(4,5-Dihydrooxazol-2-yl)phenyl)-2-methyl-3-phenylocta-2,4-dienamide (7c)

Substrate 6c was alkenylated with alkenyl boronate (2) following the general procedure $\mathbf{C}(6 \mathrm{~h})$. After purification by preparative thin-layer chromatography (petroleum ether/ethyl acetate $=20 / 1), 7 \mathbf{c}$ was obtained as a white solid $(24.5 \mathrm{mg}, 66 \%)$. $\mathrm{mp} 83-85{ }^{\circ} \mathrm{C} ;{ }^{1} \mathrm{H}$ NMR $\left(400 \mathrm{MHz}, \mathrm{CDCl}_{3}\right) \delta 12.45(\mathrm{~s}, 1 \mathrm{H}), 8.90(\mathrm{~d}, J=8.6$ $\mathrm{Hz}, 1 \mathrm{H}), 7.88(\mathrm{~d}, J=7.9 \mathrm{~Hz}, 1 \mathrm{H}), 7.51(\mathrm{t}, J=7.9 \mathrm{~Hz}, 1 \mathrm{H}), 7.40(\mathrm{t}, J=7.3 \mathrm{~Hz}, 2 \mathrm{H}), 7.32(\mathrm{t}, J=7.4 \mathrm{~Hz}, 1 \mathrm{H})$, $7.17(\mathrm{~d}, J=7.0 \mathrm{~Hz}, 2 \mathrm{H}), 7.11(\mathrm{t}, J=7.6 \mathrm{~Hz}, 1 \mathrm{H}), 6.80(\mathrm{~d}, J=15.4 \mathrm{~Hz}, 1 \mathrm{H}), 5.24-5.15(\mathrm{~m}, 1 \mathrm{H}), 4.37$ (t, $J=9.4$ $\mathrm{Hz}, 2 \mathrm{H}), 4.08(\mathrm{t}, J=9.6 \mathrm{~Hz}, 2 \mathrm{H}), 1.98(\mathrm{q}, J=7.1 \mathrm{~Hz}, 2 \mathrm{H}), 1.84(\mathrm{~s}, 3 \mathrm{H}), 1.33-1.19(\mathrm{~m}, 2 \mathrm{H}), 0.78$ (t, $J=7.4 \mathrm{~Hz}$, $3 \mathrm{H}) ;{ }^{13} \mathrm{C}$ NMR $\left(100 \mathrm{MHz}, \mathrm{CDCl}_{3}\right) \delta 170.2,164.5,141.8,139.9,139.2,136.8,132.5,130.2,130.1,129.3,129.2$, 128.1, 126.9, 122.3, 119.9, 113.4, 66.2, 54.6, 35.1, 22.3, 18.2, 13.6; HRMS (ESI-TOF) $\mathrm{m} / \mathrm{z}$ Calcd for $\mathrm{C}_{24} \mathrm{H}_{27} \mathrm{~N}_{2} \mathrm{O}_{2}[\mathrm{M}+\mathrm{H}]^{+}: 375.2067$, found: 375.2076 .<smiles>CCC/C=C/C1=C(C(=O)Nc2ccccc2C2=NCCO2)CCCC1</smiles>

$7 d$ 


\section{(E)-N-(2-(4,5-Dihydrooxazol-2-yl)phenyl)-2-(pent-1-en-1-yl)cyclohex-1-enecarboxamide (7d)}

Substrate 6d was alkenylated with alkenyl boronate (2) following the general procedure $\mathbf{C}(12 \mathrm{~h})$. After purification by preparative thin-layer chromatography (petroleum ether/ethyl acetate $=30 / 1), \mathbf{7 d}$ was obtained as a white solid (19.1 mg, 57\%). mp 124-126 ${ }^{\circ} \mathrm{C} ;{ }^{1} \mathrm{H}$ NMR $\left(400 \mathrm{MHz}, \mathrm{CDCl}_{3}\right) \delta 12.16(\mathrm{~s}, 1 \mathrm{H}), 8.86(\mathrm{~d}, J=8.4$ $\mathrm{Hz}, 1 \mathrm{H}), 7.86(\mathrm{~d}, J=7.9 \mathrm{~Hz}, 1 \mathrm{H}), 7.48(\mathrm{t}, J=7.9 \mathrm{~Hz}, 1 \mathrm{H}), 7.08(\mathrm{t}, J=7.6 \mathrm{~Hz}, 1 \mathrm{H}), 6.58(\mathrm{~d}, J=15.5 \mathrm{~Hz}, 1 \mathrm{H})$, 5.83-5.73 (m, 1H), $4.35(\mathrm{t}, J=9.5 \mathrm{~Hz}, 2 \mathrm{H}), 4.07(\mathrm{t}, J=9.5 \mathrm{~Hz}, 2 \mathrm{H}), 2.47(\mathrm{~s}, 2 \mathrm{H}), 2.30(\mathrm{~s}, 2 \mathrm{H}), 2.09-2.00(\mathrm{~m}$, 2H), 1.78-1.68 (m, 4H), 1.42-1.32 (m, 2H), $0.86(\mathrm{t}, J=7.4 \mathrm{~Hz}, 3 \mathrm{H}) ;{ }^{13} \mathrm{C}$ NMR $\left(100 \mathrm{MHz}, \mathrm{CDCl}_{3}\right) \delta 170.5$, $164.3,139.9,135.3,132.4,132.3,130.7,129.2,129.1,122.2,119.9,113.3,66.1,54.7,35.2,27.8,25.4,22.7$, 22.4, 22.2, 13.7; HRMS (ESI-TOF) $m / z$ Calcd for $\mathrm{C}_{21} \mathrm{H}_{27} \mathrm{~N}_{2} \mathrm{O}_{2}[\mathrm{M}+\mathrm{H}]^{+}: 339.2067$, found: 339.2075.

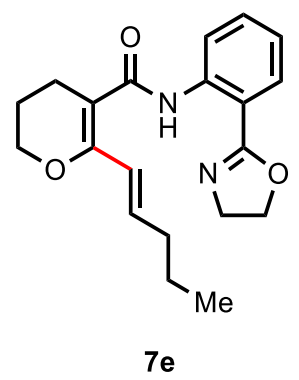

(E)- $N$-(2-(4,5-Dihydrooxazol-2-yl)phenyl)-6-(pent-1-en-1-yl)-3,4-dihydro-2H-pyran-5-carboxamide (7e)

Substrate 6e was alkenylated with alkenyl boronate (2) following the general procedure $\mathbf{C}(3 \mathrm{~h})$. After purification by preparative thin-layer chromatography (petroleum ether/ethyl acetate $=15 / 1), 7$ e was obtained as a white solid (14.8 mg, 44\%). $\mathrm{mp} 101-103{ }^{\circ} \mathrm{C} ;{ }^{1} \mathrm{H}$ NMR $\left(400 \mathrm{MHz}, \mathrm{CDCl}_{3}\right) \delta 12.10(\mathrm{~s}, 1 \mathrm{H}), 8.83(\mathrm{~d}, J=8.4$ $\mathrm{Hz}, 1 \mathrm{H}), 7.85(\mathrm{dd}, J=7.9,1.5 \mathrm{~Hz}, 1 \mathrm{H}), 7.46(\mathrm{t}, J=7.3 \mathrm{~Hz}, 1 \mathrm{H}), 7.05(\mathrm{t}, J=7.6 \mathrm{~Hz}, 1 \mathrm{H}), 6.92(\mathrm{~d}, J=15.4 \mathrm{~Hz}$, $1 \mathrm{H}), 6.37-6.24(\mathrm{~m}, 1 \mathrm{H}), 4.37(\mathrm{t}, J=9.5 \mathrm{~Hz}, 2 \mathrm{H}), 4.14-4.06(\mathrm{~m}, 4 \mathrm{H}), 2.56(\mathrm{t}, J=6.5 \mathrm{~Hz}, 2 \mathrm{H}), 2.12(\mathrm{q}, J=7.2$ $\mathrm{Hz}, 2 \mathrm{H}), 2.01-1.91(\mathrm{~m}, 2 \mathrm{H}), 1.48-1.38(\mathrm{~m}, 2 \mathrm{H}), 0.90(\mathrm{t}, J=7.4 \mathrm{~Hz}, 3 \mathrm{H}) ;{ }^{13} \mathrm{C} \mathrm{NMR}\left(100 \mathrm{MHz}, \mathrm{CDCl}_{3}\right) \delta 168.4$, 164.5, 156.4, 140.4, 134.9, 132.4, 129.1, 123.8, 121.8, 119.9, 113.2, 107.1, 66.1, 65.9, 54.7, 34.9, 22.8, 22.3, 22.0, 13.8; HRMS (ESI-TOF) $m / z$ Calcd for $\mathrm{C}_{20} \mathrm{H}_{24} \mathrm{~N}_{2} \mathrm{O}_{3} \mathrm{Na}[\mathrm{M}+\mathrm{Na}]^{+}: 363.1679$, found: 363.1687.<smiles>CCC/C=C/C1=C(C(=O)Nc2ccccc2C2=NCCO2)CCOC1</smiles>

(E)- $N$-(2-(4,5-Dihydrooxazol-2-yl)phenyl)-4-(pent-1-en-1-yl)-5,6-dihydro-2H-pyran-3-carboxamide (7f)

Substrate $\mathbf{6 f}$ was alkenylated with alkenyl boronate (2) following the general procedure $\mathbf{C}(6 \mathrm{~h})$. After purification by preparative thin-layer chromatography (petroleum ether/ethyl acetate $=15 / 1)$, $7 \mathbf{f}$ was obtained as a white solid (14.4 mg, 43\%). mp 95-97 ${ }^{\circ} \mathrm{C} ;{ }^{1} \mathrm{H}$ NMR $\left(400 \mathrm{MHz}, \mathrm{CDCl}_{3}\right) \delta 12.33(\mathrm{~s}, 1 \mathrm{H}), 8.83(\mathrm{~d}, J=8.4$ $\mathrm{Hz}, 1 \mathrm{H}), 7.87(\mathrm{dd}, J=7.9,1.3 \mathrm{~Hz}, 1 \mathrm{H}), 7.48(\mathrm{t}, J=8.0 \mathrm{~Hz}, 1 \mathrm{H}), 7.10(\mathrm{t}, J=8.0 \mathrm{~Hz}, 1 \mathrm{H}), 6.73(\mathrm{~d}, J=15.6 \mathrm{~Hz}$, $1 \mathrm{H}), 5.93-5.77(\mathrm{~m}, 1 \mathrm{H}), 4.52(\mathrm{~s}, 2 \mathrm{H}), 4.36(\mathrm{t}, J=9.5 \mathrm{~Hz}, 2 \mathrm{H}), 4.07(\mathrm{t}, J=9.5 \mathrm{~Hz}, 2 \mathrm{H}), 3.88(\mathrm{t}, J=5.6 \mathrm{~Hz}, 2 \mathrm{H})$, 2.44-2.38 (m, 2H), 2.09 (q, $J=7.2 \mathrm{~Hz}, 2 \mathrm{H}), 1.46-1.34$ (m, 2H), 0.88 (t, $J=7.4 \mathrm{~Hz}, 3 \mathrm{H}) ;{ }^{13} \mathrm{C}$ NMR $(100 \mathrm{MHz}$, $\left.\mathrm{CDCl}_{3}\right) \delta 166.6,164.4,139.6,134.4,132.6,132.5,130.2,129.2,127.9,122.5,120.0,113.4,66.3,66.2,64.2$, 
54.6, 35.1, 25.2, 22.5, 13.7; HRMS (ESI-TOF) $\mathrm{m} / z$ Calcd for $\mathrm{C}_{20} \mathrm{H}_{24} \mathrm{~N}_{2} \mathrm{O}_{3} \mathrm{Na}[\mathrm{M}+\mathrm{Na}]^{+}: 363.1679$, found: 363.1677 .

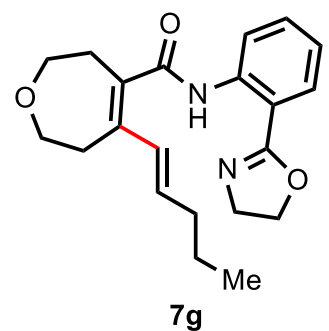

(E)-N-(2-(4,5-Dihydrooxazol-2-yl)phenyl)-5-(pent-1-en-1-yl)-2,3,6,7-tetrahydrooxepine-4-carboxamide (7g)

Substrate $\mathbf{6 g}$ was alkenylated with alkenyl boronate (2) following the general procedure $\mathbf{C}(6 \mathrm{~h})$. After purification by preparative thin-layer chromatography (petroleum ether/ethyl acetate $=10 / 1), 7 \mathrm{~g}$ was obtained as a white solid (10.5 mg, 30\%). mp 97-99 ${ }^{\circ} \mathrm{C}$; ${ }^{1} \mathrm{H}$ NMR (400 MHz, $\left.\mathrm{CDCl}_{3}\right) \delta 12.27(\mathrm{~s}, 1 \mathrm{H}), 8.82(\mathrm{~d}, J=8.4$ $\mathrm{Hz}, 1 \mathrm{H}), 7.86(\mathrm{dd}, J=7.9,1.3 \mathrm{~Hz}, 1 \mathrm{H}), 7.49(\mathrm{t}, J=7.9 \mathrm{~Hz}, 1 \mathrm{H}), 7.10(\mathrm{t}, J=7.6 \mathrm{~Hz}, 1 \mathrm{H}), 6.54(\mathrm{~d}, J=15.6 \mathrm{~Hz}$, $1 \mathrm{H}), 5.85-5.72(\mathrm{~m}, 1 \mathrm{H}), 4.37(\mathrm{t}, J=9.5 \mathrm{~Hz}, 2 \mathrm{H}), 4.06(\mathrm{t}, J=9.4 \mathrm{~Hz}, 2 \mathrm{H}), 3.83-3.79(\mathrm{~m}, 2 \mathrm{H}), 3.78-3.71(\mathrm{~m}$, $2 \mathrm{H}), 2.83-2.76(\mathrm{~m}, 2 \mathrm{H}), 2.74-2.68(\mathrm{~m}, 2 \mathrm{H}), 2.06(\mathrm{q}, J=7.4 \mathrm{~Hz}, 2 \mathrm{H}), 1.41-1.31(\mathrm{~m}, 2 \mathrm{H}), 0.86(\mathrm{t}, J=7.4 \mathrm{~Hz}$, $3 \mathrm{H}) ;{ }^{13} \mathrm{C}$ NMR $\left(100 \mathrm{MHz}, \mathrm{CDCl}_{3}\right) \delta 170.2,164.5,141.7,139.9,136.2,132.5,132.5,129.2,129.0,122.3,119.7$, 113.3, 69.3, 68.5, 66.2, 54.6, 35.2, 34.9, 32.4, 22.6, 13.7; HRMS (ESI-TOF) $m / z$ Calcd for $\mathrm{C}_{21} \mathrm{H}_{26} \mathrm{~N}_{2} \mathrm{O}_{3} \mathrm{Na}$ $[\mathrm{M}+\mathrm{Na}]^{+}: 377.1836$, found: 377.1842 .<smiles>CCC/C=C/C1=C(C(=O)Nc2ccccc2C2=NCCO2)CCN(Cc2ccccc2)C1</smiles>

$7 \mathrm{~h}$

(E)-1-Benzyl- $N$-(2-(4,5-dihydrooxazol-2-yl)phenyl)-5-(pent-1-en-1-yl)-1,2,3,6-tetrahydropyridine-4carboxamide (7h)

Substrate $\mathbf{6 h}$ was alkenylated with alkenyl boronate (2) following the general procedure $\mathbf{C}(6 \mathrm{~h})$. After purification by preparative thin-layer chromatography (petroleum ether/ethyl acetate $=10 / 1), \mathbf{7 h}$ was obtained as a white solid (27.3 mg, 64\%). mp 85-87 ${ }^{\circ} \mathrm{C} ;{ }^{1} \mathrm{H}$ NMR $\left(400 \mathrm{MHz}, \mathrm{CDCl}_{3}\right) \delta 12.25(\mathrm{~s}, 1 \mathrm{H}), 8.86(\mathrm{~d}, J=8.4$ $\mathrm{Hz}, 1 \mathrm{H}), 7.86(\mathrm{~d}, J=8.0 \mathrm{~Hz}, 1 \mathrm{H}), 7.47(\mathrm{t}, J=7.3 \mathrm{~Hz}, 1 \mathrm{H}), 7.42-7.32(\mathrm{~m}, 4 \mathrm{H}), 7.31-7.25(\mathrm{~m}, 1 \mathrm{H}), 7.08(\mathrm{t}, J=$ $7.6 \mathrm{~Hz}, 1 \mathrm{H}), 6.65(\mathrm{~d}, J=15.8 \mathrm{~Hz}, 1 \mathrm{H}), 5.75-5.62(\mathrm{~m}, 1 \mathrm{H}), 4.34(\mathrm{t}, J=9.5 \mathrm{~Hz}, 2 \mathrm{H}), 4.06(\mathrm{t}, J=9.5 \mathrm{~Hz}, 2 \mathrm{H})$, $3.68(\mathrm{~s}, 2 \mathrm{H}), 3.30(\mathrm{~s}, 2 \mathrm{H}), 2.68-2.58(\mathrm{~m}, 4 \mathrm{H}), 2.03(\mathrm{q}, J=7.1 \mathrm{~Hz}, 2 \mathrm{H}), 1.42-1.29(\mathrm{~m}, 2 \mathrm{H}), 0.85(\mathrm{t}, J=7.4 \mathrm{~Hz}$, $3 \mathrm{H}) ;{ }^{13} \mathrm{C} \mathrm{NMR}\left(100 \mathrm{MHz}, \mathrm{CDCl}_{3}\right) \delta 168.8,164.3,139.8,138.0,134.6,132.4,131.1,129.7,129.2,129.1,128.3$, 127.1, 127.0, 122.3, 120.0, 113.4, 66.1, 62.5, 54.7, 53.2, 49.1, 35.3, 27.9, 22.5, 13.7; HRMS (ESI-TOF) $\mathrm{m} / \mathrm{z}$ Calcd for $\mathrm{C}_{27} \mathrm{H}_{32} \mathrm{~N}_{3} \mathrm{O}_{2}[\mathrm{M}+\mathrm{H}]^{+}: 430.2489$, found: 430.2492 . 
<smiles>CCC/C=C/C1=C(C(=O)Nc2ccccc2C2=NCCO2)CN(C)CC1</smiles>

$7 \mathbf{i}$

(E)- $N$-(2-(4,5-Dihydrooxazol-2-yl)phenyl)-1-methyl-4-(pent-1-en-1-yl)-1,2,5,6-tetrahydropyridine-3carboxamide (7i)

Substrate 6i was alkenylated with alkenyl boronate (2) following the general procedure $\mathbf{C}(6 \mathrm{~h})$. After purification by column chromatography (neutral alumina, petroleum ether/ethyl acetate $=1 / 1$ ), $7 \mathbf{i}$ was obtained as a white solid (27.6 mg, 78\%). mp 111-113 ${ }^{\circ} \mathrm{C} ;{ }^{1} \mathrm{H}$ NMR $\left(400 \mathrm{MHz}, \mathrm{CDCl}_{3}\right) \delta 12.32(\mathrm{~s}, 1 \mathrm{H}), 8.85(\mathrm{~d}, J=8.5$ $\mathrm{Hz}, 1 \mathrm{H}), 7.86(\mathrm{~d}, J=7.9 \mathrm{~Hz}, 1 \mathrm{H}), 7.48$ (t, $J=8.0 \mathrm{~Hz}, 1 \mathrm{H}), 7.09$ (t, $J=8.0 \mathrm{~Hz}, 1 \mathrm{H}), 6.68$ (d, $J=15.8 \mathrm{~Hz}, 1 \mathrm{H})$, 5.89-5.78 (m, 1H), $4.35(\mathrm{t}, J=9.4 \mathrm{~Hz}, 2 \mathrm{H}), 4.04(\mathrm{t}, J=9.5 \mathrm{~Hz}, 2 \mathrm{H}), 3.35(\mathrm{~s}, 2 \mathrm{H}), 2.65-2.60(\mathrm{~m}, 2 \mathrm{H}), 2.50-2.44$ $(\mathrm{m}, 2 \mathrm{H}), 2.42(\mathrm{~s}, 3 \mathrm{H}), 2.10-2.00(\mathrm{~m}, 2 \mathrm{H}), 1.44-1.31(\mathrm{~m}, 2 \mathrm{H}), 0.86(\mathrm{t}, J=7.4 \mathrm{~Hz}, 3 \mathrm{H}) ;{ }^{13} \mathrm{C}$ NMR $(100 \mathrm{MHz}$, $\left.\mathrm{CDCl}_{3}\right) \delta 167.9,164.3,139.7,134.2,132.4,132.1,129.7,129.1,128.2,122.4,119.9,113.4,66.1,55.9,54.6$, 51.5, 45.5, 35.1, 26.3, 22.6, 13.7; HRMS (ESI-TOF) $m / z$ Calcd for $\mathrm{C}_{21} \mathrm{H}_{27} \mathrm{~N}_{3} \mathrm{O}_{2} \mathrm{Na}[\mathrm{M}+\mathrm{Na}]^{+}: 376.1995$, found: 376.1994 .<smiles>C=C(NC(=O)c1ccccc1C1=NCCO1)/C(C)=C(/C)c1ccccc1</smiles>

$7 \mathbf{j}$

(E)-N-(2-(4,5-Dihydrooxazol-2-yl)phenyl)-2,4-dimethyl-3-phenylpenta-2,4-dienamide (7j)

Substrate 6c was alkenylated with alkenyl boronate (4l) following the general procedure $\mathbf{C}(6 \mathrm{~h})$. After purification by preparative thin-layer chromatography (petroleum ether/ethyl acetate $=20 / 1), 7 \mathbf{j}$ was obtained as a white solid (10.4 mg, 30\%). mp $117-119^{\circ} \mathrm{C} ;{ }^{1} \mathrm{H}$ NMR $\left(400 \mathrm{MHz}, \mathrm{CDCl}_{3}\right) \delta 12.23(\mathrm{~s}, 1 \mathrm{H}), 8.83(\mathrm{~d}, J=8.3$ $\mathrm{Hz}, 1 \mathrm{H}), 7.88(\mathrm{~d}, J=8.0 \mathrm{~Hz}, 1 \mathrm{H}), 7.48(\mathrm{t}, J=7.9 \mathrm{~Hz}, 1 \mathrm{H}), 7.38(\mathrm{t}, J=7.2 \mathrm{~Hz}, 2 \mathrm{H}), 7.34-7.23(\mathrm{~m}, 3 \mathrm{H}), 7.08(\mathrm{t}$, $J=7.6 \mathrm{~Hz}, 1 \mathrm{H}), 5.13(\mathrm{~s}, 1 \mathrm{H}), 4.92(\mathrm{~s}, 1 \mathrm{H}), 4.37$ (t, $J=9.5 \mathrm{~Hz}, 2 \mathrm{H}), 4.11(\mathrm{t}, J=9.4 \mathrm{~Hz}, 2 \mathrm{H}), 1.96(\mathrm{~s}, 3 \mathrm{H}), 1.68$ $(\mathrm{s}, 3 \mathrm{H}) ;{ }^{13} \mathrm{C} \mathrm{NMR}\left(100 \mathrm{MHz}, \mathrm{CDCl}_{3}\right) \delta 171.3,164.5,145.2,144.4,140.3,138.7,132.6,131.6,129.2,128.9$, 128.2, 127.4, 122.1, 119.6, 116.1, 113.1, 66.1, 54.7, 22.0, 17.9; HRMS (ESI-TOF) $m / z$ Calcd for $\mathrm{C}_{22} \mathrm{H}_{22} \mathrm{~N}_{2} \mathrm{O}_{2} \mathrm{Na}$ $[\mathrm{M}+\mathrm{Na}]^{+}:$369.1573, found: 369.1576 .<smiles>CC(C)=CC(=C(C)C(=O)Nc1ccccc1C1=NCCO1)c1ccccc1</smiles>

$7 \mathrm{k}$

(E)- $N$-(2-(4,5-Dihydrooxazol-2-yl)phenyl)-2,5-dimethyl-3-phenylhexa-2,4-dienamide (7k) 
Substrate $\mathbf{6 c}$ was alkenylated with alkenyl boronate (4m) following the general procedure $\mathbf{C}(6 \mathrm{~h})$. After purification by preparative thin-layer chromatography (petroleum ether/ethyl acetate $=20 / 1$ ), $7 \mathbf{k}$ was obtained as a white solid (23.1 mg, 64\%). mp 82-84 ${ }^{\circ} \mathrm{C} ;{ }^{1} \mathrm{H}$ NMR $\left(400 \mathrm{MHz}, \mathrm{CDCl}_{3}\right) \delta 12.28(\mathrm{~s}, 1 \mathrm{H}), 8.87(\mathrm{~d}, J=8.4$ $\mathrm{Hz}, 1 \mathrm{H}), 7.86(\mathrm{~d}, J=7.9 \mathrm{~Hz}, 1 \mathrm{H}), 7.48(\mathrm{t}, J=7.9 \mathrm{~Hz}, 1 \mathrm{H}), 7.37(\mathrm{t}, J=7.6 \mathrm{~Hz}, 2 \mathrm{H}), 7.32-7.22(\mathrm{~m}, 3 \mathrm{H}), 7.07$ (t, $J=7.6 \mathrm{~Hz}, 1 \mathrm{H}), 6.04(\mathrm{~s}, 1 \mathrm{H}), 4.36(\mathrm{t}, J=9.5 \mathrm{~Hz}, 2 \mathrm{H}), 4.07(\mathrm{t}, J=9.5 \mathrm{~Hz}, 2 \mathrm{H}), 2.01(\mathrm{~s}, 3 \mathrm{H}), 1.65(\mathrm{~s}, 3 \mathrm{H}), 1.41$ $(\mathrm{s}, 3 \mathrm{H}) ;{ }^{13} \mathrm{C}$ NMR $\left(100 \mathrm{MHz}, \mathrm{CDCl}_{3}\right) \delta 170.8,164.3,141.0,140.1,140.0,137.3,132.5,132.3,129.1,128.1$, 127.0, 125.2, 122.1, 119.7, 113.3, 66.1, 54.7, 26.4, 19.7, 17.8; HRMS (ESI-TOF) $m / z$ Calcd for $\mathrm{C}_{23} \mathrm{H}_{24} \mathrm{~N}_{2} \mathrm{O}_{2} \mathrm{Na}$ $[\mathrm{M}+\mathrm{Na}]^{+}: 383.1730$, found: 383.1716 .<smiles>O=C(Nc1ccccc1C1=NCCO1)C1=C(C2=CCOCC2)OCCC1</smiles>

7I

$N$-(2-(4,5-Dihydrooxazol-2-yl)phenyl)-3',5,6,6'-tetrahydro-2' $H, 4 H$-[2,4'-bipyran]-3-carboxamide (7l)

Substrate 6e was alkenylated with alkenyl boronate (4u) following the general procedure $\mathbf{C}(3 \mathrm{~h})$. After purification by column chromatography (neutral alumina, petroleum ether/ethyl acetate $=10 / 1), 7 \mathbf{l}$ was obtained as a white solid $(22.8 \mathrm{mg}, 64 \%) . \mathrm{mp} 99-101{ }^{\circ} \mathrm{C} ;{ }^{1} \mathrm{H}$ NMR $\left(400 \mathrm{MHz}, \mathrm{CDCl}_{3}\right) \delta 11.98(\mathrm{~s}, 1 \mathrm{H}), 8.76(\mathrm{~d}, J=8.4$ $\mathrm{Hz}, 1 \mathrm{H}), 7.84(\mathrm{~d}, J=7.8 \mathrm{~Hz}, 1 \mathrm{H}), 7.44(\mathrm{t}, J=7.6 \mathrm{~Hz}, 1 \mathrm{H}), 7.04(\mathrm{t}, J=7.6 \mathrm{~Hz}, 1 \mathrm{H}), 6.00(\mathrm{~s}, 1 \mathrm{H}), 4.37(\mathrm{t}, J=9.5$ $\mathrm{Hz}, 2 \mathrm{H}), 4.14-4.00(\mathrm{~m}, 6 \mathrm{H}), 3.69(\mathrm{t}, J=5.3 \mathrm{~Hz}, 2 \mathrm{H}), 2.50(\mathrm{t}, J=6.5 \mathrm{~Hz}, 2 \mathrm{H}), 2.33(\mathrm{~s}, 2 \mathrm{H}), 1.97-1.86(\mathrm{~m}, 2 \mathrm{H})$; ${ }^{13} \mathrm{C} \mathrm{NMR}\left(100 \mathrm{MHz}, \mathrm{CDCl}_{3}\right) \delta 169.4,164.5,157.0,140.2,132.5,131.4,129.3,128.3,121.9,119.4,113.0,109.0$, 66.4, 66.0, 65.3, 64.0, 54.8, 26.6, 22.7, 21.9; HRMS (ESI-TOF) $m / z$ Calcd for $\mathrm{C}_{20} \mathrm{H}_{22} \mathrm{~N}_{2} \mathrm{O}_{4} \mathrm{Na}[\mathrm{M}+\mathrm{Na}]^{+}$: 377.1472, found: 377.1471 .<smiles>O=C(Nc1ccccc1C1=NCCO1)C1=C(C2=CCOCC2)CCOC1</smiles>

$7 \mathrm{~m}$

$N$-(2-(4,5-Dihydrooxazol-2-yl)phenyl)-3',5,6,6'-tetrahydro-2H,2' $H$-[4,4'-bipyran]-3-carboxamide (7m)

Substrate $\mathbf{6 f}$ was alkenylated with alkenyl boronate $(\mathbf{4 u})$ following the general procedure $\mathbf{C}(4.5 \mathrm{~h})$. After purification by column chromatography (neutral alumina, petroleum ether/ethyl acetate $=10 / 1$ ), $7 \mathbf{m}$ was obtained as a white solid (17.3 mg, 49\%). mp 95-97 ${ }^{\circ} \mathrm{C} ;{ }^{1} \mathrm{H}$ NMR $\left(400 \mathrm{MHz}, \mathrm{CDCl}_{3}\right) \delta 12.10(\mathrm{~s}, 1 \mathrm{H}), 8.74(\mathrm{~d}$, $J=8.4 \mathrm{~Hz}, 1 \mathrm{H}), 7.86(\mathrm{dd}, J=7.9,1.4 \mathrm{~Hz}, 1 \mathrm{H}), 7.45(\mathrm{t}, J=7.9 \mathrm{~Hz}, 1 \mathrm{H}), 7.08(\mathrm{t}, J=8.0 \mathrm{~Hz}, 1 \mathrm{H}), 5.74(\mathrm{~s}, 1 \mathrm{H})$, $4.45(\mathrm{~s}, 2 \mathrm{H}), 4.37$ (t, $J=9.4 \mathrm{~Hz}, 2 \mathrm{H}), 4.10-4.01(\mathrm{~m}, 4 \mathrm{H}), 3.87(\mathrm{t}, J=5.5 \mathrm{~Hz}, 2 \mathrm{H}), 3.68(\mathrm{t}, J=5.4 \mathrm{~Hz}, 2 \mathrm{H}), 2.35$ $2.29(\mathrm{~m}, 2 \mathrm{H}), 2.28-2.23(\mathrm{~m}, 2 \mathrm{H}) ;{ }^{13} \mathrm{C} \mathrm{NMR}\left(100 \mathrm{MHz}, \mathrm{CDCl}_{3}\right) \delta 167.5,164.5,139.6,137.4,134.6,132.6,130.8$, 129.3, 124.4, 122.5, 119.6, 113.2, 66.2, 66.1, 65.4, 64.2, 64.0, 54.7, 27.8, 27.2; HRMS (ESI-TOF) $\mathrm{m} / z$ Calcd for $\mathrm{C}_{20} \mathrm{H}_{22} \mathrm{~N}_{2} \mathrm{O}_{4} \mathrm{Na}[\mathrm{M}+\mathrm{Na}]^{+}: 377.1472$, found: 377.1469 . 
<smiles>CC(C)(C)OC(=O)N1CCC=C(C2=C(C(=O)Nc3ccccc3C3=NCCO3)COCC2)C1</smiles>

tert-Butyl 3-(5-((2-(4,5-dihydrooxazol-2-yl)phenyl)carbamoyl)-3,6-dihydro-2H-pyran-4-yl)-5,6-dihydropyridine-1 $(2 H)$-carboxylate $(7 n)$

Substrate $\mathbf{6 f}$ was alkenylated with alkenyl boronate $(\mathbf{4 x})$ following the general procedure $\mathbf{C}(4.5 \mathrm{~h})$. After purification by column chromatography (neutral alumina, petroleum ether/ethyl acetate $=10 / 1$ ), $7 \mathbf{n}$ was obtained as a white solid $(32.0 \mathrm{mg}, 71 \%)$, rotameric mixture. $\mathrm{mp} 73-75{ }^{\circ} \mathrm{C} ;{ }^{1} \mathrm{H} \mathrm{NMR}\left(400 \mathrm{MHz}, \mathrm{CDCl}_{3}\right) \delta 12.11$ $(\mathrm{s}, 1 \mathrm{H}), 8.75(\mathrm{~d}, J=8.5 \mathrm{~Hz}, 1 \mathrm{H}), 7.84(\mathrm{~d}, J=7.9 \mathrm{~Hz}, 1 \mathrm{H}), 7.44$ (t, $J=7.8 \mathrm{~Hz}, 1 \mathrm{H}), 7.07$ (t, $J=7.6 \mathrm{~Hz}, 1 \mathrm{H}), 5.86$ (s, 1H), 4.46 (s, 2H), 4.36 (t, J=9.4 Hz, 2H), 4.11 (brs, 2H), 3.99 (brs, 2H), 3.87 (t, $J=5.4 \mathrm{~Hz}, 2 \mathrm{H}), 3.26$ (s, 2H), 2.32 (s, 2H), 2.00 (brs, 2H), 1.42 (s, 9H); ${ }^{13} \mathrm{C} \mathrm{NMR}\left(125 \mathrm{MHz}, \mathrm{CDCl}_{3}\right) \delta$ 167.5, 167.0, 164.2, 154.8, 139.6, 136.2, 135.0, 132.5, 132.2, 129.2, 124.3, 122.4, 119.5, 113.0, 79.6, 66.1, 64.1, 54.6, 44.7, 43.9, 40.3, 39.0, 28.8, 28.4, 27.9, 25.1; HRMS (ESI-TOF) $m / z$ Calcd for $\mathrm{C}_{25} \mathrm{H}_{31} \mathrm{~N}_{3} \mathrm{O}_{5} \mathrm{Na}[\mathrm{M}+\mathrm{Na}]^{+}: 476.2156$, found: 476.2155 .<smiles>CC(C)(C)OC(=O)N1CC=C(C2=C(C(=O)Nc3ccccc3C3=NCCO3)COCC2)CC1</smiles>

tert-Butyl 4-(5-((2-(4,5-dihydrooxazol-2-yl)phenyl)carbamoyl)-3,6-dihydro-2H-pyran-4-yl)-5,6-dihydropyridine-1(2H)-carboxylate (7o)

Substrate $6 \mathbf{f}$ was alkenylated with alkenyl boronate (4w) following the general procedure $\mathbf{C}(6 \mathrm{~h})$. After purification by column chromatography (neutral alumina, petroleum ether/ethyl acetate $=10 / 1), 7 \mathbf{o}$ was obtained as a white solid $(23.3 \mathrm{mg}, 52 \%)$, rotameric mixture. mp $73-75^{\circ} \mathrm{C} ;{ }^{1} \mathrm{H} \mathrm{NMR}\left(400 \mathrm{MHz}, \mathrm{CDCl}_{3}\right) \delta 12.06$ (brs, $1 \mathrm{H}), 8.75(\mathrm{~d}, J=8.3 \mathrm{~Hz}, 1 \mathrm{H}), 7.85(\mathrm{~d}, J=7.9 \mathrm{~Hz}, 1 \mathrm{H}), 7.45(\mathrm{t}, J=7.9 \mathrm{~Hz}, 1 \mathrm{H}), 7.08(\mathrm{t}, J=7.6 \mathrm{~Hz}, 1 \mathrm{H}), 5.67$ (brs, 1H), 4.45 (s, 2H), 4.37 (t, $J=9.5 \mathrm{~Hz}, 2 \mathrm{H}), 4.07$ (t, $J=9.4 \mathrm{~Hz}, 2 \mathrm{H}), 3.87$ (t, $J=5.5 \mathrm{~Hz}, 2 \mathrm{H}), 3.84-3.70$ (s, 2H), 3.40 (brs, 2H), 2.36-2.18 (m, 4H), $1.38(\mathrm{~s}, 9 \mathrm{H}) ;{ }^{13} \mathrm{C} \mathrm{NMR}\left(125 \mathrm{MHz}, \mathrm{CDCl}_{3}\right) \delta 167.5,164.4,154.7,139.6$, 137.9, 135.6, 132.6, 130.8, 129.3, 122.9, 122.4, 119.5, 113.0, 79.6, 66.1, 66.1, 64.1, 54.7, 43.6, 42.9, 40.7, 39.2, 28.3, 27.6, 27.0; HRMS (ESI-TOF) $m / z$ Calcd for $\mathrm{C}_{25} \mathrm{H}_{31} \mathrm{~N}_{3} \mathrm{O}_{5} \mathrm{Na}[\mathrm{M}+\mathrm{Na}]^{+}: 476.2156$, found: 476.2158. 
<smiles>CN1CCC(C2=CCCCO2)=C(C(=O)Nc2ccccc2C2=NCCO2)C1</smiles>

$7 p$

4-(3,4-Dihydro-2H-pyran-6-yl)- $N$-(2-(4,5-dihydrooxazol-2-yl)phenyl)-1-methyl-1,2,5,6tetrahydropyridine-3-carboxamide (7p)

Substrate 6i was alkenylated with alkenyl boronate $(4 \mathbf{y})$ following the general procedure $\mathbf{C}(5 \mathrm{~h})$. After purification by column chromatography (neutral alumina, petroleum ether/ethyl acetate $=1.5 / 1$ ), $7 \mathbf{p}$ was obtained as a white solid (24.3 mg, 67\%). mp 68-70 ${ }^{\circ} \mathrm{C} ;{ }^{1} \mathrm{H}$ NMR $\left(400 \mathrm{MHz}, \mathrm{CDCl}_{3}\right) \delta 12.07(\mathrm{~s}, 1 \mathrm{H}), 8.78(\mathrm{~d}$, $J=8.6 \mathrm{~Hz}, 1 \mathrm{H}), 7.83(\mathrm{dd}, J=7.9,1.5 \mathrm{~Hz}, 1 \mathrm{H}), 7.44(\mathrm{t}, J=8.0 \mathrm{~Hz}, 1 \mathrm{H}), 7.05(\mathrm{t}, J=7.6 \mathrm{~Hz}, 1 \mathrm{H}), 4.90(\mathrm{t}, J=3.9$ $\mathrm{Hz}, 1 \mathrm{H}), 4.35(\mathrm{t}, J=9.5 \mathrm{~Hz}, 2 \mathrm{H}), 4.07(\mathrm{t}, J=9.5 \mathrm{~Hz}, 2 \mathrm{H}), 3.74(\mathrm{t}, J=8.0 \mathrm{~Hz}, 2 \mathrm{H}), 3.30(\mathrm{~s}, 2 \mathrm{H}), 2.60(\mathrm{t}, J=5.7$ $\mathrm{Hz}, 2 \mathrm{H}), 2.43-2.37(\mathrm{~m}, 5 \mathrm{H}), 2.00-1.92(\mathrm{~m}, 2 \mathrm{H}), 1.69-1.60(\mathrm{~m}, 2 \mathrm{H}) ;{ }^{13} \mathrm{C}$ NMR $\left(100 \mathrm{MHz}, \mathrm{CDCl}_{3}\right) \delta 169.4$, 164.0, 151.7, 140.0, 132.4, 131.3, 130.5, 129.0, 121.9, 119.7, 113.0, 100.0, 66.1, 65.9, 55.7, 54.7, 51.5, 45.3, 27.3, 22.0, 20.7; HRMS (ESI-TOF) $m / z$ Calcd for $\mathrm{C}_{21} \mathrm{H}_{26} \mathrm{~N}_{3} \mathrm{O}_{3}[\mathrm{M}+\mathrm{H}]^{+}: 368.1969$, found: 368.1966.<smiles>CN1CCC(C2=C(C(=O)Nc3ccccc3C3=NCCO3)C3=C(COCC3)C2)=C(C2=CCOCC2)C1</smiles>

$7 q$

4-(3,6-Dihydro-2H-pyran-4-yl)- $N$-(2-(4,5-dihydrooxazol-2-yl)phenyl)-1-methyl-1,2,5,6tetrahydropyridine-3-carboxamide (7q)

Substrate $\mathbf{6} \mathbf{i}$ was alkenylated with alkenyl boronate $(\mathbf{4 u})$ following the general procedure $\mathbf{C}(5 \mathrm{~h})$. After purification by column chromatography (neutral alumina, petroleum ether/ethyl acetate $=1 / 1), \mathbf{7 q}$ was obtained as a white solid (22.8 mg, 63\%). mp 82-84 ${ }^{\circ} \mathrm{C} ;{ }^{1} \mathrm{H}$ NMR $\left(400 \mathrm{MHz}, \mathrm{CDCl}_{3}\right) \delta 12.04(\mathrm{~s}, 1 \mathrm{H}), 8.76(\mathrm{~d}, J=8.4$ $\mathrm{Hz}, 1 \mathrm{H}), 7.85(\mathrm{~d}, J=7.9 \mathrm{~Hz}, 1 \mathrm{H}), 7.45(\mathrm{t}, J=7.9 \mathrm{~Hz}, 1 \mathrm{H}), 7.07(\mathrm{t}, J=7.6 \mathrm{~Hz}, 1 \mathrm{H}), 5.69(\mathrm{~s}, 1 \mathrm{H}), 4.36(\mathrm{t}, J=9.5$ $\mathrm{Hz}, 2 \mathrm{H}), 4.09-3.97(\mathrm{~m}, 4 \mathrm{H}), 3.66(\mathrm{t}, J=5.3 \mathrm{~Hz}, 2 \mathrm{H}), 3.30(\mathrm{~s}, 2 \mathrm{H}), 2.61(\mathrm{t}, J=5.7 \mathrm{~Hz}, 2 \mathrm{H}), 2.45-2.34(\mathrm{~m}, 5 \mathrm{H})$, $2.24(\mathrm{~s}, 2 \mathrm{H}) ;{ }^{13} \mathrm{C} \mathrm{NMR}\left(100 \mathrm{MHz}, \mathrm{CDCl}_{3}\right) \delta 168.5,164.3,139.8,138.1,135.0,132.5,129.8,129.3,123.8,122.3$, 119.6, 113.1, 66.0, 65.3, 64.0, 55.3, 54.7, 51.5, 45.4, 29.2, 27.4; HRMS (ESI-TOF) $m / z$ Calcd for $\mathrm{C}_{21} \mathrm{H}_{26} \mathrm{~N}_{3} \mathrm{O}_{3}$ $[\mathrm{M}+\mathrm{H}]^{+}: 368.1969$, found: 368.1968 . 
<smiles>CN(C)N1CCC(C2=CCCN(C(=O)OC(C)(C)C)C2)=C(C(=O)Nc2ccccc2C2=NCCO2)C1</smiles>

tert-Butyl 5'-((2-(4,5-dihydrooxazol-2-yl)phenyl)carbamoyl)-1'-methyl-1',2',3',5,6,6'-hexahydro-[3,4'bipyridine]-1(2H)-carboxylate (7r)

Substrate 6i was alkenylated with alkenyl boronate $(\mathbf{4 x})$ following the general procedure $\mathbf{C}(5 \mathrm{~h})$. After purification by column chromatography (neutral alumina, petroleum ether/ethyl acetate $=1 / 1$ ), $7 \mathbf{r}$ was obtained as a white solid $(44.0 \mathrm{mg}, 87 \%)$, rotameric mixture. $\mathrm{mp} 78-80{ }^{\circ} \mathrm{C}$; ${ }^{1} \mathrm{H} \mathrm{NMR}\left(400 \mathrm{MHz}, \mathrm{CDCl}_{3}\right) \delta 12.05(\mathrm{~s}, 1 \mathrm{H})$, $8.77(\mathrm{~d}, J=8.4 \mathrm{~Hz}, 1 \mathrm{H}), 7.83(\mathrm{~d}, J=7.8 \mathrm{~Hz}, 1 \mathrm{H}), 7.44(\mathrm{t}, J=7.8 \mathrm{~Hz}, 1 \mathrm{H}), 7.06(\mathrm{t}, J=7.6 \mathrm{~Hz}, 1 \mathrm{H}), 5.82(\mathrm{~s}, 1 \mathrm{H})$, $4.34(\mathrm{t}, J=9.5 \mathrm{~Hz}, 2 \mathrm{H}), 4.10$ (brs, 2H), 3.98 (brs, 2H), 3.30 (s, 2H), 3.23 (s, 2H), 2.61 (t, $J=5.6 \mathrm{~Hz}, 2 \mathrm{H}), 2.46-$ $2.36(\mathrm{~m}, 5 \mathrm{H}), 1.96$ (brs, 2H), 1.42 (s, 9H); ${ }^{13} \mathrm{C} \mathrm{NMR}\left(125 \mathrm{MHz}, \mathrm{CDCl}_{3}\right) \delta$ 168.6, 168.0, 164.1, 154.8, 139.7, 136.6, 135.4, 132.4 131.3, 129.2, 123.8, 122.2, 119.5, 113.0, 79.5, 66.1, 55.3, 54.6, 51.5, 45.4, 44.9, 44.0, 40.3, 39.0, 30.4, 29.3, 28.4, 25.1; HRMS (ESI-TOF) $\mathrm{m} / z$ Calcd for $\mathrm{C}_{26} \mathrm{H}_{34} \mathrm{~N}_{4} \mathrm{O}_{4} \mathrm{Na}[\mathrm{M}+\mathrm{Na}]^{+}: 489.2472$, found: 489.2468 .<smiles>CC(C)(C)OC(=O)N1CCC=C(C2=C(C(=O)Nc3ccccc3C3=NCCO3)CCN(Cc3ccccc3)C2)C1</smiles>

tert-Butyl 1'-benzyl-4'-((2-(4,5-dihydrooxazol-2-yl)phenyl)carbamoyl)-1',2',5,5',6,6'-hexahydro-[3,3'bipyridine]-1(2H)-carboxylate (7s)

Substrate $\mathbf{6 h}$ was alkenylated with alkenyl boronate $(\mathbf{4 x})$ following the general procedure $\mathbf{C ~}(5 \mathrm{~h})$. After purification by column chromatography (neutral alumina, petroleum ether/ethyl acetate $=10 / 1$ ), $7 \mathbf{s}$ was obtained as a white solid (50.0 mg, 92\%), rotameric mixture. $\mathrm{mp} 87-89{ }^{\circ} \mathrm{C} ;{ }^{1} \mathrm{H} \mathrm{NMR}\left(400 \mathrm{MHz}, \mathrm{CDCl}_{3}\right) \delta 12.04(\mathrm{~s}, 1 \mathrm{H})$, $8.80(\mathrm{~d}, J=8.4 \mathrm{~Hz}, 1 \mathrm{H}), 7.83(\mathrm{dd}, J=7.9,1.4 \mathrm{~Hz}, 1 \mathrm{H}), 7.43$ (t, $J=7.4 \mathrm{~Hz}, 1 \mathrm{H}), 7.40-7.31(\mathrm{~m}, 4 \mathrm{H}), 7.30-7.26$ (m, 1H), 7.05 (t, $J=7.5 \mathrm{~Hz}, 1 \mathrm{H}), 5.80(\mathrm{~s}, 1 \mathrm{H}), 4.34$ (t, $J=9.5 \mathrm{~Hz}, 2 \mathrm{H}), 4.10$ (brs, 2H), 3.91 (brs, 2H), 3.65 (s, 2H), 3.23 (brs, 2H), 3.14 (s, 2H), 2.65 (brs, 2H), 2.57 (brs, 2H), 1.99 (brs, 2H), 1.39 (s, 9H); ${ }^{13} \mathrm{C}$ NMR (125 $\left.\mathrm{MHz}, \mathrm{CDCl}_{3}\right) \delta 169.2,168.6,164.1,154.7,139.7,137.5,137.3,134.2,132.4,131.0,129.2,128.3,127.2,124.7$, 122.2, 119.6, 113.1, 79.5, 66.0, 62.2, 56.3, 55.5, 54.7, 48.9, 45.2, 44.4, 40.2, 38.9, 28.3, 27.1, 25.1, 24.9; HRMS (ESI-TOF) $\mathrm{m} / \mathrm{z}$ Calcd for $\mathrm{C}_{32} \mathrm{H}_{39} \mathrm{~N}_{4} \mathrm{O}_{4}[\mathrm{M}+\mathrm{H}]^{+}: 543.2966$, found: 543.2963 . 
<smiles>CC(C)(C)OC(=O)N1C2CCC1CC(C1=C(C(=O)Nc3ccccc3C3=NCCO3)CCN(Cc3ccccc3)C1)C2</smiles>

(1R,5S)-tert-Butyl 3-(1-benzyl-4-((2-(4,5-dihydrooxazol-2-yl)phenyl)carbamoyl)-1,2,5,6-tetrahydropyridin-3-yl)-8-azabicyclo[3.2.1]oct-2-ene-8-carboxylate (7t)

Substrate $\mathbf{6 h}$ was alkenylated with alkenyl boronate $(\mathbf{4 z})$ following the general procedure $\mathbf{C}(5 \mathrm{~h})$. After purification by column chromatography (neutral alumina, petroleum ether/ethyl acetate $=10 / 1$ ), $7 \mathbf{t}$ was obtained as a white solid $(19.0 \mathrm{mg}, 34 \%)$, rotameric mixture. mp 83-85 ${ }^{\circ} \mathrm{C} ;{ }^{1} \mathrm{H} \mathrm{NMR}\left(400 \mathrm{MHz}, \mathrm{CDCl}_{3}\right) \delta 12.00$ (brs, $1 \mathrm{H}), 8.78(\mathrm{~d}, J=8.5 \mathrm{~Hz}, 1 \mathrm{H}), 7.85(\mathrm{~d}, J=7.9 \mathrm{~Hz}, 1 \mathrm{H}), 7.45(\mathrm{t}, J=7.8 \mathrm{~Hz}, 1 \mathrm{H}), 7.35-7.25(\mathrm{~m}, 5 \mathrm{H}), 7.06(\mathrm{t}, J=$ $7.6 \mathrm{~Hz}, 1 \mathrm{H}), 5.97(\mathrm{~s}, 1 \mathrm{H}), 4.37(\mathrm{t}, J=9.5 \mathrm{~Hz}, 2 \mathrm{H}), 4.30-4.05(\mathrm{~m}, 4 \mathrm{H}), 3.70-3.55(\mathrm{~m}, 2 \mathrm{H}), 3.20-3.07(\mathrm{~m}, 1 \mathrm{H})$, 3.00-2.75 (m, 2H), 2.70-2.49 (m, 4H), 2.10-1.60 (m, 5H), 1.37 (s, 9H); ${ }^{13} \mathrm{C}$ NMR (125 MHz, $\left.\mathrm{CDCl}_{3}\right) \delta 169.5$, 169.2, 164.3, 154.1, 139.8, 138.7, 138.3, 137.5, 133.9, 133.6, 132.6, 131.3, 130.8, 130.3, 129.3, 129.3, 128.3, $127.2,122.3,119.6,113.0,79.2,66.1,62.2,55.6,55.0,54.8,53.3,52.5,52.4,51.7,48.9,36.6,35.7,34.4,33.8$, 29.4, 28.7, 28.3, 27.0; HRMS (ESI-TOF) $\mathrm{m} / z$ Calcd for $\mathrm{C}_{34} \mathrm{H}_{40} \mathrm{~N}_{4} \mathrm{O}_{4} \mathrm{Na}[\mathrm{M}+\mathrm{Na}]^{+}:$591.2942, found: 591.2942.<smiles>CN1CCC(/C=C\CCCCOc2ccc3c(c2)CC[C@@H]2[C@@H]3CC[C@]3(C)C(=O)CC[C@H]23)=C(C(=O)Nc2ccccc2C2=NCCO2)C1</smiles>

$N$-(2-(4,5-Dihydrooxazol-2-yl)phenyl)-1-methyl-4-((E)-6-(((8R,9S,13S,14S)-13-methyl-17-oxo7,8,9,11,12,13,14,15,16,17-decahydro-6H-cyclopenta[a]phenanthren-3-yl)oxy)hex-1-en-1-yl)-1,2,5,6tetrahydropyridine-3-carboxamide $(7 \mathrm{u})$

Substrate 6i was alkenylated with alkenyl boronate (4aa) following the general procedure $\mathbf{C}(9 \mathrm{~h})$. After purification by column chromatography (neutral alumina, petroleum ether/ethyl acetate $=2 / 1$ ), $7 \mathbf{u}$ was obtained as a white solid (31.2 mg, 49\%). mp 103-105 ${ }^{\circ} \mathrm{C} ;{ }^{1} \mathrm{H} \mathrm{NMR}\left(400 \mathrm{MHz}, \mathrm{CDCl}_{3}\right) \delta 12.33(\mathrm{~s}, 1 \mathrm{H}), 8.84(\mathrm{~d}, J=8.4$ $\mathrm{Hz}, 1 \mathrm{H}), 7.86(\mathrm{~d}, J=7.7 \mathrm{~Hz}, 1 \mathrm{H}), 7.48(\mathrm{t}, J=7.8 \mathrm{~Hz}, 1 \mathrm{H}), 7.16(\mathrm{~d}, J=8.5 \mathrm{~Hz}, 1 \mathrm{H}), 7.09$ (t, $J=7.6 \mathrm{~Hz}, 1 \mathrm{H})$, 7.73-6.63 (m, 2H), $6.59(\mathrm{~s}, 1 \mathrm{H}), 5.89-5.79(\mathrm{~m}, 1 \mathrm{H}), 4.33(\mathrm{t}, J=9.5 \mathrm{~Hz}, 2 \mathrm{H}), 4.04(\mathrm{t}, J=9.4 \mathrm{~Hz}, 2 \mathrm{H}), 3.87(\mathrm{t}, J$ $=6.3 \mathrm{~Hz}, 2 \mathrm{H}), 3.36(\mathrm{~s}, 2 \mathrm{H}), 2.90-2.83(\mathrm{~m}, 2 \mathrm{H}), 2.62(\mathrm{t}, J=5.8 \mathrm{~Hz}, 2 \mathrm{H}), 2.55-2.32(\mathrm{~m}, 7 \mathrm{H}), 2.28-1.91(\mathrm{~m}, 8 \mathrm{H})$, $1.76-1.70(\mathrm{~m}, 2 \mathrm{H}), 1.60-1.45(\mathrm{~m}, 7 \mathrm{H}), 0.90(\mathrm{~s}, 3 \mathrm{H}) ;{ }^{13} \mathrm{C} \mathrm{NMR}\left(100 \mathrm{MHz}, \mathrm{CDCl}_{3}\right) \delta 221.0,167.8,164.3,157.0$, 139.7, 137.6, 134.1, 132.4, 131.8, 131.7, 129.9, 129.1, 128.5, 126.2, 122.4, 119.9, 114.4, 113.4, 112.0, 67.5, 66.1, 55.8, 54.6, 51.4, 50.4, 48.0, 45.4, 43.9, 38.3, 35.8, 32.7, 31.5, 29.6, 28.7, 26.5, 26.2, 25.9, 25.8, 21.5, 13.8; HRMS (ESI-TOF) $m / z$ Calcd for $\mathrm{C}_{40} \mathrm{H}_{50} \mathrm{~N}_{3} \mathrm{O}_{4}[\mathrm{M}+\mathrm{H}]^{+}: 636.3796$, found: 636.3792 . 


\subsection{Gram-Scale Reaction}

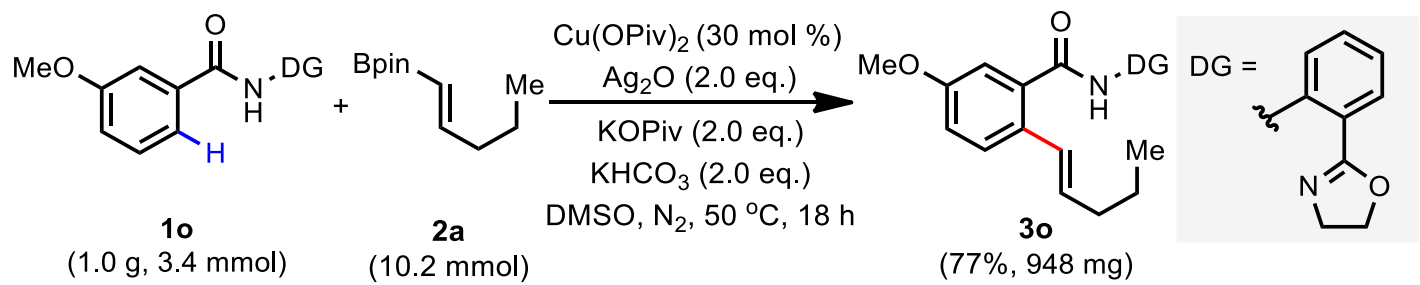

A stirring bar, substrate 10 (1.006 g, $3.4 \mathrm{mmol}), \mathrm{Cu}(\mathrm{OPiv})_{2}(272 \mathrm{mg}, 1.02 \mathrm{mmol}), \mathrm{Ag}_{2} \mathrm{O}(1.57 \mathrm{~g}, 6.8 \mathrm{mmol})$, KOPiv (952 mg, $6.8 \mathrm{mmol})$, and $\mathrm{KHCO}_{3}(680 \mathrm{mg}, 6.8 \mathrm{mmol})$ were added to an oven dried $500 \mathrm{~mL}$ Schlenk tube. The tube was capped and back-filled with nitrogen (3 times). Under the nitrogen flow, 2a (1.96 g, 10.2 $\mathrm{mmol})$ and DMSO $(68 \mathrm{~mL})$ were added to the mixture, and the tube was capped tightly again. Then the reaction mixture was placed into a pre-heated oil bath and stirred at $50{ }^{\circ} \mathrm{C}$ for $18 \mathrm{~h}$ (monitored by TLC). After cooled to room temperature, EtOAc $(250 \mathrm{~mL})$ was added to quench the reaction, and organic layer was washed with $\mathrm{NH}_{3} \cdot \mathrm{H}_{2} \mathrm{O}(250 \mathrm{~mL})$, and the water phase was extracted by EtOAc $(250 \mathrm{~mL})$. The combined organic layers were washed with saturated brine, dried over $\mathrm{Na}_{2} \mathrm{SO}_{4}$, concentrated under vacuum. The crude product was purified by column chromatography (neutral alumina, petroleum ether/ethyl acetate $=15 / 1$ ) to afford the desired product 3o (948 mg, 77\%). 


\subsection{Directing Group Removal}<smiles>CCC/C=C\c1ccc(OC)cc1C(=O)Nc1ccccc1C1=NCCO1</smiles>

30

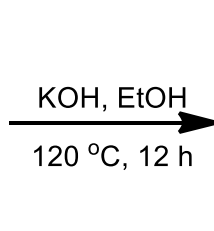<smiles>Nc1ccccc1C1=NCCO1</smiles>

9, $70 \%$

To a $15 \mathrm{~mL}$ sealed tube was added $30(36.4 \mathrm{mg}, 0.1 \mathrm{mmol}), \mathrm{KOH}(336 \mathrm{mg}, 6.0 \mathrm{mmol})$, EtOH $(2 \mathrm{~mL})$, and the tube was capped tightly. The reaction mixture was placed into a pre-heated oil bath and stirred at $120^{\circ} \mathrm{C}$ for 12 $\mathrm{h}$ under air. Upon completion (monitored by TLC), the reaction mixture was evaporated to remove solvent. Then the mixture was washed with $\mathrm{H}_{2} \mathrm{O}(25 \mathrm{~mL})$ and extracted with EtOAc $(3 \times 25 \mathrm{~mL})$. The combined organic phases were dried over $\mathrm{Na}_{2} \mathrm{SO}_{4}$ and concentrated under vacuum. The products were purified by flash column chromatography on silica gel (petroleum ether/ethyl acetate $=10 / 1)$ to give the directing group $9(11.3 \mathrm{mg}, 70 \%)$. The water fraction was treated with $1 \mathrm{~N} \mathrm{HCl}$ to adjust the $\mathrm{PH} \approx 3-4$, and extracted with EtOAc $(3 \times 25 \mathrm{~mL})$. The organic fractions were dried over $\mathrm{Na}_{2} \mathrm{SO}_{4}$ and concentrated. The product were purified by flash column chromatography on silica gel (petroleum ether/ethyl acetate $=5 / 1$ ) to give the desired product 8 as white solid (20.8 mg, 95\%). mp 54-56 ${ }^{\circ} \mathrm{C} ;{ }^{1} \mathrm{H}$ NMR (400 MHz, $\left.\mathrm{CDCl}_{3}\right) \delta 7.53-7.48(\mathrm{~m}, 2 \mathrm{H}), 7.18(\mathrm{~d}, J=15.8 \mathrm{~Hz}, 1 \mathrm{H})$, $7.06(\mathrm{dd}, J=8.7,2.8 \mathrm{~Hz}, 1 \mathrm{H}), 6.11-6.01(\mathrm{~m}, 1 \mathrm{H}), 3.84(\mathrm{~s}, 3 \mathrm{H}), 2.23(\mathrm{q}, J=7.2 \mathrm{~Hz}, 2 \mathrm{H}), 1.59-1.44(\mathrm{~m}, 2 \mathrm{H})$, $1.00-0.92(\mathrm{~m}, 3 \mathrm{H}) ;{ }^{13} \mathrm{C}$ NMR $\left(100 \mathrm{MHz}, \mathrm{CDCl}_{3}\right) \delta 173.0,158.0,133.5,132.6,128.8,128.1,127.5,119.9,114.8$, 55.5, 35.2, 22.5, 13.7; HRMS (ESI-TOF) $m / z$ Calcd for $\mathrm{C}_{13} \mathrm{H}_{15} \mathrm{O}_{3}$ [M-H]: 219.1027 , found: 219.1020 . 


\subsection{Transformations of Alkenylated Benzoic Acid}

\section{Hydrogenation of Alkenylated Benzoic Acid}<smiles>CCC/C=C/c1ccc(OC)cc1C(=O)O</smiles>

8

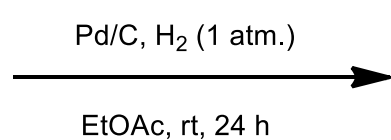

EtOAc, rt, $24 \mathrm{~h}$

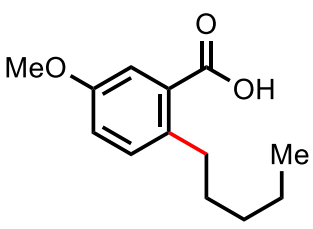

$10(91 \%)$

To the oven dried $25 \mathrm{~mL}$ round-bottom flask was added 8 (20.8 $\mathrm{mg}, 0.095 \mathrm{mmol}), \mathrm{Pd} / \mathrm{C}$ (10 wt. \% loading on carbon, $6.0 \mathrm{mg})$, and EtOAc $(2 \mathrm{~mL})$. The reaction flask was back-filled with $\mathrm{H}_{2}$ (3 times, balloon), and stirred at room temperature for $24 \mathrm{~h}$. Upon completion (monitored by TLC), the reaction mixture was filtered through a short pad of Celite, washed with other EtOAc, and concentrated under reduced pressure. The product were purified by flash column chromatography on silica gel (petroleum ether/ethyl acetate $=5 / 1$ ) to give the desired product 10 as white solid (19.0 mg, 91\%). mp 53-55 ${ }^{\circ} \mathrm{C}$; ${ }^{1} \mathrm{H}$ NMR $\left(400 \mathrm{MHz}, \mathrm{CDCl}_{3}\right) \delta 8.26$ (brs, $\left.1 \mathrm{H}\right), 7.47$ (s, $1 \mathrm{H}), 7.14(\mathrm{~d}, J=8.4 \mathrm{~Hz}, 1 \mathrm{H}), 6.97(\mathrm{~d}, J=8.1 \mathrm{~Hz}, 1 \mathrm{H}), 3.76(\mathrm{~s}, 3 \mathrm{H}), 2.89$ (t, $J=7.3 \mathrm{~Hz}, 2 \mathrm{H}), 1.60-1.48(\mathrm{~m}, 2 \mathrm{H})$, $1.34-1.18(\mathrm{~m}, 4 \mathrm{H}), 0.88-0.72(\mathrm{~m}, 3 \mathrm{H}) ;{ }^{13} \mathrm{C} \mathrm{NMR}\left(100 \mathrm{MHz}, \mathrm{CDCl}_{3}\right) \delta 173.9,157.2,137.5,132.0,130.1,118.9$, 115.3, 55.3, 33.6, 31.7, 31.5, 22.5, 14.0; HRMS (ESI-TOF) $\mathrm{m} / z$ Calcd for $\mathrm{C}_{13} \mathrm{H}_{17} \mathrm{O}_{3}[\mathrm{M}-\mathrm{H}]^{-}: 221.1183$, found: 221.1181 .

\section{Lactonization of Alkenylated Benzoic Acid}<smiles>CCC/C=C/c1ccc(OC)cc1C(=O)O</smiles>

8

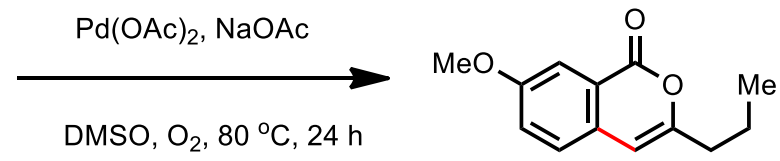

$11,51 \%$

An oven dried $25 \mathrm{~mL}$ Schlenk tube was charged with substrate 8 ( $22 \mathrm{mg}, 0.1 \mathrm{mmol}), \mathrm{Pd}(\mathrm{OAc})_{2}(2.3 \mathrm{mg}, 0.01$ mmol), and $\mathrm{NaOAc}\left(16.2 \mathrm{mg}, 0.2 \mathrm{mmol}\right.$ ) and tube was capped and back-filled with $\mathrm{O}_{2}$ (3 times). Under the $\mathrm{O}_{2}$ flow, DMSO $(2 \mathrm{~mL})$ were added to the mixture, and the tube was capped tightly again. Then the reaction mixture was placed into a pre-heated oil bath and stirred at $80^{\circ} \mathrm{C}$ for $24 \mathrm{~h}$. After cooled to room temperature, EtOAc (20 $\mathrm{mL}$ ) was added to quench the reaction, and organic layer was washed with water $(20 \mathrm{~mL})$, and the water phase was extracted by EtOAc $(20 \mathrm{~mL})$. The combined organic layers were washed with saturated brine, dried over $\mathrm{Na}_{2} \mathrm{SO}_{4}$, concentrated under vacuum. The product were purified by flash column chromatography on silica gel (petroleum ether/ethyl acetate $=10 / 1)$ to give the desired product 11 as white solid $(11.1 \mathrm{mg}, 51 \%) . \mathrm{mp} 40-42$ ${ }^{\circ} \mathrm{C} ;{ }^{1} \mathrm{H}$ NMR $\left(400 \mathrm{MHz}, \mathrm{CDCl}_{3}\right) \delta 7.67(\mathrm{~d}, J=1.8 \mathrm{~Hz}, 1 \mathrm{H}), 7.32-7.23(\mathrm{~m}, 2 \mathrm{H}), 6.23(\mathrm{~s}, 1 \mathrm{H}), 3.90(\mathrm{~s}, 3 \mathrm{H}), 2.50$ $(\mathrm{t}, J=7.5 \mathrm{~Hz}, 2 \mathrm{H}), 1.80-1.68(\mathrm{~m}, 2 \mathrm{H}), 0.99(\mathrm{t}, J=7.4 \mathrm{~Hz}, 3 \mathrm{H}) ;{ }^{13} \mathrm{C} \mathrm{NMR}\left(100 \mathrm{MHz}, \mathrm{CDCl}_{3}\right) \delta 163.3,159.0$, 155.9, 131.3, 126.6, 124.6, 121.1, 109.7, 102.6, 55.7, 35.2, 20.3, 13.5; HRMS (ESI-TOF) $\mathrm{m} / \mathrm{z}$ Calcd for $\mathrm{C}_{13} \mathrm{H}_{15} \mathrm{O}_{3}[\mathrm{M}+\mathrm{H}]^{+}:$219.1016, found: 219.1013 . 


\section{Ozonolysis of Alkenylated Benzoic Acid}

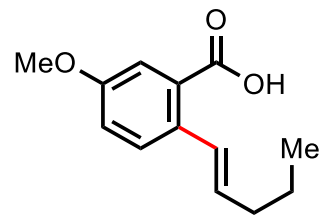

8

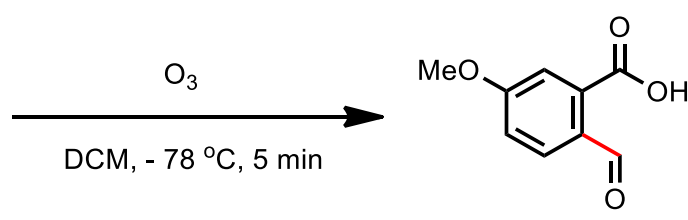

$12,73 \%$

An oven dried $10 \mathrm{~mL}$ digestion tube was charged with substrate $8(22 \mathrm{mg}, 0.1 \mathrm{mmol})$, and DCM (2 mL) and cooled to $-78{ }^{\circ} \mathrm{C}$. Then ozone was introduced into the reaction mixture for $5 \mathrm{~min}$, and the reaction system turned to dark green. Upon completion (monitored by TLC), dimethyl sulfide ( $2.0 \mathrm{~mL}$ ) was added to the mixture, and stirred at the room temperature for $4 \mathrm{~h}$. Then EtOAc $(20 \mathrm{~mL})$ and water $(20 \mathrm{~mL})$ were added. The mixture was extracted, and the water phase was extracted by other EtOAc $(20 \mathrm{~mL})$. The combined organic layers were washed with saturated brine, dried over $\mathrm{Na}_{2} \mathrm{SO}_{4}$, concentrated under vacuum. The product were purified by preparative thin-layer chromatography (petroleum ether/ethyl acetate $=1 / 1$ ) to give the desired product $\mathbf{1 2}^{13}$ as white solid (13.0 mg, 73\%). ${ }^{1} \mathrm{H}$ NMR (400 MHz, CD $\left.{ }_{3} \mathrm{OD}\right) \delta 10.31(\mathrm{~s}, 1 \mathrm{H}), 7.83(\mathrm{~d}, J=8.3 \mathrm{~Hz}, 1 \mathrm{H}), 7.16(\mathrm{~s}$, $1 \mathrm{H}), 6.98(\mathrm{~d}, J=8.0 \mathrm{~Hz}, 1 \mathrm{H}), 3.89$ (s, 3H); HRMS (ESI-TOF) $\mathrm{m} / z$ Calcd for $\mathrm{C}_{9} \mathrm{H}_{7} \mathrm{O}_{4}[\mathrm{M}-\mathrm{H}]^{-}: 179.0339$, found: 179.0335 . 


\subsection{Hydrogenation of Heterocyclic Alkenes}<smiles>COc1ccc(C2=CCOCC2)c(C(=O)N[O+])c1</smiles>

$5 \mathrm{u}$

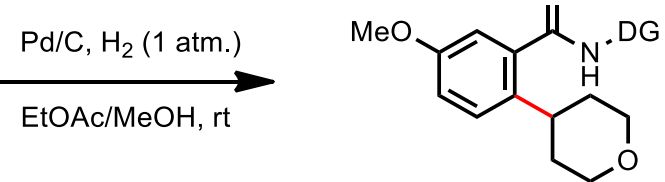

$13,81 \%$

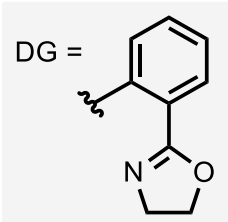

To a $25 \mathrm{~mL}$ round-bottom flask was added $5 \mathbf{u}(26.1 \mathrm{mg}, 0.069 \mathrm{mmol}), \mathrm{Pd} / \mathrm{C}(10 \mathrm{wt}$. \% loading on carbon, 40.0 $\mathrm{mg}$ ), and EtOAc/MeOH (1.5/0.5 mL). The reaction flask was back-filled with $\mathrm{H}_{2}$ (3 times, balloon), and stirred at room temperature for $48 \mathrm{~h}$. Upon completion (monitored by TLC), the reaction mixture was filtered through a short pad of Celite, washed with other EtOAc, and concentrated. The product were purified by flash column chromatography on neutral alumina (petroleum ether/ethyl acetate $=8 / 1$ ) to give the desired product $\mathbf{1 3}$ as white solid (21.3 mg, 81\%). mp 177-179 ${ }^{\circ} \mathrm{C} ;{ }^{1} \mathrm{H}$ NMR (400 MHz, $\left.\mathrm{CDCl}_{3}\right) \delta 12.55(\mathrm{~s}, 1 \mathrm{H}), 8.93(\mathrm{~d}, J=8.4 \mathrm{~Hz}, 1 \mathrm{H})$, $7.91(\mathrm{dd}, J=8.0,1.4 \mathrm{~Hz}, 1 \mathrm{H}), 7.54(\mathrm{t}, J=7.9 \mathrm{~Hz}, 1 \mathrm{H}), 7.33(\mathrm{~d}, J=8.6 \mathrm{~Hz}, 1 \mathrm{H}), 7.18-7.10$ (m, 2H), $7.01(\mathrm{dd}, J$ $=8.6,2.7 \mathrm{~Hz}, 1 \mathrm{H}), 4.37(\mathrm{t}, J=9.5 \mathrm{~Hz}, 2 \mathrm{H}), 4.09-4.00(\mathrm{~m}, 4 \mathrm{H}), 3.83(\mathrm{~s}, 3 \mathrm{H}), 3.58-3.45(\mathrm{~m}, 2 \mathrm{H}), 3.43-3.33(\mathrm{~m}$, $1 \mathrm{H}), 1.85-1.77(\mathrm{~m}, 4 \mathrm{H}) ;{ }^{13} \mathrm{C} \mathrm{NMR}\left(100 \mathrm{MHz}, \mathrm{CDCl}_{3}\right) \delta 168.5,164.6,157.5,139.9,137.4,136.6,132.6,129.3$, 128.1, 122.7, 119.8, 116.5, 113.5, 112.4, 68.5, 66.2, 55.4, 54.7, 36.7, 34.3; HRMS (ESI-TOF) $\mathrm{m} / \mathrm{z}$ Calcd for $\mathrm{C}_{22} \mathrm{H}_{25} \mathrm{~N}_{2} \mathrm{O}_{4}[\mathrm{M}+\mathrm{H}]^{+}: 381.1809$, found: 381.1799 .<smiles>COc1ccc(C2=CCN(C(=O)OCc3ccccc3)CC2)c(C(=O)N[O+])c1</smiles>

$5 w$

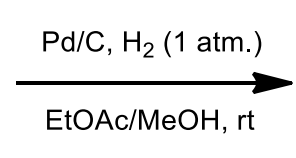

EtOAc/MeOH, rt

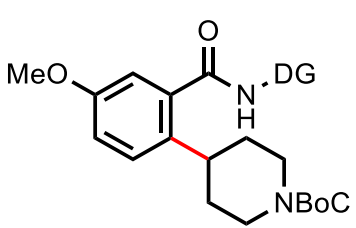

$14,95 \%$

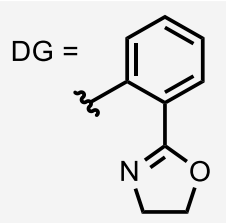

To a $25 \mathrm{~mL}$ round-bottom flask was added $\mathbf{5 w}(38 \mathrm{mg}, 0.08 \mathrm{mmol}), \mathrm{Pd} / \mathrm{C}(10 \mathrm{wt}$ \% loading on carbon, $10 \mathrm{mg})$, and $\mathrm{EtOAc} / \mathrm{MeOH}(1.5 / 0.5 \mathrm{~mL})$. The reaction flask was back-filled with $\mathrm{H}_{2}$ (3 times, balloon), and stirred at room temperature for $36 \mathrm{~h}$. Upon completion (monitored by TLC), the reaction mixture was filtered through a short pad of Celite, washed with other EtOAc, and concentrated. The product were purified by flash column chromatography on neutral alumina (petroleum ether/ethyl acetate $=5 / 1$ ) to give the desired product 14 as white solid (36.2 mg, 95\%), rotameric mixture. $\mathrm{mp} 218-220{ }^{\circ} \mathrm{C} ;{ }^{1} \mathrm{H}$ NMR $\left(400 \mathrm{MHz}, \mathrm{CDCl}_{3}\right) \delta 12.55(\mathrm{~s}, 1 \mathrm{H}), 8.92(\mathrm{~d}$, $J=8.3 \mathrm{~Hz}, 1 \mathrm{H}), 7.91(\mathrm{~d}, J=6.7 \mathrm{~Hz}, 1 \mathrm{H}), 7.54(\mathrm{t}, J=7.2 \mathrm{~Hz}, 1 \mathrm{H}), 7.30-7.26(\mathrm{~m}, 1 \mathrm{H}), 7.18-7.10(\mathrm{~m}, 2 \mathrm{H}), 6.99$ (dd, $J=8.6,2.6 \mathrm{~Hz}, 1 \mathrm{H}), 4.37$ (t, $J=9.5 \mathrm{~Hz}, 2 \mathrm{H}), 4.19$ (brs, 2H), 4.04 (t, $J=9.5 \mathrm{~Hz}, 2 \mathrm{H}), 3.83(\mathrm{~s}, 3 \mathrm{H}), 3.27$ (tt, $J=12.1 \mathrm{~Hz}, 4.0,1 \mathrm{H}), 2.77$ (brs, 2H), 1.87 (d, $J=12.4 \mathrm{~Hz}, 2 \mathrm{H}), 1.62-1.54$ (m, 2H), 1.47 (s, 9H); ${ }^{13} \mathrm{C}$ NMR $(125$ $\left.\mathrm{MHz}, \mathrm{CDCl}_{3}\right) \delta 168.5,164.6,157.5,154.9,139.9,137.5,136.5,132.6,129.3,128.0,122.7,119.8,116.4,113.5$, 112.4, 79.3, 66.2 55.4, 54.7, 44.8, 44.1, 37.7, 33.5, 28.5; HRMS (ESI-TOF) $m / z$ Calcd for $\mathrm{C}_{27} \mathrm{H}_{34} \mathrm{~N}_{3} \mathrm{O}_{5}[\mathrm{M}+\mathrm{H}]^{+}$: 480.2493, found: 480.2496 . 


\subsection{X-Ray Structure of 3a (mono)}

General procedure for crystal preparation: Compound 3a (mono) (around $15 \mathrm{mg}$ ) was dissolved in dichloromethane $(1.0 \mathrm{~mL}) /$ hexane $(5.0 \mathrm{~mL})$ in a screw cap vial $(5.0 \mathrm{~mL})$, and the vial was capped with an opentop cap. The single crystals were grown in the vial via slow evaporation of solvents at room temperature. X-ray data was collected with a Bruker APEX-II CCD diffractometer. The molecular structure and X-ray diffractional data/refinement of $\mathbf{3 a}$ (mono) were shown below.

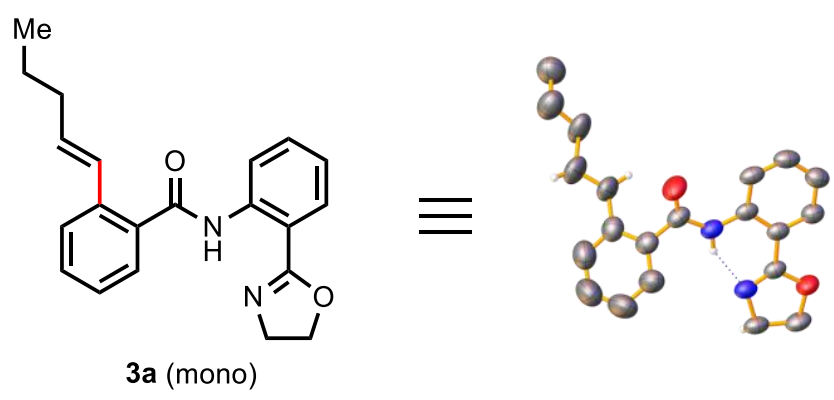

Figure S1. Molecular structure of $\mathbf{3 a}$ (mono) with thermal ellipsoids at $30 \%$ probability

\begin{tabular}{|c|c|c|c|}
\hline Bond precision: & \multicolumn{2}{|c|}{$\mathrm{C}-\mathrm{C}=0.0036 \mathrm{~A}$} & Wavelength $=0$ \\
\hline \multirow[t]{2}{*}{ Cell: } & $a=7.2548(3)$ & $b=9.1568(4)$ & $c=13.7284(7)$ \\
\hline & alpha $=86.674(2)$ & beta $=89.179(2)$ & gamma=86.906(2) \\
\hline \multirow[t]{2}{*}{ Temperature: } & $293 \mathrm{~K}$ & & \\
\hline & \multicolumn{2}{|l|}{ Calculated } & Reported \\
\hline Volume & \multicolumn{2}{|l|}{$909.07(7)$} & $909.07(7)$ \\
\hline Space group & \multicolumn{2}{|l|}{$\mathrm{P}-1$} & $\mathrm{P}-1$ \\
\hline Hall group & \multicolumn{2}{|l|}{$-\mathrm{P} 1$} & $-\mathrm{P} 1$ \\
\hline Moiety formula & \multicolumn{2}{|c|}{ C19.50 H18.50 N2 O2, 0.5(C3 H7) } & $?$ \\
\hline Sum formula & \multicolumn{2}{|c|}{$\mathrm{C} 21 \mathrm{H} 22 \mathrm{~N} 2 \mathrm{O} 2$} & $\mathrm{C} 21 \mathrm{H} 22 \mathrm{~N} 2 \mathrm{O} 2$ \\
\hline $\mathrm{Mr}$ & \multicolumn{2}{|l|}{334.41} & 334.40 \\
\hline Dx, g cm-3 & \multicolumn{2}{|l|}{1.222} & 1.222 \\
\hline $\mathrm{Z}$ & \multicolumn{2}{|l|}{2} & 2 \\
\hline $\mathrm{Mu}(\mathrm{mm}-1)$ & \multicolumn{2}{|l|}{0.079} & 0.079 \\
\hline F000 & \multicolumn{2}{|l|}{356.0} & 356.0 \\
\hline F000' & \multicolumn{2}{|l|}{356.15} & \\
\hline $\mathrm{h}, \mathrm{k}, \operatorname{lmax}$ & \multicolumn{2}{|l|}{$8,11,16$} & $8,11,16$ \\
\hline Nref & \multicolumn{2}{|l|}{3395} & 3363 \\
\hline Tmin,Tmax & \multicolumn{2}{|c|}{$0.985,0.990$} & $0.673,0.746$ \\
\hline Tmin' & \multicolumn{2}{|l|}{0.985} & \\
\hline
\end{tabular}

Correction method $=$ \# Reported $\mathrm{T}$ Limits: $\mathrm{Tmin}=0.673 \mathrm{Tmax}=0.746$ AbsCorr $=$ MULTI -

SCAN

Data completeness $=0.991$

Theta $(\max )=25.499$

$\mathrm{R}$ (reflections $)=0.0616(2626)$

$w R 2($ reflections $)=0.1799(3363)$

$\mathrm{S}=1.043$

Npar $=278$ 
Table 1. Crystal data and structure refinement for mo_d8v19243_0m.

Identification code

Empirical formula

Formula weight

Temperature

Wavelength

Crystal system

Space group

Unit cell dimensions

Volume

Z

Density (calculated)

Absorption coefficient

$\mathrm{F}(000)$

Crystal size

Theta range for data collection

Index ranges

Reflections collected

Independent reflections

Completeness to theta $=25.242^{\circ}$

Absorption correction

Max. and min. transmission

Refinement method

Data / restraints / parameters

Goodness-of-fit on $\mathrm{F}^{2}$

Final $R$ indices [I $>2 \operatorname{sigma}(\mathrm{I})]$

$\mathrm{R}$ indices (all data)

Largest diff. peak and hole mo_d8v19243_0m

$\mathrm{C} 21 \mathrm{H} 22 \mathrm{~N} 2 \mathrm{O} 2$

334.40

293(2) K

$0.71073 \AA$

Triclinic

P -1

$\mathrm{a}=7.2548(3) \AA \quad \alpha=86.674(2)^{\circ}$.

$\mathrm{b}=9.1568(4) \AA \quad \beta=89.179(2)^{\circ}$.

$\mathrm{c}=13.7284(7) \AA \quad \gamma=86.906(2)^{\circ}$.

909.07(7) $\AA^{3}$

2

$1.222 \mathrm{Mg} / \mathrm{m}^{3}$

$0.079 \mathrm{~mm}^{-1}$

356

$0.190 \times 0.160 \times 0.130 \mathrm{~mm}^{3}$

2.973 to $25.499^{\circ}$.

$-8<=\mathrm{h}<=8,-11<=\mathrm{k}<=11,-16<=\mathrm{l}<=16$

12851

$3363[\mathrm{R}(\mathrm{int})=0.0309]$

$99.0 \%$

Semi-empirical from equivalents

0.7456 and 0.6727

Full-matrix least-squares on $\mathrm{F}^{2}$

3363 / 58 / 278

1.043

$\mathrm{R} 1=0.0616, \mathrm{wR} 2=0.1662$

$\mathrm{R} 1=0.0758, \mathrm{wR} 2=0.1799$

0.352 and -0.248 e. $\AA^{-3}$ 
Table 2. Atomic coordinates $\left(\mathrm{x} 10^{4}\right)$ and equivalent isotropic displacement parameters $\left(\AA^{2} \times 10^{3}\right)$ for mo_d8v19243_0m. U(eq) is defined as one third of the trace of the orthogonalized Uij tensor.

\begin{tabular}{|c|c|c|c|c|}
\hline & $\mathrm{x}$ & $\mathrm{y}$ & $\mathrm{z}$ & $\mathrm{U}(\mathrm{eq})$ \\
\hline $\mathrm{N}(1)$ & $1889(2)$ & $6282(2)$ & $5590(1)$ & $60(1)$ \\
\hline $\mathrm{N}(2)$ & $3146(2)$ & $3471(2)$ & $5862(1)$ & $58(1)$ \\
\hline $\mathrm{O}(1)$ & $1671(2)$ & $7185(1)$ & $4043(1)$ & $74(1)$ \\
\hline $\mathrm{O}(2)$ & $3795(3)$ & $1105(2)$ & $6391(1)$ & $94(1)$ \\
\hline $\mathrm{C}(1)$ & 1198(3) & $7806(2)$ & $5660(2)$ & $63(1)$ \\
\hline $\mathrm{C}(2)$ & 1098(3) & $8417(2)$ & $4609(2)$ & $71(1)$ \\
\hline$C(3)$ & $2105(2)$ & $6057(2)$ & $4696(2)$ & $52(1)$ \\
\hline $\mathrm{C}(4)$ & $2803(2)$ & $4696(2)$ & $4268(1)$ & $52(1)$ \\
\hline$C(5)$ & $3019(3)$ & $4680(2)$ & $3261(2)$ & $63(1)$ \\
\hline$C(6)$ & $3673(3)$ & $3438(3)$ & $2821(2)$ & $72(1)$ \\
\hline$C(7)$ & $4102(3)$ & $2186(2)$ & $3390(2)$ & $70(1)$ \\
\hline $\mathrm{C}(8)$ & $3921(2)$ & $2162(2)$ & $4385(2)$ & $61(1)$ \\
\hline $\mathrm{C}(9)$ & $3285(2)$ & $3415(2)$ & $4847(2)$ & $52(1)$ \\
\hline$C(10)$ & $3431(3)$ & $2383(2)$ & $6564(2)$ & $65(1)$ \\
\hline $\mathrm{C}(11)$ & $3222(3)$ & $2881(2)$ & $7577(2)$ & $69(1)$ \\
\hline$C(12)$ & $1722(4)$ & $3801(3)$ & $7802(2)$ & $93(1)$ \\
\hline$C(13)$ & $1451(6)$ & $4253(4)$ & $8738(3)$ & $128(1)$ \\
\hline$C(14)$ & $2703(6)$ & $3799(5)$ & $9445(3)$ & $134(1)$ \\
\hline$C(15)$ & $4185(5)$ & $2906(4)$ & $9230(2)$ & $110(1)$ \\
\hline$C(16)$ & 4494(4) & $2406(3)$ & $8301(2)$ & $79(1)$ \\
\hline$C(17)$ & $6171(14)$ & 1511(14) & $8077(7)$ & $88(2)$ \\
\hline$C(18)$ & 7021(9) & $522(8)$ & $8705(5)$ & $103(2)$ \\
\hline C(19) & 9309(9) & 190(9) & $8243(5)$ & $107(2)$ \\
\hline $\mathrm{C}(20)$ & $9912(10)$ & $-1138(10)$ & $8736(5)$ & $120(2)$ \\
\hline$C(21)$ & $11996(15)$ & $-1308(15)$ & $8435(12)$ & $118(3)$ \\
\hline$C\left(17^{\prime}\right)$ & $6130(30)$ & $1450(30)$ & $8085(18)$ & $124(3)$ \\
\hline $\mathrm{C}\left(18^{\prime}\right)$ & $7593(12)$ & 1106(9) & $8610(8)$ & $131(2)$ \\
\hline $\mathrm{C}\left(19^{\prime}\right)$ & $8481(11)$ & $-591(9)$ & $8615(8)$ & $142(2)$ \\
\hline$C\left(20^{\prime}\right)$ & $10293(13)$ & $-104(12)$ & $8506(9)$ & $156(2)$ \\
\hline$C\left(21^{\prime}\right)$ & $11570(20)$ & $-1635(17)$ & $8478(18)$ & $159(4)$ \\
\hline
\end{tabular}


Table 3. Bond lengths $[\AA]$ and angles $\left[{ }^{\circ}\right]$ for mo_d8v19243_0m.

\begin{tabular}{|c|c|}
\hline $\mathrm{N}(1)-\mathrm{C}(3)$ & $1.262(2)$ \\
\hline $\mathrm{N}(1)-\mathrm{C}(1)$ & $1.466(2)$ \\
\hline $\mathrm{N}(2)-\mathrm{C}(10)$ & $1.355(3)$ \\
\hline $\mathrm{N}(2)-\mathrm{C}(9)$ & $1.399(3)$ \\
\hline $\mathrm{N}(2)-\mathrm{H}(2)$ & $0.87(2)$ \\
\hline $\mathrm{O}(1)-\mathrm{C}(3)$ & $1.355(2)$ \\
\hline $\mathrm{O}(1)-\mathrm{C}(2)$ & $1.445(2)$ \\
\hline $\mathrm{O}(2)-\mathrm{C}(10)$ & $1.222(2)$ \\
\hline$C(1)-C(2)$ & $1.518(3)$ \\
\hline $\mathrm{C}(1)-\mathrm{H}(1 \mathrm{~A})$ & 0.9700 \\
\hline $\mathrm{C}(1)-\mathrm{H}(1 \mathrm{~B})$ & 0.9700 \\
\hline $\mathrm{C}(2)-\mathrm{H}(2 \mathrm{~A})$ & 0.9700 \\
\hline $\mathrm{C}(2)-\mathrm{H}(2 \mathrm{~B})$ & 0.9700 \\
\hline$C(3)-C(4)$ & $1.472(2)$ \\
\hline$C(4)-C(5)$ & $1.389(3)$ \\
\hline$C(4)-C(9)$ & $1.409(3)$ \\
\hline$C(5)-C(6)$ & $1.377(3)$ \\
\hline $\mathrm{C}(5)-\mathrm{H}(5)$ & 0.9300 \\
\hline$C(6)-C(7)$ & $1.374(3)$ \\
\hline $\mathrm{C}(6)-\mathrm{H}(6)$ & 0.9300 \\
\hline$C(7)-C(8)$ & $1.370(3)$ \\
\hline C(7)-H(7) & 0.9300 \\
\hline$C(8)-C(9)$ & $1.396(3)$ \\
\hline $\mathrm{C}(8)-\mathrm{H}(8)$ & 0.9300 \\
\hline $\mathrm{C}(10)-\mathrm{C}(11)$ & $1.491(3)$ \\
\hline $\mathrm{C}(11)-\mathrm{C}(12)$ & $1.383(3)$ \\
\hline$C(11)-C(16)$ & $1.401(3)$ \\
\hline $\mathrm{C}(12)-\mathrm{C}(13)$ & $1.380(4)$ \\
\hline $\mathrm{C}(12)-\mathrm{H}(12)$ & 0.9300 \\
\hline $\mathrm{C}(13)-\mathrm{C}(14)$ & $1.370(5)$ \\
\hline $\mathrm{C}(13)-\mathrm{H}(13)$ & 0.9300 \\
\hline $\mathrm{C}(14)-\mathrm{C}(15)$ & $1.355(4)$ \\
\hline $\mathrm{C}(14)-\mathrm{H}(14)$ & 0.9300 \\
\hline$C(15)-C(16)$ & $1.391(4)$ \\
\hline $\mathrm{C}(15)-\mathrm{H}(15)$ & 0.9300 \\
\hline$C(16)-C(17)$ & $1.468(8)$ \\
\hline
\end{tabular}




\begin{tabular}{|c|c|}
\hline$C(16)-C\left(17^{\prime}\right)$ & $1.474(16)$ \\
\hline C(17)-C(18) & $1.345(11)$ \\
\hline $\mathrm{C}(17)-\mathrm{H}(17)$ & 0.9300 \\
\hline C(18)-C(19) & $1.785(9)$ \\
\hline C(18)-H(18) & 0.9300 \\
\hline$C(19)-C(20)$ & $1.408(9)$ \\
\hline $\mathrm{C}(19)-\mathrm{H}(19 \mathrm{~A})$ & 0.9700 \\
\hline $\mathrm{C}(19)-\mathrm{H}(19 \mathrm{~B})$ & 0.9700 \\
\hline$C(20)-C(21)$ & $1.563(12)$ \\
\hline $\mathrm{C}(20)-\mathrm{H}(20 \mathrm{~A})$ & 0.9700 \\
\hline $\mathrm{C}(20)-\mathrm{H}(20 \mathrm{~B})$ & 0.9700 \\
\hline $\mathrm{C}(21)-\mathrm{H}(21 \mathrm{~A})$ & 0.9600 \\
\hline $\mathrm{C}(21)-\mathrm{H}(21 \mathrm{~B})$ & 0.9600 \\
\hline $\mathrm{C}(21)-\mathrm{H}(21 \mathrm{C})$ & 0.9600 \\
\hline C(17')-C(18') & $1.306(19)$ \\
\hline C(17')-H(17') & 0.9300 \\
\hline $\mathrm{C}\left(18^{\prime}\right)-\mathrm{C}\left(19^{\prime}\right)$ & $1.649(11)$ \\
\hline $\mathrm{C}\left(18^{\prime}\right)-\mathrm{H}\left(18^{\prime}\right)$ & 0.9300 \\
\hline $\mathrm{C}\left(19^{\prime}\right)-\mathrm{C}\left(20^{\prime}\right)$ & $1.414(11)$ \\
\hline $\mathrm{C}\left(19^{\prime}\right)-\mathrm{H}(19 \mathrm{C})$ & 0.9700 \\
\hline $\mathrm{C}\left(19^{\prime}\right)-\mathrm{H}(19 \mathrm{D})$ & 0.9700 \\
\hline $\mathrm{C}\left(20^{\prime}\right)-\mathrm{C}\left(21^{\prime}\right)$ & $1.639(15)$ \\
\hline $\mathrm{C}\left(20^{\prime}\right)-\mathrm{H}(20 \mathrm{C})$ & 0.9700 \\
\hline $\mathrm{C}\left(20^{\prime}\right)-\mathrm{H}(20 \mathrm{D})$ & 0.9700 \\
\hline $\mathrm{C}\left(21^{\prime}\right)-\mathrm{H}(21 \mathrm{D})$ & 0.9600 \\
\hline $\mathrm{C}\left(21^{\prime}\right)-\mathrm{H}(21 \mathrm{E})$ & 0.9600 \\
\hline $\mathrm{C}\left(21^{\prime}\right)-\mathrm{H}(21 \mathrm{~F})$ & 0.9600 \\
\hline $\mathrm{C}(3)-\mathrm{N}(1)-\mathrm{C}(1)$ & $107.52(16)$ \\
\hline $\mathrm{C}(10)-\mathrm{N}(2)-\mathrm{C}(9)$ & $129.30(17)$ \\
\hline $\mathrm{C}(10)-\mathrm{N}(2)-\mathrm{H}(2)$ & $119.7(14)$ \\
\hline $\mathrm{C}(9)-\mathrm{N}(2)-\mathrm{H}(2)$ & $111.0(14)$ \\
\hline $\mathrm{C}(3)-\mathrm{O}(1)-\mathrm{C}(2)$ & $106.22(16)$ \\
\hline $\mathrm{N}(1)-\mathrm{C}(1)-\mathrm{C}(2)$ & $104.44(16)$ \\
\hline $\mathrm{N}(1)-\mathrm{C}(1)-\mathrm{H}(1 \mathrm{~A})$ & 110.9 \\
\hline $\mathrm{C}(2)-\mathrm{C}(1)-\mathrm{H}(1 \mathrm{~A})$ & 110.9 \\
\hline $\mathrm{N}(1)-\mathrm{C}(1)-\mathrm{H}(1 \mathrm{~B})$ & 110.9 \\
\hline $\mathrm{C}(2)-\mathrm{C}(1)-\mathrm{H}(1 \mathrm{~B})$ & 110.9 \\
\hline
\end{tabular}




\begin{tabular}{|c|c|}
\hline $\mathrm{H}(1 \mathrm{~A})-\mathrm{C}(1)-\mathrm{H}(1 \mathrm{~B})$ & 108.9 \\
\hline $\mathrm{O}(1)-\mathrm{C}(2)-\mathrm{C}(1)$ & $104.21(15)$ \\
\hline $\mathrm{O}(1)-\mathrm{C}(2)-\mathrm{H}(2 \mathrm{~A})$ & 110.9 \\
\hline $\mathrm{C}(1)-\mathrm{C}(2)-\mathrm{H}(2 \mathrm{~A})$ & 110.9 \\
\hline $\mathrm{O}(1)-\mathrm{C}(2)-\mathrm{H}(2 \mathrm{~B})$ & 110.9 \\
\hline $\mathrm{C}(1)-\mathrm{C}(2)-\mathrm{H}(2 \mathrm{~B})$ & 110.9 \\
\hline $\mathrm{H}(2 \mathrm{~A})-\mathrm{C}(2)-\mathrm{H}(2 \mathrm{~B})$ & 108.9 \\
\hline $\mathrm{N}(1)-\mathrm{C}(3)-\mathrm{O}(1)$ & $117.57(16)$ \\
\hline $\mathrm{N}(1)-\mathrm{C}(3)-\mathrm{C}(4)$ & $127.36(17)$ \\
\hline $\mathrm{O}(1)-\mathrm{C}(3)-\mathrm{C}(4)$ & $115.07(17)$ \\
\hline $\mathrm{C}(5)-\mathrm{C}(4)-\mathrm{C}(9)$ & 119.01(17) \\
\hline$C(5)-C(4)-C(3)$ & $118.93(17)$ \\
\hline C(9)-C(4)-C(3) & $122.05(17)$ \\
\hline$C(6)-C(5)-C(4)$ & $121.4(2)$ \\
\hline $\mathrm{C}(6)-\mathrm{C}(5)-\mathrm{H}(5)$ & 119.3 \\
\hline $\mathrm{C}(4)-\mathrm{C}(5)-\mathrm{H}(5)$ & 119.3 \\
\hline$C(7)-C(6)-C(5)$ & 119.2(2) \\
\hline $\mathrm{C}(7)-\mathrm{C}(6)-\mathrm{H}(6)$ & 120.4 \\
\hline $\mathrm{C}(5)-\mathrm{C}(6)-\mathrm{H}(6)$ & 120.4 \\
\hline$C(8)-C(7)-C(6)$ & 121.07(19) \\
\hline $\mathrm{C}(8)-\mathrm{C}(7)-\mathrm{H}(7)$ & 119.5 \\
\hline $\mathrm{C}(6)-\mathrm{C}(7)-\mathrm{H}(7)$ & 119.5 \\
\hline $\mathrm{C}(7)-\mathrm{C}(8)-\mathrm{C}(9)$ & $120.65(19)$ \\
\hline $\mathrm{C}(7)-\mathrm{C}(8)-\mathrm{H}(8)$ & 119.7 \\
\hline $\mathrm{C}(9)-\mathrm{C}(8)-\mathrm{H}(8)$ & 119.7 \\
\hline $\mathrm{C}(8)-\mathrm{C}(9)-\mathrm{N}(2)$ & $123.07(18)$ \\
\hline C(8)-C(9)-C(4) & $118.64(19)$ \\
\hline $\mathrm{N}(2)-\mathrm{C}(9)-\mathrm{C}(4)$ & $118.27(16)$ \\
\hline $\mathrm{O}(2)-\mathrm{C}(10)-\mathrm{N}(2)$ & $123.6(2)$ \\
\hline $\mathrm{O}(2)-\mathrm{C}(10)-\mathrm{C}(11)$ & $122.6(2)$ \\
\hline $\mathrm{N}(2)-\mathrm{C}(10)-\mathrm{C}(11)$ & $113.77(18)$ \\
\hline$C(12)-C(11)-C(16)$ & $119.9(2)$ \\
\hline $\mathrm{C}(12)-\mathrm{C}(11)-\mathrm{C}(10)$ & $119.0(2)$ \\
\hline$C(16)-C(11)-C(10)$ & 121.1(2) \\
\hline $\mathrm{C}(13)-\mathrm{C}(12)-\mathrm{C}(11)$ & $120.8(3)$ \\
\hline $\mathrm{C}(13)-\mathrm{C}(12)-\mathrm{H}(12)$ & 119.6 \\
\hline $\mathrm{C}(11)-\mathrm{C}(12)-\mathrm{H}(12)$ & 119.6 \\
\hline$C(14)-C(13)-C(12)$ & $119.4(3)$ \\
\hline
\end{tabular}




\begin{tabular}{|c|c|}
\hline $\mathrm{C}(14)-\mathrm{C}(13)-\mathrm{H}(13)$ & 120.3 \\
\hline $\mathrm{C}(12)-\mathrm{C}(13)-\mathrm{H}(13)$ & 120.3 \\
\hline $\mathrm{C}(15)-\mathrm{C}(14)-\mathrm{C}(13)$ & $120.4(3)$ \\
\hline $\mathrm{C}(15)-\mathrm{C}(14)-\mathrm{H}(14)$ & 119.8 \\
\hline $\mathrm{C}(13)-\mathrm{C}(14)-\mathrm{H}(14)$ & 119.8 \\
\hline$C(14)-C(15)-C(16)$ & $122.2(3)$ \\
\hline $\mathrm{C}(14)-\mathrm{C}(15)-\mathrm{H}(15)$ & 118.9 \\
\hline $\mathrm{C}(16)-\mathrm{C}(15)-\mathrm{H}(15)$ & 118.9 \\
\hline$C(15)-C(16)-C(11)$ & $117.5(3)$ \\
\hline$C(15)-C(16)-C(17)$ & $120.8(5)$ \\
\hline$C(11)-C(16)-C(17)$ & $121.5(4)$ \\
\hline$C(15)-C(16)-C\left(17^{\prime}\right)$ & $121.0(10)$ \\
\hline $\mathrm{C}(11)-\mathrm{C}(16)-\mathrm{C}\left(17^{\prime}\right)$ & $121.5(10)$ \\
\hline $\mathrm{C}(18)-\mathrm{C}(17)-\mathrm{C}(16)$ & $124.8(8)$ \\
\hline $\mathrm{C}(18)-\mathrm{C}(17)-\mathrm{H}(17)$ & 117.6 \\
\hline $\mathrm{C}(16)-\mathrm{C}(17)-\mathrm{H}(17)$ & 117.6 \\
\hline $\mathrm{C}(17)-\mathrm{C}(18)-\mathrm{C}(19)$ & $106.8(7)$ \\
\hline $\mathrm{C}(17)-\mathrm{C}(18)-\mathrm{H}(18)$ & 126.6 \\
\hline $\mathrm{C}(19)-\mathrm{C}(18)-\mathrm{H}(18)$ & 126.6 \\
\hline$C(20)-C(19)-C(18)$ & $103.7(6)$ \\
\hline $\mathrm{C}(20)-\mathrm{C}(19)-\mathrm{H}(19 \mathrm{~A})$ & 111.0 \\
\hline $\mathrm{C}(18)-\mathrm{C}(19)-\mathrm{H}(19 \mathrm{~A})$ & 111.0 \\
\hline $\mathrm{C}(20)-\mathrm{C}(19)-\mathrm{H}(19 \mathrm{~B})$ & 111.0 \\
\hline $\mathrm{C}(18)-\mathrm{C}(19)-\mathrm{H}(19 \mathrm{~B})$ & 111.0 \\
\hline H(19A)-C(19)-H(19B) & 109.0 \\
\hline$C(19)-C(20)-C(21)$ & $103.2(8)$ \\
\hline $\mathrm{C}(19)-\mathrm{C}(20)-\mathrm{H}(20 \mathrm{~A})$ & 111.1 \\
\hline$C(21)-C(20)-H(20 A)$ & 111.1 \\
\hline $\mathrm{C}(19)-\mathrm{C}(20)-\mathrm{H}(20 \mathrm{~B})$ & 111.1 \\
\hline $\mathrm{C}(21)-\mathrm{C}(20)-\mathrm{H}(20 \mathrm{~B})$ & 111.1 \\
\hline $\mathrm{H}(20 \mathrm{~A})-\mathrm{C}(20)-\mathrm{H}(20 \mathrm{~B})$ & 109.1 \\
\hline $\mathrm{C}(20)-\mathrm{C}(21)-\mathrm{H}(21 \mathrm{~A})$ & 109.5 \\
\hline $\mathrm{C}(20)-\mathrm{C}(21)-\mathrm{H}(21 \mathrm{~B})$ & 109.5 \\
\hline $\mathrm{H}(21 \mathrm{~A})-\mathrm{C}(21)-\mathrm{H}(21 \mathrm{~B})$ & 109.5 \\
\hline $\mathrm{C}(20)-\mathrm{C}(21)-\mathrm{H}(21 \mathrm{C})$ & 109.5 \\
\hline $\mathrm{H}(21 \mathrm{~A})-\mathrm{C}(21)-\mathrm{H}(21 \mathrm{C})$ & 109.5 \\
\hline $\mathrm{H}(21 \mathrm{~B})-\mathrm{C}(21)-\mathrm{H}(21 \mathrm{C})$ & 109.5 \\
\hline$C\left(18^{\prime}\right)-C\left(17^{\prime}\right)-C(16)$ & $130(2)$ \\
\hline
\end{tabular}




$\begin{array}{ll}\mathrm{C}\left(18^{\prime}\right)-\mathrm{C}\left(17^{\prime}\right)-\mathrm{H}\left(17^{\prime}\right) & 115.2 \\ \mathrm{C}(16)-\mathrm{C}\left(17^{\prime}\right)-\mathrm{H}\left(17^{\prime}\right) & 115.2 \\ \mathrm{C}\left(17^{\prime}\right)-\mathrm{C}\left(18^{\prime}\right)-\mathrm{C}\left(19^{\prime}\right) & 118.1(13) \\ \mathrm{C}\left(17^{\prime}\right)-\mathrm{C}\left(18^{\prime}\right)-\mathrm{H}\left(18^{\prime}\right) & 121.0 \\ \mathrm{C}\left(19^{\prime}\right)-\mathrm{C}\left(18^{\prime}\right)-\mathrm{H}\left(18^{\prime}\right) & 121.0 \\ \mathrm{C}\left(20^{\prime}\right)-\mathrm{C}\left(19^{\prime}\right)-\mathrm{C}\left(18^{\prime}\right) & 91.7(8) \\ \mathrm{C}\left(20^{\prime}\right)-\mathrm{C}\left(19^{\prime}\right)-\mathrm{H}(19 \mathrm{C}) & 113.3 \\ \mathrm{C}\left(18^{\prime}\right)-\mathrm{C}\left(19^{\prime}\right)-\mathrm{H}(19 \mathrm{C}) & 113.3 \\ \mathrm{C}\left(20^{\prime}\right)-\mathrm{C}\left(19^{\prime}\right)-\mathrm{H}(19 \mathrm{D}) & 113.3 \\ \mathrm{C}\left(18^{\prime}\right)-\mathrm{C}\left(19^{\prime}\right)-\mathrm{H}(19 \mathrm{D}) & 113.3 \\ \mathrm{H}(19 \mathrm{C})-\mathrm{C}\left(19^{\prime}\right)-\mathrm{H}(19 \mathrm{D}) & 110.7 \\ \mathrm{C}\left(19^{\prime}\right)-\mathrm{C}\left(20^{\prime}\right)-\mathrm{C}\left(21^{\prime}\right) & 103.2(10) \\ \mathrm{C}\left(19^{\prime}\right)-\mathrm{C}\left(20^{\prime}\right)-\mathrm{H}(20 \mathrm{C}) & 111.1 \\ \mathrm{C}\left(21^{\prime}\right)-\mathrm{C}\left(20^{\prime}\right)-\mathrm{H}(20 \mathrm{C}) & 111.1 \\ \mathrm{C}\left(19^{\prime}\right)-\mathrm{C}\left(20^{\prime}\right)-\mathrm{H}(20 \mathrm{D}) & 111.1 \\ \mathrm{C}\left(21^{\prime}\right)-\mathrm{C}\left(20^{\prime}\right)-\mathrm{H}(20 \mathrm{D}) & 111.1 \\ \mathrm{H}(20 \mathrm{C})-\mathrm{C}\left(20^{\prime}\right)-\mathrm{H}(20 \mathrm{D}) & 109.1 \\ \mathrm{C}\left(20^{\prime}\right)-\mathrm{C}\left(21^{\prime}\right)-\mathrm{H}(21 \mathrm{D}) & 109.5 \\ \mathrm{C}\left(20^{\prime}\right)-\mathrm{C}\left(21^{\prime}\right)-\mathrm{H}(21 \mathrm{E}) & 109.5 \\ \mathrm{H}(21 \mathrm{D})-\mathrm{C}\left(21^{\prime}\right)-\mathrm{H}(21 \mathrm{E}) & 109.5 \\ \mathrm{C}\left(20^{\prime}\right)-\mathrm{C}\left(21^{\prime}\right)-\mathrm{H}(21 \mathrm{~F}) & 109.5 \\ \mathrm{H}(21 \mathrm{D})-\mathrm{C}\left(21^{\prime}\right)-\mathrm{H}(21 \mathrm{~F}) & 109.5 \\ \mathrm{H}(21 \mathrm{E})-\mathrm{C}\left(21^{\prime}\right)-\mathrm{H}(21 \mathrm{~F}) & 109.5 \\ & \end{array}$

Symmetry transformations used to generate equivalent atoms: 
Table 4. Anisotropic displacement parameters $\left(\AA^{2} \times 10^{3}\right)$ for mo_d8v19243_0m. The anisotropic displacement factor exponent takes the form: $\quad-2 \pi^{2}\left[h^{2} a^{* 2} U^{11}+\ldots+2 h k a^{*} b^{*} U^{12}\right]$

\begin{tabular}{|c|c|c|c|c|c|c|}
\hline & $\mathrm{U}^{11}$ & $\mathrm{U}^{22}$ & $\mathrm{U}^{33}$ & $\mathrm{U}^{23}$ & $\mathrm{U}^{13}$ & $\mathrm{U}^{12}$ \\
\hline $\mathrm{N}(1)$ & $55(1)$ & $49(1)$ & $75(1)$ & $-11(1)$ & $-2(1)$ & $3(1)$ \\
\hline $\mathrm{N}(2)$ & $54(1)$ & $44(1)$ & $76(1)$ & $-4(1)$ & $-4(1)$ & $2(1)$ \\
\hline $\mathrm{O}(1)$ & $92(1)$ & $47(1)$ & $79(1)$ & $-2(1)$ & $-1(1)$ & $14(1)$ \\
\hline $\mathrm{O}(2)$ & $132(2)$ & $51(1)$ & $96(1)$ & $0(1)$ & $-15(1)$ & $13(1)$ \\
\hline $\mathrm{C}(1)$ & $52(1)$ & $49(1)$ & $88(2)$ & $-17(1)$ & $-3(1)$ & $5(1)$ \\
\hline $\mathrm{C}(2)$ & $70(1)$ & $43(1)$ & $100(2)$ & $-11(1)$ & $-4(1)$ & $6(1)$ \\
\hline$C(3)$ & $39(1)$ & $42(1)$ & $74(1)$ & $-4(1)$ & $-3(1)$ & $-2(1)$ \\
\hline $\mathrm{C}(4)$ & $38(1)$ & $46(1)$ & $73(1)$ & $-9(1)$ & $-2(1)$ & $-3(1)$ \\
\hline$C(5)$ & $58(1)$ & $57(1)$ & $75(1)$ & $-6(1)$ & $1(1)$ & $0(1)$ \\
\hline$C(6)$ & $69(1)$ & $72(1)$ & $75(1)$ & $-19(1)$ & $3(1)$ & $2(1)$ \\
\hline$C(7)$ & $57(1)$ & $60(1)$ & $94(2)$ & $-25(1)$ & $0(1)$ & $5(1)$ \\
\hline $\mathrm{C}(8)$ & $47(1)$ & $45(1)$ & $92(2)$ & $-11(1)$ & $-4(1)$ & $2(1)$ \\
\hline $\mathrm{C}(9)$ & $35(1)$ & $46(1)$ & $76(1)$ & $-8(1)$ & $-3(1)$ & $-3(1)$ \\
\hline$C(10)$ & $60(1)$ & $50(1)$ & $84(2)$ & $1(1)$ & $-9(1)$ & $1(1)$ \\
\hline$C(11)$ & $70(1)$ & $57(1)$ & $80(2)$ & $4(1)$ & $2(1)$ & $-5(1)$ \\
\hline$C(12)$ & $90(2)$ & $92(2)$ & $94(2)$ & $3(1)$ & $15(1)$ & $13(1)$ \\
\hline$C(13)$ & $138(3)$ & $128(3)$ & $111(3)$ & $-5(2)$ & $41(2)$ & $30(2)$ \\
\hline$C(14)$ & $175(4)$ & $137(3)$ & $85(2)$ & $-10(2)$ & $19(2)$ & $27(3)$ \\
\hline$C(15)$ & $138(3)$ & $112(2)$ & $79(2)$ & $3(2)$ & $-7(2)$ & $3(2)$ \\
\hline$C(16)$ & $90(2)$ & $70(1)$ & $76(2)$ & $5(1)$ & $-5(1)$ & $-7(1)$ \\
\hline$C(17)$ & $81(3)$ & 101(3) & $79(3)$ & $1(3)$ & $-26(2)$ & $21(3)$ \\
\hline$C(18)$ & $89(3)$ & 117(3) & $98(3)$ & $11(3)$ & $-35(2)$ & $19(3)$ \\
\hline$C(19)$ & $73(3)$ & $138(4)$ & $103(3)$ & $37(3)$ & $-18(2)$ & $15(3)$ \\
\hline$C(20)$ & $97(3)$ & $140(4)$ & $118(4)$ & $24(3)$ & $-2(3)$ & $14(3)$ \\
\hline$C(21)$ & $96(5)$ & $145(7)$ & 111(5) & $-10(5)$ & $6(4)$ & $6(5)$ \\
\hline $\mathrm{C}\left(17^{\prime}\right)$ & $107(5)$ & $110(5)$ & $157(6)$ & $-4(5)$ & $-30(5)$ & $-25(5)$ \\
\hline$C\left(18^{\prime}\right)$ & $114(4)$ & $117(4)$ & $164(5)$ & $3(4)$ & $-28(4)$ & $-27(4)$ \\
\hline $\mathrm{C}\left(19^{\prime}\right)$ & $124(4)$ & $120(4)$ & $183(5)$ & $7(4)$ & $-29(4)$ & $-29(4)$ \\
\hline $\mathrm{C}\left(20^{\prime}\right)$ & $138(5)$ & $139(4)$ & 192(6) & $3(4)$ & $8(4)$ & $-34(4)$ \\
\hline$C\left(21^{\prime}\right)$ & $125(8)$ & $167(8)$ & 187(8) & $-18(7)$ & $14(7)$ & $-14(6)$ \\
\hline
\end{tabular}


Table 5. Hydrogen coordinates ( $\times 10^{4}$ ) and isotropic displacement parameters $\left(\AA^{2} \times 10^{3}\right)$ for mo_d8v19243_0m.

\begin{tabular}{|c|c|c|c|c|}
\hline & $\mathrm{x}$ & $\mathrm{y}$ & $\mathrm{z}$ & $\mathrm{U}(\mathrm{eq})$ \\
\hline $\mathrm{H}(2)$ & $2850(30)$ & $4360(20)$ & $6028(15)$ & $66(6)$ \\
\hline $\mathrm{H}(1 \mathrm{~A})$ & 2035 & 8345 & 6031 & 75 \\
\hline $\mathrm{H}(1 \mathrm{~B})$ & -12 & 7855 & 5970 & 75 \\
\hline $\mathrm{H}(2 \mathrm{~A})$ & -149 & 8774 & 4447 & 85 \\
\hline $\mathrm{H}(2 \mathrm{~B})$ & 1922 & 9211 & 4495 & 85 \\
\hline $\mathrm{H}(5)$ & 2715 & 5525 & 2877 & 76 \\
\hline $\mathrm{H}(6)$ & 3823 & 3448 & 2147 & 86 \\
\hline $\mathrm{H}(7)$ & 4523 & 1340 & 3095 & 84 \\
\hline $\mathrm{H}(8)$ & 4224 & 1304 & 4757 & 74 \\
\hline $\mathrm{H}(12)$ & 886 & 4119 & 7318 & 112 \\
\hline $\mathrm{H}(13)$ & 429 & 4859 & 8887 & 153 \\
\hline $\mathrm{H}(14)$ & 2534 & 4105 & 10075 & 160 \\
\hline $\mathrm{H}(15)$ & 5023 & 2618 & 9720 & 132 \\
\hline $\mathrm{H}(17)$ & 6682 & 1637 & 7454 & 105 \\
\hline $\mathrm{H}(18)$ & 6515 & 95 & 9272 & 123 \\
\hline $\mathrm{H}(19 \mathrm{~A})$ & 9305 & 96 & 7543 & 129 \\
\hline $\mathrm{H}(19 \mathrm{~B})$ & 10090 & 974 & 8388 & 129 \\
\hline $\mathrm{H}(20 \mathrm{~A})$ & 9236 & -1948 & 8527 & 144 \\
\hline $\mathrm{H}(20 \mathrm{~B})$ & 9769 & -1084 & 9436 & 144 \\
\hline $\mathrm{H}(21 \mathrm{~A})$ & 12167 & -862 & 7792 & 177 \\
\hline $\mathrm{H}(21 \mathrm{~B})$ & 12373 & -2329 & 8438 & 177 \\
\hline $\mathrm{H}(21 \mathrm{C})$ & 12725 & -837 & 8890 & 177 \\
\hline $\mathrm{H}\left(17^{\prime}\right)$ & 6115 & 1020 & 7487 & 149 \\
\hline $\mathrm{H}\left(18^{\prime}\right)$ & 8120 & 1800 & 8972 & 158 \\
\hline $\mathrm{H}(19 \mathrm{C})$ & 8279 & -1151 & 9225 & 170 \\
\hline $\mathrm{H}(19 \mathrm{D})$ & 8093 & -1119 & 8067 & 170 \\
\hline $\mathrm{H}(20 \mathrm{C})$ & 10616 & 454 & 9053 & 187 \\
\hline $\mathrm{H}(20 \mathrm{D})$ & 10427 & 496 & 7906 & 187 \\
\hline $\mathrm{H}(21 \mathrm{D})$ & 11457 & -2186 & 9090 & 239 \\
\hline $\mathrm{H}(21 \mathrm{E})$ & 12835 & -1415 & 8364 & 239 \\
\hline $\mathrm{H}(21 \mathrm{~F})$ & 11160 & -2199 & 7962 & 239 \\
\hline
\end{tabular}


Table 6. Torsion angles $\left[{ }^{\circ}\right]$ for mo_d8v19243_0m.

\begin{tabular}{|c|c|}
\hline $\mathrm{C}(3)-\mathrm{N}(1)-\mathrm{C}(1)-\mathrm{C}(2)$ & $-1.0(2)$ \\
\hline $\mathrm{C}(3)-\mathrm{O}(1)-\mathrm{C}(2)-\mathrm{C}(1)$ & $-2.2(2)$ \\
\hline $\mathrm{N}(1)-\mathrm{C}(1)-\mathrm{C}(2)-\mathrm{O}(1)$ & $2.0(2)$ \\
\hline $\mathrm{C}(1)-\mathrm{N}(1)-\mathrm{C}(3)-\mathrm{O}(1)$ & $-0.5(2)$ \\
\hline $\mathrm{C}(1)-\mathrm{N}(1)-\mathrm{C}(3)-\mathrm{C}(4)$ & $178.86(16)$ \\
\hline $\mathrm{C}(2)-\mathrm{O}(1)-\mathrm{C}(3)-\mathrm{N}(1)$ & $1.8(2)$ \\
\hline $\mathrm{C}(2)-\mathrm{O}(1)-\mathrm{C}(3)-\mathrm{C}(4)$ & $-177.60(15)$ \\
\hline $\mathrm{N}(1)-\mathrm{C}(3)-\mathrm{C}(4)-\mathrm{C}(5)$ & $-177.22(17)$ \\
\hline $\mathrm{O}(1)-\mathrm{C}(3)-\mathrm{C}(4)-\mathrm{C}(5)$ & $2.1(2)$ \\
\hline $\mathrm{N}(1)-\mathrm{C}(3)-\mathrm{C}(4)-\mathrm{C}(9)$ & $1.8(3)$ \\
\hline $\mathrm{O}(1)-\mathrm{C}(3)-\mathrm{C}(4)-\mathrm{C}(9)$ & $-178.87(15)$ \\
\hline$C(9)-C(4)-C(5)-C(6)$ & $0.7(3)$ \\
\hline$C(3)-C(4)-C(5)-C(6)$ & $179.73(17)$ \\
\hline$C(4)-C(5)-C(6)-C(7)$ & $0.7(3)$ \\
\hline$C(5)-C(6)-C(7)-C(8)$ & $-1.2(3)$ \\
\hline $\mathrm{C}(6)-\mathrm{C}(7)-\mathrm{C}(8)-\mathrm{C}(9)$ & $0.3(3)$ \\
\hline $\mathrm{C}(7)-\mathrm{C}(8)-\mathrm{C}(9)-\mathrm{N}(2)$ & $-177.26(16)$ \\
\hline$C(7)-C(8)-C(9)-C(4)$ & $1.1(3)$ \\
\hline $\mathrm{C}(10)-\mathrm{N}(2)-\mathrm{C}(9)-\mathrm{C}(8)$ & $-5.4(3)$ \\
\hline$C(10)-N(2)-C(9)-C(4)$ & $176.17(17)$ \\
\hline $\mathrm{C}(5)-\mathrm{C}(4)-\mathrm{C}(9)-\mathrm{C}(8)$ & $-1.6(2)$ \\
\hline $\mathrm{C}(3)-\mathrm{C}(4)-\mathrm{C}(9)-\mathrm{C}(8)$ & $179.42(15)$ \\
\hline $\mathrm{C}(5)-\mathrm{C}(4)-\mathrm{C}(9)-\mathrm{N}(2)$ & $176.88(15)$ \\
\hline $\mathrm{C}(3)-\mathrm{C}(4)-\mathrm{C}(9)-\mathrm{N}(2)$ & $-2.1(2)$ \\
\hline $\mathrm{C}(9)-\mathrm{N}(2)-\mathrm{C}(10)-\mathrm{O}(2)$ & $-3.6(3)$ \\
\hline $\mathrm{C}(9)-\mathrm{N}(2)-\mathrm{C}(10)-\mathrm{C}(11)$ & $177.62(17)$ \\
\hline $\mathrm{O}(2)-\mathrm{C}(10)-\mathrm{C}(11)-\mathrm{C}(12)$ & $-133.2(3)$ \\
\hline $\mathrm{N}(2)-\mathrm{C}(10)-\mathrm{C}(11)-\mathrm{C}(12)$ & $45.6(3)$ \\
\hline $\mathrm{O}(2)-\mathrm{C}(10)-\mathrm{C}(11)-\mathrm{C}(16)$ & $45.4(3)$ \\
\hline $\mathrm{N}(2)-\mathrm{C}(10)-\mathrm{C}(11)-\mathrm{C}(16)$ & $-135.8(2)$ \\
\hline$C(16)-C(11)-C(12)-C(13)$ & $-0.5(4)$ \\
\hline$C(10)-C(11)-C(12)-C(13)$ & $178.2(3)$ \\
\hline $\mathrm{C}(11)-\mathrm{C}(12)-\mathrm{C}(13)-\mathrm{C}(14)$ & $1.0(5)$ \\
\hline $\mathrm{C}(12)-\mathrm{C}(13)-\mathrm{C}(14)-\mathrm{C}(15)$ & $-0.5(6)$ \\
\hline $\mathrm{C}(13)-\mathrm{C}(14)-\mathrm{C}(15)-\mathrm{C}(16)$ & $-0.5(6)$ \\
\hline$C(14)-C(15)-C(16)-C(11)$ & $1.1(5)$ \\
\hline
\end{tabular}




$\begin{array}{lc}\mathrm{C}(14)-\mathrm{C}(15)-\mathrm{C}(16)-\mathrm{C}(17) & 176.3(7) \\ \mathrm{C}(14)-\mathrm{C}(15)-\mathrm{C}(16)-\mathrm{C}\left(17^{\prime}\right) & 179.4(12) \\ \mathrm{C}(12)-\mathrm{C}(11)-\mathrm{C}(16)-\mathrm{C}(15) & -0.5(4) \\ \mathrm{C}(10)-\mathrm{C}(11)-\mathrm{C}(16)-\mathrm{C}(15) & -179.1(2) \\ \mathrm{C}(12)-\mathrm{C}(11)-\mathrm{C}(16)-\mathrm{C}(17) & -175.8(7) \\ \mathrm{C}(10)-\mathrm{C}(11)-\mathrm{C}(16)-\mathrm{C}(17) & 5.6(8) \\ \mathrm{C}(12)-\mathrm{C}(11)-\mathrm{C}(16)-\mathrm{C}\left(17^{\prime}\right) & -178.9(12) \\ \mathrm{C}(10)-\mathrm{C}(11)-\mathrm{C}(16)-\mathrm{C}\left(17^{\prime}\right) & 2.5(12) \\ \mathrm{C}(15)-\mathrm{C}(16)-\mathrm{C}(17)-\mathrm{C}(18) & 34.7(15) \\ \mathrm{C}(11)-\mathrm{C}(16)-\mathrm{C}(17)-\mathrm{C}(18) & -150.3(9) \\ \mathrm{C}\left(17^{\prime}\right)-\mathrm{C}(16)-\mathrm{C}(17)-\mathrm{C}(18) & -60(28) \\ \mathrm{C}(16)-\mathrm{C}(17)-\mathrm{C}(18)-\mathrm{C}(19) & -161.5(10) \\ \mathrm{C}(17)-\mathrm{C}(18)-\mathrm{C}(19)-\mathrm{C}(20) & -162.2(9) \\ \mathrm{C}(18)-\mathrm{C}(19)-\mathrm{C}(20)-\mathrm{C}(21) & -172.6(8) \\ \mathrm{C}(15)-\mathrm{C}(16)-\mathrm{C}\left(17^{\prime}\right)-\mathrm{C}\left(18^{\prime}\right) & -11(3) \\ \mathrm{C}(11)-\mathrm{C}(16)-\mathrm{C}\left(17^{\prime}\right)-\mathrm{C}\left(18^{\prime}\right) & 167.0(18) \\ \mathrm{C}(17)-\mathrm{C}(16)-\mathrm{C}\left(17^{\prime}\right)-\mathrm{C}\left(18^{\prime}\right) & 76(28) \\ \mathrm{C}(16)-\mathrm{C}\left(17^{\prime}\right)-\mathrm{C}\left(18^{\prime}\right)-\mathrm{C}\left(19^{\prime}\right) & 141.4(19) \\ \mathrm{C}\left(17^{\prime}\right)-\mathrm{C}\left(18^{\prime}\right)-\mathrm{C}\left(19^{\prime}\right)-\mathrm{C}\left(20^{\prime}\right) & 135.4(15) \\ \mathrm{C}\left(18^{\prime}\right)-\mathrm{C}\left(19^{\prime}\right)-\mathrm{C}\left(20^{\prime}\right)-\mathrm{C}\left(21^{\prime}\right) & -179.6(11) \\ & \\ & \end{array}$

Symmetry transformations used to generate equivalent atoms: 


\section{References}

1. (a) Fu, X.-P.; Xuan, Q.-Q.; Liu, L.; Wang, D.; Chen, Y.-J.; Li, C.-J. Tetrahedron 2013, 69, 4436-4444. (b) Wang, C.-P.; Dong, G.-B. J. Am. Chem. Soc. 2018, 140, 6057-606. (b) Yu, J.-F.; Li, J.-J.; Wang, P.; Yu, J.-Q. Angew. Chem. Int. Ed. 2019, 10.1002/anie.201910395.

2. (a) Shang, M.; Sun, S.-Z.; Dai, H.-X.; Yu, J.-Q. J. Am. Chem. Soc. 2014, 136, 3354-3357. (b) Shang, M.; Wang, M.-M.; Saint-Denis, T.-G.; Li, M.-H.; Dai, H.-X.; Yu, J.-Q. Angew. Chem. Int. Ed. 2017, 56, 5317-5321.

3. Song S.; Zhu, S.-F.; Pu, L.-Y.; Zhou, Q.-L. Angew. Chem. Int. Ed. 2013, 52, 6072-6075.

4. Zhao, J.; Niu, Z.-Q.; Fu, H.; Li, Y.-D. Chem. Commun. 2014, 50, 2058-2060.

5. Gao, G.-L.; Yan, J.-X.; Yang, K.; Fener Chen, F.-E.; Song, Q.-L. Green Chem. 2017, 19, 3997-4001.

6. Lawson, J.-R; Wilkins, L.-C; Melen, R.-L Chem. Eur. J. 2017, 23, 10997-11000.

7. Nakajima, K.; Kato, T.; Nishibayashi, Y. Org. Lett. 2017, 19, 4323-4326.

8. Nakamura, M.; Hatakeyama, T.; Hara, K.; Fukudome, H.i; Nakamura, E. J. Am. Chem. Soc. 2004, 126, 14344-14345.

9. Crestey, F.; Hooyberghs, G.; Kristensen, J. L. Tetrahedron 2012, 68, 1417-1421.

10. Kovalenko, M.; Yarmoliuk, D. V.; Serhiichuk, D.; Chernenko, D.; Smyrnov, V.; Breslavskyi, A.; Hryshchuk, O. V.; Kleban, I.; Rassukana, Y.; Tymtsunik, A. V.; Tolmachev, A. A.; Kuchkovska, Y. O.; Grygorenko, O. O. Eur. J. Org. Chem. 2019, 5624-5635.

11. Silvi, M.; Sandford, C.; Aggarwal, V. K. J. Am. Chem. Soc. 2017, 139, 5736-5739.

12. Kondoh, A.; Jamison, T. F. Chem. Commun. 2010, 46, 907-909.

13. Koeberle, S. C.; Fischer, S.; Schollmeyer, D.; Schattel, V.; Grütter, C.; Rauh, D.; Laufer, S. A. J. Med. Chem. 2012, 55, 5868-5877. 


\section{NMR Spectra}

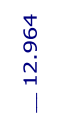

$\int$
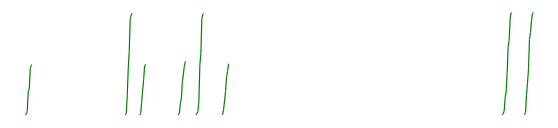<smiles>O=C(Nc1ccccc1C1=NCCO1)c1ccc(Cl)cc1</smiles>

${ }^{1} \mathrm{HNMR}\left(400 \mathrm{MHz}, \mathrm{CDCl}_{3}\right)$
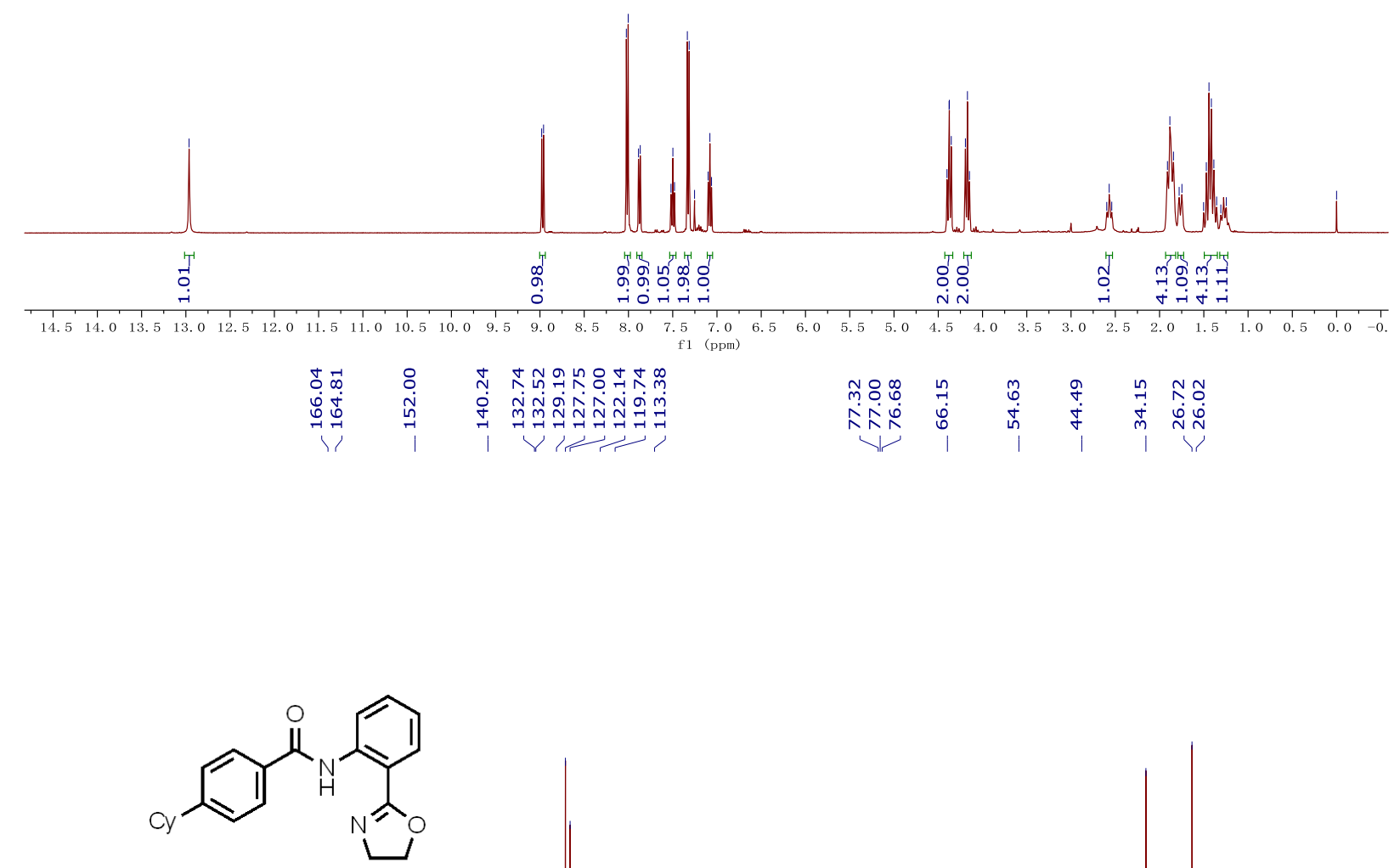

1d

${ }^{13} \mathrm{C}$ NMR $\left(100 \mathrm{MHz}, \mathrm{CDCl}_{3}\right)$

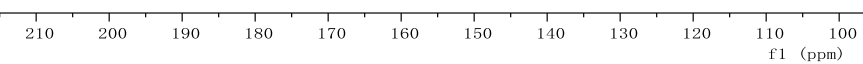




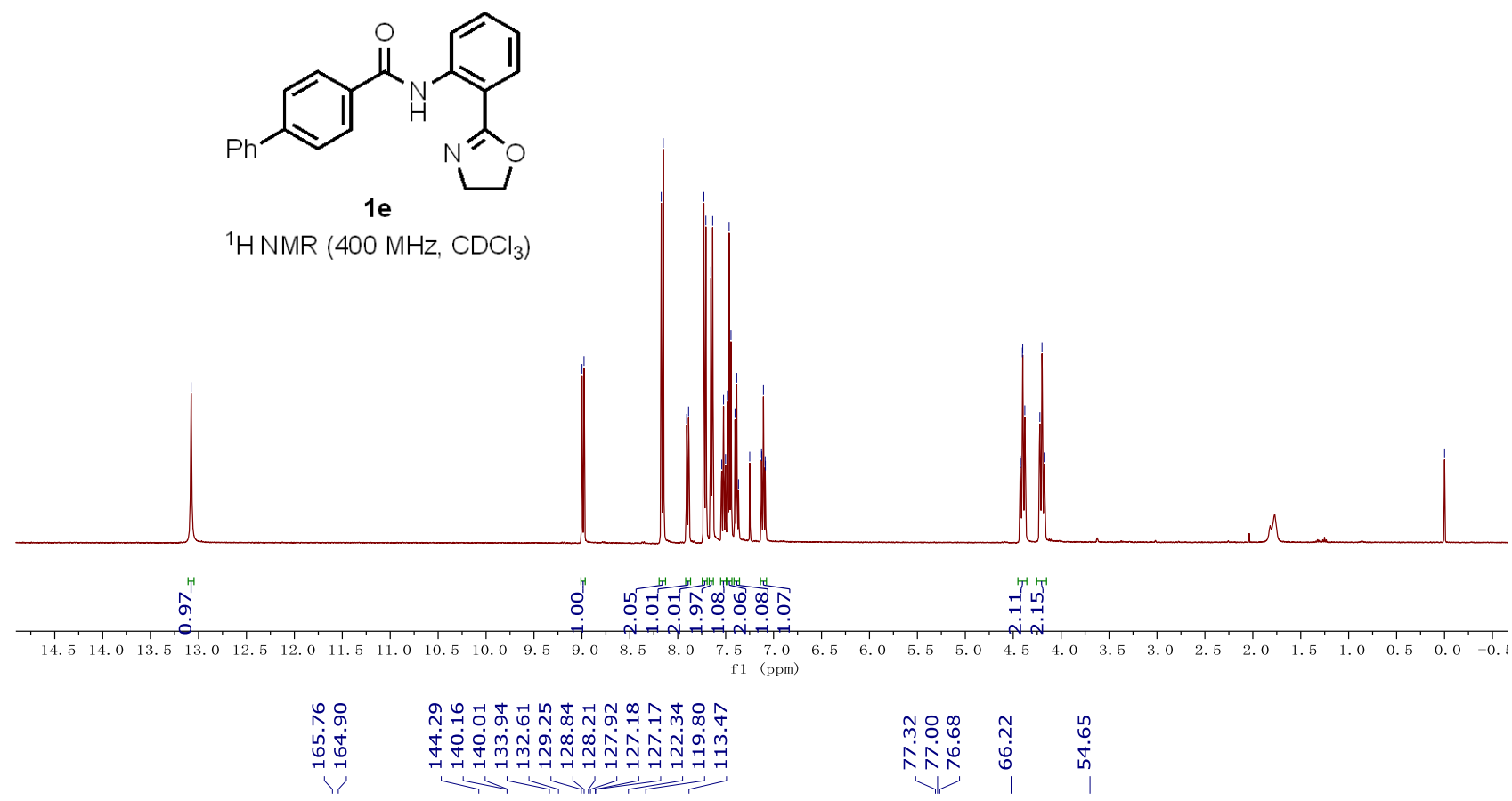

$1 e$

${ }^{1} \mathrm{HNMR}\left(400 \mathrm{MHz}, \mathrm{CDCl}_{3}\right)$
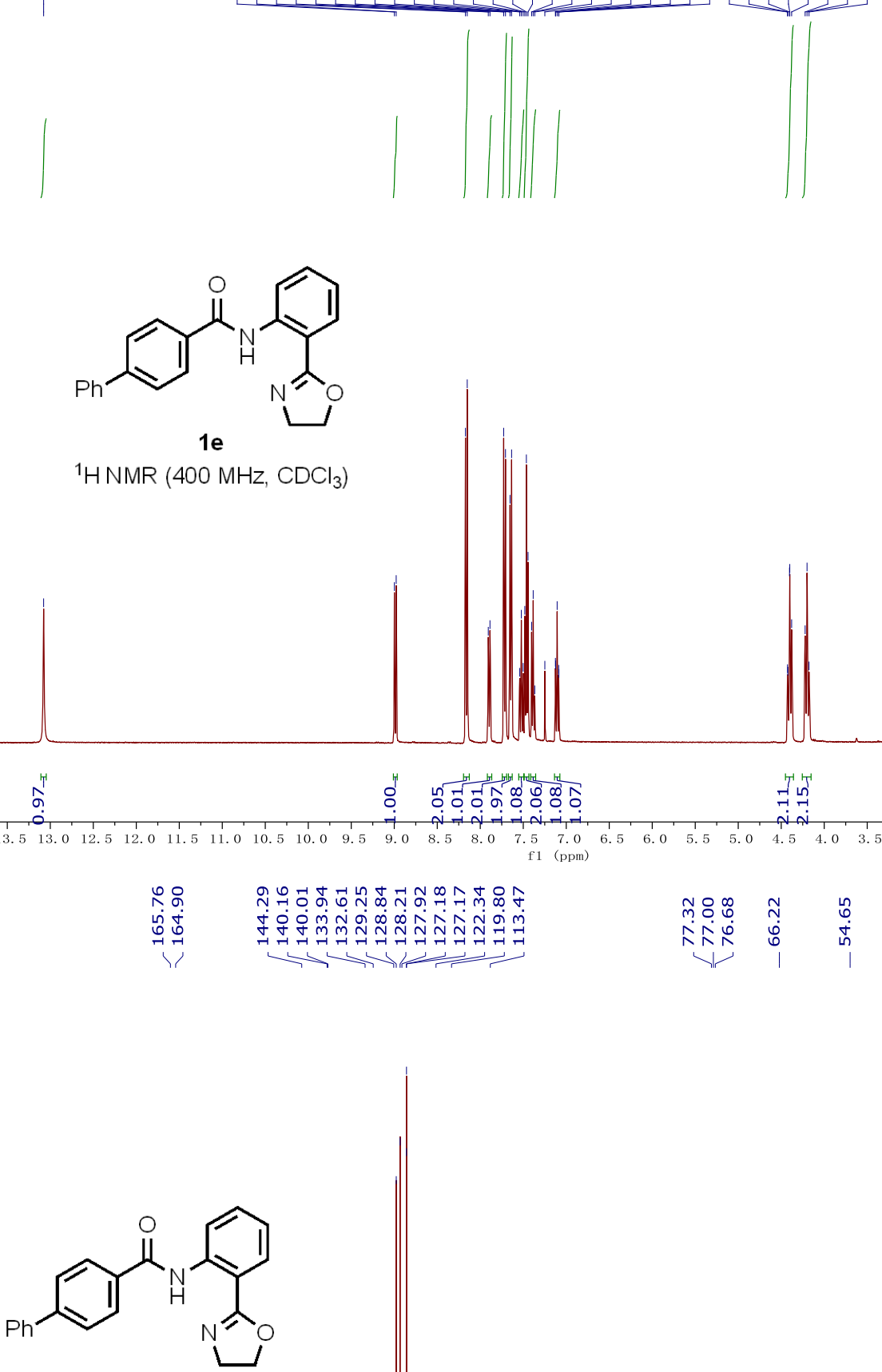

$1 e$

${ }^{13} \mathrm{C}$ NMR $\left(100 \mathrm{MHz}, \mathrm{CDCl}_{3}\right)$

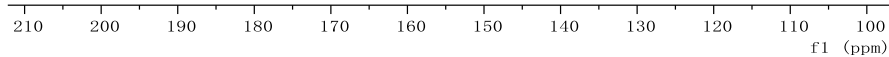


<smiles>CSc1ccc(C(=O)Nc2ccccc2C2=NCCO2)cc1</smiles>

${ }^{1} \mathrm{HNMR}\left(400 \mathrm{MHz}, \mathrm{CDCl}_{3}\right)$

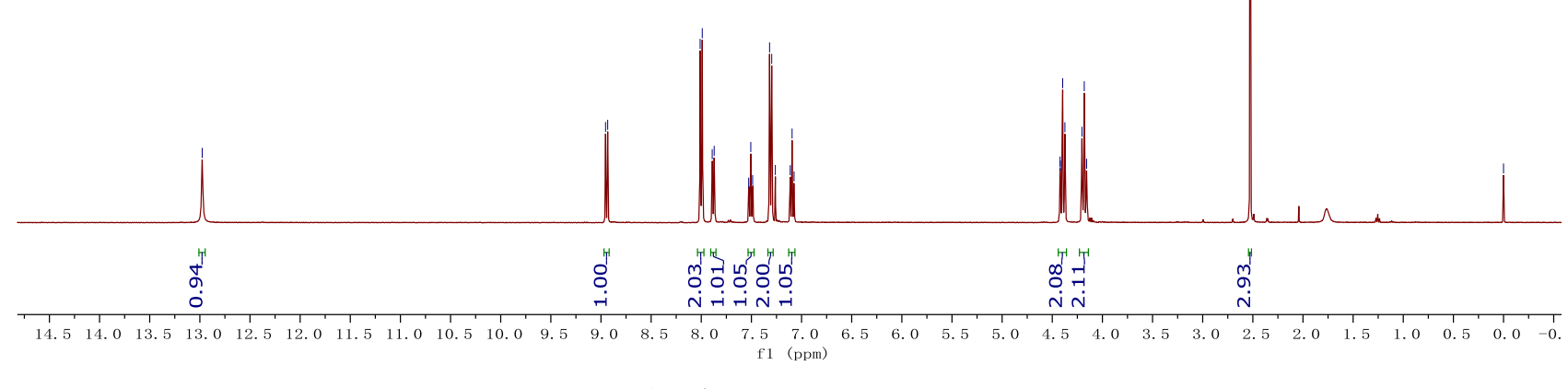

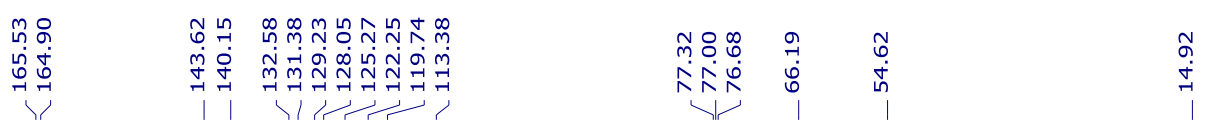

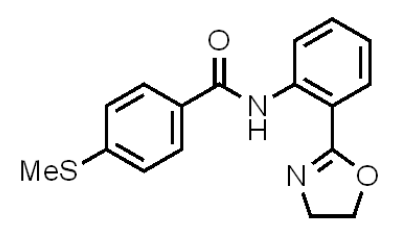

1h

${ }^{13} \mathrm{C}$ NMR $\left(100 \mathrm{MHz}, \mathrm{CDCl}_{3}\right.$ )
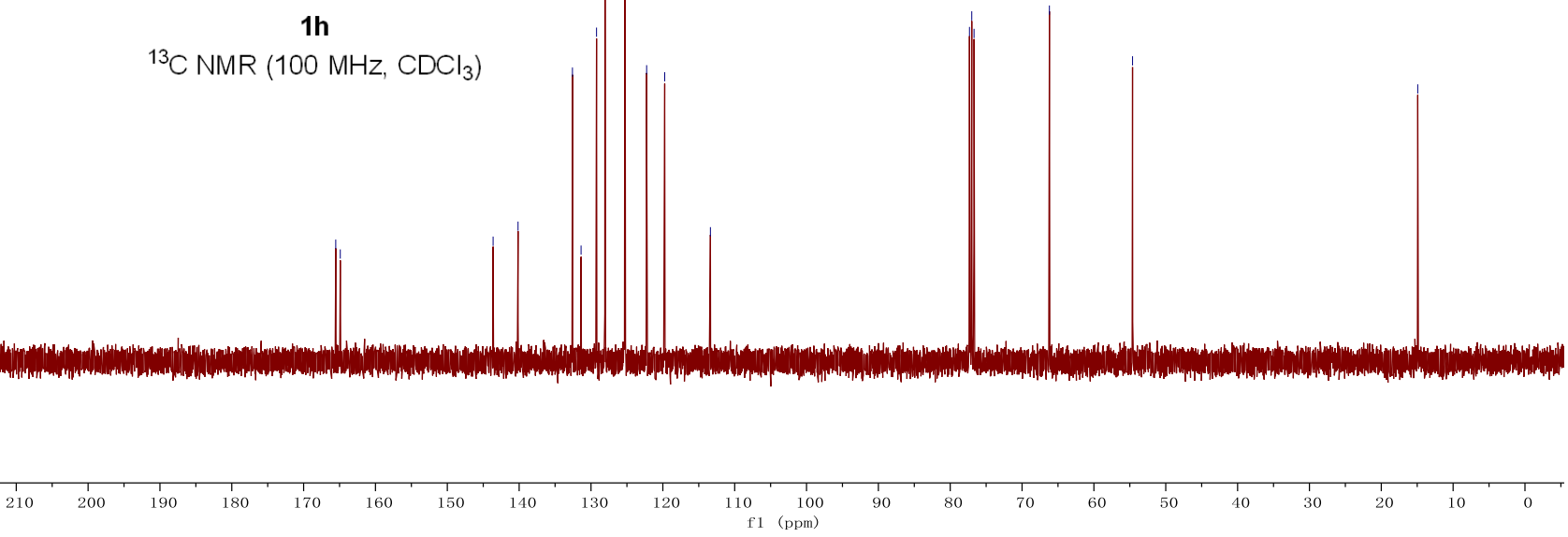
<smiles>CCNc1ccc(C(=O)Nc2ccccc2C2=NCCO2)cc1</smiles>

$1 \mathrm{i}$

${ }^{1} \mathrm{HNMR}\left(400 \mathrm{MHz}, \mathrm{CDCl}_{3}\right)$

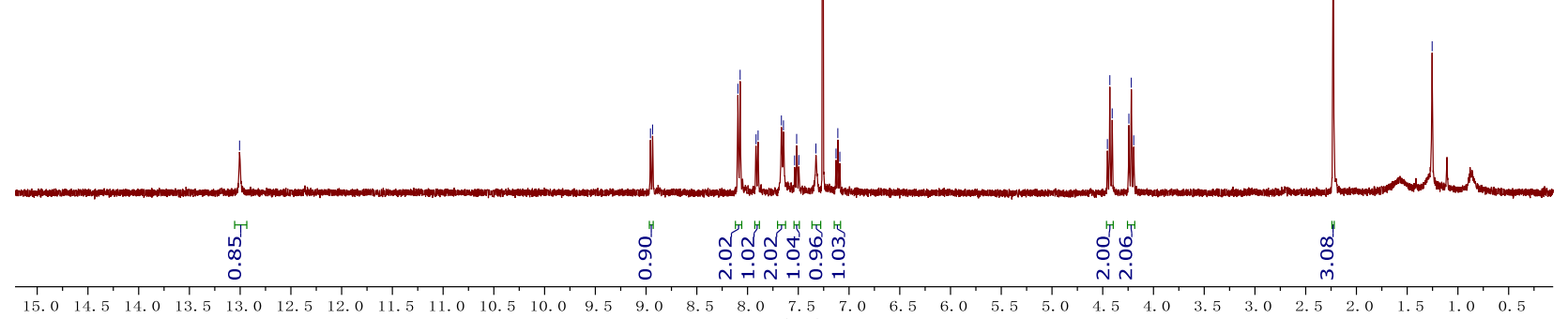

\begin{tabular}{llllllllllllllllllll} 
& & & \\
\hline
\end{tabular}

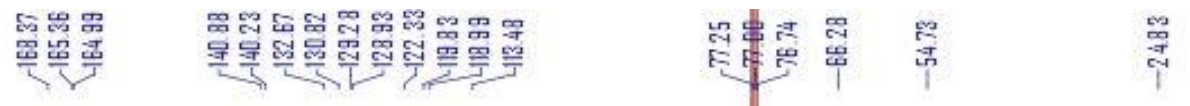<smiles>CNc1ccc(C(=O)Nc2ccccc2C2=NCCO2)cc1</smiles>
$1 \mathrm{i}$

${ }^{13} \mathrm{C}$ NMR $\left(125 \mathrm{MHz}, \mathrm{CDCl}_{3}\right)$

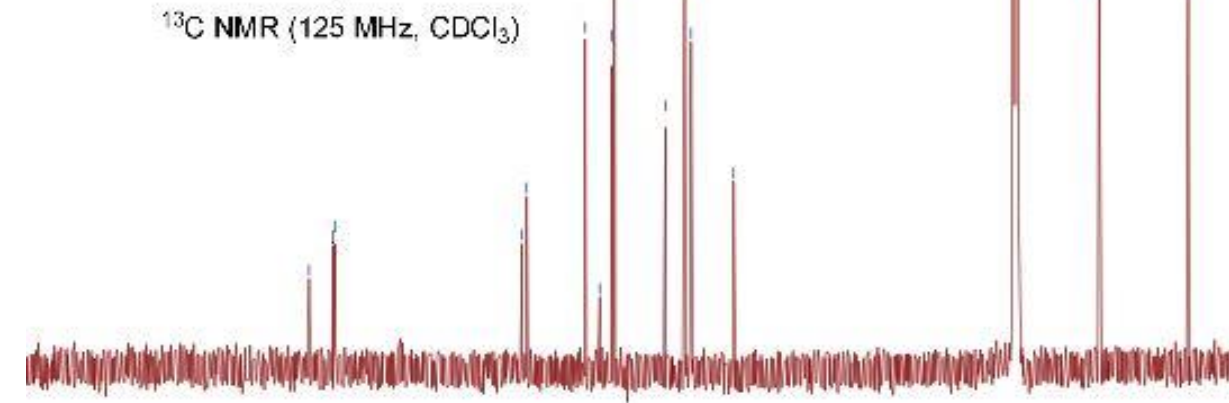
$\begin{array}{lllllllllll}200 & 190 & 150 & 170 & 160 & 150 & 140 & 130 & 120 & 110 & 100\end{array}$ 

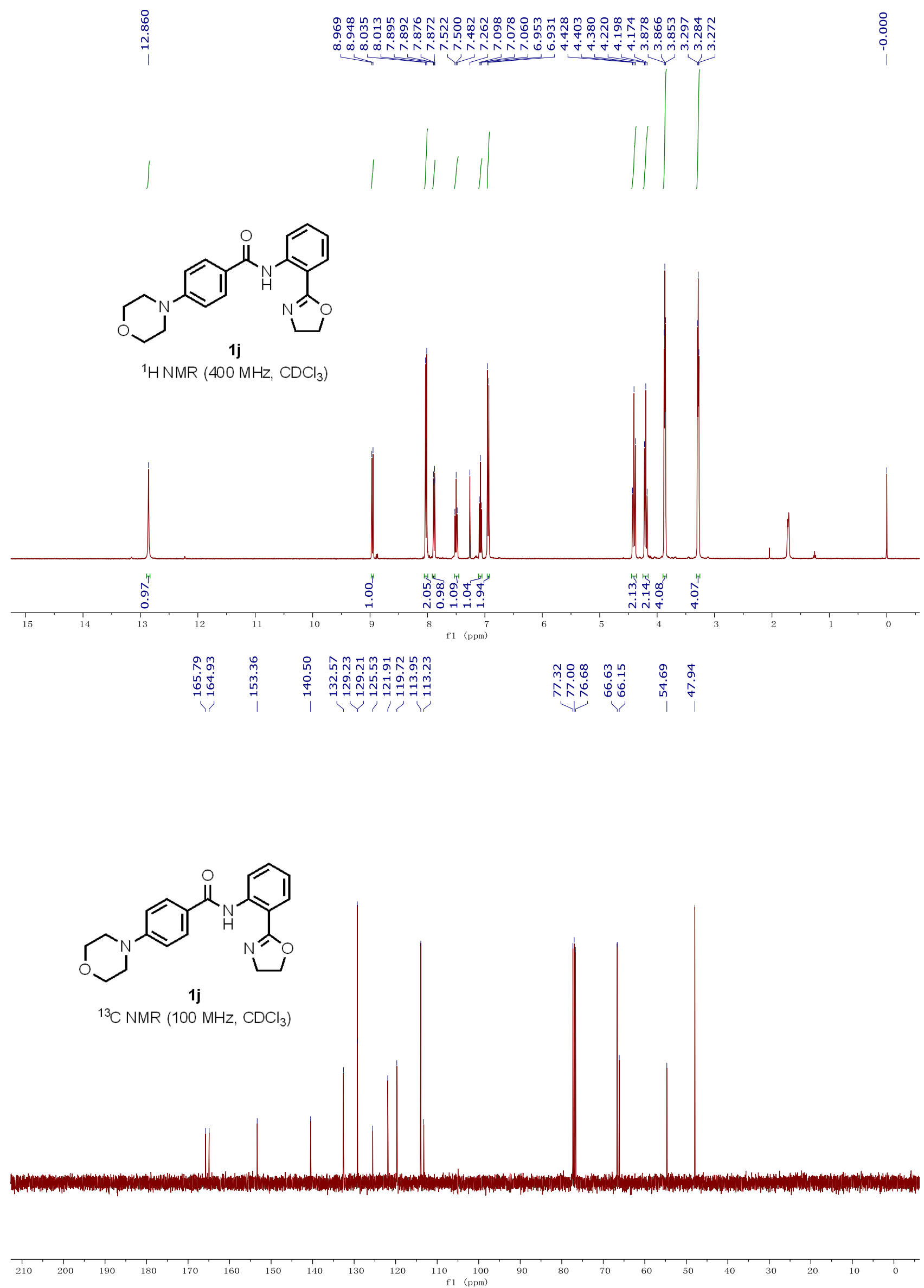
<smiles>C1CCC1</smiles>

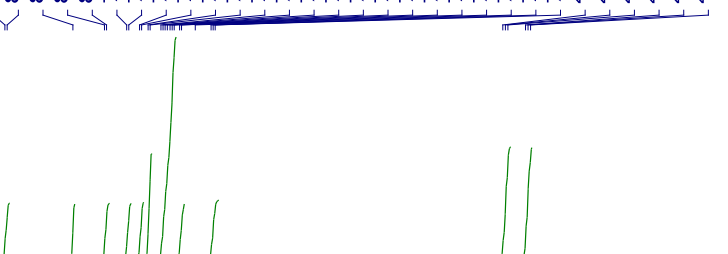<smiles>O=C(Nc1ccccc1C1=NCCO1)c1cccc(-c2ccccc2)c1</smiles>

1n

${ }^{1} \mathrm{HNMR}\left(400 \mathrm{MHz}, \mathrm{CDCl}_{3}\right)$

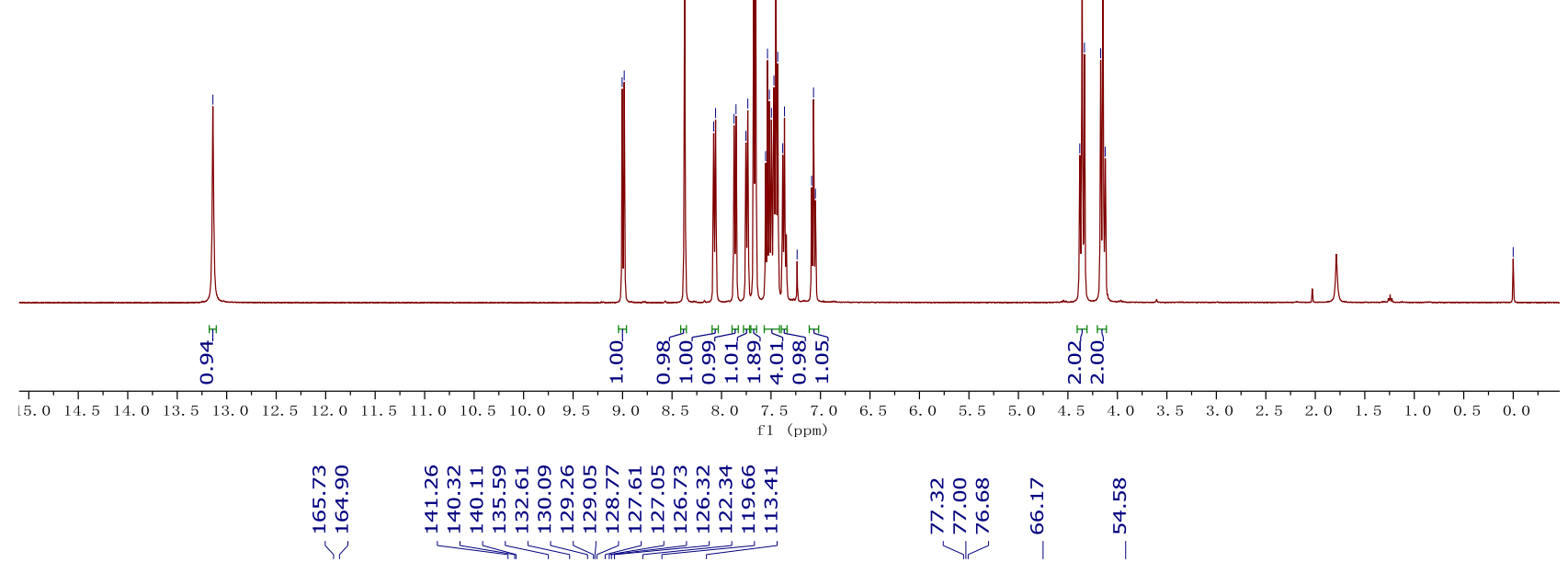<smiles>O=C(Nc1ccccc1C1=NCCO1)c1cccc(-c2ccccc2)c1</smiles>

1n

${ }^{13} \mathrm{C} \mathrm{NMR}\left(100 \mathrm{MHz}, \mathrm{CDCl}_{3}\right)$

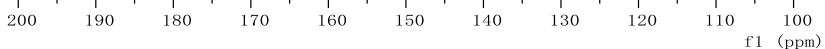



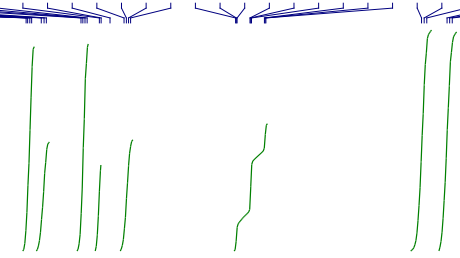<smiles>CC(F)(F)Oc1cccc(C(=O)Nc2ccccc2C2=NCCO2)c1</smiles>

$1 \mathrm{p}$

${ }^{1} \mathrm{HNMR}\left(400 \mathrm{MHz}, \mathrm{CDCl}_{3}\right)$
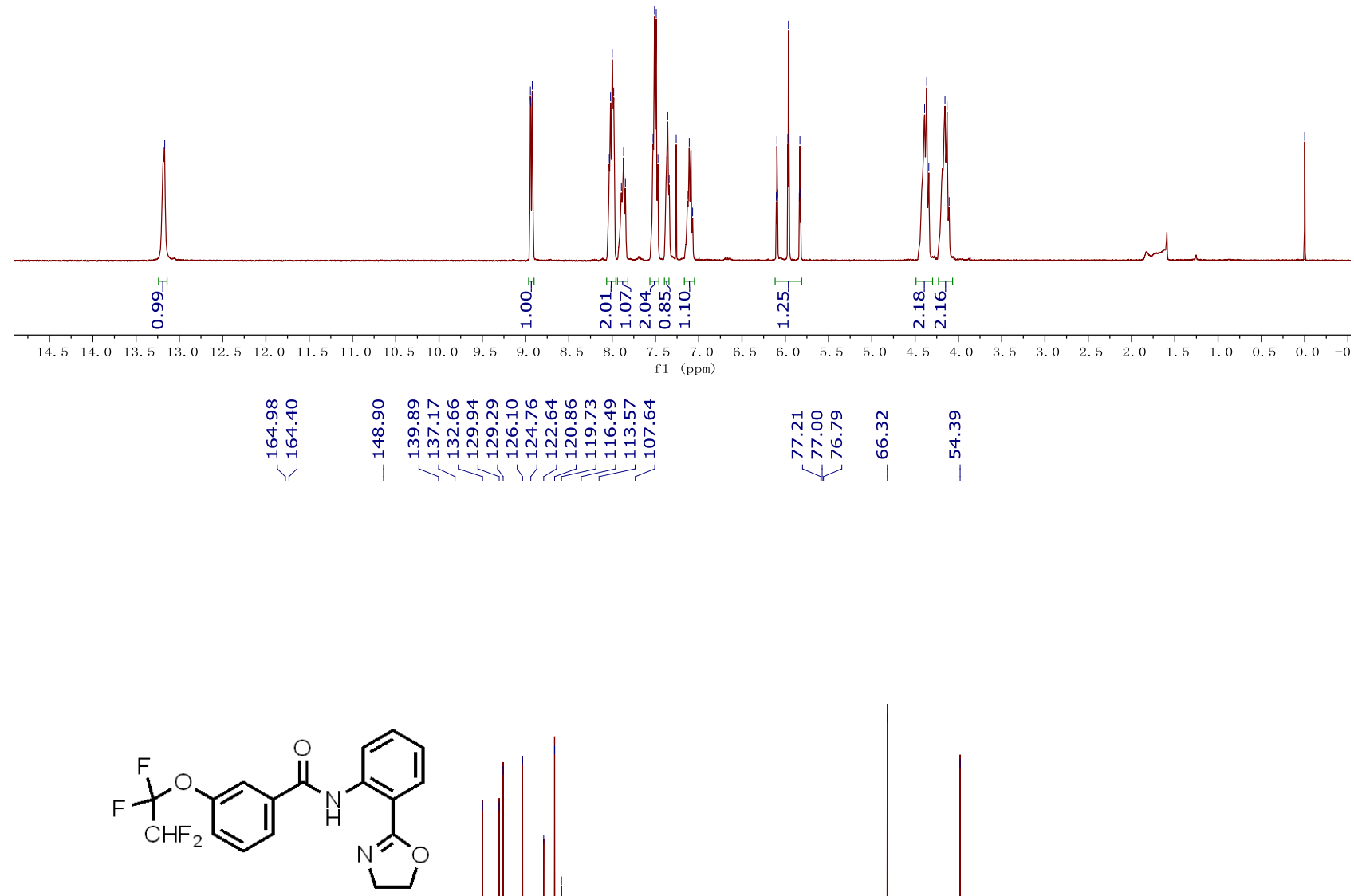

$1 \mathrm{p}$

${ }^{13} \mathrm{C} \mathrm{NMR}(150 \mathrm{MHz}$, decoupled for $\left.{ }^{19} \mathrm{~F}, \mathrm{CDCl}_{3}\right)$

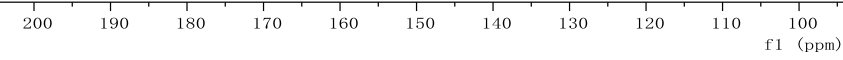



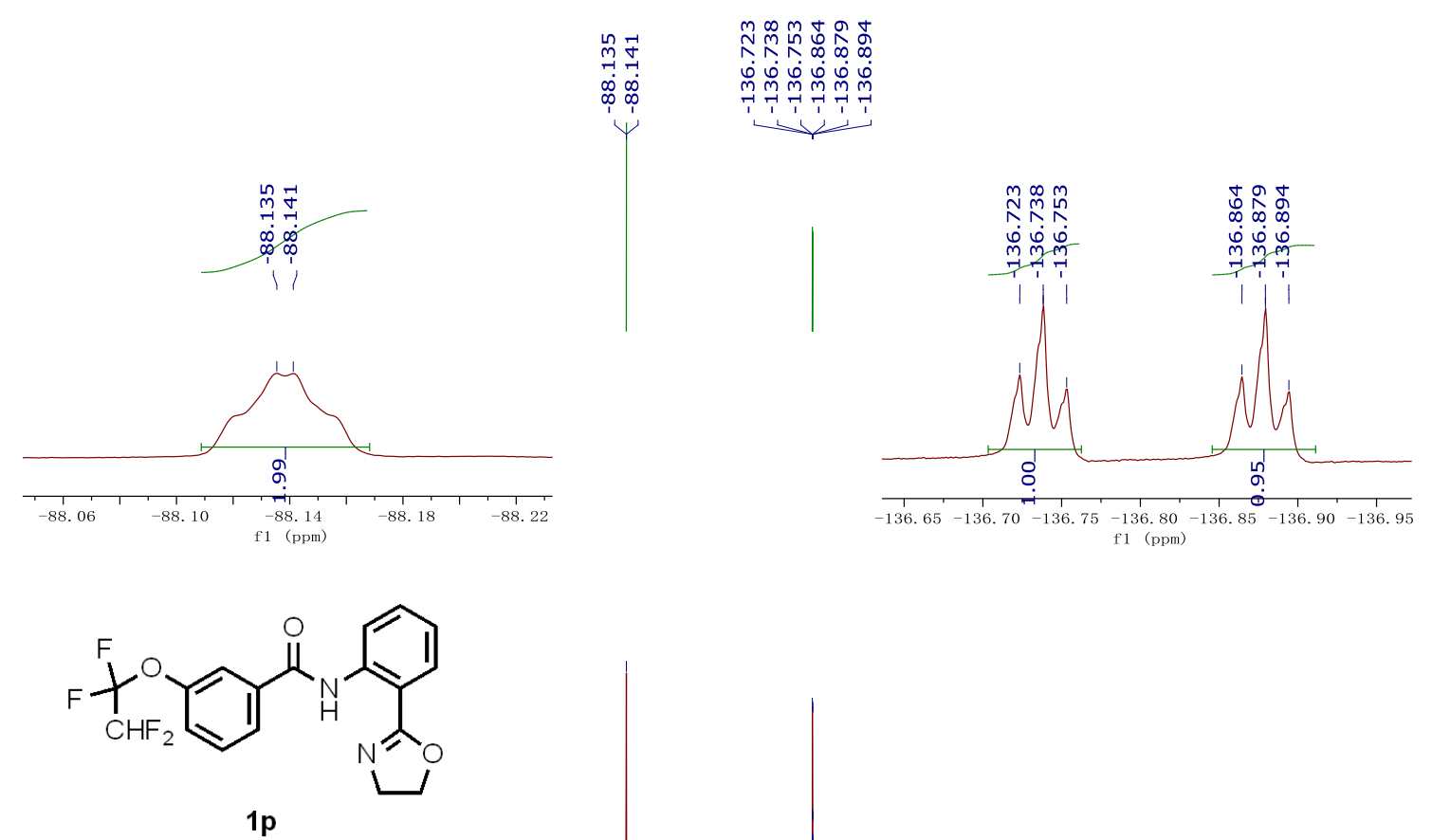

${ }^{19} \mathrm{~F} \mathrm{NMR}\left(375 \mathrm{MHz}, \mathrm{CDCl}_{3}\right)$
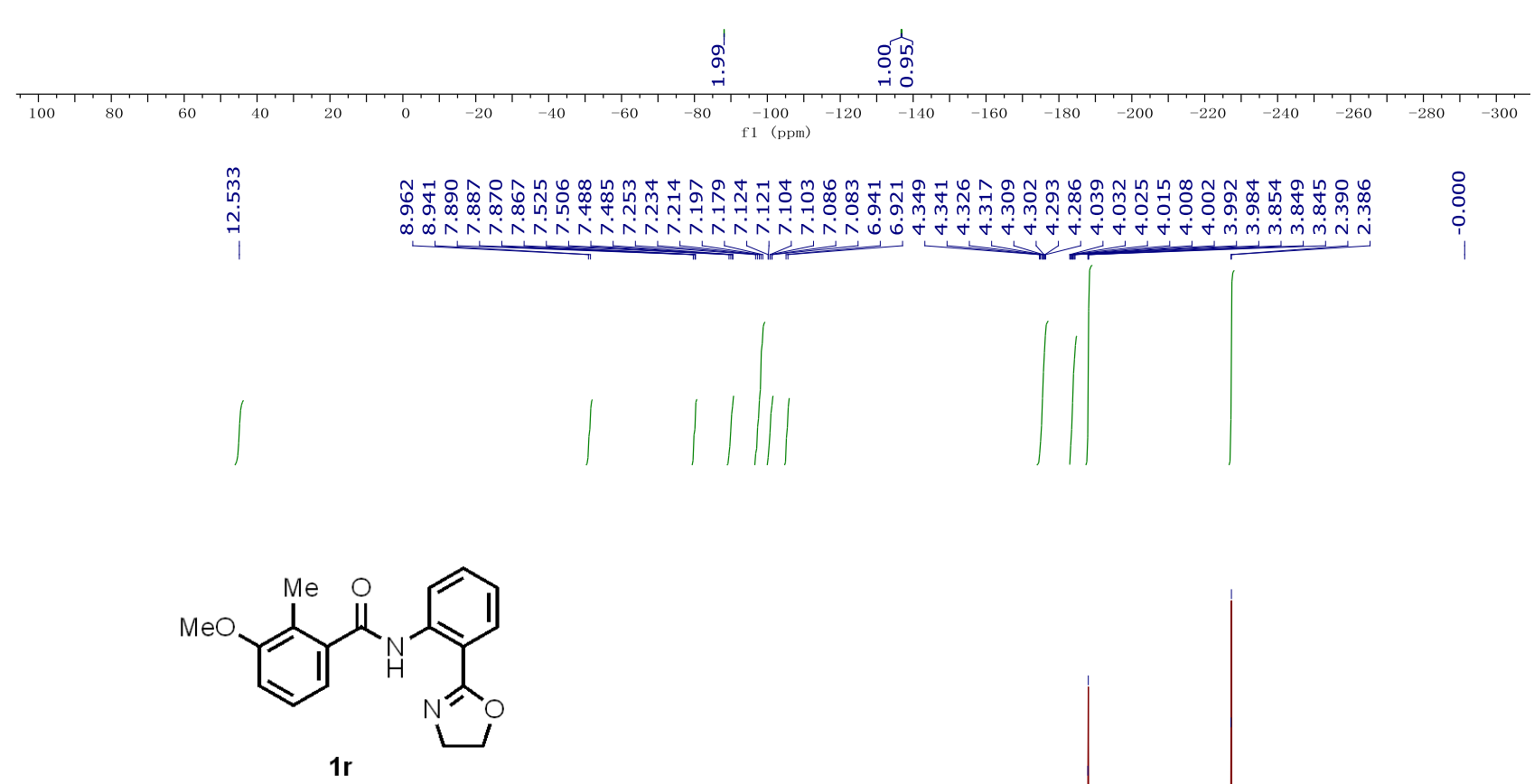

${ }^{1} \mathrm{H} \mathrm{NMR}\left(400 \mathrm{MHz}, \mathrm{CDCl}_{3}\right)$

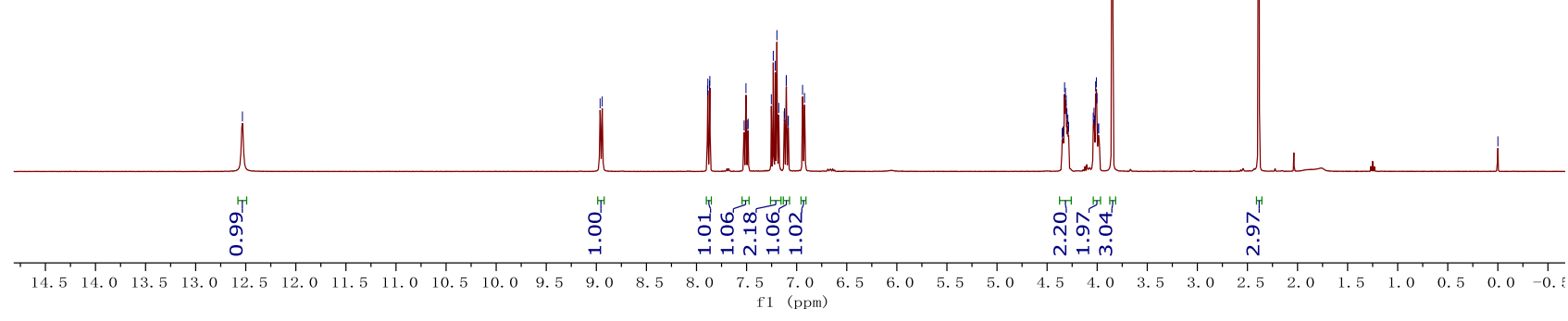




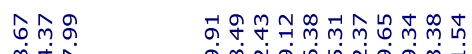

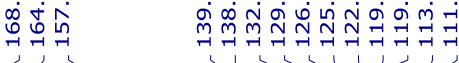

m்ְ

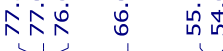

$\underset{\substack{\text { N } \\ \stackrel{n}{N}}}{1}$

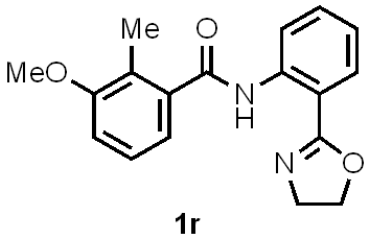

${ }^{13} \mathrm{C} \mathrm{NMR}\left(100 \mathrm{MHz}, \mathrm{CDCl}_{3}\right)$
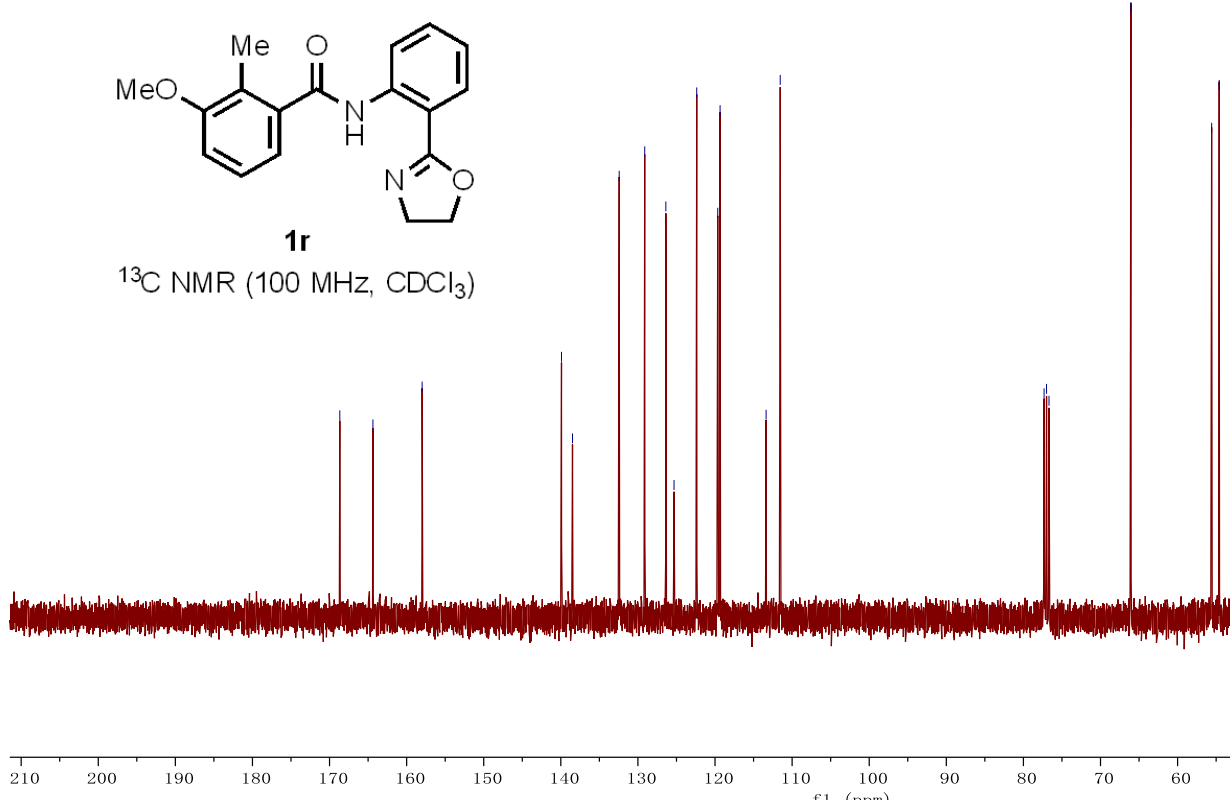

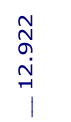

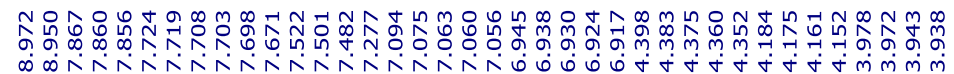
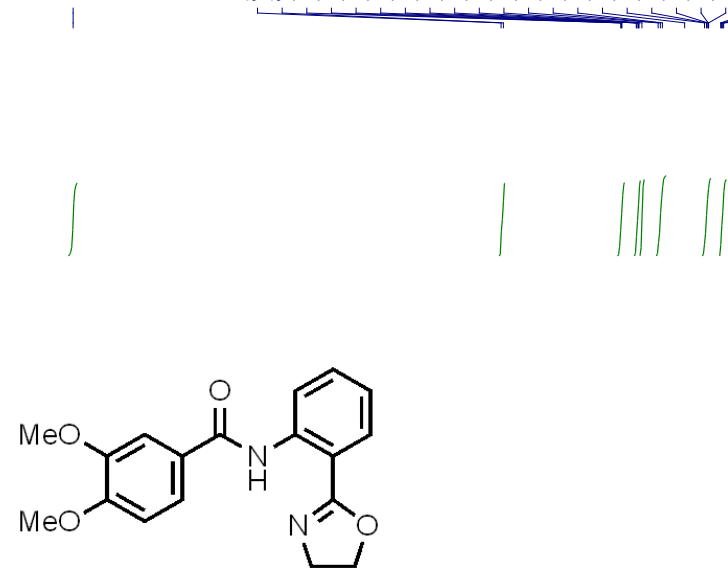

$1 \mathrm{~s}$

${ }^{1} \mathrm{H}$ NMR (400 MHz, $\mathrm{CDCl}_{3}$ )

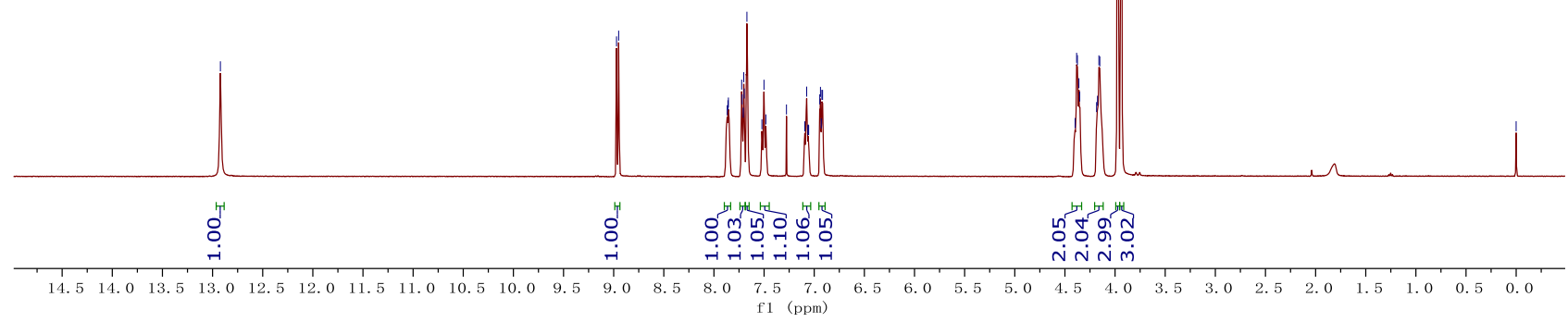




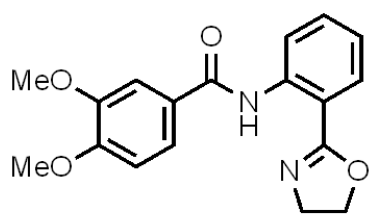

$1 \mathrm{~s}$

${ }^{13} \mathrm{C} \mathrm{NMR}\left(100 \mathrm{MHz}, \mathrm{CDCl}_{3}\right)$
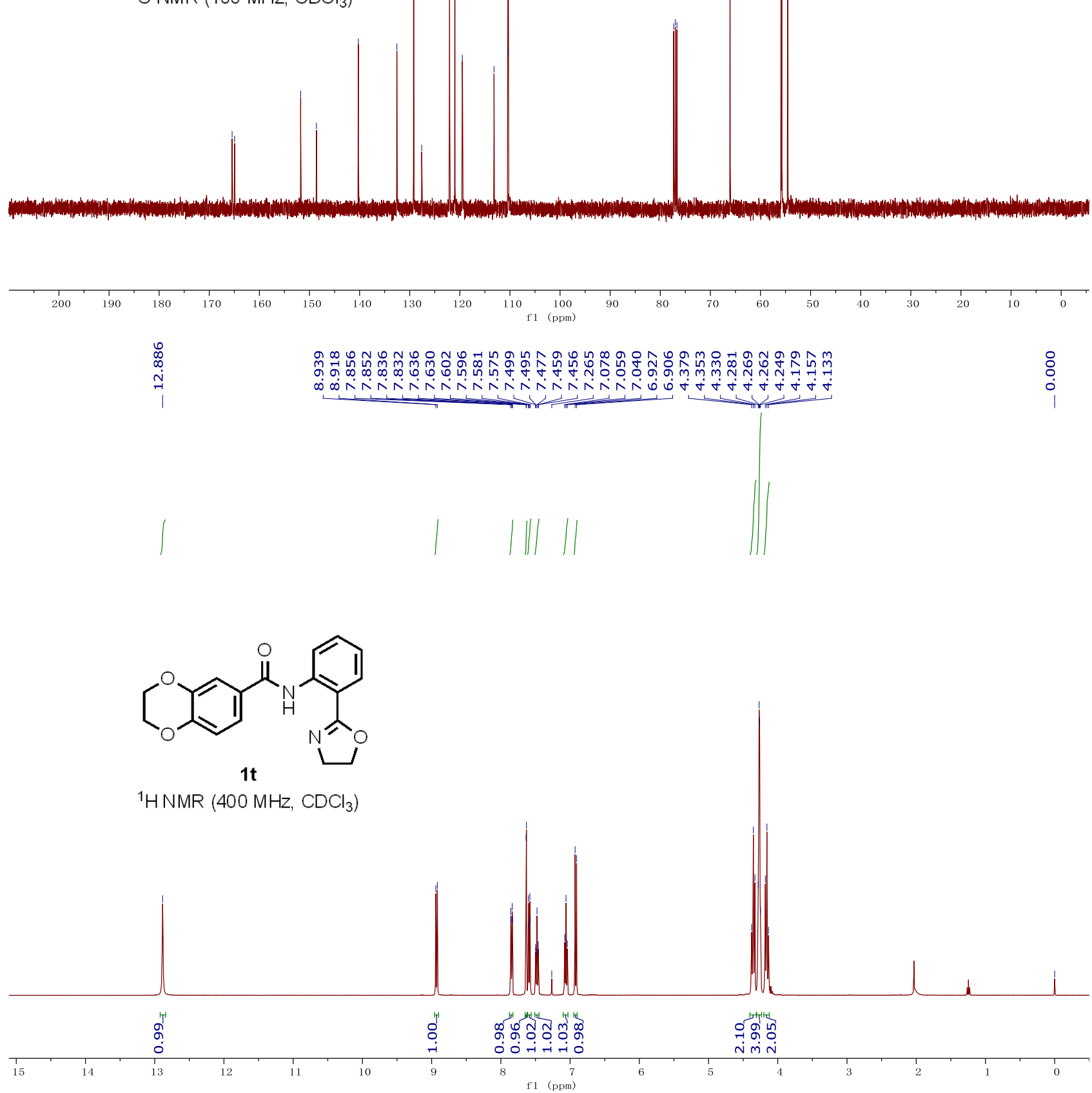


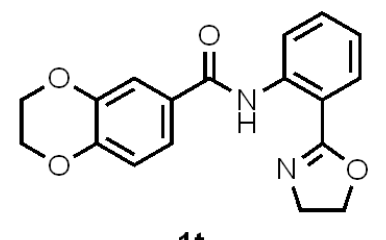

$1 t$

${ }^{13} \mathrm{C} \mathrm{NMR}\left(100 \mathrm{MHz}, \mathrm{CDCl}_{3}\right.$ )

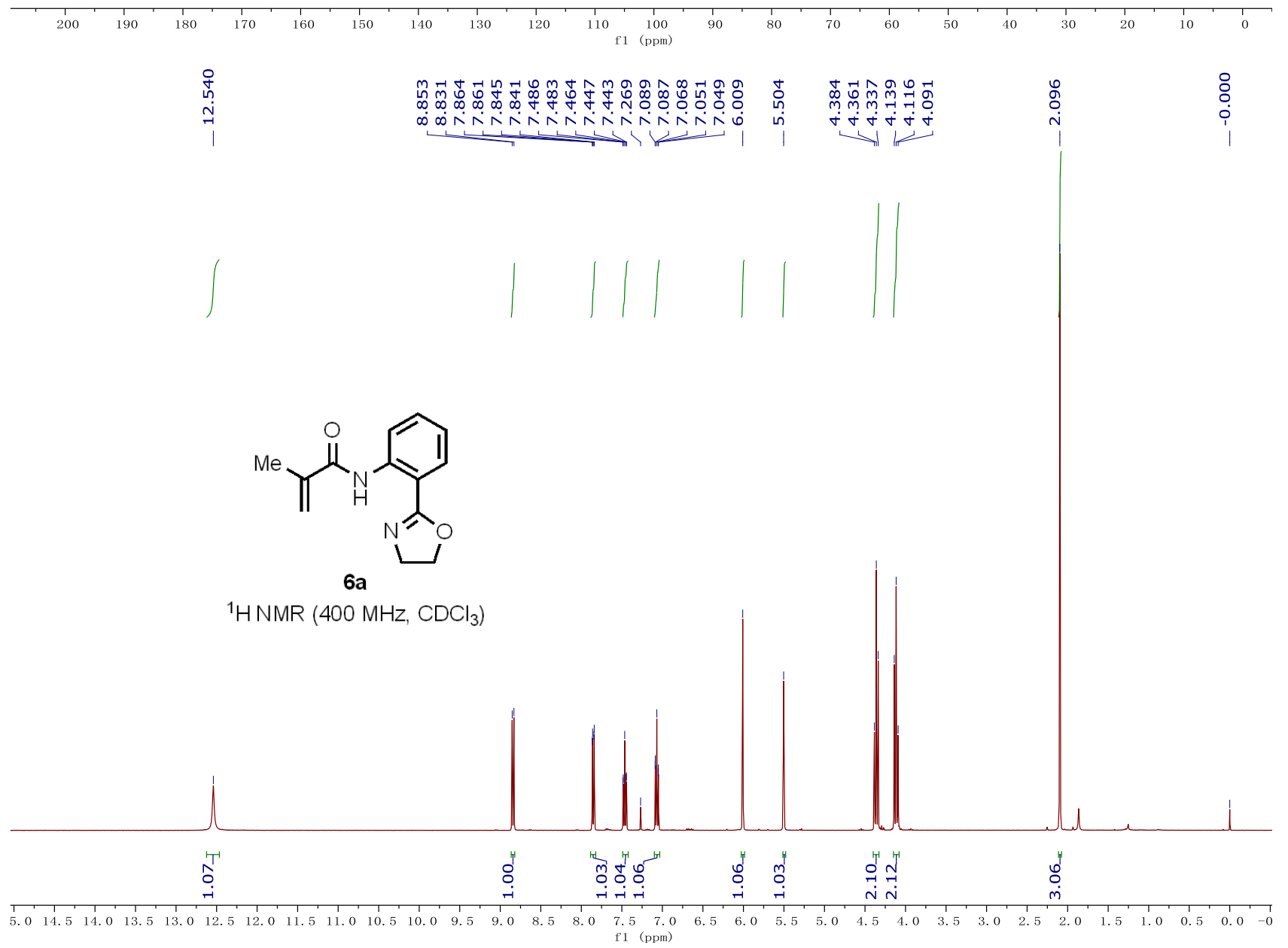




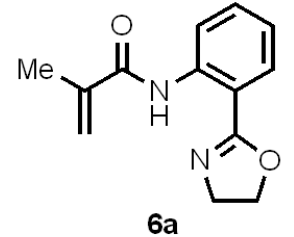

${ }^{13} \mathrm{C}$ NMR $\left(100 \mathrm{MHz}, \mathrm{CDCl}_{3}\right)$

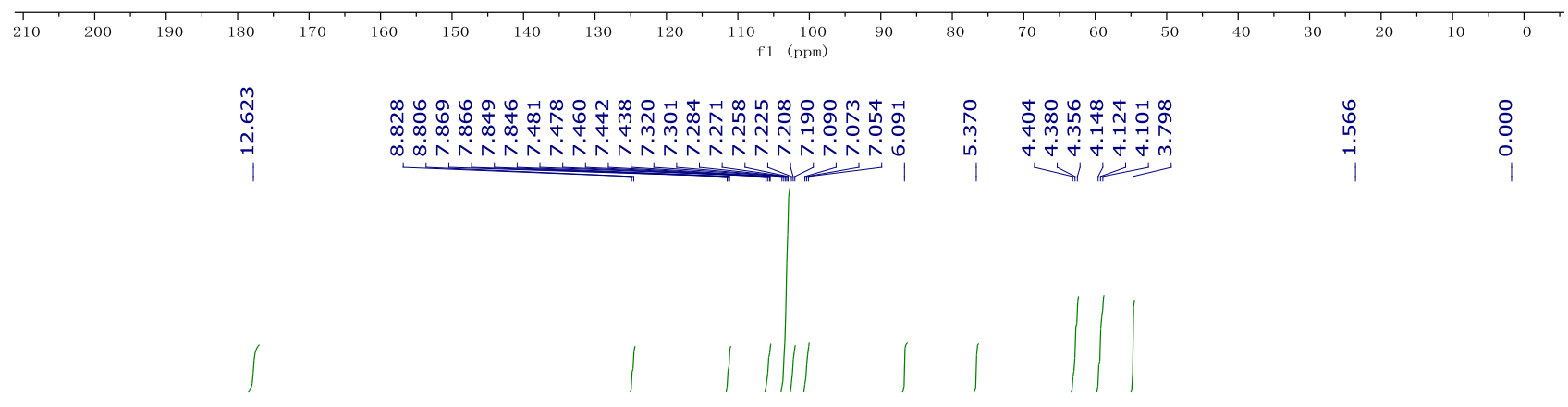<smiles>C=C(Cc1ccccc1)C(=O)Nc1ccccc1C1=NCCO1</smiles>

$6 b$

${ }^{1} \mathrm{HNMR}\left(400 \mathrm{MHz}, \mathrm{CDCl}_{3}\right)$

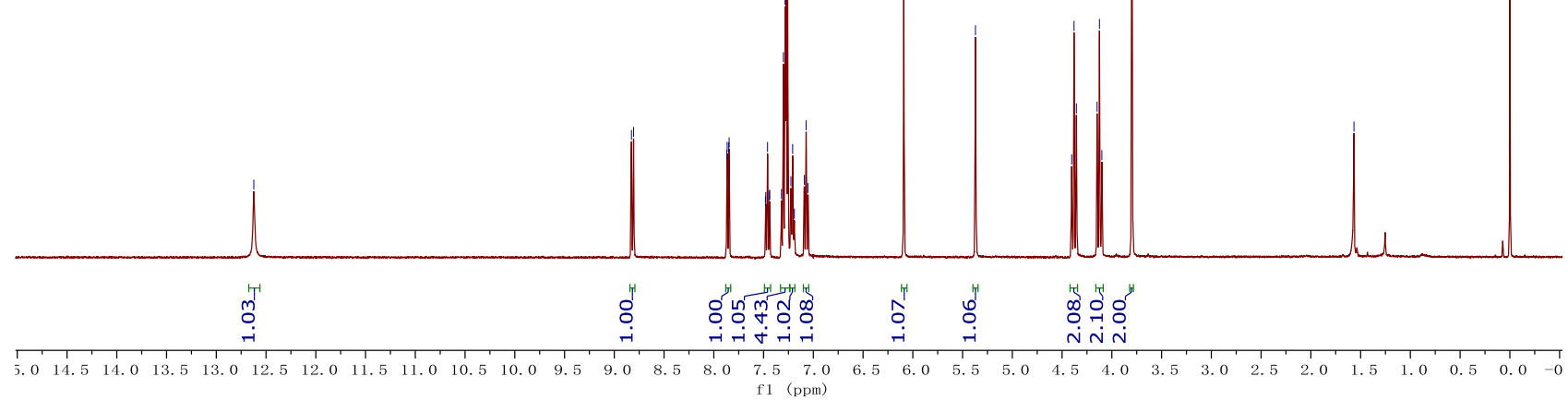




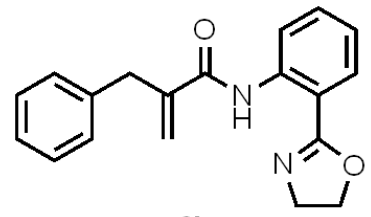

6b

${ }^{13} \mathrm{C}$ NMR (100 MHz, $\mathrm{CDCl}_{3}$ )
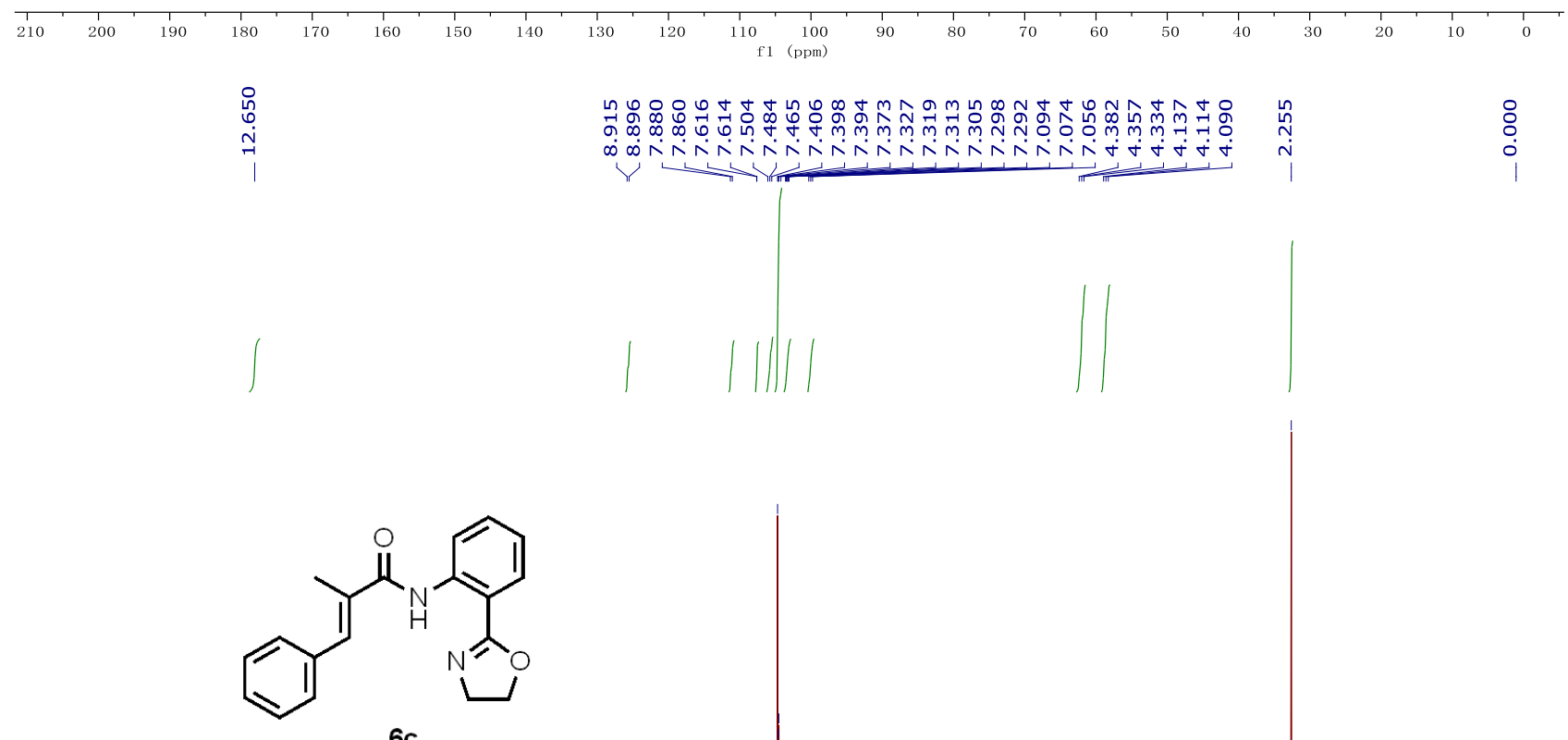

${ }^{1} \mathrm{HNMR}\left(400 \mathrm{MHz}, \mathrm{CDCl}_{3}\right.$ )

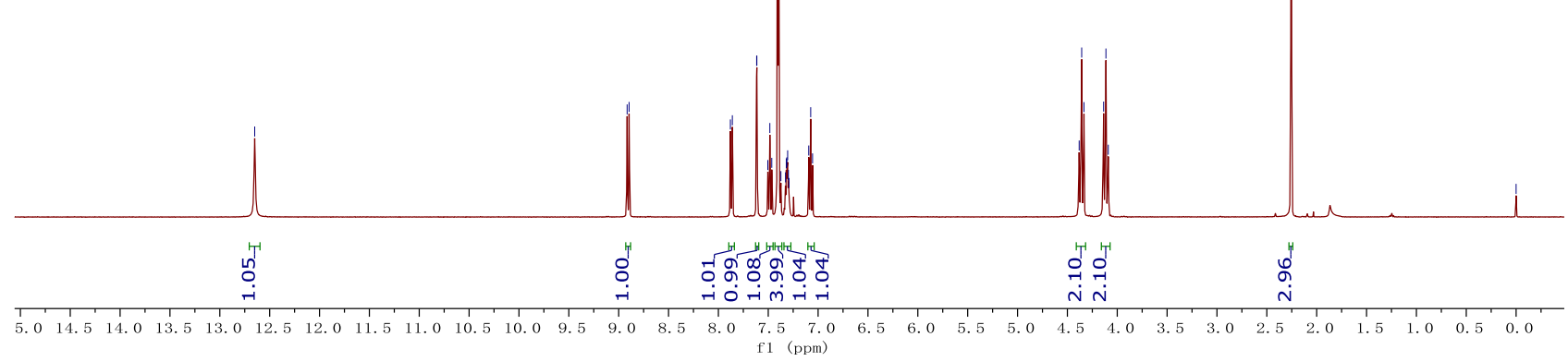




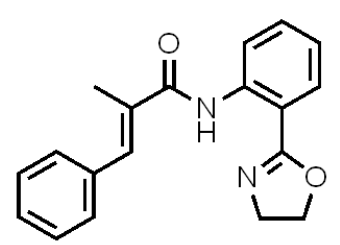

$6 c$

${ }^{13} \mathrm{C}$ NMR $\left(100 \mathrm{MHz}, \mathrm{CDCl}_{3}\right)$
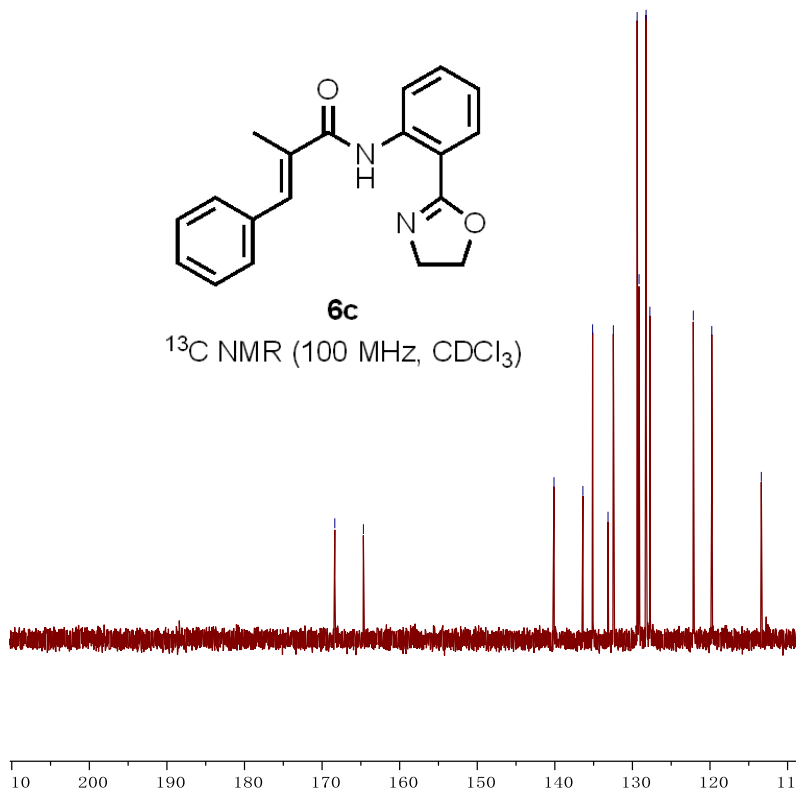

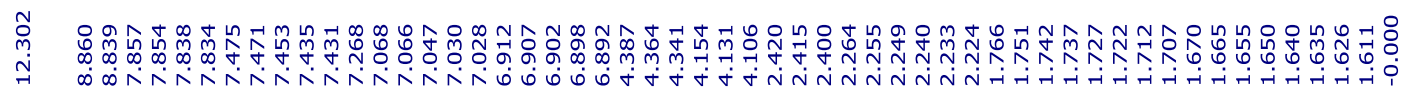

$\infty$
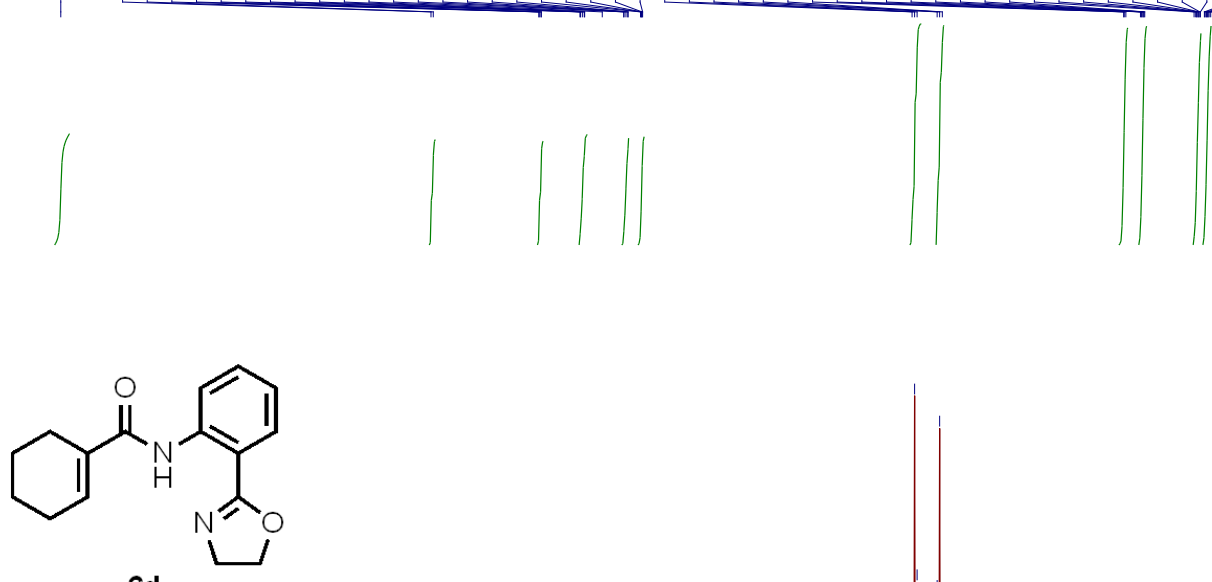

6d

${ }^{1} \mathrm{HNMR}\left(400 \mathrm{MHz}, \mathrm{CDCl}_{3}\right)$

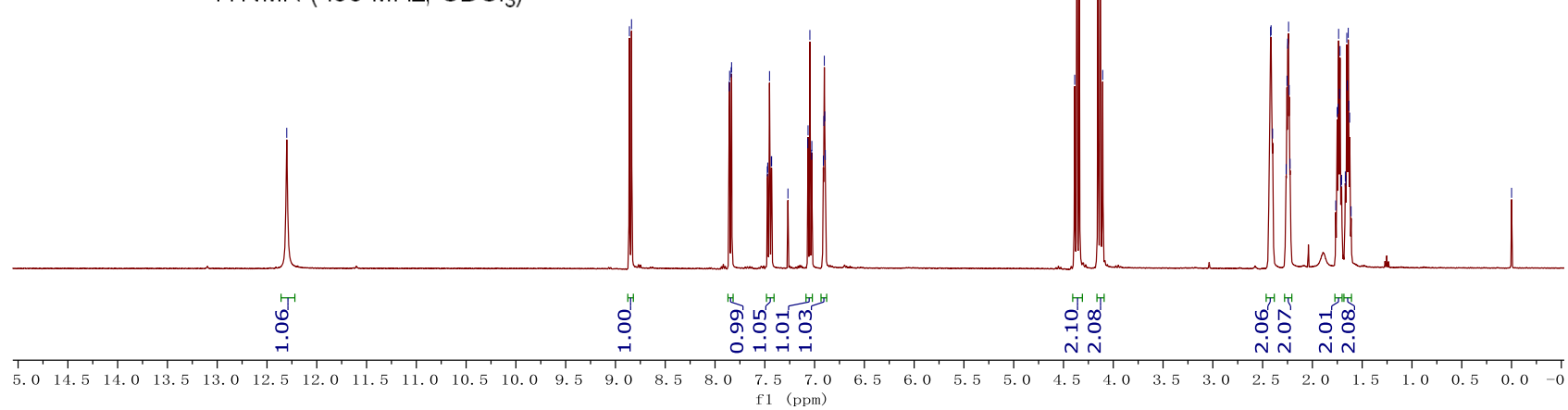




\begin{tabular}{|c|c|c|c|c|}
\hline 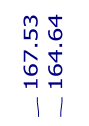 & 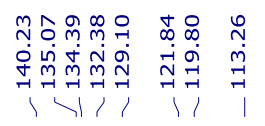 & 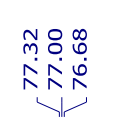 & $\begin{array}{l}\stackrel{0}{0} \\
\dot{0} \\
1 \\
1\end{array}$ & \\
\hline
\end{tabular}
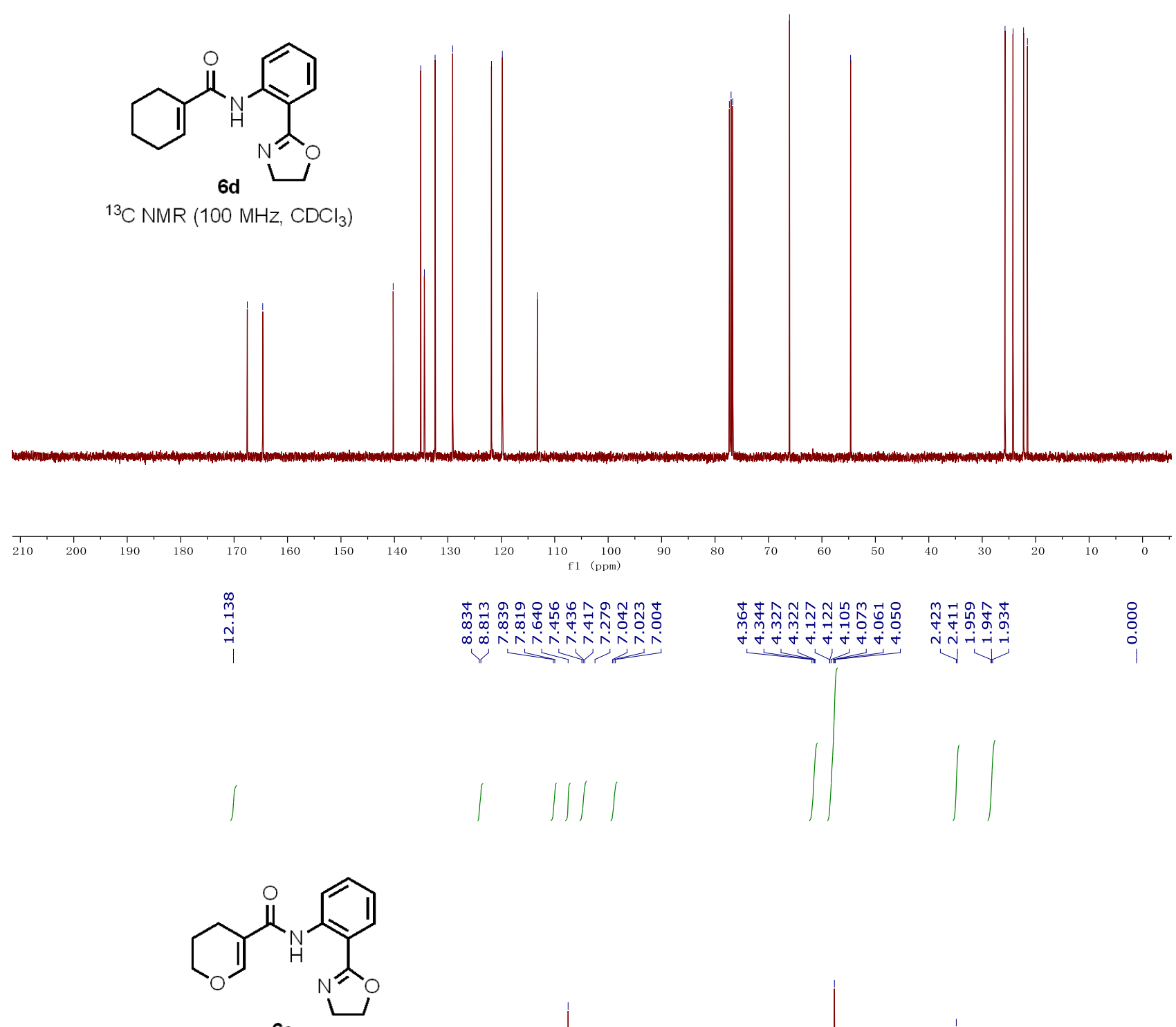

${ }^{1} \mathrm{HNMR}\left(400 \mathrm{MHz}, \mathrm{CDCl}_{3}\right)$

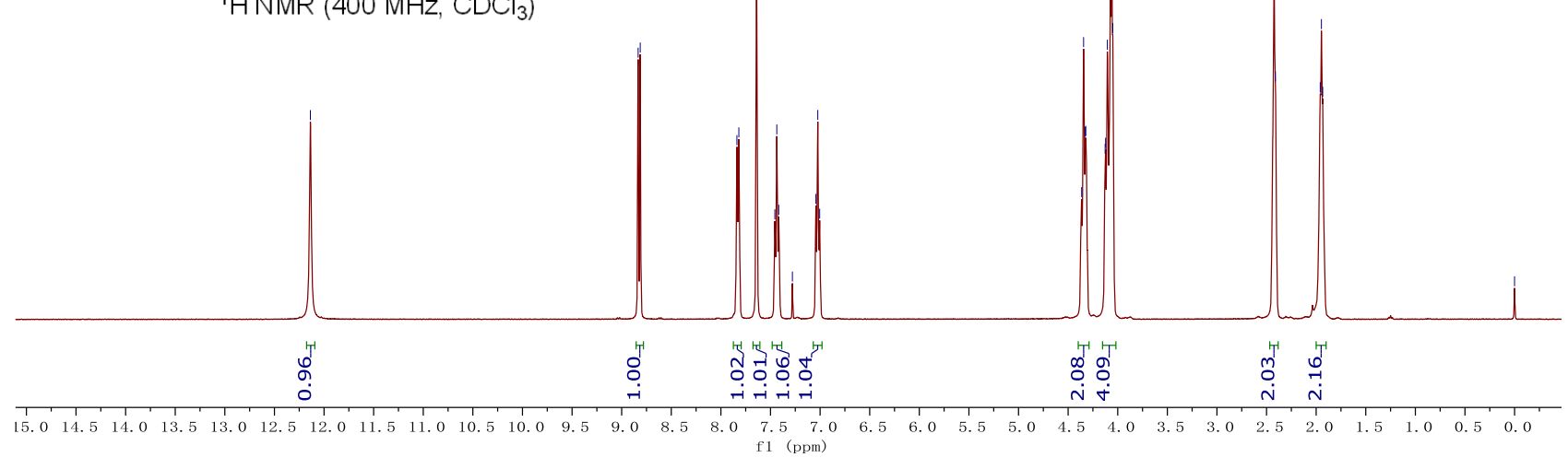




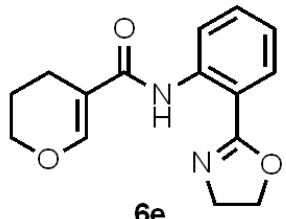

${ }^{13} \mathrm{C} \mathrm{NMR}\left(100 \mathrm{MHz}, \mathrm{CDCl}_{3}\right)$
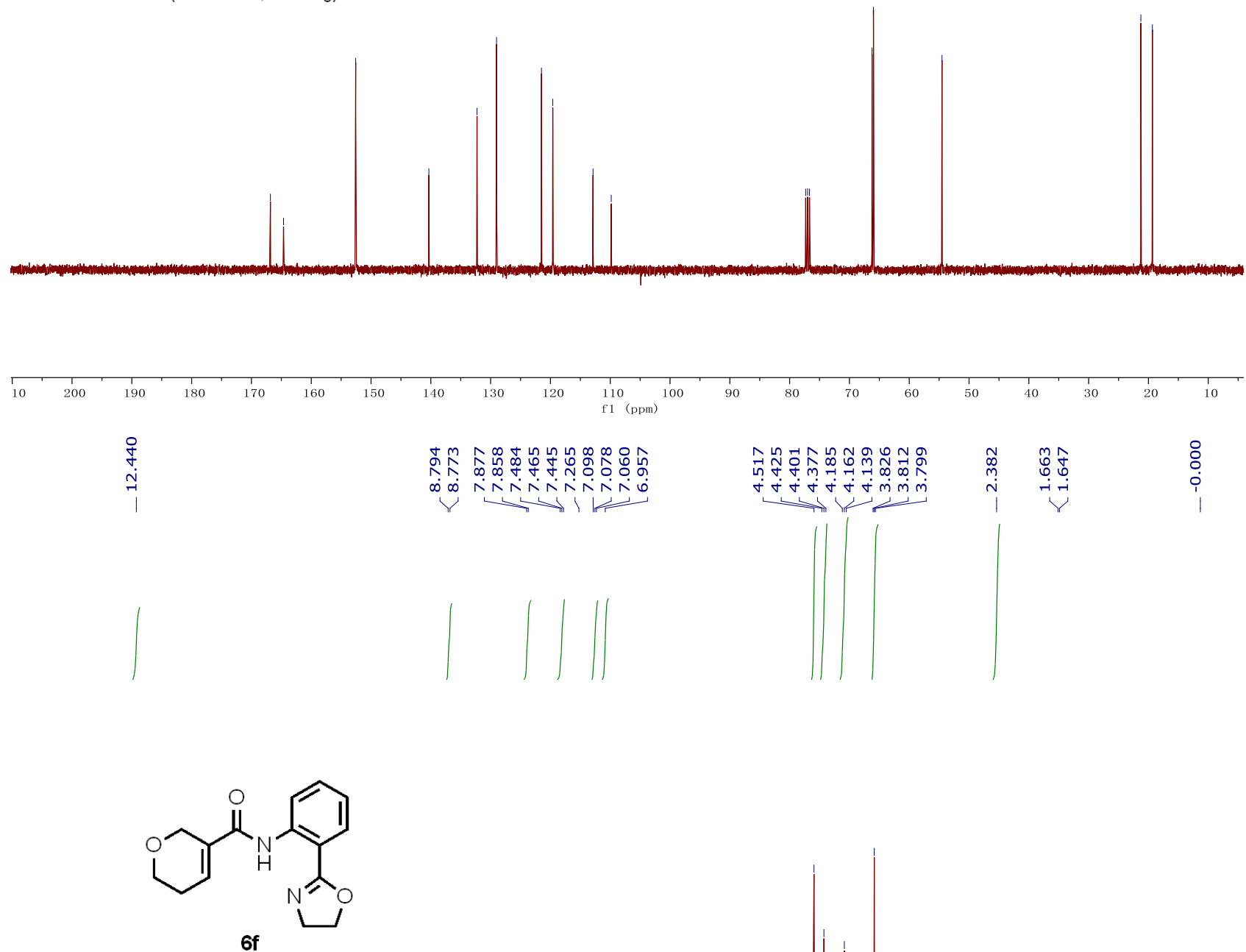

${ }^{1} \mathrm{HNMR}\left(400 \mathrm{MHz}, \mathrm{CDCl}_{3}\right.$ )

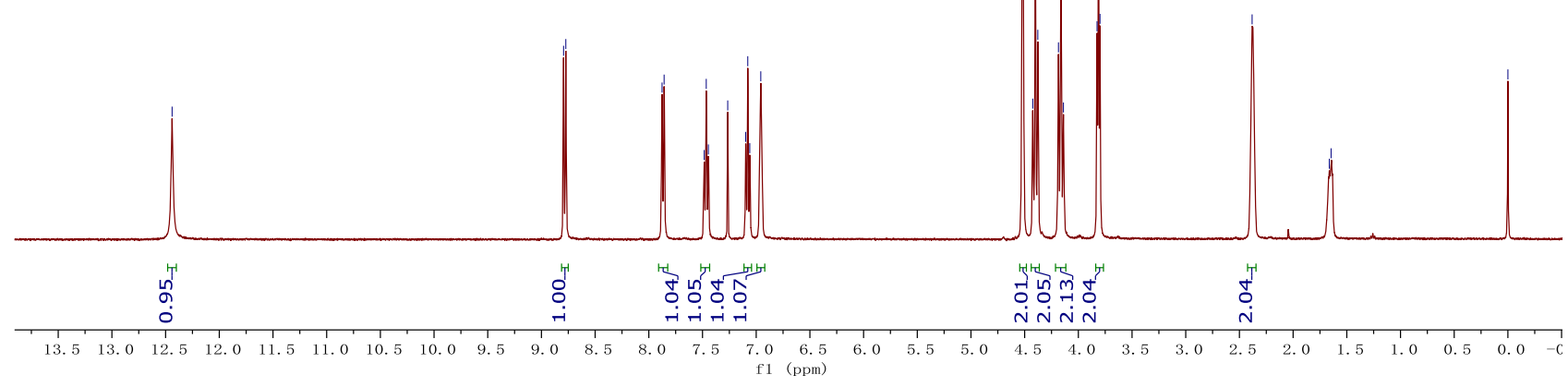




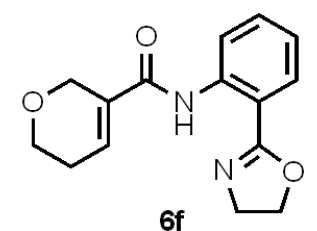

${ }^{13} \mathrm{C} \mathrm{NMR} \mathrm{(100} \mathrm{MHz,} \mathrm{CDCl}_{3}$ )
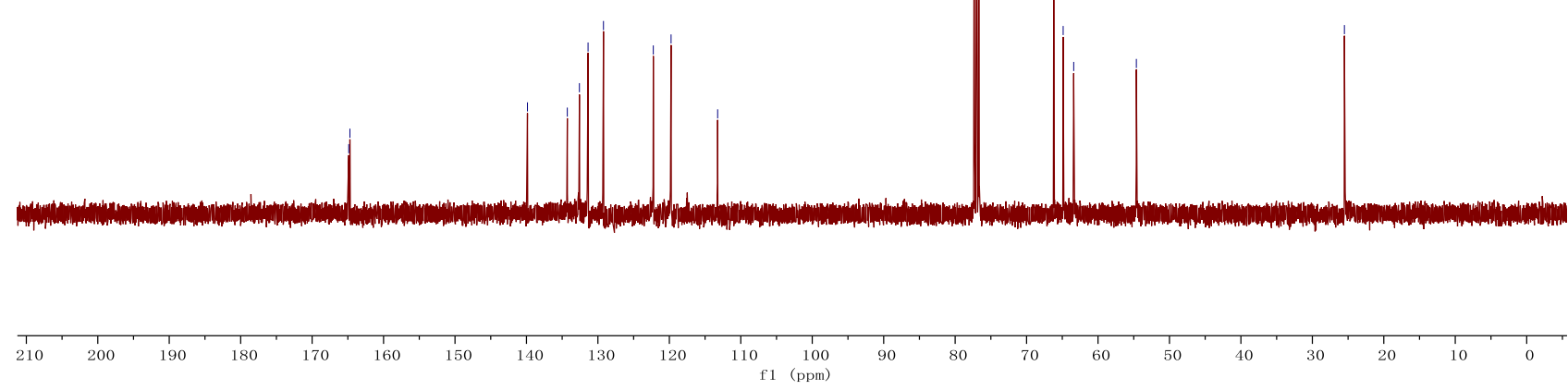

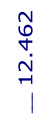

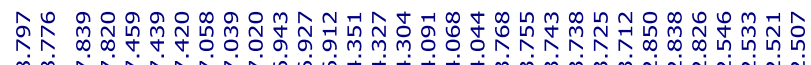

ninninan
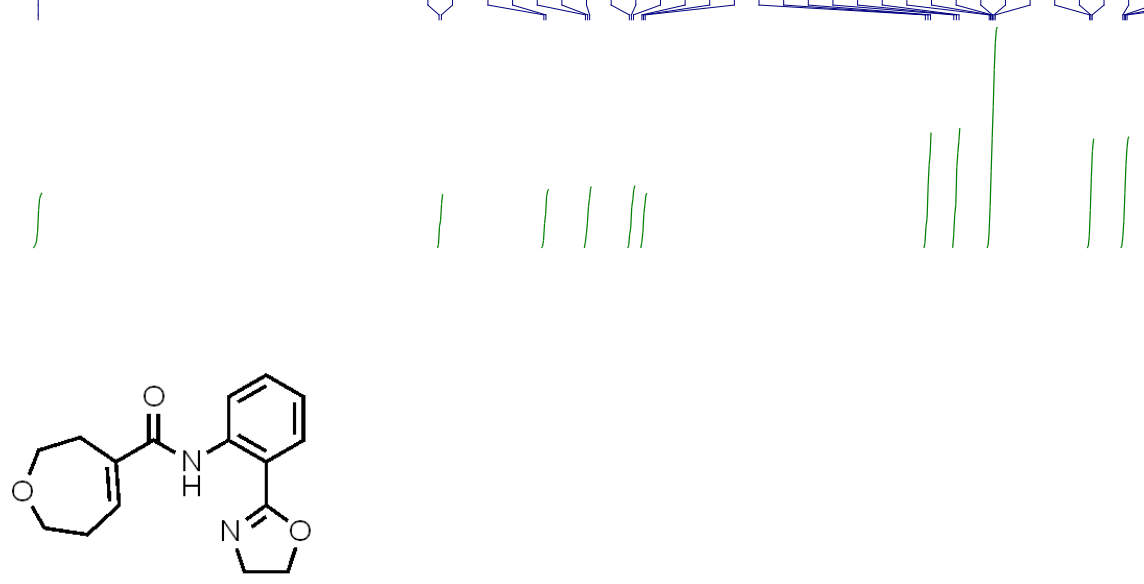

$6 \mathrm{~g}$

${ }^{1} \mathrm{HNMR}\left(400 \mathrm{MHz}, \mathrm{CDCl}_{3}\right)$

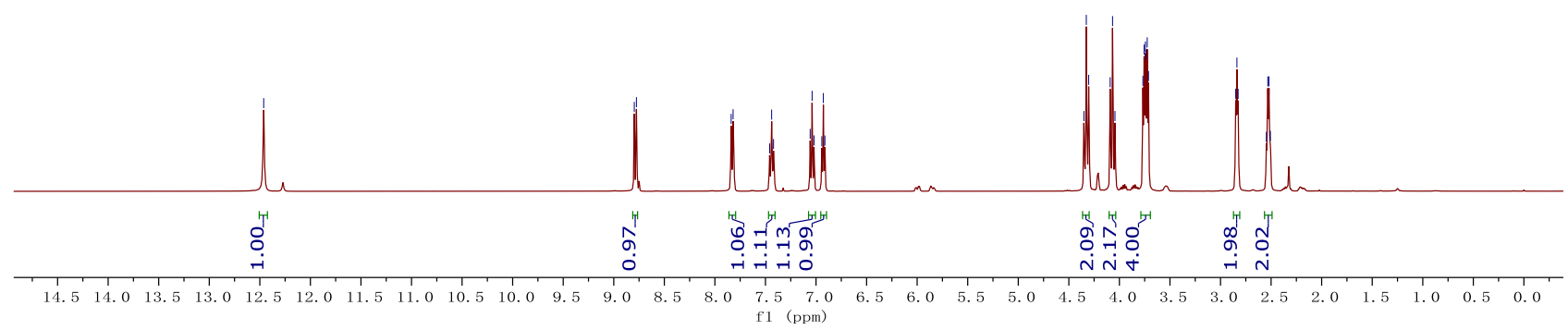




\begin{tabular}{|c|c|c|}
\hline 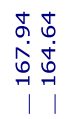 & 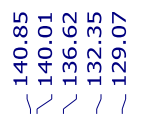 & 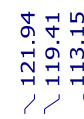 \\
\hline
\end{tabular}

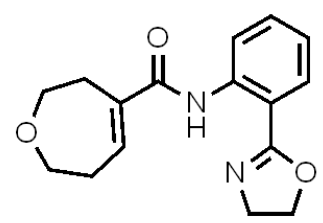

$6 \mathrm{~g}$

${ }^{13} \mathrm{C}$ NMR $\left(100 \mathrm{MHz}, \mathrm{CDCl}_{3}\right)$
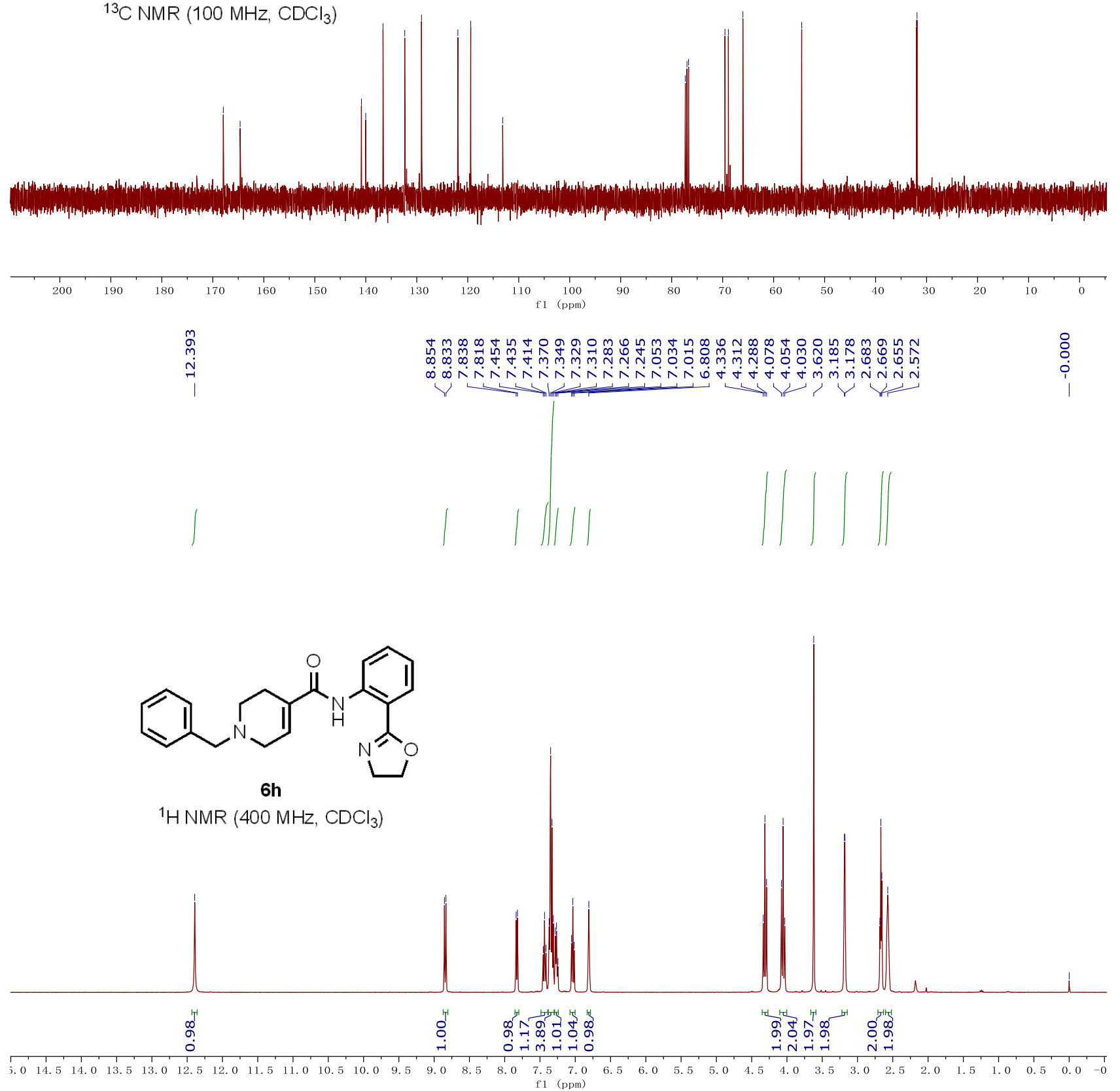


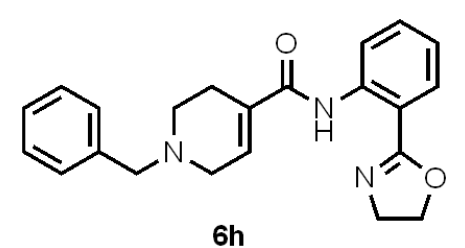

${ }^{13} \mathrm{C} \mathrm{NMR}\left(100 \mathrm{MHz}, \mathrm{CDCl}_{3}\right)$
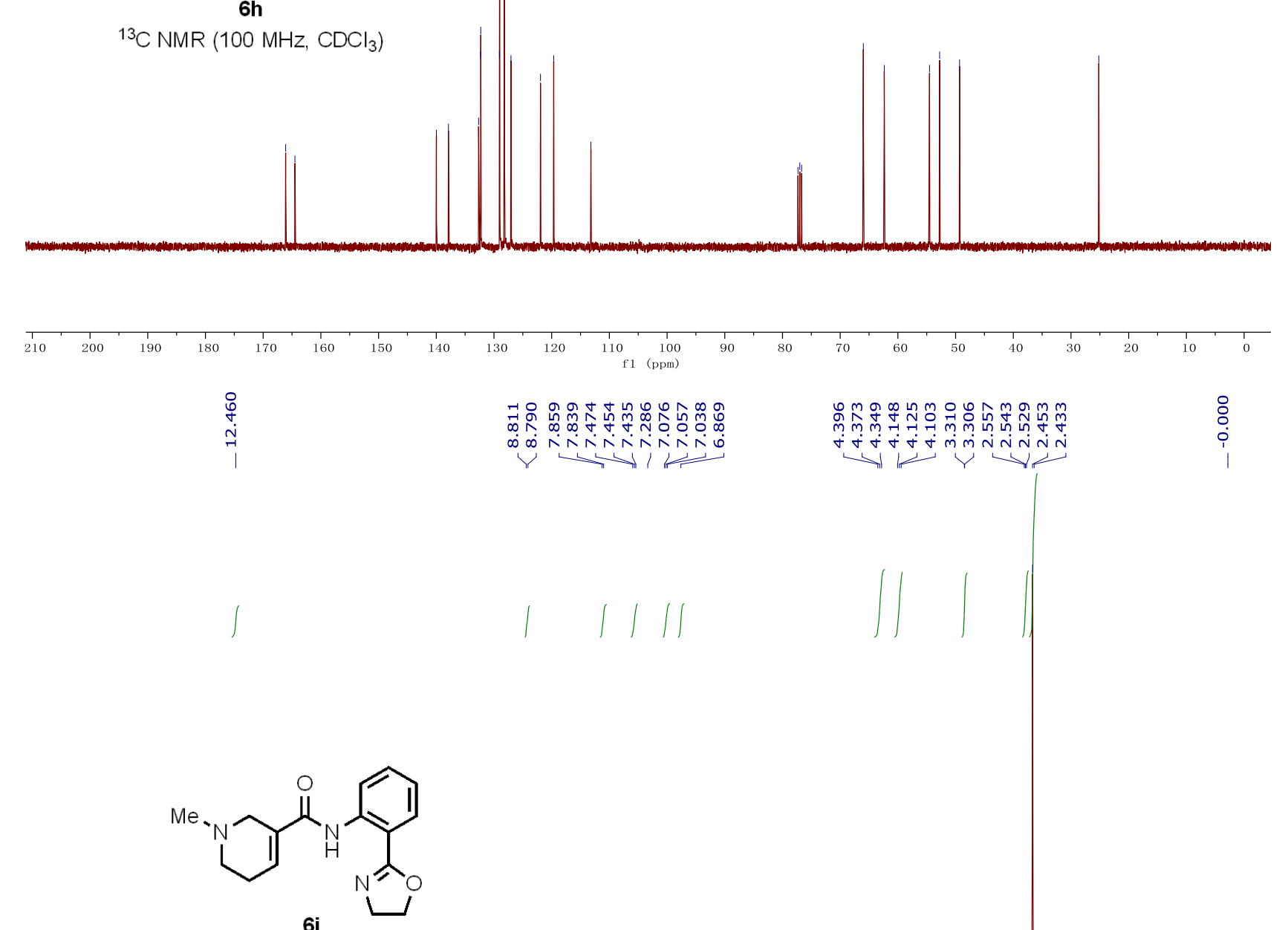

${ }^{1} \mathrm{H}$ NMR $\left(400 \mathrm{MHz}, \mathrm{CDCl}_{3}\right)$

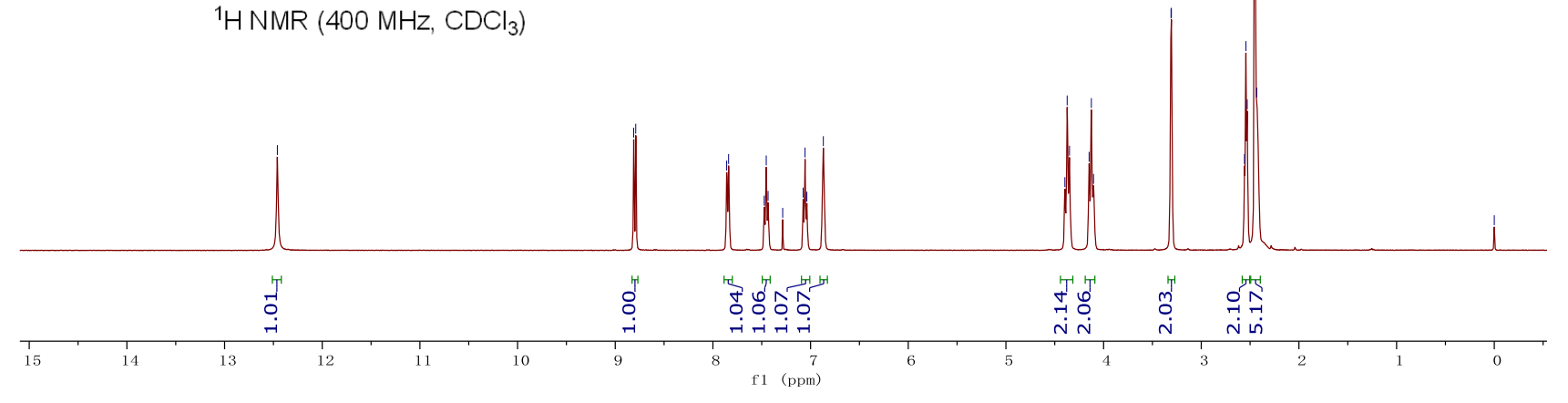




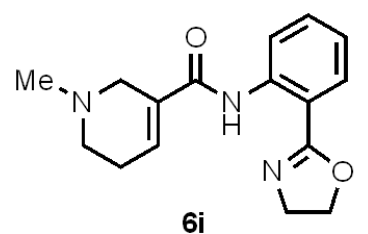

${ }^{13} \mathrm{C} \mathrm{NMR}\left(100 \mathrm{MHz}, \mathrm{CDCl}_{3}\right)$
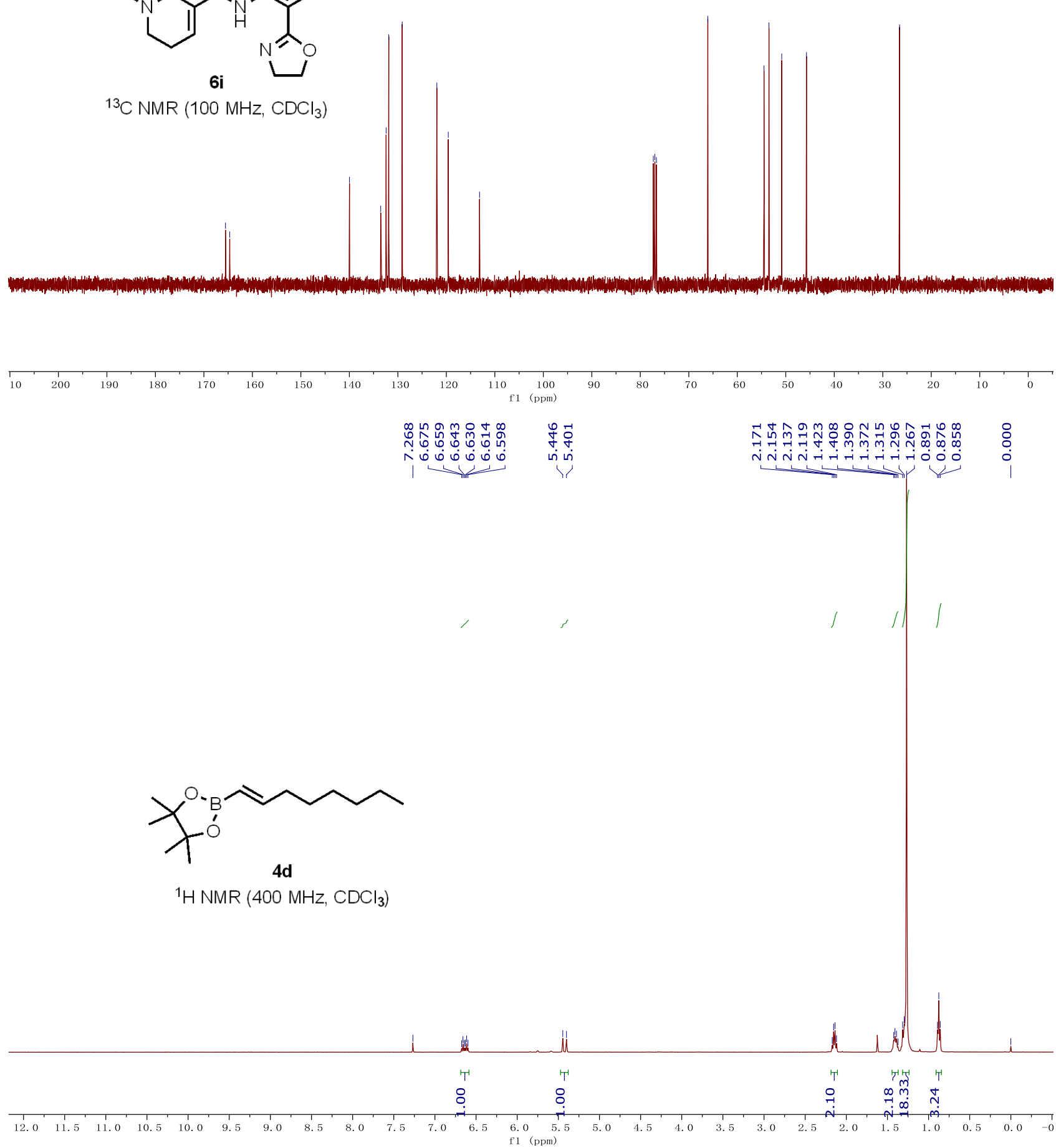


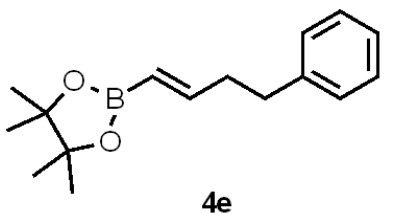

${ }^{1} \mathrm{H}$ NMR $\left(400 \mathrm{MHz}, \mathrm{CDCl}_{3}\right)$

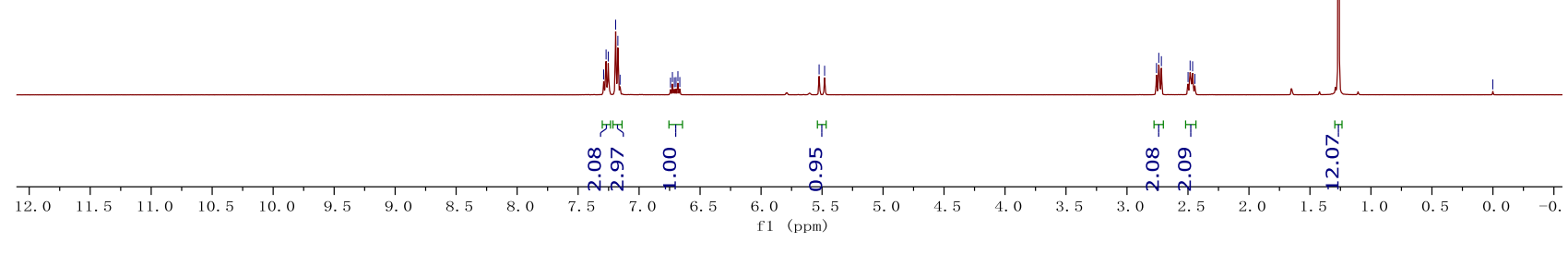

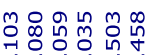

ம்

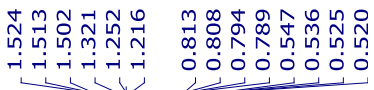

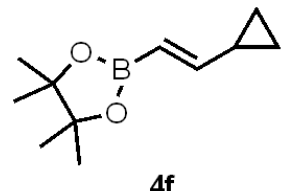

${ }^{1} \mathrm{H} \mathrm{NMR}\left(400 \mathrm{MHz}, \mathrm{CDCl}_{3}\right.$ )

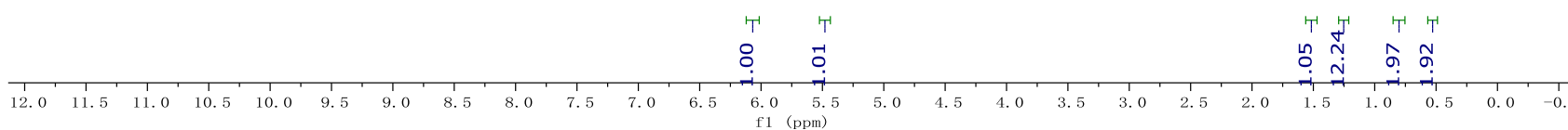




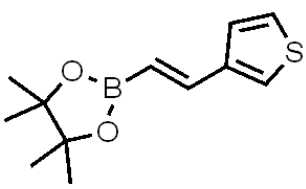

4h

${ }^{1} \mathrm{H}$ NMR (400 MHz, $\mathrm{CDCl}_{3}$ )

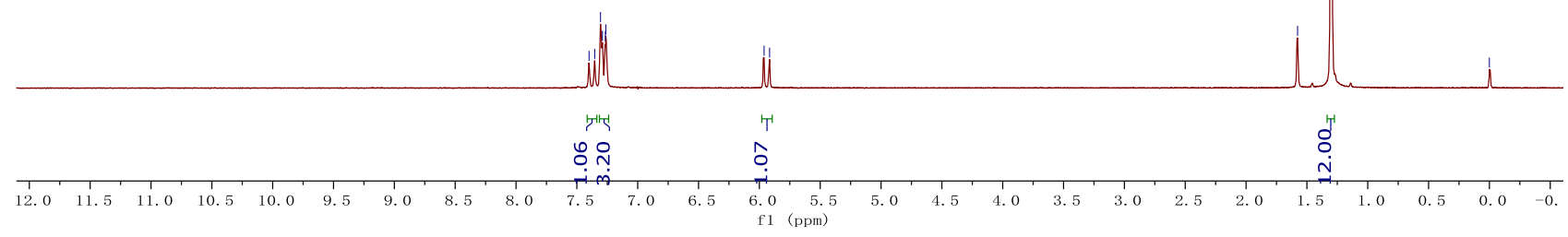

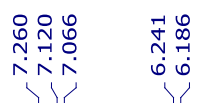

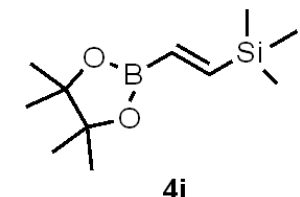

${ }^{1} \mathrm{H} \mathrm{NMR}\left(400 \mathrm{MHz}, \mathrm{CDCl}_{3}\right)$

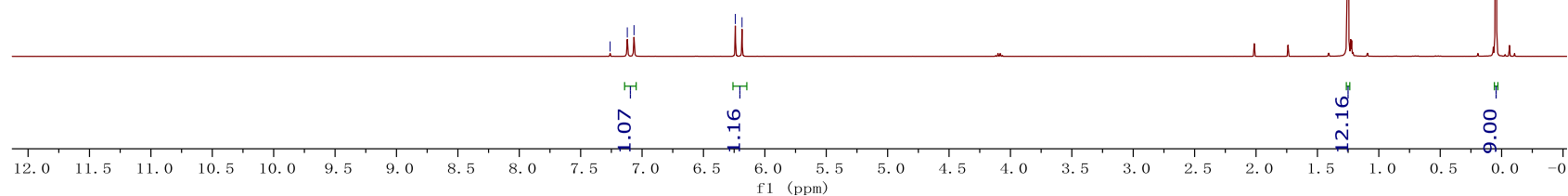




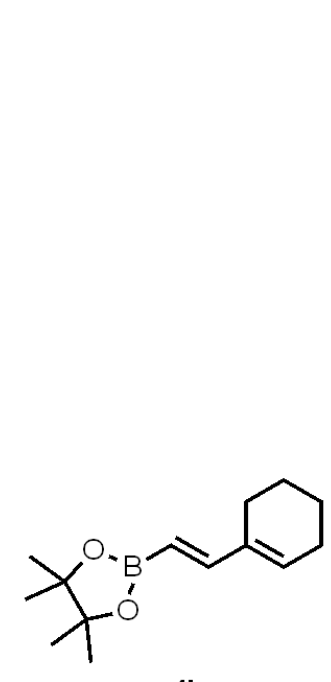

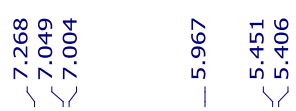

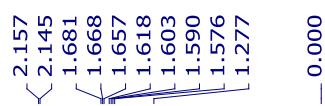

4k

${ }^{1} \mathrm{H}$ NMR (400 MHz, CDCl ${ }_{3}$ )

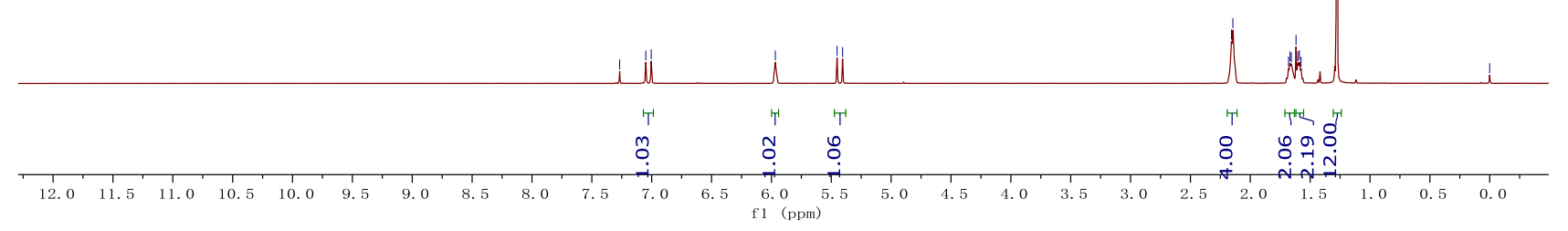

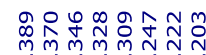

ヘヘヘヘヘヘヘヘ

난

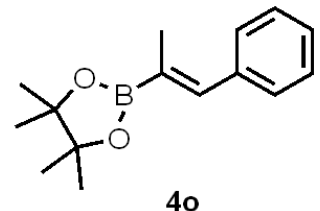

${ }^{1} \mathrm{H} \mathrm{NMR}\left(400 \mathrm{MHz}, \mathrm{CDCl}_{3}\right)$

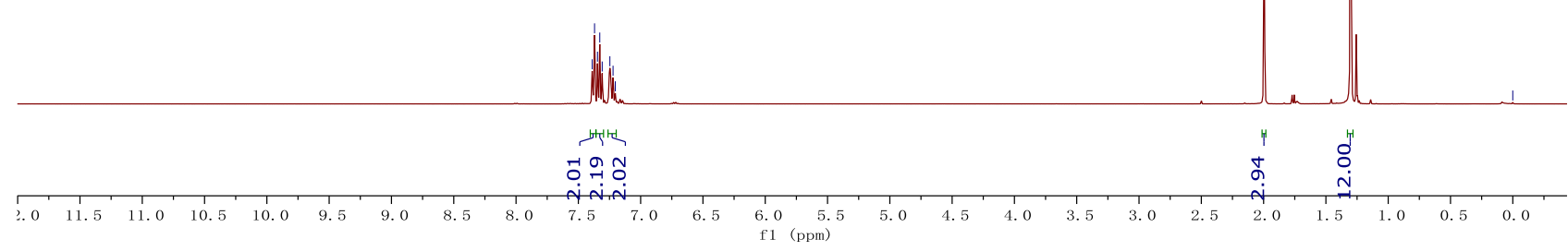




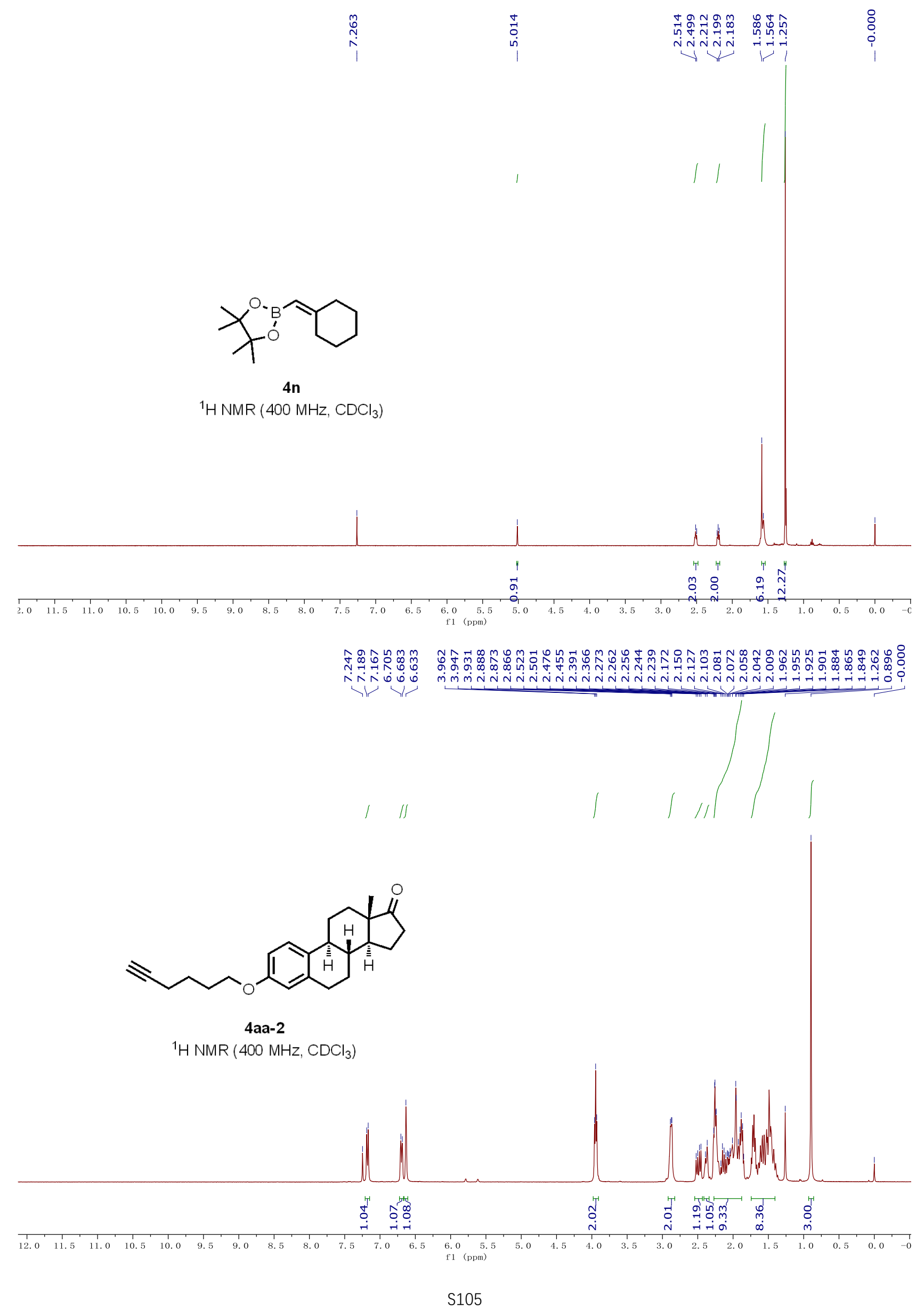




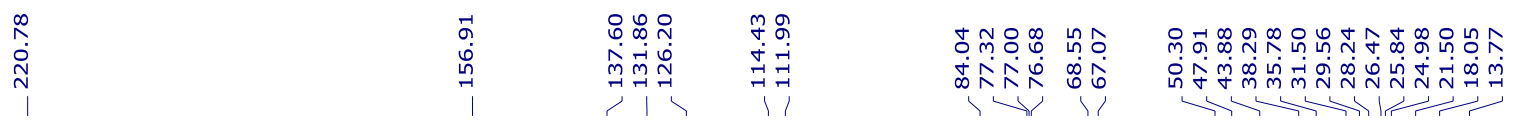

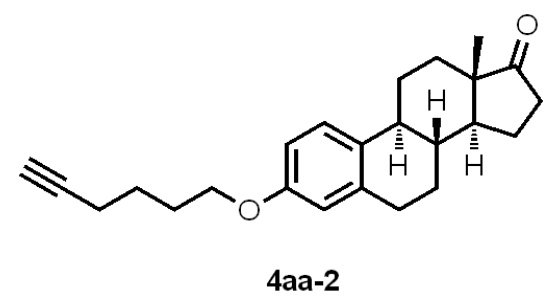

${ }^{13} \mathrm{C} \mathrm{NMR}\left(100 \mathrm{MHz}, \mathrm{CDCl}_{3}\right)$

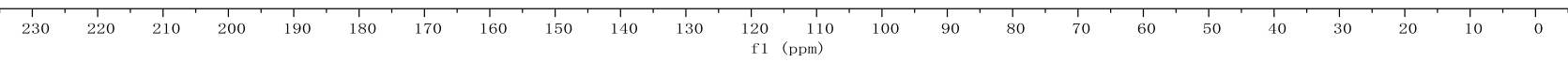

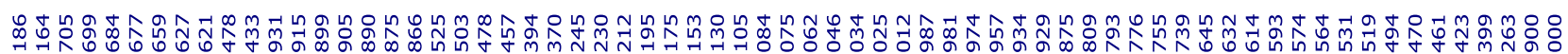
तヘ

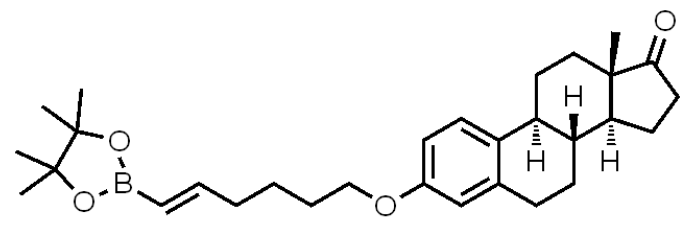

4aa

${ }^{1} \mathrm{H} \mathrm{NMR}\left(400 \mathrm{MHz}, \mathrm{CDCl}_{3}\right)$

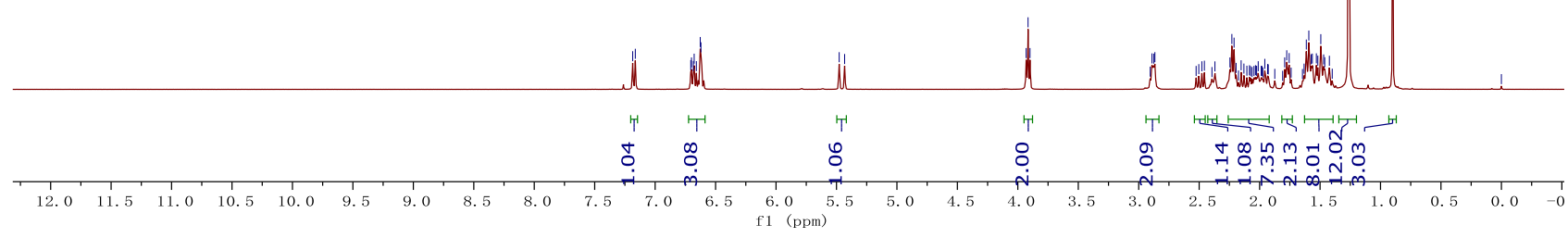



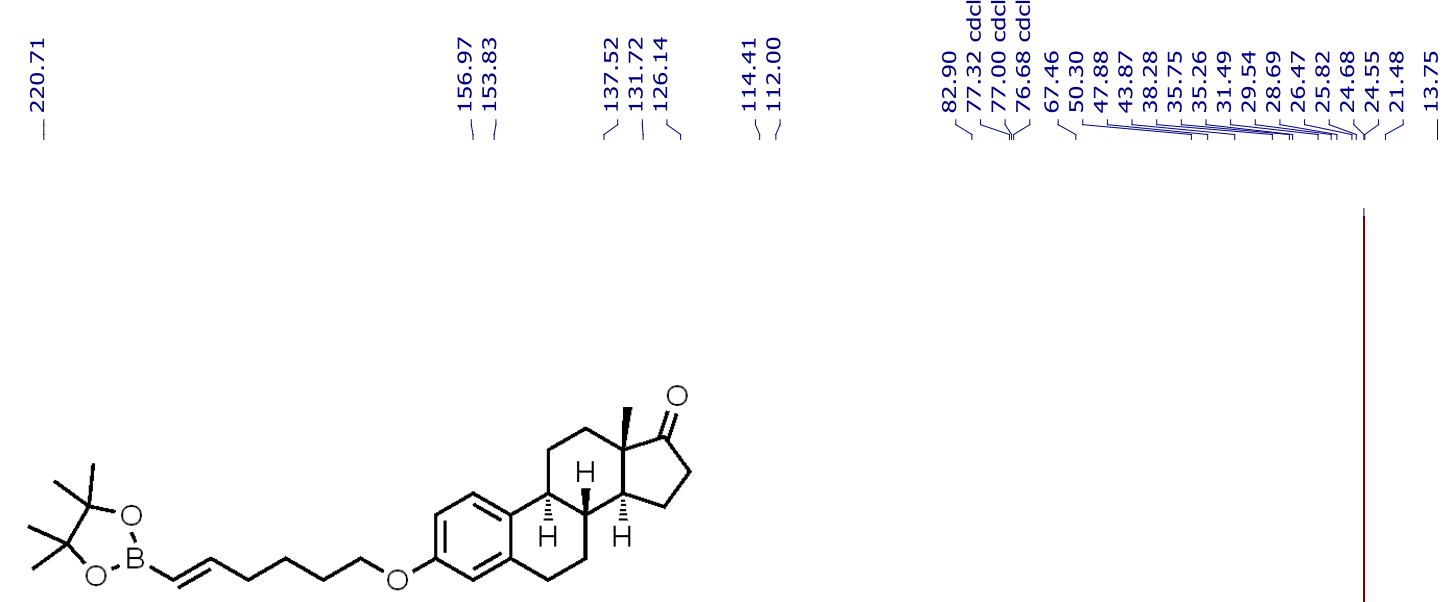

$4 a a$

${ }^{13} \mathrm{C}$ NMR $\left(100 \mathrm{MHz}, \mathrm{CDCl}_{3}\right)$

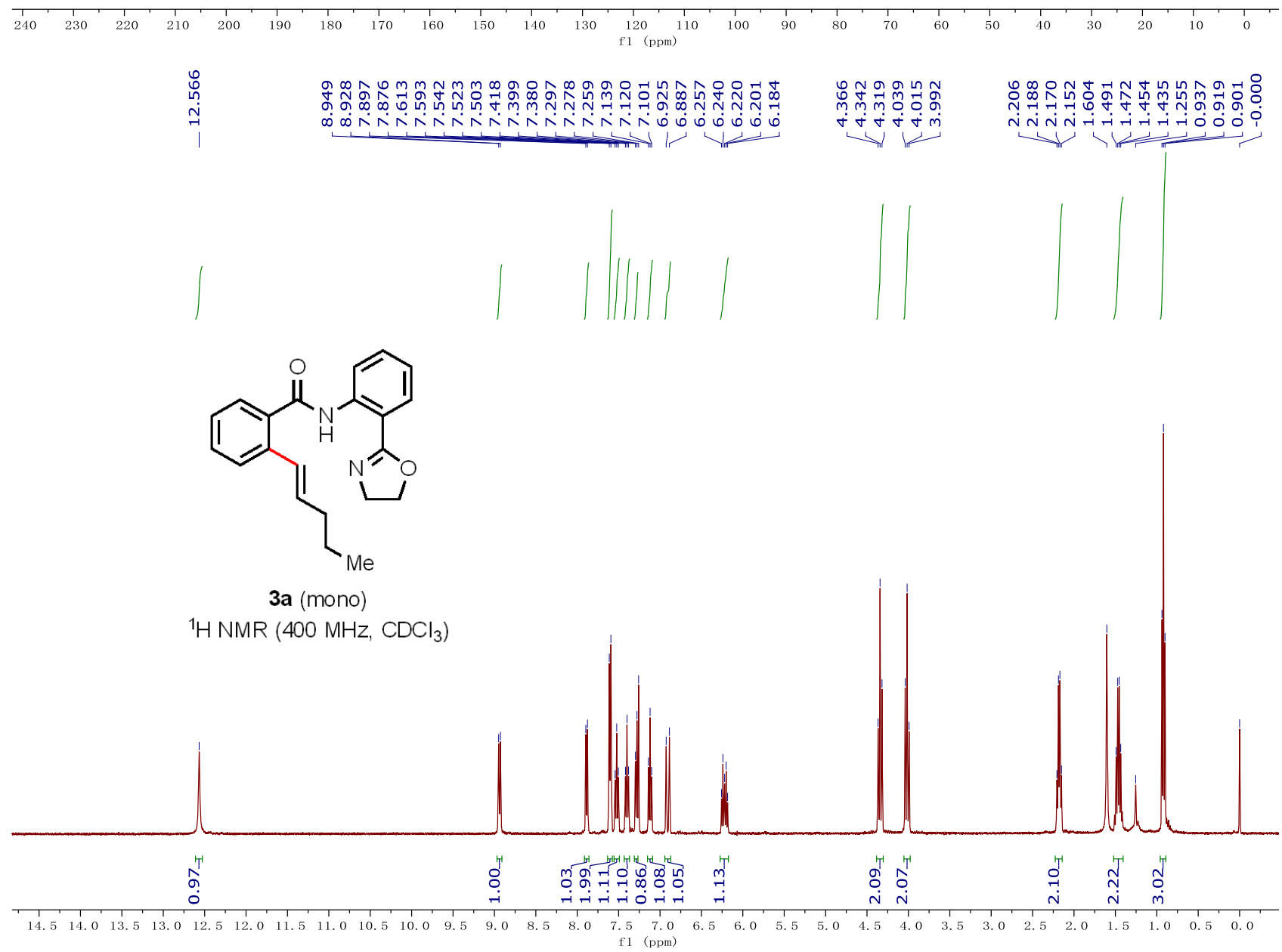




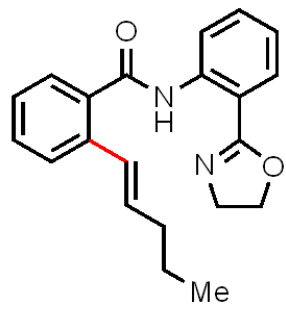

3a (mono)

${ }^{13} \mathrm{C}$ NMR $\left(100 \mathrm{MHz}, \mathrm{CDCl}_{3}\right)$

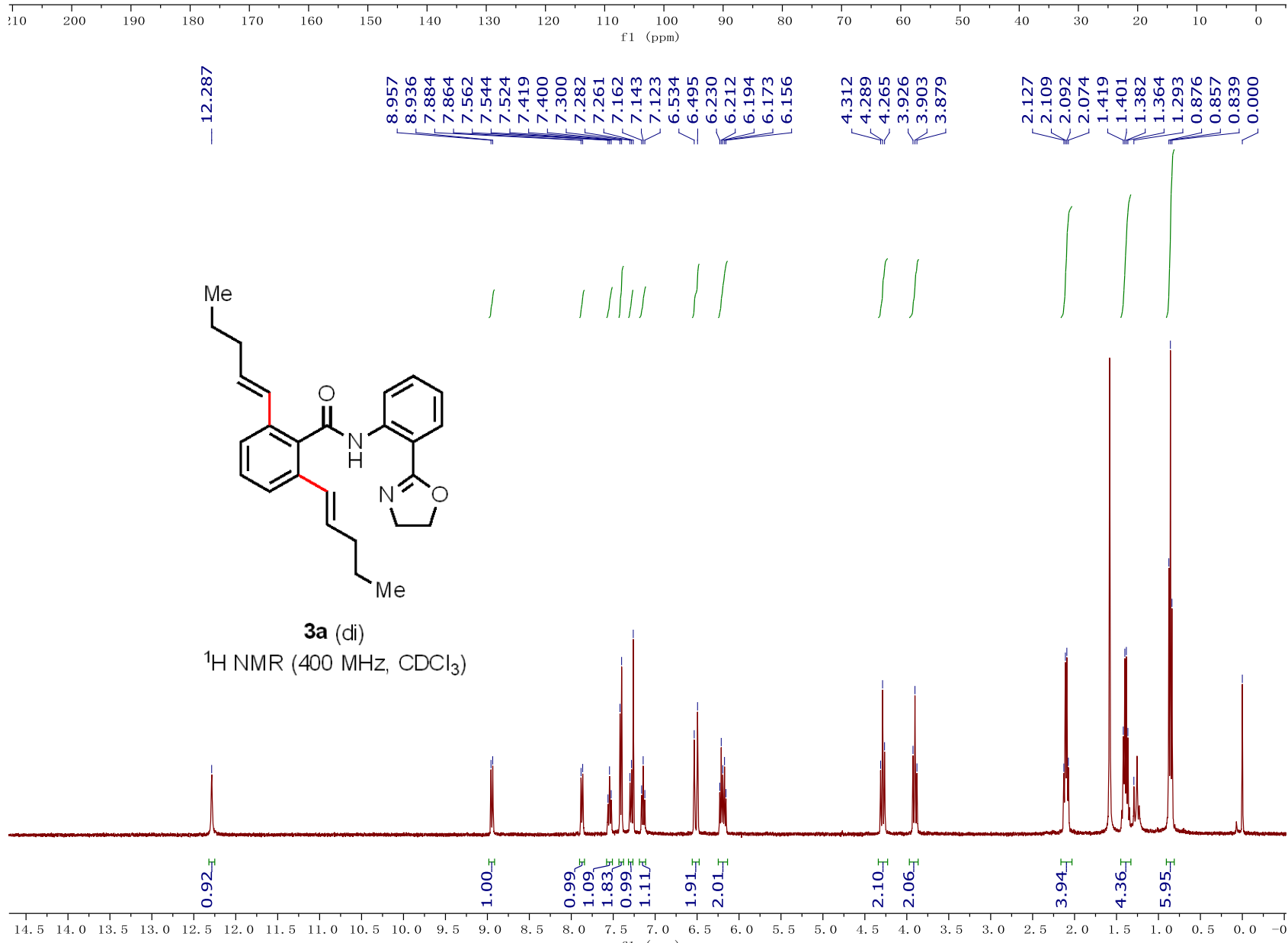

1 (ppm) 


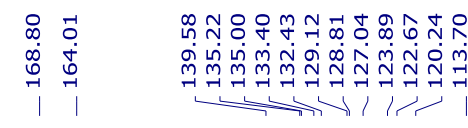<smiles>CCC/C=C/c1cccc(/C=C/CCC)c1C(=O)Nc1ccccc1C1=NCCO1</smiles>

3a (di)

${ }^{13} \mathrm{C}$ NMR $\left(100 \mathrm{MHz}, \mathrm{CDCl}_{3}\right)$

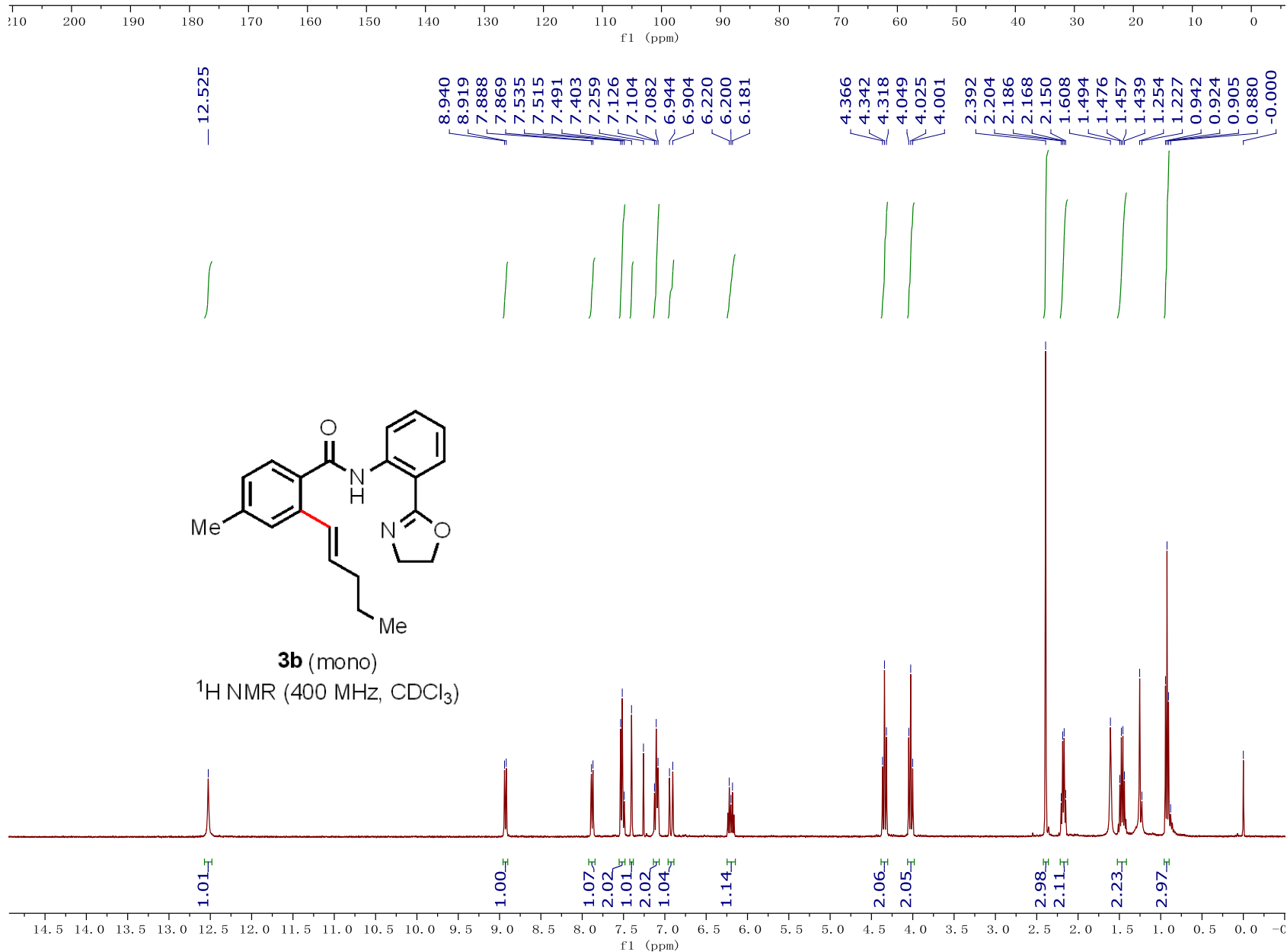




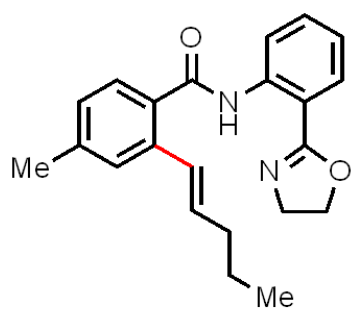

3b (mono)

${ }^{13} \mathrm{C}$ NMR $\left(100 \mathrm{MHz}, \mathrm{CDCl}_{3}\right)$

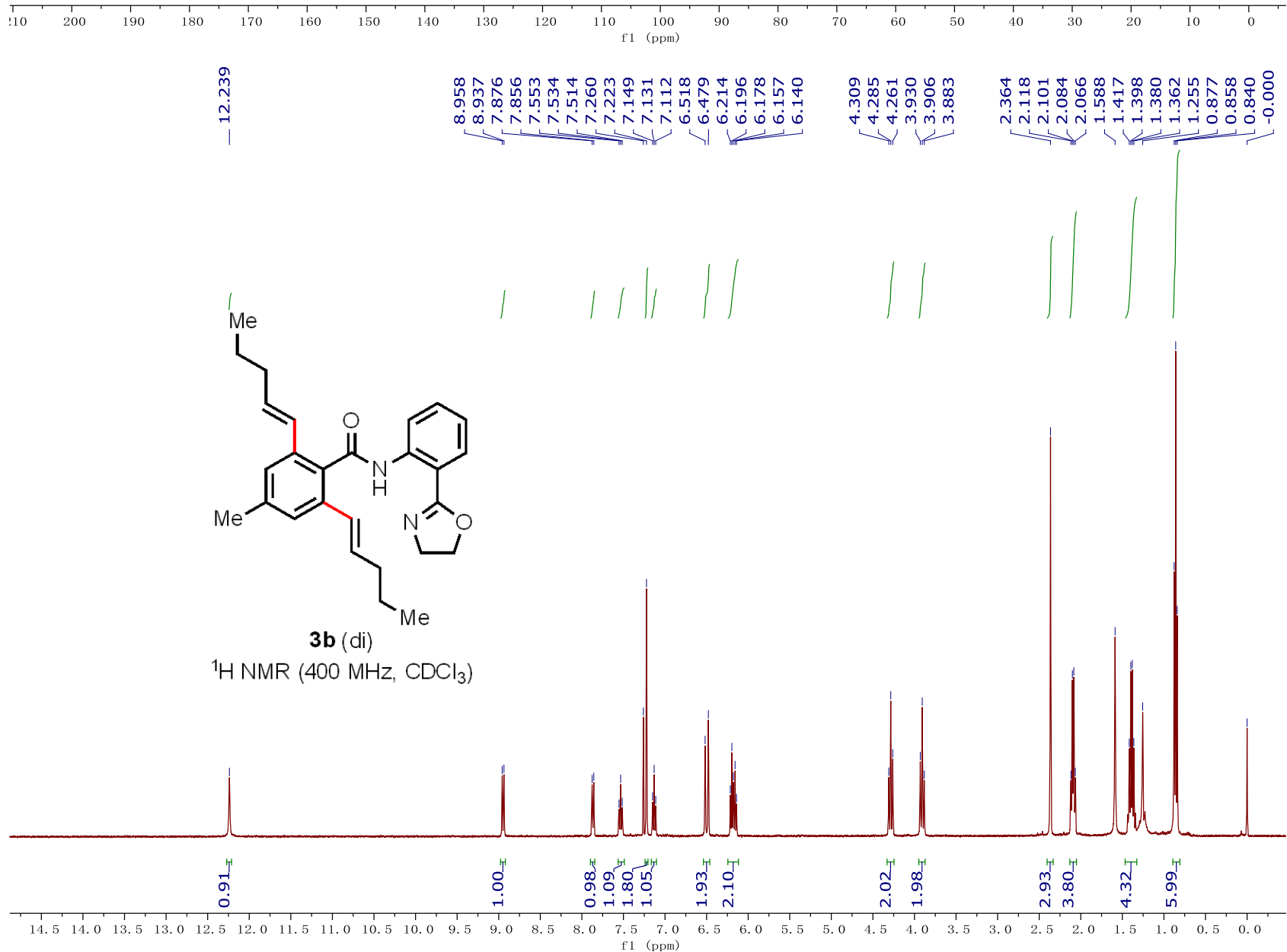



$\begin{array}{lll}2 & \infty \\ 0 & 0 \\ 0 & 0 \\ 0 & 0 \\ -1 & 0\end{array}$

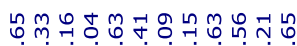
:

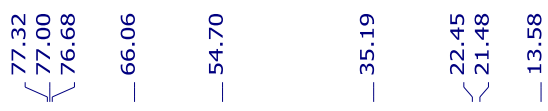

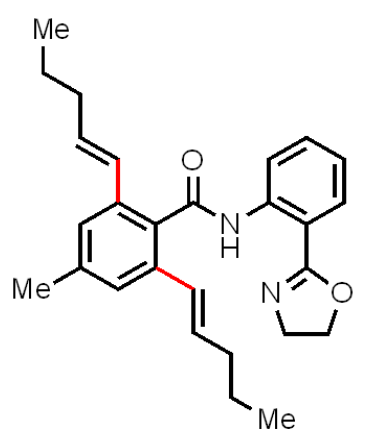

3b (di)

${ }^{13} \mathrm{C} \mathrm{NMR}\left(100 \mathrm{MHz}, \mathrm{CDCl}_{3}\right)$
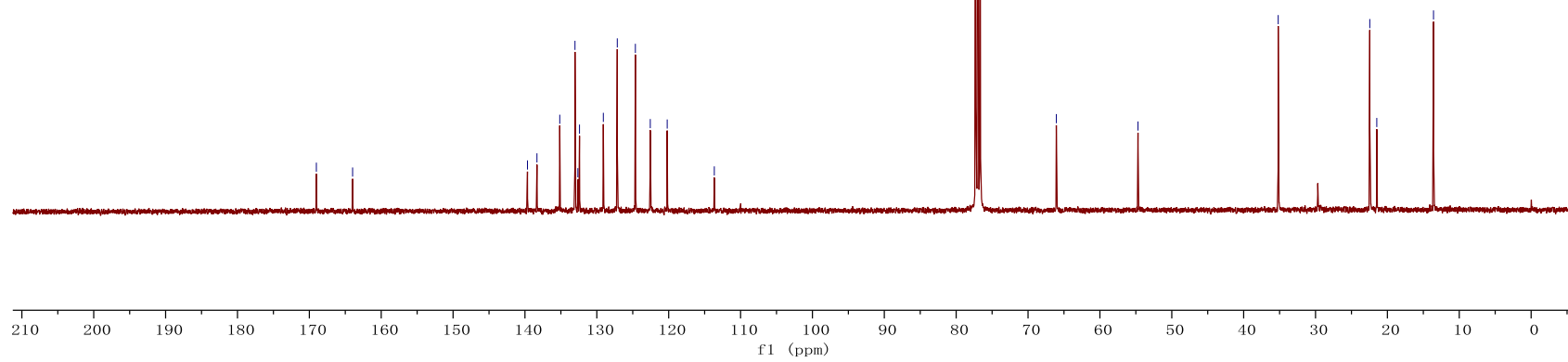

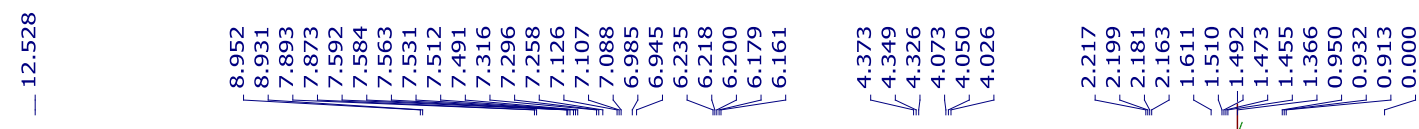

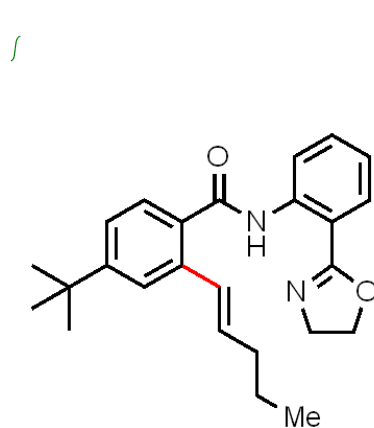

3c (mono)

${ }^{1} \mathrm{HNMR}\left(400 \mathrm{MHz}, \mathrm{CDCl}_{3}\right)$

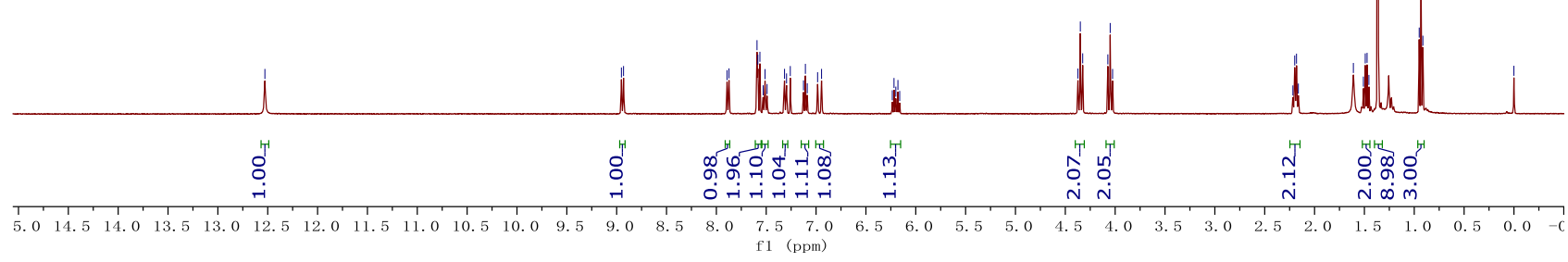




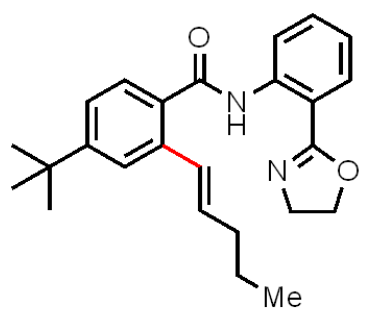

3c (mono)

${ }^{13} \mathrm{C}$ NMR $\left(100 \mathrm{MHz}, \mathrm{CDCl}_{3}\right)$

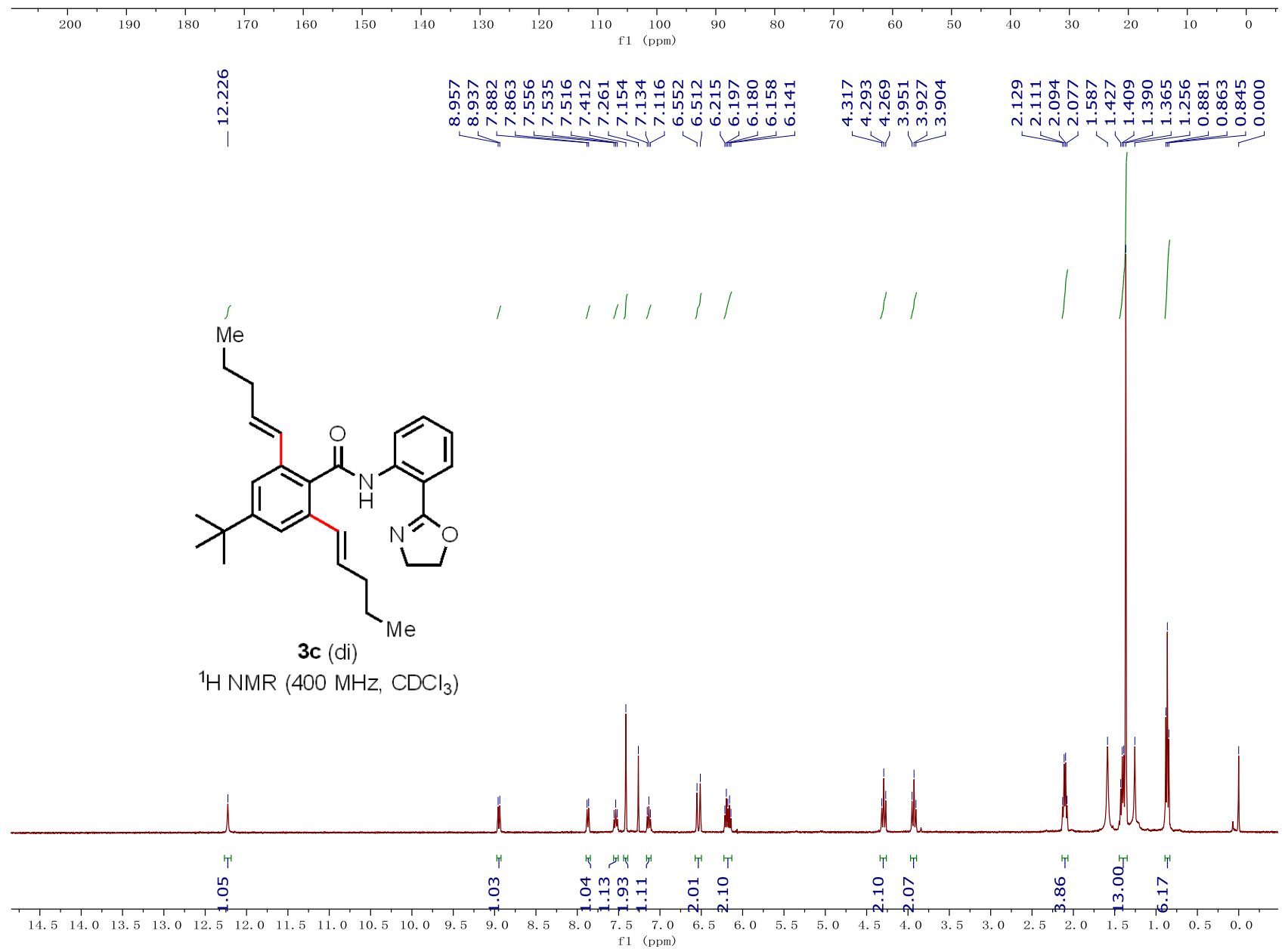




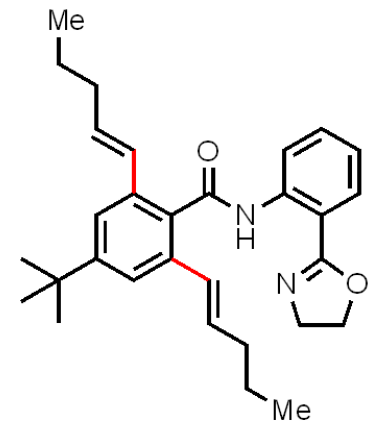

$3 c(\mathrm{di})$

${ }^{13} \mathrm{C} \mathrm{NMR}\left(100 \mathrm{MHz}, \mathrm{CDCl}_{3}\right)$

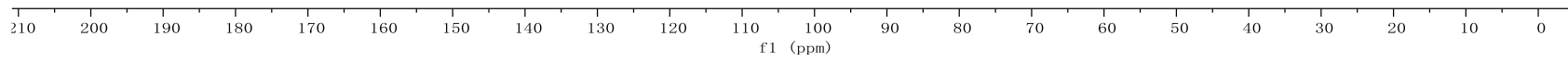

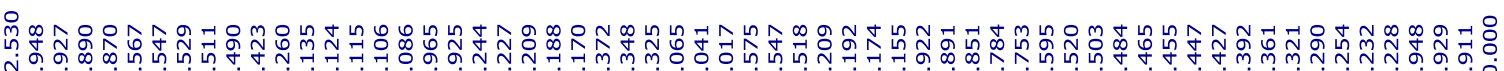

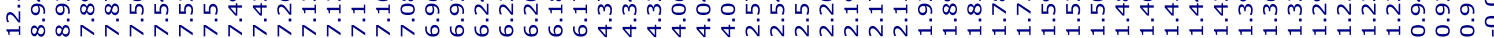<smiles>CCC/C=C/c1cc(C2CCCCC2)ccc1C(=O)Nc1ccccc1C1=NCCO1</smiles>

3d (mono)

${ }^{1} \mathrm{HNMR}\left(400 \mathrm{MHz}, \mathrm{CDCl}_{3}\right)$

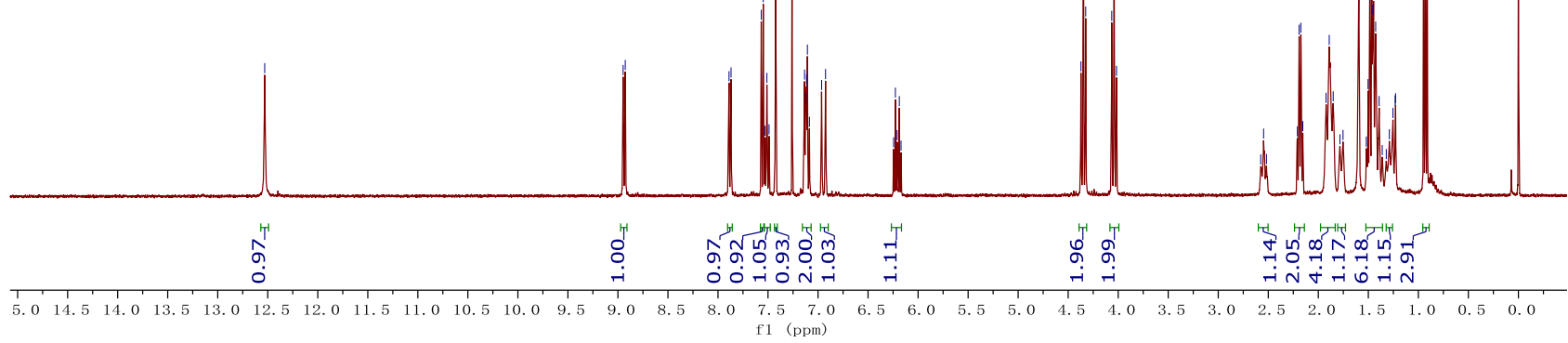




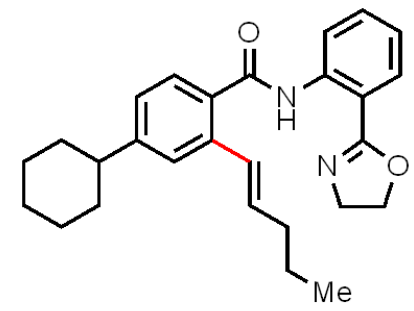

3d (mono)

${ }^{13} \mathrm{C} \mathrm{NMR}\left(100 \mathrm{MHz}, \mathrm{CDCl}_{3}\right)$

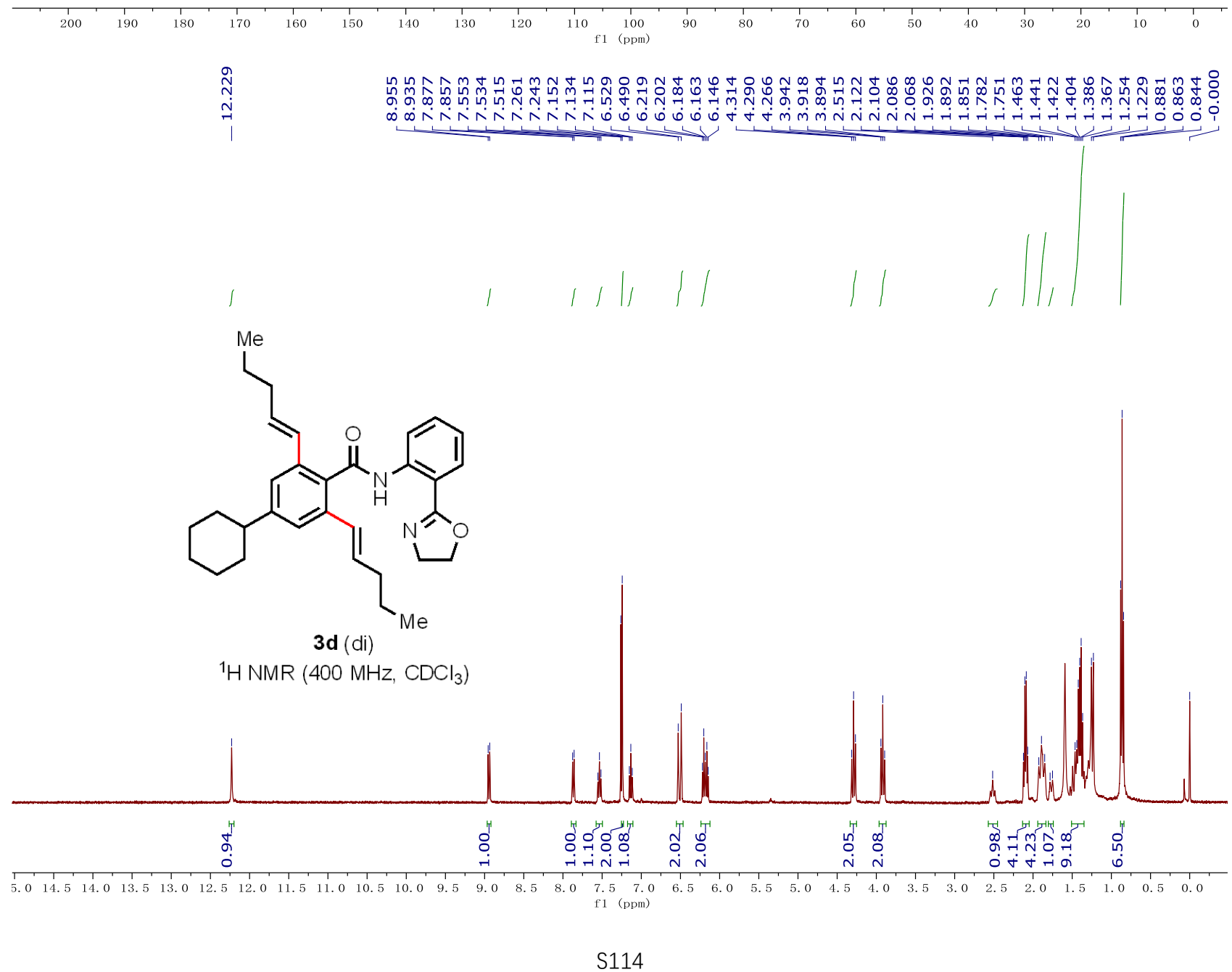




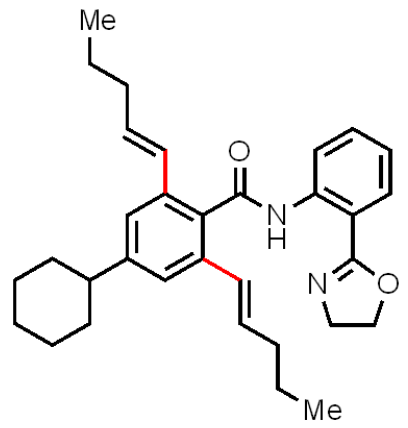

3d (di)

${ }^{13} \mathrm{C} \mathrm{NMR}\left(100 \mathrm{MHz}, \mathrm{CDCl}_{3}\right)$

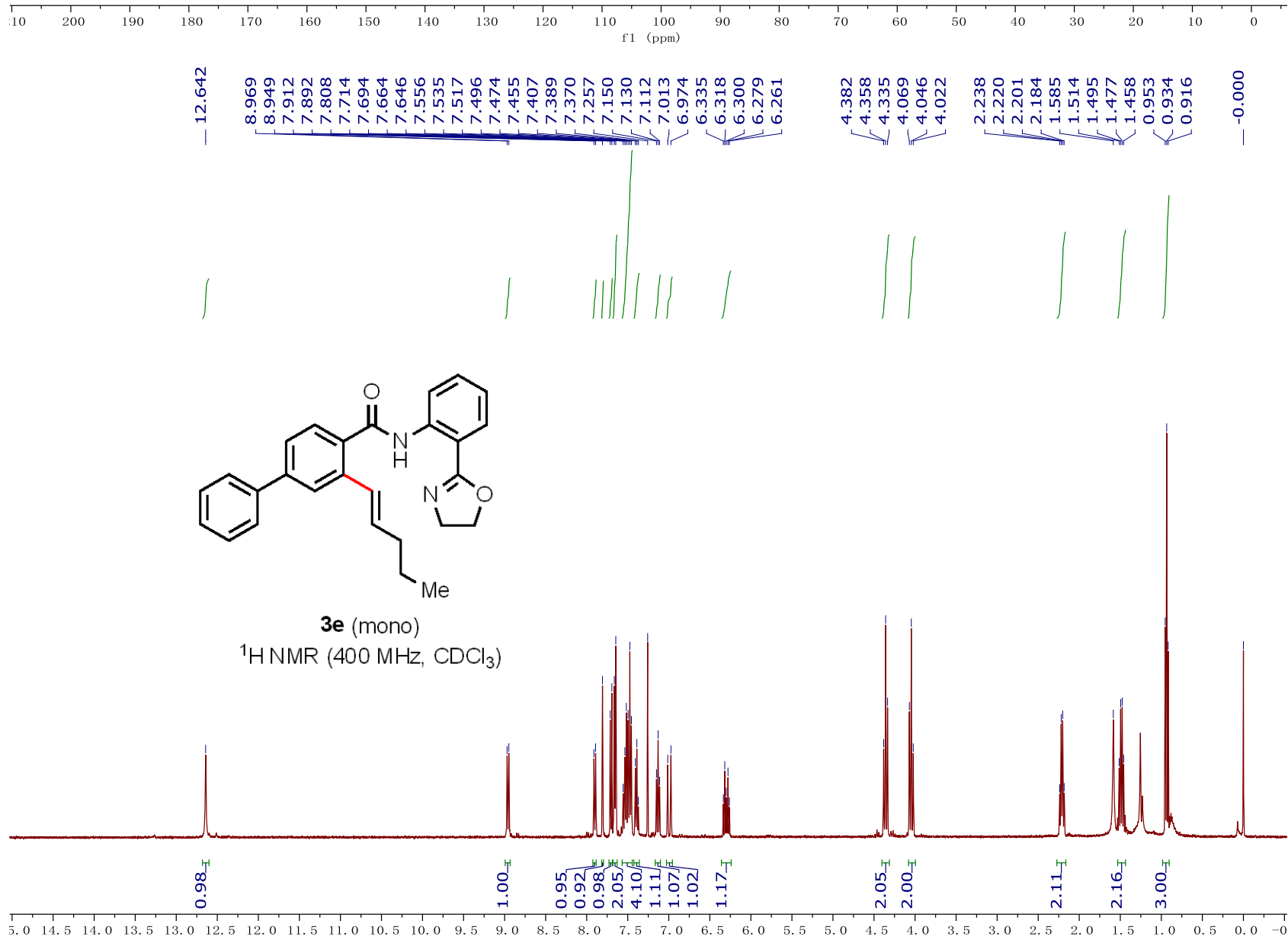




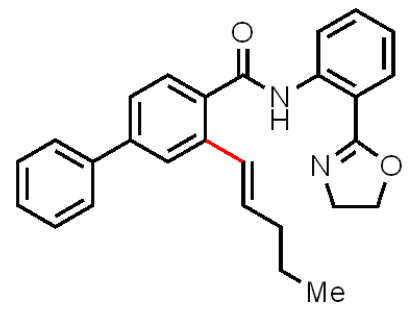

3 e (mono)

${ }^{13} \mathrm{C}$ NMR $\left(100 \mathrm{MHz}, \mathrm{CDCl}_{3}\right)$

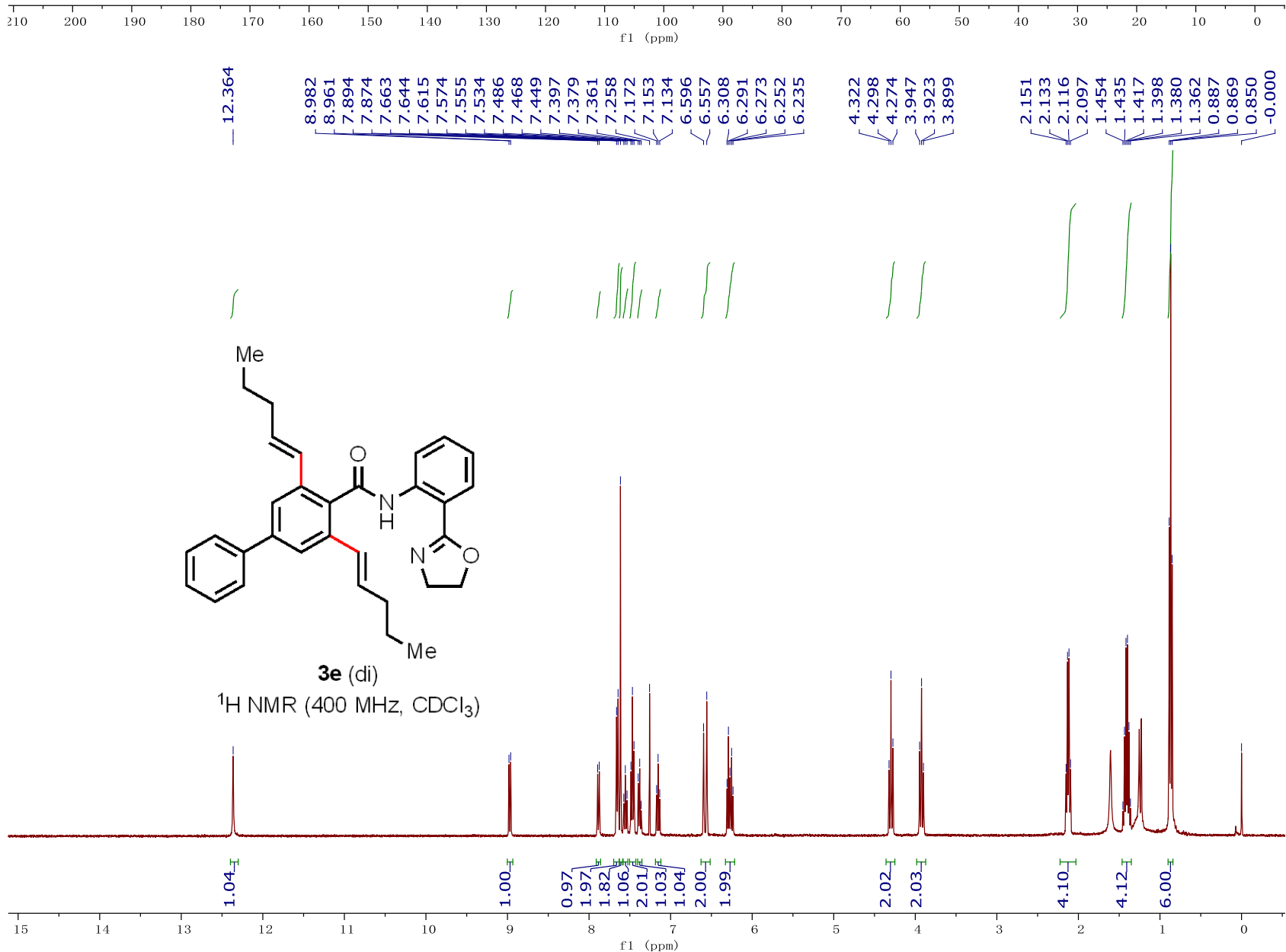




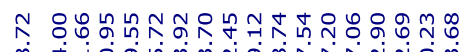

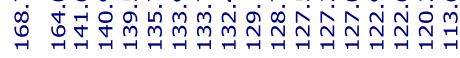

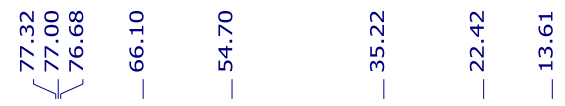<smiles>CCC/C=C/c1cc(-c2ccccc2)cc(/C=C/CCC)c1C(=O)Nc1ccccc1C1=NCCO1</smiles>

$3 e(d i)$

${ }^{13} \mathrm{C} \mathrm{NMR}\left(100 \mathrm{MHz}, \mathrm{CDCl}_{3}\right)$

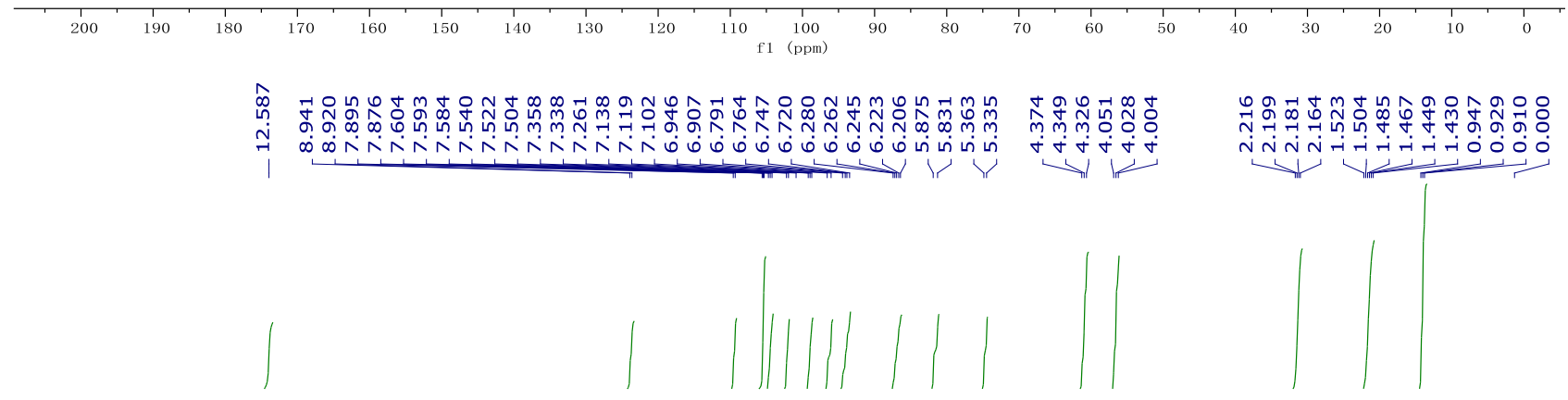<smiles>C=Cc1ccc(C(=O)Nc2ccccc2C2=NCCO2)c(/C=C/CCC)c1</smiles>

$3 f$ (mono)

${ }^{1} \mathrm{HNMR}\left(400 \mathrm{MHz}, \mathrm{CDCl}_{3}\right)$

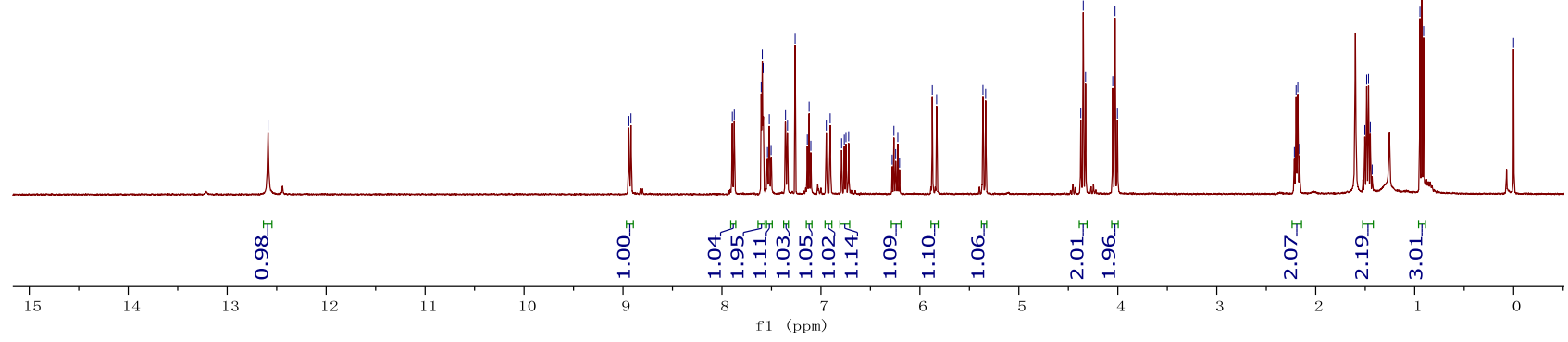




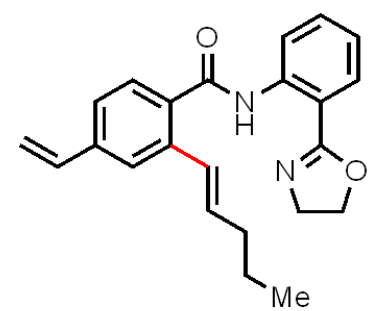

3f (mono)

$\left.{ }^{13} \mathrm{C} \mathrm{NMR} \mathrm{(100} \mathrm{MHz,} \mathrm{CDCl}_{3}\right)$
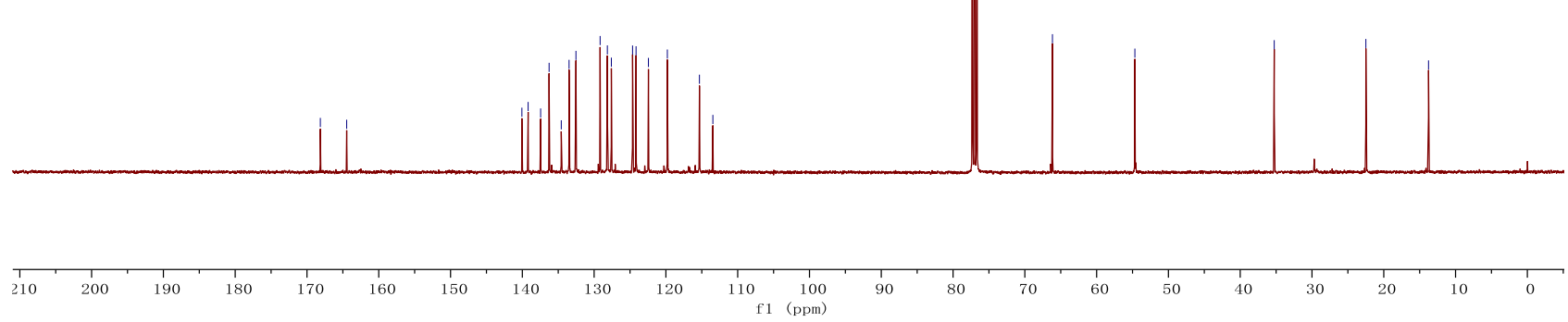

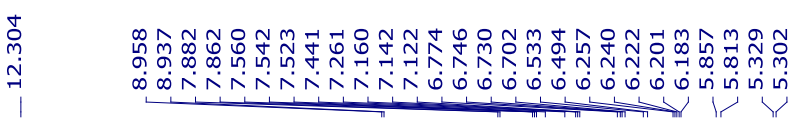

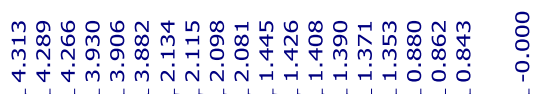

$\int$
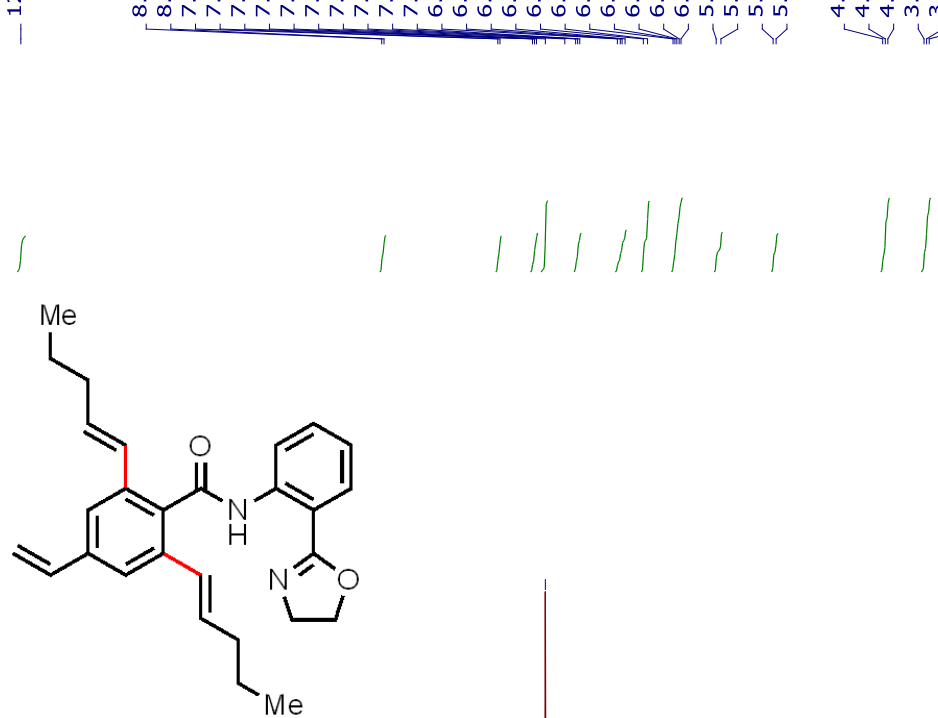

$3 \mathbf{f}$ (di)

${ }^{1} \mathrm{H}$ NMR (400 $\mathrm{MHz}, \mathrm{CDCl}_{3}$ )

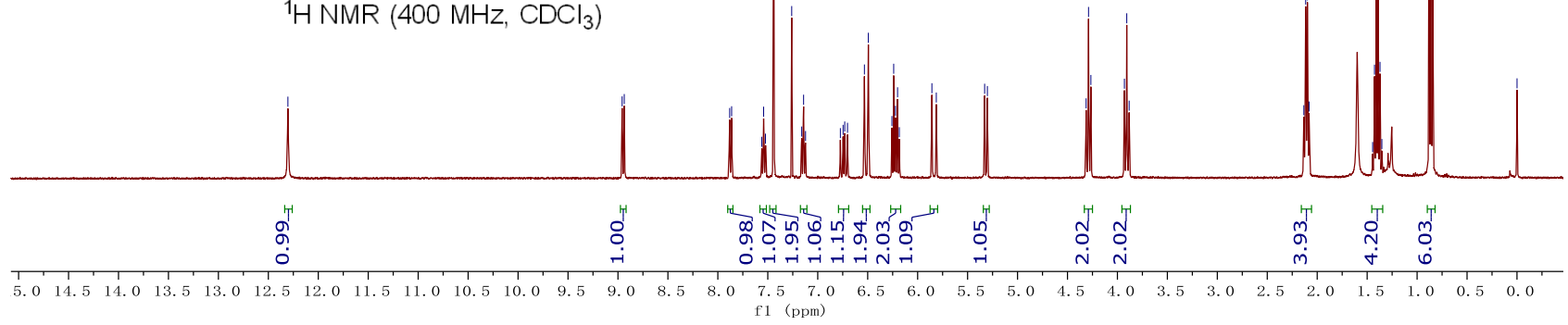




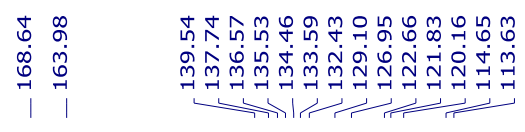<smiles>C=Cc1cc(/C=C/CCC)c(C(=O)Nc2ccccc2C2=NCCO2)c(/C=C/CCC)c1</smiles>

$3 f$ (di)

${ }^{13} \mathrm{C}$ NMR $\left(100 \mathrm{MHz}, \mathrm{CDCl}_{3}\right)$

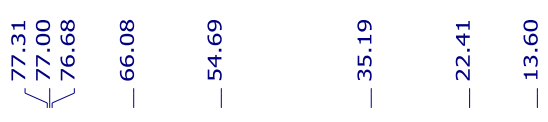
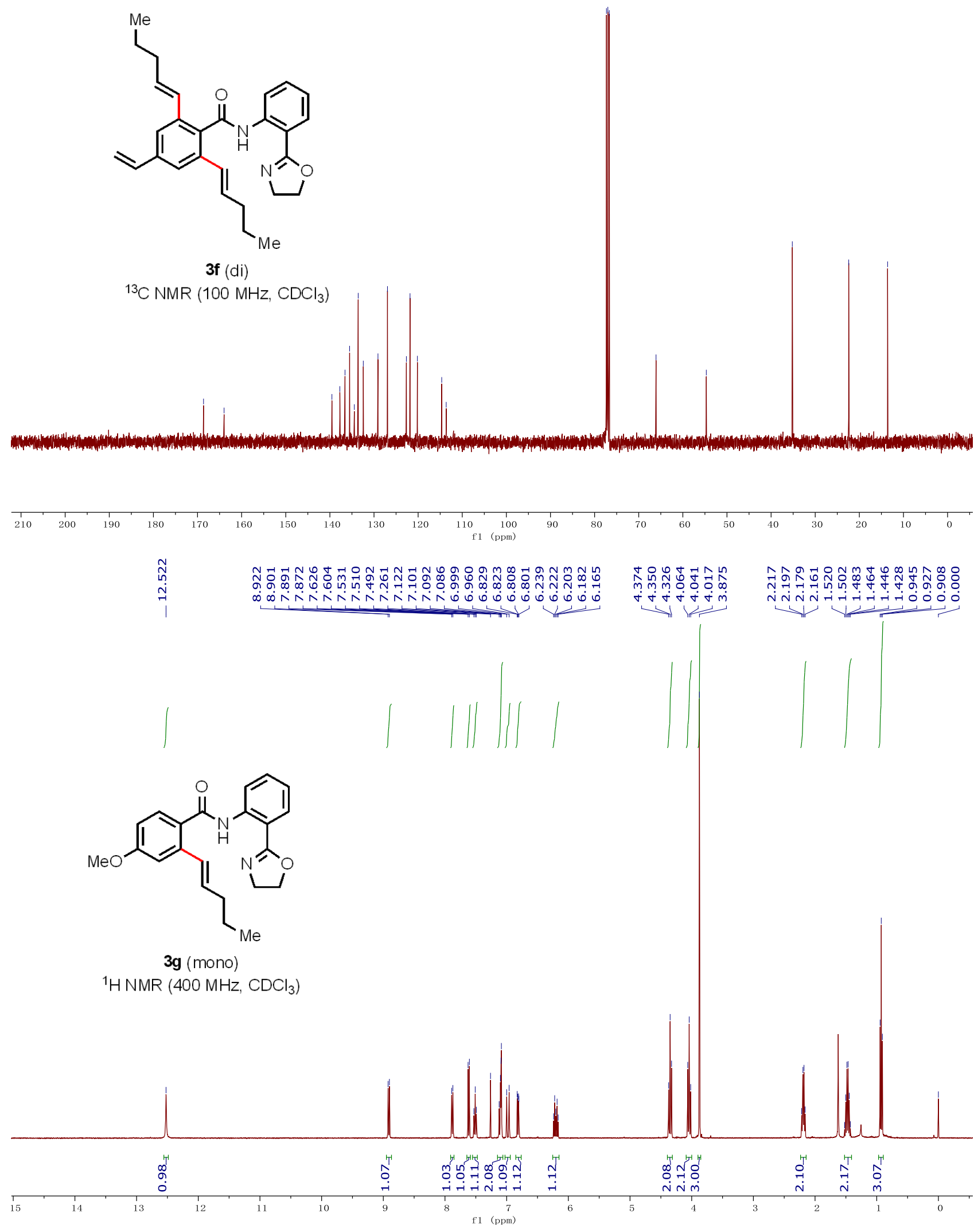


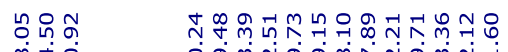

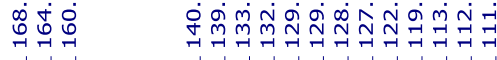

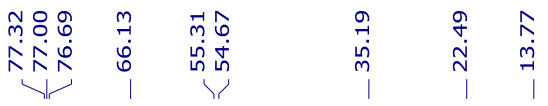

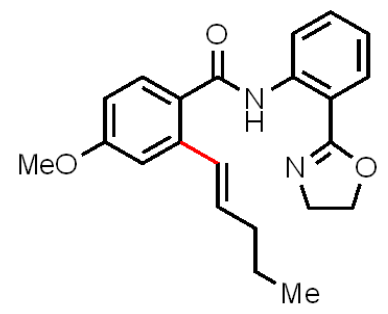

$\mathbf{3 g}$ (mono)

${ }^{13} \mathrm{C} \mathrm{NMR}\left(100 \mathrm{MHz}, \mathrm{CDCl}_{3}\right)$

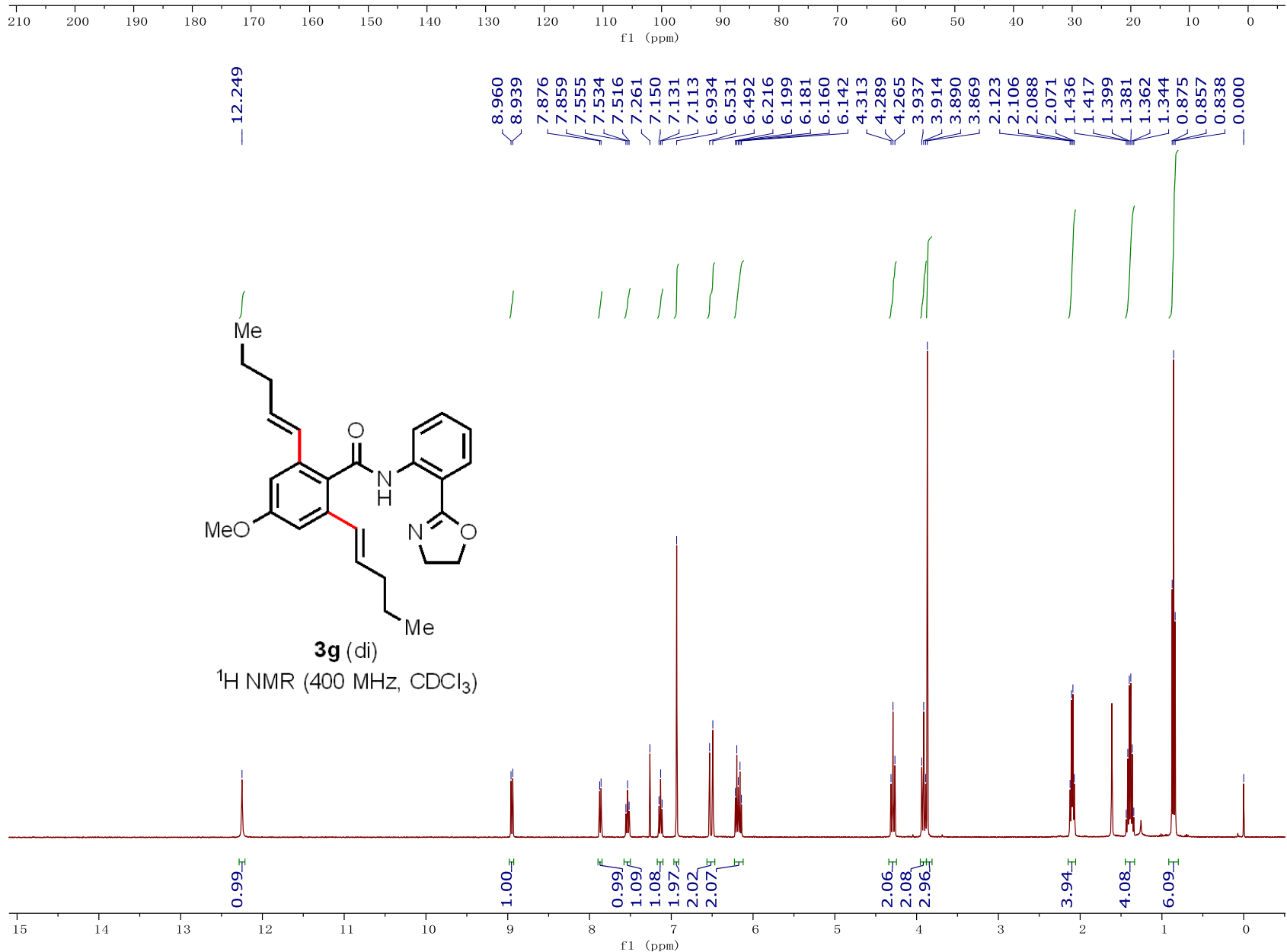




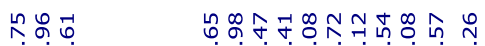

舟弟

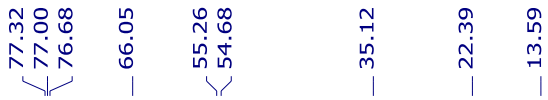<smiles>CCC/C=C/c1cc(OC)cc(/C=C/CCC[Na])c1C(=O)Nc1ccccc1C1=NCCO1</smiles>

$\mathbf{3 g}$ (di)

${ }^{13} \mathrm{C} \mathrm{NMR}\left(100 \mathrm{MHz}, \mathrm{CDCl}_{3}\right)$

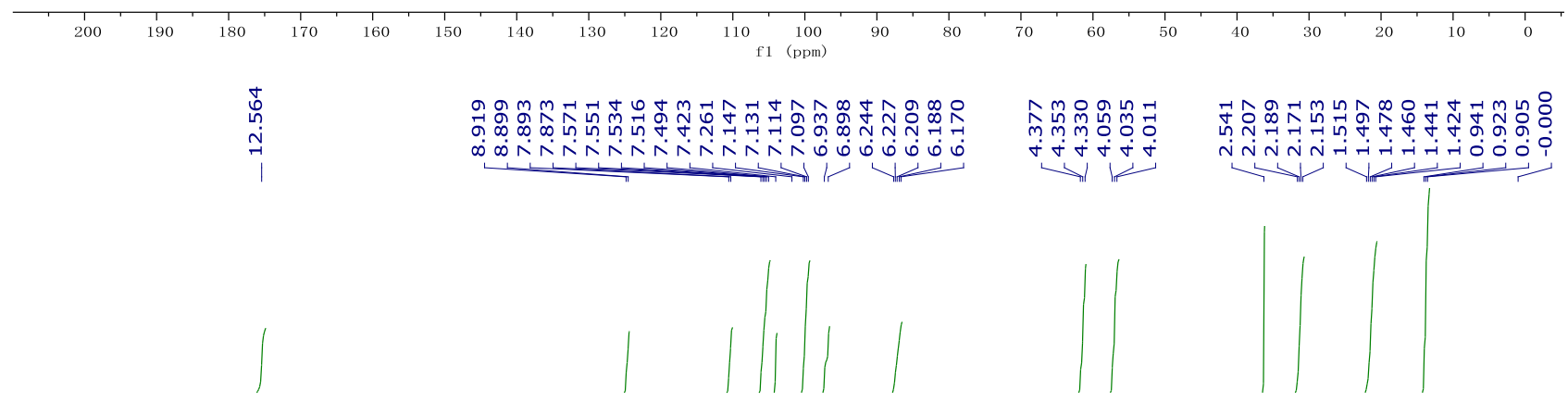<smiles>CCC/C=C/c1cc(S(C)(=O)=O)ccc1C(=O)Nc1ccccc1C1=NCCO1</smiles>

3h (mono)

${ }^{1} \mathrm{HNMR}\left(400 \mathrm{MHz}, \mathrm{CDCl}_{3}\right.$ )

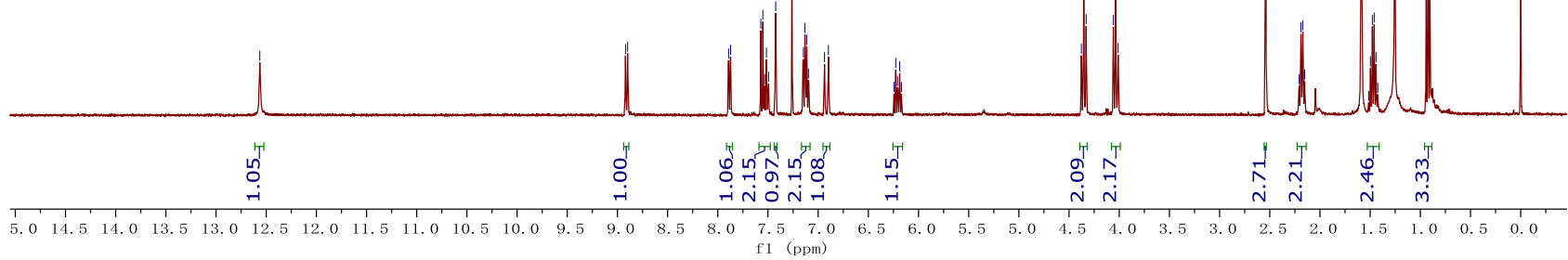




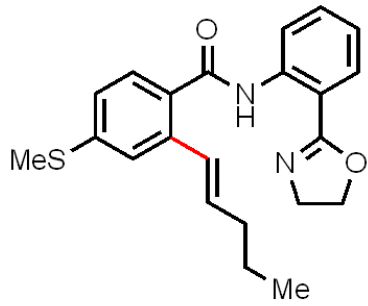

3h (mono)

${ }^{13} \mathrm{C} \mathrm{NMR}\left(100 \mathrm{MHz}, \mathrm{CDCl}_{3}\right)$

\section{ii Ui II}

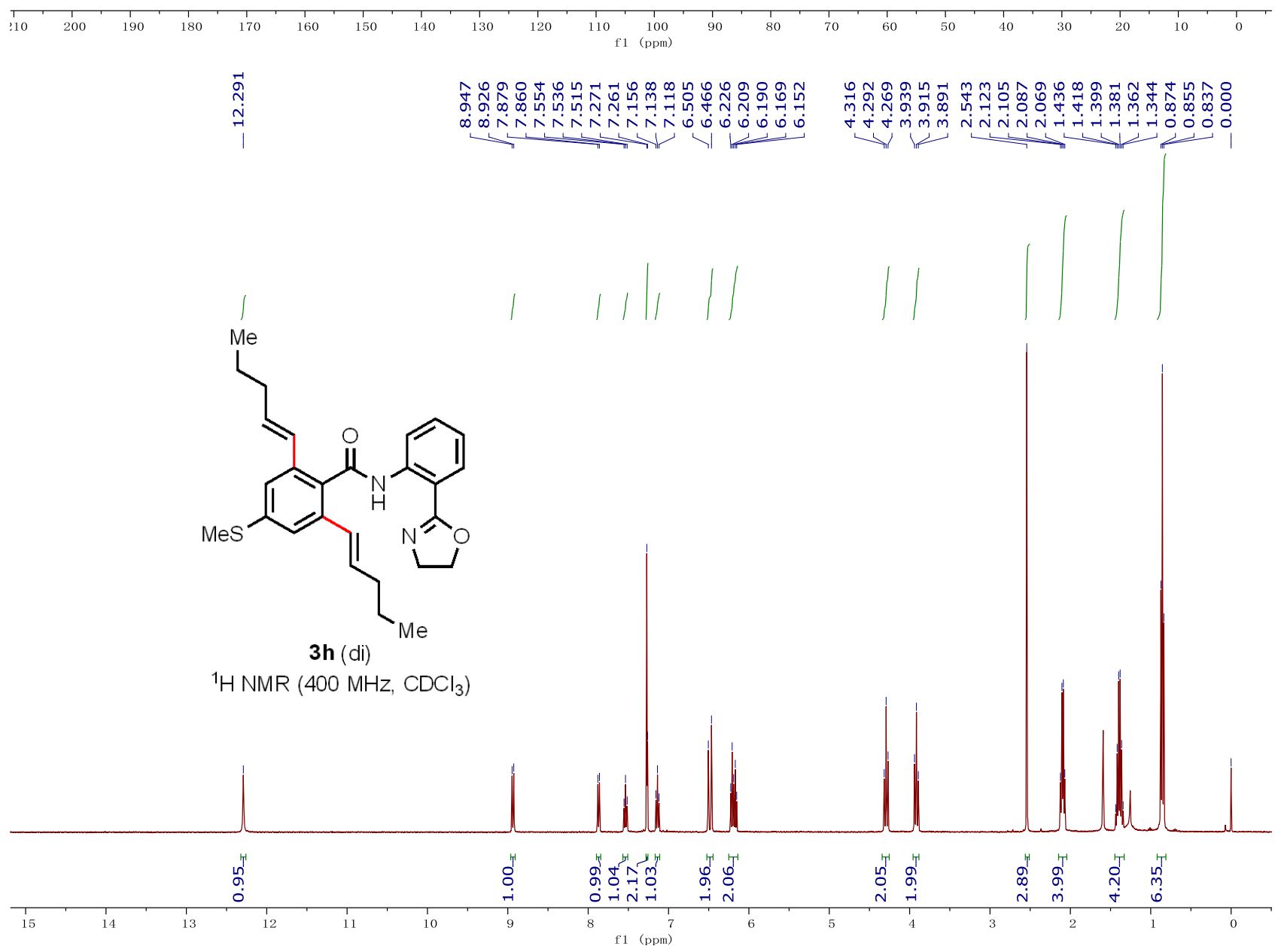




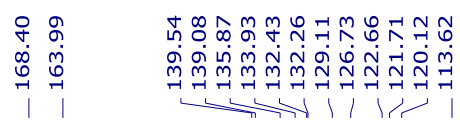

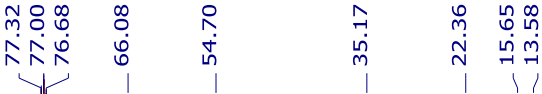

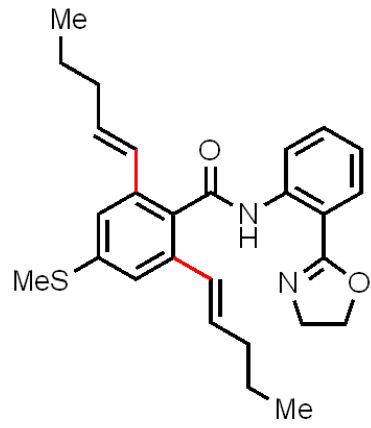

3h (di)

${ }^{13} \mathrm{C}$ NMR $\left(100 \mathrm{MHz}, \mathrm{CDCl}_{3}\right)$

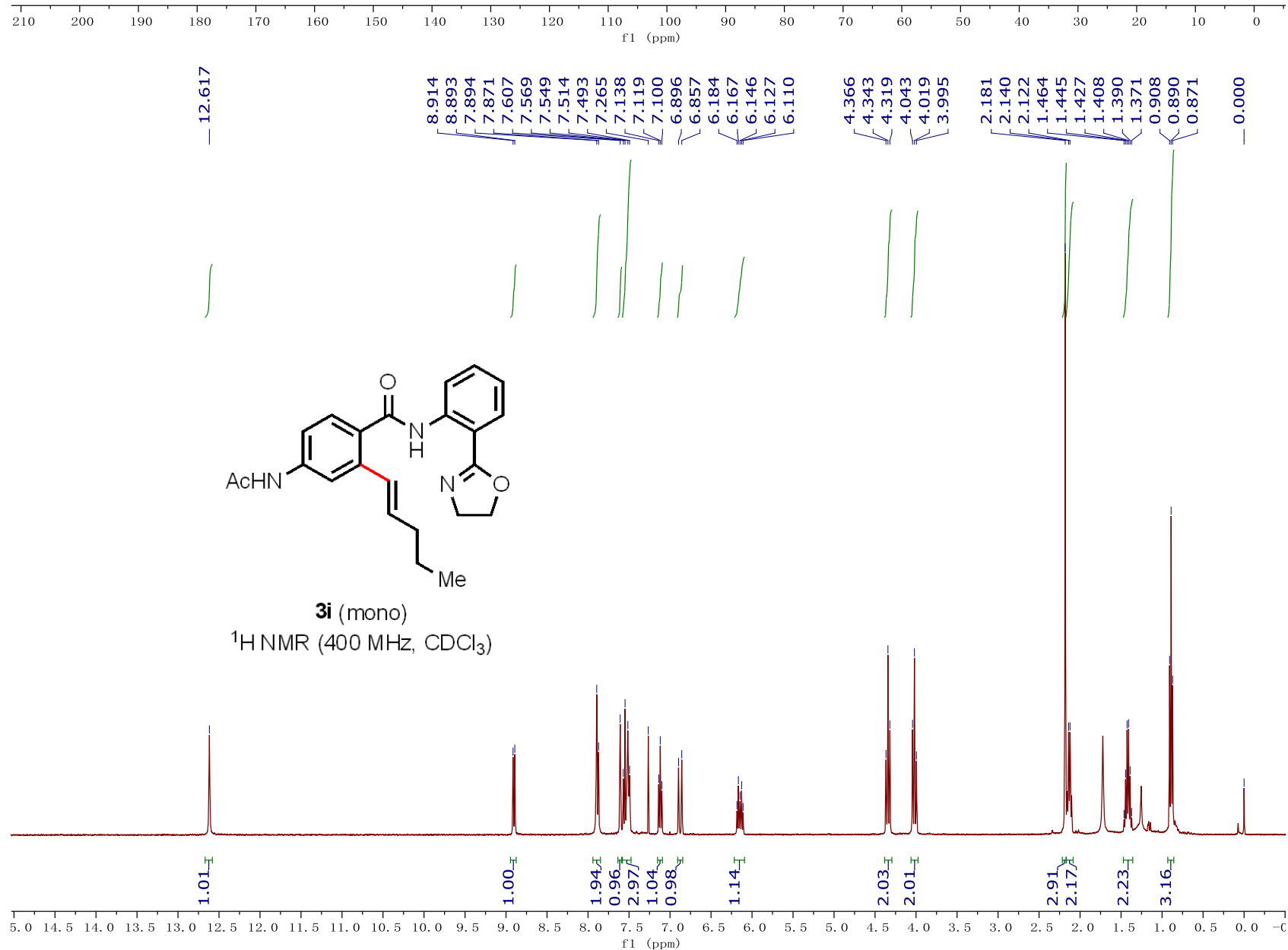


<smiles>C=CNc1ccc(C(=O)Nc2ccccc2C2=NCCO2)c(C=CCCC)c1</smiles>

$3 \mathbf{i}$ (mono)

$\left.{ }^{13} \mathrm{C} \mathrm{NMR} \mathrm{(100} \mathrm{MHz,} \mathrm{CDCl}_{3}\right)$

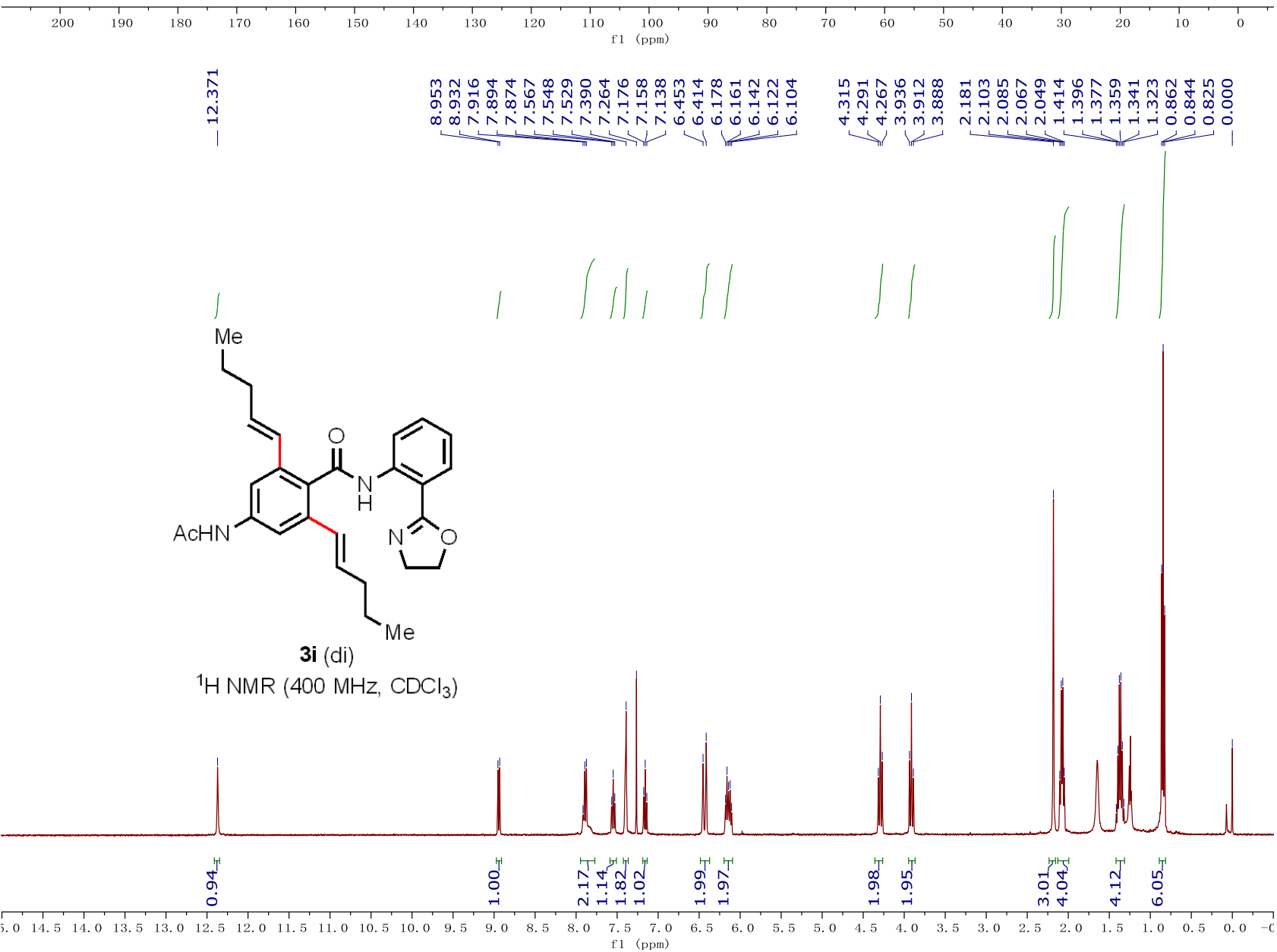




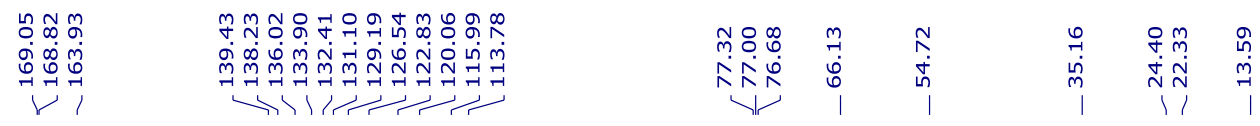

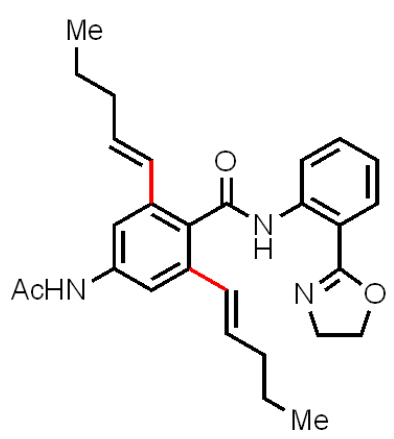

3i (di)

${ }^{13} \mathrm{C} \mathrm{NMR}\left(100 \mathrm{MHz}, \mathrm{CDCl}_{3}\right)$

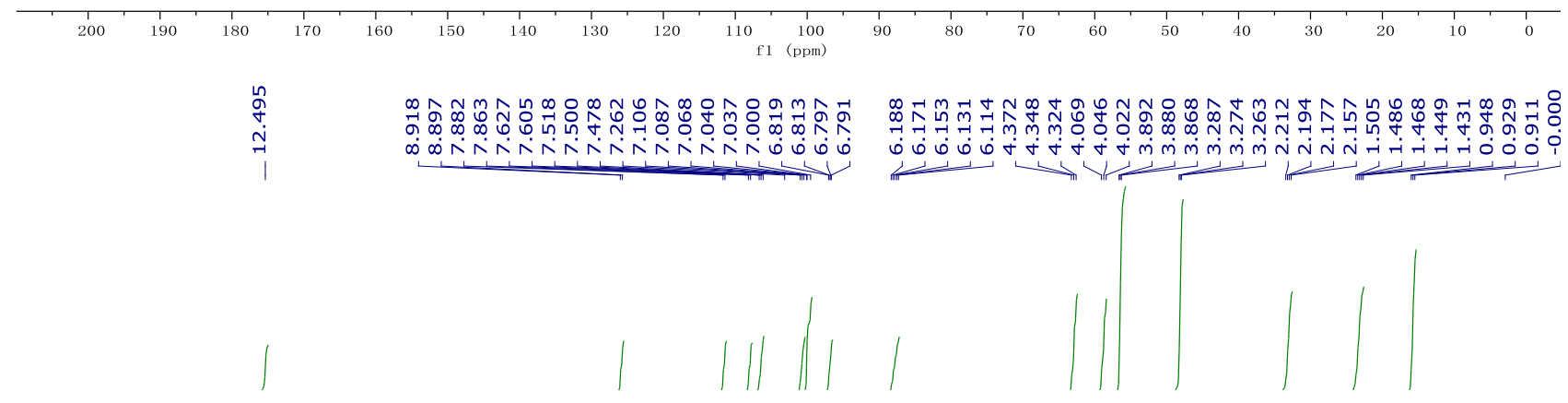<smiles>CCC/C=C/c1cc(N2CCOCC2)ccc1C(=O)Nc1ccccc1C1=NCCO1</smiles>

3j (mono)

${ }^{1} \mathrm{H}$ NMR $\left(400 \mathrm{MHz}, \mathrm{CDCl}_{3}\right)$

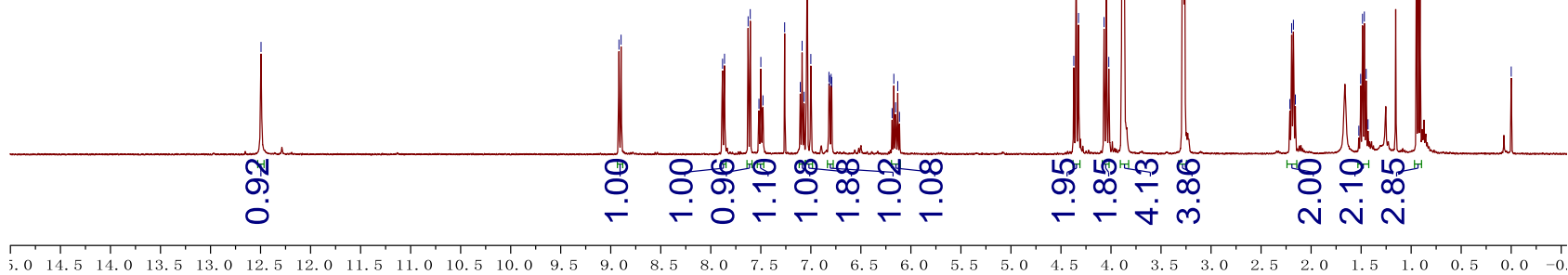

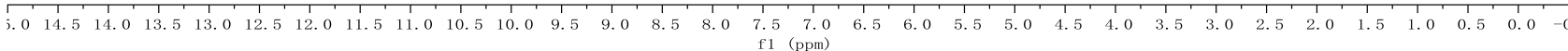




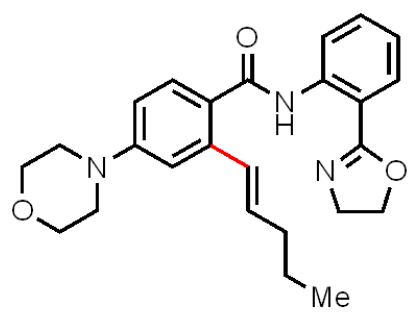

3j (mono)

${ }^{13} \mathrm{C}$ NMR $\left(100 \mathrm{MHz}, \mathrm{CDCl}_{3}\right)$

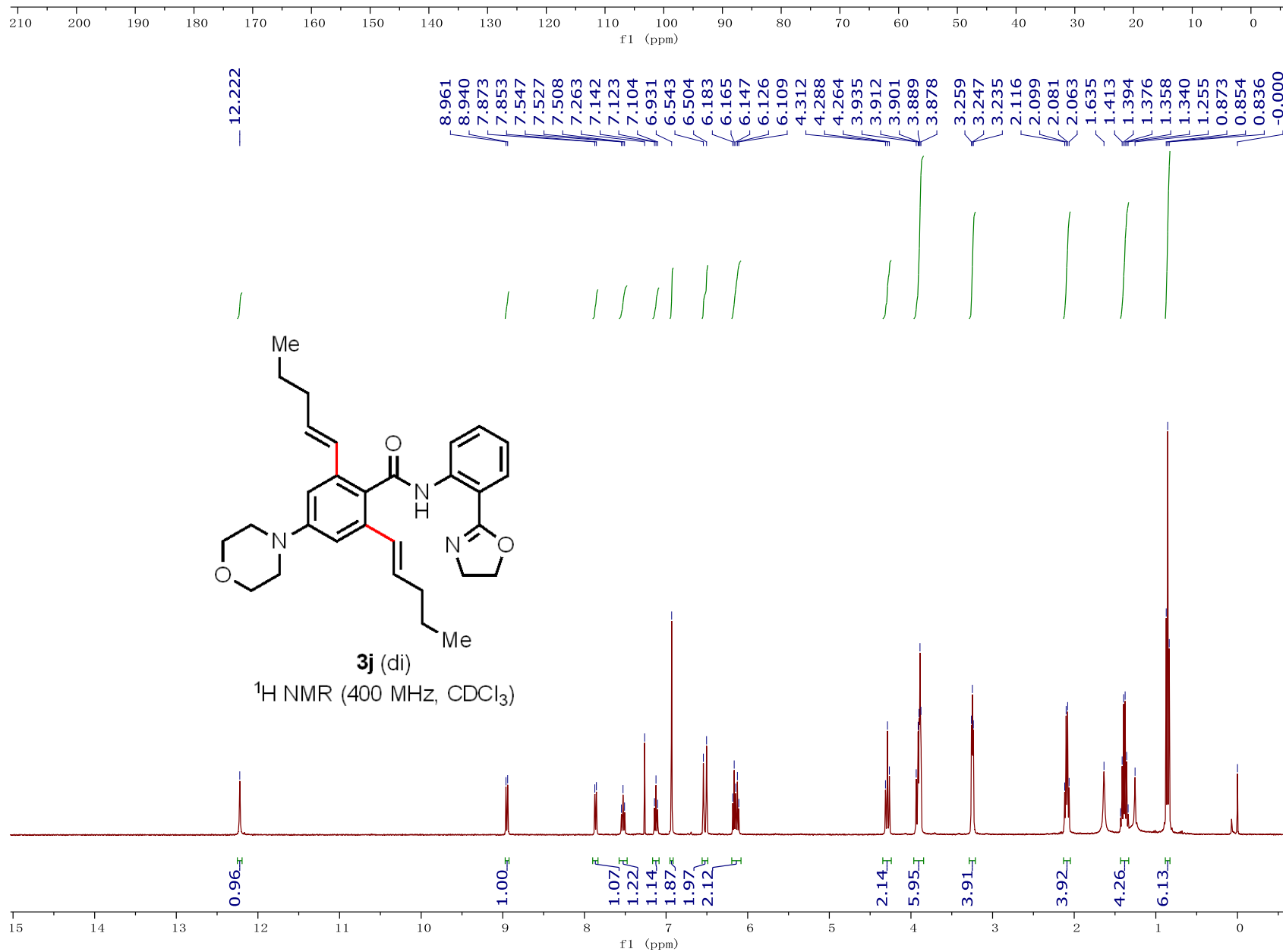


<smiles>CCCC=Cc1cc(N2CCOCC2)cc(C=CCCC)c1C(=O)Nc1ccccc1C1=NCCO1</smiles>

3j (di)

$\left.{ }^{13} \mathrm{C} \mathrm{NMR} \mathrm{(100} \mathrm{MHz,} \mathrm{CDCl}_{3}\right)$

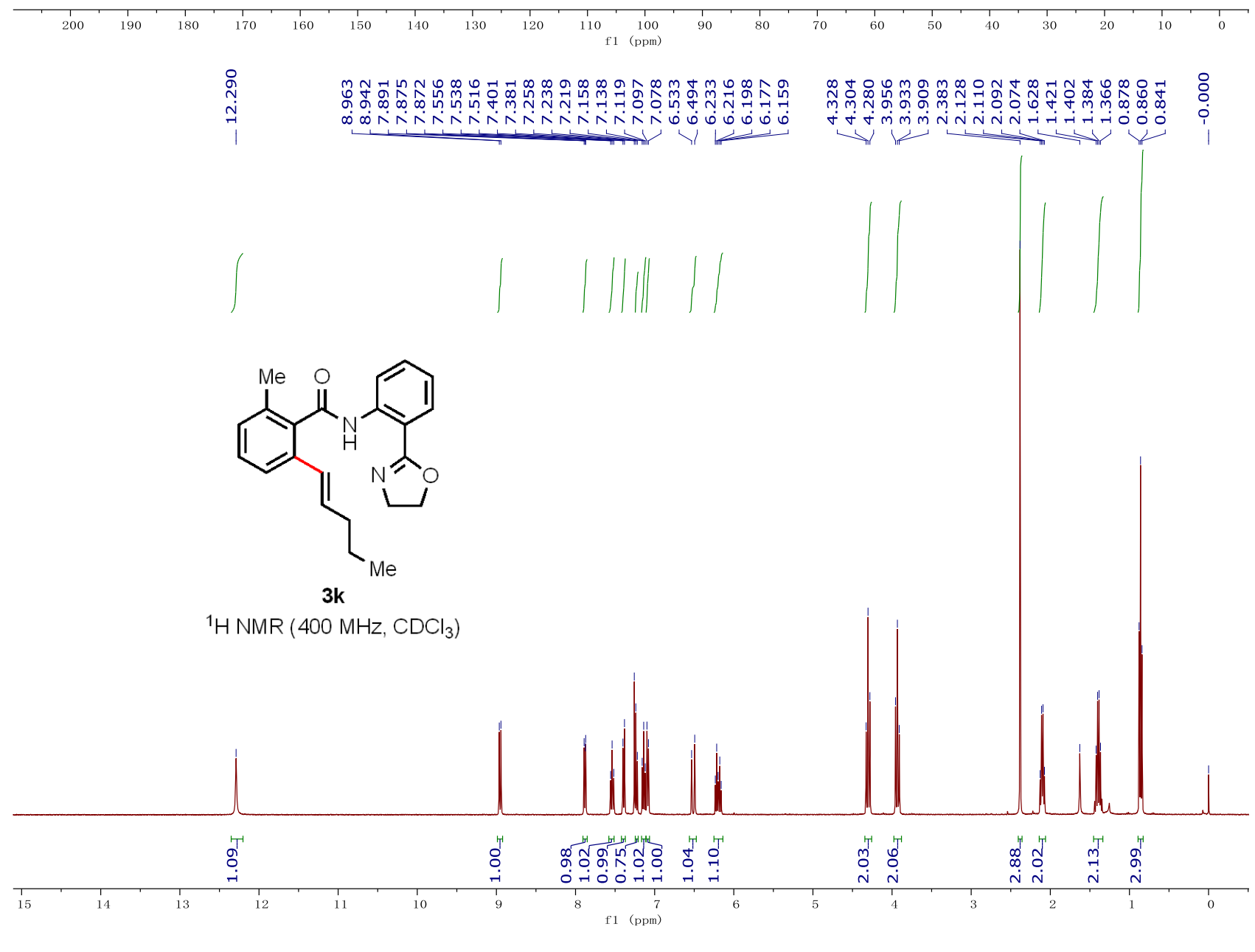


<smiles>CCC/C=C/c1cccc(C)c1C(=O)Nc1ccccc1C1=NCCO1</smiles>

3k

${ }^{13} \mathrm{CNMR}\left(100 \mathrm{MHz}, \mathrm{CDCl}_{3}\right)$

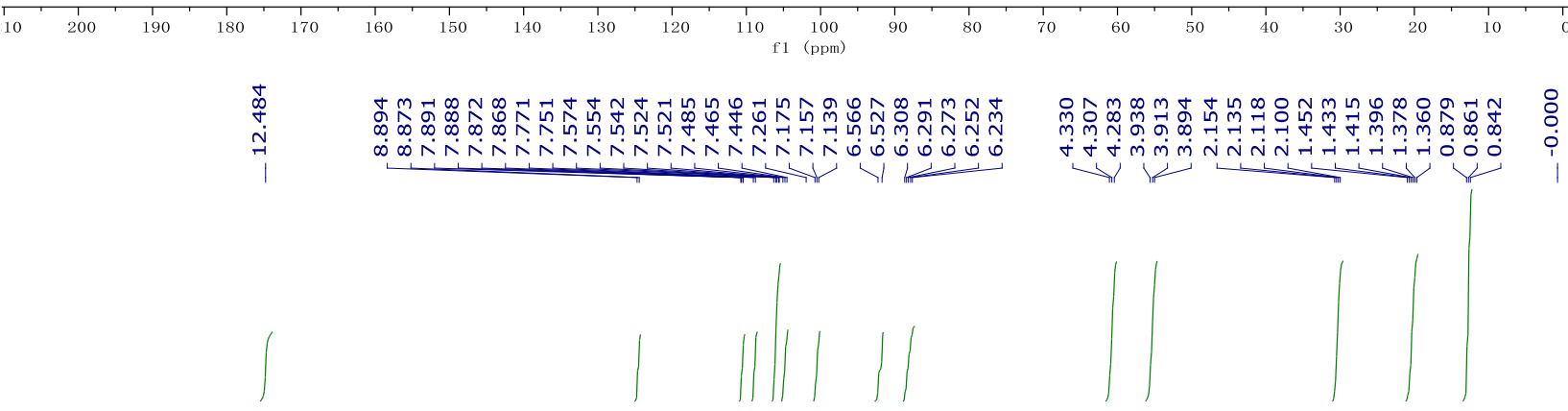<smiles>CCC/C=C/c1cccc(C(F)(F)F)c1C(=O)Nc1ccccc1C1=NCCO1</smiles>

3I

${ }^{1} \mathrm{H} \operatorname{NMR}\left(400 \mathrm{MHz}, \mathrm{CDCl}_{3}\right)$

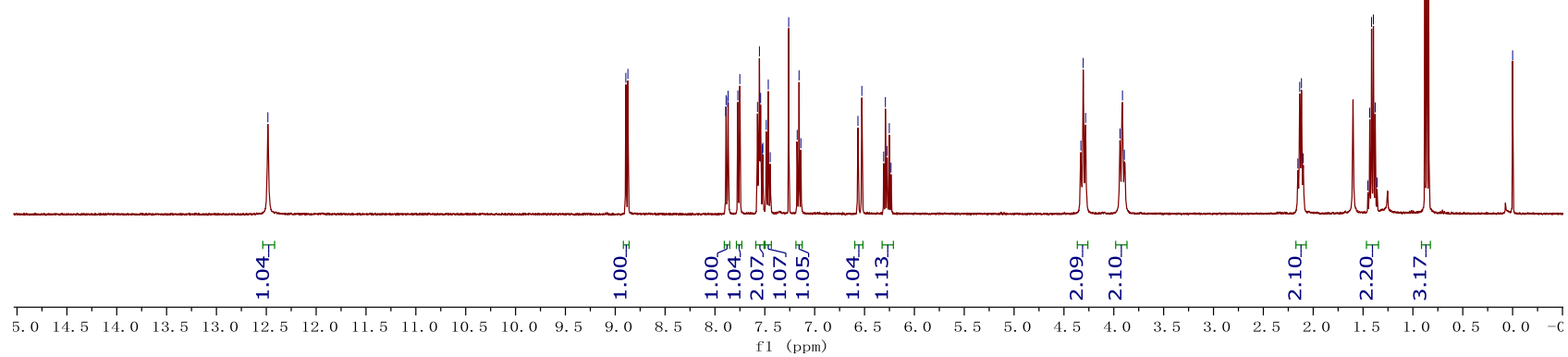




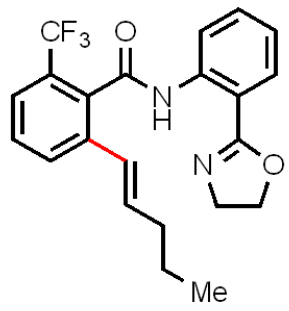

$$
31
$$

${ }^{13} \mathrm{C}$ NMR $\left(100 \mathrm{MHz}, \mathrm{CDCl}_{3}\right)$
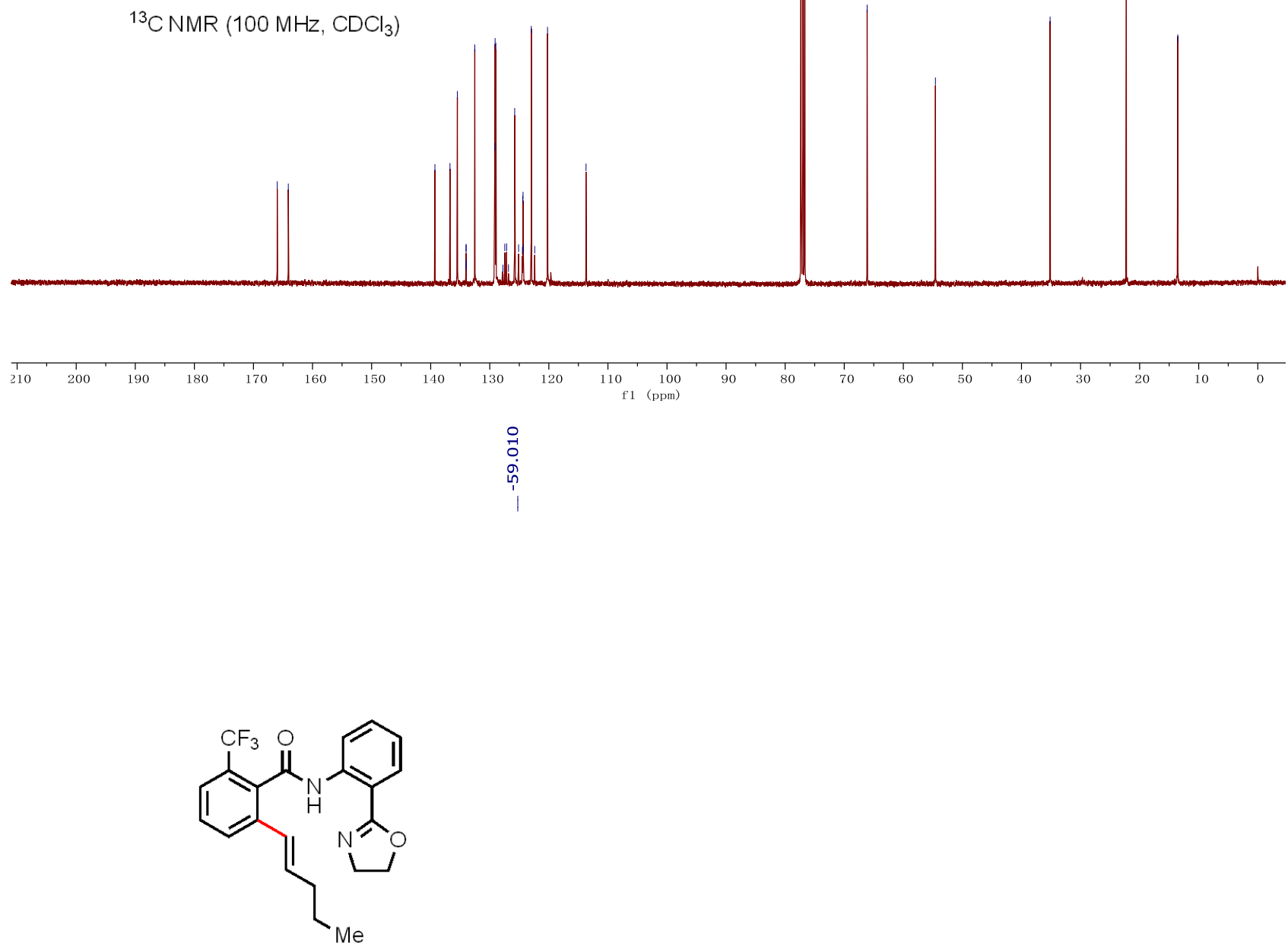

3I

${ }^{19} \mathrm{~F}$ NMR (375 MHz, CDCl 3 )

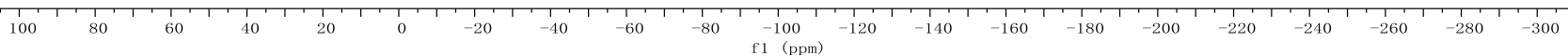




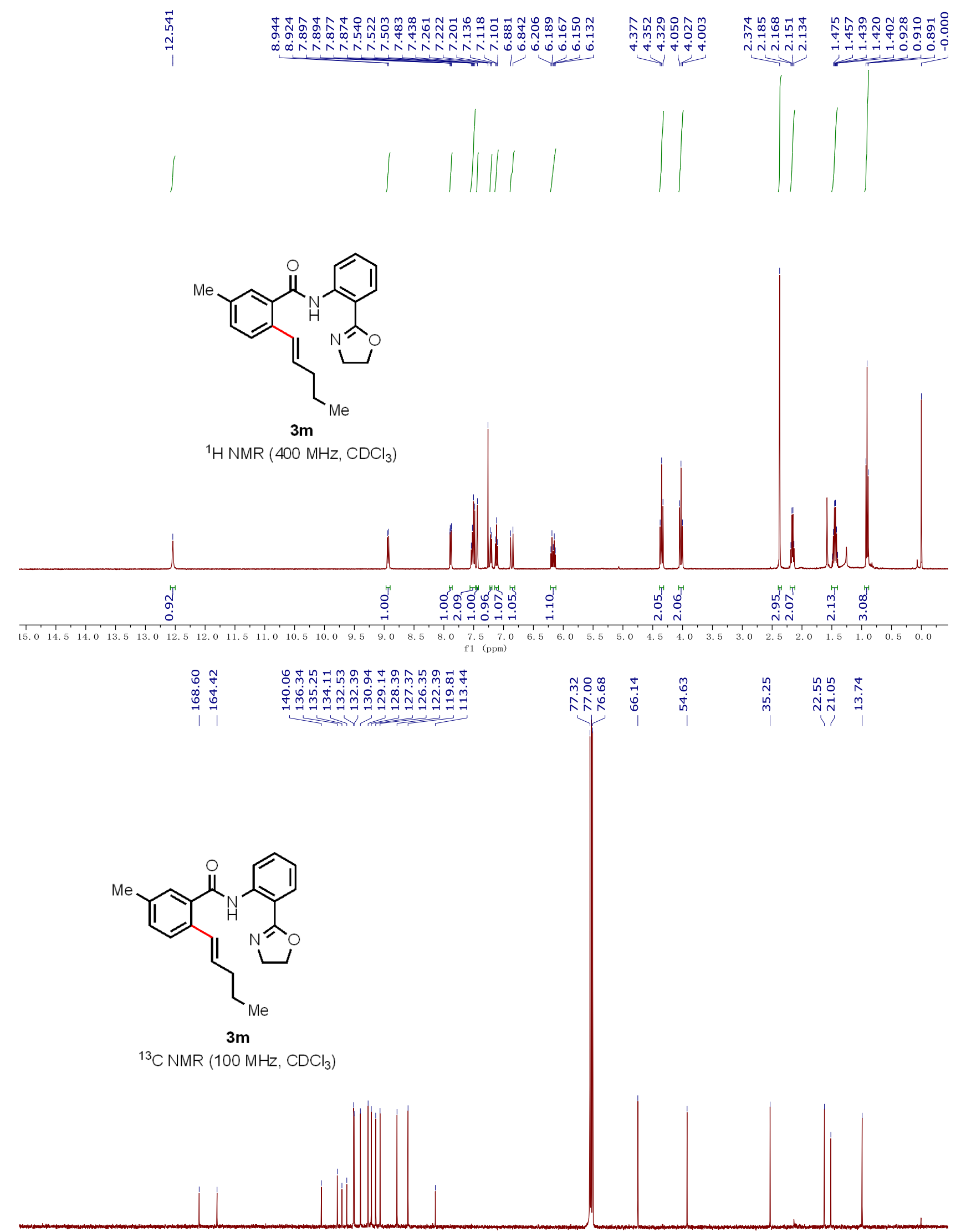

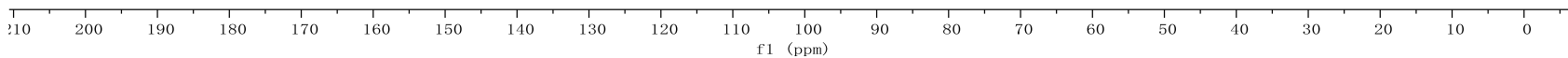



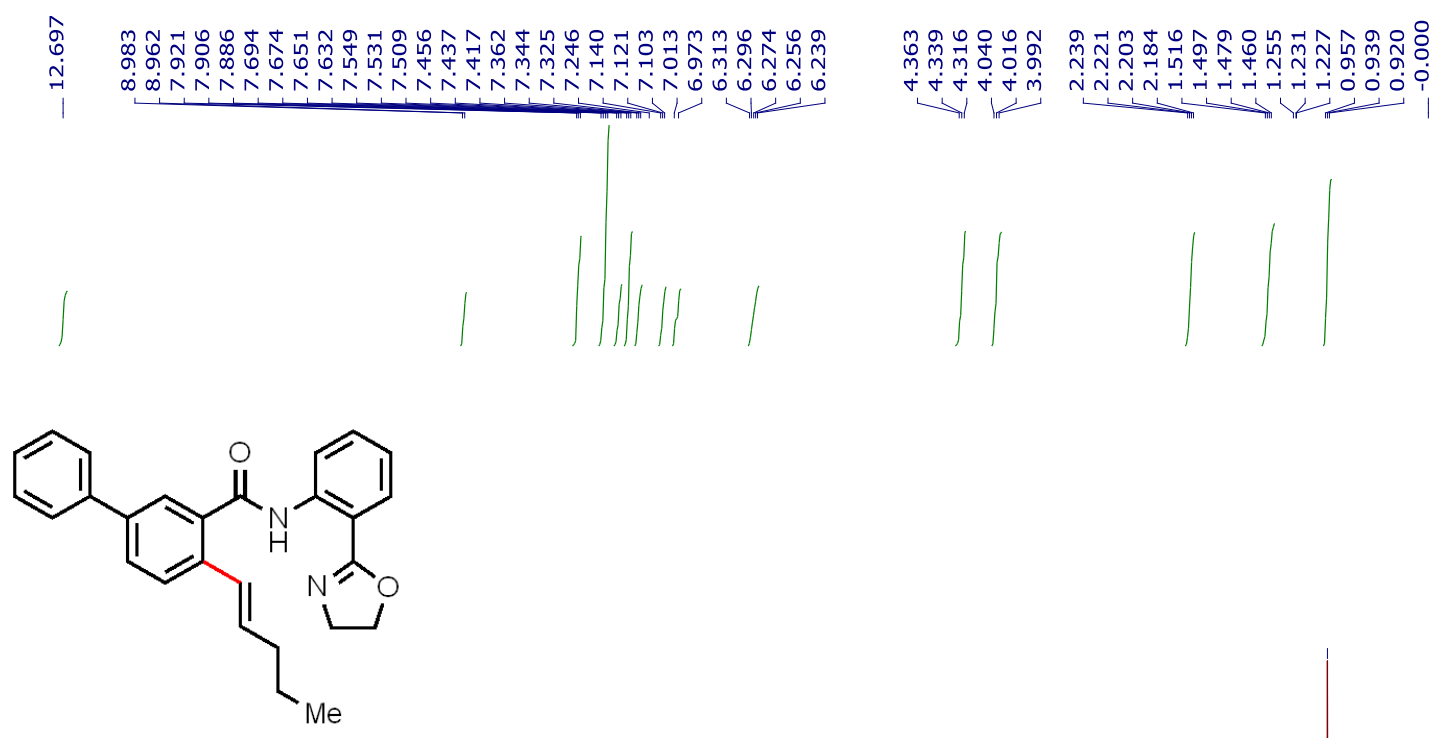

3n

${ }^{1} \mathrm{H} \mathrm{NMR}\left(400 \mathrm{MHz}, \mathrm{CDCl}_{3}\right)$
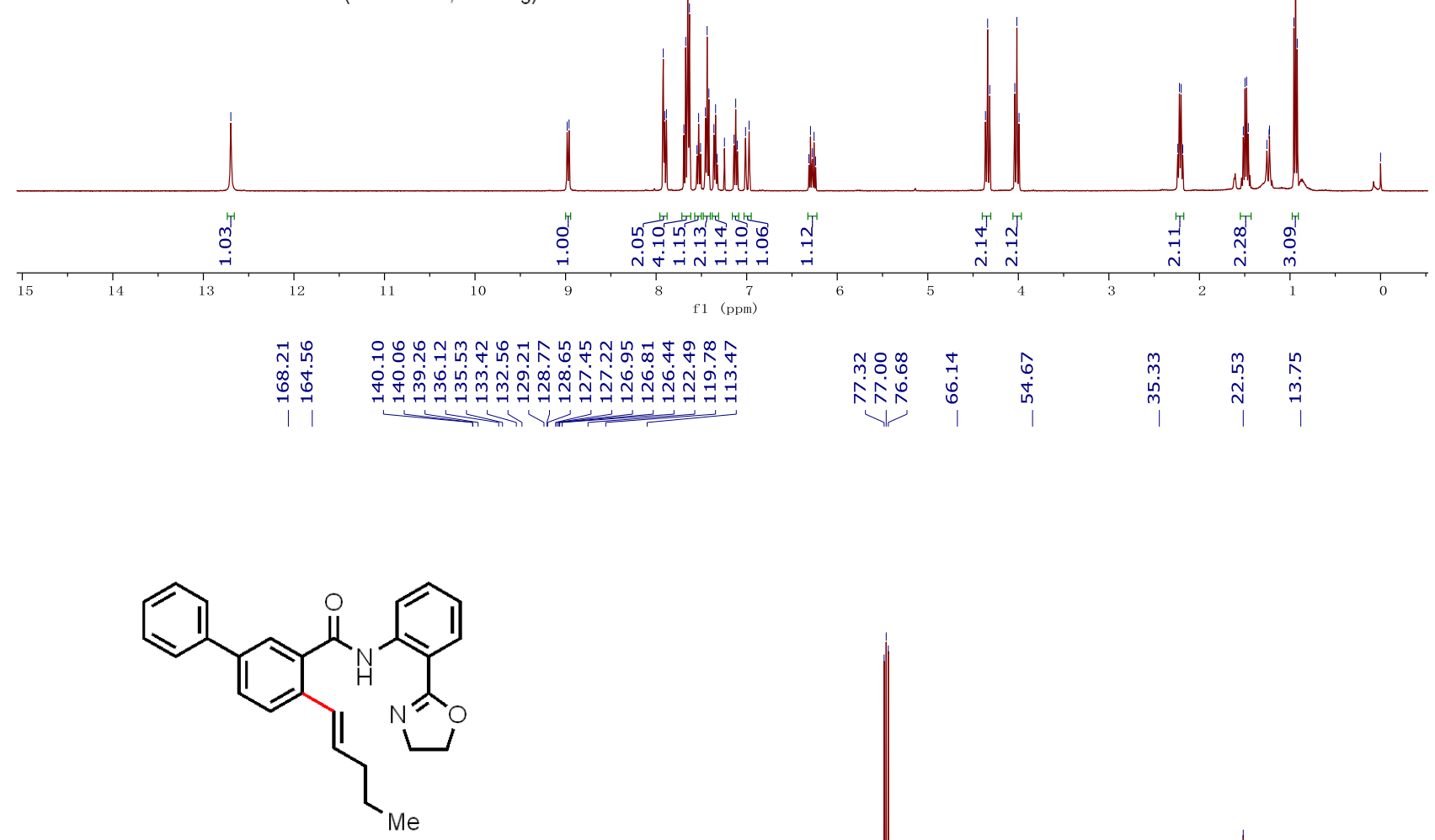

3n

${ }^{13} \mathrm{C} \mathrm{NMR}\left(100 \mathrm{MHz}, \mathrm{CDCl}_{3}\right)$

$\begin{array}{lllllllllll}200 & 190 & 180 & 170 & 160 & 150 & 140 & 130 & 120 & 110 & 100 \\ f 1 & & & & & & & & & & 100 m)\end{array}$ 


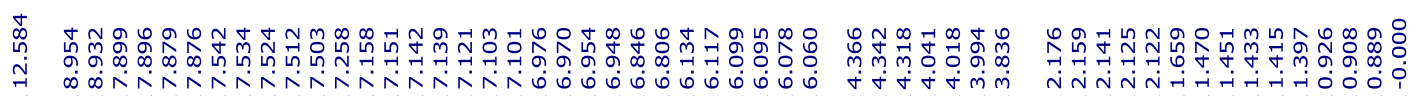
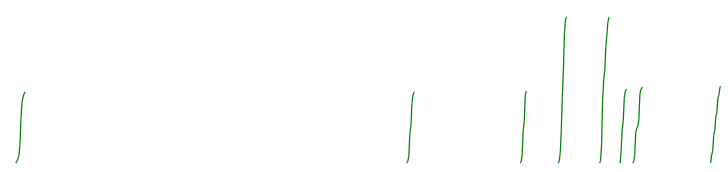<smiles>CCC/C=C/c1ccc(OC)cc1C(=O)Nc1ccccc1C1=NCCO1</smiles>

30

${ }^{1} \mathrm{HNMR}\left(400 \mathrm{MHz}, \mathrm{CDCl}_{3}\right)$

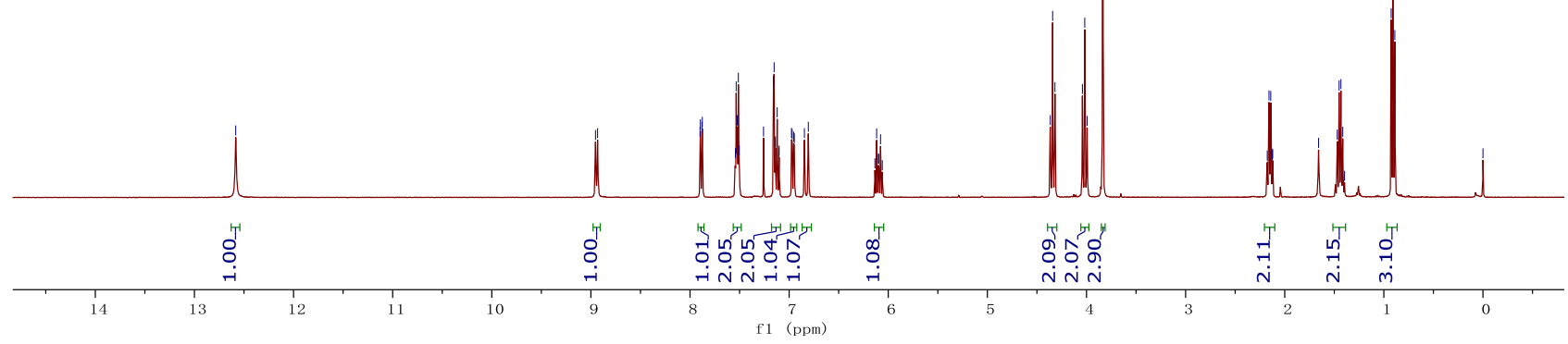

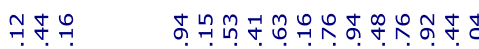

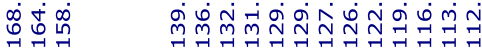

1,1

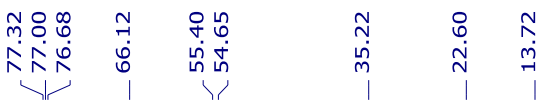

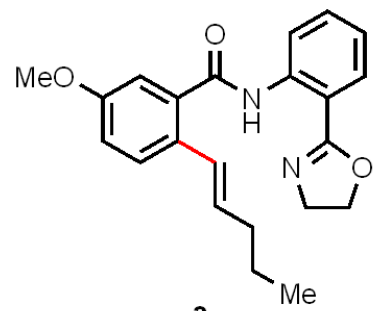

${ }^{13} \mathrm{C}$ NMR $\left(100 \mathrm{MHz}, \mathrm{CDCl}_{3}\right)$

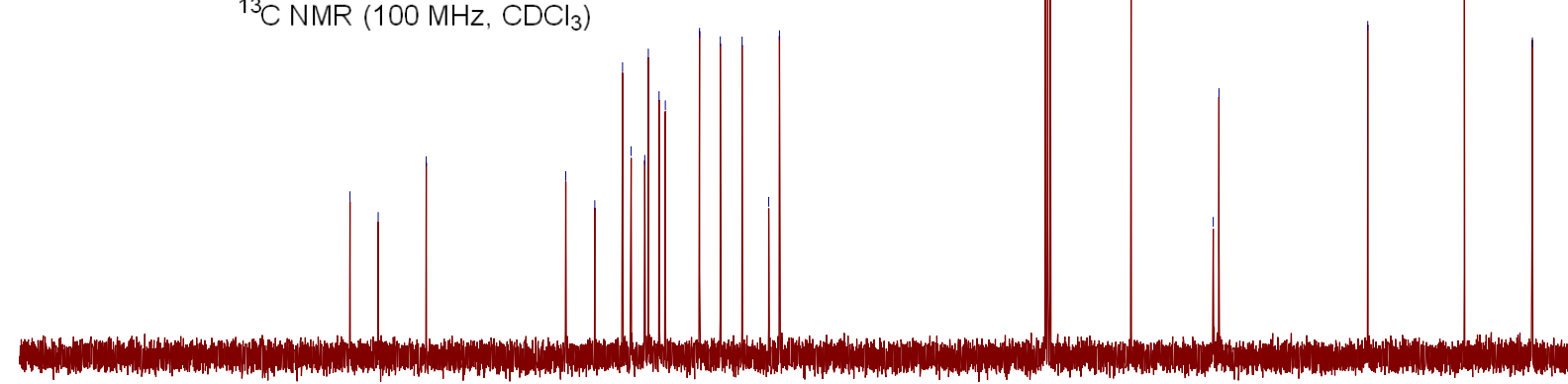

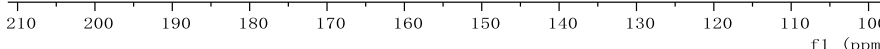




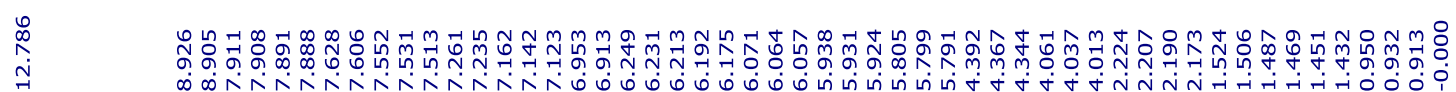
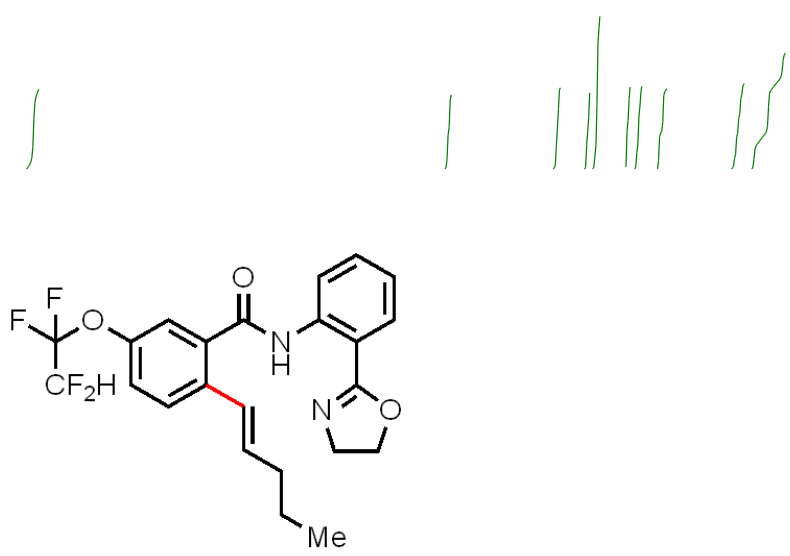

$3 p$

${ }^{1} \mathrm{H}$ NMR $\left(400 \mathrm{MHz}, \mathrm{CDCl}_{3}\right)$

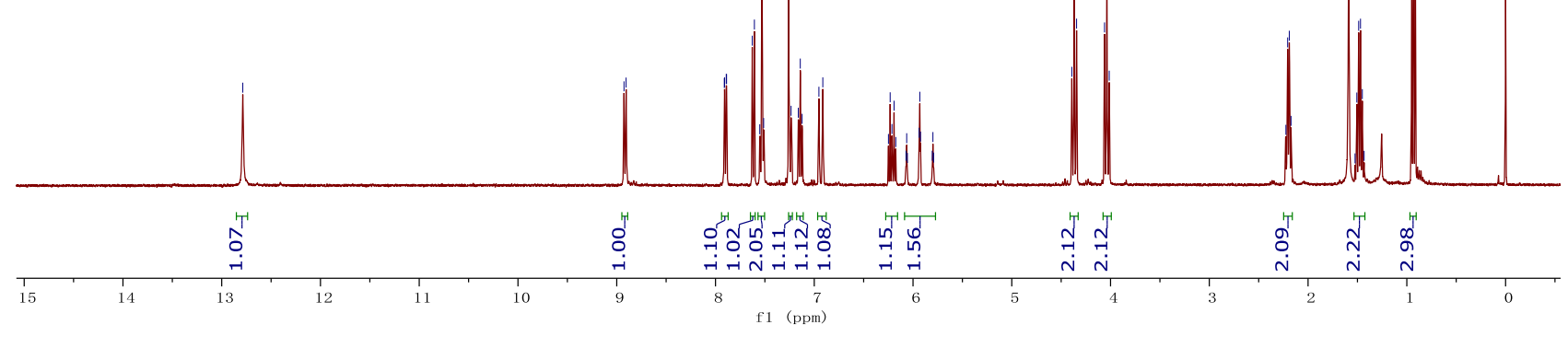

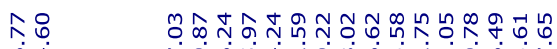

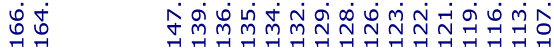

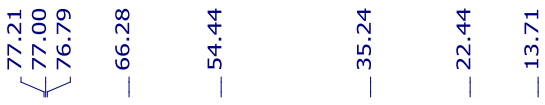

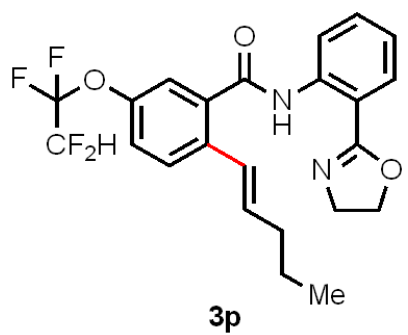

${ }^{13} \mathrm{C} \mathrm{NMR}(150 \mathrm{MHz}$ decoupled for ${ }^{19} \mathrm{~F}, \mathrm{CDCl}_{3}$ )

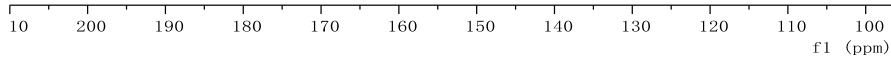



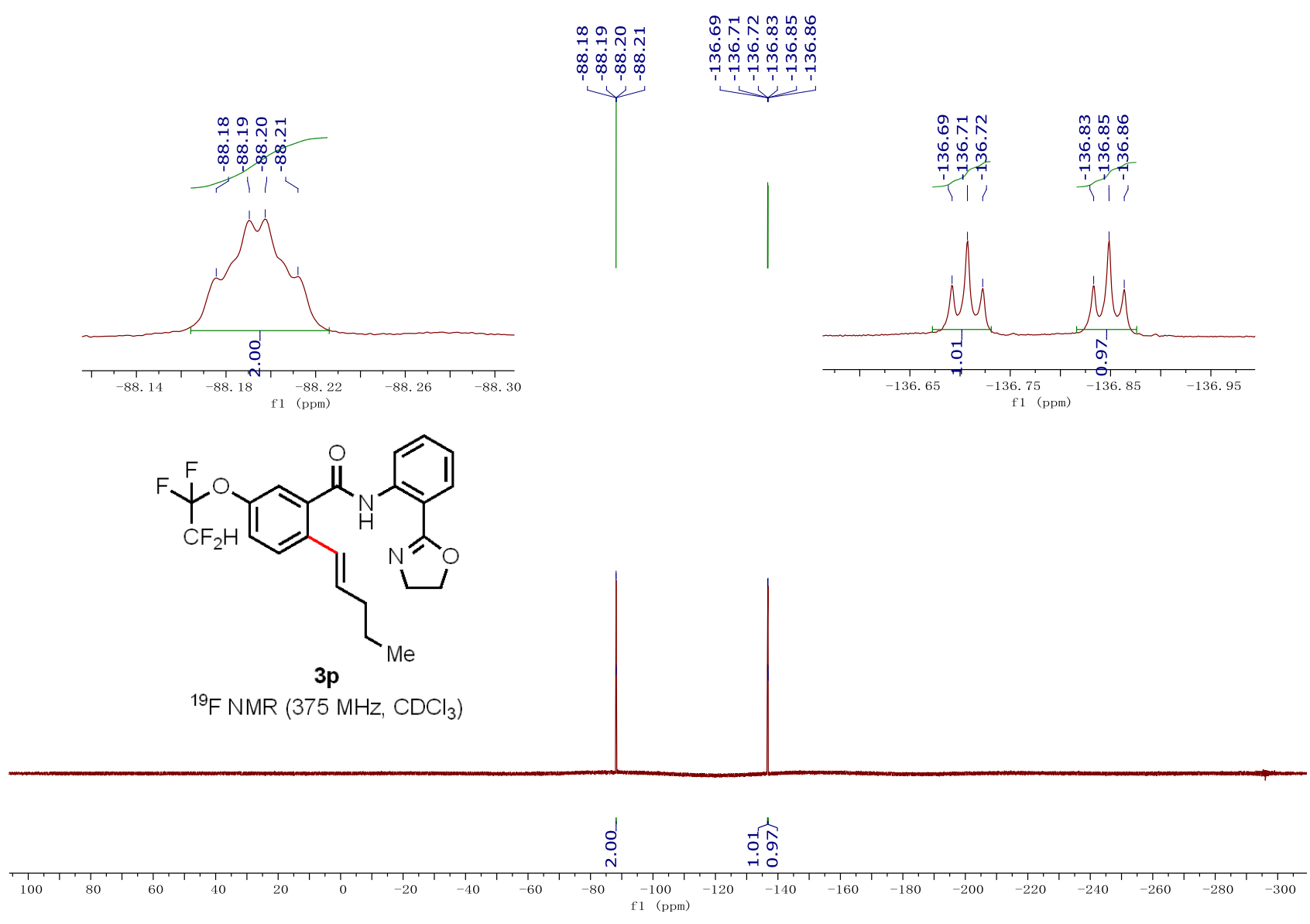

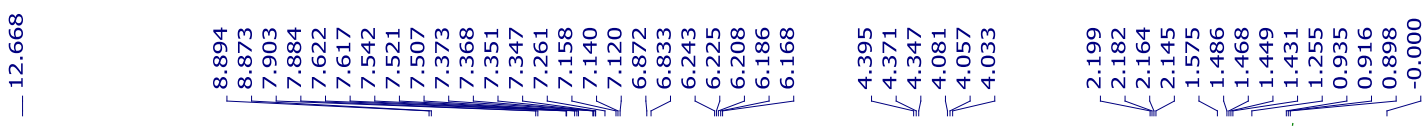
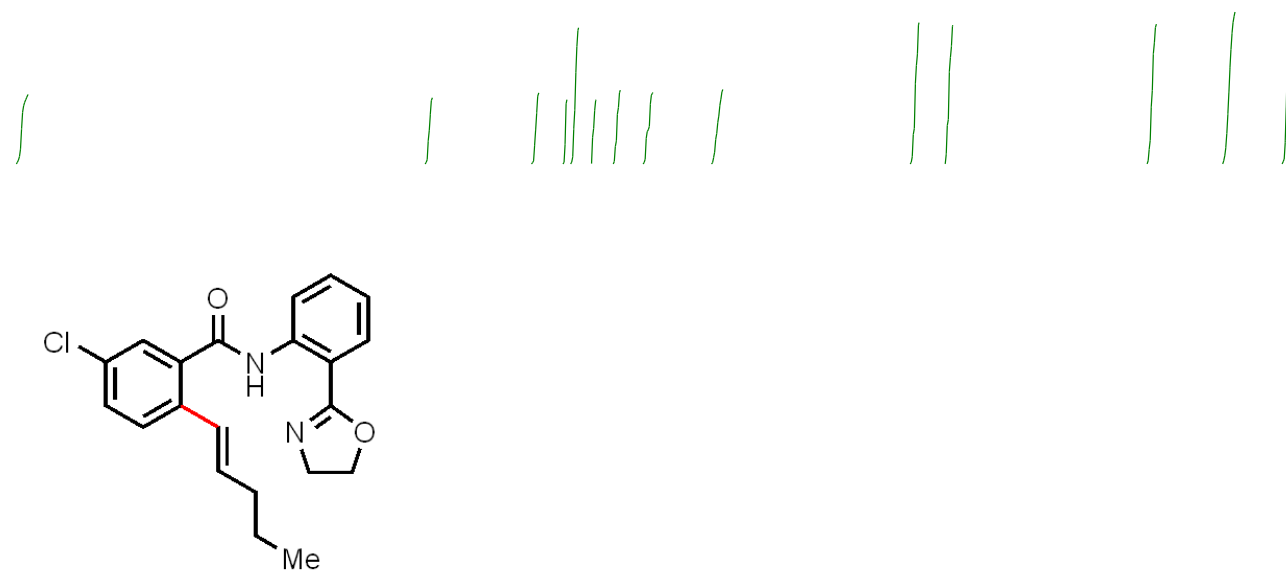

$3 q$

${ }^{1} \mathrm{H}$ NMR (400 MHz, $\left.\mathrm{CDCl}_{3}\right)$

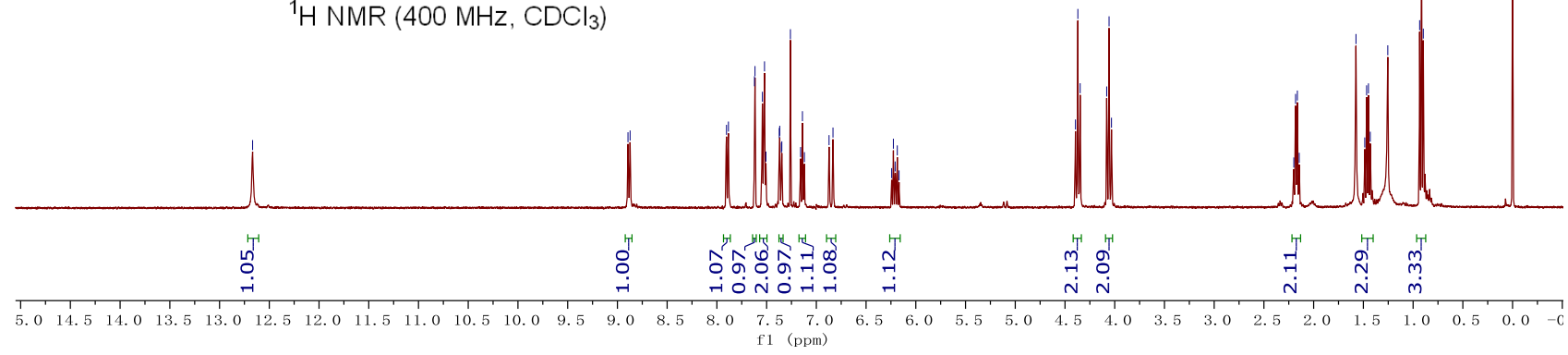




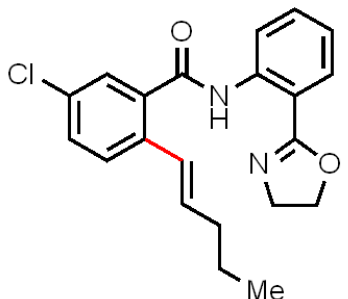

$3 q$

${ }^{13} \mathrm{C} \mathrm{NMR}\left(100 \mathrm{MHz}, \mathrm{CDCl}_{3}\right)$
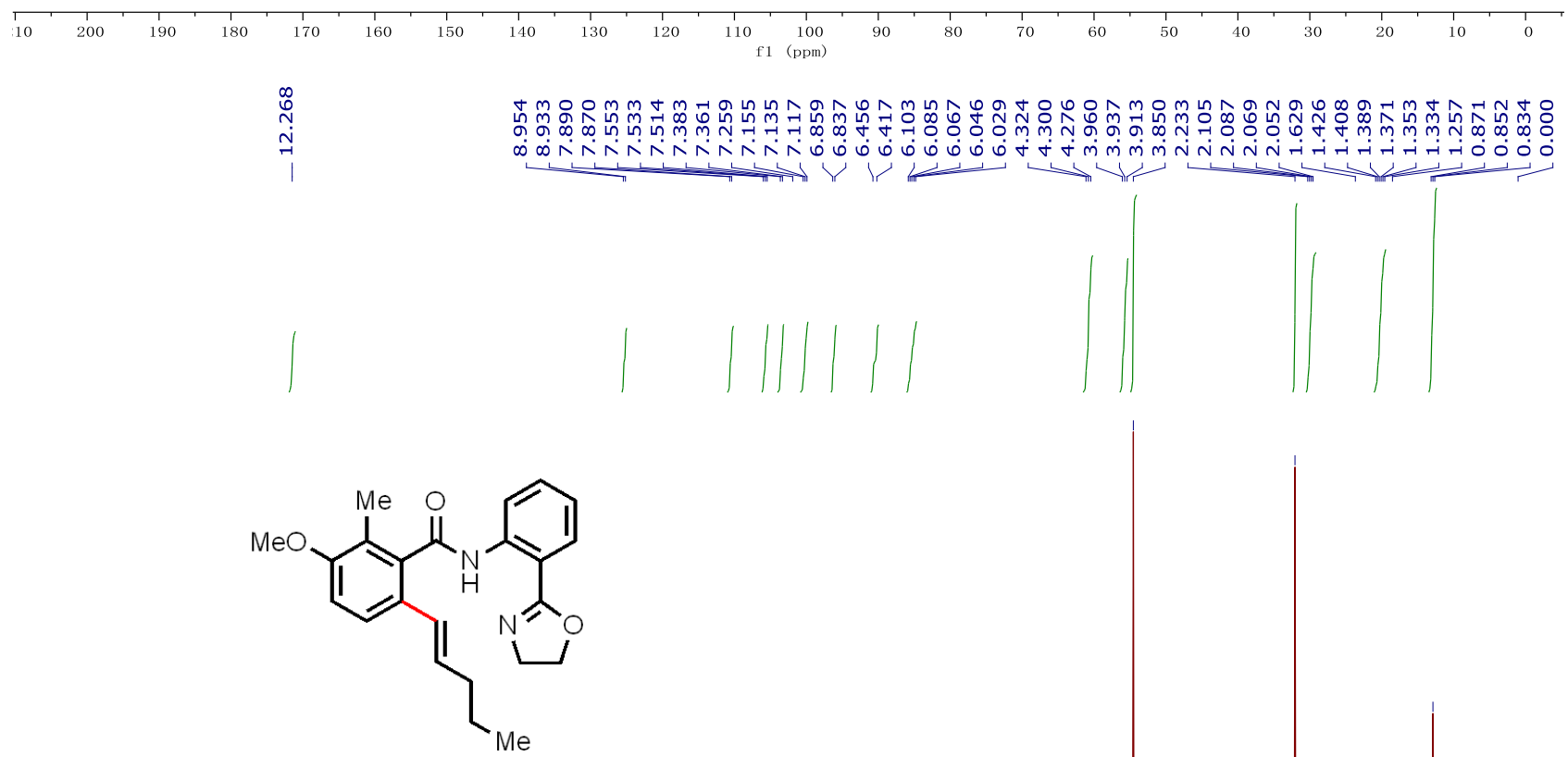

$3 r$

${ }^{1} \mathrm{HNMR}\left(400 \mathrm{MHz}, \mathrm{CDCl}_{3}\right)$

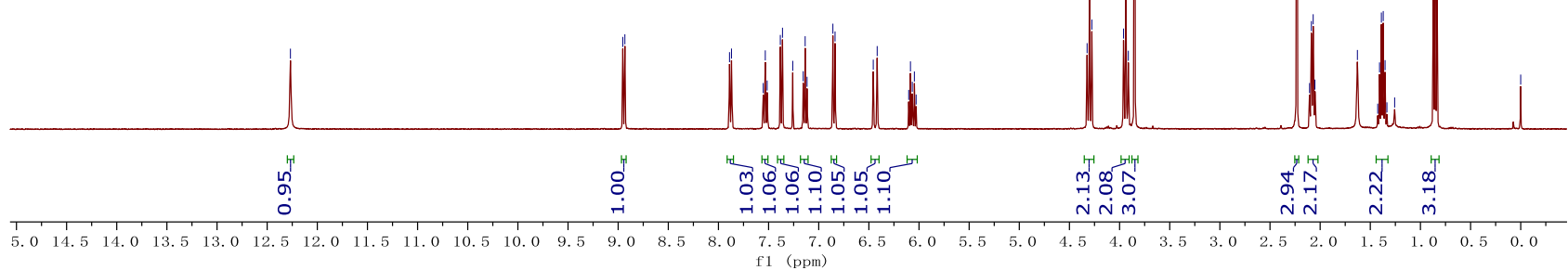




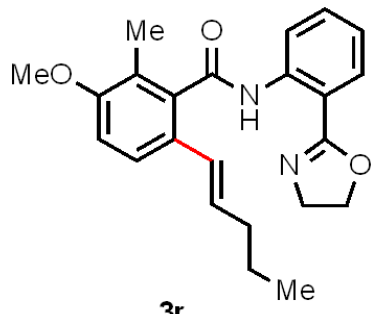

${ }^{13} \mathrm{C} \mathrm{NMR}\left(100 \mathrm{MHz}, \mathrm{CDCl}_{3}\right)$
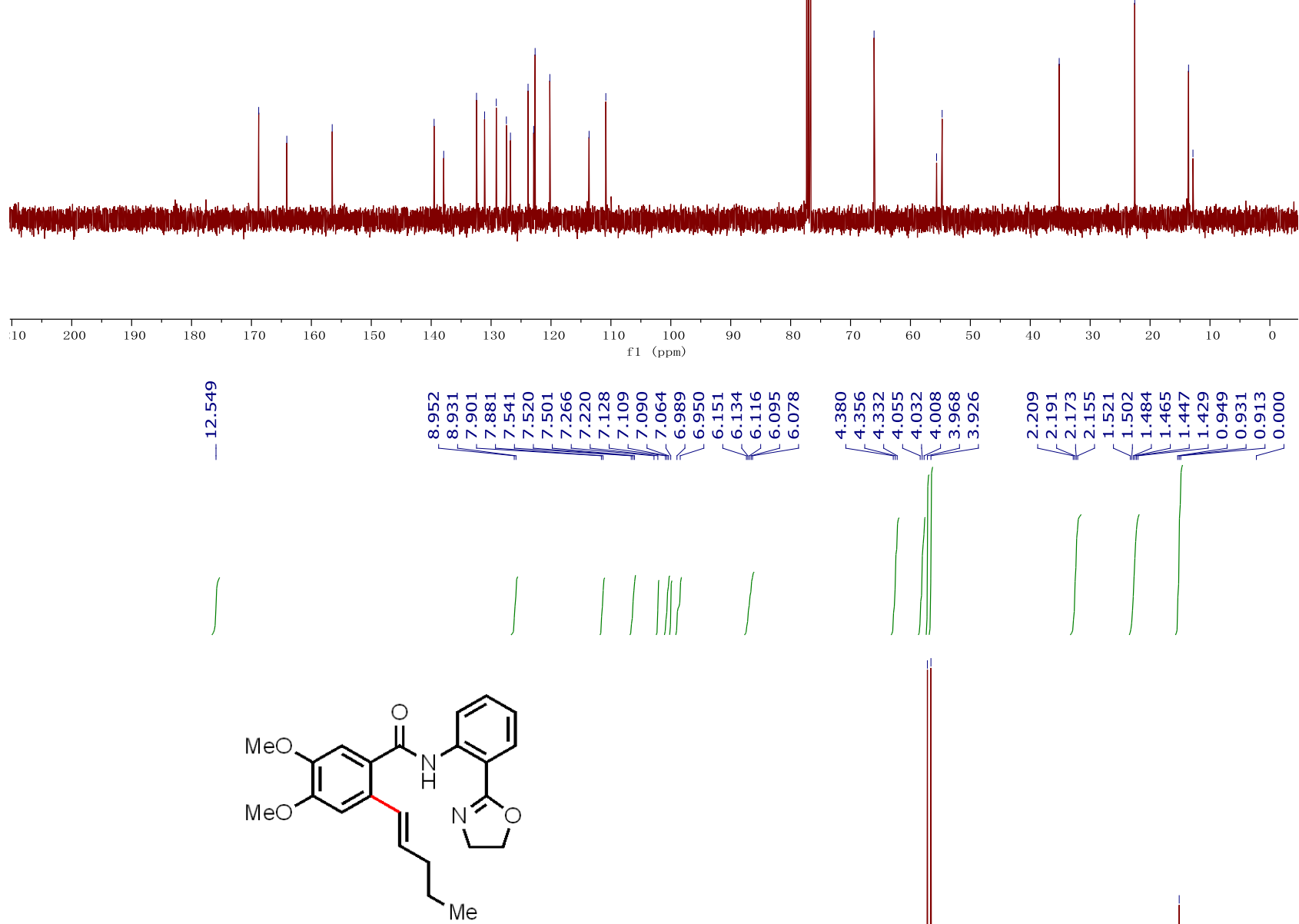

$3 s$

${ }^{1} \mathrm{HNMR}\left(400 \mathrm{MHz}, \mathrm{CDCl}_{3}\right)$

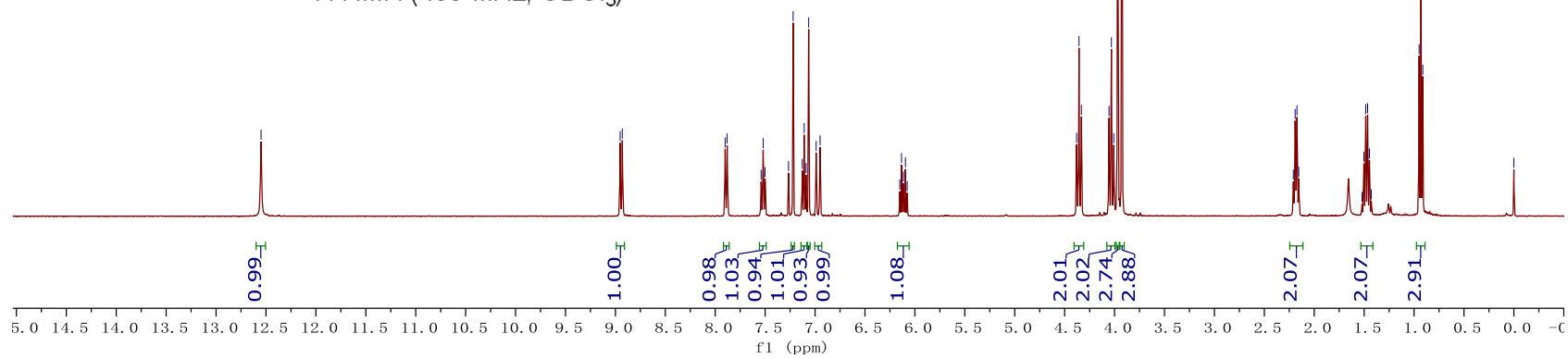




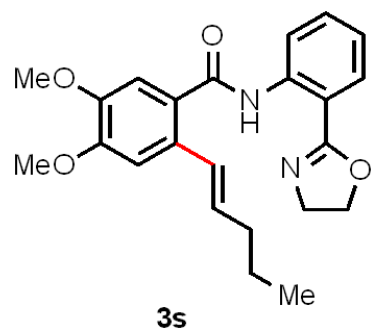

${ }^{13} \mathrm{C} \mathrm{NMR}\left(100 \mathrm{MHz}, \mathrm{CDCl}_{3}\right)$
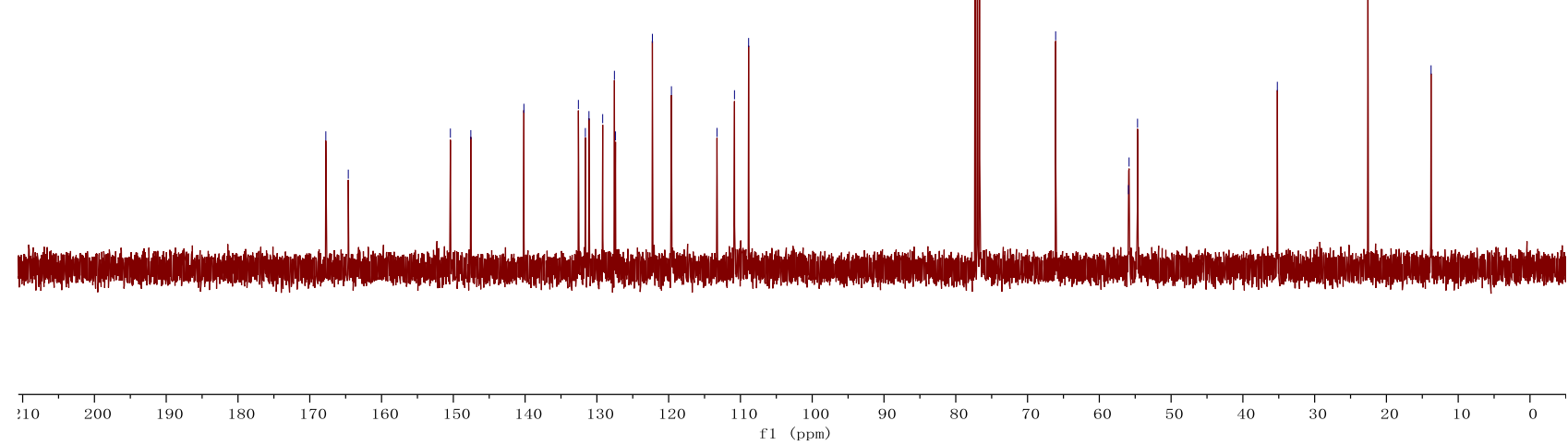

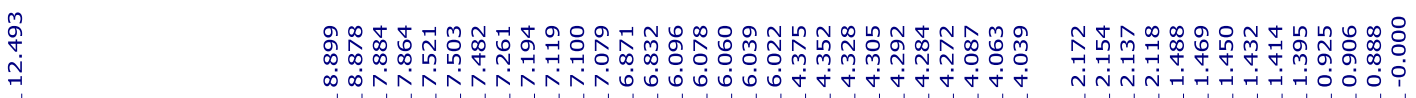

$\underbrace{\infty}_{\text {miा }}$

倠
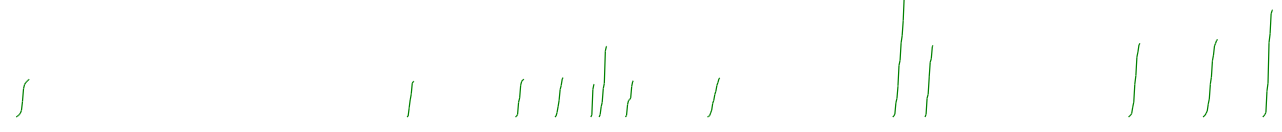<smiles>CCC/C=C/c1cc2c(cc1C(=O)Nc1ccccc1C1=NCCO1)OCCO2</smiles>

3t $\left(\mathrm{C}_{7}\right)$

${ }^{1} \mathrm{H} \mathrm{NMR}\left(400 \mathrm{MHz}, \mathrm{CDCl}_{3}\right)$

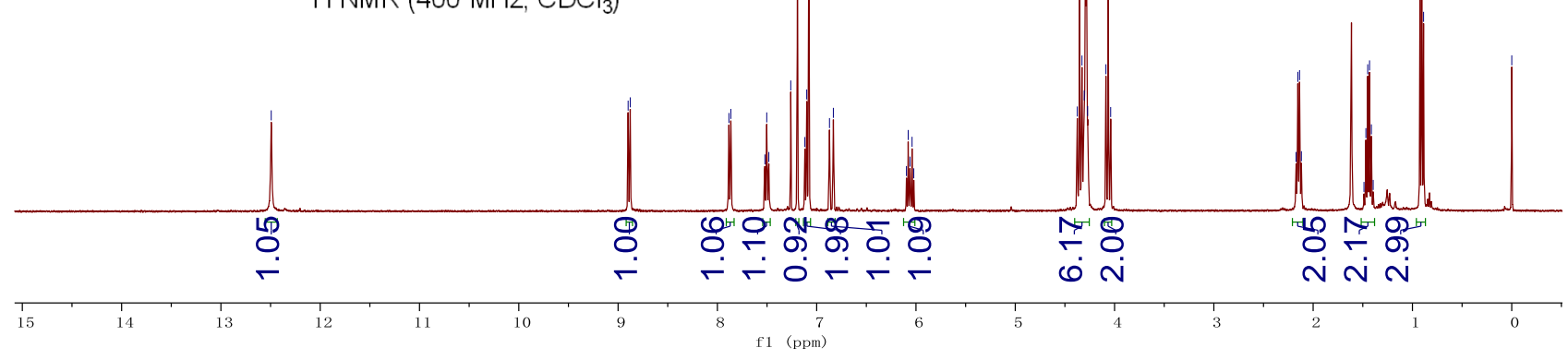




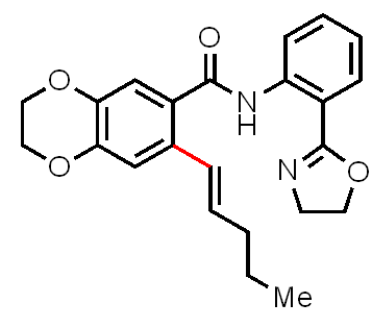

3t $\left(\mathrm{C}_{7}\right)$

${ }^{13} \mathrm{C} \mathrm{NMR}\left(100 \mathrm{MHz}, \mathrm{CDCl}_{3}\right)$
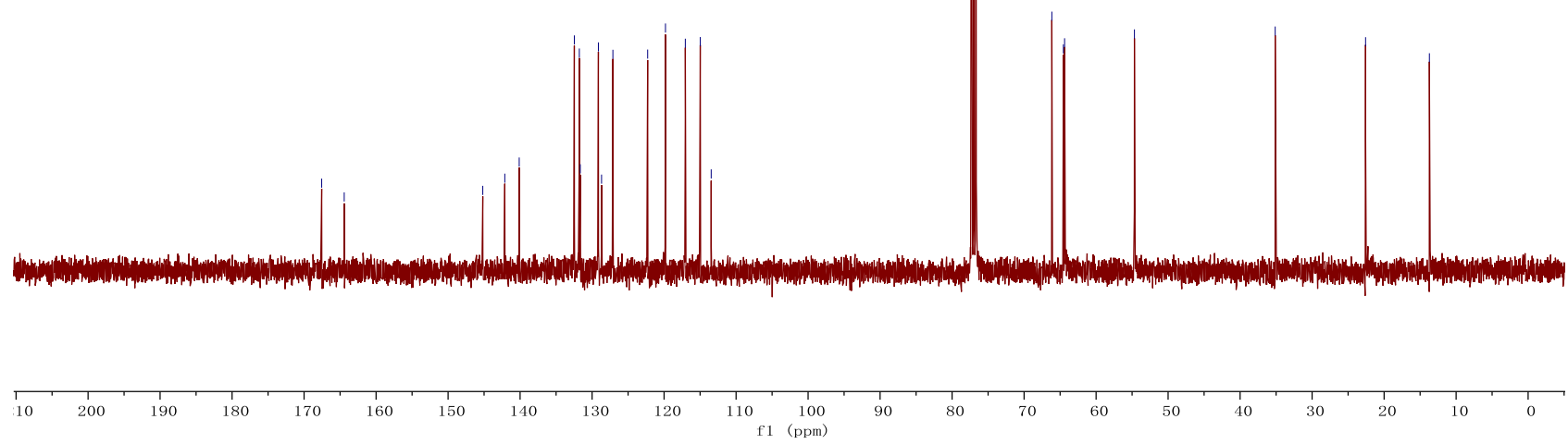

$\underset{\substack{n \\ \stackrel{n}{N}}}{\stackrel{\sim}{N}}$

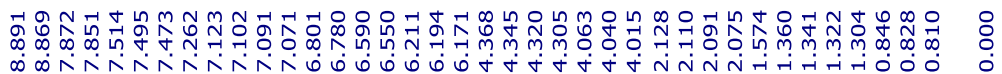

l

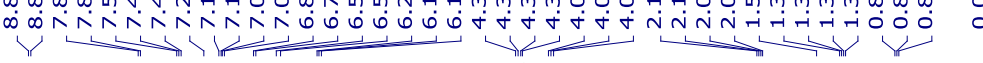<smiles>CCC/C=C/c1c(C(=O)Nc2ccccc2C2=NCCO2)ccc2c1OCCO2</smiles>

3t $\left(\mathrm{C}_{5}\right)$

${ }^{1} \mathrm{H}$ NMR $\left(400 \mathrm{MHz}, \mathrm{CDCl}_{3}\right)$

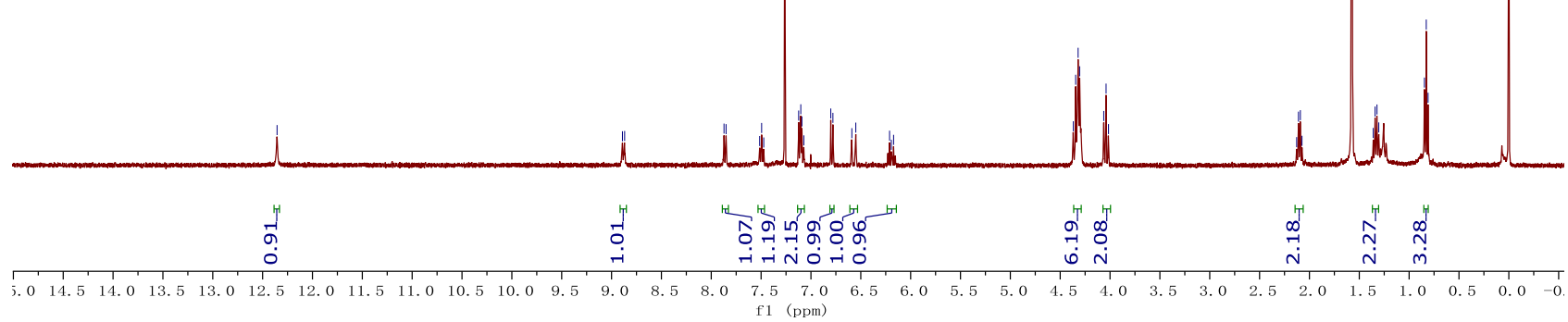




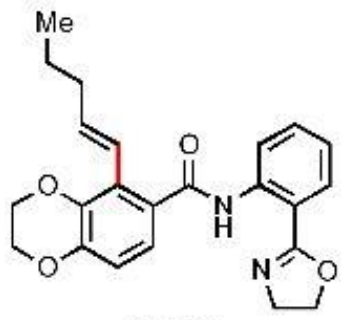

$3 t\left\langle C_{5}\right)$

${ }^{13} \mathrm{C} \mathrm{NMR}\left(100 \mathrm{MHz}^{\mathrm{CDCl}}\right)_{3}$
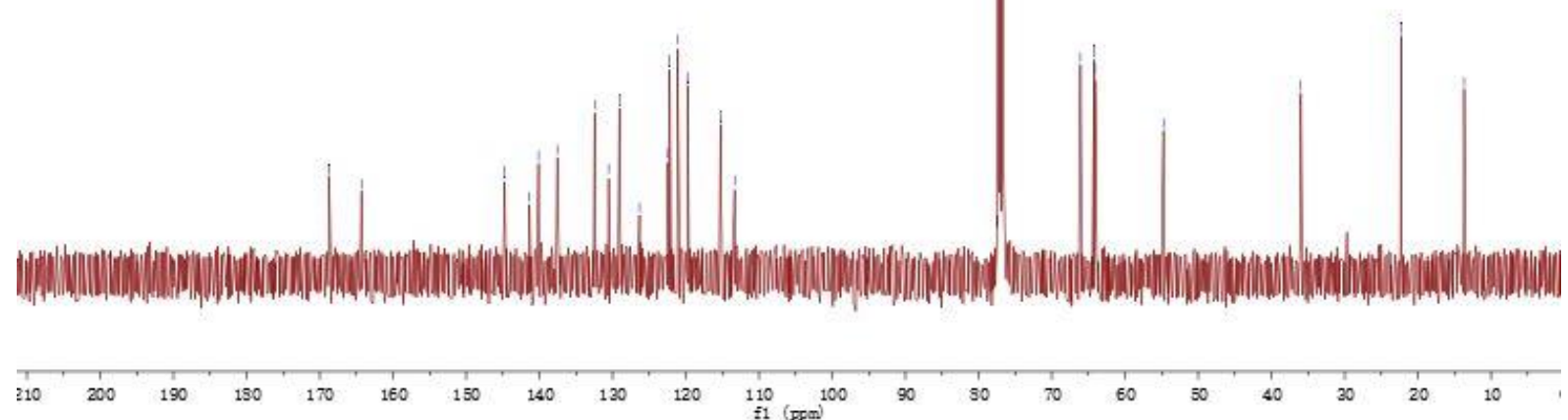

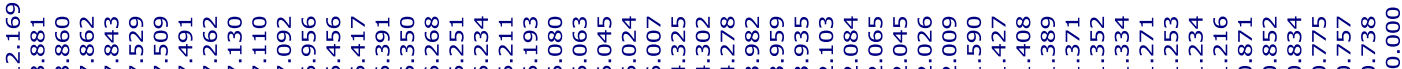
ન
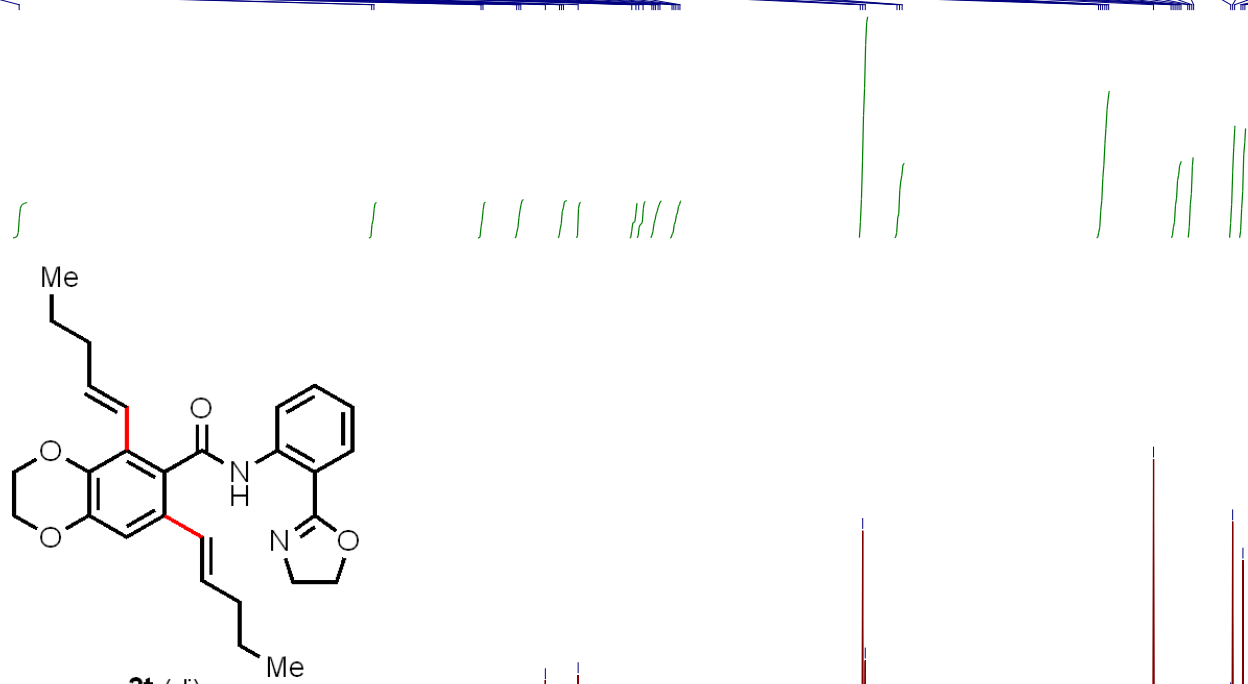

3t (di)

${ }^{1} \mathrm{HNMR}\left(400 \mathrm{MHz}, \mathrm{CDCl}_{3}\right)$
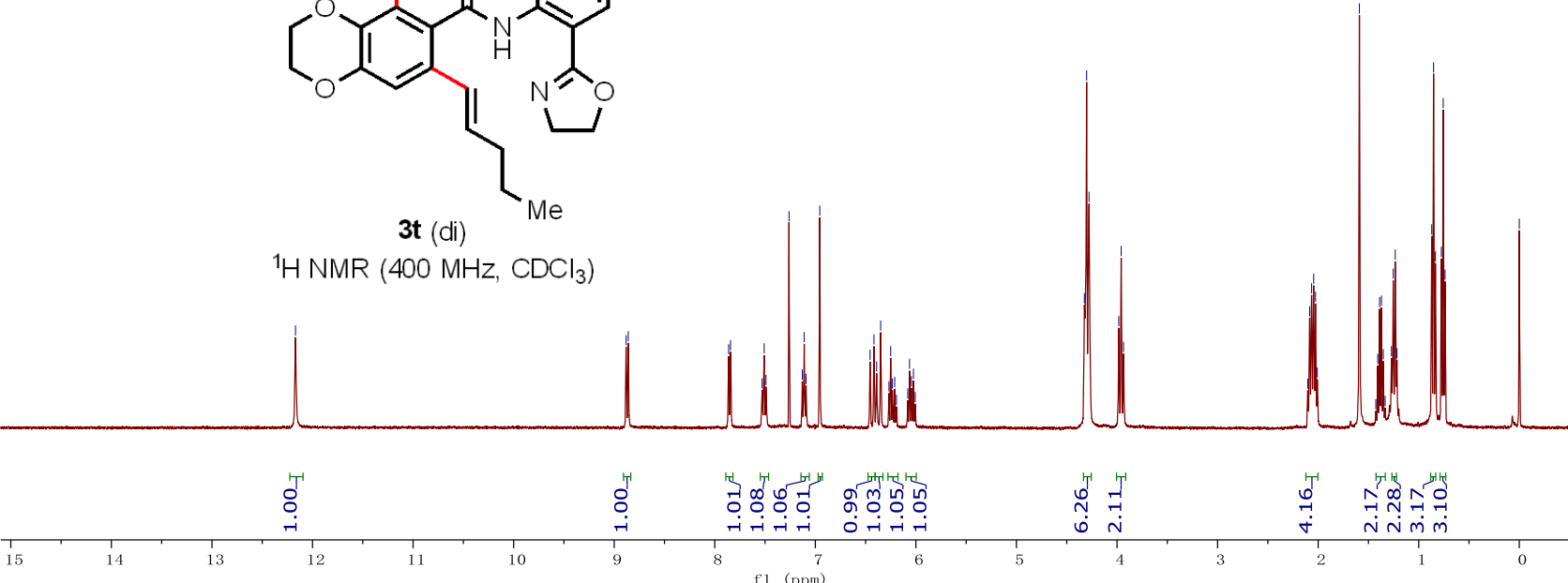

ग़่

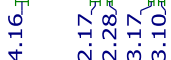




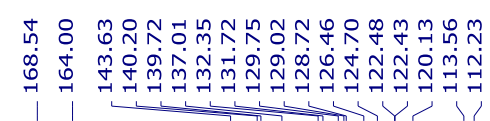

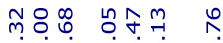

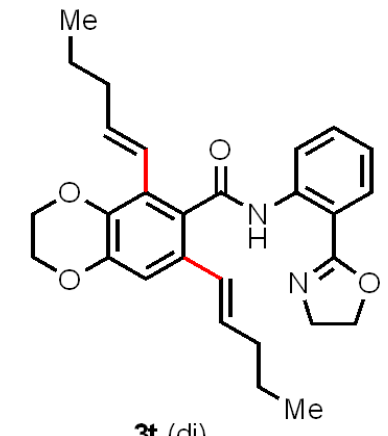

3t (di)

${ }^{13} \mathrm{C}$ NMR $\left(100 \mathrm{MHz}, \mathrm{CDCl}_{3}\right)$

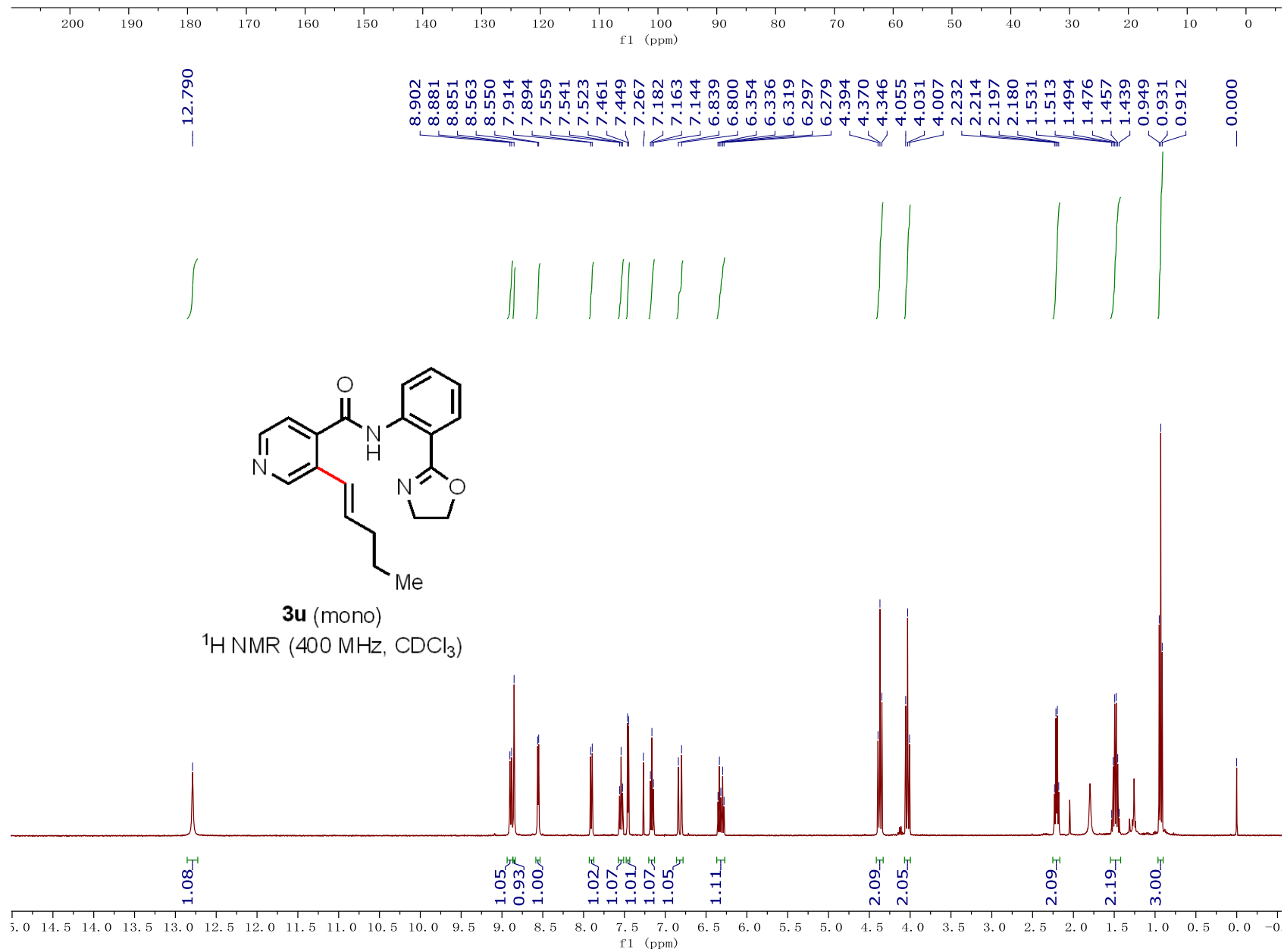




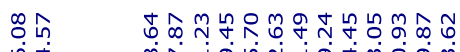

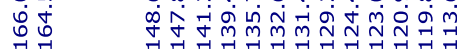

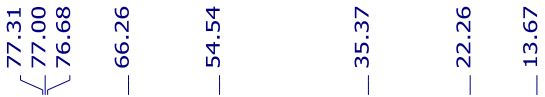

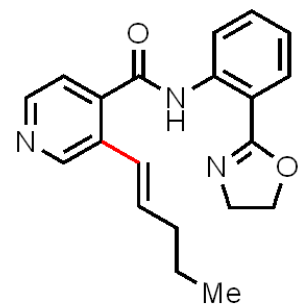

3u (mono)

${ }^{13} \mathrm{C} \mathrm{NMR}\left(100 \mathrm{MHz}, \mathrm{CDCl}_{3}\right)$

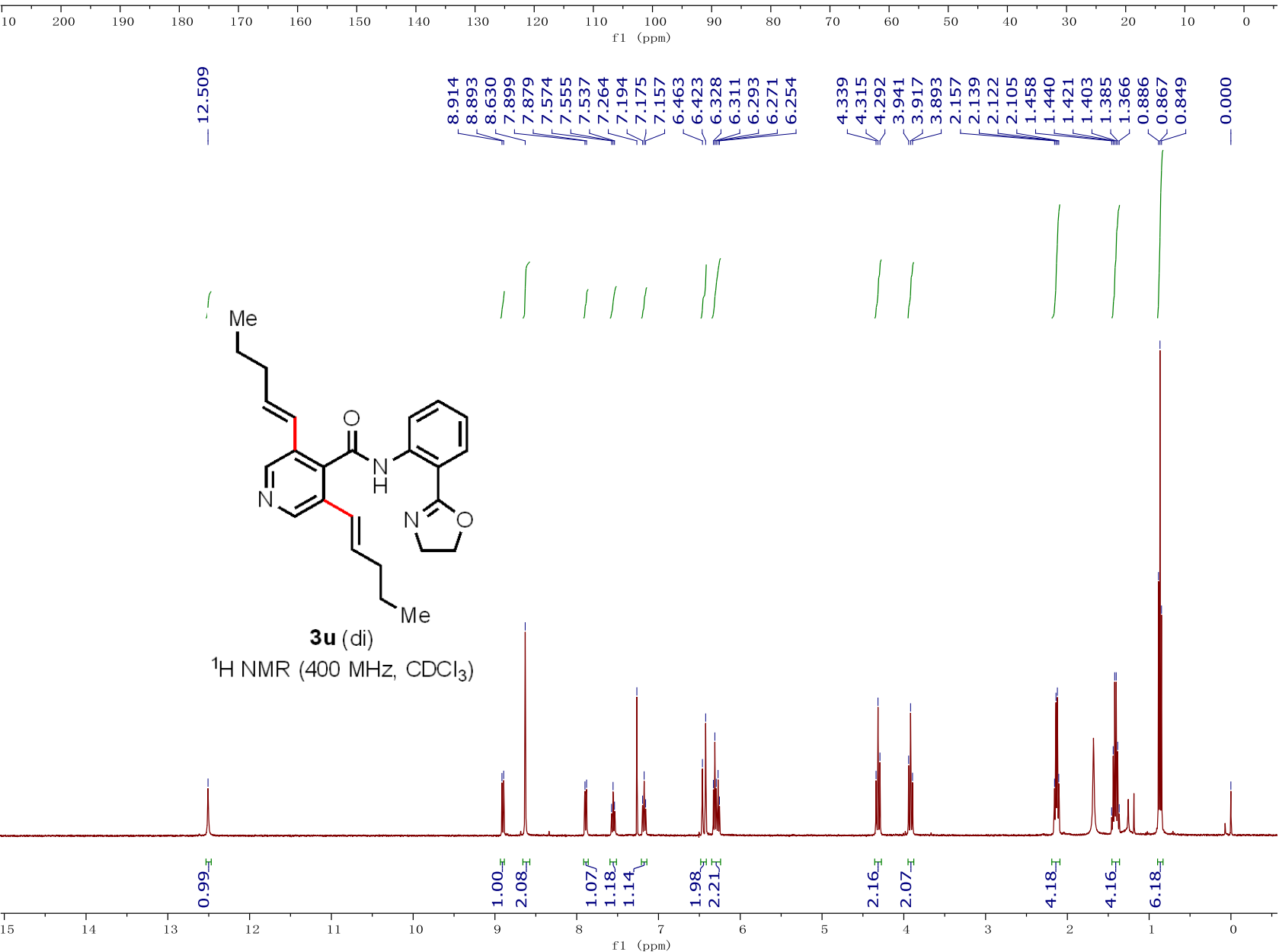




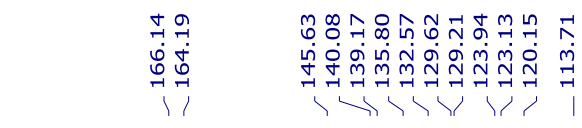

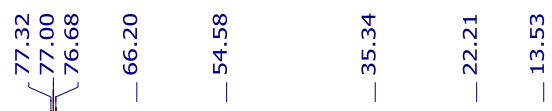

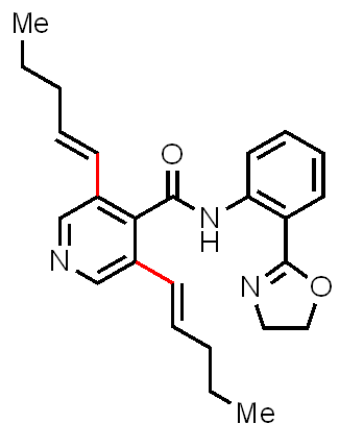

3u (di)

${ }^{13} \mathrm{C} \mathrm{NMR}\left(100 \mathrm{MHz}, \mathrm{CDCl}_{3}\right)$
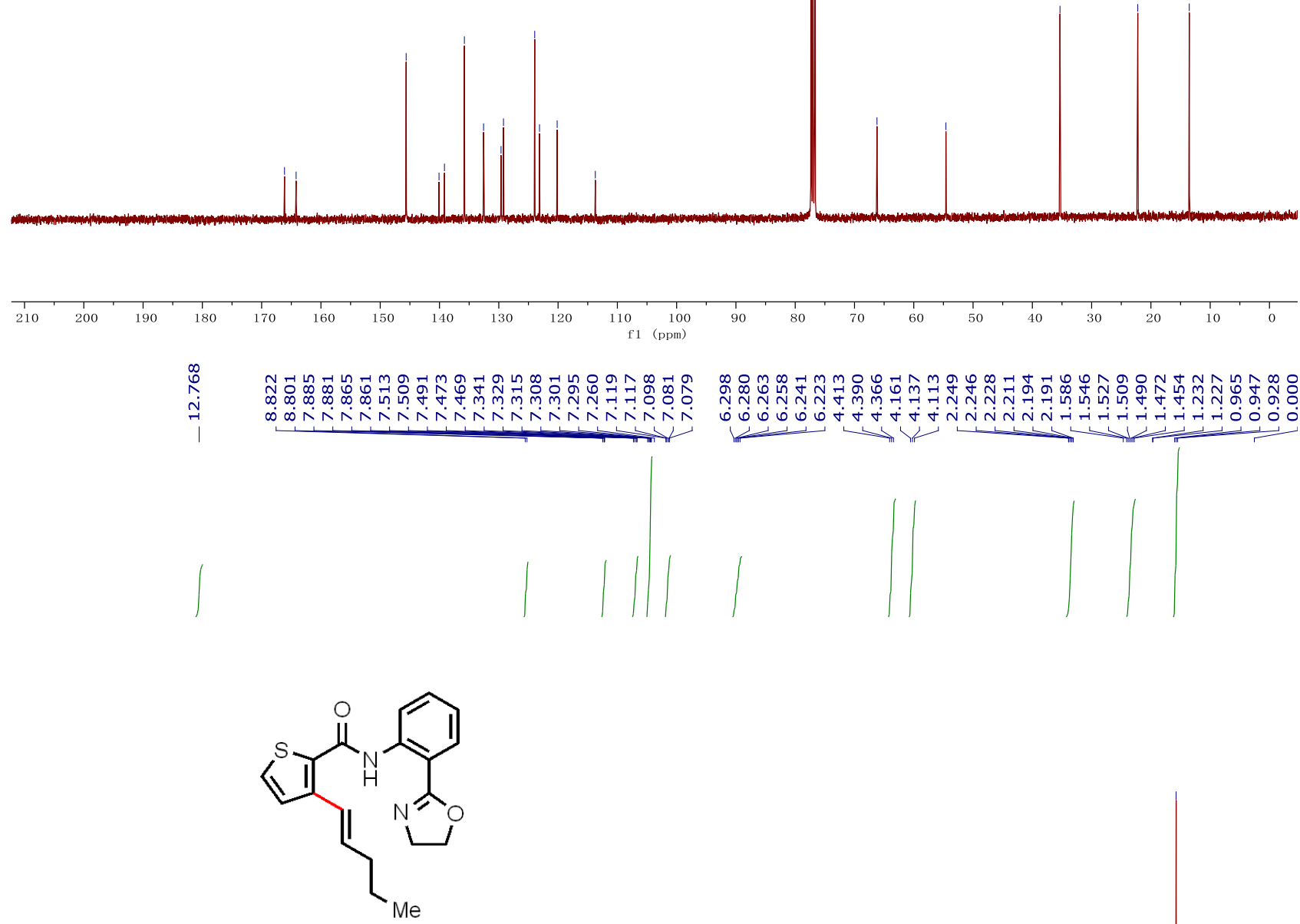

$3 v$

${ }^{1} \mathrm{H}$ NMR $\left(400 \mathrm{MHz}, \mathrm{CDCl}_{3}\right)$

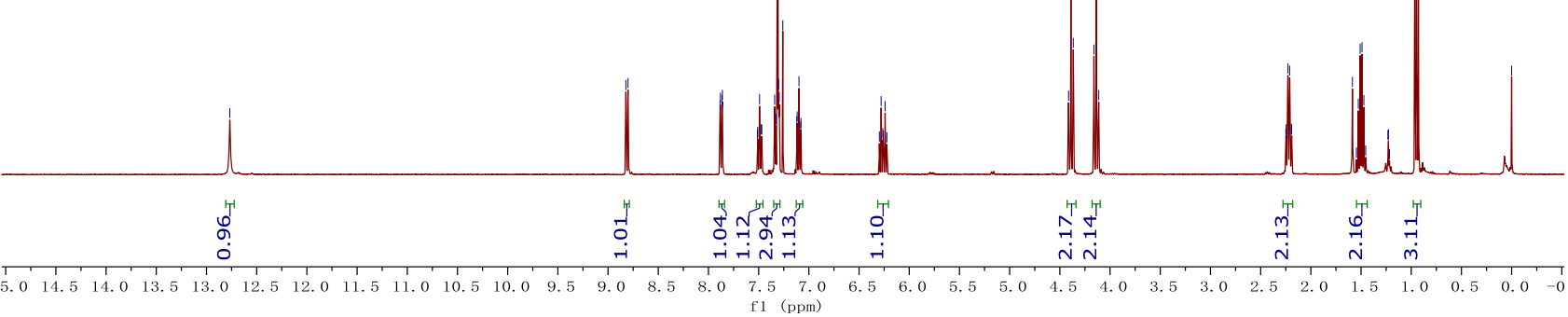


<smiles>CCC/C=C/c1ccsc1C(=O)Nc1ccccc1C1=NCCO1</smiles>

3v

${ }^{13} \mathrm{C}$ NMR $\left(100 \mathrm{MHz}, \mathrm{CDCl}_{3}\right)$

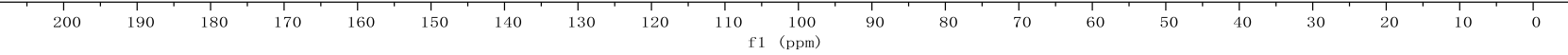

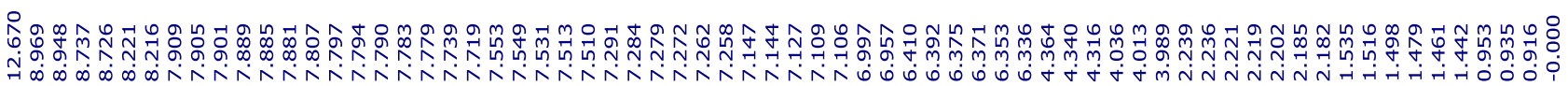

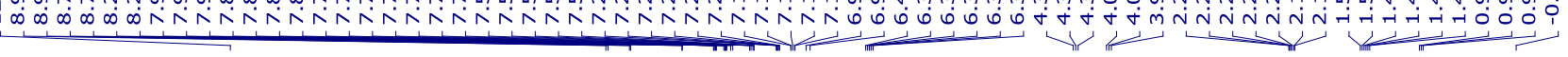<smiles>CCC/C=C/c1cc(-c2ccccn2)ccc1C(=O)Nc1ccccc1C1=NCCO1</smiles>
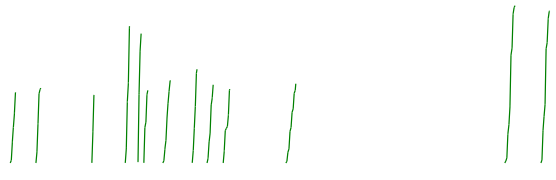

3w (mono)

${ }^{1} \mathrm{HNMR}\left(400 \mathrm{MHz}, \mathrm{CDCl}_{3}\right)$

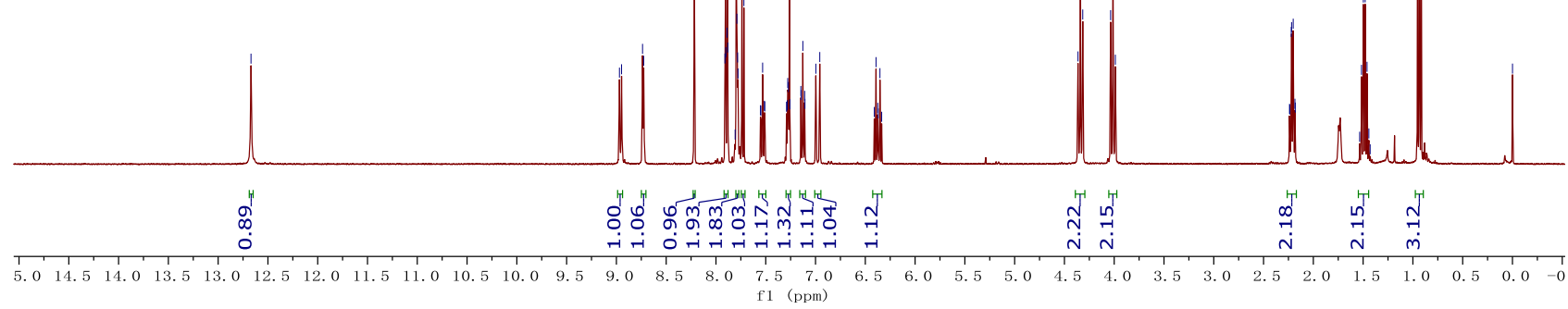




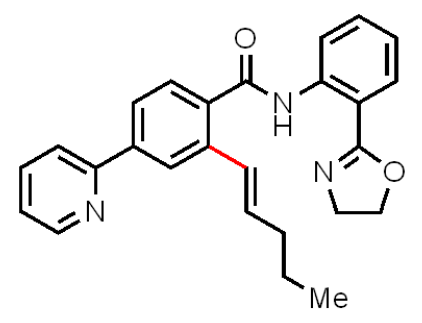

$3 \mathbf{w}$ (mono)

${ }^{13} \mathrm{C} \mathrm{NMR}\left(100 \mathrm{MHz}, \mathrm{CDCl}_{3}\right)$
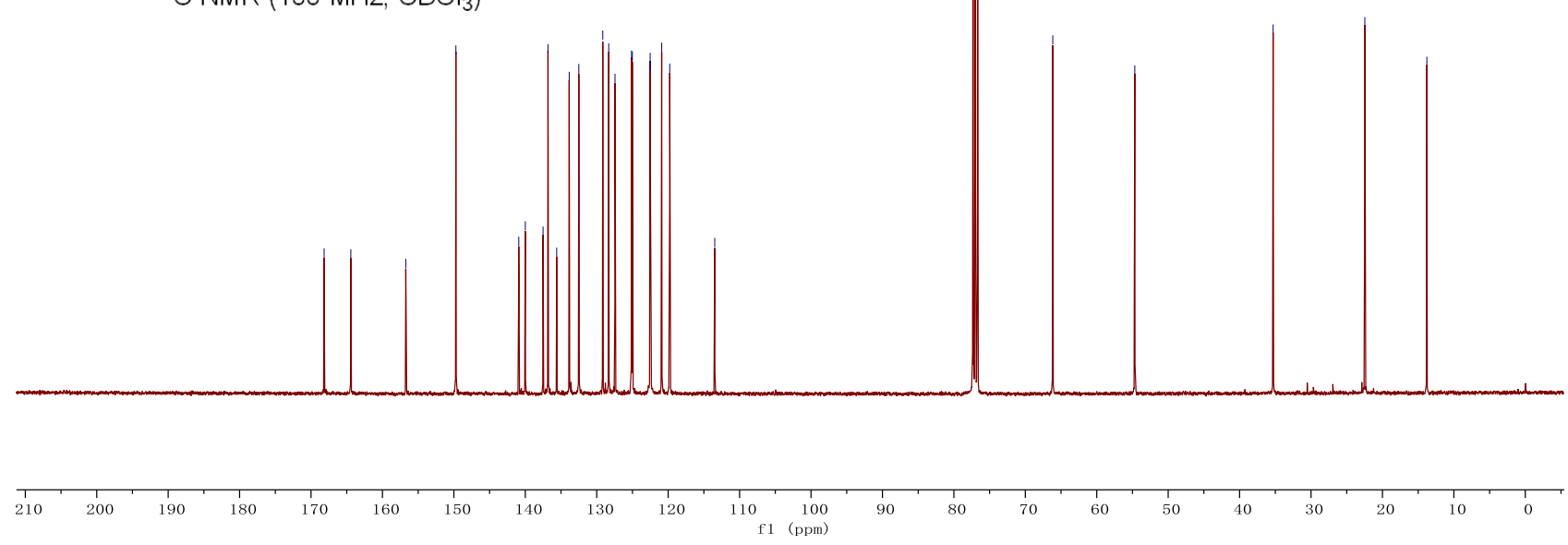

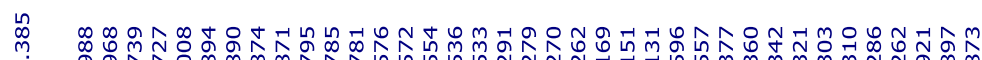

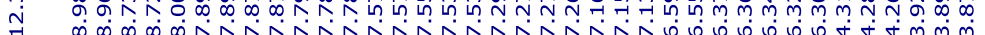

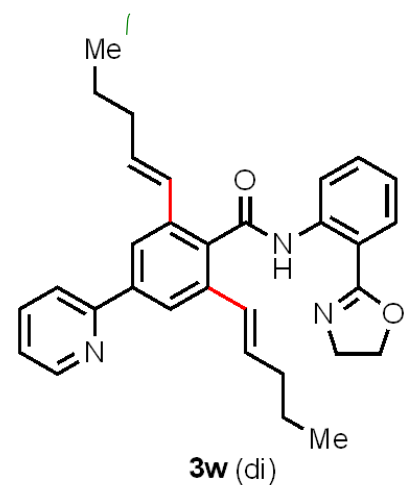

${ }^{1} \mathrm{H}$ NMR $\left(400 \mathrm{MHz}, \mathrm{CDCl}_{3}\right)$

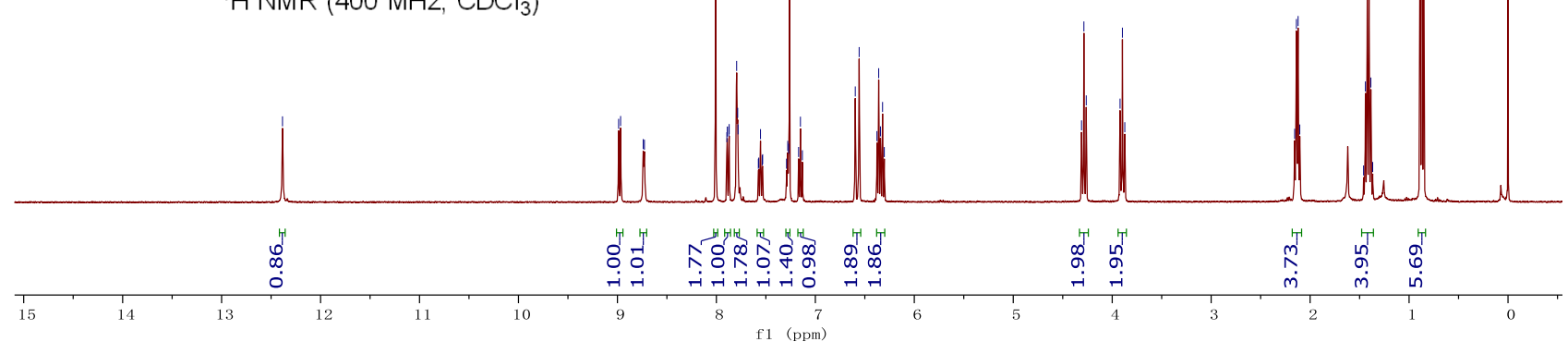




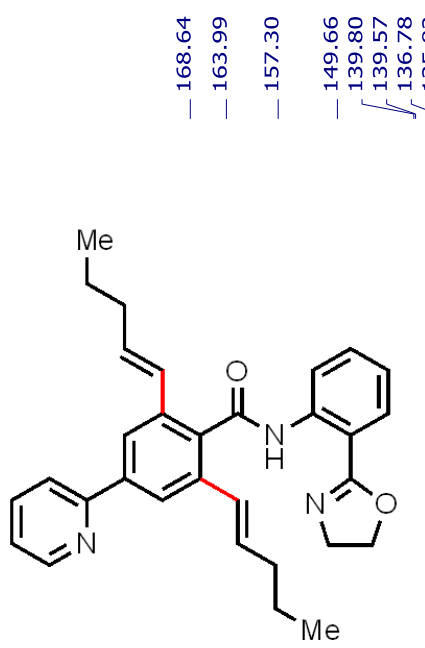

$3 \mathbf{w}$ (di)

${ }^{13} \mathrm{C} \mathrm{NMR}\left(100 \mathrm{MHz}, \mathrm{CDCl}_{3}\right)$

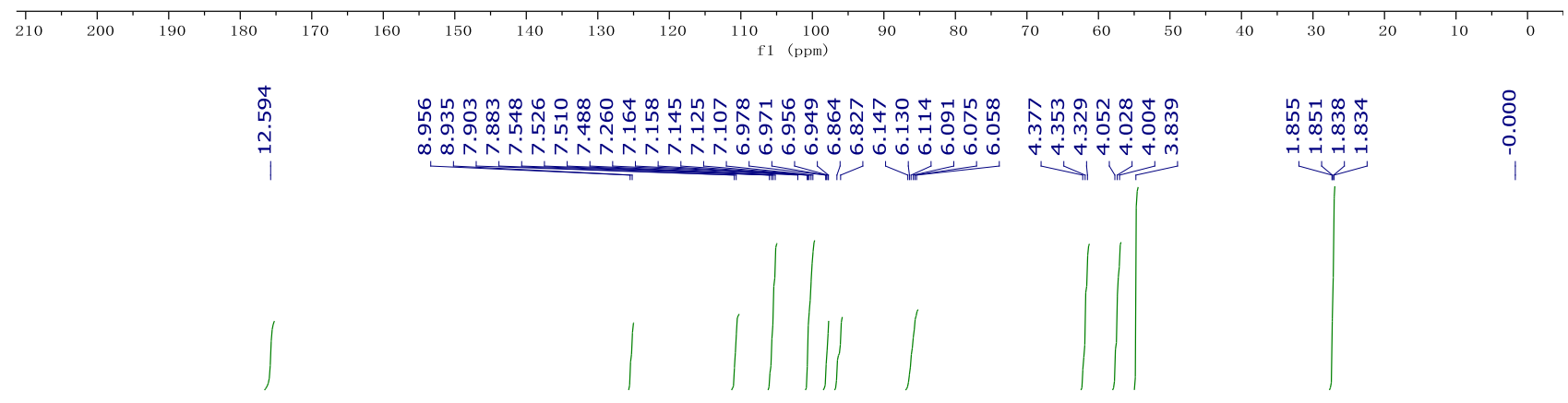<smiles>C/C=C/c1ccc(OC)cc1C(=O)Nc1ccccc1C1=NCCO1</smiles>

$5 a$

${ }^{1} \mathrm{H}$ NMR (400 MHz, $\mathrm{CDCl}_{3}$ )

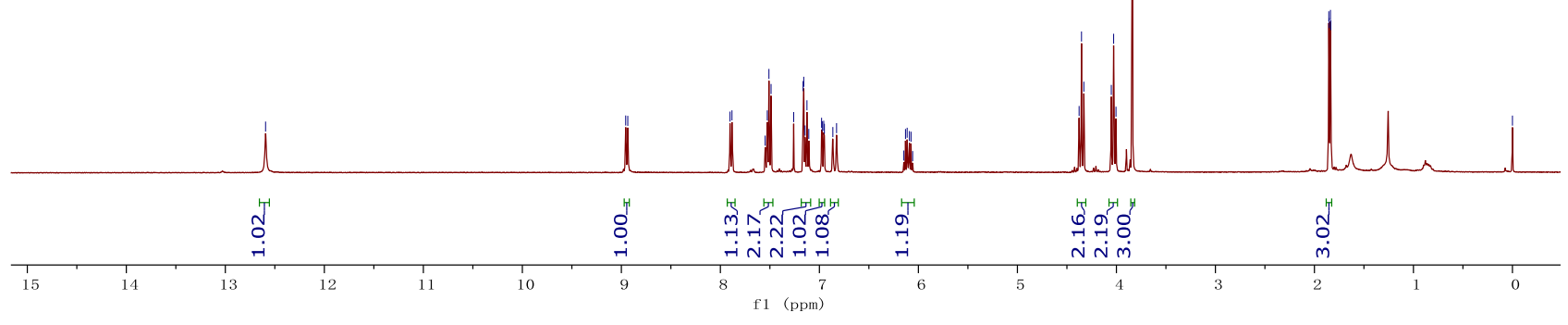




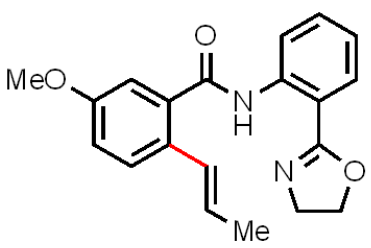

$5 a$

${ }^{13} \mathrm{C} \mathrm{NMR}\left(100 \mathrm{MHz}, \mathrm{CDCl}_{3}\right)$
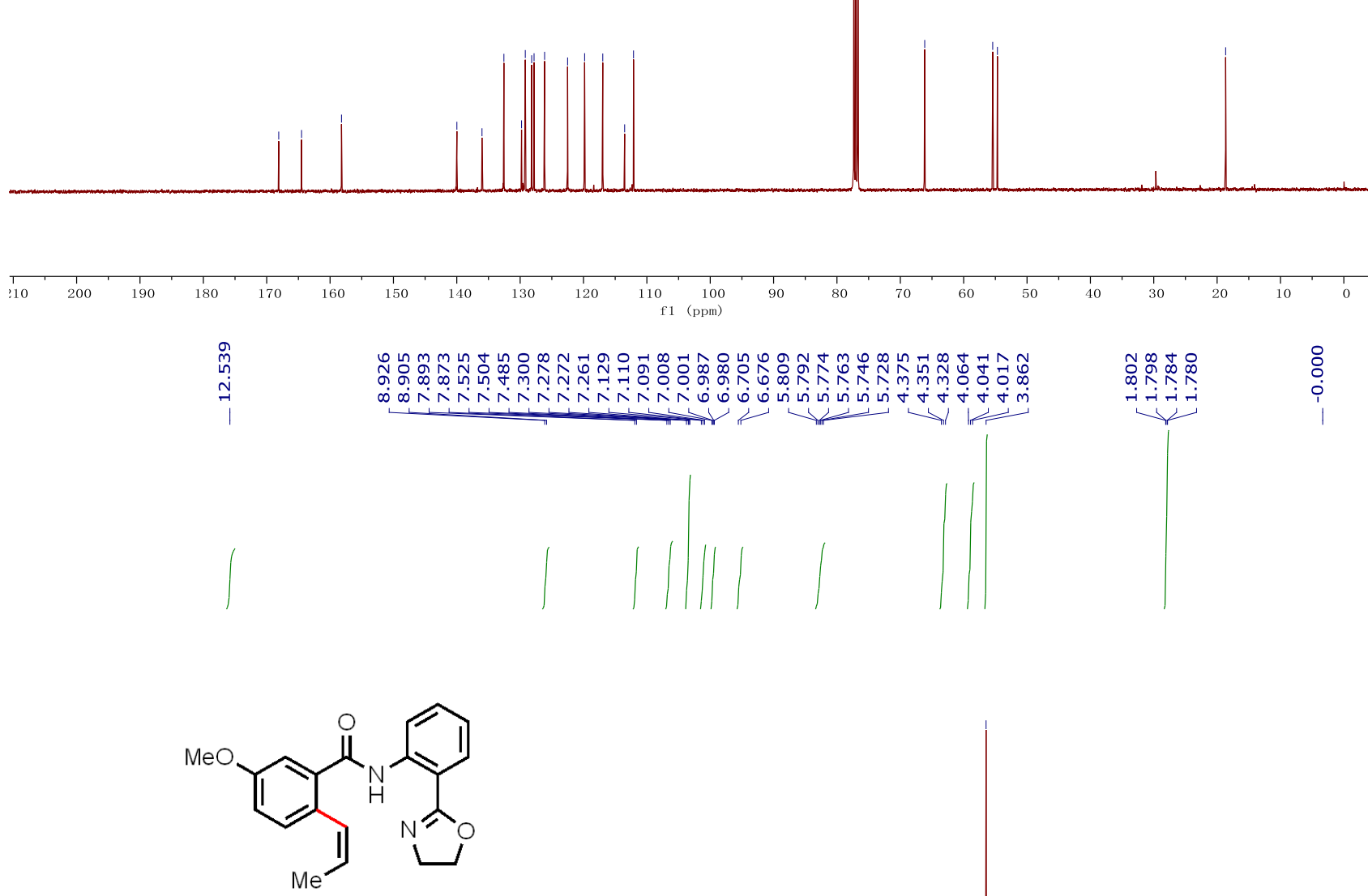

5b

${ }^{1} \mathrm{H}$ NMR $\left(400 \mathrm{MHz}, \mathrm{CDCl}_{3}\right)$

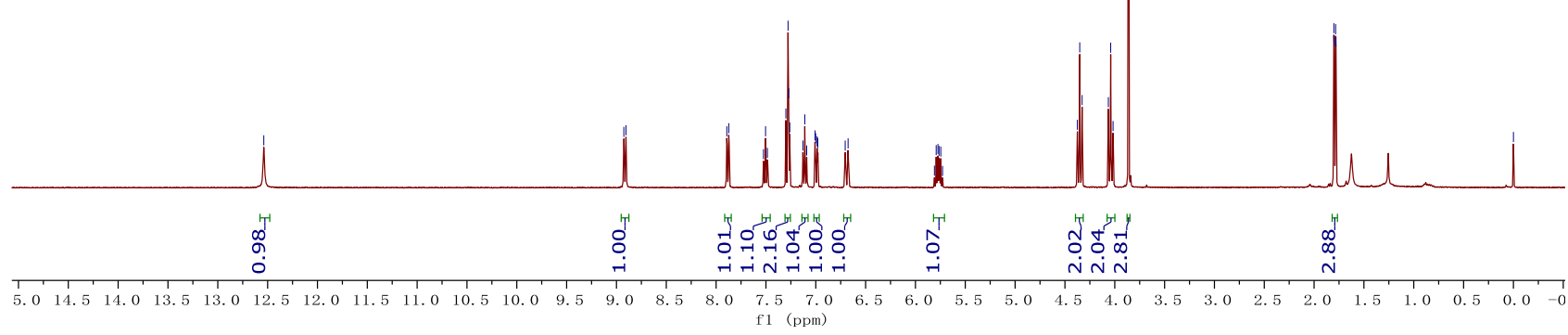


<smiles>C/C=C\c1ccc(OC)cc1C(=O)Nc1ccccc1C1=NCCO1</smiles>

$5 b$

${ }^{13} \mathrm{C}$ NMR $\left(100 \mathrm{MHz}, \mathrm{CDCl}_{3}\right)$

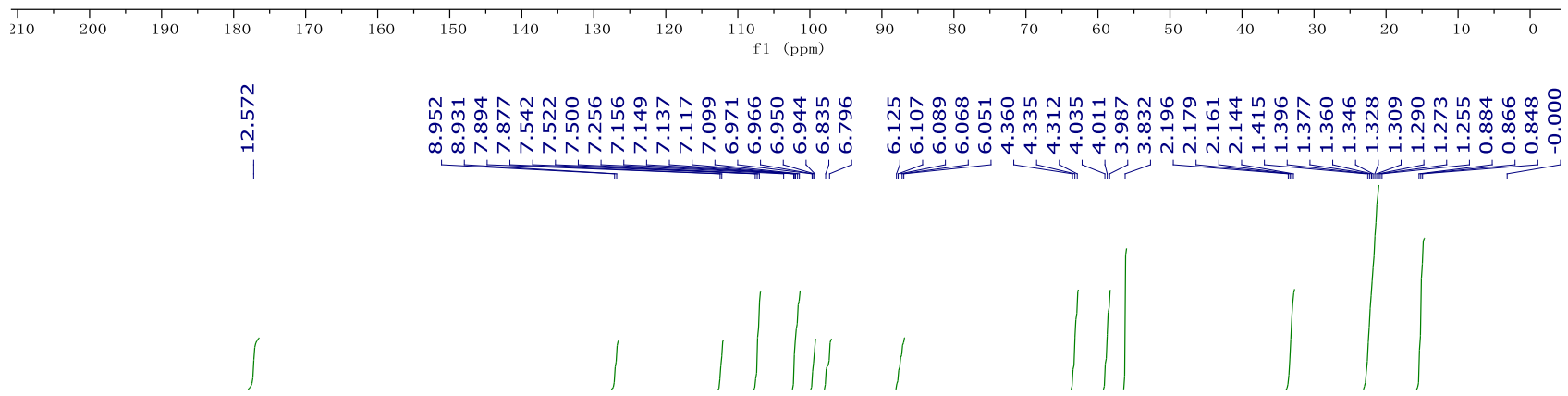<smiles>CCCC/C=C/c1ccc(OC)cc1C(=O)Nc1ccccc1C1=NCCO1</smiles>

${ }^{1} \mathrm{H}$ NMR $\left(400 \mathrm{MHz}, \mathrm{CDCl}_{3}\right)$

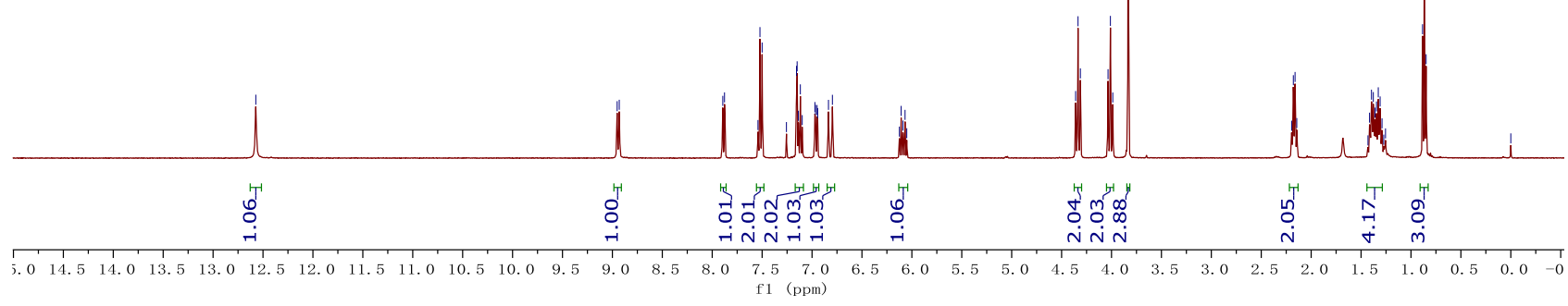


<smiles>CCCC/C=C/c1ccc(OC)cc1C(=O)Nc1ccccc1C1=NCCO1</smiles>

$5 c$

${ }^{13} \mathrm{C} \mathrm{NMR}\left(100 \mathrm{MHz}, \mathrm{CDCl}_{3}\right)$
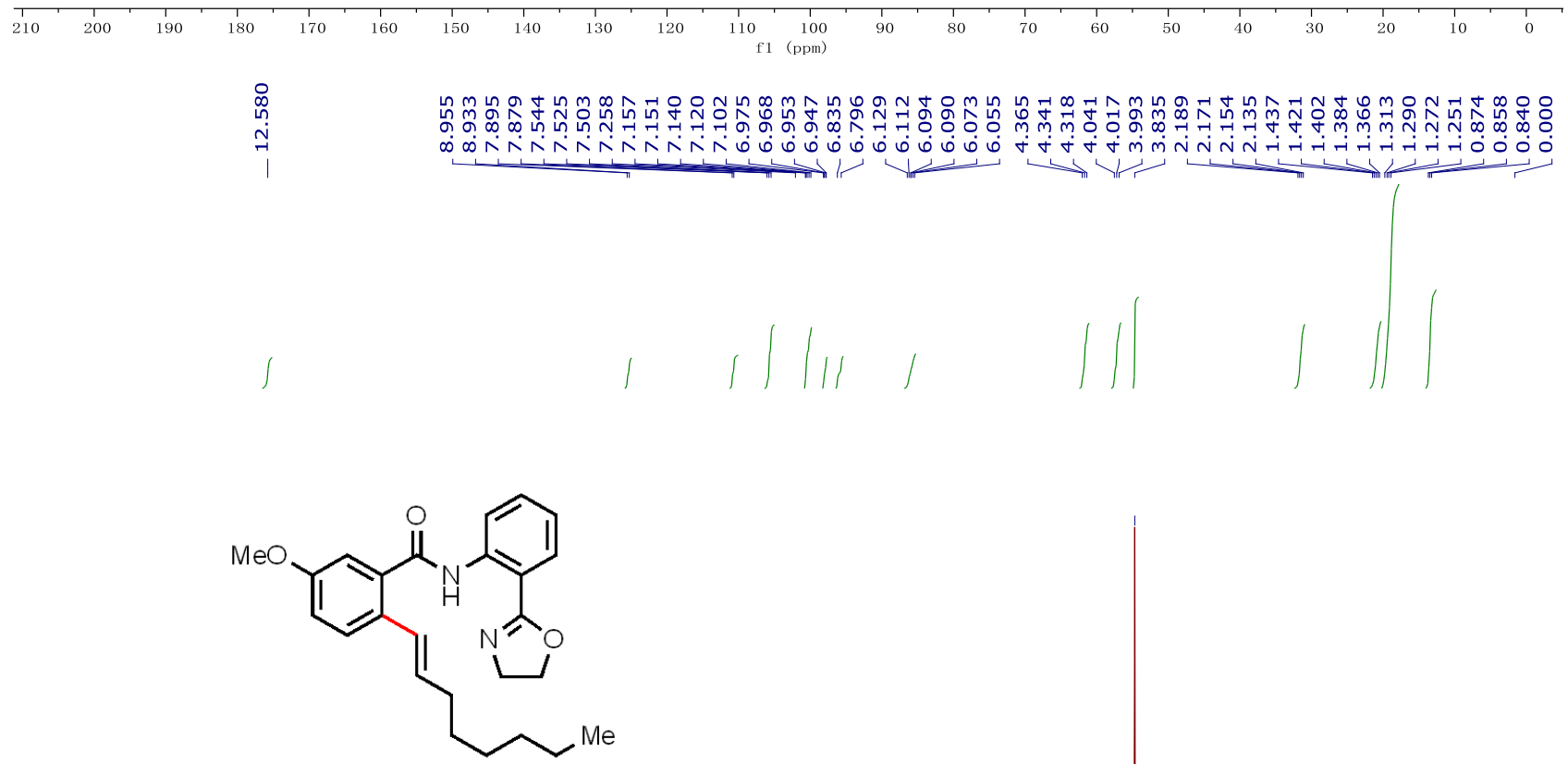

5d

${ }^{1} \mathrm{H}$ NMR (400 MHz, $\mathrm{CDCl}_{3}$ )

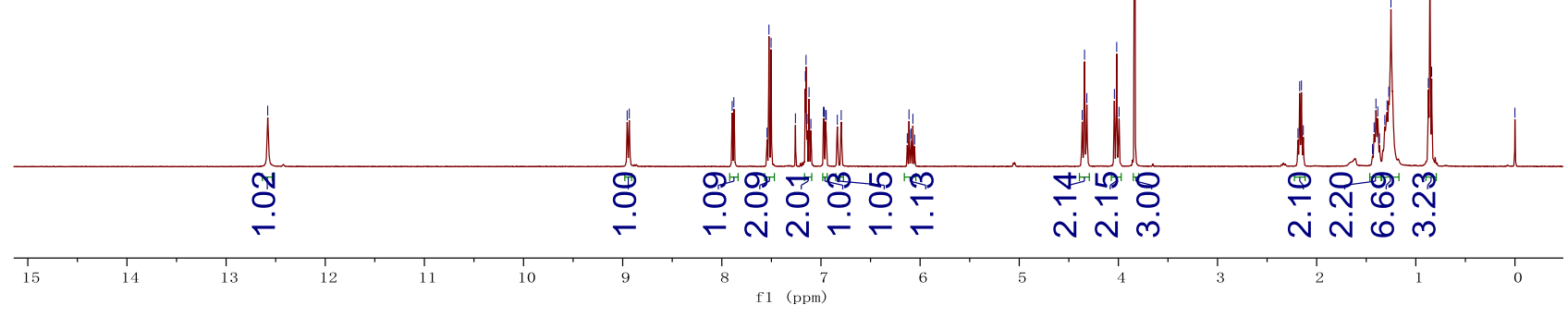




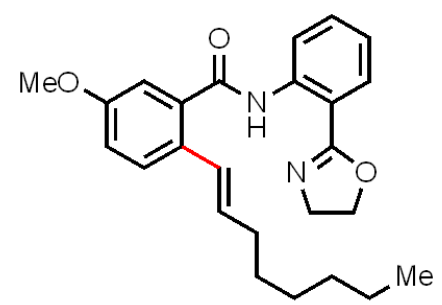

5d

${ }^{13} \mathrm{C} \mathrm{NMR}\left(100 \mathrm{MHz}, \mathrm{CDCl}_{3}\right)$

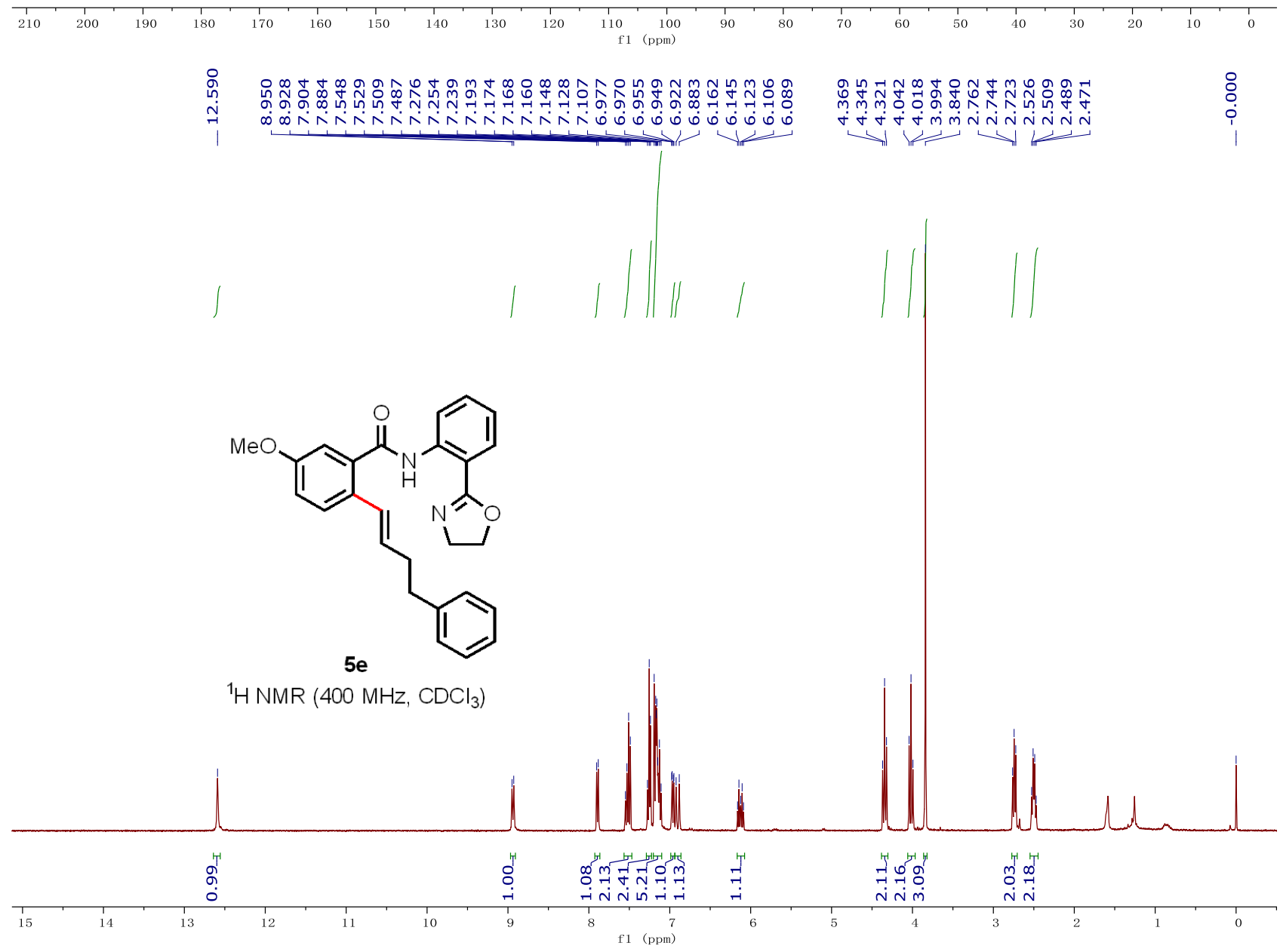




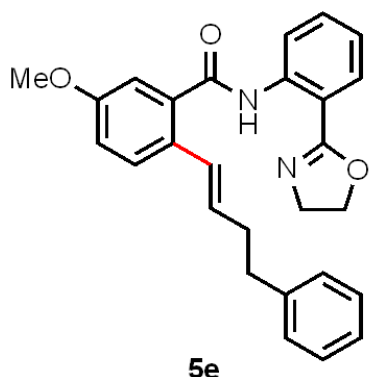

${ }^{13} \mathrm{C} \mathrm{NMR}\left(100 \mathrm{MHz}, \mathrm{CDCl}_{3}\right)$
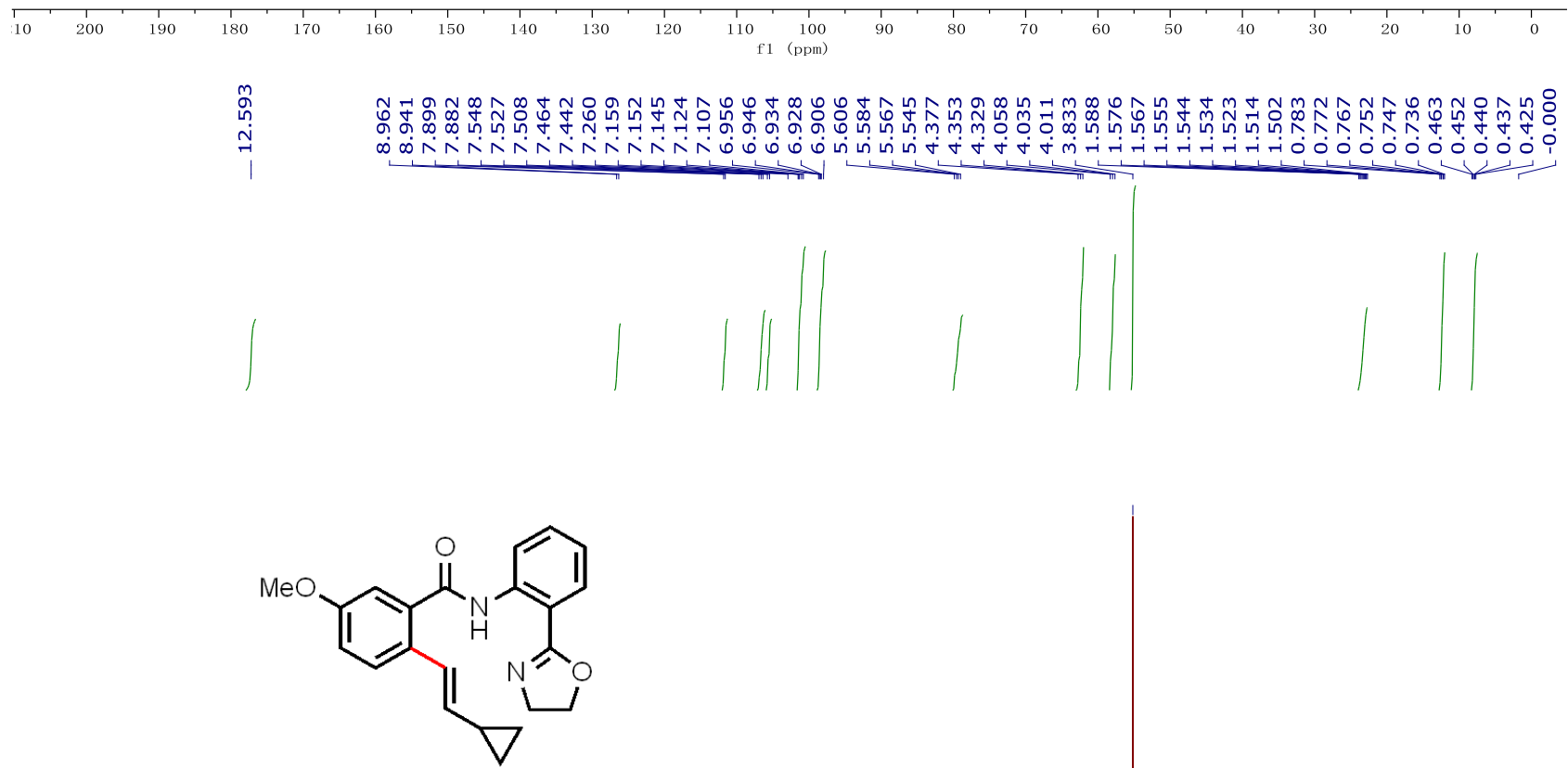

$5 f$

${ }^{1} \mathrm{H}$ NMR $\left(400 \mathrm{MHz}, \mathrm{CDCl}_{3}\right)$

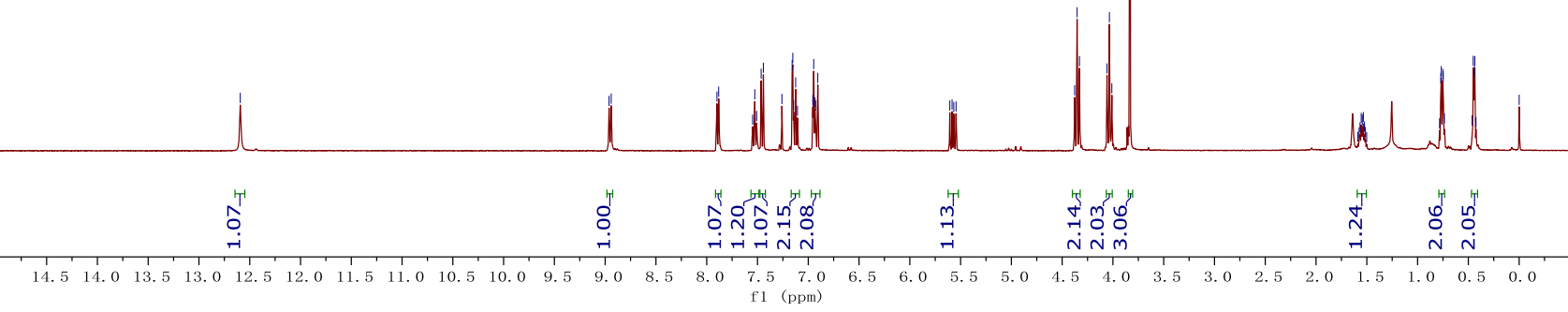




\begin{tabular}{|c|c|c|c|}
\hline 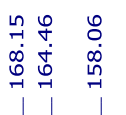 & 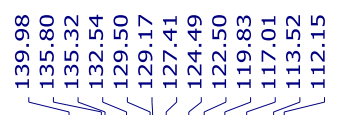 & 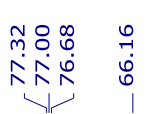 & 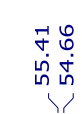 \\
\hline
\end{tabular}

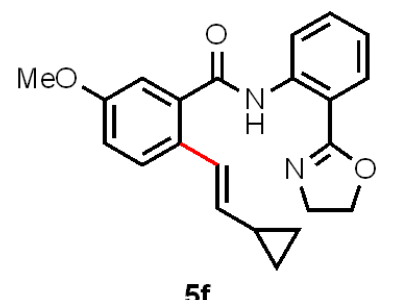

${ }^{13} \mathrm{C} \mathrm{NMR}\left(100 \mathrm{MHz}, \mathrm{CDCl}_{3}\right)$
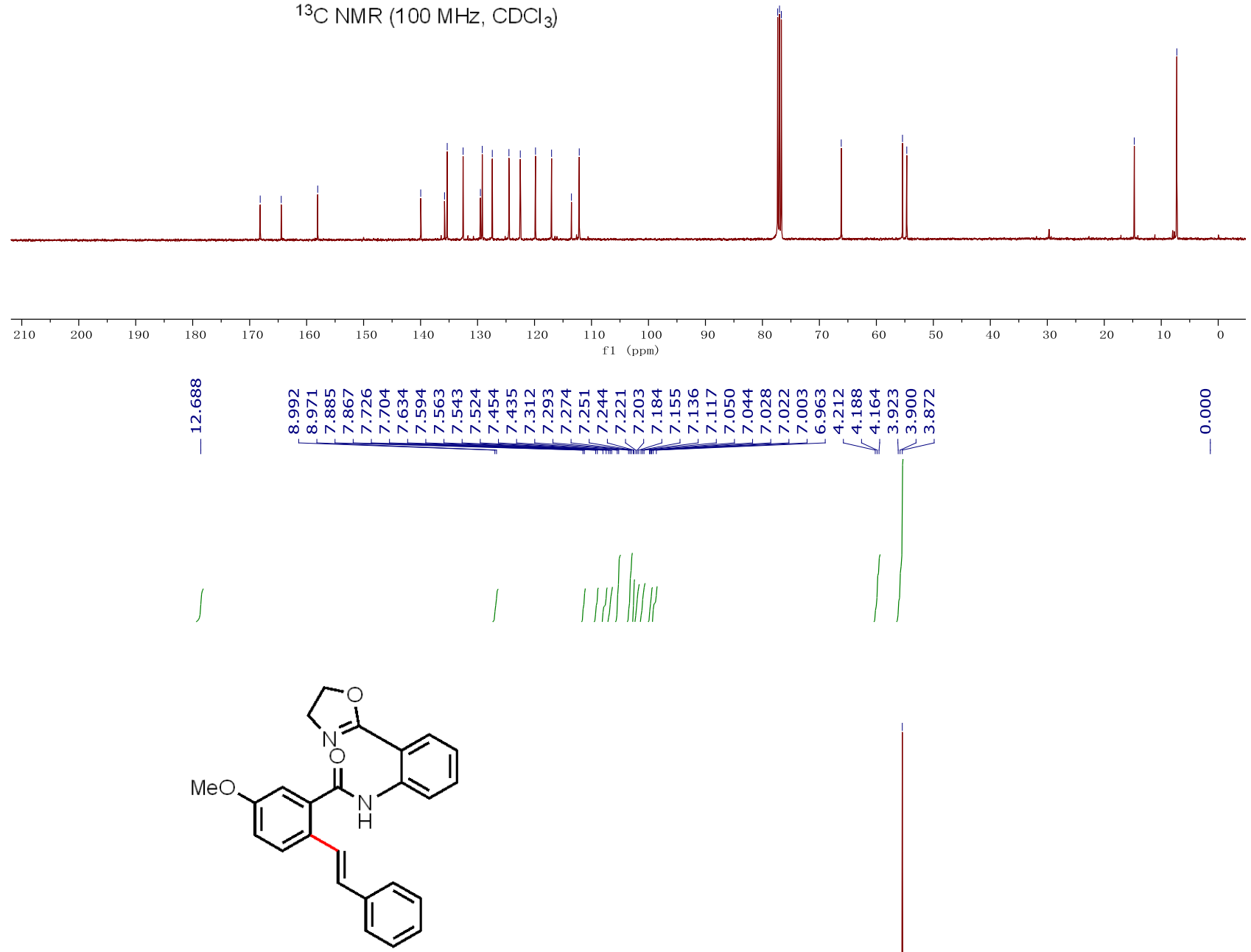

$5 \mathrm{~g}$

${ }^{1} \mathrm{H} \mathrm{NMR}\left(400 \mathrm{MHz}, \mathrm{CDCl}_{3}\right)$

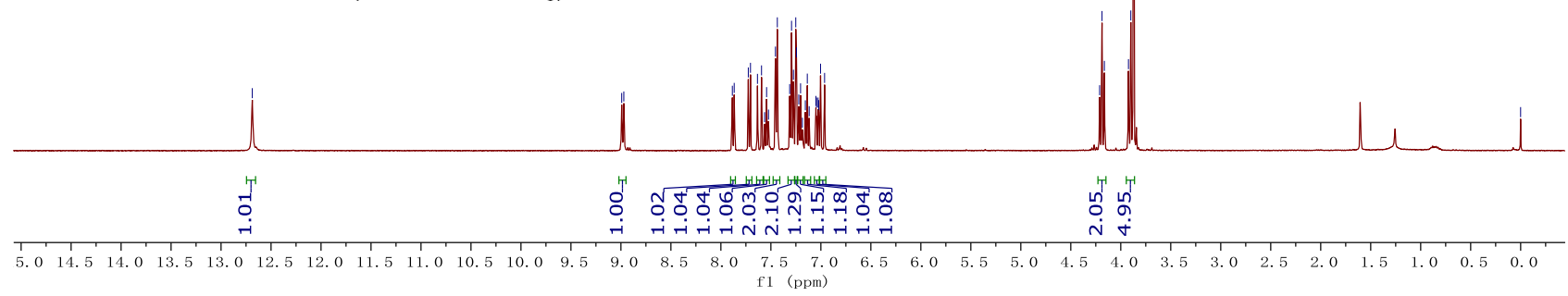


<smiles>COc1ccc(/C=C/c2ccccc2)c(C(=O)Nc2ccccc2C2=NCCO2)c1</smiles>

$5 \mathrm{~g}$

${ }^{13} \mathrm{C}$ NMR $\left(100 \mathrm{MHz}, \mathrm{CDCl}_{3}\right)$

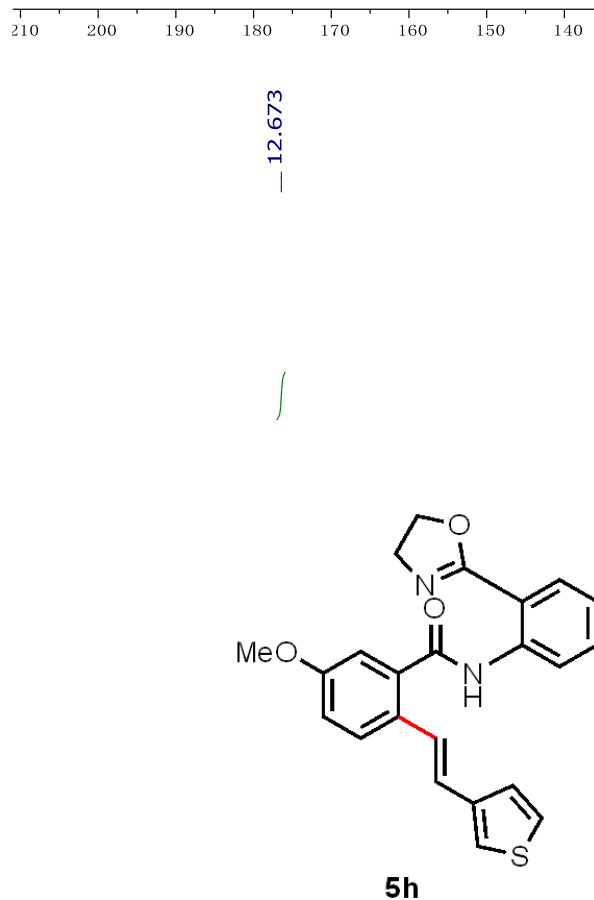

${ }^{1} \mathrm{H} \mathrm{NMR}\left(400 \mathrm{MHz}, \mathrm{CDCl}_{3}\right)$
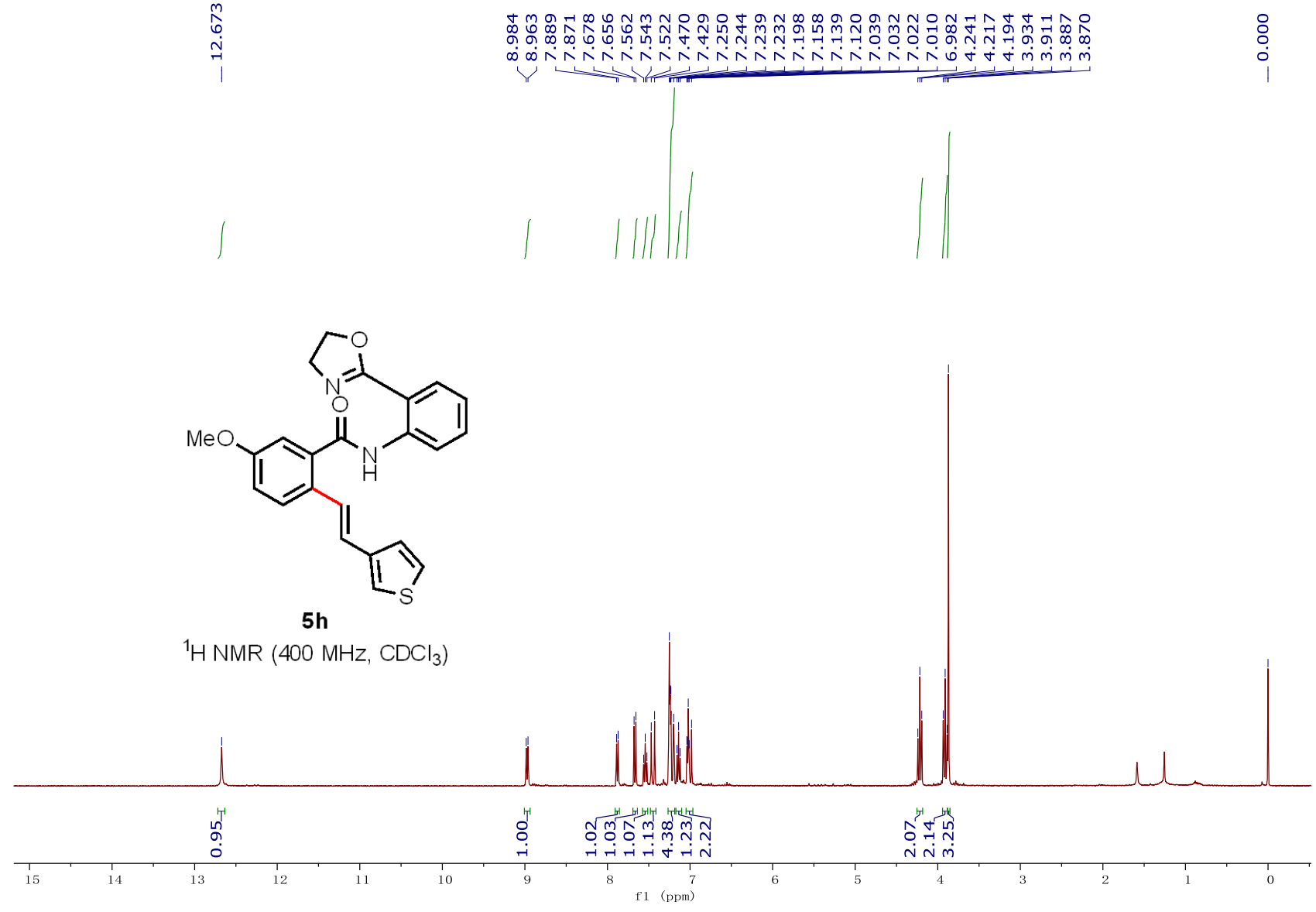


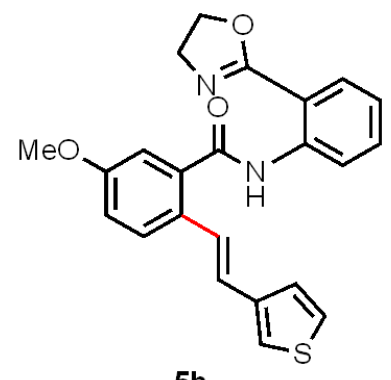

$5 \mathrm{~h}$

${ }^{13} \mathrm{C}$ NMR $\left(100 \mathrm{MHz}, \mathrm{CDCl}_{3}\right)$

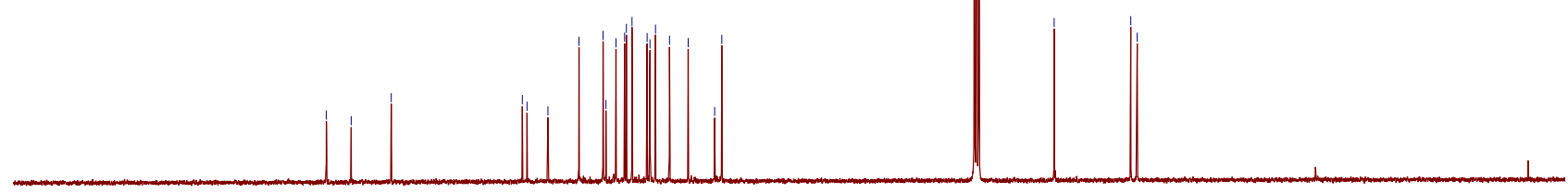

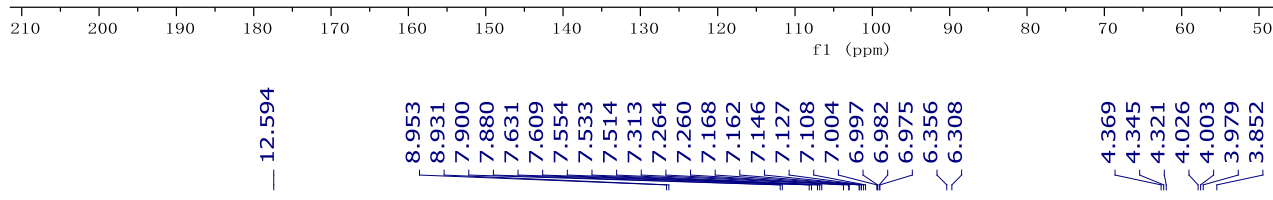<smiles>COc1ccc(/C=C/[Si](C)(C)C)c(C(=O)Nc2ccccc2C2=NCCO2)c1</smiles>

$5 i$

${ }^{1} \mathrm{H}$ NMR (400 MHz, $\mathrm{CDCl}_{3}$ )

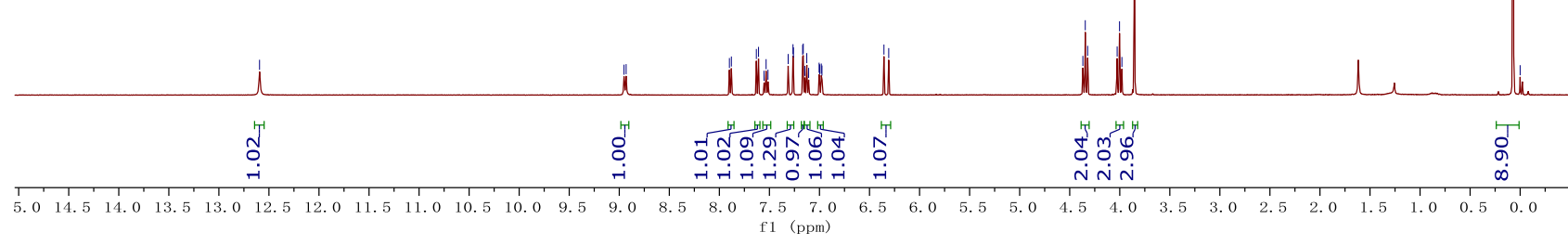




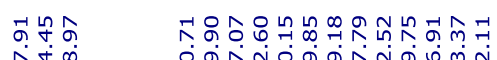

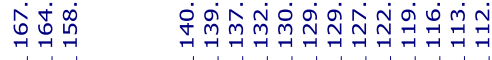

mํํำ

र쇼

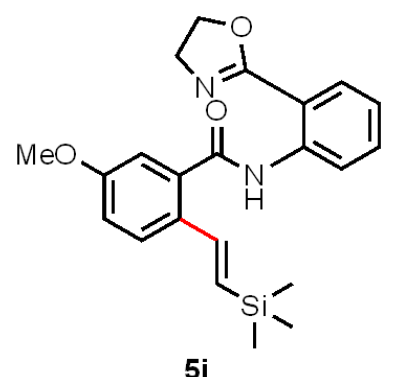

${ }^{13} \mathrm{C} \mathrm{NMR}\left(100 \mathrm{MHz}, \mathrm{CDCl}_{3}\right)$

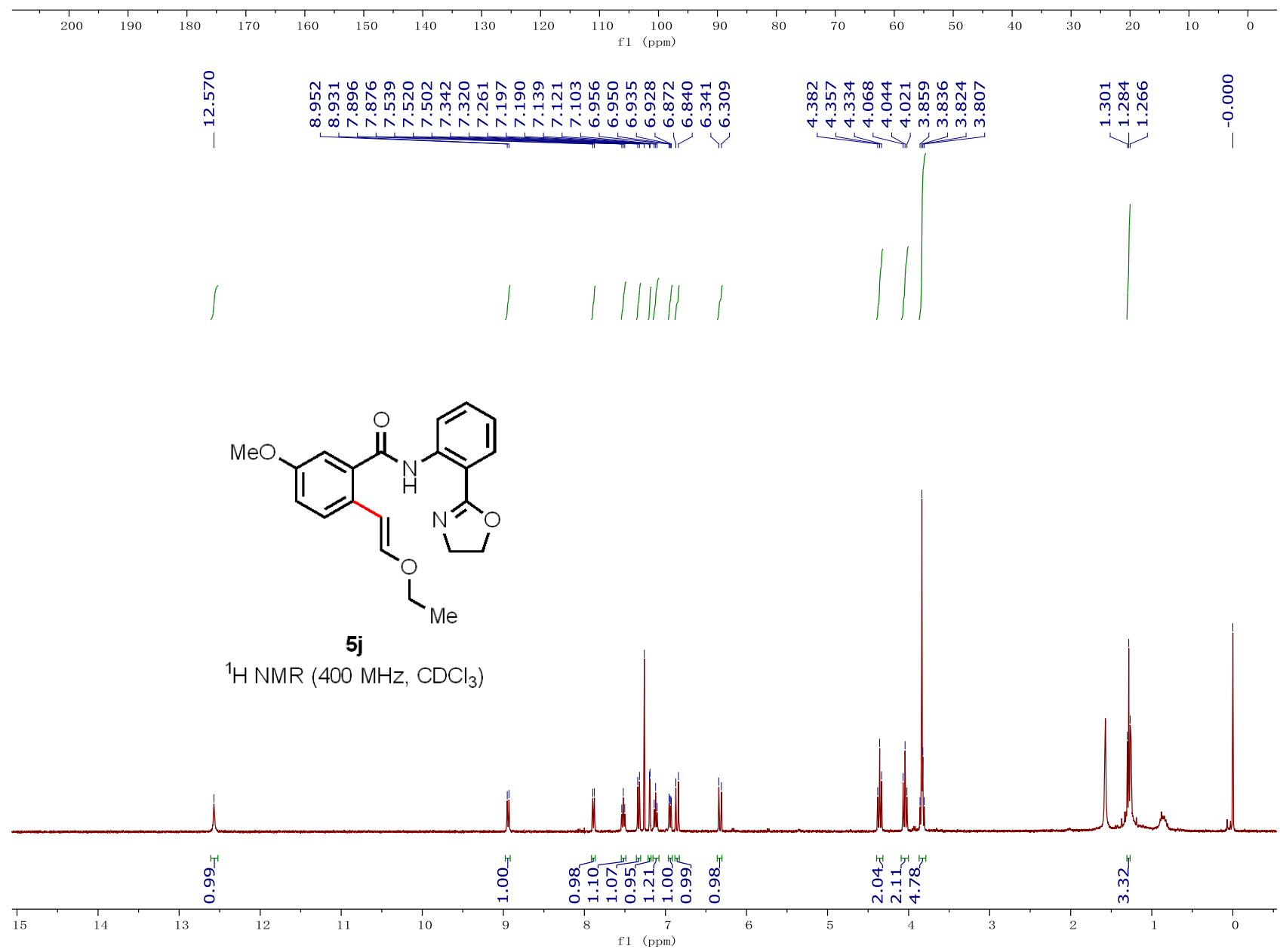




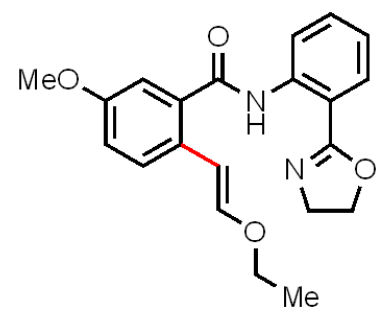

$5 \mathbf{j}$

${ }^{13} \mathrm{C}$ NMR $\left(100 \mathrm{MHz}, \mathrm{CDCl}_{3}\right)$

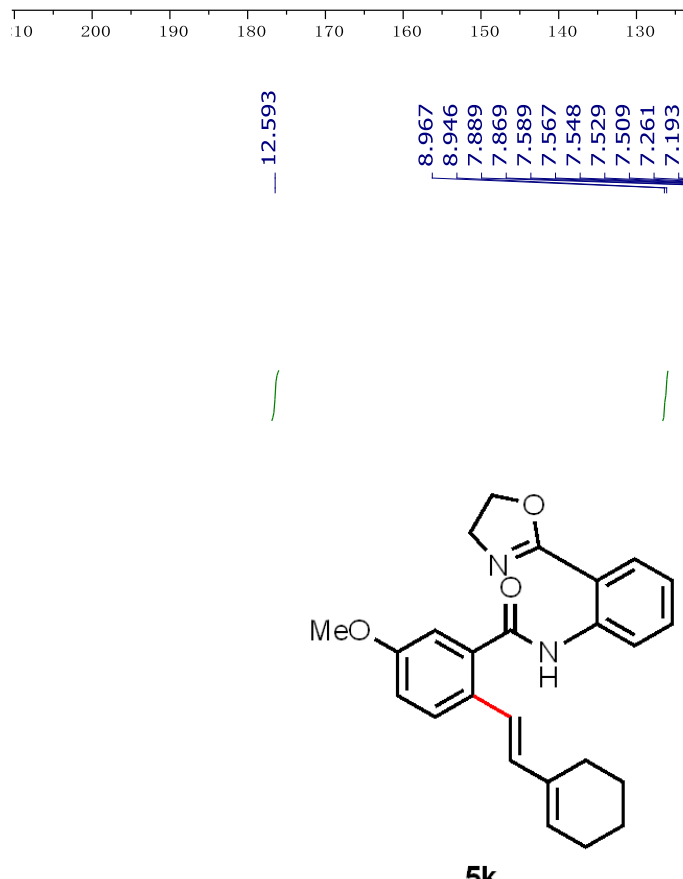

$5 \mathbf{k}$

${ }^{1} \mathrm{H}$ NMR $\left(400 \mathrm{MHz}, \mathrm{CDCl}_{3}\right)$

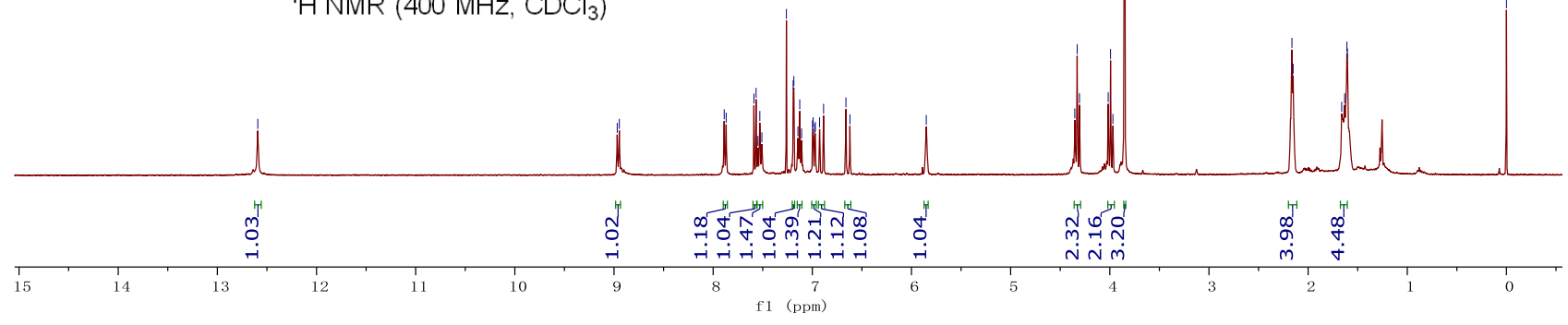


<smiles>COc1ccc(/C=C/C2=CCCCC2)c(C(=O)Nc2ccccc2C2=NCCO2)c1</smiles>

$5 \mathbf{k}$

${ }^{13} \mathrm{C} \mathrm{NMR}\left(100 \mathrm{MHz}, \mathrm{CDCl}_{3}\right)$
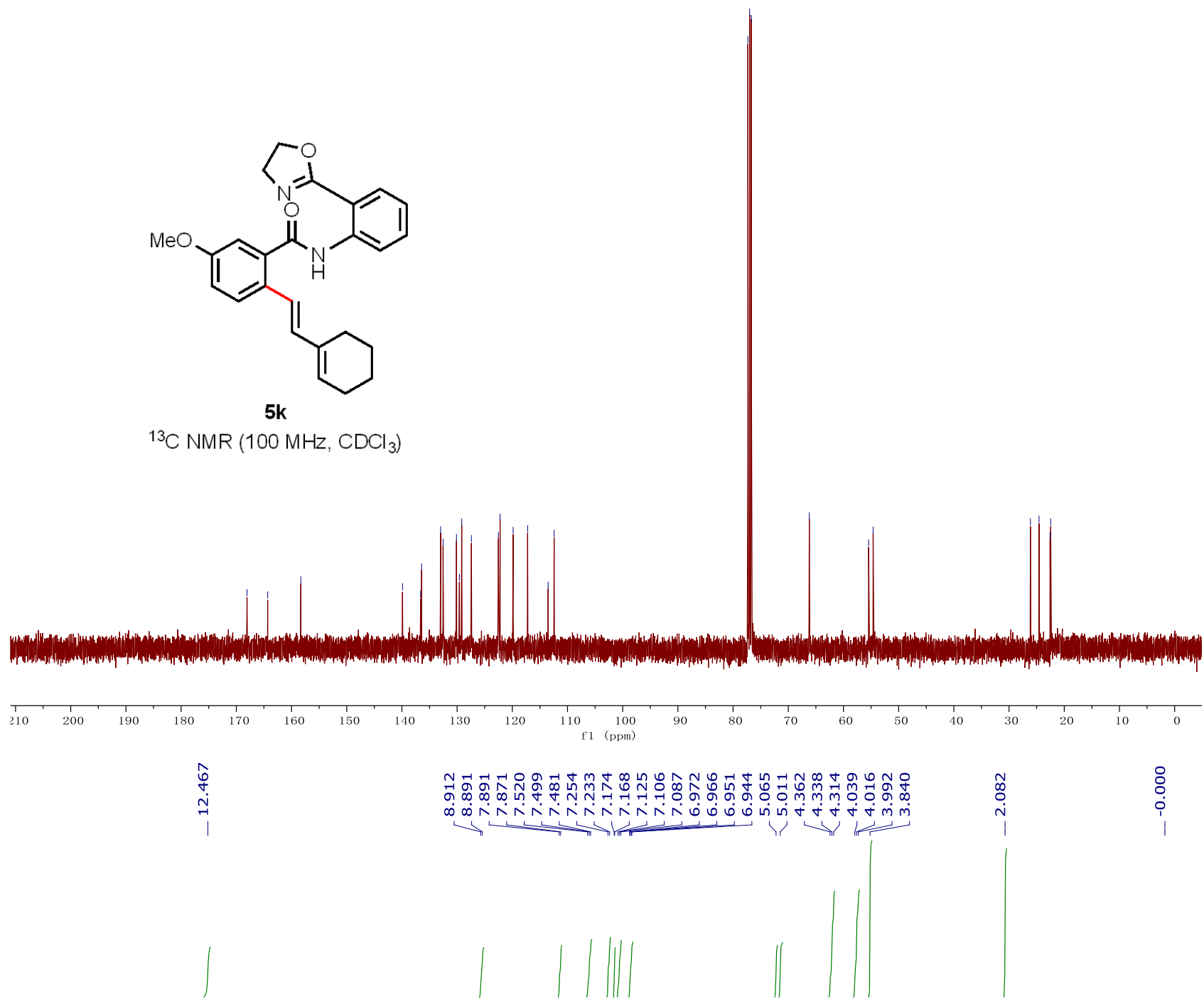<smiles>C=C(C)c1ccc(OC)cc1C(=O)Nc1ccccc1C1=NCCO1</smiles>

5

${ }^{1} \mathrm{H} \mathrm{NMR}\left(400 \mathrm{MHz}, \mathrm{CDCl}_{3}\right)$

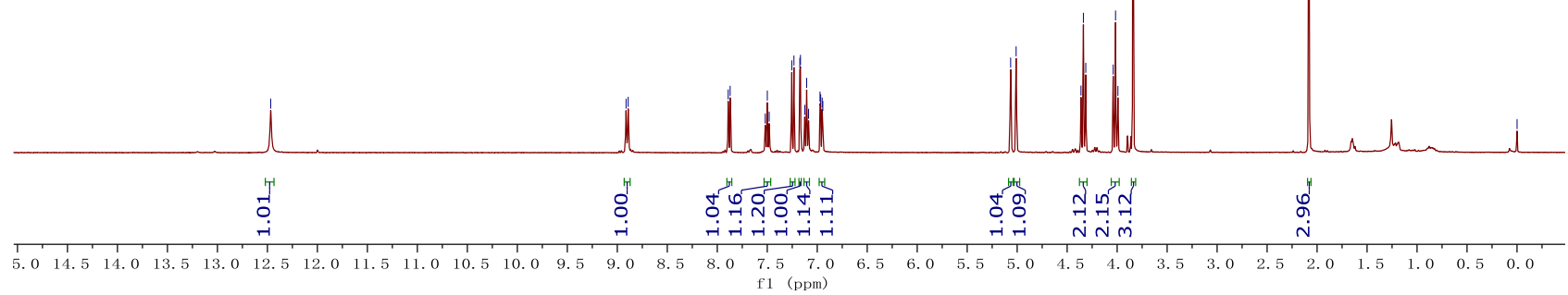


<smiles>C=C(C)c1ccc(OC)cc1C(=O)Nc1ccccc1C1=NCCO1</smiles>

5

${ }^{13} \mathrm{C} \mathrm{NMR}\left(100 \mathrm{MHz}, \mathrm{CDCl}_{3}\right)$
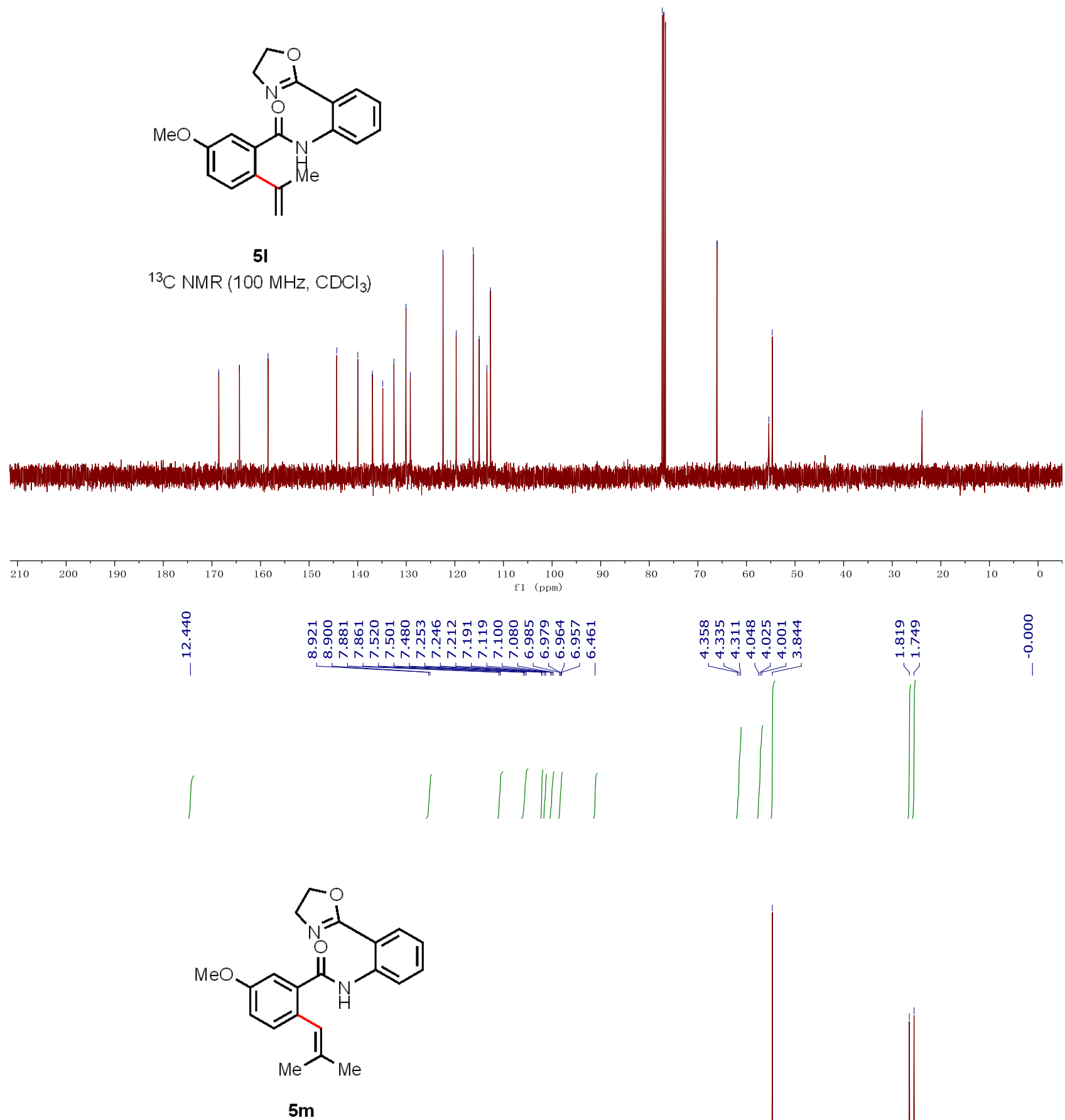

${ }^{1} \mathrm{HNMR}\left(400 \mathrm{MHz}, \mathrm{CDCl}_{3}\right)$

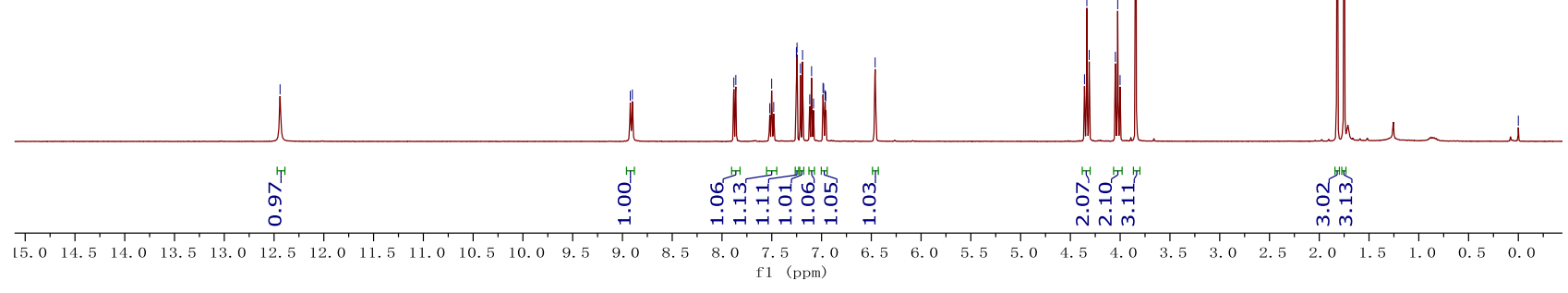


<smiles>COc1ccc(C=C(C)C)c(C(=O)Nc2ccccc2C2=NCCO2)c1</smiles>

$5 \mathrm{~m}$

${ }^{13} \mathrm{C}$ NMR $\left(100 \mathrm{MHz}, \mathrm{CDCl}_{3}\right)$
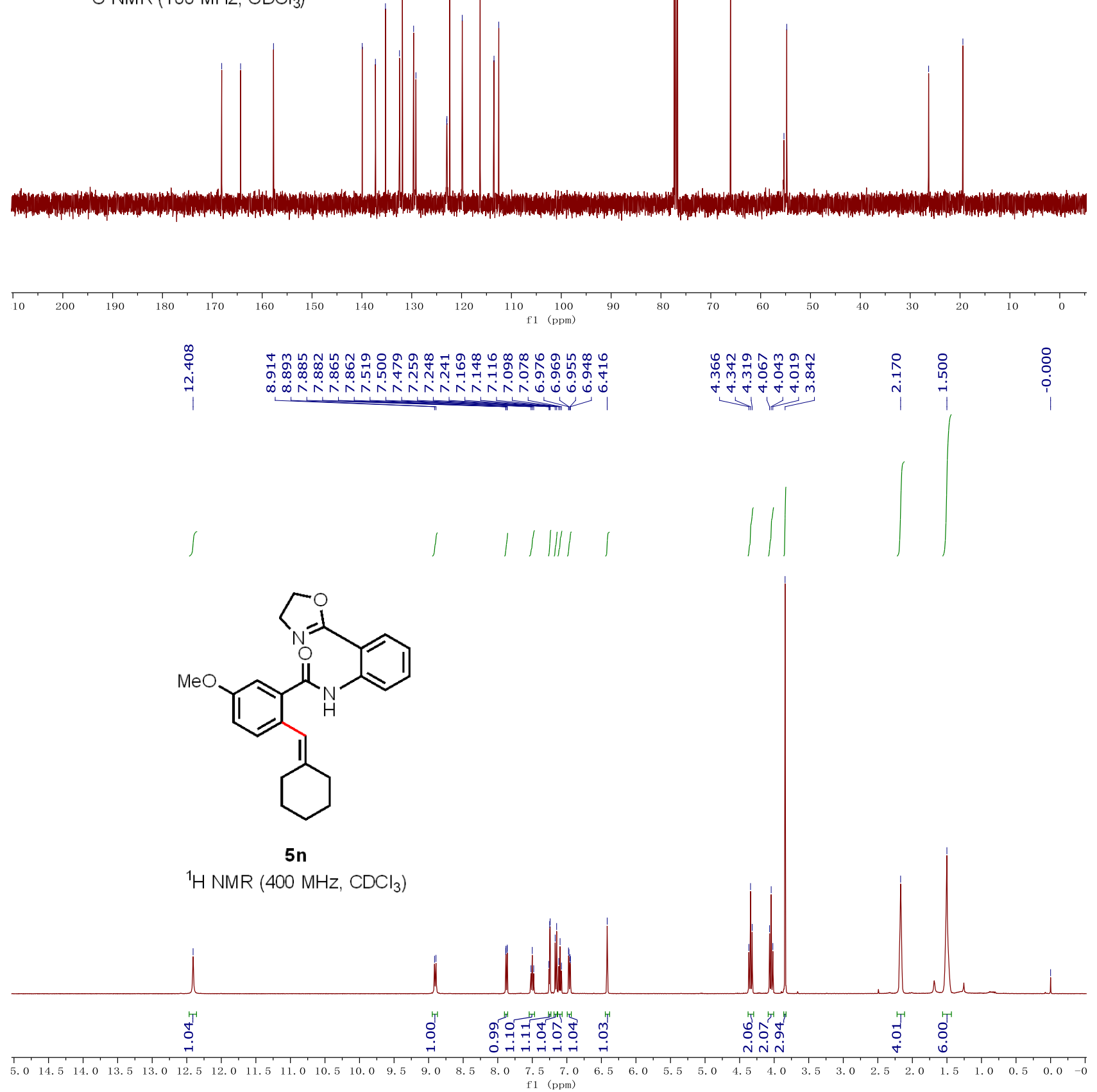


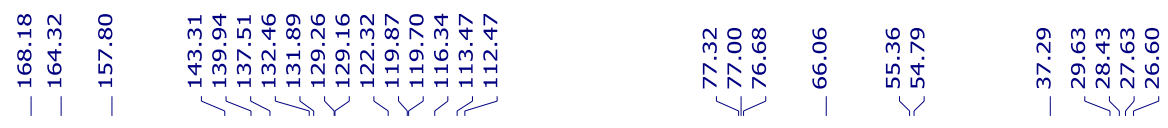<smiles></smiles>

$5 n$

${ }^{13} \mathrm{C} \mathrm{NMR}\left(100 \mathrm{MHz}, \mathrm{CDCl}_{3}\right)$

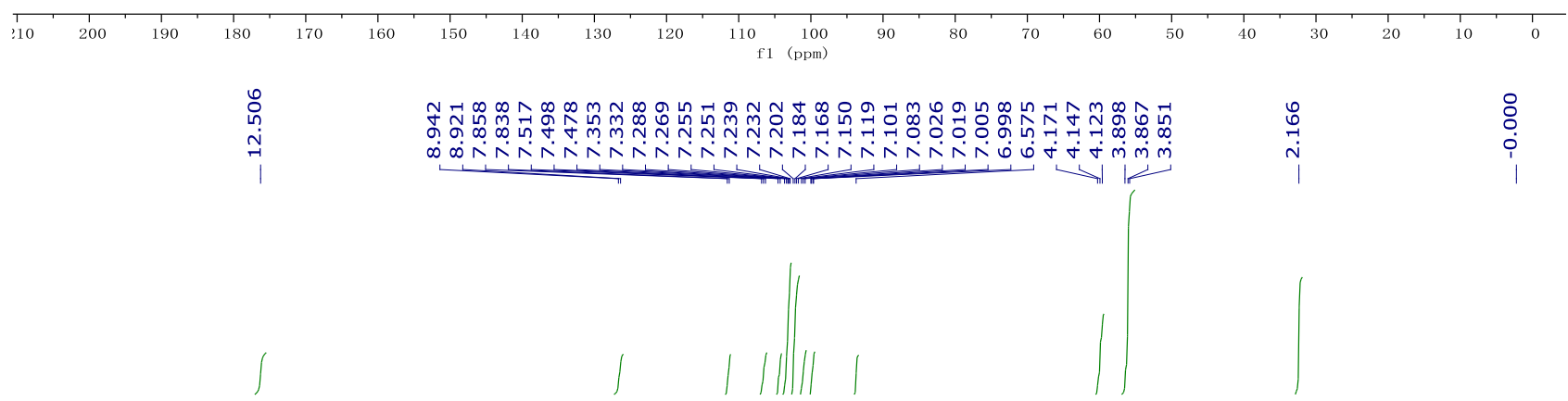<smiles>COc1ccc(/C(=C/c2ccccc2)c2cccc(C(=O)Nc3ccccc3C3=NCCO3)c2)c(C)c1</smiles>

50

${ }^{1} \mathrm{H}$ NMR $\left(400 \mathrm{MHz}, \mathrm{CDCl}_{3}\right.$ )

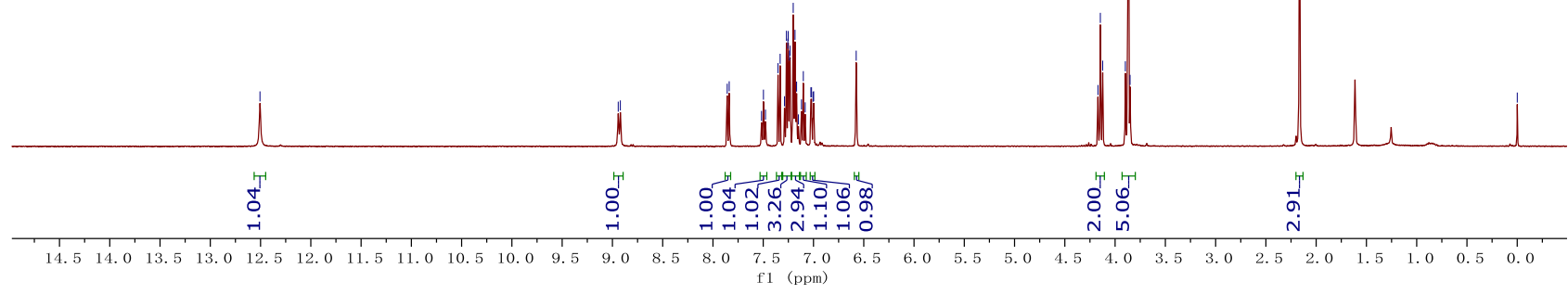


<smiles>COc1ccc(/C(=C/c2ccccc2)c2cc(OC)ccc2C(=O)Nc2ccccc2C2=NCCO2)c(C)c1</smiles>

50

${ }^{13} \mathrm{C} \mathrm{NMR}\left(100 \mathrm{MHz}, \mathrm{CDCl}_{3}\right)$

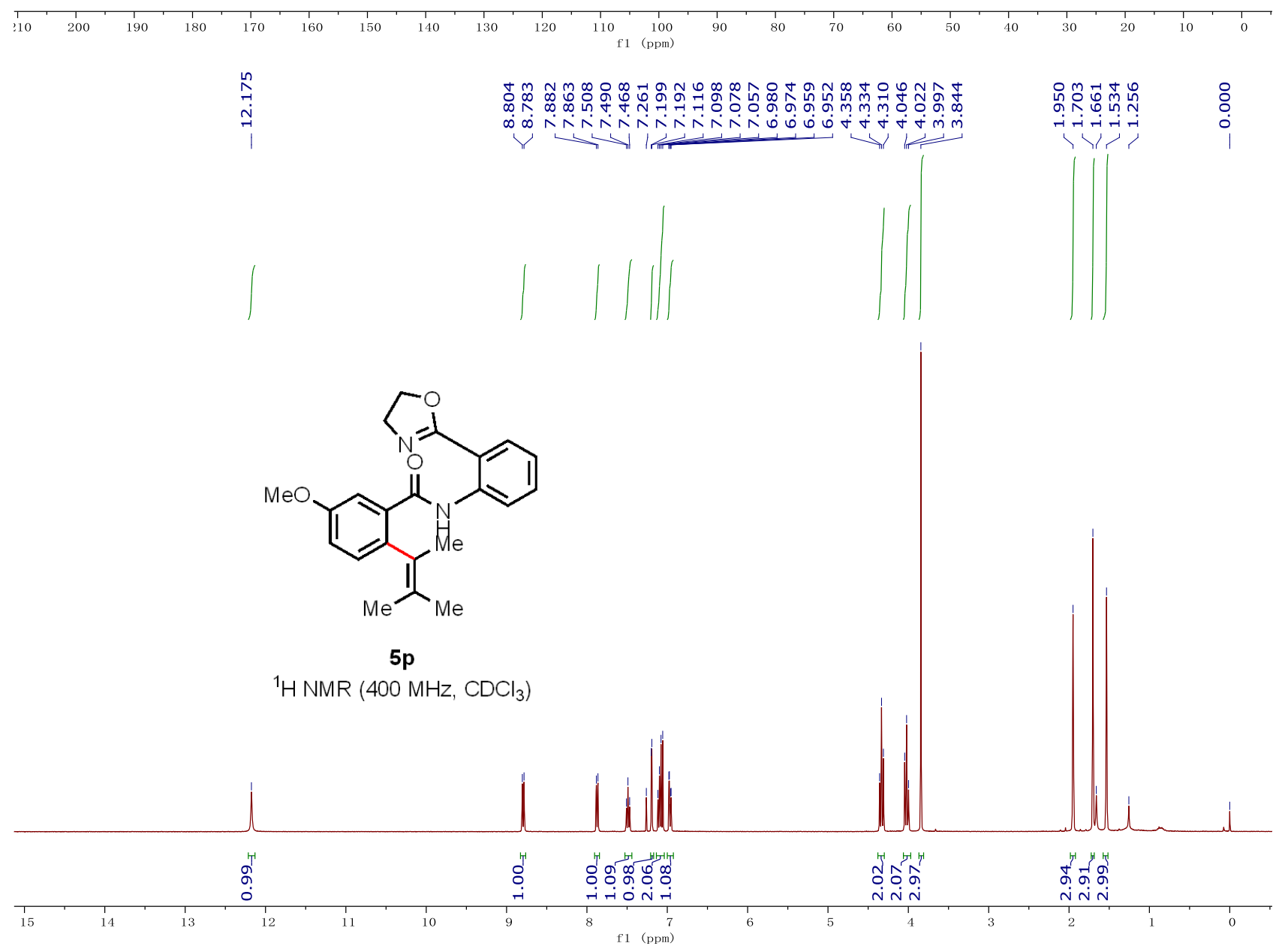




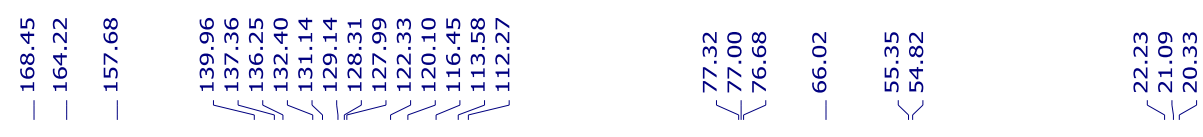<smiles>COc1ccc(C(C)=C(C)C)c(C(=O)Nc2ccccc2C2=NCCO2)c1</smiles>

$5 p$

${ }^{13} \mathrm{C} \mathrm{NMR}\left(100 \mathrm{MHz}, \mathrm{CDCl}_{3}\right)$
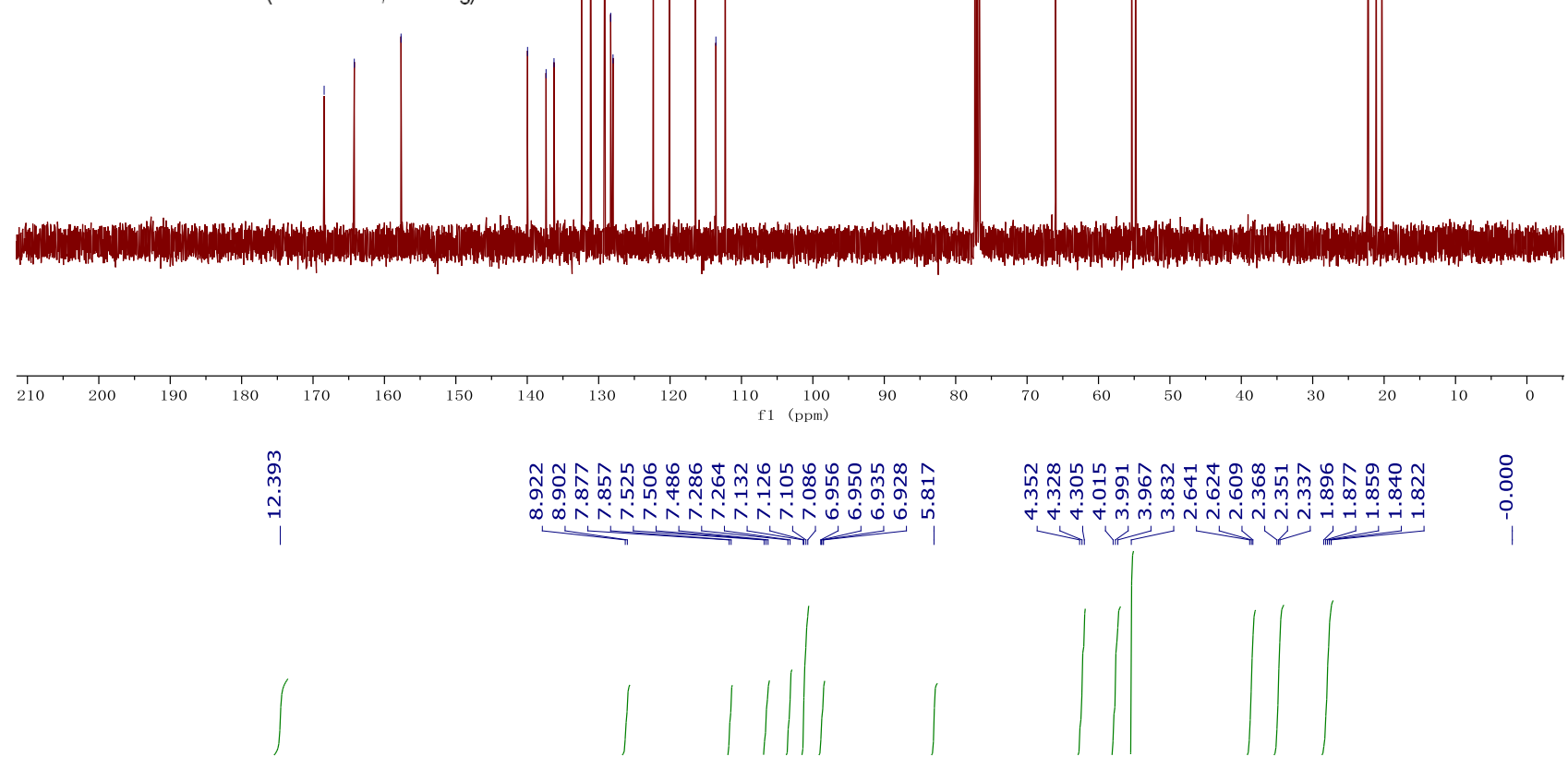<smiles>COc1ccc(C2=CCCC2)c(C(=O)Nc2ccccc2C2=NCCO2)c1</smiles>

$5 q$

${ }^{1} \mathrm{H} \mathrm{NMR}\left(400 \mathrm{MHz}, \mathrm{CDCl}_{3}\right)$

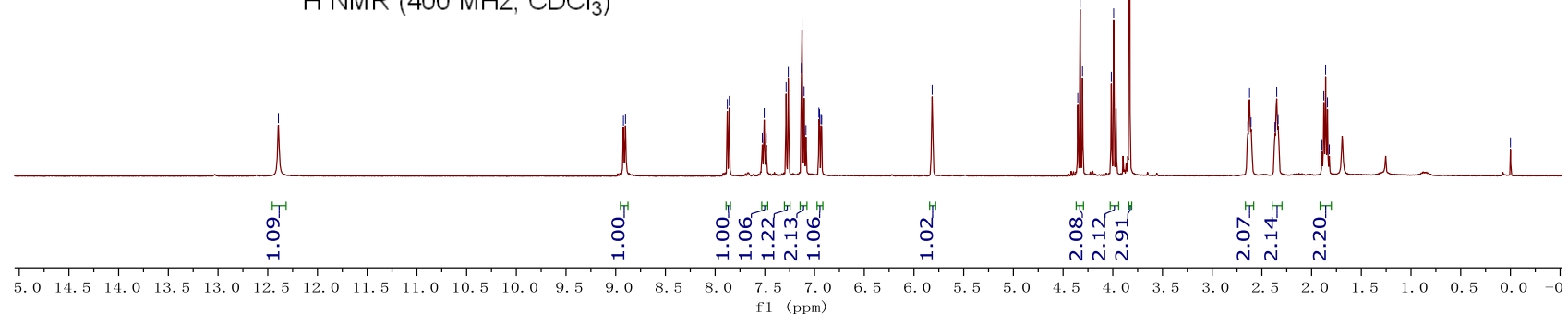


कำ

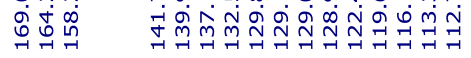

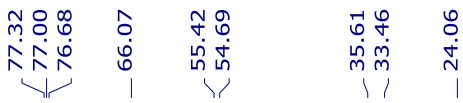

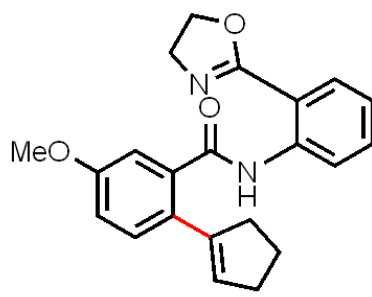

$5 q$

${ }^{13} \mathrm{C} \mathrm{NMR}\left(100 \mathrm{MHz}, \mathrm{CDCl}_{3}\right)$

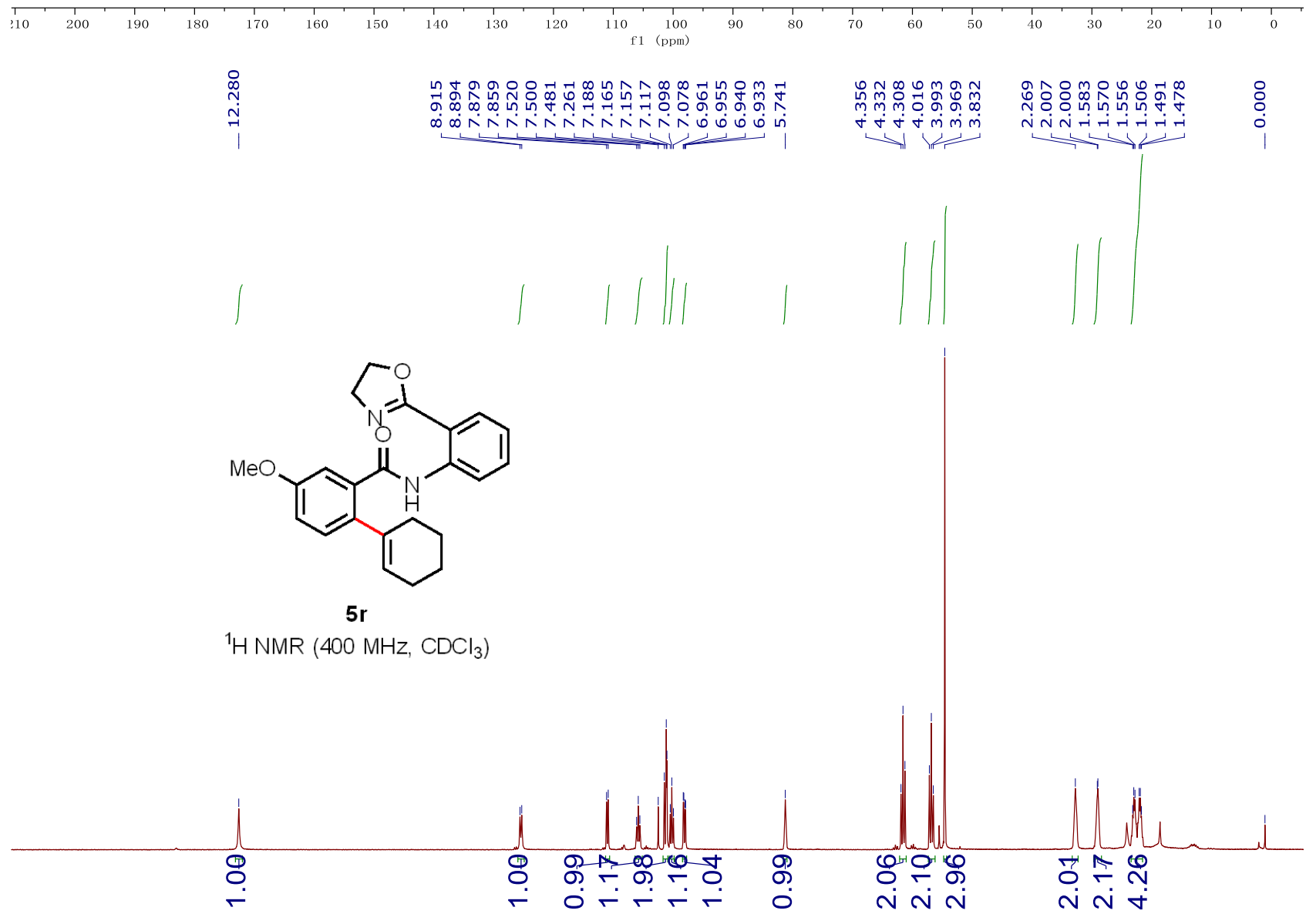

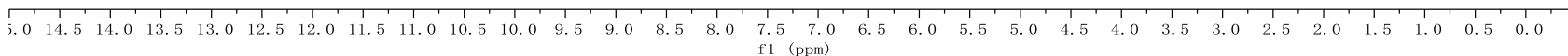




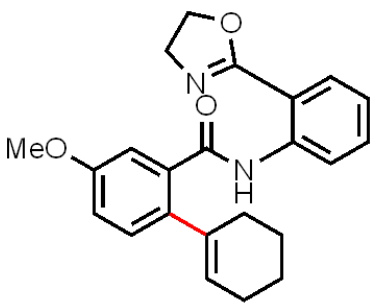

$5 r$

${ }^{13} \mathrm{C} \mathrm{NMR}\left(100 \mathrm{MHz}, \mathrm{CDCl}_{3}\right)$

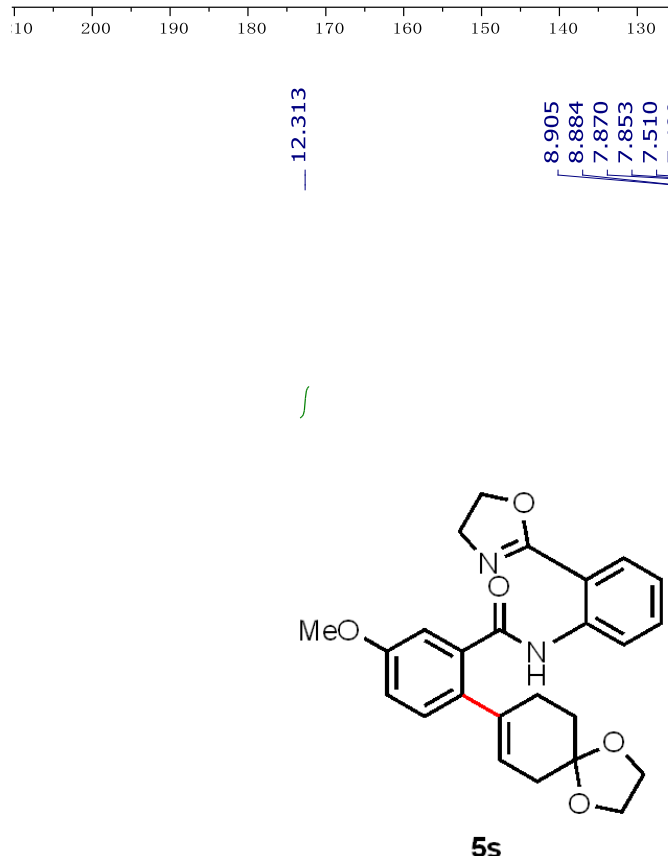

${ }^{1} \mathrm{H} \mathrm{NMR}\left(400 \mathrm{MHz}, \mathrm{CDCl}_{3}\right)$

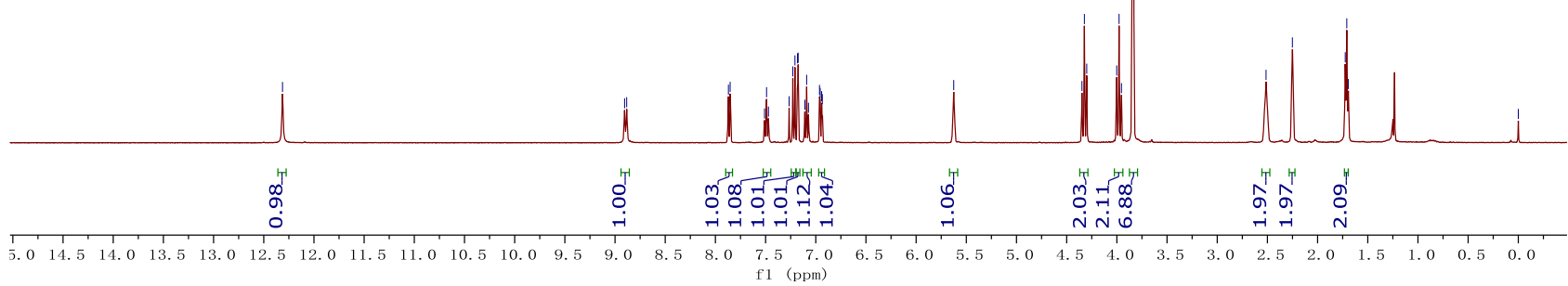




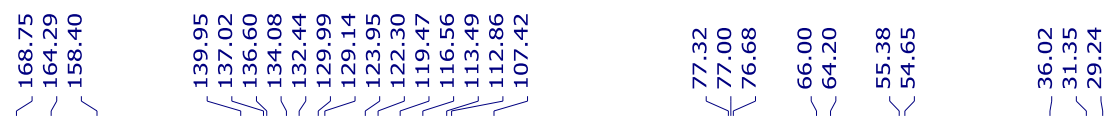<smiles>COc1ccc(C2=CCC3(CC2)OCCO3)c(C(=O)Nc2ccccc2C2=NCCO2)c1</smiles>

5s

${ }^{13} \mathrm{C} \mathrm{NMR}\left(100 \mathrm{MHz}, \mathrm{CDCl}_{3}\right)$

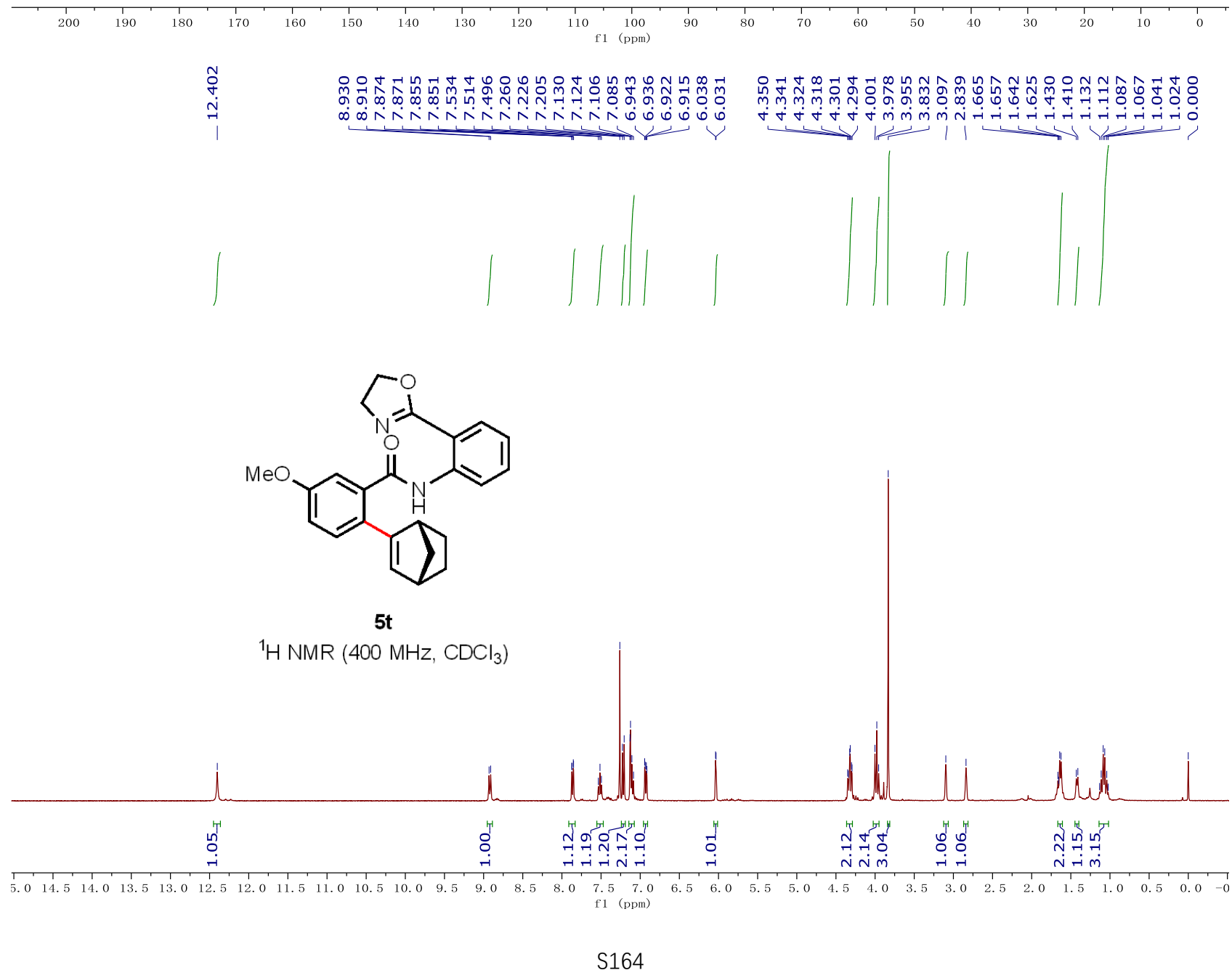


<smiles>COc1ccc(C2=CC3CCC2C3)c(C(=O)Nc2ccccc2C2=NCCO2)c1</smiles>

$5 t$

${ }^{13} \mathrm{C} \mathrm{NMR}\left(100 \mathrm{MHz}, \mathrm{CDCl}_{3}\right)$
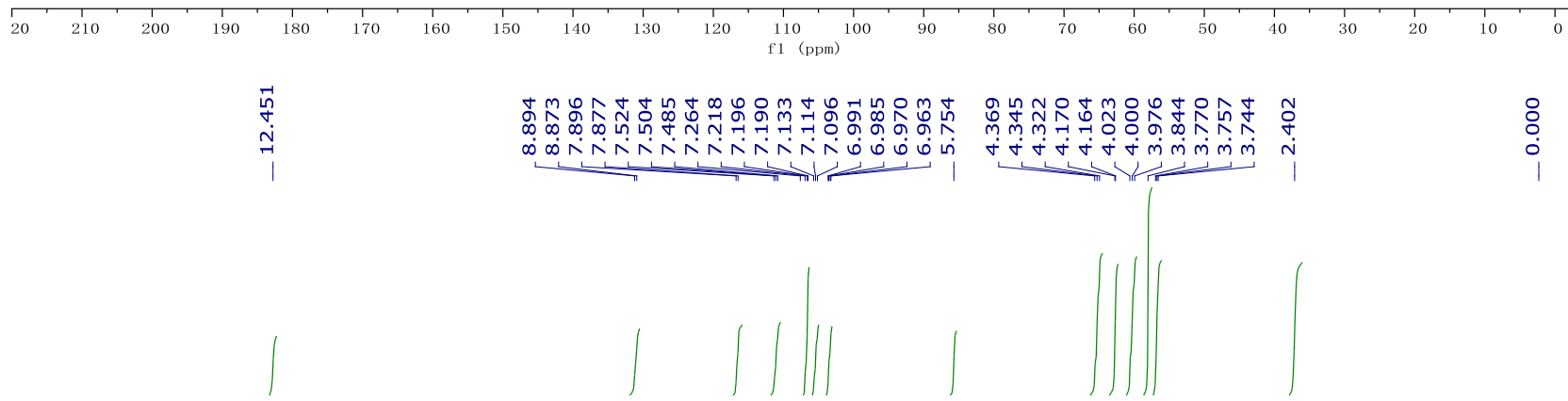<smiles>COc1ccc(C2=CCOCC2)c(C(=O)Nc2ccccc2C2=NCCO2)c1</smiles>

$5 u$

${ }^{1} \mathrm{H}$ NMR $\left(400 \mathrm{MHz}, \mathrm{CDCl}_{3}\right)$

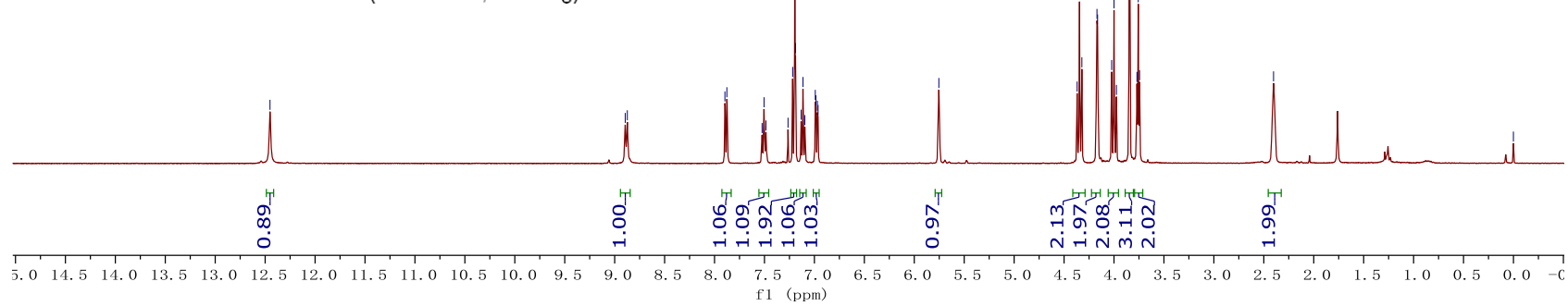




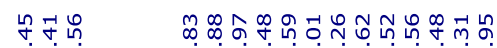

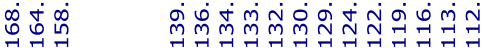

mิํำ

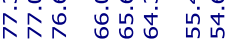

ஸे<smiles>COc1ccc(C2=CCOCC2)c(C(=O)Nc2ccccc2C2=NCCO2)c1</smiles>

5u

${ }^{13} \mathrm{C}$ NMR $\left(100 \mathrm{MHz}, \mathrm{CDCl}_{3}\right)$

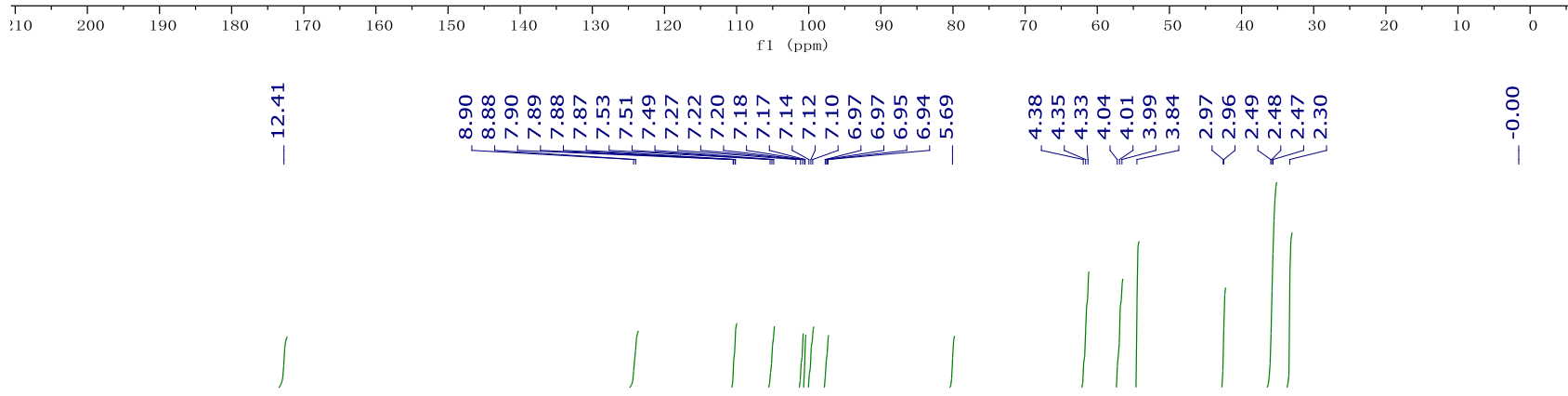<smiles>COc1ccc(C2=CCN(C)CC2)c(C(=O)Nc2ccccc2C2=NCCO2)c1</smiles>

$5 \mathrm{v}$

${ }^{1} \mathrm{H}$ NMR $\left(400 \mathrm{MHz}, \mathrm{CDCl}_{3}\right)$

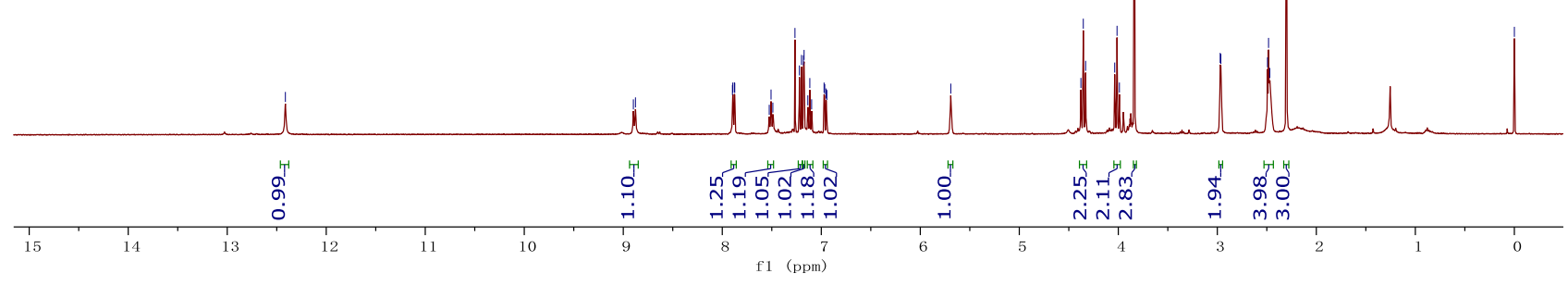


<smiles>COc1ccc(C2=CCN(S(C)(=O)=O)CC2)c(C(=O)Nc2ccccc2C2=NCCO2)c1</smiles>

$5 v$

${ }^{13} \mathrm{C} \mathrm{NMR}\left(100 \mathrm{MHz}, \mathrm{CDCl}_{3}\right)$

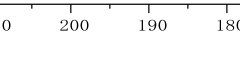

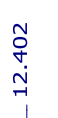<smiles>COc1ccc(C2=CCN(C(=O)OC(C)(C)C)CC2)c(C(=O)Nc2ccccc2C2=NCCO2)c1</smiles>

${ }^{1} \mathrm{H}$ NMR $\left(400 \mathrm{MHz}, \mathrm{CDCl}_{3}\right)$

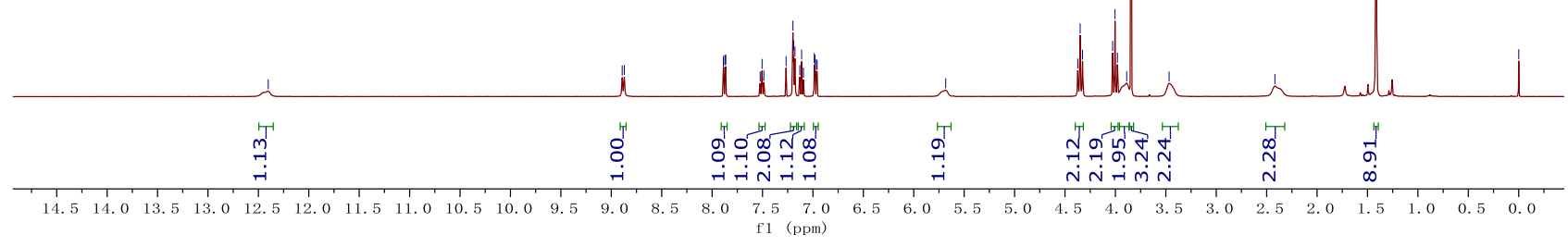



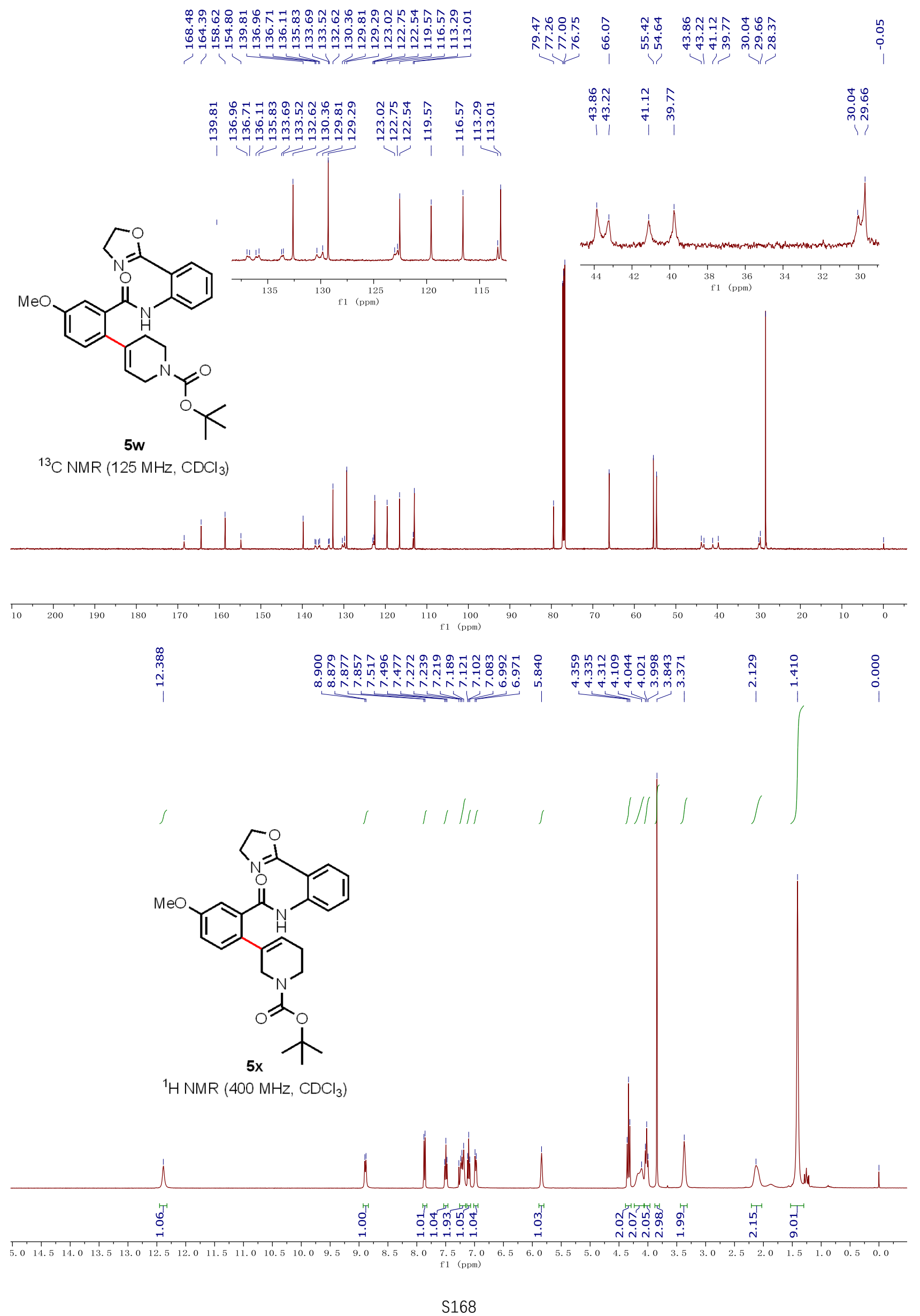
<smiles>COc1ccc(C2=CCCN(C(=O)OC(C)(C)C)C2)c(C(=O)Nc2ccccc2C2=NCCO2)c1</smiles>

$5 x$
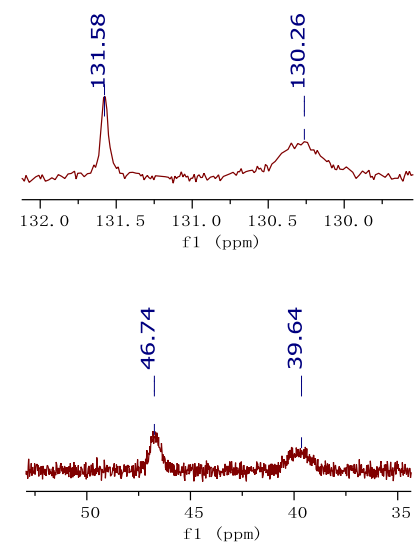

${ }^{13} \mathrm{C} \mathrm{NMR}\left(150 \mathrm{MHz}, \mathrm{CDCl}_{3}\right)$
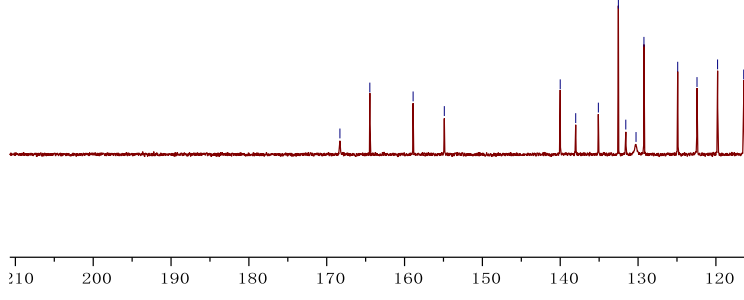

\section{$\underset{\substack{n \\ \mathfrak{n}}}{\mathfrak{r}}$}

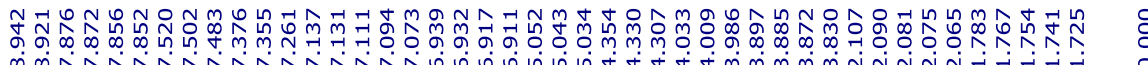
itid<smiles>C1CCC1</smiles><smiles>C1=CC2=CC=C1C2</smiles><smiles>COc1ccc(C2=CCCCO2)c(C(=O)Nc2ccccc2C2=NCCO2)c1</smiles>

$5 y$

${ }^{1} \mathrm{H} \mathrm{NMR}\left(400 \mathrm{MHz}, \mathrm{CDCl}_{3}\right)$

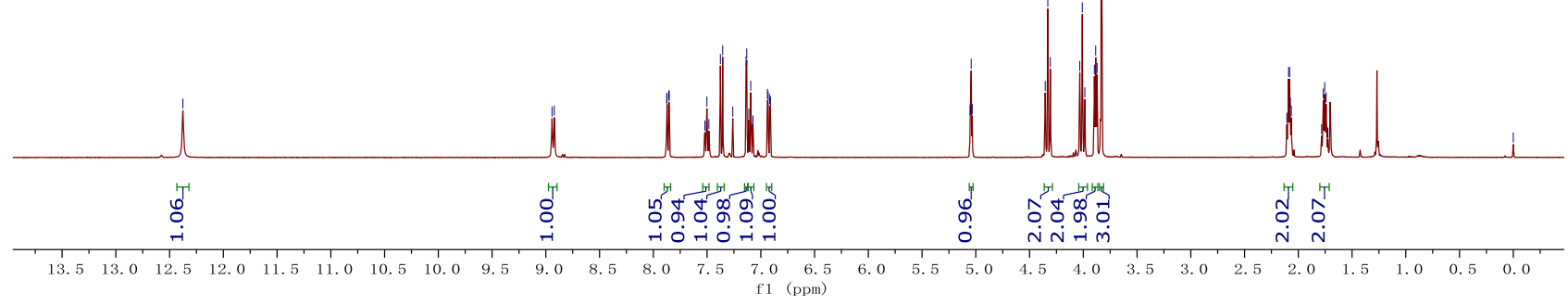




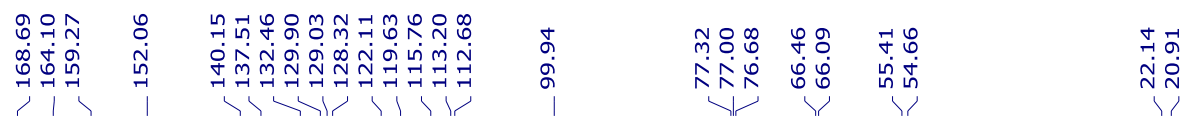<smiles>COc1ccc(C2=CCCCO2)c(C(=O)Nc2ccccc2C2=NCCO2)c1</smiles>

$5 y$

${ }^{13} \mathrm{C} \mathrm{NMR}\left(100 \mathrm{MHz}, \mathrm{CDCl}_{3}\right)$
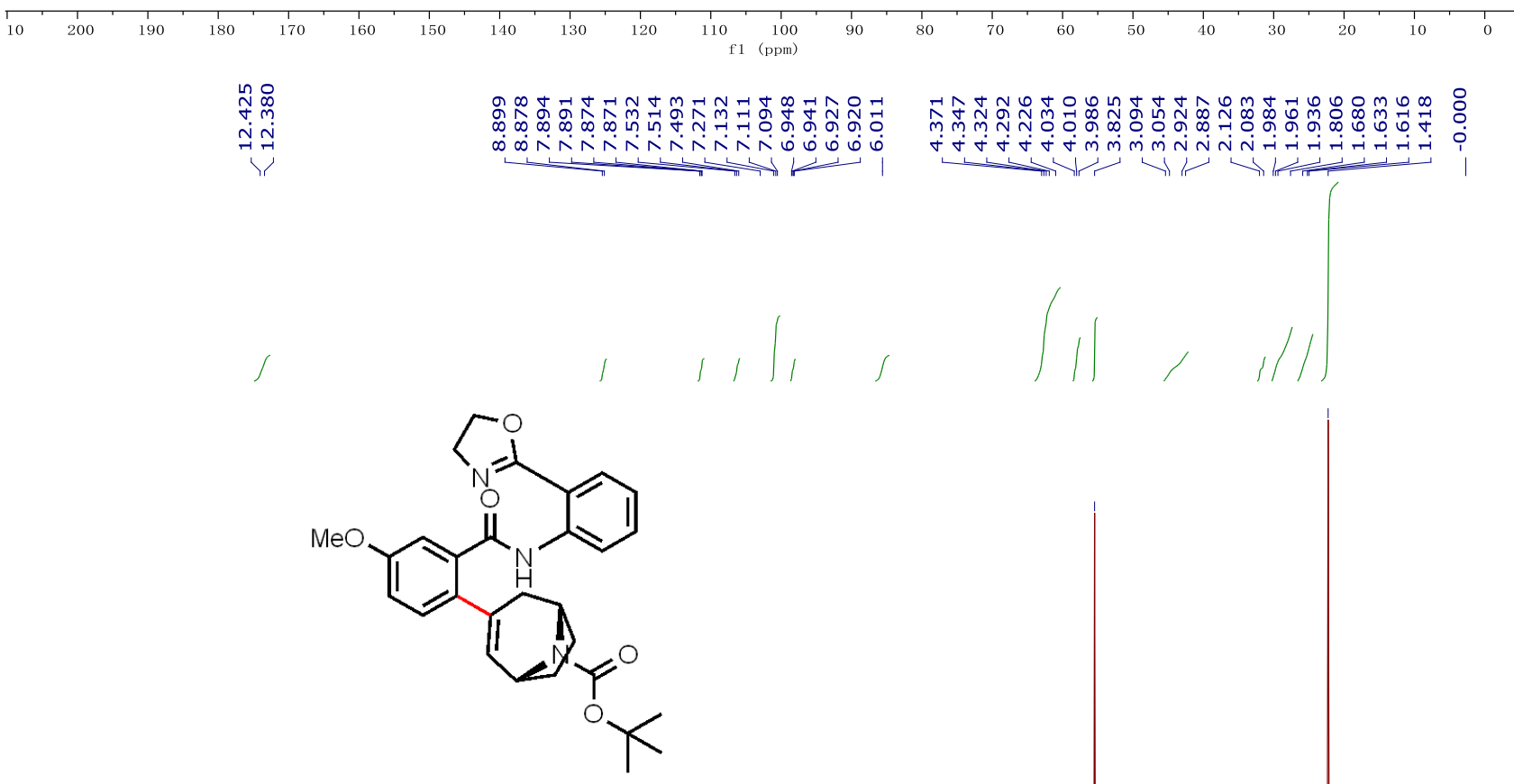

$5 z$

${ }^{1} \mathrm{H}$ NMR $\left(400 \mathrm{MHz}, \mathrm{CDCl}_{3}\right)$

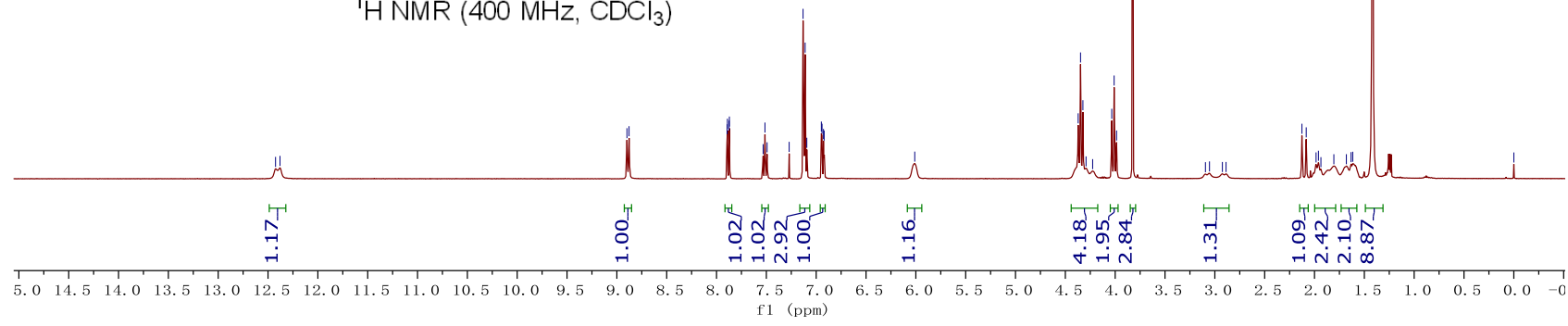




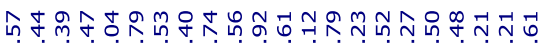

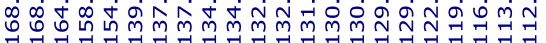

in $\frac{1}{d}$

$\stackrel{\infty}{\infty}$

${ }^{13} \mathrm{C} \mathrm{NMR}\left(125 \mathrm{MHz}, \mathrm{CDCl}_{3}\right)$

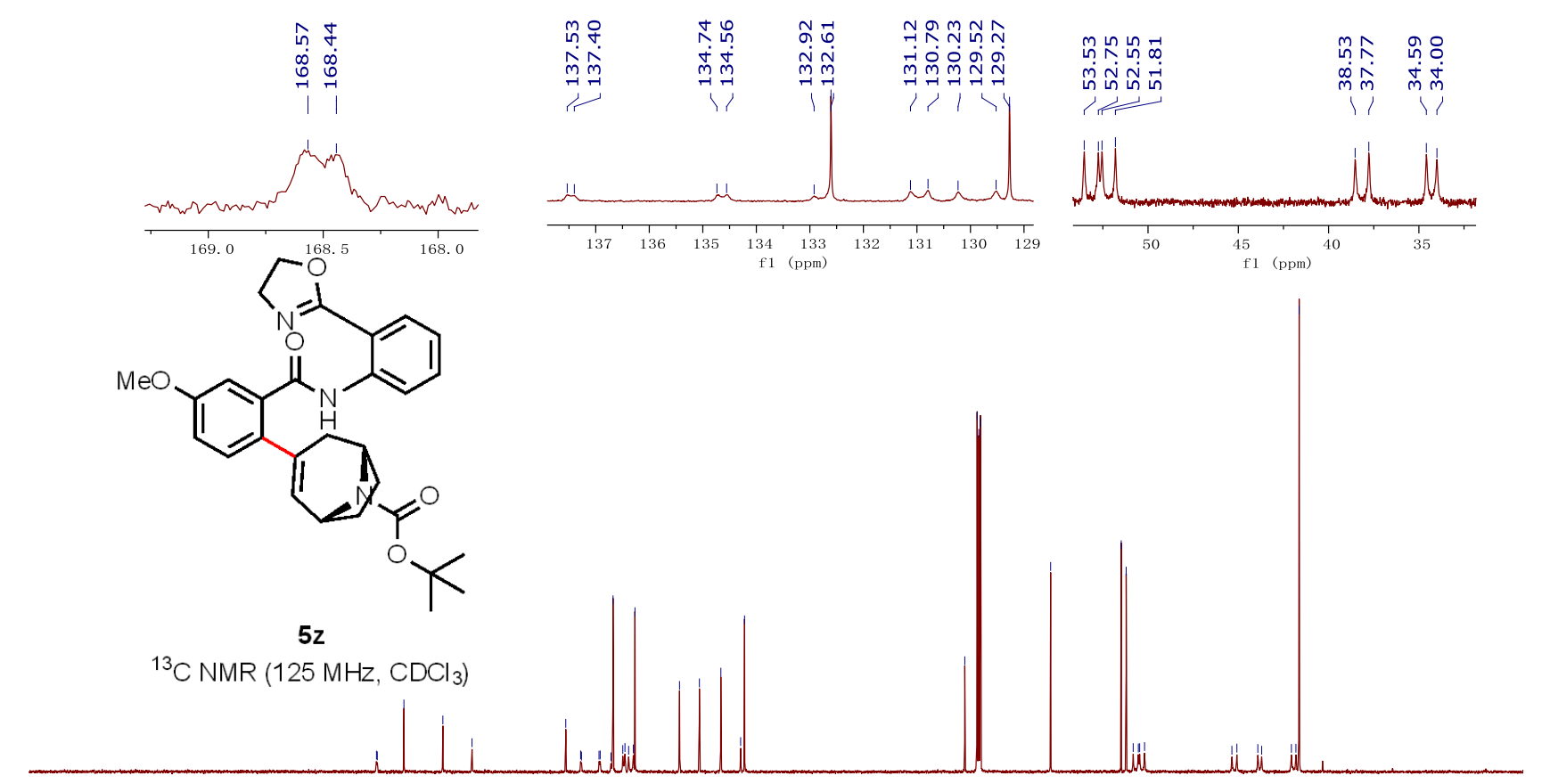

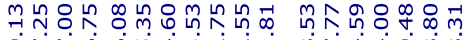

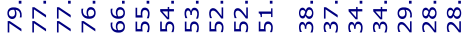

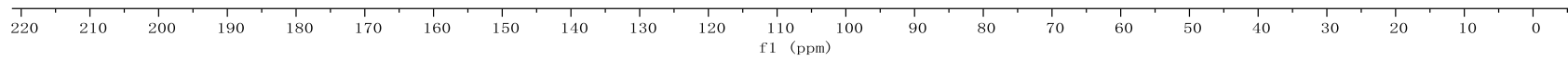

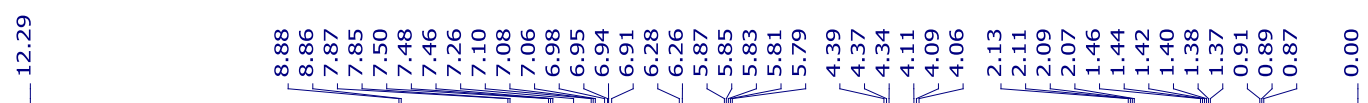<smiles>CCC/C=C/C=C(/C)C(=O)Nc1ccccc1C1=NCCO1</smiles>

$7 a$

${ }^{1} \mathrm{H} \mathrm{NMR}\left(400 \mathrm{MHz}, \mathrm{CDCl}_{3}\right)$

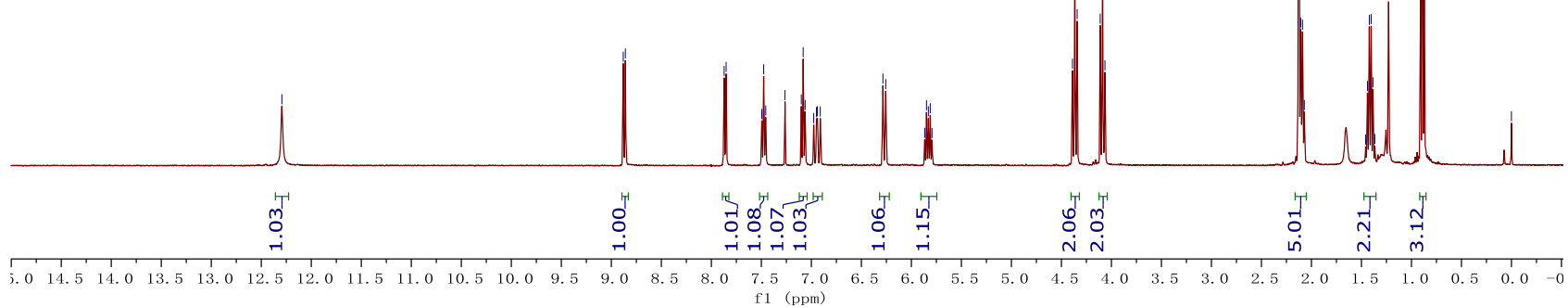




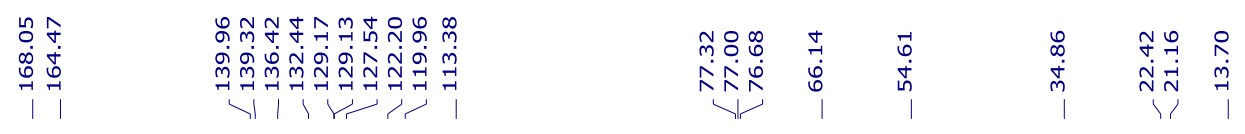<smiles>CCC/C=C/C=C(/C)C(=O)Nc1ccccc1C1=NCCO1</smiles>

$7 a$

${ }^{13} \mathrm{C} \mathrm{NMR}\left(100 \mathrm{MHz}, \mathrm{CDCl}_{3}\right)$
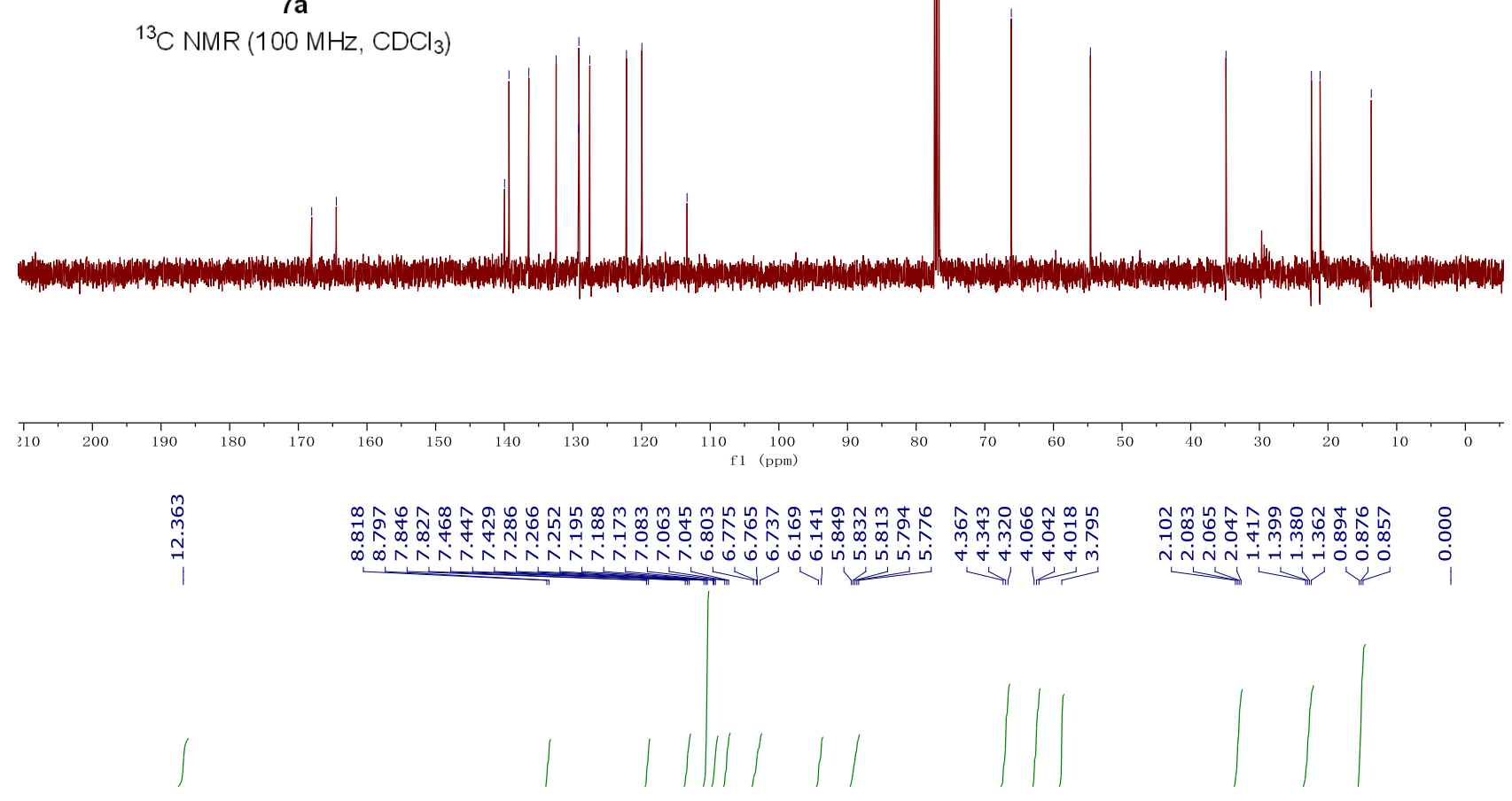<smiles>CCC/C=C/C=C(/Cc1ccccc1)C(=O)Nc1ccccc1C1=NCCO1</smiles>

7b

${ }^{1} \mathrm{H}$ NMR $\left(400 \mathrm{MHz}, \mathrm{CDCl}_{3}\right)$

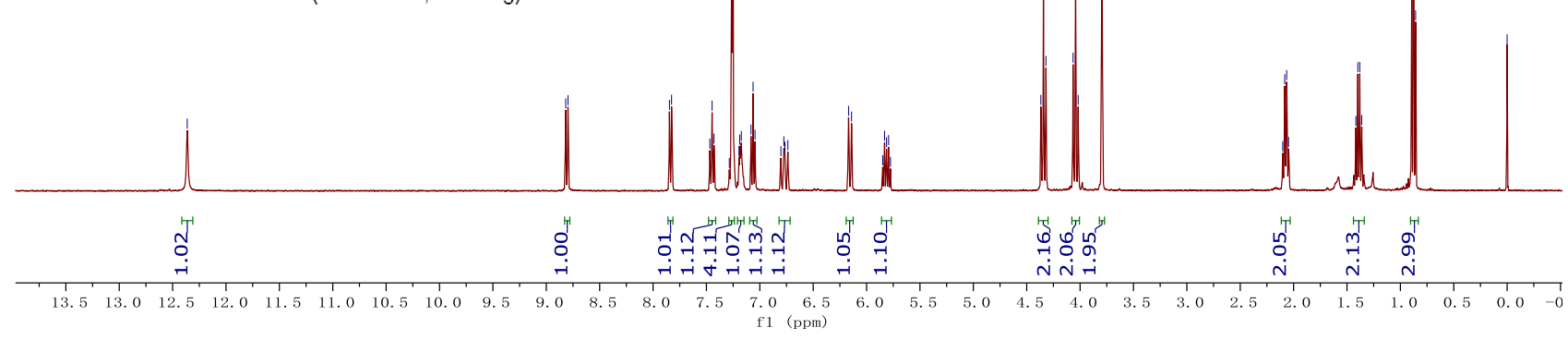


<smiles>CCC/C=C/C=C(/Cc1ccccc1)C(=O)Nc1ccccc1C1=NCCO1</smiles>

7b

${ }^{13} \mathrm{C} \mathrm{NMR}\left(100 \mathrm{MHz}, \mathrm{CDCl}_{3}\right)$

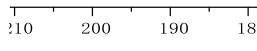

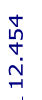

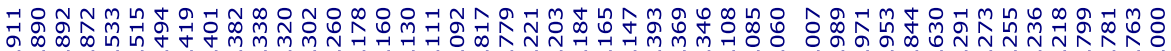

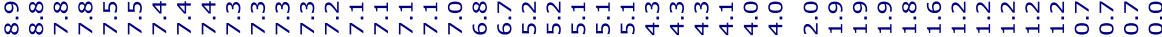

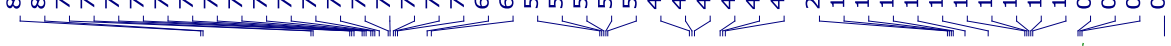<smiles>CCC/C=C/C(=C(/C)C(=O)Nc1ccccc1C1=NCCO1)c1ccccc1</smiles>

7c

${ }^{1} \mathrm{H}$ NMR $\left(400 \mathrm{MHz}, \mathrm{CDCl}_{3}\right)$
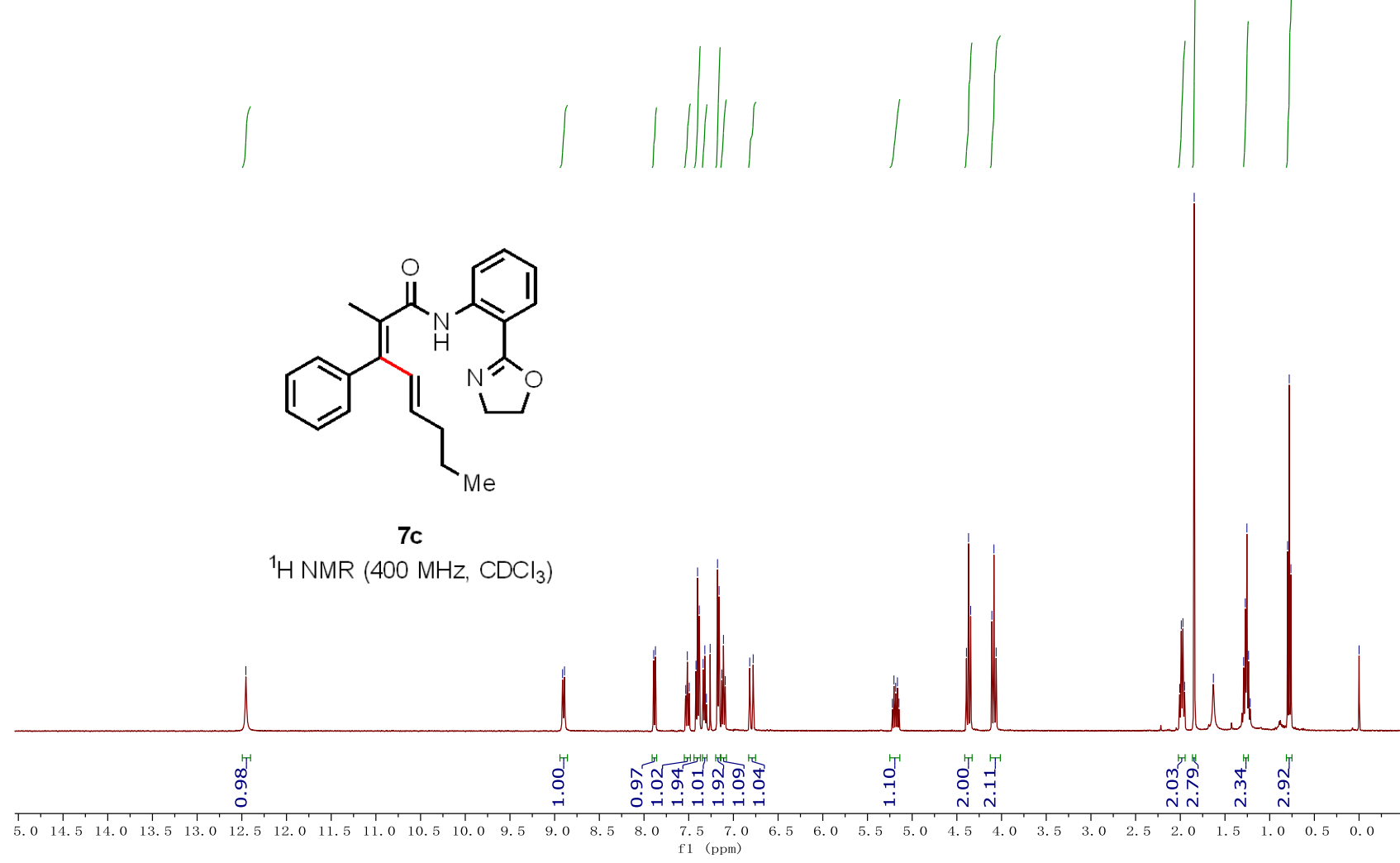


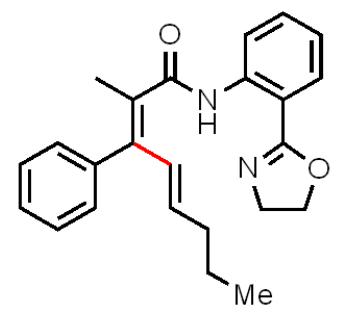

7c

${ }^{13} \mathrm{C} \mathrm{NMR}\left(100 \mathrm{MHz}, \mathrm{CDCl}_{3}\right)$

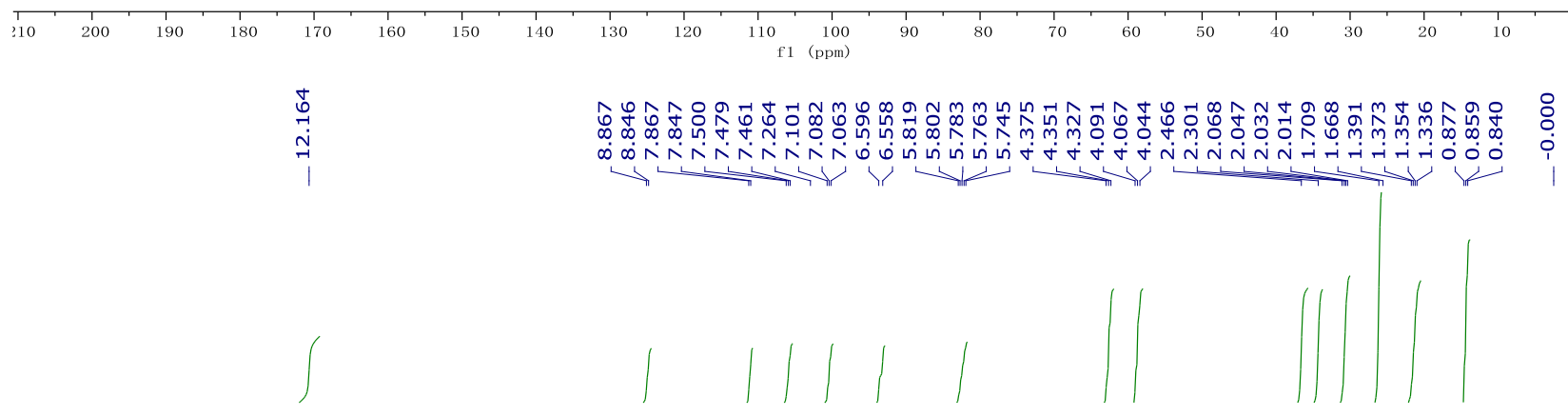<smiles>CCC/C=C/C1=C(C(=O)Nc2ccccc2C2=NCCO2)CCCC1</smiles>

7d

${ }^{1} \mathrm{H}$ NMR $\left(400 \mathrm{MHz}, \mathrm{CDCl}_{3}\right)$

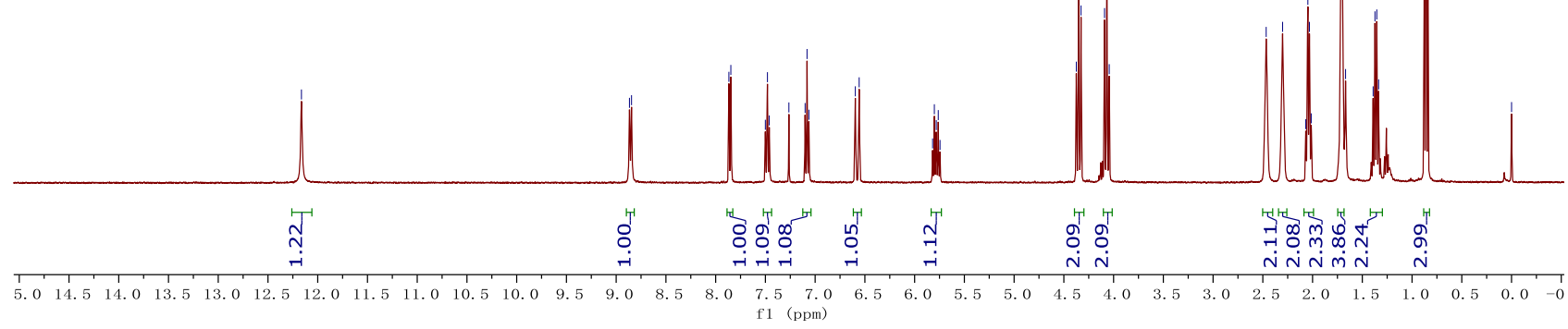




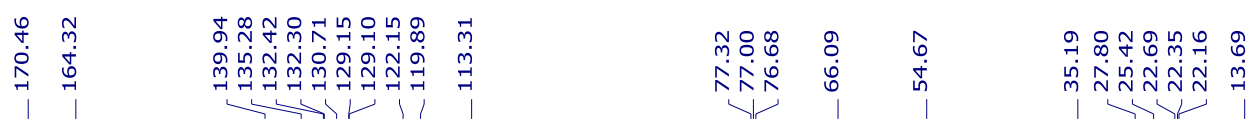<smiles>CCC/C=C/C1=C(C(=O)Nc2ccccc2C2=NCCO2)CCCC1</smiles>

7d

${ }^{13} \mathrm{C} \mathrm{NMR}\left(100 \mathrm{MHz}, \mathrm{CDCl}_{3}\right)$

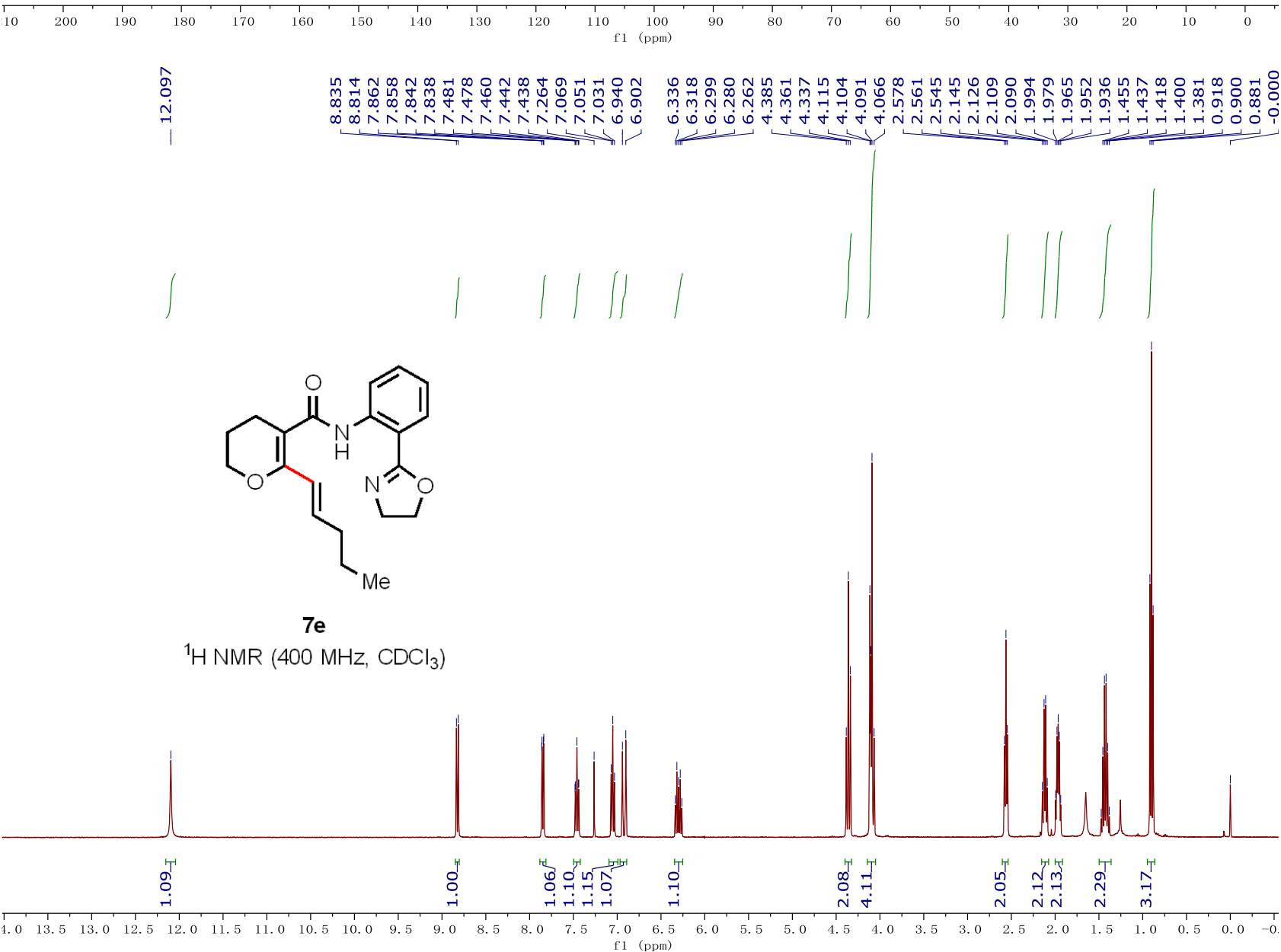




\begin{tabular}{|c|c|c|c|c|c|c|c|}
\hline 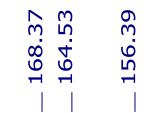 & 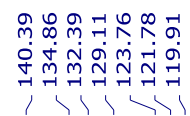 & $\begin{array}{l}\stackrel{P}{N} \\
\stackrel{m}{7} \\
1\end{array}$ & \begin{tabular}{l}
\multirow{N}{*}{} \\
$\hat{1}$ \\
0 \\
-1
\end{tabular} & 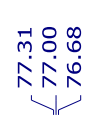 & 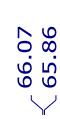 & & in \\
\hline
\end{tabular}<smiles>CCC/C=C/C1=C(C(=O)Nc2ccccc2C2=NCCO2)CCCO1</smiles>

$7 e$

${ }^{13} \mathrm{C} \mathrm{NMR}\left(100 \mathrm{MHz}, \mathrm{CDCl}_{3}\right)$

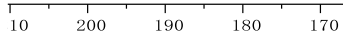

$\underset{\substack{m \\ \stackrel{7}{N}}}{1}$

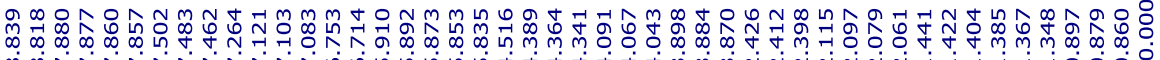
-i

$\underbrace{\infty}$
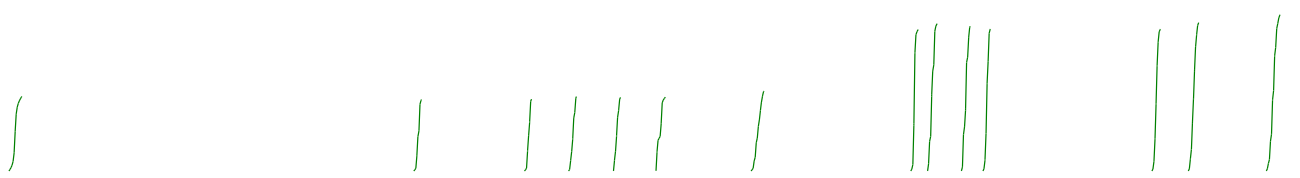<smiles>CCC/C=C/C1=C(C(=O)Nc2ccccc2C2=NCCO2)COCC1</smiles>

$7 f$

${ }^{1} \mathrm{H}$ NMR $\left(400 \mathrm{MHz}, \mathrm{CDCl}_{3}\right)$

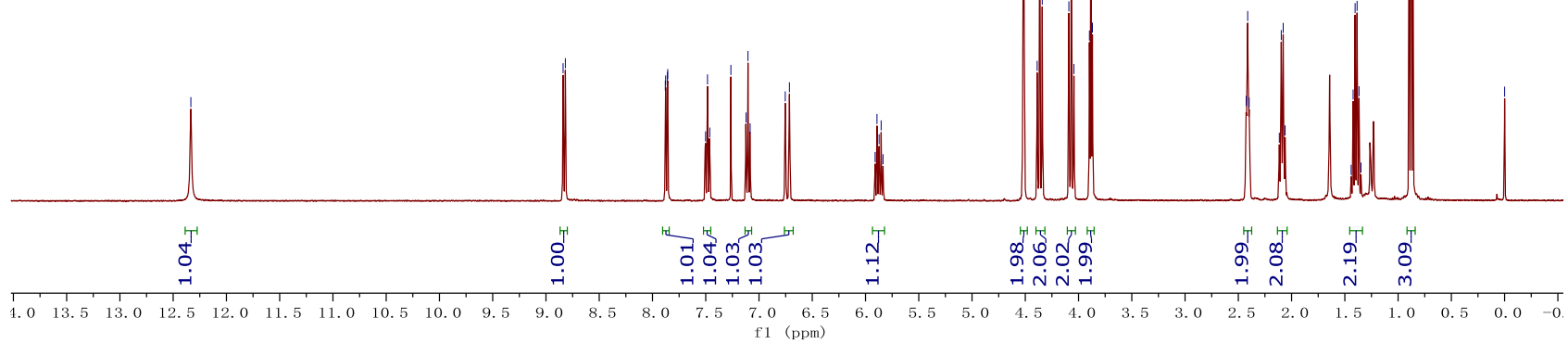




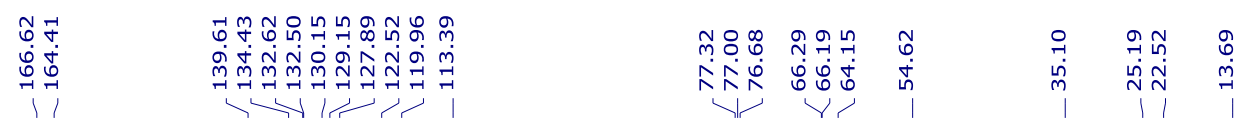<smiles>CCC/C=C/C1=C(C(=O)Nc2ccccc2C2=NCCO2)COCC1</smiles>

$7 f$

${ }^{13} \mathrm{C} \mathrm{NMR}\left(100 \mathrm{MHz}, \mathrm{CDCl}_{3}\right)$
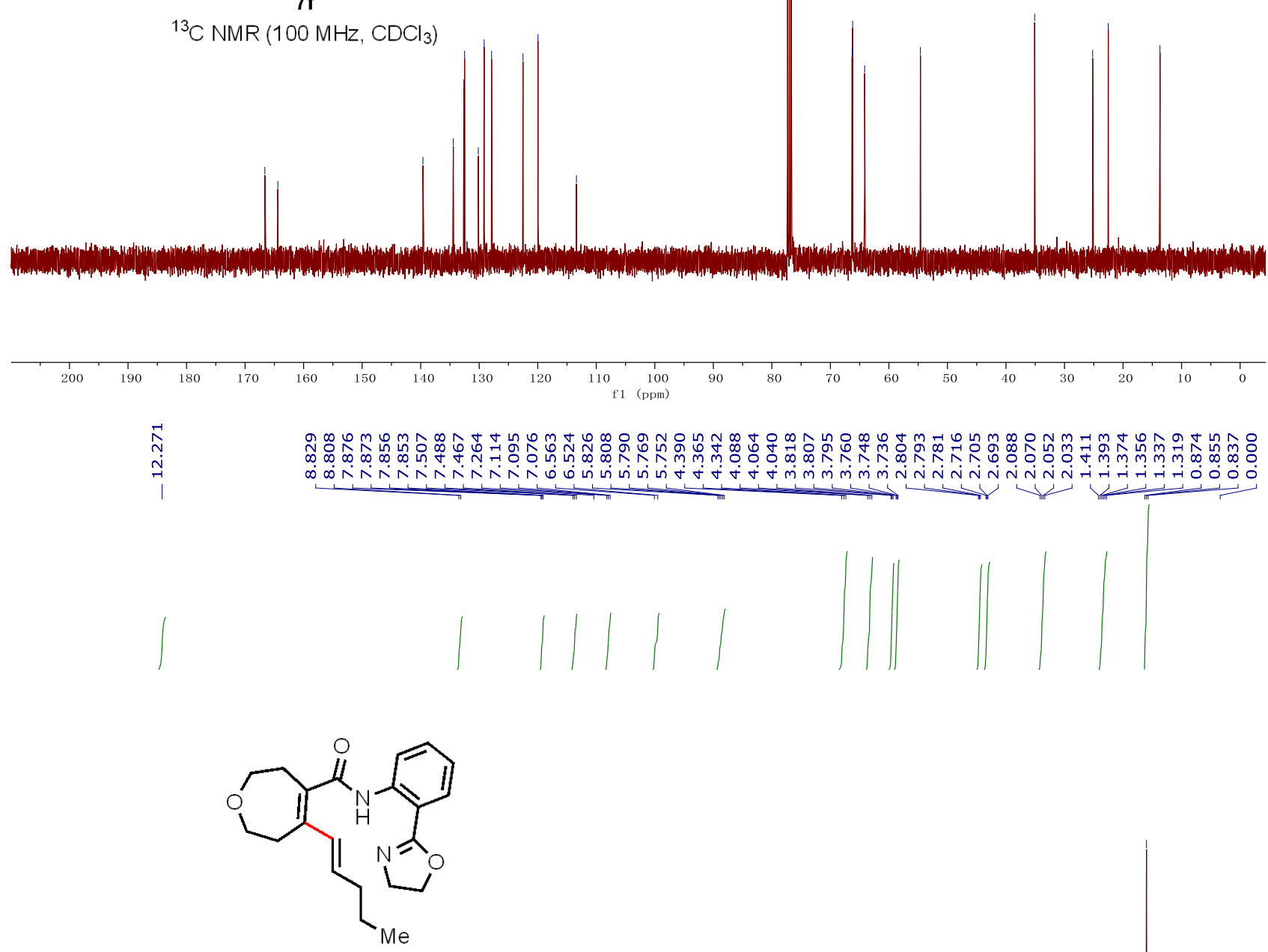

$7 \mathrm{~g}$

${ }^{1} \mathrm{H} \mathrm{NMR}\left(400 \mathrm{MHz}, \mathrm{CDCl}_{3}\right)$

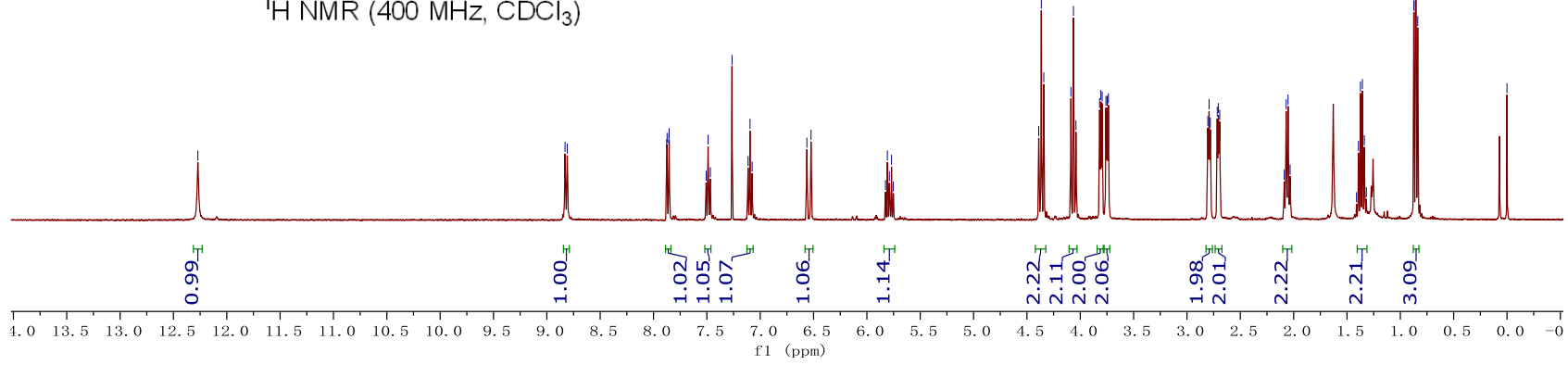




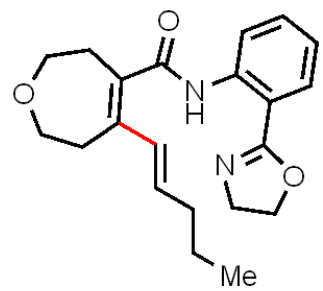

$7 \mathrm{~g}$

${ }^{13} \mathrm{C} \mathrm{NMR}\left(100 \mathrm{~Hz}, \mathrm{CDCl}_{3}\right)$

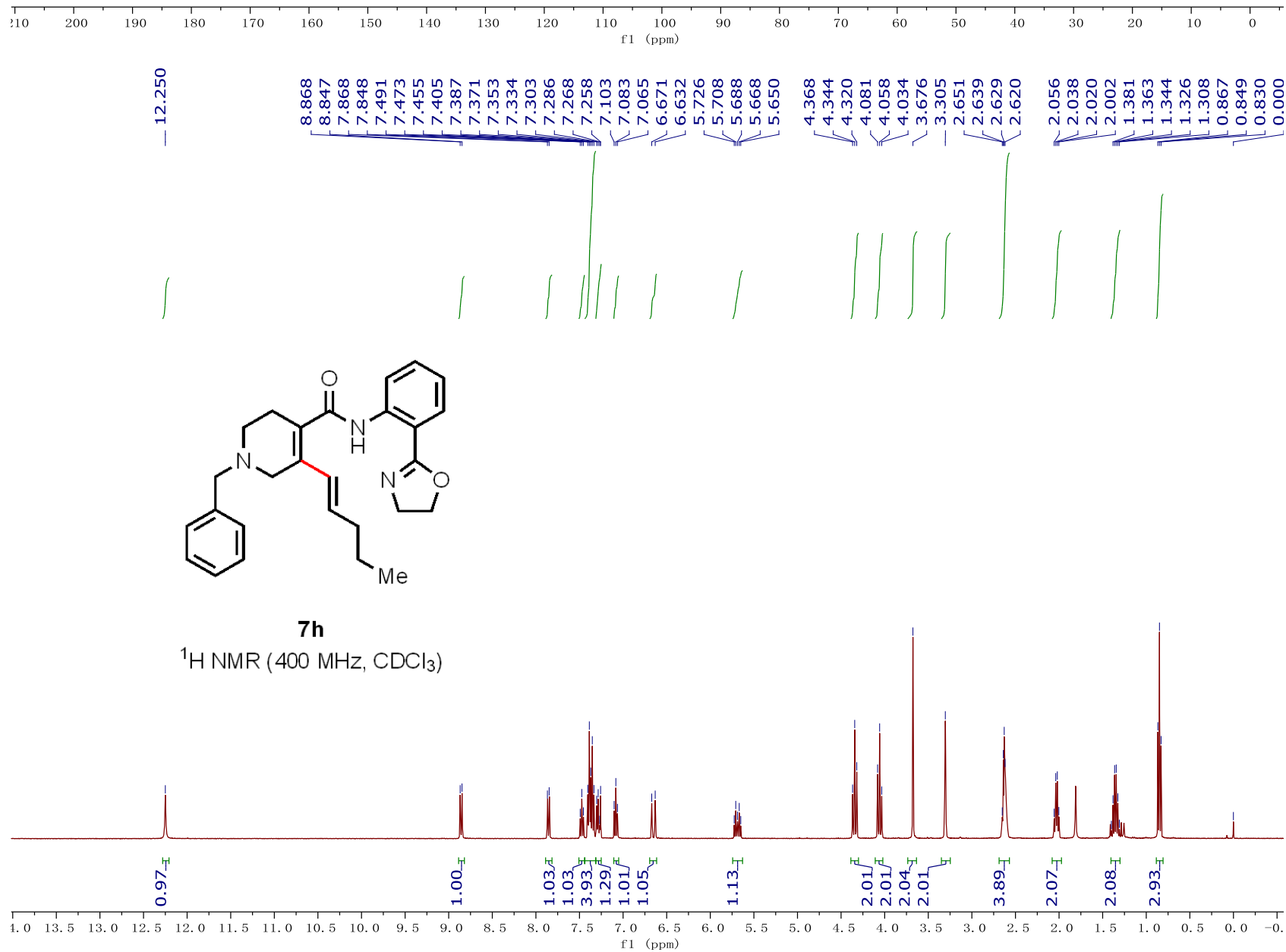




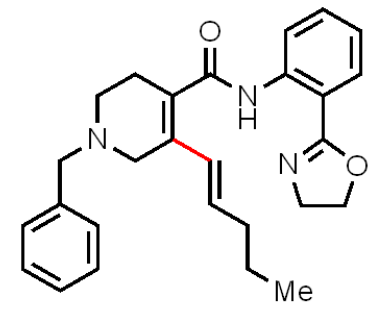

$7 \mathrm{~h}$

${ }^{13} \mathrm{C} \mathrm{NMR}\left(100 \mathrm{MHz}, \mathrm{CDCl}_{3}\right)$
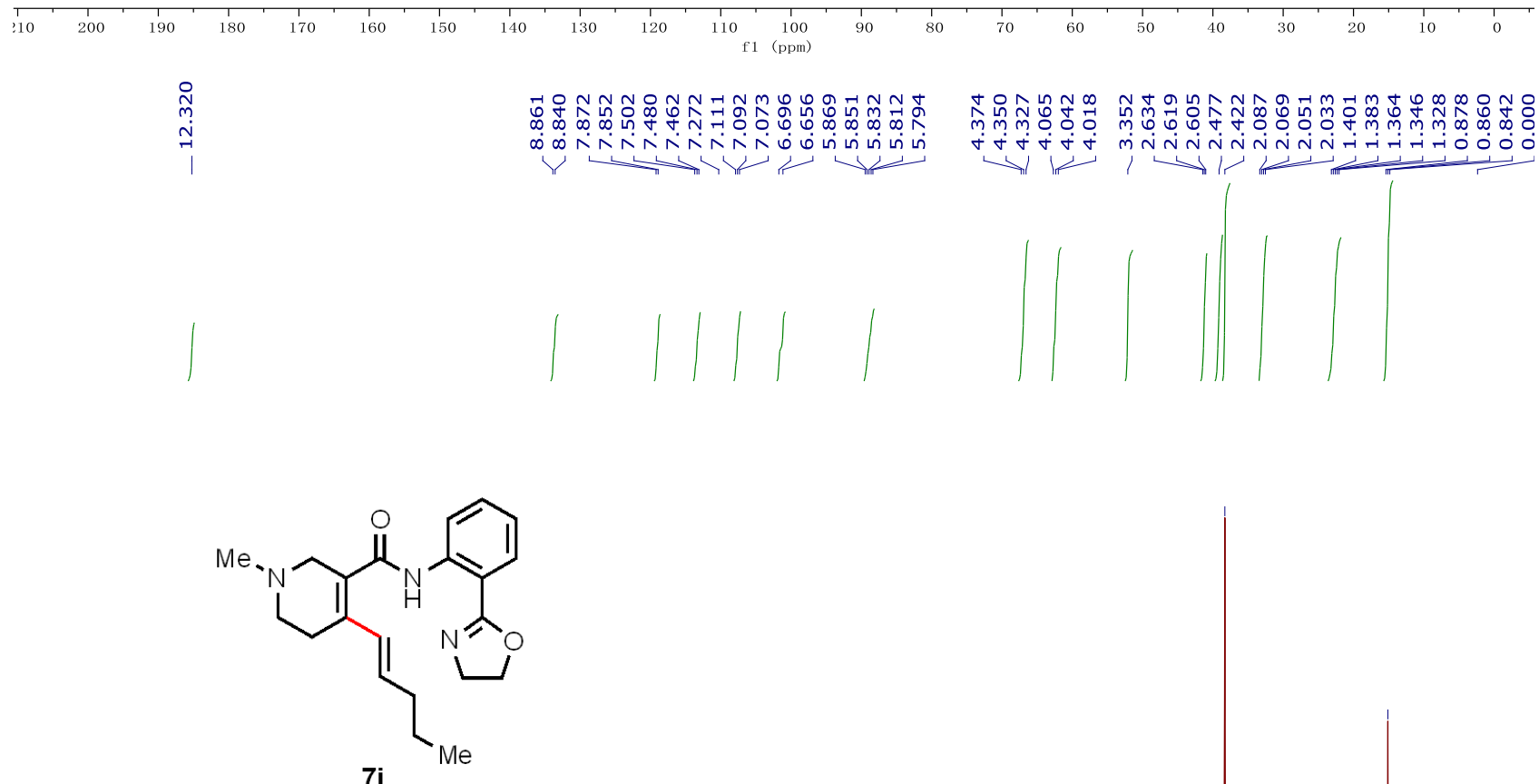

${ }^{1} \mathrm{H}$ NMR (400 MHz, $\mathrm{CDCl}_{3}$ )

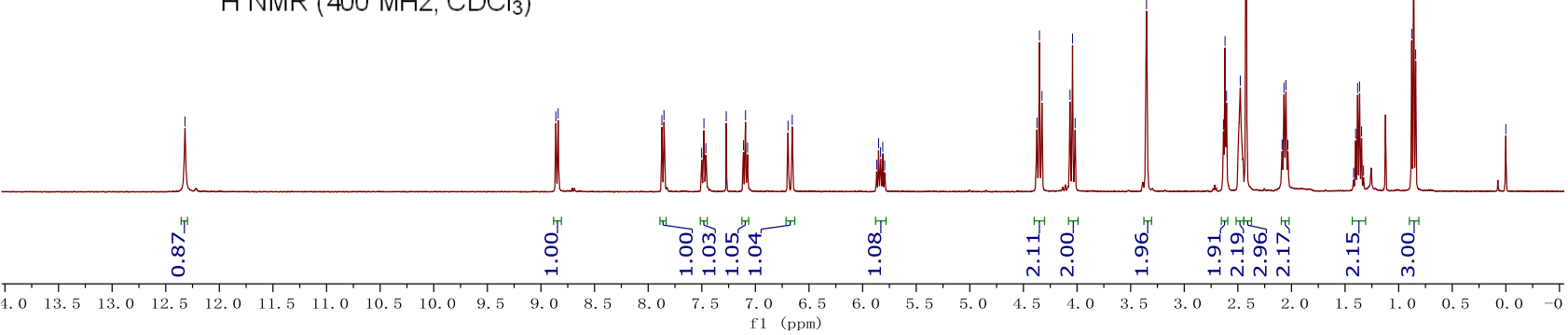




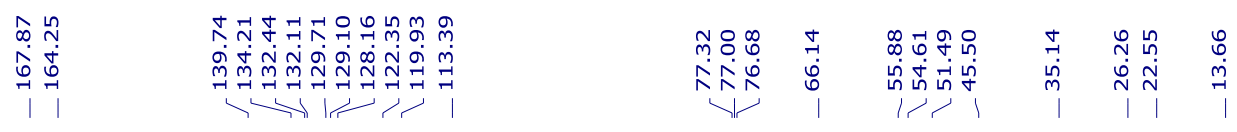

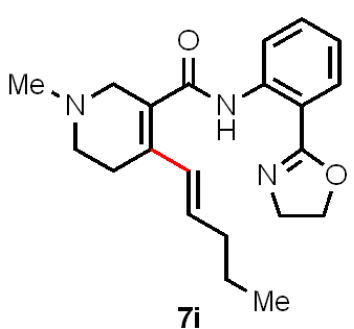

${ }^{13} \mathrm{C}$ NMR $\left(100 \mathrm{MHz}, \mathrm{CDCl}_{3}\right)$

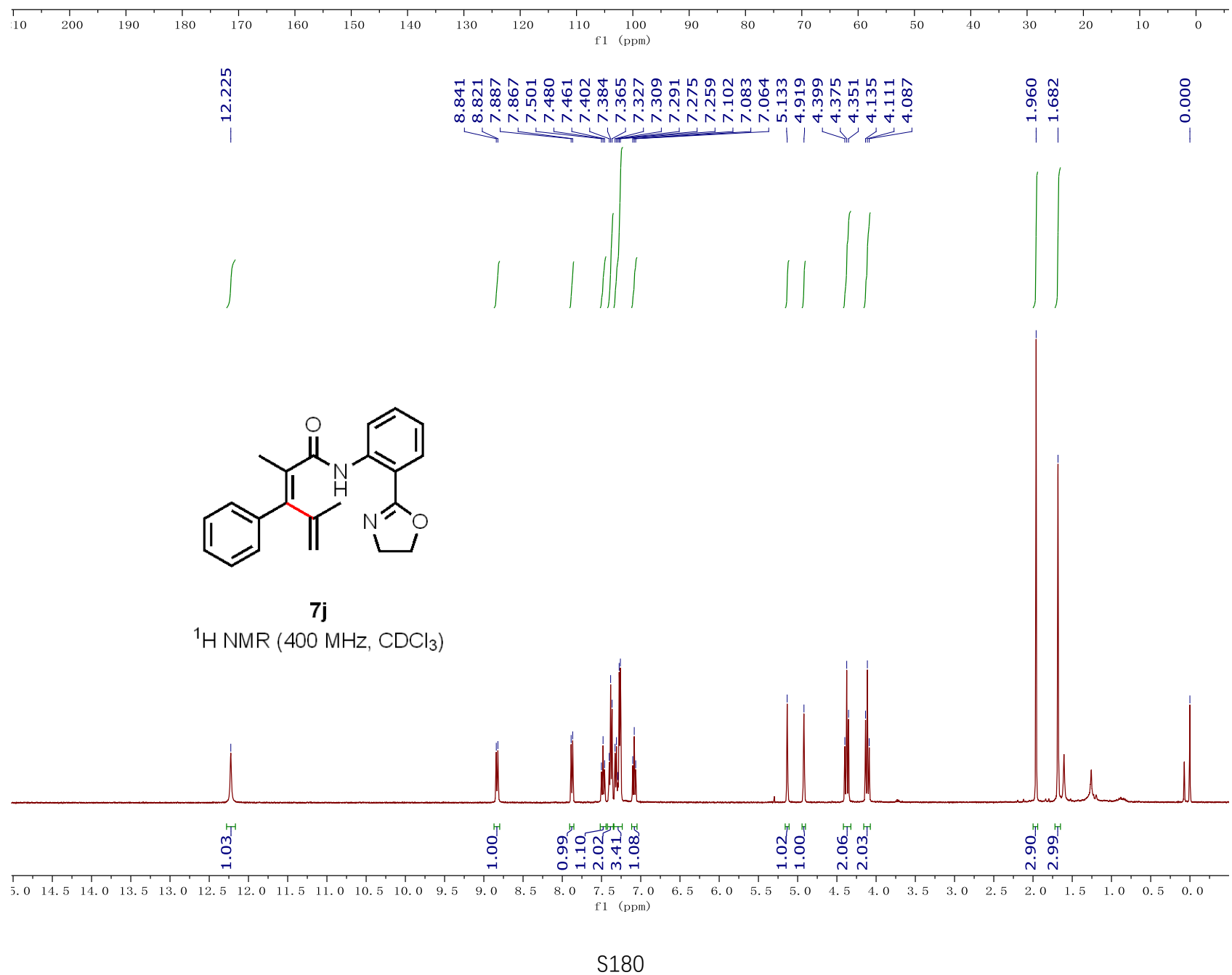




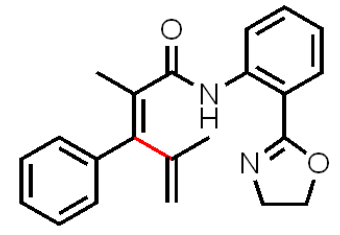

7j

${ }^{13} \mathrm{C}$ NMR $\left(100 \mathrm{MHz}, \mathrm{CDCl}_{3}\right)$

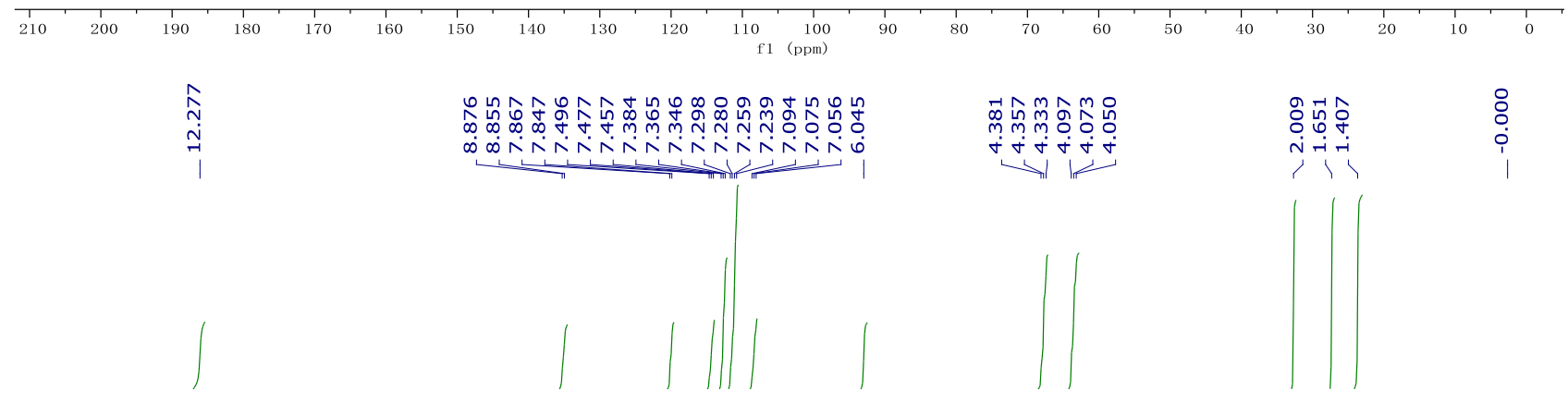<smiles>CC(C)=C/C(=C(/C)C(=O)Nc1ccccc1C1=NCCO1)c1ccccc1</smiles>

$7 k$

${ }^{1} \mathrm{H}$ NMR (400 MHz, $\mathrm{CDCl}_{3}$ )

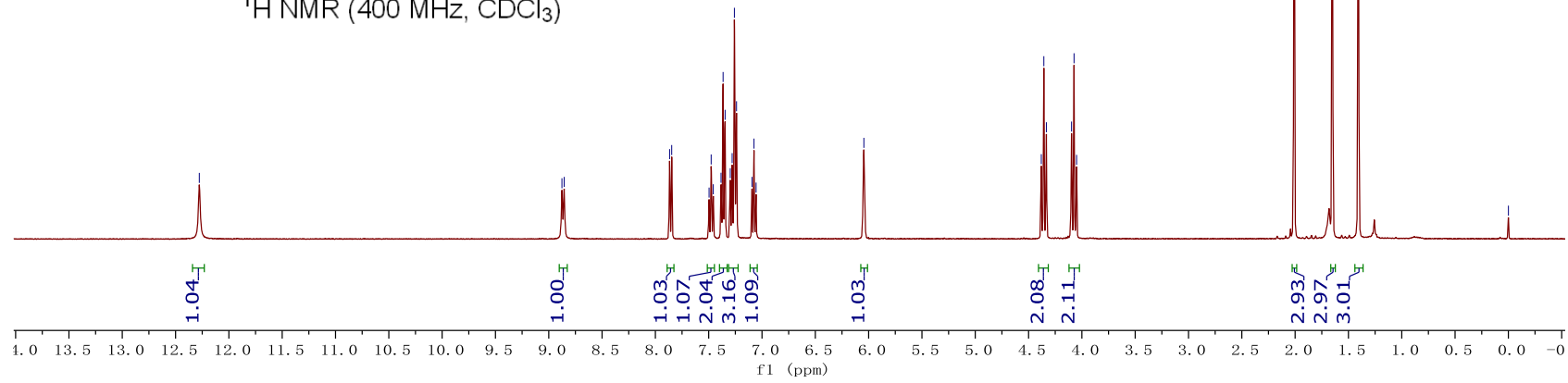




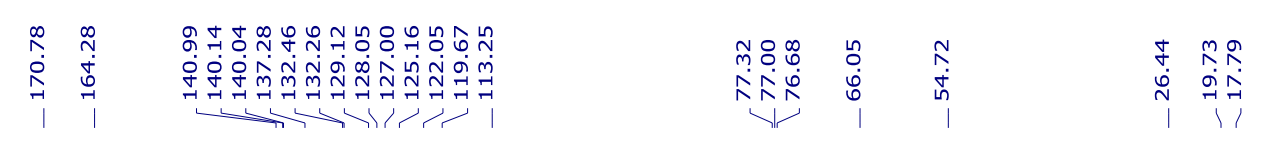

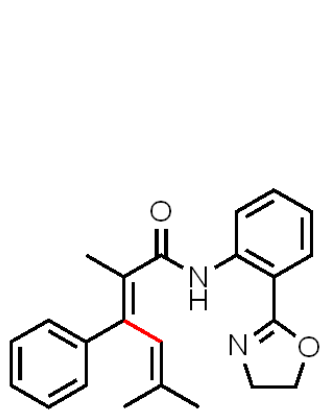

$7 k$

${ }^{13} \mathrm{C}$ NMR $\left(100 \mathrm{MHz}, \mathrm{CDCl}_{3}\right)$

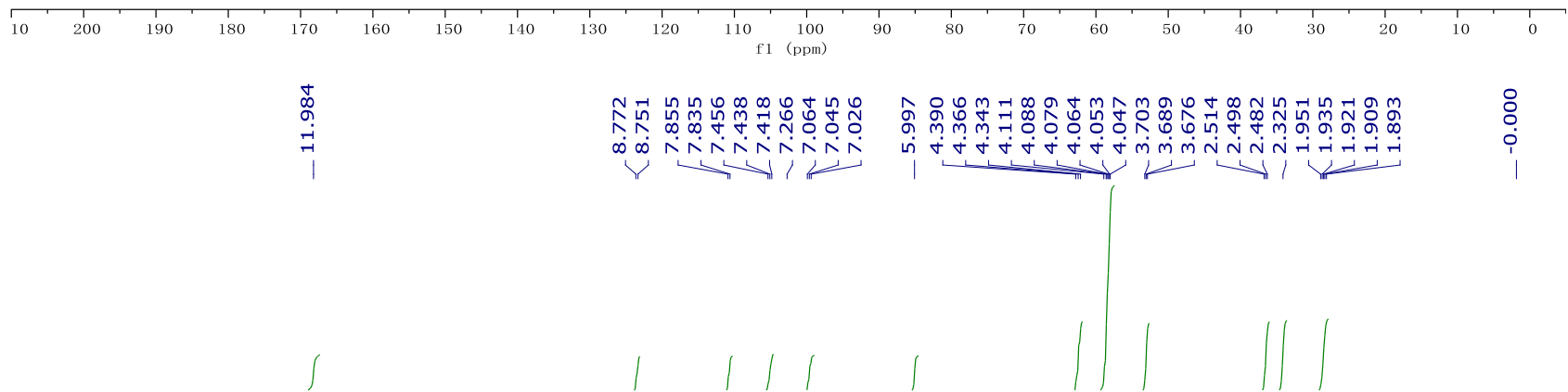<smiles>O=C(Nc1ccccc1C1=NCCO1)C1=C(C2=CCOCC2)OCCC1</smiles>

${ }^{1} \mathrm{H} \mathrm{NMR}\left(400 \mathrm{MHz}, \mathrm{CDCl}_{3}\right)$

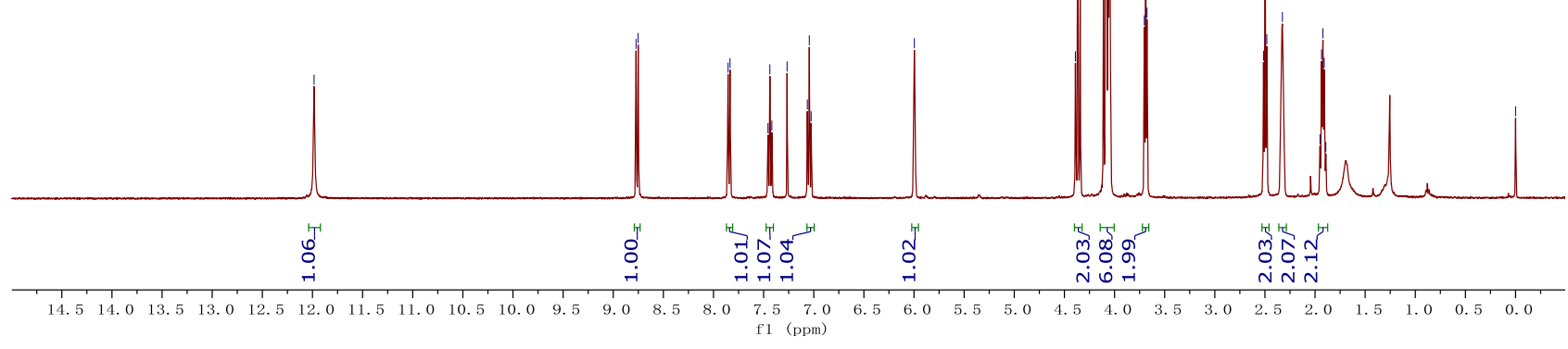


<smiles>O=C(Nc1ccccc1C1=NCCO1)C1=C(C2=CCOCC2)OCCC1</smiles>

71

${ }^{13} \mathrm{C} \mathrm{NMR}\left(100 \mathrm{MHz}, \mathrm{CDCl}_{3}\right)$
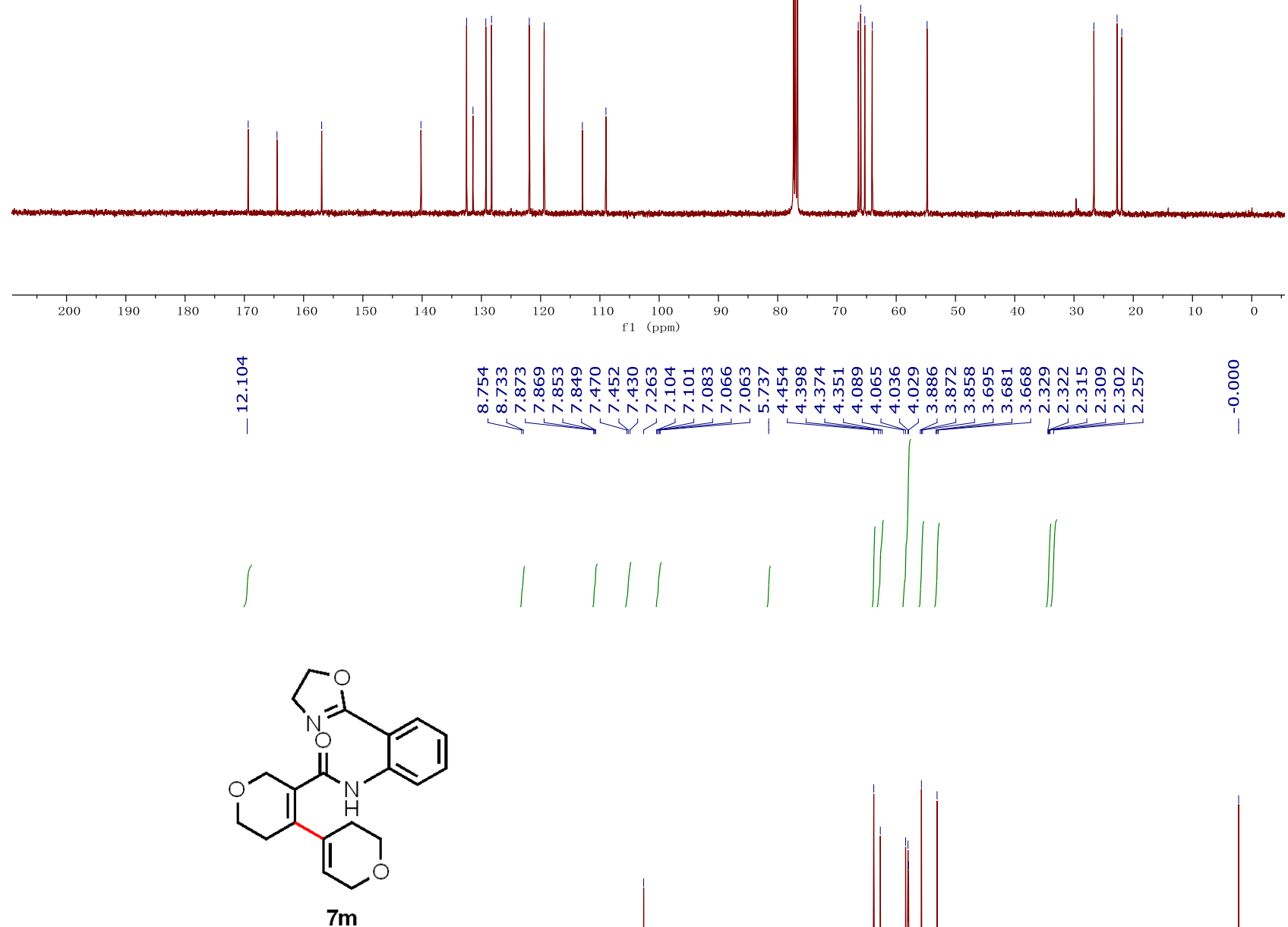

${ }^{1} \mathrm{H}$ NMR $\left(400 \mathrm{MHz}, \mathrm{CDCl}_{3}\right)$

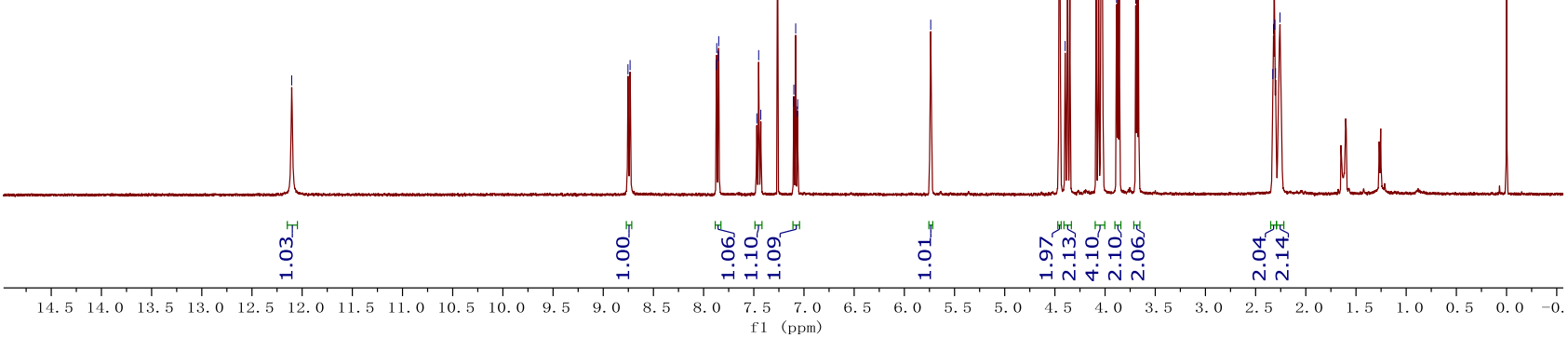




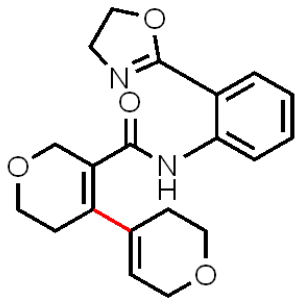

$7 \mathrm{~m}$

${ }^{13} \mathrm{C} \mathrm{NMR}\left(100 \mathrm{MHz}, \mathrm{CDCl}_{3}\right)$
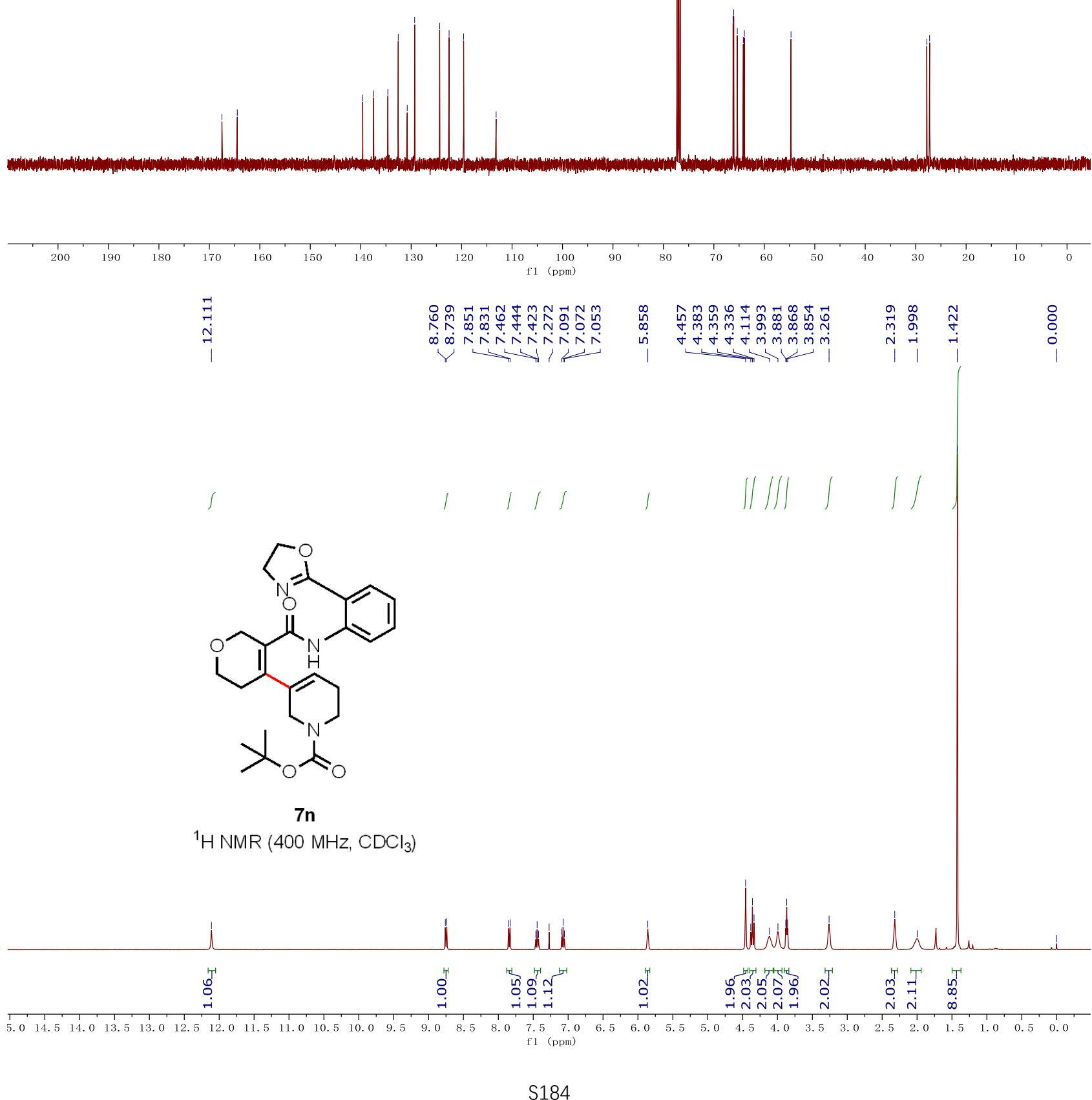

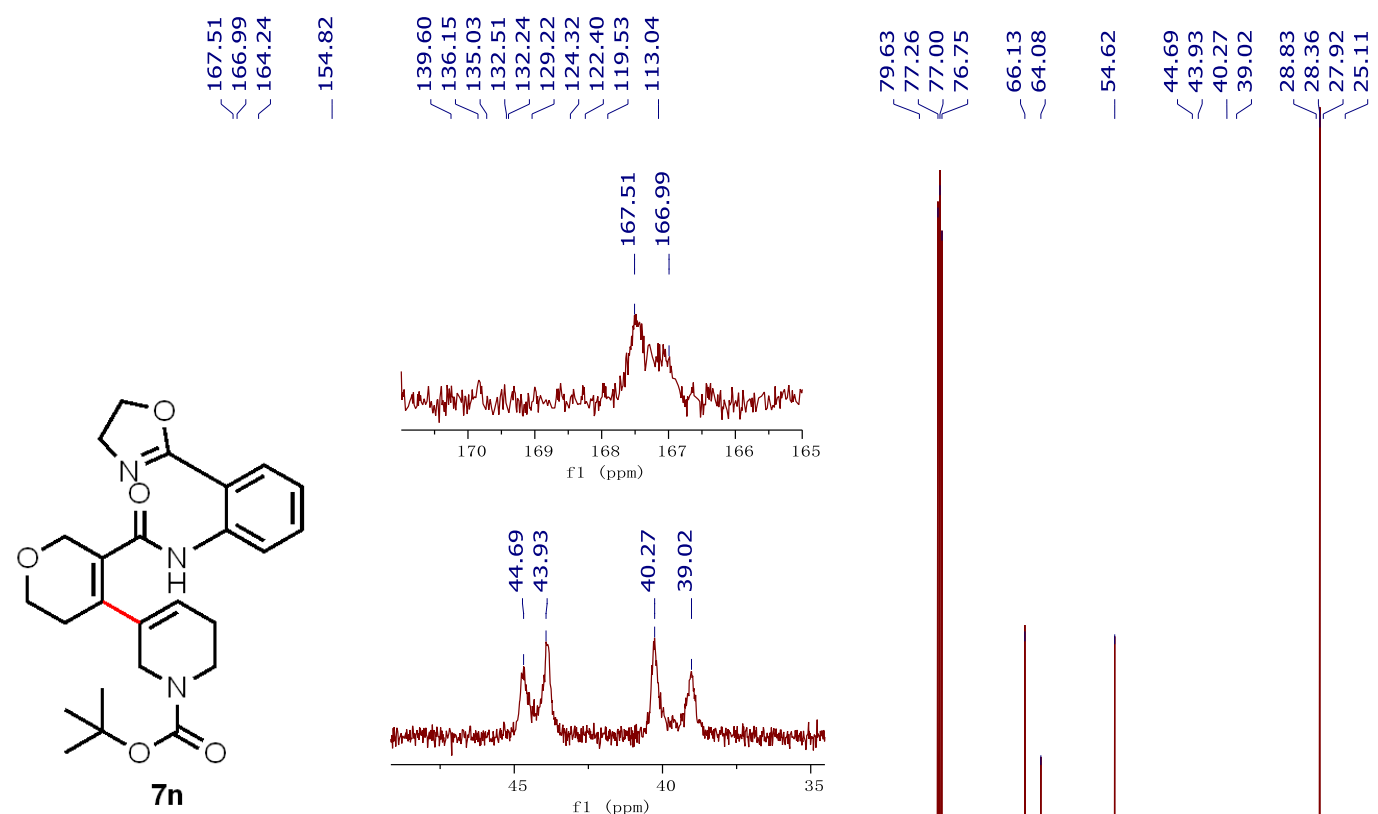

${ }^{13} \mathrm{C}$ NMR $\left(125 \mathrm{MHz}, \mathrm{CDCl}_{3}\right)$
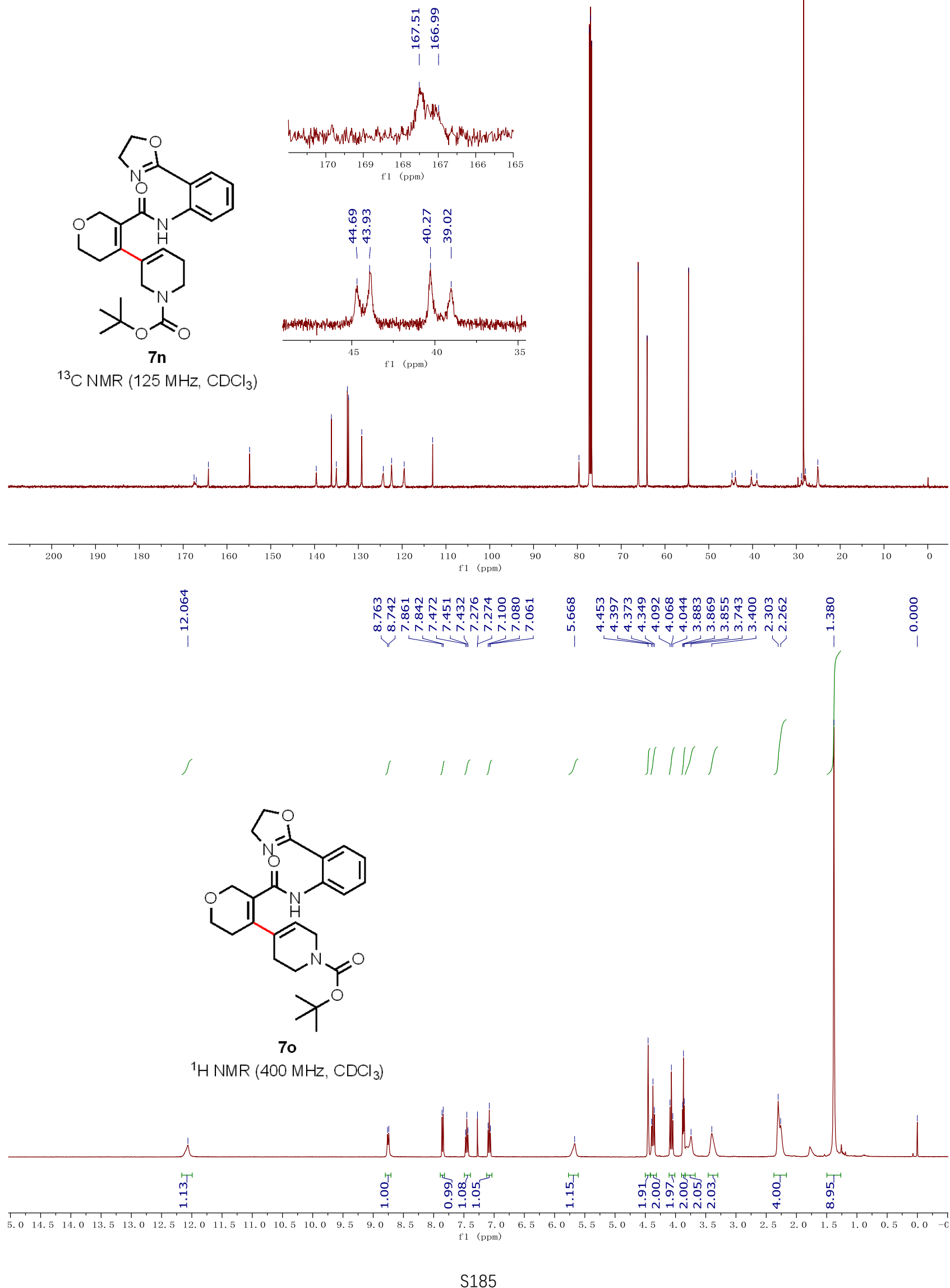
<smiles>CC(C)(C)OC(=O)N1CC=C(C2=C(C(=O)Nc3ccccc3C3=NCCO3)COCC2)CC1</smiles>

7o

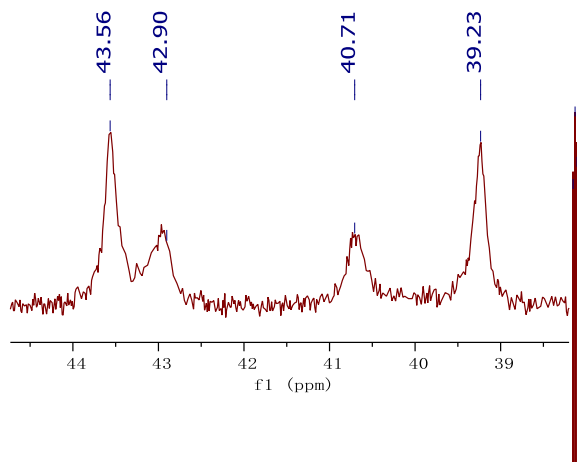

${ }^{13} \mathrm{C} \mathrm{NMR}\left(125 \mathrm{MHz}, \mathrm{CDCl}_{3}\right)$
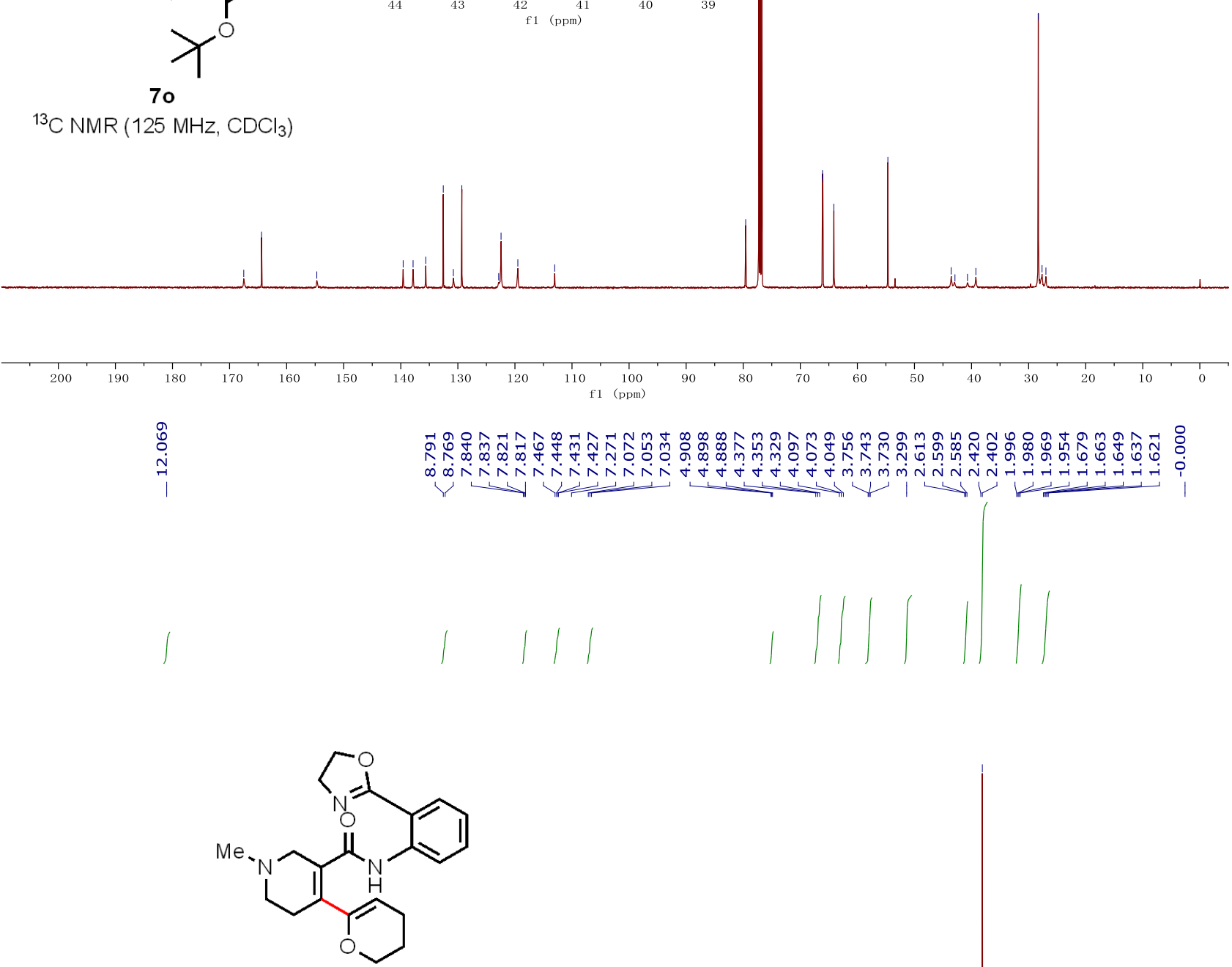

$7 \mathrm{p}$

${ }^{1} \mathrm{HNMR}\left(400 \mathrm{MHz}, \mathrm{CDCl}_{3}\right)$

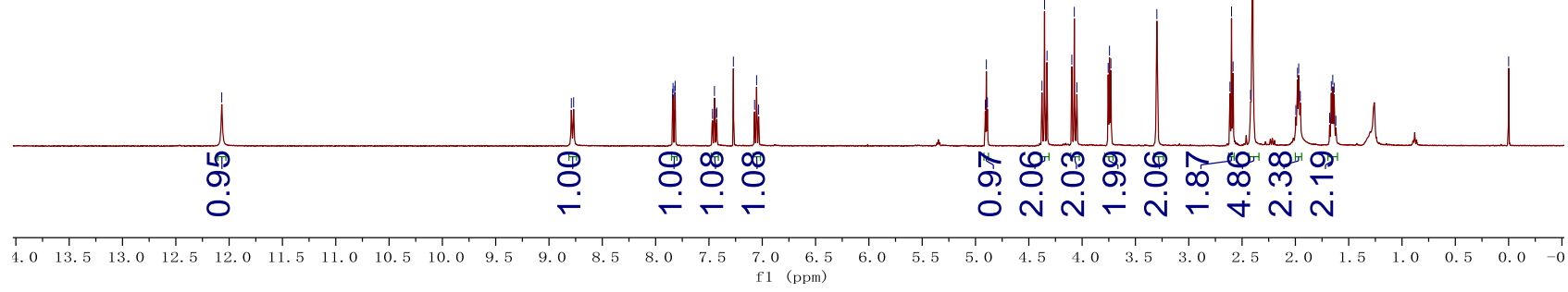




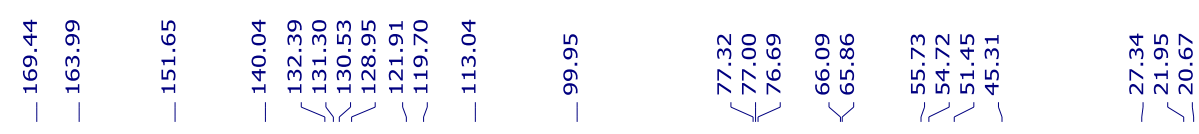<smiles>CN1CCC(C2=CCCCO2)=C(C(=O)Nc2ccccc2C2=NCCO2)C1</smiles>

$7 \mathrm{p}$

${ }^{13} \mathrm{C} \mathrm{NMR} \mathrm{(100} \mathrm{MHz,} \mathrm{CDCl}_{3}$ )

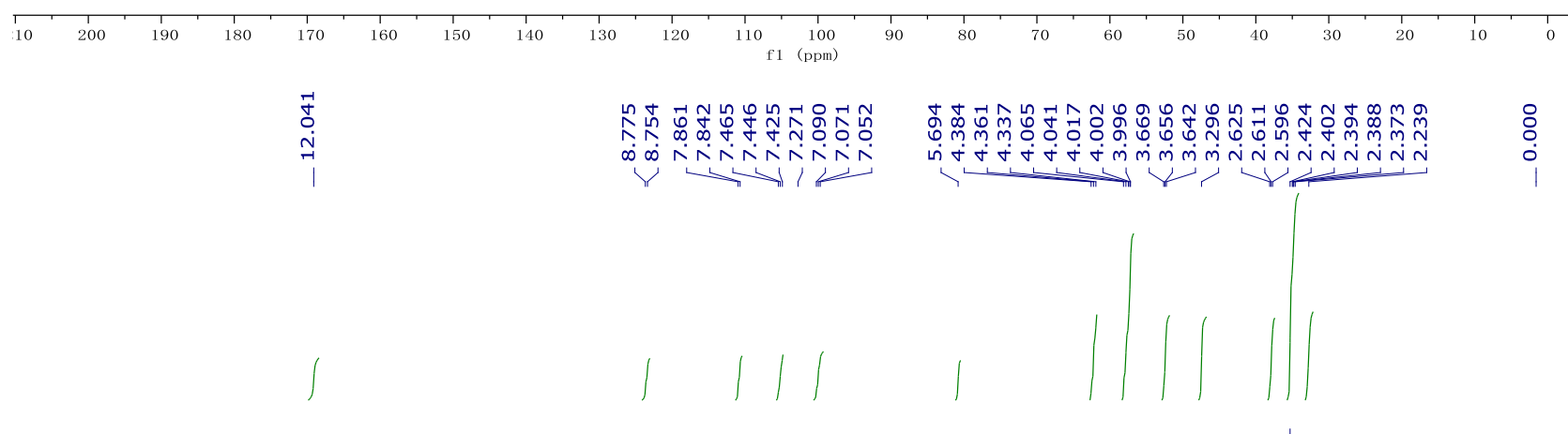<smiles>CN1CCC(C2=CCOCC2)=C(C(=O)Nc2ccccc2C2=NCCO2)C1</smiles>

$7 q$

${ }^{1} \mathrm{HNMR}\left(400 \mathrm{MHz}, \mathrm{CDCl}_{3}\right)$

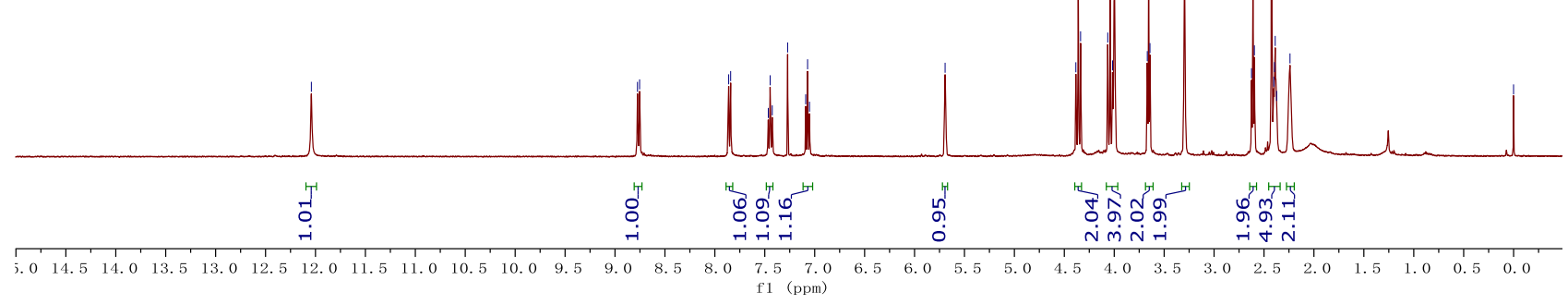




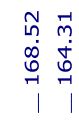

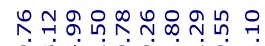

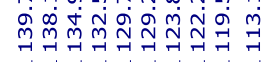

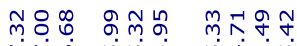

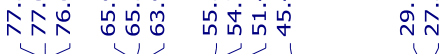<smiles>CN1CCC(C2=CCOCC2)=C(C(=O)Nc2ccccc2C2=NCCO2)C1</smiles>

$7 q$

${ }^{13} \mathrm{C}$ NMR $\left(100 \mathrm{MHz}, \mathrm{CDCl}_{3}\right)$
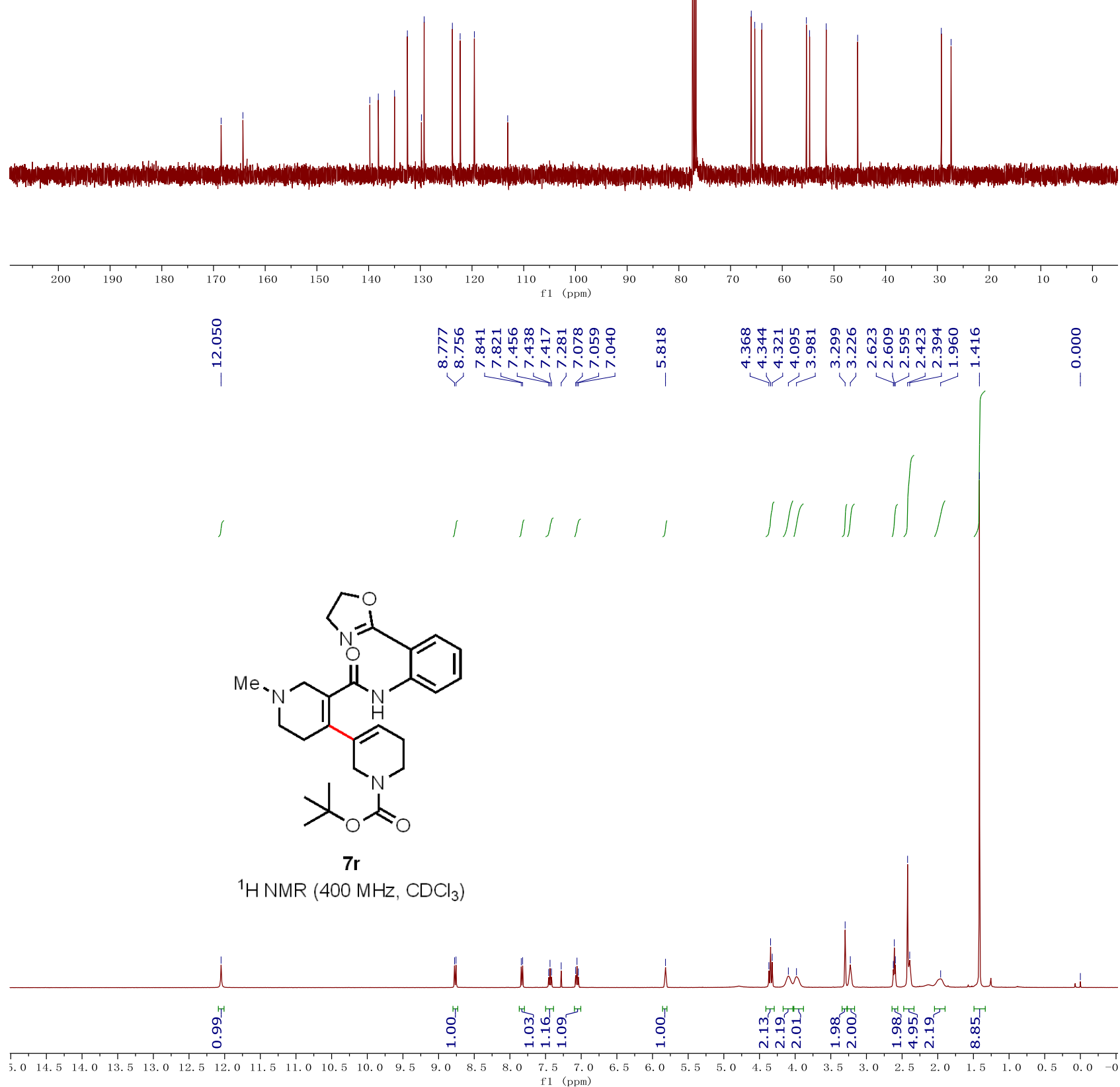

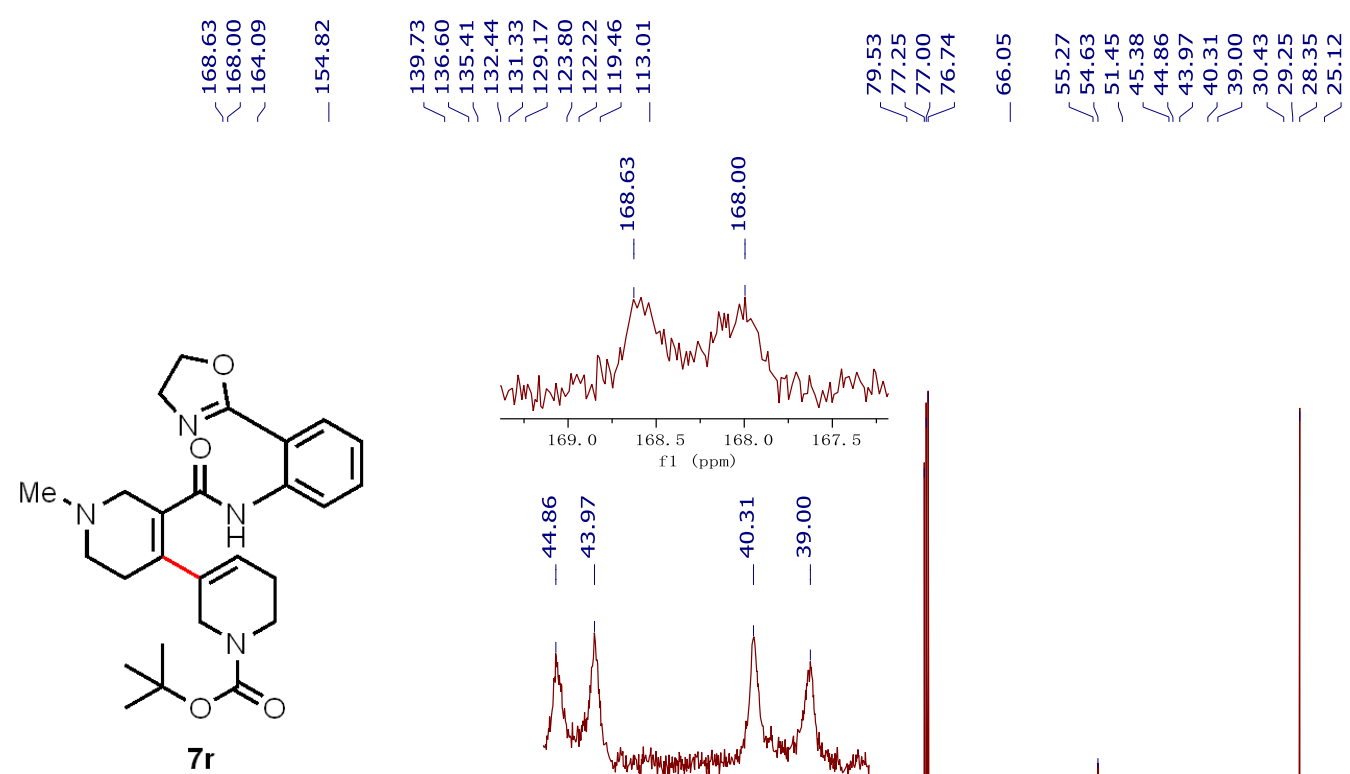

${ }^{13} \mathrm{C} \mathrm{NMR}\left(125 \mathrm{MHz}, \mathrm{CDCl}_{3}\right)$

$$
\begin{array}{ll}
0 & 8 \\
0 & 0 \\
\infty & \infty \\
0 & 0 \\
0 & 0 \\
1 &
\end{array}
$$
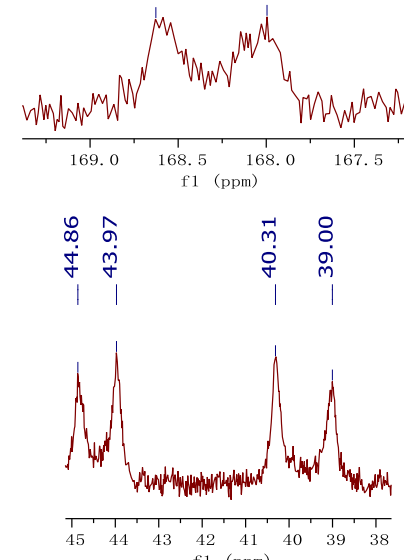

f1 (ppm)
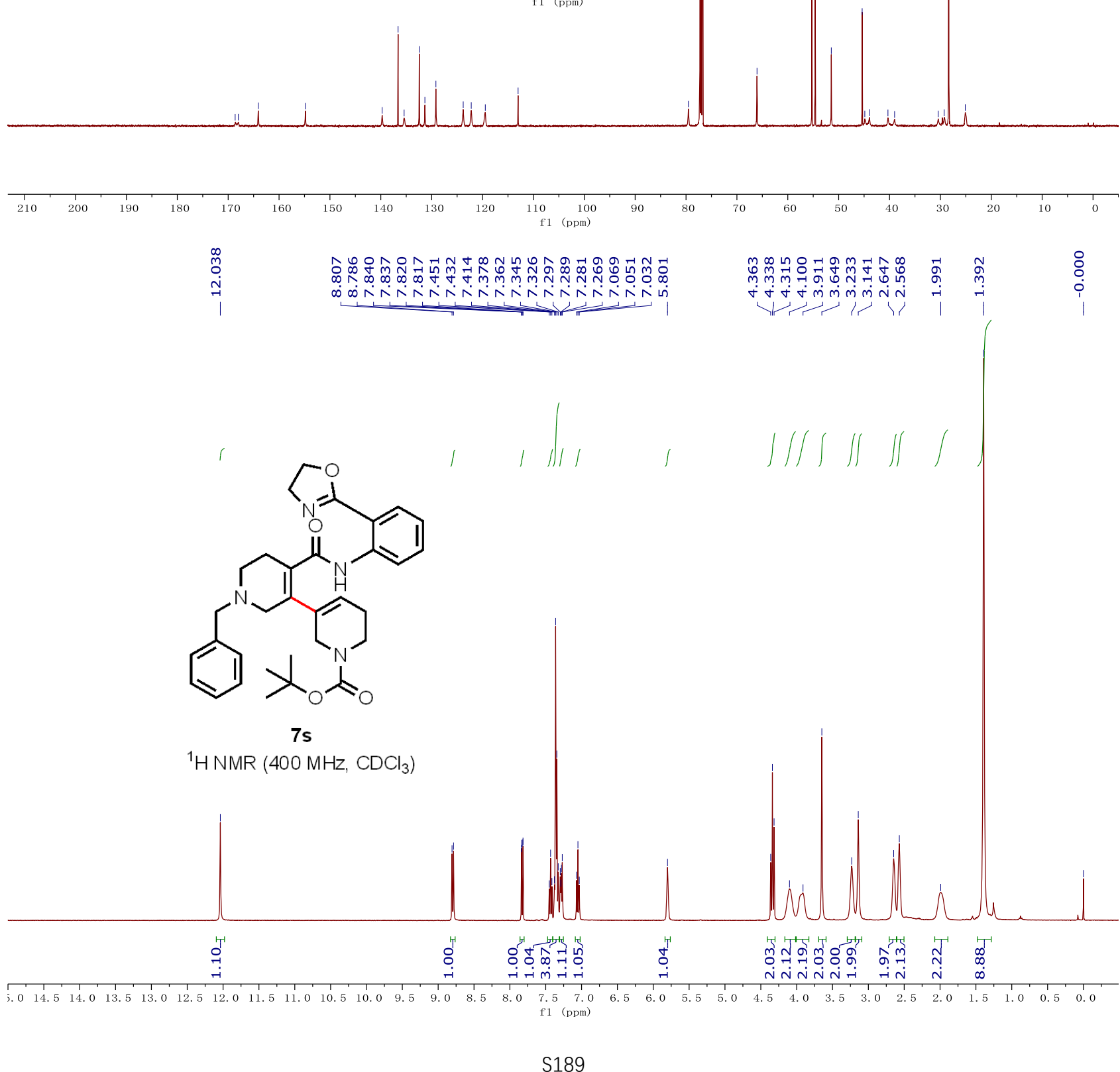


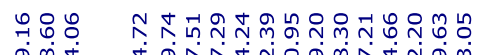

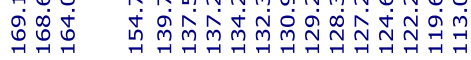
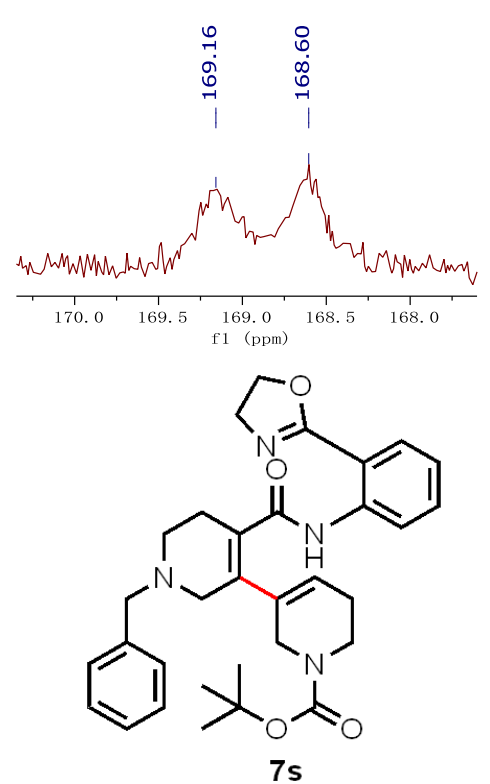

${ }^{13} \mathrm{C} \mathrm{NMR}\left(125 \mathrm{MHz}, \mathrm{CDCl}_{3}\right)$

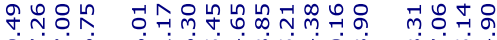

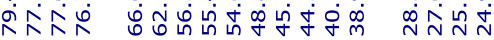
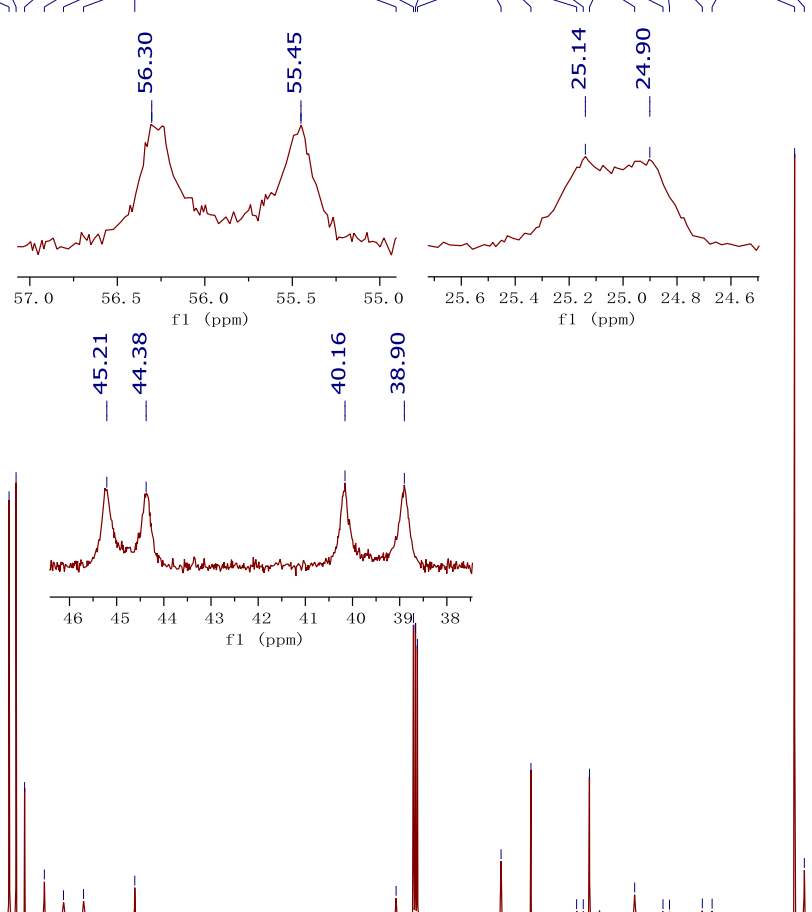

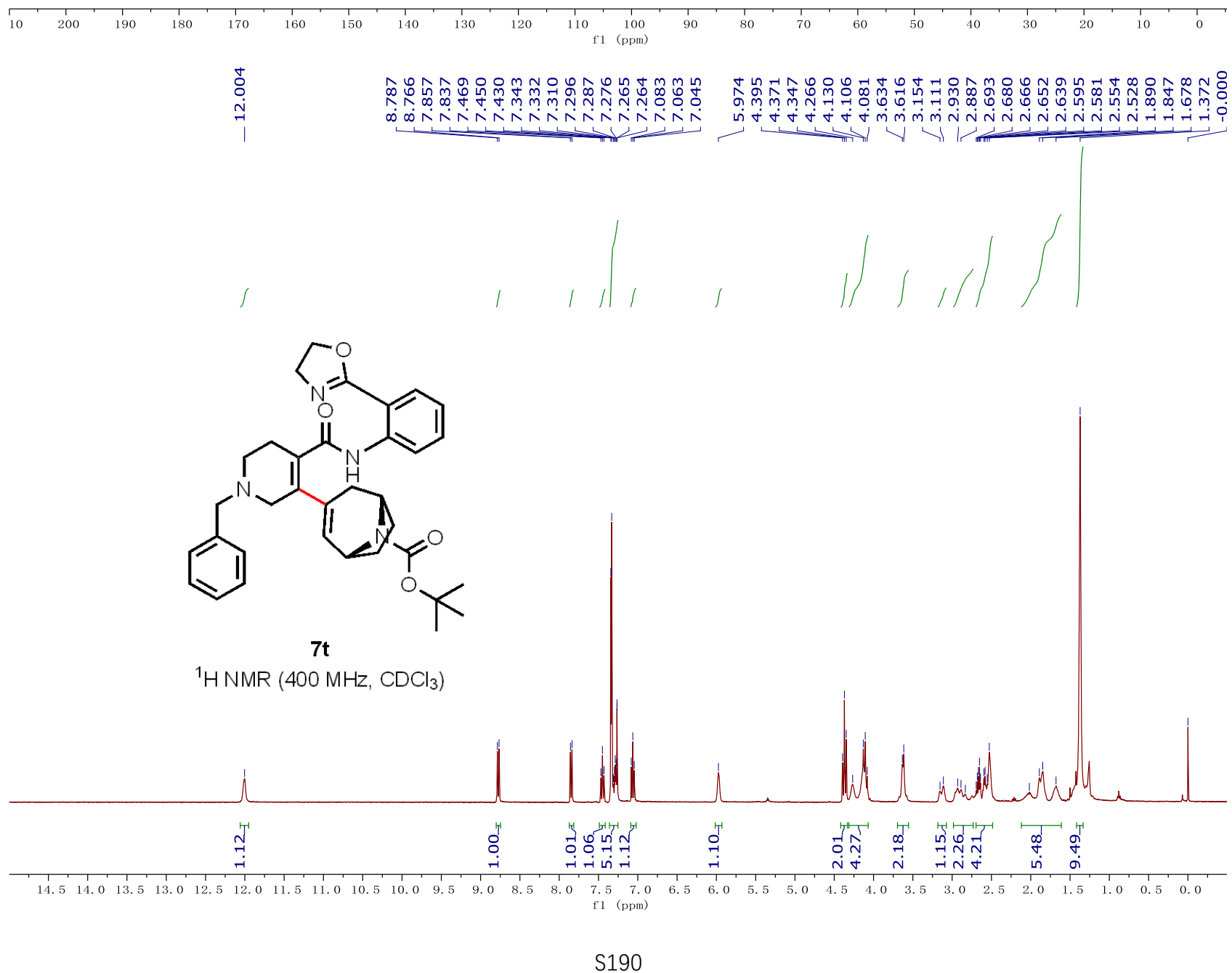



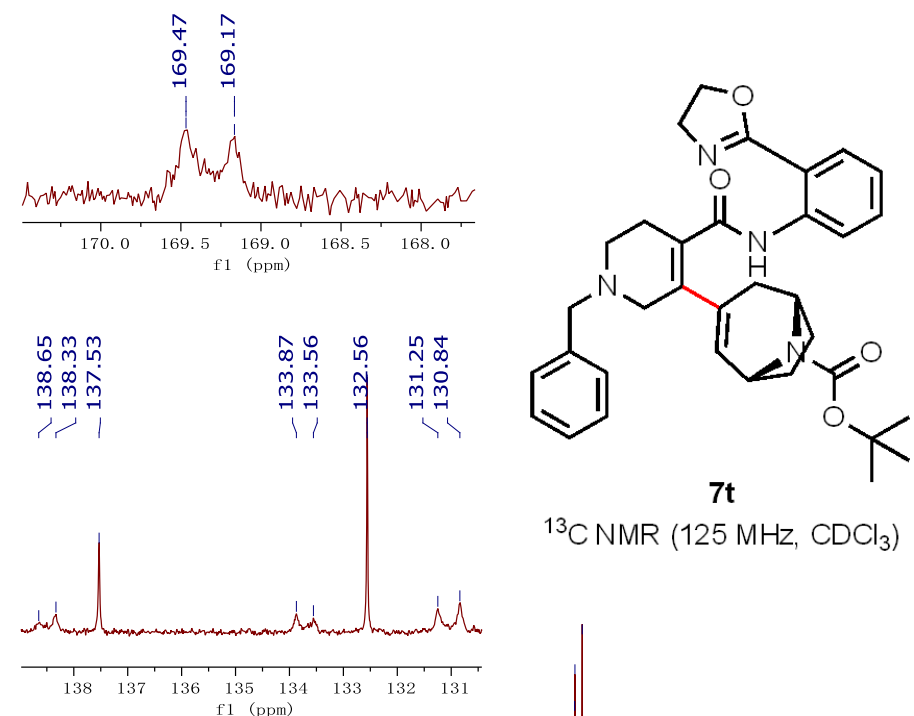

${ }^{13} \mathrm{C} \mathrm{NMR}\left(125 \mathrm{MHz}, \mathrm{CDCl}_{3}\right)$

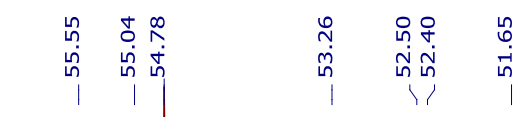
$\begin{array}{llllllllll} & & & & & & & & & \\ 6.0 & 55.5 & 55.0 & 54.5 & 54.0 & 53.5 & 53.0 & 52.5 & 52.0 & 51.5\end{array}$

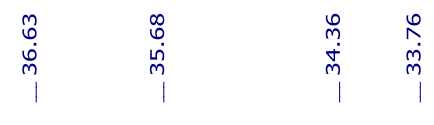
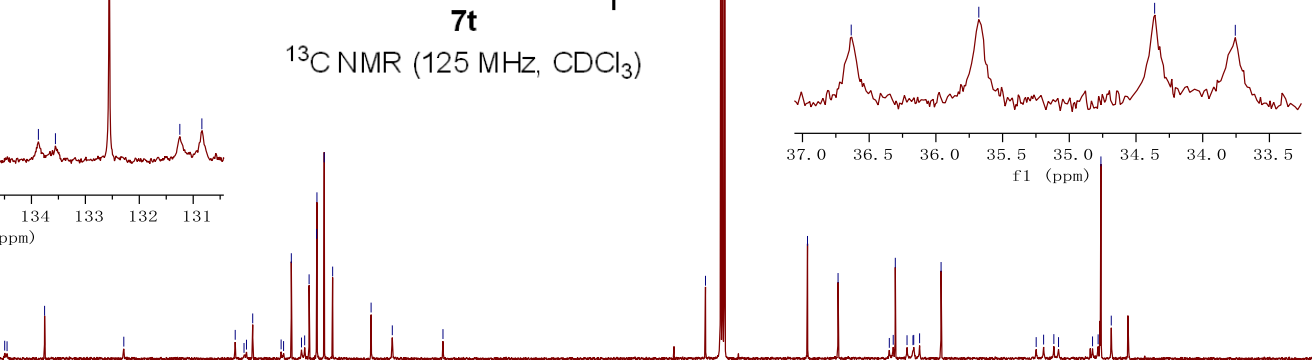

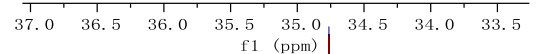

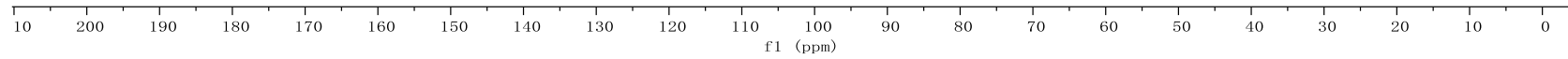

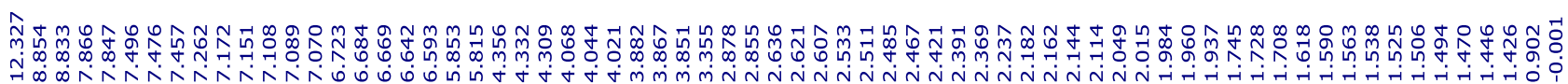<smiles>CN1CCC(/C=C/CCCCOc2ccc3c(c2)CC[C@H]2[C@@H]4CCC(=O)[C@@]4(C)CC[C@@H]32)=C(C(=O)Nc2ccccc2C2=NCCO2)C1</smiles>

${ }^{1} \mathrm{H} \mathrm{NMR}\left(400 \mathrm{MHz}, \mathrm{CDCl}_{3}\right)$

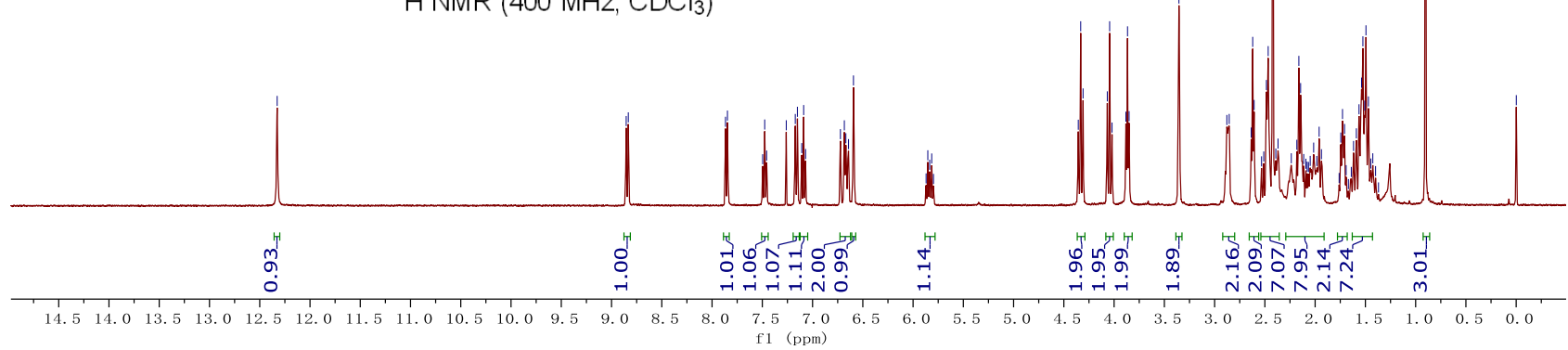


<smiles>CN1CCC(/C=C/CCCCOc2ccc3c(c2)CC[C@@H]2[C@@H]3CC[C@]3(C)C(=O)CC[C@@H]23)=C(C(=O)Nc2ccccc2C2=NCCO2)C1</smiles>

$7 u$

${ }^{13} \mathrm{CNMR}\left(100 \mathrm{MHz}, \mathrm{CDCl}_{3}\right)$
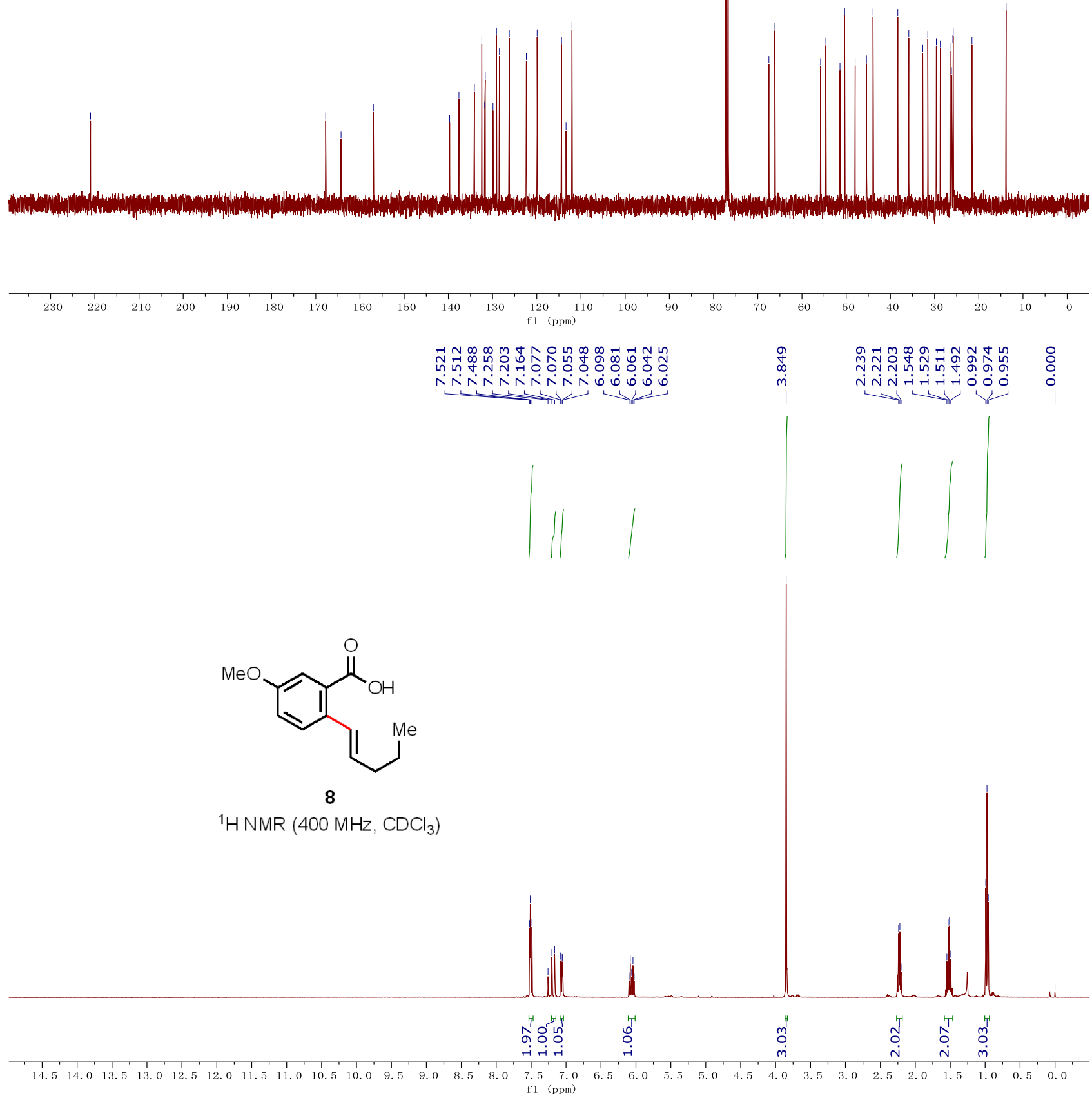


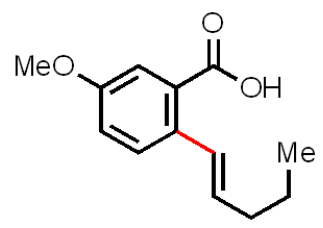

8

${ }^{13} \mathrm{C} \mathrm{NMR}\left(100 \mathrm{MHz}, \mathrm{CDCl}_{3}\right.$ )
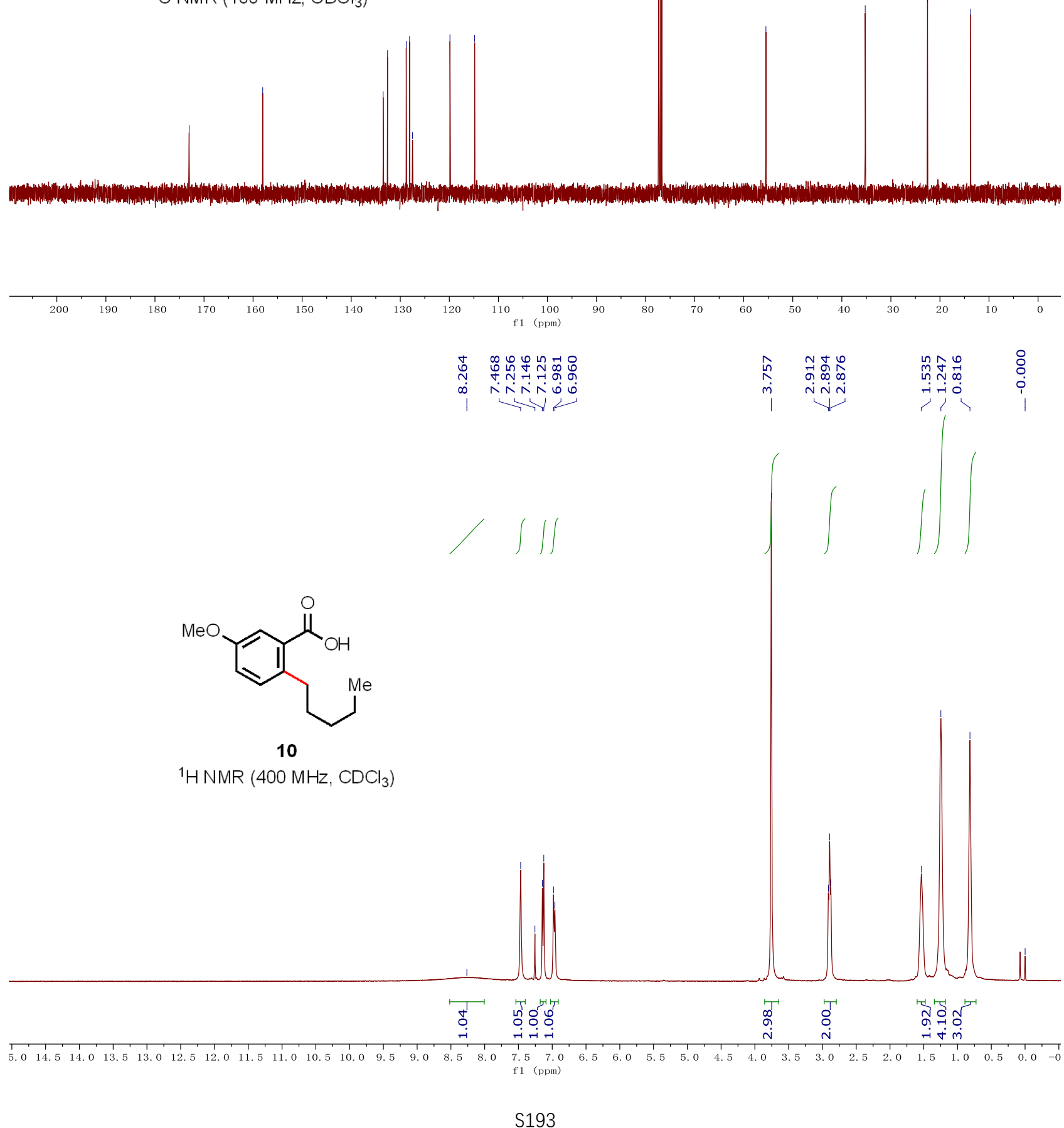


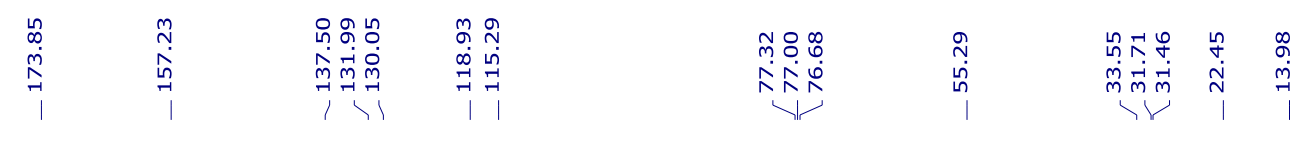

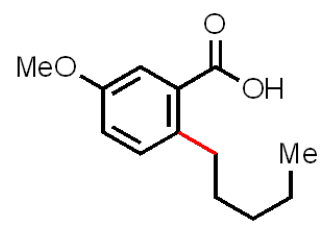

10

${ }^{13} \mathrm{C} \mathrm{NMR}\left(100 \mathrm{MHz}, \mathrm{CDCl}_{3}\right)$

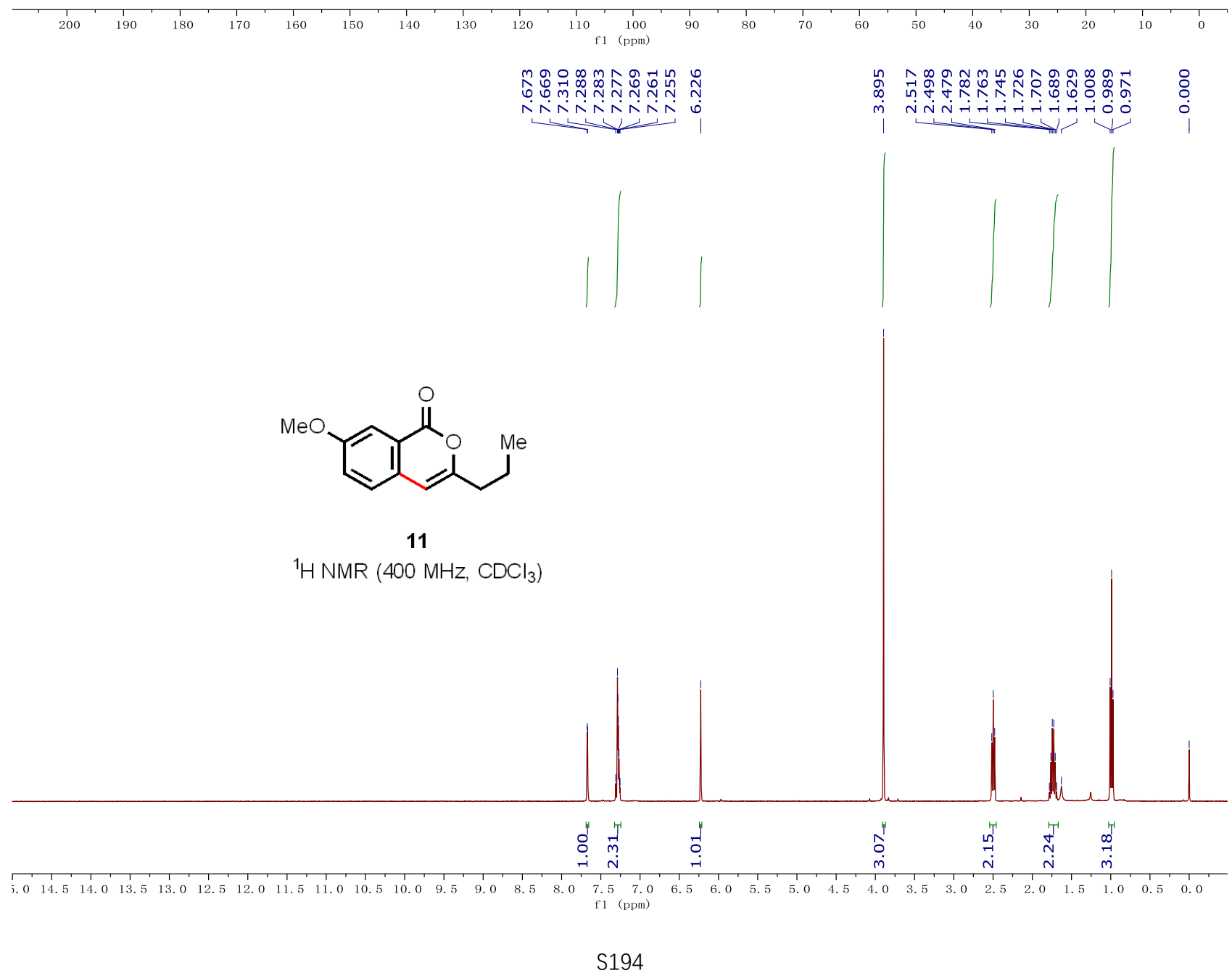




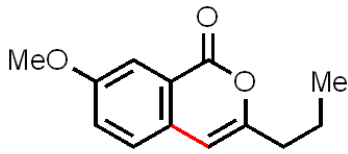

11

${ }^{13} \mathrm{CNMR}\left(100 \mathrm{MHz}, \mathrm{CDCl}_{3}\right)$
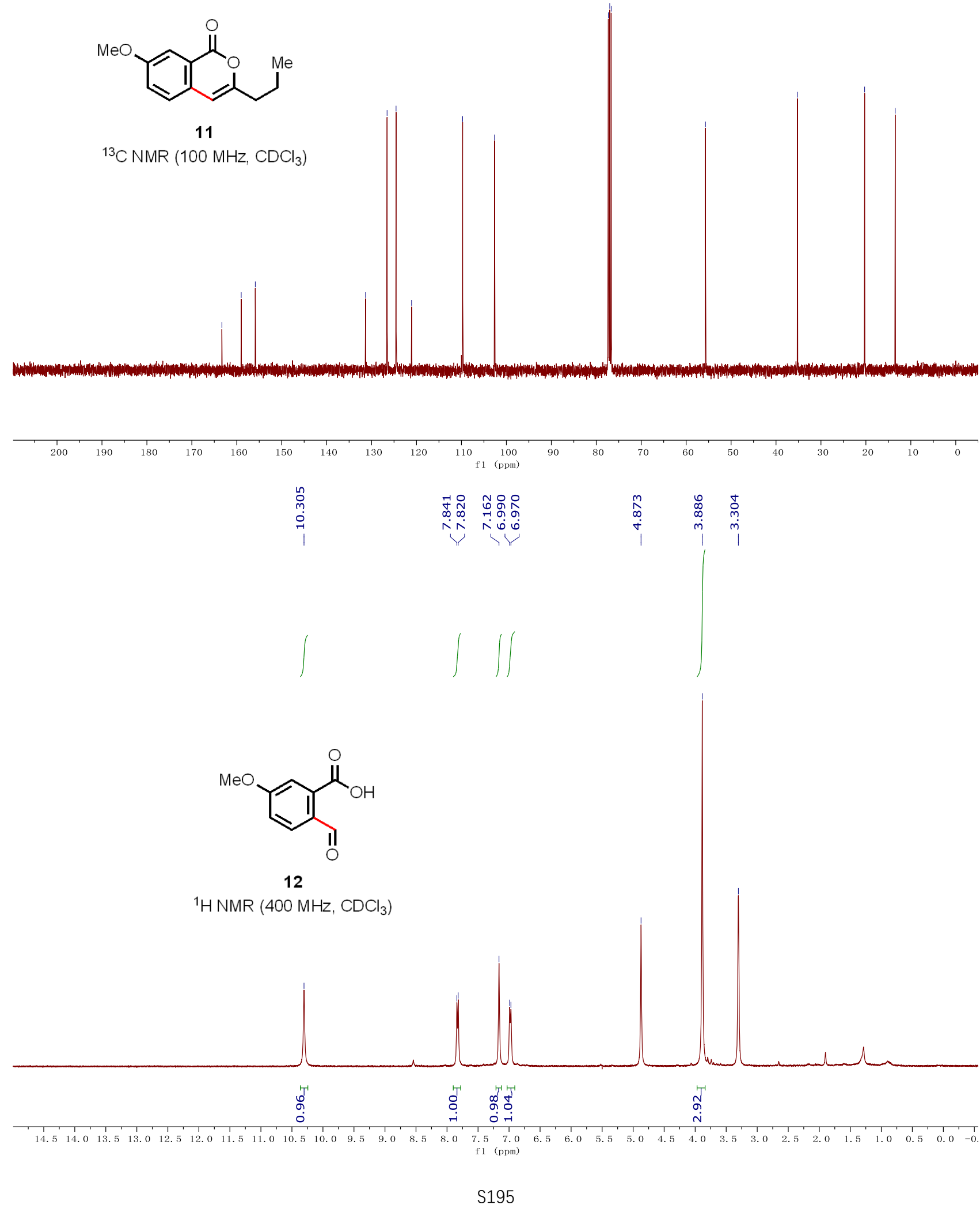


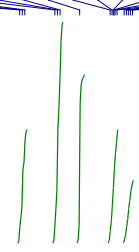<smiles>COc1ccc(C2CCOCC2)c(C(=O)Nc2ccccc2C2=NCCO2)c1</smiles>

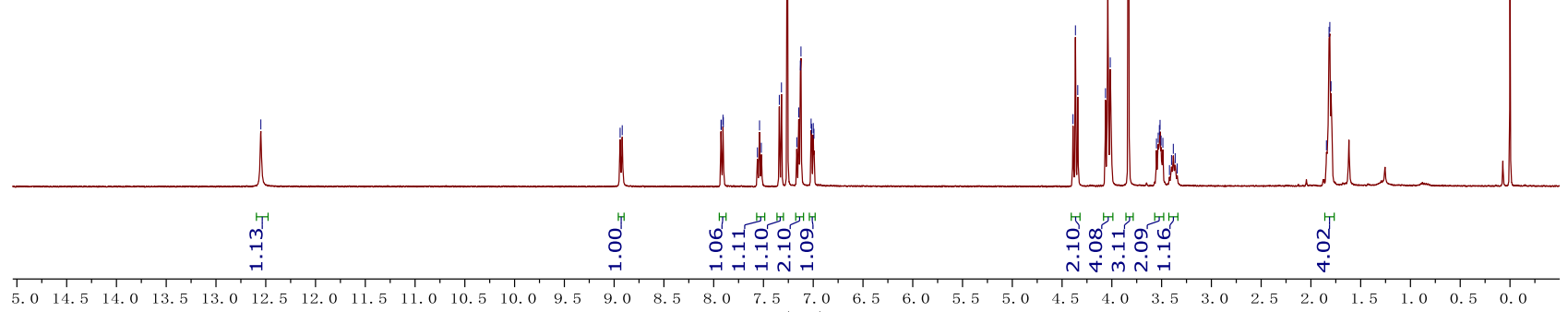

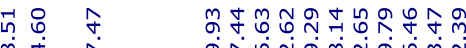

总

रा 1,1111

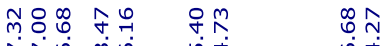

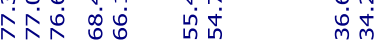<smiles>COc1ccc(C2CCOCC2)c(C(=O)Nc2ccccc2C2=NCCO2)c1</smiles>

13

${ }^{13} \mathrm{C} \mathrm{NMR}\left(100 \mathrm{MHz}, \mathrm{CDCl}_{3}\right)$

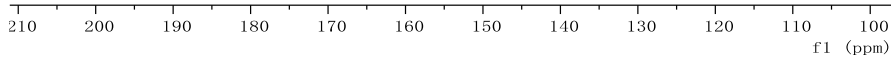




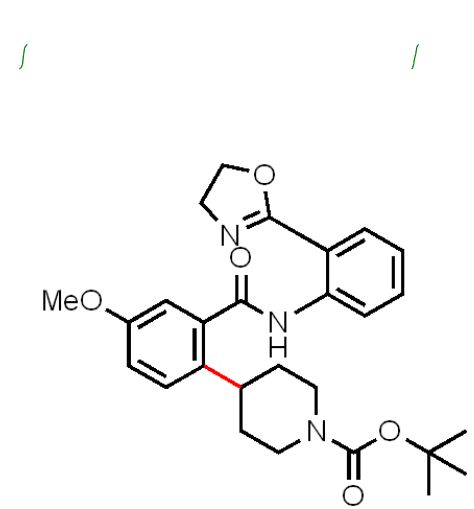

14

${ }^{1} \mathrm{HNMR}\left(400 \mathrm{MHz}, \mathrm{CDCl}_{3}\right)$
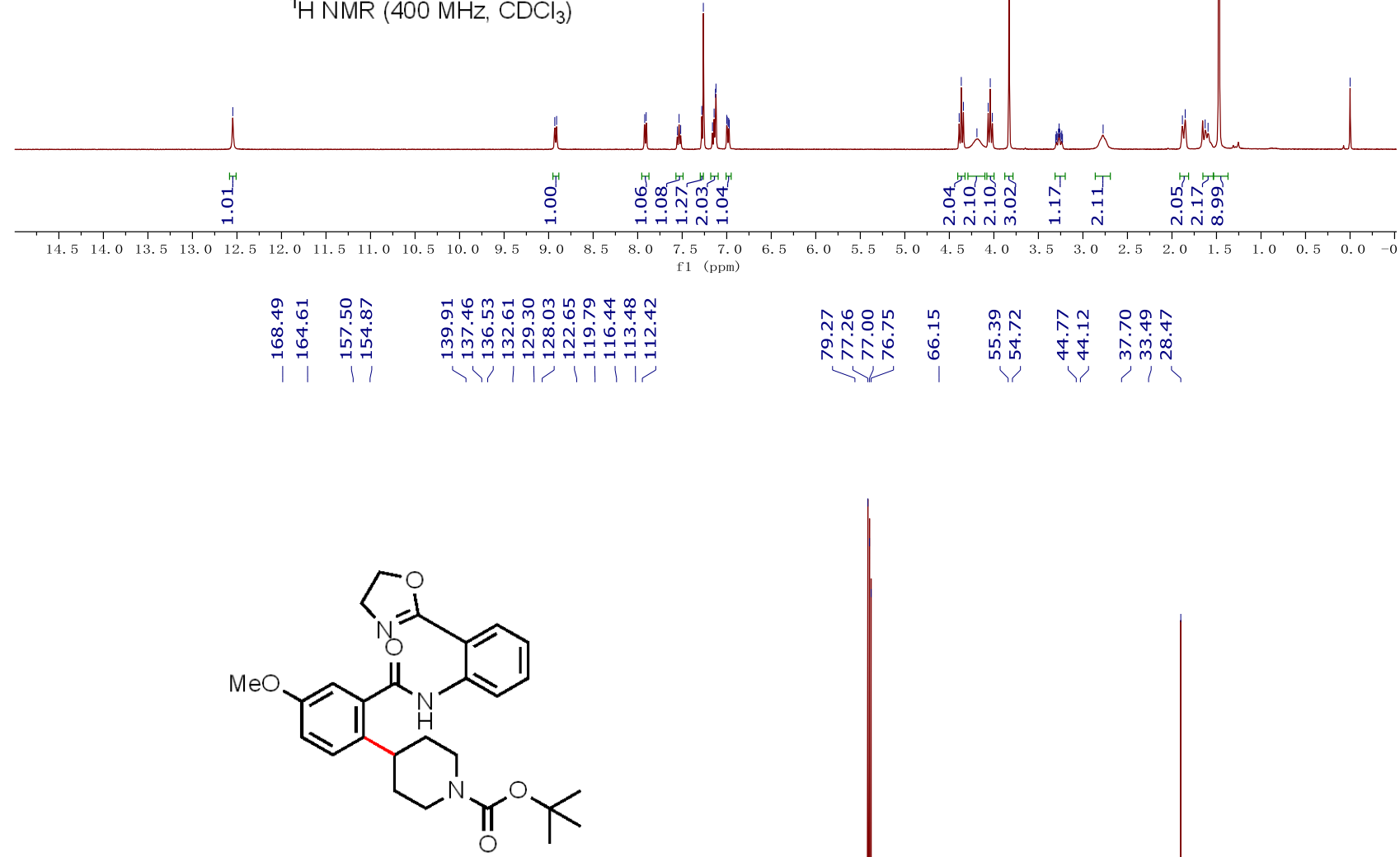

14

${ }^{13} \mathrm{C} \mathrm{NMR}\left(125 \mathrm{MHz}, \mathrm{CDCl}_{3}\right)$

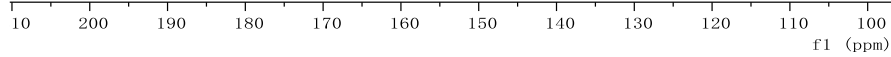

